


\section{LIBRARY}

UNIVERSITY OF

CALIFORNIA

SANTA CRUZ

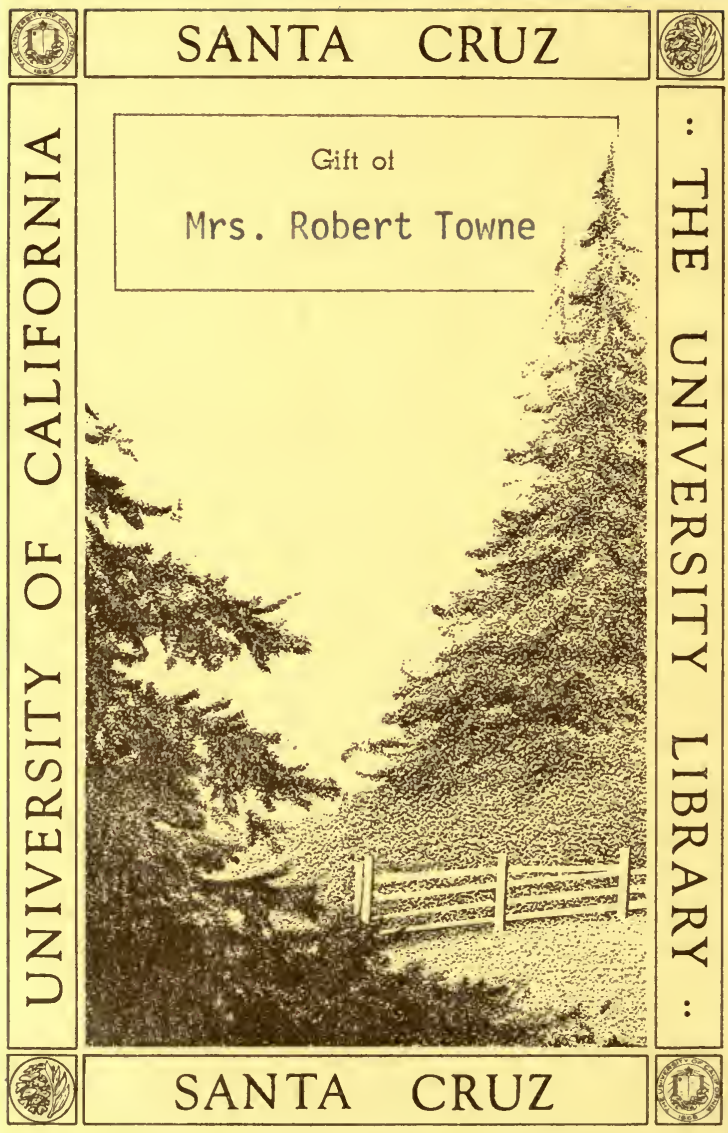



Digitized by the Internet Archive in 2007 with funding from Microsoft Corporation 


\section{THE PRINCIPLES OF CHEMISTRY}

BY

D. MENDELÉEFF

PART TWO

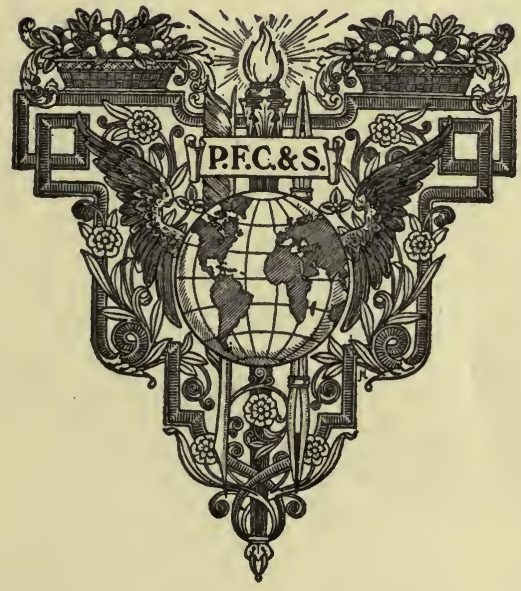

NEW YORK

P. F. COLLIER \& SON 



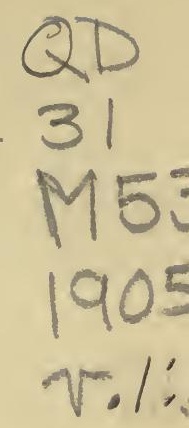

\section{PRINCIPLES OF CHEMISTRY}

\section{(PART TWO)}





\title{
PRINCIPLES OF CHEMISTRY
}

\author{
CHAPTER VII \\ MOLECULES AND A'TOMS. THE LAWS OF GAY-LUSSAC AND \\ AVOGADRO-GERHARDT
}

HYDROGEN combines with oxygen in the proportion of two volumes to one. The composition by volume of nitrous oxide is exactly similarit is composed of two volumes of nitrogen and one volume of oxygen. By decomposing ammonia by the action of an electric spark it is easy to prove that it contains one volume of nitrogen to three volumes of hydrogen. So, similarly, it is found, whenever a compound is decomposed and the volurnes of the gases proceeding from it are measured, that the volumes of the gases or vapours entering into combination are in a very simple proportion to one another. With water, nitrous oxide, itc., this may be proved by direct observation ; but in the majority of cases, and especially with substances which, although volatile-that is, capable of passing into a gaseous (or vaporous) state-are liquid at the ordinary temperature, such a direct method of observation presents many difficulties. But, then, if the densities of the vapours and gases be known, the same simplicity in their ratio is shown by calculation. The volume of a substance is proportional to its weight, and inversely proportional to its density, and therefore by dividing the amount by weight of each substance entering into the composition of a compound by its density in the gaseous or vaporous state we shall obtain factors which will be in the same proportion as the volumes of the substances entering into the composition of the compound. ${ }^{1}$ So, for example,

1 If the weight be indicated by $\mathrm{P}$, the density by $\mathrm{D}$, and the volume by $\mathrm{V}$, then

$$
\mathrm{D}=K \mathrm{~V}
$$

where $K$ is a coefficient depending on the system of the expressions $P, D$, and $V$ If $D$ be the weight of a cubic measure of a substance referred to the weight of the same measure of water-if, as in the metrical system. (Chapter I., Note 9), the cubic measure of one part by weight of water be taken as a unit of volume-then $K=1$. But, whatever it be, it is cancelled in dealing with the comparison of volumes, because comparative and not absolute measures of volumes are taken. In this chapter, as throughout the book, the weight $\mathrm{P}$ is given in grams in dealing with absolute weights; and if comparative, as in 
water contains eight parts by weight of oxygen to one part by weight of hydrogen, and their densities are 16 and 1, consequently their volumes (or the above-mentioned factors) are 1 and $\frac{1}{2}$, and therefore it is seen without direct experiment that water contains two rolumes of hydrogen for every one volume of oxygen. So adso, knowing that nitric oxide contains fourteen parts of nitrogen and sixteen parts of oxygen, and knowing that the specific gravities of these last two gases are fourteen and sixteen, we find that the rolumes in which nitrogen and oxygen combine for the formation of nitric oxide are in the proportion of $1: 1$. We will cite another example. In the last chapter we saw that the density of $\mathrm{NO}_{2}$ only becomes cunstaut and equal to twenty. three (referred to hydrogen) above $135^{\circ}$, and as a matter of fact a method of direct observation of the volumetric composition of this substance would be very difficult at so high a temperature. But it may be easily calculated. $\mathrm{NO}_{2}$, as is seen from its formula and analysis, contains thirty-two parts by weight of oxygen to fourteen parts by weight of nitrogen, forming forty-six parts by weight of $\mathrm{NO}_{2,}$, and knowing the densities of these gases we find that one volume of nitrogen with two volumes of oxygen gives two volumes of nitrogen peroxide. Therefore, knowing the amounts by weight of the substances participating in a reaction or forming a given substance, and knowing the density of the gas or vapour, ${ }^{2}$ the volumetric relations of the substances acting in a

the expression of chemical composition, then the weight of an atom is taken as unity. The density of gases, D, is also taken in reference to the density of hydrogen, and the volume $\mathrm{V}$ in metrical units (cubic centimetres), if it be a matter of absolute magnitudes of volumes, and if it be a matter of chemical transformations-that is, of relative volumes -then the volume of an atom of hydrogen, or of one part by weight of hydrogen, is taken as unity, and all volumes are expressed according to these units.

2 As the volumetric relations of vapours and gases, next to the relations of substances by weight, form the most important province of chemistry, and a most important meaus for the attainment of chemical conclusions, and inasmuch as these volumetrio relations are determined by the densities of gases and vapours, necessarily the methods of determining the densities of vapours (and also of gases) are important factors in chemical research. These methods are described in detail in works on physics and physical and analytical chemistry, and therefore we here only touch on the general principles of the subject.

If we know the weight $p$ and volume $v$, occupied by the vapour of a given substance at a temperature $t$ and pressure $h$, then its density may be directly obtained by dividing $p$ by the weight of a volume $v$ of hydrogen (if the density be expressed according to hydrogen, see Chapter II., Note 23) at $t$ and $h$. Hence, the methods of determining the density of vapours and gases are based on the determination of $p, v, t$, and $h$. The two last data (the temperature $t$ and pressure $h$ ) are given by the thermometer and barometer and the heights of mercury or other liquid-confining the gas, and therefore do not require further explanation. It need only be remarked that: (1) In the case of easily volatile liquids there is no difficulty in procuring a bath with a constant temperature, but that it is nevertheless best (especially considering the inaccuracy of thermometers) to have a medium of absolutely constant temperature, and therefore to take either a bath in which some substance is melting-such as melting ice at $0^{\circ}$ or crystals of 
reaction or entering into the composition of a compound, may be also determined.

sodium acetate, melting at $+56^{\circ}$-or, as is more generally practised, to place the vessel containing the substance to be experimented with in the vapour of a liquid boiling at a definite temperature, and knowing the pressure under which it is boiling, to determine the temperature of the vapour. For this purpose the boiling points of water at different pressures are given in Chapter I., Note 11, and the boiling points of certain easily procurable liquids at various pressures are given in Chapter II., Note 27. (2) With respect to tempera. tures above $800^{\circ}$ (below which mercurial thermometers may be conveniently employed), they are most simply obtained constant (to give time for the weight and volume of a substance being observed in a given space, and to allow that space to attain the calculated temperature $t$ ) by means of substances boiling at a high temperature. Thus, for instance, at the ordinary atmospheric pressure the temperature $t$ of the vapour of sulphur is about $445^{\circ}$, of phosphorus pentasulphide $518^{\circ}$, of tin chloride $606^{\circ}$, of cadmium $770^{\circ}$, of zinc $930^{\circ}$ (according to Violle and others), or $1040^{\circ}$ (according to Deville), \&c. (3) The indications of the hydrogen thermometer must be considered as the most exact (but as hydrogen diffuses through incandescent platinum, nitrogen is usually employed). (4) The temperature of the vapours used as the bath should in every case be several degrees higher than the boiling point of the liquid whose density is to be determined, in order that no portion should remain in a liquid state. But even in this case, as is seen from the example of nitric peroxide (Chapter VI.), the vapour density does not always remain constant with a change of $t$, as it should were the law of the expansion of gases and vapours absolutely exact (Chapter II., Note 26). If variations of a chemical and physical nature similar to that which we saw in nitric peroxide take place in the vapours, the main interest is centred in constant densities, which do not vary with $t$, and therefore the possible effect of $t$ on the density must always be kept in mind in having recourse to this means of investigation. (5) Usually, for the sake of convenience of observation, the vapour density is determined at the atmospheric pressure which is read on the barometer; but in the case of substances which are volatilised with difficulty, and also of substances which decompose, or, in general, vary at temperatures near their boiling points, it is best or even indispensable to conduct the determination at low pressures, whilst for substances which decompose at low pressures the observations have to be conducted under a more or less considerably increased pressure. (6) In many cases it is convenient to determine the vapour density of a substance in admixture with other gases, and consequently under the partial pressure, which may be calculated from the volume of the mixture and that of the intermixed gas (see Chapter I., Note 1). This method is especially important for substances which are easily decom. posable, because, as shown by the phenomena of dissociation, a substance is able to remain unchanged in the atmosphere of one of its products of decomposition. Thus, Wurtz delermined the density of phosphorio chloride, $\mathrm{PCl}_{5}$, in admixture with the vapour of phosphorous chloride, $\mathrm{PCl}_{3}$. (7) It is evident, from the example of nitric peroxide, that a change of pressure may alter the density and aid decomposition, and therefore identical results are sometimes obtained (if the density be variable) by raising $t$ and lowering $h$; but if the density does not vary under these variable conditions (at least, to an extent appreciably exceeding the limits of experimental error), then this constant density indicates the gaseous and invariable state of a substance. The laws hereafter laid down refer only to such vapour densities. But the majority of volatile substances show such a constant density at a certain degree above their boiling points up to the starting point of decomposition. Thus, the density of aqueous vapour does not vary for $t$ between the ordinary temperature and $1000^{\circ}$ (there are no trustworthy determinations beyond this) and for pressures varying from fractions of an atmosphere up to several atmospheres. If, however, the density does vary considerably with a variation of $h$ and $t$, the fact may serve as a guide for the investigation of the chemical changes which are undergone by the substance in a state of vapour, or at least as an indication of a deviation from the laws of Boyle, Mariotte, and Gay-Lussac (for the expansion of gases 
Such an investigation (either direct, or by calculation from the densities and composition) of every chemical reaction, resulting in the forma-

with $t$ ). In certain cases the separation of one form of deviation from the other may be explained by special hypotheses.

With respect to the means of determining $p$ and $v$, with a view to finding the rapour density, we may distinguish three chief methods: (a) by weight, by ascertaining the weight of a definite volume of vapour; (b) by volume, by measuring the volume occupied by the vapour of a definite weight of a substance; and (c) by displacement. The last.

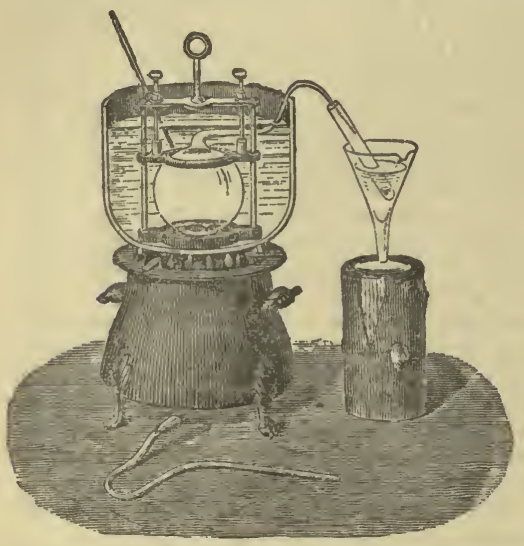

FIG. 52.-Apparatus for determining the vapour density by Dumas' method. A small quantity of the liquid whose vapour density is to be determined is placed in the glass globe, and heated in a water or oil bath to a temperature above the boiling point of the liquid. When all the liquid has been converted into vapour and has displaced all the air from the glone, the latter is sealed up and weighed. The capacity of the globe is then mensured, and in this manner the volume occu. pied by a known weight of vapour at a known tem. perature is determined.

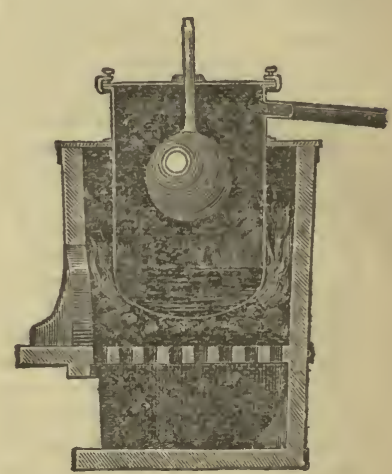

F1G. 53.-Devil'e and Troost's apparatus for deterniuing the vapour densities, according to Dumas' method, of sub. stances which boil at. high temperatures. A porcelain globe containing the sub. stance whose vapour density is to be determined is heated in the vapour of mercury $\left(350^{\circ}\right)$, sulphnr $\left(410^{\circ}\right)$, cad. mium $\left(850^{\circ}\right)$, or zine $\left(1,040^{\circ}\right)$. The globe is sealed up in an oxybydrogen flame.

mentioned is essentially volumetric, because a known weight of a substance is taken, and the volume of the air displaced by the vapour at a given $t$ and $h$ is determined.

The method by weight $(a)$ is the most trustworthy and historically important. Dumas' method is typical. An ordinary spherical glass or porcelain vessel, like those shown respectively in figs. 52 and 53 , is taken, and an excess of the substance to be experimented upon is introduced into it. The vessel is heated to $a$ temperature $t$ higher than the boiling point of the liquid: this gives a vapour which displaces the air, and fills the spherical space. When the air and vapour cease escaping from the sphere, it is fused up or closed by some means; and when cool, the weight of the vapour remaining in the sphere is determined (either by direct weighing of the vessel with the vapour and introducing the necessary corrections for the weight of the air and of the vapour itself, or the weight of the volatilised substance 18 determined by chemical methods), and the volume of the vapour at $t$ and the barometric pressure $h$ are then calculated.

The volumetric method $(b)$ originally employed by Clay.Lussac and then modified by Hofmann and others is based on the principle that a weighed quantity of the liquid to be experimented with (placed in a small closed ressel, which is sometimes fused up before 
tion of definite chemical compounds, shows that the volumes of the reacting substances in a gaseous or vaporous state are either equal or are in

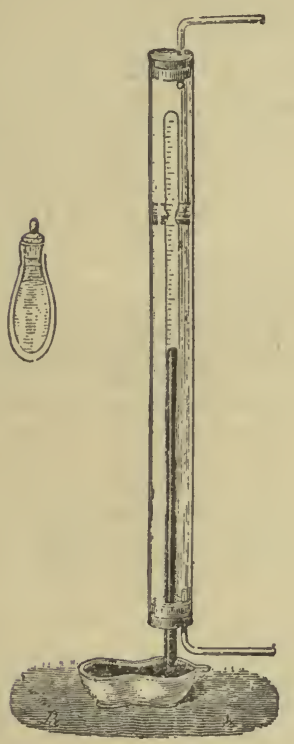

Fı. 54.-Hofmann's apparntus tor determining vapour densities. The internal tube, about one metre long, which is calibrated and graduated. is filled with mercury and inverted in a mercury bath. A small bottle (depicted in its natural size on the left) containing a weighed quantity of the liquid whose va pour density is to be determined, is introduced in to the Torricellian vacuum. Steam, or the vapour of amyl alcohol, \&ec., is passed through the outer tube, and heats the internal tube to the temperature $f$, at which the volume of vayour is measured.

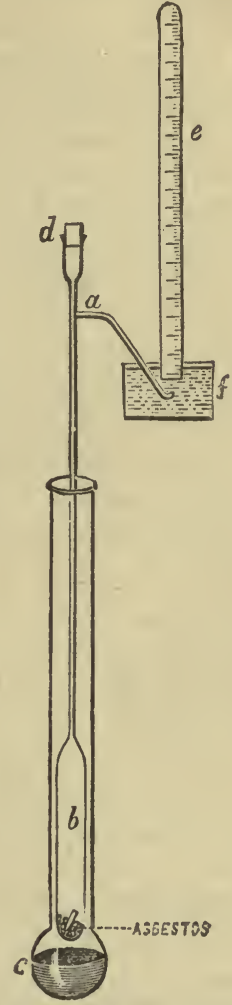

Fiv. 55. - Victor Meser's apparatus for cletermining vapour densities, The tube $b$ is heated in the vapour of a liquid of constant bolling point. A. glass tube, containing the liquiil to be experimented upon, is causel to fall froun $d$. The alr displaced is collected in the cylinder $e$, in the trough $f$

weighing, and, if quite full of the liquid, breaks when heated in a vacuum) is intro. duced into a graduated cylinder heated to $t$, or simply into a Torricellian vacuum, as shown in fig. 54, and the number of volumes occupied by the vapour noted when the space holding it is heated to the desired temperature $t$.

The method of displacement $(c)$ proposed by Victor Meyer is based on the fact that a space $b$ is heated to a constant temperature $t$ (by the surrounding vapours of a liquid of constant boiling point), and the air. (or other gas enclosed in this space) is allowed to attrin this temperature, and when it has done so a glass bulb containing a weighed quall- 
simple multiple proportion. ${ }^{3}$ This forms the first law of those discovered by Gay-Lussac. It may be formulated as follows: The amounts of sub. stances entering into chemical reaction occupy under similar physical conditions, in a gaseous or vaporous state, equal or simple multiple volumes. This law refers not only to elements, but also to compounds entering into mutual chemical combination; thus, for example, one volume of ammonia gas combines with one volume of hydrogen chloride. For in the formation of sal-ammoniac, $\mathrm{NH}_{4} \mathrm{Cl}$, there enter into reaction 17 parts by weight of ammonia, $\mathrm{NH}_{3}$, which is 8.5 times denser than hydrogen, and 36.5 parts by weight of hydrogen chloride, whose vapour density is 18.25 times that of hydrogen, as has been proved by direct experiment. By dividing the weights by the respective densities we find that the volume of ammonia, $\mathrm{NH}_{3}$, is equal to two, and so also the volume of hydrogen chloride. Hence the volumes of the compounds which here combine together are equal to each other. Taking into consideration that the law of Gay-Lussac holds good, not only for elements, but also for compounds, it should be expressed as follows : Substances interact with one another in commensurable volumes of their vapours. ${ }^{4}$

tity of the substance to be experimented with is dropped into the space. The substance is immediately converted into vapour, and displaces the air into the graduated cylinder $e$. The amount of this air is calculated from its volume, and hence the volume at $t$, and therefore also the volume occupied by the vapour, is found. The general arrangement of the apparatus is given in fig. 55 .

3 Vapours and gases, as already explained in the second chapter, are subject to the same laws, which are, however, only approximate. It is evident that for the deduction of the laws which will presently be enunciated it is only possible to take into consideration a perfect gaseous state (far removed from the liquid state) and chemical invariability in which the vapour density is constant-that is, the volume of a given gas or vapour varies like a volume of hydrogen, air, or other gas, with the pressure and temperature.

It is necessary to make this statement in order that it may be clearly seen that the laws of gaseous volumes, which we shall describe presently, are in the most intimate connection with the laws of the variations of volumes with pressure and temperature. And as these latter laws (Chapter II.) are not infallible, but only approximately exact, the same, therefore, applies to the laws about to be described. And as it is possible to find more exact laws (a second approximation) for the variation of $v$ with $p$ and $t$ (for example, van der Waals' formula, Chapter II., Note 33), so also a.more exact expression of the relation between the composition and the density of vapours and gases is also possible. But to prevent any doubt arising at the very beginning as to the breadth and general application of the laws of volumes, it will be sufficient to mention that the density of such gases as oxygen, nitrogen, and carbonic anhydride is already known to remain constant (within the limits of experimental error) between the ordinary temperature and a white heat; whilst, judging from what is said in my work on the 'Tension of Gases' (vol. i. p. 9), it may be said that, as regards pressure, the relative density remains very constant, even when the deviations from Mariotte's law are very considerable. However, in this respect the number of data is as yet too small to arrive at an exact conclusion.

4 We must recollect that this law is only approximate, like Boyle and Mariotte's law, and that, therefore, like the latter, a more exact expression may be found for the exceptions. 
The law of combining volumes and the law of multiple proportion were discovered independently of each other-the one in France by Gay-Lussac, the other in England by Dalton-almost simultaneously. In the language of the atomic hypothesis it may be said that atomic quantities of elements occupy equal or multiple volumes.

The first law of Gay-Lussao expresses the relation between the volumes of the component parts of a compound. Let us now consider the relation existing between the volumes of the component parts and of the compounds which proceed from them. This may sometimes be determined by direct observation. Thus the volume occupied by water, formed by two volumes of hydrogen and one volume of oxygen, may be determined by the aid of the apparatus shown in fig. 56 . The long glass tube is closed at the top and open at the bottom, which is immersed in a cylinder containing mercury. The closed end is furnished with wires like a eudioneter. The tube is filled with mercury, and then a certain volume of detonating gas is introduced. This gas is obtained from the decomposition of water, and therefore in every three volumes contains two volumes of hydrogen and one volume of oxygen. The tube is surrounded by a second and wider glass tube, and the vapour of a substance boiling above $100^{\circ}$ - that is, whose boiling point is higher than that of water-is passed through the annular space between them. Amyl alcohol, whose boiling point is $132^{\circ}$, may be taken for this purpose. The amyl alcohol is boiled in the vessel to the right hand and its vapour passed between the walls of the two tubes. In the case of amyl alcohol the outer glass tube should be connected with a condenser to prevent the escape into the air of the unpleasant. smelling vapour. The detonating gas is thus heated up to a temperature of $132^{\circ}$ When its volume becomes constant it is measured, the height of the column of mercury in the tube above the level of the mercury in the cylinder being noted. Let this volume equal $v$; it will therefore contain $\frac{1}{3} v$ of oxygen and $\frac{2}{3} v$ of hydrogen. The current of vapour is then stopped, and the gas exploded; water is formed, which condenses into a liquid. The volume occupied by the vapour of the water formed has now to be determined. For this purpose the vapour of the amyl alcohol is again passed between the tubes, and thus the whole of the water' formed is converted into vapour at the same temperature as that at which the detonating gas was measured, and the cylinder of mercury being raised until the column of mercury in the tube stands at, the same height above the surface of the mercury in the cylinder as it did before the explosion, it is found that the volume of the water formed is equal to $\frac{2}{3} v$-that is, it is equal to the volume of the hydrogen contained 


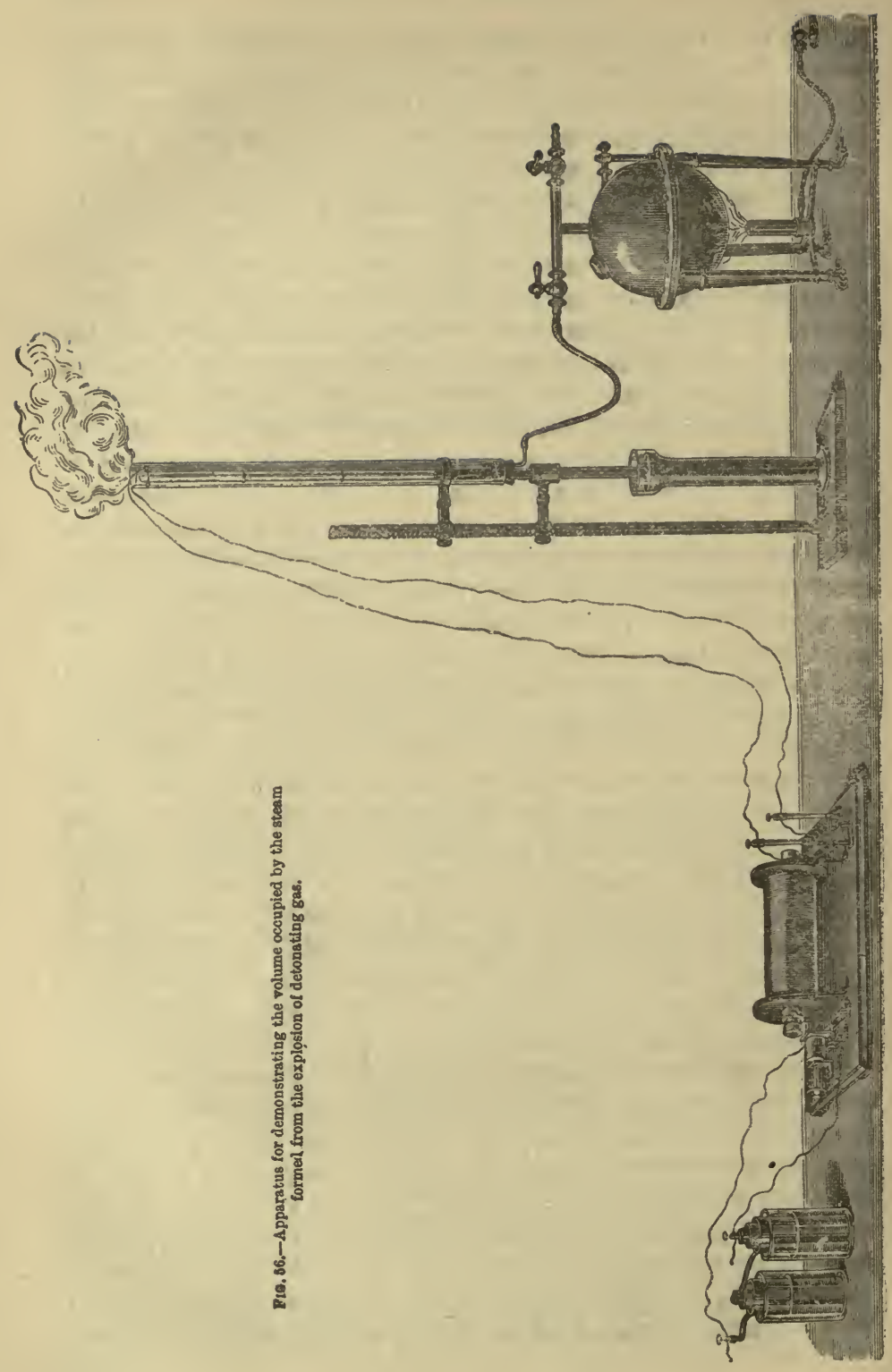


in it. Consequently the volumetric composition of water is expressed in the following terms: Two volumes of hydrogen combine with one volume of oxygen to form two volumes of aqueous vapour. For substances which are gaseous at the ordinary temperature, this direct method of observation is sometimes very easily conducted; for instance, with ammonia, nitric and nitrous oxides. Thus to determine the composition by volume of nitrous oxide, the above-described apparatus may be employed. Nitrous oxide is introduced into the tube, and after measuring its volume electric sparks are passed through the gas ; it is then found that two volumes of nitrous oxide have given three volumes of gases-namely, two volumes of nitrogen and one volume of oxygen. Consequently the composition of nitrous oxide is similar to that of water; two volumes of nitrogen and one volume of oxygen give two volumes of nitrous oxide. By decomposing ammonia it is found to be composed in such a manner that two volumes give one volume of nitrogen and three volumes of hydrogen; also two volumes of nitric oxide are formed by the union of one volume of oxygen with one volume of nitrogen. The same relations may be proved by calculation from the vapour densities, as was described above.

Comparisons of various results made by the aid of direct observations or calculation, an example of which has just been cited, led GayLussac to the conclusion that the volume of a compound in a gaseous or vaporous state is always in simple multiple proportion to the volume of each of the component parts of which it is formed (and consequently to the sum of the volumes of the elements of which it is formed). This is the second law of Gay-Lussac; it extends the simplicity of the volumetric relations to compounds, and is of the same nature as that presented by the elements entering into mutual combination. Hence not only the substances forming a given compound, but also the substances formed, exhibit a simple relation of volume when measured as vapour or gas. ${ }^{5}$

When a compound is formed from two or more components, there may or may not be a contraction; the volume of the reacting substances is in this case either equal to or greater than the volume of the resultant

5 This second law of volumes may be considered as a consequence of the first law. The first law requires simple ratios between the volumes of the combining substances $A$ and $B$. A substance $A B$ is produced by their combination. It may, according to the law of multiple proportion, combine, not only with substances $C, D, \& c$., but also with $A$ and with $B$. In this new combination the volume of $A B$, combining with the volume of $A$, should be in simple multiple proportion with the volume of $A$; hence the volume of the compound $A B$ is in simple proportion to the volume of its component parts. There. fore only one law of volumes need be accepted. We shall afterwards see that there is a third law of volumes embracing also the two first laws. 
compound. The reverse is naturally observed in the case of decom. positions, when from one substance there are produced several of simpler nature. Therefore in the future we shall term combination a reaction in which a contraction is observed-that is, a diminution in the volume of the component bodies in a state of vapour or gas ; and we shall term decomposition a reaction in which an expansion is produced: while those reactions in which the volumes in a gaseous or vaporous state remain constant (the volumes being naturally compared at the same temperature and pressure) we shall term reactions of substitution or of double decomposition. Thus the transition of oxygen into ozone is a reaction of combination, the formation of nitrous oxide from oxygen and nitrogen will also be a combination, the formation of nitric oxide from the same will be a reaction of substitution, the action of oxygen on nitric oxide a combination, and so on.

The degree of contraction produced in the formation of chemical compounds not unfrequently leads to the possibility of distinguishing the degree of change which takes place in the chemical character of the components when combined. In those cases in which a contrac. tion occurs, the-properties of the resultant compound are very dif ferent from the properties of the substances of which it is composed. Thus ammonia bears no resemblance in its physical or chemical properties to the elements from which it is derived; a contraction takes place in a state of vapour, indicating a proximation. of the elementsthe distance between the atoms is diminished, and from gaseous substances there is formed a liquid substance, or at any rate one which is easily liquefied. For this reason nitrous oxide formed by the condensation of two permanent gases is a substance which is somewhat easily converted into a liquid again, nitric acid, which is formed from elements which are permanent gases, is a liquid, whilst, on the contrary, nitric oxide, which is formed without contraction and is decomposed without expansion, remains a gas which is as difficult to liquefy as nitrogen and oxygen. In order to obtain a still more complete idea of the dependence of the properties of a compound on the properties of the component substances, it is further necessary to know the quantity of heat which is developed in the formation of the compound. If this quantity be large-as, for example, in the formation of water-then the amount of energy in the resultant compound will be considerably less than the energy of the elements entering into its composition; whilst, on the contrary, if the amount of heat evolved in the formation of a compound be small, or if there even be an absorption of heat, as in the formation of nitrous oxide, then the energy of the elements is 
not destroyed, or is only altered to a slight extent; hence, notwithstanding the contraction (compression) involved in its formation, nitrous oxide supports combustion.

The preceding laws were deduced froni purely experimental and empirical data and as such evoke further consequences, as the law of multiple proportions gave rise to the atomic theory and the law of equivalents (Chapter IV.) In view of the atomic conception of the constitution of substances, the question naturally arises as to what, then, are the relative volumes proper to those physically indivisible molecules which chemically react on each other and consist of the atoms of elements. The simplest possible hypothesis in this respect would be that the volumes of the molecules of substances are equal ; or, what is the same thing, to suppose that equal volumes of vapours and gases contain an equal number of molecules. This proposition was first enunciated by the Italian savant Avogadro in 1810. It was also admitted by the French physico-mathematician Ampere (1815) for the sake of simplifying all kinds of physico-mathematical conceptions respecting gases. But Avogadro and Ampère's propositions were not generally received in science until Gerhardt in the forties had applied them to the generalisation of chemical reactions, and had demonstrated, by aid of a series of phenomena, that the reactions of substances actually take place with the greatest simplicity, and more especially that such reactions take place between those quantities of substances which occupy equal volumes, and until he had stated the hypothesis in an exact manner and deduced the consequences that necessarily follow from it. Following Gerhardt, Clausius, in the fifties, placed this hypothesis of the equality of the number of molecules in equal volumes of gases and vapours on the basis of the kinetic theory of gases. At the present day the hypothesis of Avogadro and Gerhardt lies at the basis of contemporary physical, mechanical, and chemical conceptions ; the consequences arising from it have often been subject to doubt, but in the end have been verified by the most diverse methods ; and now, when all efforts to' refute those consequences have proved fruitless, the hypothesis must be considered as verified, ${ }^{6}$ and the law of Avogadro-Gerhardt must be spoken of as fundamental, and as of great importance for the comprehension of the phenomena of nature: The

- It must not be forgotten that Newton's law of gravity was fixst a hypothesis, but it became a trustworthy, perfect theory, and acquired the qualities of a furdamental law owing to the concord between its deductions and actual facts. Alr laws, all theories, of natural phenomena, are at first hypotheses. Some are rapidly established by, their consequences exactly agreeing with facts; others only take root by slow degrees; and there are many which are destined to be refuted owing to their conseguiences being found to be et vaxiance with facts. 
law may now be formulated from two points of riew In the first place, from a physical aspect : equal volumes of gases (or vapours) at equal tem. peratures and pressures contain the same number of molecules-or of particles of matter which are neither mechanically nor physically divisible-previous to chemical change. In the second place, from a chemical aspect, the same law may be expressed thus : the quantities of substances entering into chemical reactions occupy, in a state of vapour, equal volumes. For our purpose the chemical aspect is the most important, and therefore, before developing the law and its consequences, we will consider the chemical phenomena from which the law is deduced or which it serves to explain.

When two isolated substances interact with each other directly and easily-as, for instance, an alkali and an acid-then it is found that the reaction is-accomplished between quantities which in a gaseous state occupy equal volumes. Thus ammonia, $\mathrm{NH}_{3}$, reacts directly with hydrochloric acid, $\mathrm{HCl}$, forming sal-ammoniac, $\mathrm{NH}_{4} \mathrm{Cl}$, and in this case the 17 parts by weight of ammonia occupy the same volume as the 36.5 parts by weight of hydrochloric acid. ${ }^{7}$ Ethylene, $\mathrm{C}_{2} \mathrm{H}_{4}$, combines with chlorine, $\mathrm{Cl}_{2}$, in only one proportion, forming ethylene dichloride, $\mathrm{C}_{2} \mathrm{H}_{4} \mathrm{Cl}_{2}$, and this combination proceeds directly and with great facility, the reacting quantities occupying equal volumes. Chlorine reacts with hydrogen in only one proportion, forming hydrochloric acid, $\mathrm{HCl}$, and in this case equal volumes in. teract with each other. If an equality of volumes is observed in cases of combination, it should be even more frequently encountered in cases of decomposition, taking place in substances which split up into two others. Indeed, acetic acid breaks up into marsh gas, $\mathrm{CH}_{4}$, and carbonic anhydride, $\mathrm{CO}_{2}$, and in the proportions in which they are formed from acetic acid they occupy equal volumes. Also from phthalic acid, $\mathrm{C}_{8} \mathrm{H}_{6} \mathrm{O}_{4}$, there way be obtained benzoic acid, $\mathrm{C}_{7} \mathrm{H}_{6} \mathrm{O}_{2}$, and carbonic anhydride, $\mathrm{CO}_{2}$, and as all the elements of phthalic acid enter into the composition of these substances, it follows that, although

This is not only seen from the above calculations, but may be proved by experiment. A glass tube, divided in the middle by a stopcock, is taken and one portion filled with dry hydrogen chloride (the dryness of the gases is very necessary, because ammonia and hydrogen chloride are both very soluble in water, so that a small trace of water may contain a large amount of these gases in solution) and the other with dry ammonia, under the atmospheric pressure. One orifice (for instance, of that portion which contains the ammonia) is firmly closed, and the other is immersed under mercury, and the cock is then opened. Solid sal-ammoniac is formed, but if the rolume of one gas be greater than that of the other, some of the first gas will remain. By immersing the tube in the mercury in order that the internal pressure shall equal the atmospheric pressure, it may easily be shown that the volume of the remaining gas is equal to the difference between the volumes of the two portions of the tube, and that this remaining gas is part of that who:e volume was the greater. 
they cannot re-form it by their direct action on each other (the reaction is not reversible), still they form the direct products of its decomposition, and they occupy equal volumes. But benzoic acid, $\mathrm{C}_{7} \mathrm{H}_{8} \mathrm{O}_{2}$, is itself composed of benzene, $\mathrm{C}_{6} \mathrm{H}_{6}$, and carbonic anhydride, $\mathrm{CO}_{2}$, which also occupy equal volumes. ${ }^{8}$ There is an immense number of similar examples anong those organic substances to whose study Gerhardt consecrated his whole life and work, and he did not allow such facts as these to escape his attention. Still more frequently in the phenomena of substitution, when two substances react on one another, and two are produced without a change of volume, it is found that the two substances acting on each other occupy equal volumes as well as each of. the two resultant substances. Thus, in general, reactions of substitution take place betwieen volatile acids, $\mathrm{HX}$, and volatile alcohols, $\mathrm{R}(\mathrm{OH})$, with the formation of ethereal salts, $\mathrm{RX}$, and water, $\mathrm{H}(\mathrm{OH})$, and the volume of the vapour of the reacting quantities, $\mathrm{HX}, \mathrm{R}(\mathrm{OH})$, and $\mathrm{RX}$, is the same as that of water $\mathrm{H}(\mathrm{OH})$, whose weight, corresponding with the formula, 18 , occupies 2 volumes, if 1 part by weight of hydrogen occupy 1 volume and the density of aqueous vapour referred to hydrogen is 9 . Such general examples, of which there are many, ${ }^{9}$ show that the reaction of equal volumes forms a chemical phenomenon of -frequent occurrence, indicating the necessity for acknowledging the law of Avogadro-Gerhardt.

But the question arises, What is the relation of volumes if the reaction of two substances takes place in more than one proportion, according to the law of multiple proportions? A definite answer can only be given in cases which have been very thoroughly studied. Thus chlorine, in acting on marsh gas, $\mathrm{CH}_{A}$, forms four compounds, $\mathrm{CH}_{3} \mathrm{Cl}, \mathrm{CH}_{2} \mathrm{Cl}_{2}$, $\mathrm{CHCl}_{3}$, and $\mathrm{CCl}_{4}$, and it may be established by direct experiment that the substance $\mathrm{CH}_{3} \mathrm{Cl}$ (methylic chloride) precedes the remainder, and that the latter proceed from it by the further action of chlorine. And this substance, $\mathrm{CH}_{3} \mathrm{Cl}$, is formed by the reaction of equal volumes of

8 Let us demonstrate this by figures: From 122 grams of benzoic acid there are obtained (a) 78 grams of benzene, whose density referred to hydrogen $=39$, hence the relative volume $=2$; and $(b) 44$ grams of carbonic anhydride, whose density $=22$, and hence the volume $=2$. It is the same in other cases.

9 A large number of such generalised reactions, showing reaction by equal volumes, occur in the case of the hydrocarbon derivatives, because many of these compounds are volatile. The reactions of alkalis on accids, or anhydrides on water, \&o., which are so frequent between mineral substances, present but few such examples, because many of these substances are not volatile and their vapour densities are unknown. But essentially the same is seen in these cases also; for instance, sulphurio aoid, $\mathrm{H}_{2} \mathrm{SO}_{4}$; breaks up into the anhydride, $\mathrm{SO}_{3}$, and water, $\mathrm{H}_{2} \mathrm{O}$, which exhibit an equality of volumes. Let us take another example where three substances combine in equal volumes : carbonic anhydride, $\mathrm{CO}_{2}$, ammonia, $\mathrm{NH}_{3}$, and water, $\mathrm{H}_{2} \mathrm{O}$ (the volumes of all are equal to 2), form acid ammonium carbonate, $\left(\mathrm{NH}_{4}\right) \mathrm{HCO}_{3}$. 
marsh gas, $\mathrm{CH}_{4}$, and chlorine, $\mathrm{Cl}_{2}$, according to the equation $\mathrm{CH}_{4}+\mathrm{Cl}_{2}$ $=\mathrm{CH}_{3} \mathrm{Cl}+\mathrm{HCl}$. A great number of similar cases are met with amongst organic-that is, carbon-compounds. Gerhardt was led to the discovery of his law by investigating many such reactions, and by observing that in them the reaction of equal volumes precedes all others.

But if nitrogen or hydrogen give several compounds with oxygen, the question proposed above cannot be answered with complete clear. ness, because the successive formations of the different combinations cannot be so strictly defined. It may be supposed, but neither definitely affirmed nor experimentally confirmed, that nitrogen and oxygen first give nitric oxide, $\mathrm{NO}$, and only subsequently the brown vapours $\mathrm{N}_{2} \mathrm{O}_{3}$ and $\mathrm{NO}_{2}$. Such a sequence in the combination of nitrogen with oxygen can only be supposed on the basis of the fact that NO forms $\mathrm{N}_{2} \mathrm{O}_{3}$ and $\mathrm{NO}_{2}$ directly with oxygen. If it be admitted that $\mathrm{NO}$ (and not $\mathrm{N}_{2} \mathrm{O}$ or $\mathrm{NO}_{2}$ ) be first formed, then this instance would also confirm the law of Avogadro-Gerhardt, because nitric oxide contains equal volumes of nitrogen and oxygen. So, also, it may be admitted that, in the combination of hydrogen with oxygen, hydrogen peroxide is first formed (equal volumes of hydrogen and oxygen), which is decomposed by the heat evolved into water and oxygen. This explains the presence of traces of hydrogen peroxide (Chapter IV.) in almost all cases of the combustion or oxidation of hydrogenous substances; for it cannot be supposed that water is first formed and then the peroxide of hydrogen, because up to now such a reaction has not been observed, whilst the formation of $\mathrm{H}_{2} \mathrm{O}$ from $\mathrm{H}_{2} \mathrm{O}_{2}$ is very easily re. produced. ${ }^{10}$

Thus a whole series of phenomena show that the chemical reaction of substances actually takes place, as a rule, between equal volumes, but this does not preclude the possibility of the frequent reaction of un-

to This opinion which I have always held (since the first editions of this work), as to the primary origin of hydrogen peroxide and of the formation of water by means of its decomposition, has in latter days become more generally accepted, thanks more especially to the work of Traube. Probably it explains most simply the necessity for the presence of traces of water in many reactions, as, for instance, in the explosion of carbonic oxide with oxygen, and perhaps the theory of the explosion of detonating gas itself and of the combustion of hydrogen will gain in clearness and truth if we take into consideration the preliminary formation of hydrogen peroxide and its decomposition. We may here point out the fact that Ettingen (at Dorpat, 1888) observed the existence of currents and waves in the explosion of detonating gas by taking photographs, which showed the periods of combustion and the waves of explosion, which should be taken into consideration in the theory of this subject. As the formation of $\mathrm{H}_{2} \mathrm{O}_{2}$ from $\mathrm{O}_{2}$ and $\mathrm{H}_{2}$ corresponds with a less amount of heat than the formation of water from $\mathrm{H}_{2}$ and $\mathrm{O}$, it may be that the temperature of the flame of detonating gas depends on the pre-formation of hydrogen peroxide. 
equal volumes, although, in this case, it is often possible to discover a preceding reaction between equal volumes. ${ }^{11}$

The law of Arogadro-Gerhardt may also be easily expressed in an algebraical form. If the weight of a molecule, or of that quantity of a substance which enters into chemical reaction and occupies in a state

11 The possibility of reactions between unequal volumes, notwithstanding the general pplication of the law of Avogadro-Gerhardt, may, in addition to what has been said abo:e, depend on the fact that the participating substances, at the moment of reaction, undergo a preliminary modification, decomposition, isomeric (polymeric) transformation, \&c. Thus, if $\mathrm{NO}_{2}$ seems to proceed from $\mathrm{N}_{2} \mathrm{O}_{4}$, if $\mathrm{O}_{2}$ is formed from $\mathrm{O}_{3}$, and the converse, then it cannot be denied that the production of molecules containing only one atom is also possible-for instance, of oxygen-as also of higher polymeric forms-as the molecule $\mathrm{N}$ from $\mathrm{N}_{2}$, or $\mathrm{H}_{3}$ from $\mathrm{H}_{2}$. In this manner it is obviously possible, by means of a series of hypotheses, to explain the cases of the formation of ammonia, $\mathrm{NH}_{3}$, from 3 vols. of hydrogen and 1 vol. of nitrogen. But it must be observed that perbaps our information in similar instances is, as yet, far from being complete. If hydrazine or diamide $\mathrm{N}_{2} \mathrm{H}_{4}$ (Chapter VI. Note 20 bls) is formed and the imide $\mathrm{N}_{2} \mathrm{H}_{2}$ in which 2 vols. of hydrogen are combined with 2 vols. of nitrogen, then the reaction here perhaps first takes place between equal volumes. If it be shown tbat diamide gives nitrogen and anmonia $\left(3 \mathrm{~N}_{2} \mathrm{H}_{4}=\mathrm{N}_{2}+4 \mathrm{NH}_{5}\right)$ under the action of sparks, heat, or the silent discharge, \&c., then it will be possible to admit that it is formed before ammonia. And perhaps the still less stable imide $\mathrm{N}_{2} \mathrm{H}_{2}$, which may also decompose with the formation of ammonia, is produced before the amide $\mathrm{N}_{2} \mathrm{H}_{4}$.

I mention this to show that the fact of apparent exceptions existing to the law of reactions between equal volumes does not prove the impossibility of their being included under the law on further study of the subject. Having put forward a certain law or hypothesis, consequences must be deduced from it, and if by their means clearness and con. sistency are attained-and especially, if by their means that which could not otherwise be known can be predicted-then the consequences verify the hypothesis. This was the case with the law now under discussion. The mere simplicity of the deduction of the weights proper to the atoms of the elements, or the mere fact that having admitted the law it follows (as will afterwards be shown) that the vis viva of the' molecules of all gases is a constant quantity, is quite sufficient reason for retaining the hypothesis, if not for believing in it as a fact beyond doubt. And such is the whole doctrine of atoms. And since by the acceptance of the law it became possible to foretell even the properties and atomic weights of elements which had not yet been discovered, and these predictions afterwards proved to be in agreement with the actual facts, it is evident that the law of Avogadro-Gerhardt penetrates deeply into the nature of the chemical relation of sub. stances. This being granted, it is possible at the present time to exhibit and deduce the truth under consideration in many ways, and in every case, like all that is highest in acience (for example, the laws of the indestructibility of matter, of the conservation of energy, of gravity, \&c.), it proves to be not an empirical conclusion from direct observation and experiment, not a direct result of analysis, but a creation, or instinctive penetration, of the inquiring mind, guided and directed by experiment and observation-a synthesis of which the exact sciences are capable equally with the.highest forms of art. Without such a synthetical process of reasoning, science would only be a mass of disconnected results of arduous labour, and would not be distinguished by that vitality with which it is realiy endowed when once it succeeds in attaining a synthesis, or concordance of outward form with the inner natare of things, without losing sight of the diversities of individual parts; in short, when it discovers by means of outward phenomena, which are apparent to the sense of touch, to observation, and to the common mind, the internal signification of things-discovering simplicity in complexity and uniformity in diversity. And this is the highest problem of science. 
of vapour, according to the law, a volume equal to that occupied by the molecules of other bodies, be indicated by the letters $M_{1}, M_{2} \ldots \ldots \ldots$. or, in general, $\mathrm{M}$, and if the letters $\mathrm{D}_{1}, \mathrm{D}_{2}, \ldots \ldots \ldots \ldots$ or, in general, $\mathrm{D}$, stand for the density or weight of a given volume of the gases or vapours of the corresponding substances under certan definite conditions of temperature and pressure, then the law requires that

$$
\frac{M_{1}}{D_{1}}=\frac{M_{2}}{D_{2}} \ldots \ldots \ldots \ldots=\frac{M}{\bar{D}}=C
$$

where $\mathrm{U}$ is a certain constant. This expression shows directly that the volumes corresponding with the weights $\mathrm{M}_{1}, \mathrm{M}_{2} \ldots \ldots \ldots \ldots \mathrm{M}$, are equal to a certain constant, because the volume is proportional to the weight and inversely proportional to the density. The magnitude of $\mathrm{C}$ is naturally conditioned by and dependent on the units taken for the expression of the weights of the molecules and the densities. The weight of a molecule (equal to the sum of the atomic weights of the elements forming it) is usually expressed by taking the weight of an atom of hydrogen as unity, and hydrogen is now also chosen as the unit for the expression of the densities of gases and vapours; it is therefore only necessary to find the magnitude of the constant for any one compound, as it will be the same for all others. Let us take. water. Its reacting mass is expressed (conditionally and relatively) by the formula or molecule $\mathrm{H}_{2} \mathrm{O}$, for which $\mathrm{M}=18$, if $\mathrm{H}=1$, as we already know from the composition of water. Its vapour density, or $\mathrm{D}$, compared to hydrogen $=9$, and consequently for water $\mathrm{C}=2$, and therefore and in general for the molecules of all substances $\mathrm{M}$ $\mathrm{D}=2$.

Consequently the weight of a molecule is equal to twice its vapour density expressed in relation to hydrogen, and conversely the density of a gas is equal to half the nolecular weight referred to hydrogen.

The truth of this may be seen from a very large number of observed vapour densities by comparing them with the results obtained by calculation. As an illustration, we may point out that for ammonia, $\mathrm{NH}_{3}$, the weight of the molecule or quantity of the reacting substance, as well as the composition and weight corresponding with the formula, is expressed by the figures $14+3=17$. Consequently $M=17$. Hence, according to the law, D =8.5. And this result is also obtained by experiment. The density, according to both formula and experiment, of nitrous oxide, $\mathrm{N}_{2} \mathrm{O}$, is 22 , of nitric acid 15 , and of nitric peroxide 23 . In the case of nitrous anhydride, $\mathrm{N}_{2} \mathrm{O}_{3}$, as a substance which dissociates into $\mathrm{NO}+\mathrm{NO}_{2}$, the density should vary between 38 (so long as the 
$\mathrm{N}_{2} \mathrm{O}_{3}$ remains unchanged) and 19 (when $\mathrm{NO}+\mathrm{NO}_{2}$ is obtained). There are no figures of constant density for $\mathrm{H}_{2} \mathrm{O}_{2}, \mathrm{NHO}_{3}, \mathrm{~N}_{2} \mathrm{O}_{4}$, and many similar compounds which are either wholly or partially decom. posed in passing into vapour. Salts and similar substances either have no vapour density because they do not pass into vapour (for instance, potassium nitrate, $\mathrm{KNO}_{3}$ ) without decomposition, or, if they pass into vapour without decomposing, their vapour density is observed with difficulty only at very high temperatures. The practical de. termination of the vapour density at these high temperatures (for example, for sodium chloride, ferrous chloride, stannous chloride, \&c.) requires special methods which have been worked out by Sainte-Claire Deville, Crafts, Nilson and Pettersson, Meyer, Scott, and others. Having overcome the difficulties of experiment, it is found that the law of Avogadro-Gerhardt holds good for such salts as potassium iodide, beryllium chloride, aluninium chloride, ferrous chloride, \&c.that is, the density obtained by experiment prores to be equal to half the molecular weight--naturally within the limits of experimental error or of possible deviation from the law.

Gerhardt deduced his law from a great number of examples of volatile carbon compounds. We shall become acquainted with certain of them in the following chapters ; their entire study, from the complexity of the subject, and from long-established custom, forms the subject of a special branch of chemistry termed 'organic' chemistry. With all these substances the observed and calculated densities are very similar.

When the consequences of a law are verified by a great number of observations, it should be considered as confirmed by experiment. But this does not exclude the possibility of apparent deviations. They may evidently be of two kinds : the fraction $\frac{M}{\bar{D}}$ may be found to be either greater or less than 2-that is, the calculated density may be either greater or less than the observed density. When the difference between the results of experiment and calculation falls within the possible errors of experiment (for example, equal to hundredths of the density), or within a possible error owing to the laws of gases having an only approximate application (as is seen from the deviations, for instance, from the law of Boyle and Mariotte), then the fraction $\frac{\mathrm{M}}{\mathrm{D}}$ proves but slightly different from 2 (between 1.9 and $2 \cdot 2$ ), and such cases as these may be classed among those which ought to be expected from the nature of the subject. It is a different matter if the quotient. of $\frac{M}{D}$ be several times, and in general a multiple, greater or less than 2. 
The application of the law must then be explained or it must be laid aside, because the laws of nature admit of no exceptions. We will therefore take two such cases, and first one in which the quotient $\frac{M}{D}$ is greater than 2, or the density obtained by experiment is less than is in accordance with the law.

It must be admitted, as a consequence of the law of Avogadro. Gerhardt, that there is a decomposition in those cases where the volume of the vapour corresponding with the weight of the amount of a substance entering into reaction is greater than the volume of two parts by weight of hydrogen. Suppose the density of the vapour of water to be determined at a temperature above that at which it is decomposed, then, if not all, at any rate a large proportion of the water will be decomposed into hydrogen and oxygen. The density of such .a mixture of gases, or of detonating gas, will be less than that of aqueous vapour; it will be equal to 6 (compared with hydrogen), because 1 volume of oxygen weighs 16, and 2 volumes of hydrogen 2; and, consequently, 3 volumes of detonating gas weigh 18 and 1 volume 6 , while the density of aqueous vapour $=9$. Hence, if the 'density of aqueous vapour be determined after its decomposition, the quotient $\frac{M}{D}$ would be found to be 3 and not 2 . This phenomenon might be considered as a deviation from Gerhardt's law, but this would not be correct, because it may be shown by means of diffusion through porous substances, as described in Chapter II., that water is decomposed at such high temperatures. In the case of water itself there can naturally be no doubt, hecause its vapour density agrees with the law at all temperatures at which it has been determined. ${ }^{12}$ But there are many substances which decompose with great ease directly they are volatilised, and therefore only exist as solids or liquids, and not in a state of vapour. There are, for example, many salts of this kind, besides all definite solutions having a constant boiling point, all the compounds of ammonia-for example, all ammonium salts-\&c. Their vapour densities, determined by Bineau, Deville, and others, show that they do not agree with Gerhardt's law. Thus the vapour density of sal-

12 As the density of aqueous vapour remains constant within the limits of experimental accuracy, even at $1,000^{\circ}$, when dissociation has certainly commenced, it would appear that only a very small amount of water is decomposed at these temperatures. If even 10 p.c. of water were decomposed, the density would be 8.57 and the quotient $\mathrm{M} / \mathrm{D}=2 \cdot 1$, but at the high temperatures here concerned the error of experiment is not greater than the difference between this quantity and 2 . And probably at $1,000^{\circ}$ the dissociation is far from being equal to 10 p.c. Hence the variation in the vapcur density of water does not give us the means of ascertaining the amount of its dissociation. 
nmmoniac, $\mathrm{NH}_{4} \mathrm{Cl}$, is nearly 14 (compared with hydrogen), whilst its molecular weight is not less than 53.5 , whence the vapour density should be nearly 27 , according to the law. The molecule of sal-ammoniac cannot be less than $\mathrm{NH}_{4} \mathrm{Cl}$, because it is formed from the molecules $\mathrm{NH}_{3}$ and $\mathrm{HCl}$, and contains single atoms of nitrogen and chlorine, and therefore cannot be divided; it further never enters into reactions with the molecules of other substances (for instance, potassium hydroxide, or nitric acid) in quantities of less than 53.5 parts by weight, \&c. The calculated density (about.27) is here double the observed density (about 13.4); hence $\frac{M}{D}=4$ and not 2 . For this reason the vapour density of sal-ammoniac for a long time served as an argument for doubting the truth of the law. But it proved otherwise, after the matter had been fully investigated. The low density depends on the decomposition of sal-ammonias; on volatilising, into ammonia and hydrogen chloride. The observed density is not that of sal-ammoniac, but of a mixture of $\mathrm{NH}_{3}$ and $\mathrm{HCl}$, which should be nearly 14 , because the density of $\mathrm{NH}_{3}=8.5$ and $\mathrm{HCl}=18 \cdot 2$, and therefore the density of their mixture (in equal volumes) should be about 13.4.13 The actual decomposition of the vapours of sal-ammoniac was demonstrated by Pebal and Than by the same method as the decomi. position of water, by passing the vapour of sal-ammoniac through a porous substance. The experiment demonstrating the decomposition during volatilisation of sal-ammoniac may be made very easily, and is a very instructive point in the history of the law of Avogadro-Gerhardt, because without its aid it would never have been imagined that salammoniac decomposed in volatilising, as this decomposition bears all the signs of simple sublimation; consequently the knowledge of the decomposition itself was forestalled by the law The whole aim and practical use of the discovery of the laws of nature consists in, and is shown by, the fact that they enable the unknown to be foretold, the unobserved to be foreseen. The arrangement of the experiment is based on the following reasoning. ${ }^{14}$ According to the law and to experiment, the density of ammonia, $\mathrm{NH}_{3}$, is $8 \frac{1}{2}$, and of hydrochloric

13 This explanation of the vapour density of sal-ammoniac, sulphuric acid, and similar substances which decompose in being distilled was the most natural to resort to as soon as the application of the law of Avogadro-Gerhardt to chemical relations was begun; it was, for instance, given in my work on Specific Volumes, 1856, p. 99. The formula, $M / D=2$, which was applied later by many other investigators, had already been made use of in that work.

14 The beginner must remember that an experiment and the mode in which it is carried out must be determined by the principle or fact which it is intended to illustrate, and not vice versa, as some suppose. The idea which determines the necessity of an experiment is the chief consideration. 
acid, $\mathrm{HCl}, 18$, if the density of hydrogen $=1$. Consequently, in a mixture of $\mathrm{NH}_{3}$ and $\mathrm{HCl}$, the ammonia will penetrate much more rapidly through a porous mass, or a fine orifice, than the heavier hydro. chloric acid, just as in a former experiment the hydrogen penetrated more rapidly than the oxygen. Therefore, if the vapour of sal-ammoniac comes into contact with a porous mass, the ammonia will pass through it in greater quantities than the hydrochloric acid, and this excess of apumonia may be detected by means of moist red litmus paper, which sheuld be turned blue. If the vapour of sal-ammoniac were not decomposed, it would pass through the porous mass as a whole, and the colour of the litmus paper would not be altered, because sal-ammoniac is a neutral salt. Thus, by testing with litmus the substances passing through the porous mass, it may be decided whether the sal-ammoniac is decomposed or not when passing into vapour. Sal-ammoniao volatilises at so moderate a temperature that the experiment may be conducted in a glass tube heated by means of a lamp, an asbestos plug being placed near the centre of the tube. ${ }^{15}$ The asbestos forms a porous mass, which is unaltered at a high temperature. A piece of dry sal-ammoniac is placed at one side of the asbestos plug, and is heated by a Bunsen burner. The vapours formed are driven by a current of air forced from a gasometer or bag through two tubes containing pieces of moist litmus paper, one blue and one red paper in each. If the sal-ammoniac be heated, then the ammonia appears on the opposite side of the asbestos plug, and the litmus there turns blue. And as an excess of hydrochloric acid remains on the side where the sal-ammoniac is heated, it turns the litmus at that end red. This proves that the sal-ammoniac, when converted into vapour, splits up into ammonia and hydrochloric acid, and at the same time gives an instance of the possibility of correctly conjecturing a fact on the basis of the law of Avogadro-Gerhardt. ${ }^{15} \mathrm{bls}$

So also the fact of a decomposition may be proved in the other instances where $\frac{M}{D}$ proved greater than 2, and hence the apparent deviations appear in reality as an excellent proof of the general applica. tion and significance of the law of Avogadro-Gerhardt.

is It is important that the tubes, asbestos, and sal-ammoniac should be dry, as other. wise the moisture retains the ammonia and hydrogen chloride.

is bis Baker (1894) showed that the decomposition of $\mathrm{NH}_{4} \mathrm{Cl}$ in the act of volatilising only takes place in the presence of water, traces of which are amply sufficient, but that in the total absence of moisture (attained by carefully drying with $\mathrm{P}_{2} \mathrm{O}_{5}$ ) there is no decomposition, and the vapour density of the sal-ammoniac is found to be normal, i.e., nearly 27. It is not yet quite clear what part the trace of moisture plays here, and it must be presumed that the phenomenon belongs to the category of electrical and contact phenomena, which have not yet been fully explained (see Chapter IX., Note 29). 
In those cases where the quotient $\frac{M}{\bar{D}}$ proves to be less than 2 , or the observed density greater than that calculated, by a multiple number of times, the matter is evidently more simple, and the fact observed only indicates that the weight of the molecule is as many times greater as that taken as the quotient obtained is less than 2. So, for instance, in the case of ethylene, whose composition is expressed by $\mathrm{CH}_{2}$, the density was found by experiment to be 14, and in the case of amylene, whose composition is also $\mathrm{CH}_{2}$, the density proved to be 35 , and consequently the quotient for ethylene $=1$, and for amylene $=z$. If the molecular weight of ethylene be taken, not as 14, as might be imagined from its composition, but as twice as great-namely, as 28and for amylene as fire times greater - that is as 70 - then the molecular composition of the first will be $\mathrm{C}_{2} \mathrm{H}_{4}$, and of the second $\mathrm{C}_{5} \mathrm{H}_{10}$, and for both of them $\frac{M}{D}$ will be equal to 2. This application of the law, which at first sight may appear perfectly arbitrary, is nevertheless strictly correct, because the amount of ethylene which reacts-for example, with sulphuric and other acids-is not equal to 14 , but to 28 parts by weight. Thus with $\mathrm{H}_{2} \mathrm{SO}_{4}, \mathrm{Br}_{2}$, or $\mathrm{HI}$, \&c., ethylene combines in a quantity $\mathrm{C}_{2} \mathrm{H}_{4}$, and amylene in a quantity $\mathrm{C}_{3} \mathrm{H}_{10}$, and not $\mathrm{CH}_{2}$. On the other hand, ethylene is a gas which liquefies with diff. culty (absolute boiling point $=+10^{\circ}$, whilst amylene is a liquid boiling at $35^{\circ}$ (absolute boiling point $=+192^{\circ}$ ), and by admitting the greater density of the molecules of amylene $(M=70)$ its difference from the Jighter molecules of ethylene $(\mathrm{M}=28)$ becomes clear. Thus, the simaller quotient $\frac{\mathrm{M}}{\mathrm{D}}$ is an indication of polymerisation, as the larger iquotient is of decomposition. The difference between the densities of oxygen and ozone is a case in point.

On turning to the elements, it is found in certain cases, especially with metals-for instance, mercury, zinc, and cadmium-that that weight of the atoms which must be acknowledged in their compounds (of which mention will be afterwards made) appears to be also the pnolecular weight. Thus, the atomic weight of mercury must be taken $a s=200$, but the vapour density $=100$, and the quotient $=2$. Conisequently the molecule of mercury contains one atom, $\mathrm{Hg}$. It is the same with sodium, cadmium, and zinc. This is the simplest possible molecule, which necessarily is only possible in the case of elements, as the molecule of a compound must contain at least two atoms. However, the molecules of many of the elements prove to be complex-for instance, the weight of an atom of oxygen $=16$, and its density $=16$, so that its 
molecule must contain two atoms, $\mathrm{O}_{2}$, which might already be concludedi by comparing its density with that of ozone, whose molecule contains) $\mathrm{O}_{3}$ (Chapter IV.) So also the inolecule of hydrogen equals $\mathrm{H}_{2}$, of chlorine $\mathrm{Cl}_{2}$, of nitrogen $\mathrm{N}_{2}$, \&c. If chlorine react with hydrogen, the volume remains unaltered after the formation of hydrochloric acid, $\mathrm{H}_{2}+\mathrm{Cl}_{2}=\mathrm{HCl}+\mathrm{HCl}$. It is a case of substitution between the one, and the other, and therefore the volumes remain constant. There are elements whose molecules are much more complex - for instance, sulphur, $\mathrm{S}_{6}$-although, by heating, the density is reduced to a third, and $\mathrm{S}_{2}$ is formed. Judging from the vapour density of phosphorus $(D=62)$ ! the molecule contains four atoms $P_{4}$. Hence many elements when polymerised appear in molecules which are more complex than the simplest possible. In carbon, as we shall afterwards find, a very complex molecule must be admitted, as'otherwise its non-volatility and other properties cannot be understood. And if compounds are decomposed by a more or less powerful heat, and if polymeric substances are depolymerised (that is, the weight of the molecule diminishes) by a rise of temperature, as $\mathrm{N}_{2} \mathrm{O}_{4}$ passes into $\mathrm{NO}_{2}$, or ozone, $\mathrm{O}_{3}$, into ordinary oxygen, $\mathrm{O}_{2}$, then we might expect to find the splitting-up of the complex molecules of elements into the simplest molecule containing a single atom only-that is to say, if $\mathrm{O}_{2}$ be obtained from $\mathrm{O}_{3}$, then the formation of $\mathrm{O}$ might also be looked for. The possibility but not proof of such a proposition is indicated by the vapour of iodine. Its normal density $=127$ (Dumas, Deville, and others), which corresponds with the molecule $I_{2}$. At temperatures above $800^{\circ}$ (up to which the density remains almost constant), this density distinctly decreases, as is seen from the verified results.obtained by Victor Meyer, Crafts, and Troost. At the ordinary pressure and $1,000^{\circ}$ it is about 100 , at $1,250^{\circ}$ about 80 , at $1,400^{\circ}$ about 75 , and apparently it strives to reduce itself to one-half-that is, to 63 . Under a reduced pressure this splitting-up, or depolymerisation, of iodine vapour actually reaches a density 16 of 66 , as Crafts demonstrated by reducing the pressure to $100 \mathrm{~mm}$. and raising the temperature to $1,500^{\circ}$ From this it may be concluded that at high temperatures and low pressures the molecule $I_{2}$ gradually passes into the molecule $I$ containing one atom like mercury, and that something similar occurs with other elements at a considerable rise of temperature, which tends to bring about the disunion of compounds and the decomposition of complex molecules. ${ }^{17}$

16 Just as we saw (Chapter VI. Note 46) an increase of the dissociation of $\mathrm{N}_{2} \mathrm{O}_{4}$ and the formation of a large proportion of $\mathrm{NO}_{2}$, with a decrease of pressure. The decomposition of $I_{2}$ into $I+I$ is a similar dissociation.

17 Although at first there appeared to be a similar phenomenon in the case of chlorine, it was afterwards proved that if there is a decrease of density it is only a small 
Besides these cases of apparent discrepancy from the law of Avogadro-Gerhardt there is yet a third, which is the last, and is very instructive. In the investigation of separate substances they have to be isolated in the purest possible form, and their chemical and physical properties, and among them the vapour density, then determined. If it be normal-that is, if $\mathrm{D}=\mathrm{M} / 2$-it often serves as a proof of the purity of the substance, i.e. of its freedom from all foreign inatter. If it be abnormal - that is, if $D$ be not equal to $M / 2$-then for those who do not believe in the law it appears as a new argument against it and nothing more; but to those who have already grasped the important significance of the law it becomes clear that there is some error in the observation, or that the density was determined under conditions in which the vapour does not follow the laws of Boyle or Gay-Lussac, or else that the substance has not been sufficiently purified, and contains other substances. The law of Avogadro.Gerhardt in that case furnishes convincing evidence of the necessity of a fresh and more exact research. And as yet the causes of error have always been found. There are not a few examples in point in the recent history of chemistry. We will cite one instance. In the case of pyrosulphuryl chloride, $\mathrm{S}_{2} \mathrm{O}_{5} \mathrm{Cl}_{2}$, $M=215$, and consequently $D$ should $=107.5$, instead of which Ogier and others obtained 53.8 -that is, a density half as great; and further, Ogier (1882) demonstrated clearly that the substance is not dissociated by distillation into $\mathrm{SO}_{3}$ and $\mathrm{SO}_{2} \mathrm{Cl}_{2}$, or any other two products, and

one. In the case of bromine it is not much greater, and is far from being equal to that for iodine.

As in general we very often involuntarily confuse chemical processes with physical, it may be that a physical process of change in the coefficient of expansion with a change of temperature participates with a change in molecular weight, and partially, if not wholly, accounts for the decrease of the density of chlorine, bromine, and iodine. Thus, I have remarked (Comptes Rendus, 1876) that the coefficient of expansion of gases increases with their molecular weight, and (Chapter II., Note 26) the results of direct experiment show the coefficient of expansion of hydrobromic acid $(M=81)$ to be 0.00386 Instead of 0.00367 , which is that of hydrogen $(M=2)$. Hence, in the case of the vapour of iodine $(M=254)$ a very large coefticient of expansion is to be expected, and from this cause alone the relative density would fall. As the molecule of chlorine $\mathrm{Cl}_{2}$ is lighter $(=71)$ than that of bromine $(=160)$, which is lighter than that of iodine $(=254)$, we see that the order in which the decomposability of the vapours of these haloids is observed corresponds with the expected rise in the coefficient of expansion. Taking the coefficient of expansion of iodine vapour as 0.004 , then at $1,000^{\circ}$ its density would be 116 . Therefore the dissociation of iodine may be only an apparent phenomenon. However, on the other: hand, the heavy rapour of mercury $(M=200, D=100)$ scarcely decreases in density at a temperature of $1,500^{\circ}(D=98$, according to Victor Meyer); but it must not be forgotten that the molecule of mercury contains only one atom, whilst that of iodine contains two, and this is very important. Questions of this kind which are diffeult to decide by experimental methods must long remain without a certain explanation, owing to the difficulty, and sometimes impossibility, of distinguishing between physical and clemical changes. 
thus the abnormal density of $\mathrm{S}_{2} \mathrm{O}_{5} \mathrm{Cl}_{2}$ remained unexplained until D $\mathbf{P}$ Konovaloff (1885) showed that the previous investigators were working with a mixture (containing $\mathrm{SO}_{3} \mathrm{HCl}$ ), and that pyrosulphuryl chloride has a normal density of approximately 107 Had not the law of Avogadro-Gerhardt served as a guide, the impure liquid would have still passed as pure; the more so since the determination of the amount of chlorine could not aid in the discovery of the impurity. Thus, by following a true law of nature we are led to true deductions.

All cases which have been studied confirm the law of Avogadro. Gerhardt, and as by it a deduction is obtained, from the determination of the vapour density (a purely physical property), as to the weight of the molecule or quantity of a substance entering into chemical reaction, this law links together the two provinces of learn. ing-physics and chemistry -in the most intimate manner. Besides which, the law of Arogadro-Gerhardt places the conceptions of molecules and atoms on a firm foundation, which was previously wanting. Although since the days of Dalton it had become evident that it was necessary to adnit the existence of the elementary atom (the chemical individual indivisible by chemical or other forces), and of the groups of atoms (or molecules) of compounds, indivisible by mechanical and physical forces; still the relative magnitude of the molecule and atom was not defined with sufficient clearness. Thus, for instance, the atomic weight of oxygen might be taken as 8 or 16 , or any multiple of these numbers, and nothing indicated a reason for the acceptation of one rather than another of these magnitudes $;^{18}$ whilst as regards the weights of the molecules of elements and compounds there was no trustworthy knowledge whatever. With the establishment of Gerhardt's law the idea of the molecule was fully defined, as well as the relative magnitude of the elementary atom.

The chenical parlicle or molecule must be considered as the

18 And so it was in the fifties. Some took $O=8$, others $O=16$. Water in the first case would be $\mathrm{HO}$ and hydrogen peroxide $\mathrm{HO}_{2}$, and in the second case, as is how gene. rally accepted, water $\mathrm{H}_{2} \mathrm{O}$ and hydrogen peroxide $\mathrm{H}_{2} \mathrm{O}_{2}$ or HO. Disagreemert and con. fusion reigned. In 1860 the chemists of the whole world met at Carloruhe for the purpose of arriving at some agreement and uniformity of opinion. I was present at this Congress, and well remember how great was the difference of opinion, and how a compromise was advocated with great acumen by many scientific men, and with what warmth the followers of Gerhardt, at whose head stood the Italian professor, Canizzaro, followed up the consequences of the law of Arogadro. In the spirit of scientific freedom, without which ecience would make no progress, and would remain petrified as in the middle ages, and with the simultaneous necessity of scientific conservatism, without which the roots of past study could give no fruit, a compromise was not arrived at, nor onght it to have been, but instead of it truth, in the form of the law of Avogadro-Gerhardt, received by means of the Congress a wider development, and soon afterwards conquered all minds. Then the new so-called Gerhardt atomic weights established themselves, and in the seventies they were already in general use. 
quantity of a substance which enters into chemical reaction with other, molevules, and occupies in a state of vapour the same volums as two parts by weight of hydrogen.

The molecular weight (which has been indicated by $\mathbf{M}$ ) of a sub. stance is determined by its composition, transformations, and vapour density.

The molecule is not divisible by the mechanical and physical changes of substances, but in chemical reaction it is either altered in its properties, or quantity, or structure, or in the nature of the motion of its parts.

An agglomeration of molecules, which are alike in all chemical respects, makes up the masses of homogeneous substances in all states. $^{19}$

Molecules consist of atoms in a certain state of distribution and motion, just as the solar system ${ }^{20}$ is made up of inseparable parts (the sun, planets, satellites, comets, \&c.) The greater the number of atoms in a molecule, the more complex is the resultant substance. The equilibrium between the dissimilar atoms may be more or less stable, and may for this reason give more or less stable substances. Physical and mechanical transformations alter the velocity of the

19 A bubble of gas, a drop of liquid, or the smallest crystal, presents an agglomera. tion of a number of molecules, in a state of continual motion (like the stars of the Milky Way), distributing themselves evenly or forming new systems. If the aggregation of all kinds of heterogeneous molecules be possible in a gaseous state, where the molecules are considerably removed from each other, then in a liquid state, where they are already close together, such an aggregation becomes possible only in the sense of the mutual reaction between them which results from their chemical attraction, and especially in the aptitude of heterogeneous molecules for combining together. Solutions and other so-called indefinite chemical compounds should be regarded in this light. According to the principles developed in this work we should regard them as containing both the compounds of the heterogeneous molecules themselves and the products of their decomposition, as in peroxide of nitrogen, $\mathrm{N}_{2} \mathrm{O}_{1}$ and $\mathrm{NO}_{2}$. And we must consider that those molecules $\mathrm{A}$, which at a given moment are combined with $\mathrm{B}$ in $\mathrm{AB}$, will in the following moment become free in order to again enter into a combined form. The laws of chemical equilibrium proper to dissociated systems cannot, be regarded in any other light.

20 This strengthens the fundamental idea of the unity and harmony of type of all creation and is one of those ideas which impress themselves on man in all ages, and give rise to a hope of arriving in time, by means of a laborious series of discoveries, observations, experiments, laws, hypotheses, and theories, at a comprehension of the internal and invisible structure of concrete substances with that same degree of clearness and exactitude which has been attained in the visible structure of the heavenly bodies. It is not many years ago since the law of Avogadro-Gerhardt took root in science. It is within the menory of many living scientific men, and of mine amongst others. It is not surprising, therefore, that as yet little progress has been made in the province of molecular mechanics; but the theory of gases alone, which is intimately connected with the conception of molecules, shows by its success that the time is approaching when our knowledge of the internal structure of matter will be defined and established. 
motion and the distances between the individual molecules, or of the atoms in the molecules, or of their sum total, but they do not alter the original equilibrium of the systen ; whilst chemical changes, on the other hand, alter the molecules themselves, that is, the velocity of motion, the relative distribution, and the quality and quantity of the atoms in the molecules.

Atoms are the smallest quantities or chemically indivisible masses of the elements forming the molecules of elements and compounds.

Atoms have weight, the sum of their weights forms the weight of the molecule, and the sum of the weights of the molecules forms the weight of masses, and is the cause of gravity, and of all the phenomena which depend on the mass of a substance.

The elements are characterised, not only by their independent existence, their incapacity of being converted into each other, \&c., but also by the weight of their atoms.

Chemical and physical properties depend on the weight, composition, and properties of the molecules forming a substance, and on the weight and properties of the atoms forming the molecules.

This is the substance of those principles of molecular mechanics which lie at the basis of all contemporary physical and chemical constructions since the establishment of the law of AvogadroGerhardt. The fecundity of the principles enunciated is seen at every step in all the particular cases forming the present store of chemical data. We will here cite a few examples of the application of the law.

As the weight of an atom must be understood as the minimum quantity of an element entering into the composition of all the molecules formed by it, therefore, in order to find the weight of an atom of oxygen, let us take the molecules of those of its compounds which have already been described, together with the molecules of certain of those carbon compounds which will be described in the following chapter :-

\begin{tabular}{lcc|lcc} 
& $\begin{array}{c}\text { Molecular } \\
\text { Weight }\end{array}$ & $\begin{array}{c}\text { Amount of } \\
\text { Oxygen }\end{array}$ & & $\begin{array}{c}\text { Molecular } \\
\text { Weight }\end{array}$ & $\begin{array}{c}\text { Amount of } \\
\text { Oxygen }\end{array}$ \\
$\mathrm{H}_{2}^{2} \mathrm{O}$ & 18 & 16 & $\mathrm{HNO}_{3}$ & 63 & 48 \\
$\mathrm{~N}_{2}^{2} \mathrm{O}$ & 44 & 16 & $\mathrm{CO}$ & 28 & 16 \\
$\mathrm{NO}^{\mathrm{O}}$ & 30 & 16 & $\mathrm{CO}_{2}$ & 44 & 32 \\
$\mathrm{NO}_{2}$ & 46 & 32 & & &
\end{tabular}

The number of substances taken might be considerably increased, but the result would be the same-that is, the molecules of the compounds of oxygen would never be found to contain less than 16 parts by weight of this element, but always $n 16$, where $n$ is a whole number. 
The molecular weights of the above compounds are found either directly from the density of their vapour or gas, or from their reactions. Thus, the vapour density of nitric acid (as a substance which easily decom. poses above its boiling point) cannot be accurately determined, but tho fact of its containing one part by weight of hydrogen, and all its properties and reactions, indicate the above molecular composition and no other. In this manner it is very easy to find the atomic weight of all the elements, knowing the molecular weight and composition of their compounds. It may, for instance, be easily proved that less than $n 12$ parts of carbon never enters into the molecules of carbon compounds, and therefore $\mathrm{C}$ must be taken as 12 , and not as 6 which was the number in use before Gerhardt. In a similar manner the atomic weights now accepted for the elements oxygen, nitrogen, carbon, chlorine, sulphur, \&c., were found and indubitably established, and they are even now termed the Gerhardt atomic weights. As regards the metals, many of which do not give a single volatile compound, we shall afterwards see that there are also methods by which their atomic weights may be established, but nevertheless the law of Arogadro-Gerhardt is here also ultimately resorted to, in order to remove any-doubt which may be encountered. Thus, for instance, although much that was known concerning the compounds of beryllium necessitated its atomic weight being taken as $\mathrm{Be}=9$-that is, the oxide as $\mathrm{BeO}$ and the chloride $\mathrm{BeCl}_{2}-$ still certain analogies gave reason for considering its atomic weight ta be $\mathrm{Be}=13.5$, in which case its oxide would be expressed by the composition $\mathrm{Be}_{2} \mathrm{O}_{3}$, and the chloride by $\mathrm{BeCl}_{3}{ }^{21}$ It was then found that the vapour density of beryllium chloride was approximately 40 , when it became quite clear that its molecular weight was 80 , and as this satisfies the formula $\mathrm{BeCl}_{2}$, but does not suit the formula $\mathrm{BeCl}_{3}$, it therefore became neces. sary to regard the atomic weight of $\mathrm{Be}$ as 9 and not as $13 \frac{1}{2}$.

21 If $\mathrm{Be}=9$, and beryllium chloride be $\mathrm{BeCl}_{2}$, then for every 9 parts of beryllium there are 71 parts of chlorine, and the molecular weight of $\mathrm{BeCl}_{2}=80$; hence the vapour density should be 40 or $n 40$. If $\mathrm{Be}=13.5$, and beryllium chloride be $\mathrm{BeCl}_{3}$, then to 13.5 of beryllium there are 106.5 of chlorine; hence the molecular weight would be 120 , and the vapour density, 60 or $n 60$. The composition is evidently the same in both cases, because $9: 71:: 13.5: 106 \cdot 5$. Thus, if the symbol of an element designate different atomic weights, apparently very different formulæ may equally well express both the percentage composition of compounds, and those properties which are required by the laws of multiple proportions and equivalents. The chemists of former days accurately expressed the composition of substances, and accurately applied Dalton's laws, by taking $\mathrm{H}=1, \mathrm{O}=8, \mathrm{C}=6, \mathrm{Si}=14$, \&c. The Gerhardt equivalents are also satisfied by them, because $\mathrm{O}=16, \mathrm{C}=12, \mathrm{Si}=28, \& \mathrm{c}$, , are multiples of them. The choice of one or the other multiple quantity for the atomic weight is impossible without a firm and concrete conception of the molecule and atom, and this is only obtained as a consequence of the law of Avogadro-Gerhardt, and hence the modern atomic weights are the results of this law (see Note 28). 


\section{With the establishment of a true conception of molecules and} atoms, chemical formulæ became direct expressions, not only of com. position, ${ }^{22}$ but álso of molecular weight or vapour density, and consequently of a series of fundamental chemical and physical data, inasmuch as a number of the properties of substances are dependent on their

23 The percentage amounts of the elements contained in a given compound may be calculated from its formula by a simple proportion. Thus, for example, to find the percentage amount of hydrogen in hydrochloric acid we reason as follows:- $\mathrm{HCl}$ shows that hydrochloric acid contains $85^{\circ} 5$ of chlorine and 1 part of hydrogen. Hence, in $86^{\circ} \cdot 6$ parts of hydrochloric acid there is 1 part by weight of hydrogen, consequently 100 parts by weight of hydrochloric acid will contain as many more units of hydrogen as 100 is greater than 36.5 ; therefore, the proportion is as follows $-x \quad 1 \cdot 100 \quad 36.5$ or $x=\frac{100}{86.5}$ =2739. Therefore 100 parts of hydrochlorio acid contain $2 \cdot 739$ parts of hydrogen. In general, when it is required to transfer a formula into its percentage composition, we must replace the symbols by their corresponding atomio weights and find their sum, and knowing the amount by weight of a given element in it, it is easy by proportion to find the amount of this element in 100 or any other quantity of parts by weight. If, on the contrary, it be required to find the formula from a given percentage composition, we must proceed as follows: Divide the percentage amount of each element entering into the composition of a substance by its atomio weight, and compare the figures thus obtained - they should be in simple multiple proportion to each other. Thus, for instance, from the percentage composition of hydrogen. peroxide. 5.88 of hydrogen and 94.12 of oxygen, it is easy to find its formula; it is only necessary to divide the amount of hydrogen by unity and the amount of oxygen by 16 . The numbers 5.88 and 5.88 are thus obtained, which are in the ratio $1: 1$, which means that in hydrogen peroxide there is one atom of hydrogen to one atom of oxygen.

The following is a proof of the practical rule given above that to find the ratio of the number of atoms from the percentage composition, it is necessary to divide the per. centage amounts by the atomic weights of the corresponding substances, and to find the ratio which these numbers bear to each other. Let us suppose that two radicles (simple or compound), whose symbols and combining weights are $\mathrm{A}$ and $\mathrm{B}$, combine together, forming a compotund composed of $x$ atoms of A and $y$ atoms of B. The formula of the substance will be $\mathrm{A} x \mathrm{~B} y$. From this formula we know that our compound contains $x \mathrm{~A}$ parts by weight of the first element, and $y \mathrm{~B}$ of the second. In 100 parts of our compound there will be (by proportion) $\frac{100 . x \mathrm{~A}}{x \mathrm{~A}+y \mathrm{~B}}$ of the first element, and $\frac{100 . y \mathrm{~B}}{x \mathrm{~A}+y \mathrm{~B}}$ of the second. Let us divide these quantities, expressing the percentage amounts by the corresponding combining weights; we then obtain $\frac{100 x}{x \mathrm{~A}+y \mathrm{~B}}$ for the first element and $\frac{100 y}{x \mathrm{~A}+y \mathrm{~B}}$ for the second element. And these numbers are in the ratio $x: y$-that is, in the ratio of the number of atoms of the two substances.

It may be further observed that even the very language or nomenclature of chemistry acquires a particular clearness and conciseness by means of the conception of molecules, because then the names of substances may directly indicate their composition. Thus the term 'carbon dioxide' tells more about and expresses $\mathrm{CO}_{2}$ better than carbonic acid gas, or even carbonic anhydride. Such nomenclature is already employed by many. But expressing the composition without an indication or even hint as to the properties, would be neglecting the advantageous side of the present momenclature. Sulphur dioxide, $\mathrm{SO}_{2}$, expresses the same as barium dioxide, $\mathrm{BaO}_{2}$, but sulphurous anhydride indicates the acid properties of $\mathrm{SO}_{2}$. Probably in time one harmonious chemical language will succeed in embracing both advantages. 
vapour density, or molecular weight and composition. The vapour density $D=\frac{M}{2}$ For instance, the formula of ethyl ether is $\mathrm{C}_{4} \mathrm{H}_{10} \mathrm{O}$, corresponding with the molecular weight 74 , and the vapour density 37 , which is the fact. Therefore, the density of vapours and gases has ceased to be an empirical magnitude obtained by experiment only, and has acquired a rational meaning. It is only necessary to remember that 2 grams of hydrogen, or the molecular weight of this primary gas in grams, occupies, at $0^{\circ}$ and $760 \mathrm{~mm}$. pressure, a volume of $22 \cdot 3$ litres (or 22,300 cubic centimetres), in order to directly determine the weights of cubical measures of gases and vapours from their formulæ, because the molecular weights in grams of all other vapours at $0^{\circ}$ and $760 \mathrm{~mm}$. occupy the same volume, 22.3 litres. Thus, for example, in the case of carbonic anhydride, $\mathrm{CO}_{2}$, the molecular weight $\mathrm{M}=44$, hence 44 grams of carbonic anhydride at $0^{\circ}$ and $760 \mathrm{~mm}$. occupy a volume of $22 \cdot 3$ litres - consequently, a litre weighs $1.97 \mathrm{gram}$. By combining the laws of gases-Gay-Lussac's, Mariotte's, and Avogadro-Gerhardt's-we obtain $^{23}$ a general formula for gases

$$
6200 s(273+t)=\mathbf{M} p
$$

where 8 is the weight in grams of a cubic centimetre of a vapour or gas at a temperature $t$ and pressure $p$ (expressed in centimetres of mercury) if the molecular weight of the gas $=M$. Thus, for instance, at $100^{\circ}$ and 760 millimetres pressure (i.e. at the atmospheric pressure) the weight of a cubic centimetre of the vapour of ether $(M=74)$ is $s=0.0024 .{ }^{24}$

23 This formula (which is given in my work on 'The Tension of Gases,' and in a somewhat modified form in the 'Comptes Rendus,' Feb. 1876) is deduced in the following manner. According to the law of Avogadro-Gerhardt, $M=2 D$ for all gases, where $M$ is the molecular weight and $D$ the density referred to hydrogen. But it is equal to the weight $s_{0}$ of a cubic centimetre of a gas in grams at $0^{\circ}$ and $76 \mathrm{~cm}$. pressure, divided by 0.0000898 , for this is the weight in grams of a cubic centimetre of hydrogen. But the weight $s$ of a cubic centimetre of a gas at a temperature $t$ and under a pressure $p$ (in centimetres) is equal to $s_{0} p_{i}^{\prime} 76(1+a t)$. Therefore, $s_{0}=8.76 \cdot(1+a t) p$; hence $\mathrm{D}=$ $76.8(1+a t) \cdot 0.0000898 p$. whence $\mathrm{M}=152 s(1+a t) / 0.0000898 p$, which gives the above expression, because $1 / a=273$, and 152 multiplied by 273 and divided by 0.0000898 is nearly 6200 . In place of $s, \mathrm{~m} / v$ may be taken, where $m$ is the weight and $v$ the volume of a vapour.

24 The above formula may be directly applied in order to. ascertain the molecular weight from the data; weight of vapour $m$ grms., its volume $v$ c.c., pressure $p \mathrm{~cm}$., and temperature $t^{\circ}$; for $s=$ the weight of vapour $m$, divided by the volume $v$, and conse. quently $\mathrm{M}=6,200 \mathrm{~m} \cdot(273+t)[\mathrm{pv}$ Therefore, instead of the formula (see Chapter II., Note 34$), p v=R(273+t)$, where $R$ varies with the mass and nature of a gas, we may apply the formula $p v=6,200(m / M)(273+t)$. These formulø simplify the calculations in many cases. For example, required the volume $v$ occupied by $5 \mathrm{grms}$. of aqueous vapour at a temperature $t=127^{\circ}$ and under a pressure $p=76 \mathrm{~cm}$. According to the formula $M=6,200 m(273+t) / p v$, we find that $v=9,064$ c.c., as in the case of water $\mathrm{M}=18, m$ in this instance $=5 \mathrm{grms}$. (These formulø, however, like the laws of gases, are only approximate.) 
As the molecules of many elements (hydrogen, oxygen, nitrogen, chlorine, bromine, sulphur - at least at high temperatures) are of uniform composition, the formulie of the compounds formed by them directly indicate the composition by volume. So, for example, the formula $\mathrm{HNO}_{3}$ directly shows that in the decomposition of nitric acid there is obtained 1 vol. of hydrogen, 1 vol. of nitrogen, and 3 vols. of oxygen.

And since a great number of mechanical, physical, and chemical properties are directly dependent on the elementary and volumetric composition, and on the vapour density, the accepted system of atoms and molecules gives the possibility of simplifying a number of most coinplex relations. For instance, it may be easily demonstrated that the vis viva of the molecules of all vapours and gases is alike. For it is proved by mechanics that the vis viva of a moving mass $=\frac{1}{2} m v^{2}$, where $m$ is the mass and $v$ the velocity. For a molecule, $m=\mathbf{M}$, or the molecular weight, and the velocity of the motion of gaseous molecules $=$ a constant which we will designate by $\mathrm{C}$, divided by the square root of the density of the gas ${ }^{25}=\mathrm{C} / \sqrt{ } \mathrm{D}$, and as $\mathrm{D}=\mathrm{M} / 2$, the vis viva of molecules $=\mathrm{C}^{2}$-that is, a constant for all molecules. Q.E.D. ${ }^{26}$ The specific heat of gases (Chapter XIV.), and many other of their properties, are determined by their density, and consequently by their molecular weight. Gases and vapours in passing into a liquid state evolve the so-called latent heat, which also proves to be in connection with the molecular weight. The observed latent heats

25 Chapter I., Note 84.

${ }_{23}$ The velocity of the transmission of sound through gases and vapours closely bears on this. It $=\sqrt{ } K p g / D(1+a t)$, where $K$ is the ratio between the two specific heats (it is approximately 1.4 for gases containing two atoms in a molecule), $p$ the pressure of the gas expressed by weight (that is, the pressure expressed by the height of a column of mercury multiplied by the density of mercury), $g$ the acceleration of gravity, D the weight of a cubic measure of the gas, $\alpha=0.00867$, and $t$ the temperature. Hence, if $K$ be known, and as D can be found from the composition of a gas, we can calculate the velocity of the transmission of sound in that gas. Or if this velocity be known, we can find $K$. The relative velocities of sound in two gases can be easily determined (Kundt).

If a horizontal glass tube (about 1 metre long and closed at both ends) be full of a gas, and be firmly fixed at its middle point, then it is easy to bring the tube and gas into a state of vibration, by rubbing it from centre to end with a damp cloth. The vibration of the gas is easily rendered visible, if the interior of the tube be dusted with lycopodiun (the yellow powder-dust or spores of the lycopodium plant is often employed in medicine), before the gas is introduced and the tube fused up. The fine lycopodium powder arranges itself in patches, whose number depends on the velocity of sound in the gas. If there be 10 patches, then the velocity of sonnd in the gas is ten timess slower than in glass. It is evident that this is an easy method of comparing the velocity of sound in gases. It has been demonstrated by experiment that the velocity of sound in oxygen is four times less than in hydrogen, and the square roots of the densities and molecular weights of hydrogen and oxygen stand in this ratio. 
of carbon bisulphide, $\mathrm{CS}_{2}=90$, of ether, $\mathrm{C}_{4} \mathrm{H}_{10} \mathrm{O},=94$, of benzene, $\mathrm{C}_{6} \mathrm{H}_{6},=109$, of alcohol, $\mathrm{C}_{2} \mathrm{H}_{6} \mathrm{O},=200$, of chloroform, $\mathrm{CHCl}_{3},=67$, dc., show the amount of heat expended in converting one part by weight of the above substances into vapour. A great uniformity is observed if the measure of this heat be referred to the weight of the molecule. For carbon bisulphide the formula $\mathrm{CS}_{2}$ expresses a weight 76 , hence the latent heat of evaporation referred to the molecular quantity $\mathrm{CS}_{2}=\mathbf{7} 6 \times 90=6,840$, for ether $=9,656$, for benzene $=8,502$, for alcohol $=9,200$, for chloroform $=8,007$, for water $=9,620$, \&cc. That is, for molecular quantities, the latent heat varies comparatively little, from 7,000 to 10,000 heat units, whilst for equal parts by weight it is ten times greater for water than for chloroform and many other substances. ${ }^{27}$

Generalising from the above, the weight of the molecule determines the properties of a substance independently of its composition-i.e. of the number and quality of the atoms entering into the moleculewhenever the substance is in a gaseous state (for instance, the density of gases and vapours, the velocity of sound in them, their specific heat, \&c.), or passes into that state, as we see in the latent heat of evaporation. This is intelligible from the point of view of the atomic theory in its present form, for, besides a rapid motion proper to the molecules of gaseous bodies, it is further necessary to postulate that these molecules are dispersed in space (filled throughout with the luminiferous ether) like the heavenly bodies distributed throughout the universe. Here, as there, it is only the degree of removal (the distance) and the masses of substances which take effect, while those peculiarities of a substance which are expressed in chemical transformations, and only come into action on near approach or on contact, are in abeyance by reason of the dispersal. Hence it is at once obvious,

भ7 If the conception of the molecular weights of substances does not give an exact law when applied to the latent heat of evaporation, at all events it brings to light a certain uniformity in figures, which otherwise only represent the simple result of obser. vation. Molecular quantities of liquids appear to expend almost equal amounts of heat in their evaporation. It may be said that the latent heat of evaporation of molecular quantities is approximately constant, because the vis viva of the motion of the molecules is, as we saw above, a constant quantity. According to thermo-dynamics the latent heat of evaporation is equal to $\frac{t+273}{\mathrm{E}},\left(n^{\prime}-n\right) \frac{d p}{d T} 13 \cdot 59$, where $t$ is the boiling point, $n^{\prime}$ the specific volume (i.e. the volume of a unit of weight) of the vapour, and $n$ the specific volume of the liquid, $d p / d \mathrm{~T}$ the variation of the tension with a rise of temperature per $1^{\circ}$, and 18.89 the density of the mercury according to which the pressure is measured. Thus the latent heat of evaporation increases not only with a decrease in the vapour density (i.e. the molecular weigitt), but also with an increase in the boiling point, and therefore depends on different factors. 
in the first place, that in the case of solids and liquids, in which the molecules are closer together than in gases and vapours, a greater complexity is to be expected, i.e. a dependence of all the properties not only upon the weight of the molecule but also upon its composition and quality, or upon the properties of the individual chemical atoms forming the molecule; and, in the second place, that, in the case of a small number of molecules of any substance being disseminated through a mass of another substance-for example, in the formation of weak (dilute) solutions (although in this case there is an act of chemical reaction-i.e. a combination, decomposition, or substitution)-the dispersed molecules will alter the properties of the mediun in which they are dissolved, almost in proportion to the molecular weight and almost independently of their composition. The greater the number of molecules disseninated-i.e. the stronger the solution-the more clearly defined will those properties become which depend upon the conıposition of the dissolved substance and its relation to the molecules of the solvent, for the distribution of one kind of molecules in the sphere of attraction of others cannot but be influenced by their mutual chemical reaction. These general considerations give a starting point for explaining why, since the appearance of Van't Hoff's memoir (1886), 'The Laws of Chemical Equilibrium in a Diffused Gaseous or Liquid State' (see Chapter I., Note 19), it has been found more and more that dilute (weak) solutions exhibit such variations of properties as depend wholly upon the weight and number of the molecules and not upon their composition, and even give the means of determining the weight of molecules by studying the variations of the properties of a solvent on the introduction of a small quantity of a substance passing into solution. Although this subject has been already partially considered in the first chapter (in speaking of solutions), and properly belongs to a special (physical) branch of chemistry, we touch upon it here because the meaning and. importance of molecular weights are seen in it in a new and peculiar light, and because it gives a method for determining them whenever it is possible to obtain dilute solutions. Among the numerous properties of dilute solutions which have been investigated (for instance, the osmotic pressure, vapour tension, boiling point, internal friction, capillarity, variation with change of temperature, specific heat, electroconductivity, index of refraction, \&c.) we will select one-the 'depression' or fall of the temperature of freezing (Raoult's cryoscopic method), not only because this method has been the most studied, but also because it is the most easily carried out and most frequently applied for determining the weight of the molecules of substances in solution, although here, owing to the novelty of the subject there are 


\section{also many experimental discrepancies which cannot as yet be ex. plained by theory. ${ }^{27}$ bls}

27 bis The osmotic pressure, vapour tension of the solvent, and several other meane applied like the cryoscopio method to dilute solutions for determining the molecular weight of a substance in solution, are more difficult to carry out in practice, and only the method of determining the rise of the boiling point of dilute solutions can from its facility be placed parallel with the cryoscopic method, to which it bears a strong resemblance, as in both the solvent changes its state and is partially separated. In the boiling point method it passes off in the form of a vapour, while in cryoscopio determinations it separates out in the form of a solid body.

Van't Hoff, starting from the second law of thermo-dynamics, showed that the dependence of the rise of pressure $(d p)$ upon a rise of temperature $(d T)$ is determined by the equation $d p=\left(k m p / 2 \mathrm{~T}^{z}\right) d \mathrm{~T}$, where $k$ is the latent heat of evaporation of the solvent, $m$ its molecular weight, $p$ the tension of the saturated vapour of the solvent at $T$, and $T$ the absolute temperature $(\mathrm{T}=273+t)$, while Raoult found that the quantity $\left(p-p^{\prime}\right) / p$ (Chapter I., Note 50) or the measure of the relative fall of tension ( $p$ the tension of the solvent or water, and $p$ ' of the solution) is found by the ratio of the number of molecules, $n$ of the substance dissolved, and $\mathrm{N}$ of the solvent, so that $\left(p-p^{\prime}\right) / p=\mathrm{C} n /(\overline{\mathrm{N}}+n)$ where $\mathrm{C}$ is a constant. With very dilute solutions $p-p^{\prime}$ may be taken as equal to $d p$, and the fraction $n /(\mathrm{N}+n)$ as equal to $n$ 'N (because in that case the value of $\mathrm{N}$ is very much greater than ( $n)$, and then, judging from experiment, $C$ is nearly unity-hence: $d p / p=n, ' \mathrm{~N}$ or $d p=n p, / \mathrm{N}$, and on substituting this in the above equation we have $\left(k m p / 2 \mathrm{~T}^{2}\right) d \mathrm{~T}=n p / \mathrm{N}$. Taking a weight of the solvent $m . \mathrm{N}=100$, and of the substance dissolved (per 100 of the solvent) $q$, where $q$ evidently $=n \mathrm{M}$, if $\mathrm{M}$ be the molecular weight of the substance dissolved, we find that $n / N=q m / 100 M$, and hence, according to the preceding equation, we have $\mathrm{M}=\frac{0.02}{k} \mathrm{~T}^{3} \quad \frac{q}{d \mathrm{~T}}$, that is, by taking a solution of $q$ grms. of a substance in 100 grms. of a solvent, and determining by experiment the rise of the boiling point $d \mathrm{~T}$, we find the molecular weight $\mathrm{M}$ of the substance dissolved, because the fraction $0.02 \mathrm{~T}^{2} / k$ is (for a given pressure and solvent) a constant; for water at $100^{\circ}\left(\mathrm{T}=373^{\circ}\right.$ ) when $k=534$ (Chapter I., Note 11 ), it is nearly $5 \cdot 2$, for etler nearly 21 , for bisulphide of carbon nearly 24 , for alcohol nearly 11.5 , \&c. As an example, we will cite from the determinations made by Professor Sakurai, of Japan (1893), that when water was the solvent and the substance dissolved, corrosive sublimate, $\mathrm{HgCl}_{2}$, was taken in the quantity $q=8.978$ and 4.258 grms:, the rise in the boiling point $d \mathrm{~T}$ was $=0^{\circ} \cdot 179$ and $0^{\circ} .084$, whence $M=261$ and 268 , and when alcohol was the solvent, $q=10.873$ and 8.765 and $d \mathrm{~T}=0^{\circ} \cdot 471$ and $0^{\circ} \cdot 380$, whence $\mathrm{M}=266$ and 265 , whilst the actual molecular weight of corrosive sublimate $=271$, which is very near to that given by this method. In the same manner for aqueous solutions of sugar $(M=342)$, when $q$ varied from 14 to $2 \cdot 4$, and the rise of the boiling point from $0^{\circ} .21$ to $0^{\circ} .035$, M was found to vary between 839 and 364. For solutions of iodine $I_{2}$ in ether, the molecular weight was found by this method to be between 255 and 262 , and $I_{2}=254$. Sakurai obtained similar results (between 247 and 262) for solutions of iodine in bisulphide of carbon.

We will here remark that in determining $\mathbf{M}$ (the molecular weight of the substance dissolved) at small but increasing concentrations (per 100 grms. of water), the results obtained by Julio Baroni (1893) show that the value of $M$ found by the formula máy either increase or decrease. An increase, for instance, takes place in aqueons solutions of $\mathrm{HgCl}_{2}$ (from 255 to 334 instead of 271 ), $\mathrm{KNO}_{3}$ (57-66 instead of 101), $\mathrm{AgNO}_{3}$ (104-107 instead of 170$), \mathrm{K}_{2} \mathrm{SO}_{4}$ (55-89 instend of 174), sugar (328-348 instead of 342$)$, \&c. On the contrary the calculated value of $\mathbf{M}$ decreases as the concentration increases, for solutions of $\mathrm{KCl}$ (40-39 instead of $74 \cdot 5), \mathrm{NaCl}(33-28$ instead of $58 \cdot 5), \mathrm{NaBr}(60-49$ instead of 103), \&c. In this case (as also for $\mathrm{LiCl}, \mathrm{NaI}, \mathrm{C}_{2} \mathrm{H}_{5} \mathrm{NaO}_{3}, \& \mathrm{c}$.) the value of $i$ (Chapter I., Note 49), or the ratio between the actual molecular weight and that found by the rise of the boiling point, was found to increase with the concentration, i.e. to be greater than 1 , and to differ more and more from unity as the strength of the solution becomes 
If 100 gram-molecules of water, i.e. $1,800 \mathrm{grms}$, be taken and $n$ gram-molecules of sugar, $\mathrm{C}_{12} \mathrm{H}_{22} \mathrm{O}_{11}$, i.e. $n 342$ grms., be dissolved in greater. For example, according to Schlamp (1894), for $\mathrm{LiCl}$, with a variation of from 1.1 to $6.7 \mathrm{grm}$. $\mathrm{LiCl}$ per 100 of water, $i$ varies from 1.63 to 1.89 . But for substances of the first series $\left(\mathrm{HgCl}_{2}, \& c\right.$.), although in very dilute solutions $i$ is greater than 1 , it approximates to 1 as the concentration increases, and this is the normal phenomenon for solutions which do not conduct an electric current, as, for instance, of sugar. And with certain electrolytes, such as $\mathrm{HgCl}_{2}, \mathrm{MgSO}_{4}$, \&c., $i$ exhibits a similar variation; thus, for $\mathrm{HgCl}_{2}$ the value of $\mathrm{M}$ is found to vary between 255 and 334 ; that is, $i$ (as the molecular weight $=271$ ) varies between 1.06 and 0.81 . Hence I do not believe that the difference between $i$ and unity (for instance, for $\mathrm{CaCl}_{2}, i$ is about 8 , for KI about 2 , and decreases with the concentration) can at present be placed at the basis of any general chemical conclusions, and it requires further experimental research. Among other methods by which the value of $i$ is now determined for dilute solutions is the study of their electroconductivity, admitting that $i=1+a(k-1)$, where $a=$ the ratio of the molecular conductivity to the limiting conductivity corresponding to an infinitely large dilution (see Physical Chemistry), and $k$ is the number of ions into which the substance dissolved can split up. Without entering upon a criticism of this method of determining $i$, I will only remark that it frequently gives values of $i$ very close to those found by the depression of the freezing point and rise of the boiling point; but that this accordance of results is sometimes very doubtful. Thus for a solution containing $5.67 \mathrm{grms} . \mathrm{CaCl}_{2}$ per 100 grms. of water, $i$, according to the vapour tension $=2.52$, according to the boiling point $=2 \cdot 71$, according to the electroconductivity $=2 \cdot 28$, while for solutions in propyl alcohol (Scllamp 1894) $i$ is near to 1.33. In a word, although these methods of determining the molecular weight of substances in solution show an undoubted progress in the general chemical principles of the molecular thenry, there are still many points which require explanation.

We will add certain general relations which apply to these problems. Isotonio (Chapter I., Note 19) solutions exhibit not only similar osmotic pressures, but also the same vapour tension, boiling point and freezing temperature. The osmotic pressure bears the same relation to the fall of the vapour tension as the specific gravity of a solution does to the specific gravity of the vapour of the solvent. The general formulis underlying the whole doctrine of the influence of the molecular weight upon the properties of solutions considered above, are : 1 . Raoult in 1886-1890 showed that

$$
\frac{p-p^{\prime}}{p} \cdot \frac{100}{a} \cdot \frac{\mathrm{M}}{m}=\text { a constant } \mathrm{C}
$$

where $p$ and $p^{\prime}$ are the rapour tensions of the solvent and substance dissolved, $a$ the amount in grms. of the substance dissolved per 100 grms. of solvent, $M$ and $m$ the molecular weights of the substance dissolved and solvent. 2. Raoult and Recoura in 1890 showed that the constant above $\mathrm{C}=$ the ratio of the actual vapour density $d^{\prime}$ of the solvent to the theoretical density $d$ calculated according to the molecular weight. This deduction may now be considered proved, becanse both the fall of tension and the ratio of the vapour densities $d^{\prime} / d$ give, for water 1.08 , for alcohol 1.02 , for ether 1.04 , for bisulphide of carbon $1 \cdot 00$, for benzene $1 \cdot 02$, for acetic acid 1.63. 3. By applying the principles of thermodynamics and calling $L_{1}$ the latent heat of fusion and $T_{1}$ the absolute $(=t+273)$ temperature of fusion of the solvent, and $\mathrm{L}_{2}$ and $\mathrm{T}_{2}$ the corresponding valucs for the boiling point, Van't Hoff in 1886-1890 deduced:-

$$
\begin{aligned}
& \frac{\text { Depression of freezing point }}{\text { Rise of boiling point }}=\frac{L_{2}}{L_{1}} \cdot \frac{T_{1}{ }^{2}}{T_{2}^{2}} \\
& \text { Depression of freezing point }=\frac{A_{T_{1}}{ }^{2} a}{L_{1} M_{1}^{-}} \\
& \text {Rise of boiling point }=\dot{A T}_{2}^{2} a \\
& \dot{L}_{2} M_{1}^{-}
\end{aligned}
$$

where $A=0.01986$ (or nearly 0.02 as we took it above), $a$ is the weight in grms. of the 
them, then the depression $d$, or fall (counting from $0^{\circ}$ ) of the temperature of the formation of ice will be (according to Pickering)

$$
\begin{array}{lccccc}
n=0 & 0.010 & 0.025 & 0.100 & 0.250 & 1.000 \\
d=0^{\circ} & 0^{\circ} .0103 & 0^{\circ} .0280 & 0^{\circ} .1115 & 0^{\circ} .2758 & 1^{\circ} .1412
\end{array}
$$

which shows that for high degrees of dilution (up to $0.25 n$ ) $d$ ap. proximately (estimating the possible errors of experiment at $\pm 0^{\circ} .005$ ) $=n 1 \cdot 10$, because then $d=0^{\circ}, v^{\circ} .0110,0^{\circ} .0275_{2} 0^{\circ} \cdot 1100,0^{\circ} \cdot 2750,1^{\circ} \cdot 1000$, and the difference between these figures and the results of experiment for very dilute solutions is less than the possible errors of experiment (for $n=1$ the difference is already greater) and therefore for dilute solutions of sugar it may be said that $n$ molecules of sugar in dis. solving in 100 molecules of water give a depression of about $1^{\circ .1} \mathrm{n}$. Similar data for acetone (Chapter I., Note 49) give a depression of $1.006 n$ for $n$ molecules of acetone per 100 molecules of water. And in general, for indifferent substances (the majority of organic bodies) the depression per $100 \mathrm{H}_{2} 0$ is nearly $n 1^{\circ} \cdot 1$ to $n 1^{\circ} .0$ (ether, for instance, gives the last numiber), and consequently in dissolving in $100 \mathrm{grms}$. of water it is about $18^{\circ} .0 n$ to $19^{\circ} .0 n$, taking this rule to apply to the case of a small number of $n$ (not over $0.2 n$ ). If instead of water, other liquid or fused solvents (for example, benzene, acetic acid, acetone, nitrobenzene or molten naplithaline, metals, \&c.) be taken and in the proportion of 100 molecules of the solvent to $n$ molecules of a dissolved indifferent (neither acid nor saline) substance, then the depression is found to be equal to from $0^{\circ} .62 n$ to $0^{\circ} .65 n$ and in general $\mathrm{K} n$. If the molecular weight of the solvent $=m$, then 100 gram-molecules will weigh $100 \mathrm{~m}$ grms., and the depression will bo approximately (taking $0.63 n$ ) equal to $m 0.63 n$ degrees for $n$ molecules of the substance dissolved in $100 \mathrm{grms}$. of the solvent, or in general the depression for $100 \mathrm{grms}$. of a given solvent $=k n$ where $k$ is alnost a constant quantity (for water nearly 18 , for acetone nearly 37, \&c.) for all dilute solutions. Thus, having found a convenient solvent for a given substance and prepared a definite (by weight) solution (i.e. knowing how many grms. $r$ of the solvent there are to $q$ grms. of the substance dissolved) and having determined the depression $d$-i.e. the fall in temperature of freezing for the solvent-it is possible to determine the molecular weight of the substance dissolved, because $d=k n$ where $d$ is found by experiment and $k$ is determined by the nature of the solvent, and therefore $n$ or the number. of molecules of the

substance dissolved per 100 grms. of the solvent, $\mathbf{M}_{\mathbf{2}}$ the molecular weight of the dissolved substance (in the solution), and $M$ the molecular weight of this substance according to its composition and vapour density, then $i=\mathrm{M} ! \mathrm{M}_{1}$. The experimental data and theoretical considerations upon which these formulæ are bused will be found in textbooks of physical and theoretical chemistry. 
substance dissolved can be found. But if $r$ grms. of the solvent and $q$ grms. of the substance dissolved are taken, then there are $100 \mathrm{q} / \mathrm{r}$ of the latter per 100 grms. of the former, and this quantity $=n \mathrm{X}$, where $n$ is found from the depression and $={ }_{k}^{d}$ and $\mathrm{X}$ is the mole. cular weight of the substance dissolved. Hence $\mathrm{X}=\frac{100 q . k}{r d}$, which gives the molecular weight, naturally only approximately, but still with sufficient accuracy to easily indicate, for instance, whether in peroxide of hydrogen the molecule contains $\mathrm{HO}$ or $\mathrm{H}_{2} \mathrm{O}_{2}$ or $\mathrm{H}_{3} \mathrm{O}_{3}$, scc. $\left(\mathrm{H}_{2} \mathrm{O}_{2}\right.$ is obtained). Moreover, attention should be drawn to the fact that a great many substances taken as solvents give per 100 molecules a depression of about $0.63 n$, whilst water gives about $1.05 n$, i.e. a larger quantity, as though the molecules of liquid water were more complex than is expressed by the formula $\mathrm{H}_{2} \mathrm{O}^{28} \mathrm{~A}$ similar phenomenon which repeats itself in the osmotic pressure, vapour tension of the solvent, $d c$. (see Chapter I., Notes 19 and 49), i.e. a variation of the constant ( $k$ for 100 grms. of the solvent or $K$ for 100 molecules of it), is also observed in passing from indifferent substances to saline (to acids, alkalis and salts) both in aqueous and other solutions as we will

28 A similar conclusion respecting the molecular weight of liquid water (i.e. that its molecule in a liquid state is more complex than in a gaseous state, or polymerized into $\mathrm{H}_{8} \mathrm{O}_{4}, \mathrm{H}_{3} \mathrm{O}_{5}$ or in general into $n \mathrm{H}_{2} \mathrm{O}$ ) is frequently met in chemico-physical literature, but as yet there is no basis for its being fully admitted, although it is possible that a polymerization or aggregation of several molecules into one takes place in the passage of water into a liquid or solid state, and that there is a converse depolymerization in the act of evaporation. Recently, particular attention has been drawn to this subject owing to the researches of Eütvös (1886) and Ramsay and Shields (1893) on the variation of the surface tension $\mathrm{N}$ with the temperature $\left(\mathrm{N}=\right.$ the capillary constant $a^{2}$ multiplied by the specific gravity and-divided by 2 , for example, for water at $0^{\circ}$ and $100^{\circ}$ the value of $a^{2}=15.41$ and $12.58 \mathrm{sq} . \mathrm{mm}$, and the surface tension 7.92 and 6.04 ). Starting from the absolute boiling point (Chapter II., Note 29) and adding $6^{\circ}$, as was necessary from all the data obtained, and calling this temperature $T$, it is found that $\mathbf{A S}=k \mathrm{~T}$, where $\mathbf{S}$ is the surface of a gram-molecule of the liquid (if $\mathrm{M}$ is its weight in grams, $s$ its $\mathrm{sp}$. gx., then its sp. volume $=\mathrm{M} / 8$, and the surface $\mathrm{S}=\sqrt{\left.\left(\mathrm{M}_{i} /\right)^{2}\right)^{2}}$, $\mathrm{A}$ the surface tension (determined by experiment at $\mathrm{T}$ ), and $k$ a constant which is independent of the composition of the molecule. The equation $A S=k T$ is in complete agreement with the well-known equation for gases $v p=\mathrm{RT}(\mathrm{p}, 110)$ which serves for deducing the molecular weight from the vapour density. Ramsay's researches led him to the conclusion that the liquid molecules of $\mathrm{CS}_{2}$, ether, benzene, and of many other substances, have the same value as in a state of vapour, whilst with other liquids this is not the case, and that to obtain an accordance, that is, that $k$ shall be a constant, it is necessary to assume the molecular weight in the liquid stute to be $n$ times as great. For the fatty alcohols and acids $n$ varies from $1 \frac{1}{3}$ to $3 \frac{1}{2}$, for water from $2 \frac{1}{4}$ to 4 , according to the temperature (at which the depolymerization takes place). Hence, although this subject offers a great theoretical interest, it cannot be regarded as firmly established, the more so since the fundamental obervations are difficult to make and not sufficiently numerous, should, however, further experiments confirm the conclusions arrived at by Professor Ramsay, this will give another method of determining molecular weights. 
show (according to Pickering's data 1892) for solutions of $\mathrm{NaCl}$ and $\mathrm{CuSO}_{4}$ in water. For

$$
\begin{array}{lllll}
n=0.01 & 0.03 & 0.05 & 0.1 & 0.5
\end{array}
$$

molecules of $\mathrm{NaCl}$ the depression is

$$
\begin{array}{llll}
d=0.0177 & 0^{\circ} .0598 & 0^{\circ} .0992 & 0^{\circ} .1958
\end{array}
$$

00.9544

which corresponds to a depression per molecule

$$
\begin{array}{lllll}
\mathrm{K}=1.77 & 1.96 & 1.98 & 1.96 & 1.91
\end{array}
$$

i.e. here in the most dilute solutions (when $n$ is nearly 0 ) $d$ is obtained about $1 \cdot 7 n$, while in the case of sugar it was about $1 \cdot 1 n$. For CuSO, for the same values of $n$, experiment gave :

$$
\begin{array}{lcccc}
d=0^{\circ} .0164 & 0^{0} .0451 & 0.0621 & 0^{0.1321}-0^{0.5245} \\
\mathrm{~K}=1.64 & 1.50 & 1.44 & 1.32 & 1.05
\end{array}
$$

i.e. here again $d$ for very dilute solutions is nearly $1.7 n$, but the value of $\mathrm{K}$ falls as the solution becomes more concentrated, while for $\mathrm{NaCl}$ it nt first increased and only fell for the more concentrated solutions. The value of $\mathrm{K}$ in the solution of $n$ molecules of a body in $100 \mathrm{H}_{2} \mathrm{O}$, when $d=\mathrm{K} n$, for very dilute solutions of $\mathrm{CaCl}_{2}$ is nearly $2 \cdot 6$, for $\mathrm{Ca}\left(\mathrm{NO}_{3}\right)_{2}$ nearly, $2 \cdot 5$, for $\mathrm{HNO}_{3}, \mathrm{KI}$ and $\mathrm{KHO}$ nearly $1.9-2 \cdot 0$, for borax $\mathrm{Na}_{2} \mathrm{~B}_{4} \mathrm{O}_{7}$ nearly $3 \cdot 7$, \&c., while for sugar and similar substances it is, as has been already mentioned, nearly $1 \cdot 0-1 \cdot 1$. Although these figures are very different ${ }^{28}$ bls still $k$ and $\mathrm{K}$ may be considered constant for analogous substances, and therefore the weight of the molecule of the body in solution can be found from $d$. And as the vapour tension of solutions and their boiling points (see Note 27 bis and Chapter I., Note 51) vary in the same manner as the freezing point depression, so they also may serve as means for determining the molecular weight of a substance in solution. ${ }^{29}$

Thus not only in vapours and gases, but also in dilute solutions of solid and liquid substances, we see that if not all, still many properties

28 bis Their variance is expressed in the same manner as was done by Van't Hoff (Chapter I., Notes 19 and 49) by the quantity $i$, taking it as $=1$ when $k=1.05$, in that case for KI, $i$ is nearly 2 , for borax about 4 , \&c.

\begin{tabular}{|c|c|c|c|c|c|}
\hline & $\begin{array}{c}\text { Molecular } \\
\text { weight }\end{array}$ & Sp. gr. at $16^{\circ}$ & & $\begin{array}{l}\text { Molecular } \\
\text { weight }\end{array}$ & Sp. gr. at $15^{\circ}$ \\
\hline $\mathrm{HCl}$ & $\quad 36.5$ & 1.0041 & $\mathrm{CaCl}_{2}$ & 111 & $1 \cdot 0286$ \\
\hline $\mathrm{NaCl}$ & $58 \cdot 5$ & $1 \cdot 0106$ & $\mathrm{NiCl}_{2}$ & 180 & 1.0328 \\
\hline $\mathrm{KCl}$ & $74 \cdot 5$ & 1.0121 & $\mathrm{ZnCl}_{2}$ & 186 & 1.0831 \\
\hline $\mathrm{BeCl}_{2}$ & $80^{4}$ & $1 \cdot 0188$ & $\mathrm{BaCl}_{2}$ & 208 & $1 \cdot 0489$ \\
\hline $\mathrm{MgCl}_{2}$ & 95 & 1.0203 & & & \\
\hline
\end{tabular}

29 We will cite one more example, showing the direct dependence of the properties of a substance on the molecular weight. If one molecular part by weight of the various chlorides-for instance, of sodium, calcium, barium, \&c.-be dissolved in 200 molecular parts by weight of water (for instance, in 8,600 grams) then it is found that the greater the molecular weight of the salt dissolved; the greater is the specific gravity of the resultant solution. 
are wholly dependent upon the molecular weight and not upon the 'quality of a substance, and that this gives the possibility of determining the weight of molecules by studying these properties (for instance, the vapour density, depression of the freezing point, \&c.) It is apparent from the foregoing that the physical and even more so the chemical properties of homogeneous substances, more especially solid and liquid, do not depend exclusively upon the weights of their molecules, but that many are in definite (sce Chapter XV.) dependence upon the weights of the atoms of the elements entering into their composition, and are determined by their quantitative and individual peculiarities. Thus the density of solids and liquids (as will afterwards be shown) is chiefly determined by the weights of the atoms of the elements entering into their composition, inasmuch as dense elements (in a free state) and compounds are only met with among substances containing elements with large atomic weights, such as gold, platinum, and uranium. And these elements themselves, in a free state, are the heaviest of all elenients. Substances containing such light elements as hydrogen, carbon, oxygen and nitrogen (like many organic substances) never have a high specific gravity; in the majority of cases it scarcely exceeds that of water. The density generally decreases with the increase of the amount of hydrogen, as the lightest element, and a substance is often obtained lighter than water. The refractive power of substances also entirely depends on the composition and the properties of the component elements. ${ }^{29}$ bis The history

29 bis With respect to the optical refractive power of substances, it must first be observed that the coefficient of refraction is determined by two methods: (a) either all the data are referred to one definite ray - for instance, to the Fraunhofer (sodium) line $\mathrm{D}$ of the solar spectrum - that is, to a ray of definite wave length, and often to that red ray (of the hydrogen spectrum) whose wave length is 656 inillionths of a millimetre; (b) or Cauchy's formula is used, showing the relation between the coefficient of refraction and dispersion to the wave length $n=A+\frac{B}{\lambda^{2}}$, where $A$ and $B$ are two constants varying for every substance but constant for all rays of the spectrum, and $\lambda$ is the wave length of that ray whose coefficient of refraction is $n$. In the latter method the investigation usually concerns the magnitudes of $\mathbf{A}$, which are independent of dispersion. We shall afterwards cite the data, investigated by the first method, by which Gladstone, Landolt, and others established the conception of the refraction equivalent.

It has long been known that the coefficient of refraction $n$ for a given substance decreases with the density of a substance $\mathrm{D}$, so that the magnitude $(n-1) \div \mathrm{D}=\mathrm{C}$ is almost constant for a given ray (having a definite wave length) and for a given sulstance. This constant is called the refractive energy, and its product with the atomic or molecular weight of a sulstance the refraction equivalent. The coefficient of refraction of oxygen is 1.00021, of hydrogen 1.00014, their densities (referred to water) are 0.00143 and 0.00009 , and their atomic weights, $\mathrm{O}=16, \mathrm{H}=1$; hence their refraction equivalents are 8 and $1 \cdot 5$. Water contains $\mathrm{H}_{2} \mathrm{O}$, consequently the sum of the equivalents of refraction is $(2 \times 1 \cdot 5)+3=6$. But as the coefficient of refraction of water $=1.331$, Its refraction equivalent $=5.958$, or nearly 6 . Comparison shows that, approximately, the sum of the refraction equivalents of the atoms forming compounds 
of chemistry presents a striking example in point-Newton foresaw from the high refractive index of the diamond that it would contain a combustible substance since so many combustible oils have a high refractive power. We shall afterwards see (Chapter $\mathrm{XV}$.) that many of those properties of substances which are in direct dependence not upon the weight of the molecules' but upon their composition, or, in other words, upon the properties and quantities of the elements entering into them, stand in a peculiar (periodic) dependence upon the atomic weight of the elements; that is, the mass (of molecules and atoms), proportional to the weight, determines the properties of substances as it also determines (with the distance) the motions of the heavenly bodies.

(or mixtures) is equal to the refraction equivalent of the compound. According to the ! researches of Gladstone, Landolt, Hagen, Bruihl and others, the refraction equivalents of the elements are- $\mathrm{H}=1 \cdot 3, \mathrm{Li}=3.8, \mathrm{~B}=4 \cdot 0, \mathrm{C}=5 \cdot 0, \mathrm{~N}=4 \cdot 1$ (in its highest state of oxidation, $5 \cdot 3), \mathrm{O}=2 \cdot 9, \mathrm{~F}=1 \cdot 4, \mathrm{Na}=4 \cdot 8, \mathrm{Mg}=7 \cdot 0, \mathrm{Al}=8 \cdot 4, \mathrm{Si}=6.8, \mathrm{P}=18: 8, \mathrm{~S}=16 \cdot 0, \mathrm{Cl}=99$ $\mathrm{K}=8 \cdot 1, \mathrm{Ca}=10 \cdot 4, \mathrm{Mn}=12 \cdot 2, \mathrm{Fe}=12.0$ (in the salts of its higher oxides, 20.1), $\mathrm{Co}=10.8$, $\mathrm{Cu}=11^{\circ} 6, \mathrm{Zn}=10^{\circ}, \mathrm{As}=15 \cdot 4, \mathrm{Bi}=15 \cdot 8, \mathrm{Ag}=15 \cdot 7, \mathrm{Cd}=13 \cdot 6, \mathrm{I}=24^{\circ} \cdot 5, \mathrm{Pt}=26 \cdot 0, \mathrm{Hg}=20^{\circ}$, $\mathrm{Pb}=24 \cdot 8$, \&c. The refraction equivalents of many elements could only be calculated from the solutions of their compounds. The composition of a solution being known it is possible to calculate the refraction equivalent of one of its component parts, those for all its other components being known. The results are founded on the acceptance of a law which cannot be strictly applied. Nevertheless the representation of the refraction equivalents gives an easy means for directly, although only approximately, obtaining the coefficient of refraction from the chemical composition of a substance. For instance, the composition of carbon bisulphide is $\mathrm{CS}_{2}=76$, and from its density, 1·27, we find its coefficient of refraction to be 1.618 (because the refraction equivalent $=5+2 \times 16=87$ ), which is very near the actual figure. It is evident that in the above representation com. pounds are looked on as simple mixtures of atoms, and the physical properties of a compound as the sum of the properties present in the elementary atoms forming it. If this representation of the presence of simple atoms in compounds had not existed, the idea of combining by a few figures a whole mass of data relating to the coefficient of refrac. tion of different substances could hardly have arisen. For further details on this subject, see works on Plysical Chemistry. 


\section{CHAPTER VIII}

\section{CARBON AND THE HYDROCARBONS}

It is necessary to clearly distinguish between the two closely-allied terms, charcoal and carbon. Charcoal is well known to everybody, although it is no easy matter to obtain it in a chemically pure state. Pure charcoal is a simple, insoluble, infusible, combustible substance produced by heating organic matter, and has the familiar aspect of a black mass, devoid of any crystalline structure, and completely in. soluble. Charcoal is a substance possessing a peculiar combination of physical and chemical properties. This substance, whilst in a state of ignition, combines directly with oxygen; in organic substances it is found in combination with hydrogen, oxygen, nitrogen, and sulphur. But in all these combinations there is no real charcoal, as in the same sense there is no ice in steam. What is found in such combinations is termed 'carbon'-that is, an element common to charcoal, to those substances which can be formed from it, and also to those substances from which it can be obtained. Carbon may take the form of charcoal, but occurs also as diamond and as graphite. Truly no other element has such a wide terminology. Oxygen is always called 'oxygen,' whether it is in a free gaseous state, or in the form of ozone, or oxygen in water, or in nitric acid or in carbonic anhydride. But here there is some confusion. In water it is evident that there is no oxygen in a gaseous form, such as can be obtained in a free state, no oxygen in the form of ozone, but a substance which is capable of producing both oxygen, ozone, and water As an element, oxygen possesses a known chemical individuality, and an influence on the properties of those combinations into which it enters. Hydrogen gas is a substanee which reacts with difficulty, but the element hydrogen represents in its combinations an easily displaceable component part. Carbon may be considered as an atom of carbon matter, and charcoal as a collection of such atoms forming a whole substance, or mass of molecules of the substance. The accepted atomic weight of carbon is 12 , because that is the least quantity of carbon which enters 
into combination in molecules of its compounds; but the weight of the molecules of charcoal is probably very much greater. This weight remains unknown because charcoal is capable of but few direct reactions and those only at a high temperature (when the weight of its molecules probably changes, as when ozone changes into oxygen), and it does not turn into vapour. Carbon exists in nature, both in a free and combined state, in most varied forms and aspects. Carbon in a free state is found in at least three different forms, as charcoal, graphite, and the diamond. In a combined state it enters into the composition of what are called organic substances-a multitude of substances which are found in all plants and animals. It exists as carbonic anhydride both in air and in water, and in the soil and crust of the earth as salts of carbonic acid and as organic remains.

The variety of the substances of which the structure of plants and animals is built up is familiar to all. Wax, oil, turpentine, and tar, cotton and albumin, the tissue of plants and the muscular fibre of animals, vinegar and starch, are all vegetable and animal matters, and all carbon compounds. ${ }^{1}$ The class of carbon compounds is so vast

1 Wood is the non-vital part of ligneous plants: the vital part of ordinary trees is situated between the bark and the lignin. Every year a layer of lignin is deposited on this part by the juices which are absorbed by the roots and drawn up by the leaves; for this reason the age of trees may be determined by the number of lignin layers deposited. The woody matter consists principally of fibrous tissue on to which the lignin or so-called incrusting matter has been deposited. The tissue has the composition $\mathrm{C}_{6} \mathrm{H}_{10} \mathrm{O}_{5}$, the substance deposited on it contains more carbon and hydrogen and less oxygen. This matter is saturated with moisture when the wood is in a fresh state. Fresh birch wood contains about 31 p.c. of water, lime wood 47 p.c., oak 35 p.c., pine and fir about 37 p.c. When dried in the air the wood loses a considerable quantity of water and not more than 19 p.c. remains. By artificial means this loss of water may be increased. If water be driven into the pores of wood the latter becomes heavier than water, as the lígnin of which it is composed has a density of about 1.6. One cubic centimetre of birch wood does not weigh more than $0.901 \mathrm{gram}$, fir 0.894 , lime tree 0.817 , poplar 765 when in a fresh state; when in a dry state birch weighs 0.622 , pine 0.550 , fir 0.355 , lime 0.430 , guaiacum $1 \cdot 342$, ebony $1 \cdot 226$. On one hectare $(2 \cdot 7$ acres $)$ of woodland the yearly growth averages the amount of 3,000 kilograms (or about 3 tons) of wood, but rarely reaches as much as 5,000 kilos. The average chemical composition of wood dried in air may be expressed as follows:- Hygroscopio water 15 p.c., carbon 42 p.c., hydrogen 5 p.c., oxygen and nitrogen 87 p.c., ash 1 p.c. Wood parts with its hygroscopic water at $150^{\circ}$, and decomposes at about $300^{\circ}$, giving a brown, brittle, so-called red charcoal; above $350^{\circ}$ black charcoal is produced. As the hydrogen contained in wood requires for its combustion about forty parts by weight of oxygen, which is present to the amount of about 86 p.c., all that burns of the wood is the carbon which it contains, 100 parts of wood only giving out as much heat as forty parts of charcoal, and therefore it would be far more profitable to use charcoal for heating purposes than wood, if it were possible to obtain it in such quantities as correspond with its percentage ratio-that is forty parts per 100 parts of wood. Generally, however, the quantity produced is far less, not more than 30 p.c., because part of the carbon is given off as gas, tar, \&c. If wood has to be transported great distances, or if 
that it forms a separate branch of chemistry, known under the name of organic chemistry-that is, the chemistry of carbon compounds, or, more strictly, of the hydrocarbons and their derivatives.

it is necessary to obtain a very high temperature by burning it, then even as little as 25 p.c. of charcoal from 100 parts of wood may be advantageous. Charcoal (from wood) develops on burning 8,000 heat units, whilst woed dried in air does not develop more than 2,800 units of heat; therefore seven parts of charcoal give as much heat as wenty parts of wood. As regards the temperature of combustion, it is far higher with charcosl than with wood, because twenty parts of burning wood give, besides the carbonic anhydride which is also formed together with charcoal, eleven parts of water, the evaporation of which requires a considerable amount of heat.

The composition of the growing parts of plants, the leaves, young branches, shoots, \&c., differs from the composition of the wood in that these vital parts contain a considerable

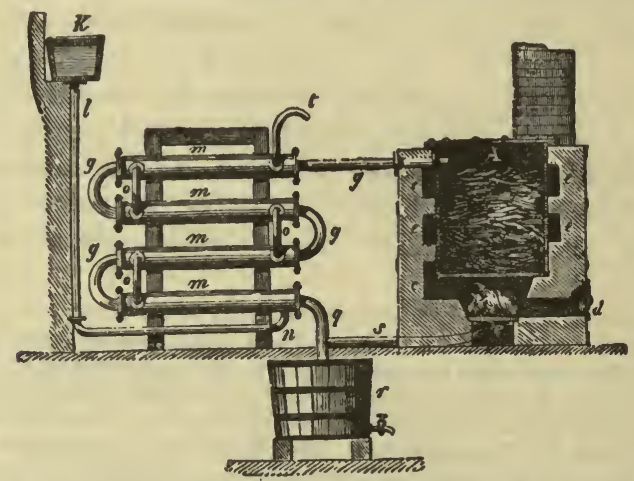

Fra. 67.-Apparatus for the dry distillation of wood. The retort a containing the wood is heated by the flues ce. The steam and volatile products of distillation pass along the tube $g$ through thie condenser $m$, where they are condensed. The form, distribution, and dimensions of the apparatus vary.

quantity of sap which contains much nitrogenous matter (in the wood itself there is very little), mineral salts, and a large amount of water. Taking, for example, the composition of clover and pasture hay in the green and dry state; in 100 parts of green clover there is about 80 p.c. of water and 20 p.c. of dry matter, in which there are about 8.5 parts of nitrogenous albuminous matter, about 9.5 parts of soluble and about 5 parts of insoluble non-nitrogenous matter, and about 2 p.c. of ash. In dry clover or clover-hay there is about 15 p.c. of water, 13 p.c. of nitrogenous matter, and 7 p.c. of ash. This composition of grassy substances shows that they are capable of forming the same sort of charcoal as wood itself. It also shows the difference of nutritive properties existing between wood and the substances mentioned. These latter serve as food for animals, because they contain those substances which are capable of being dissolved (entering into the blood) and forming the body of animals; such sabstances are proteids, starch, \&c. Let us remark here that with a good harvest an acre of land gives in the forn of grass as nuch organic substance as it yields in the form of wood.

One hundred parts of dry wood are capable of giving, on dry distillation, besides 25 p.c. of charcoal and 30 p.c. or more of tar, 40 p.c. of watery liquid, containing acetic acid and wood spirit, and about 25 p.c. of gases, which may be used for heating or lighting purposes, becnuse they do not differ from ordinary illuminating gas, which can 
If any one of these organic compounds be strongly heated without free access of air-or, better still, in a vacuum-it decomposes with more or less facility. If the supply of air be insufficient, or the temperature be too low for combustion (see Chapter III.), and if the first volatile products of transformation of the organic matter are subjected to conden. sation (for example, if the door of a stove be opened), an imperfect combustion takes place, and smoke, with charcoal or soot, is formed. ${ }^{2}$

indeed be obtained from wood. As wood-charcoal and tar are valuable products, in somo cases the dry distillation of wood is carried on principally for producing them. For this purpose those kinds of woods are particularly advantageous which contain resinous substances, especially coniferous trees, such as fir, pine, \&o.; birch, oak, and ash give much less tar, but on the other hand they yield more aqueous liquor. The latter is used for the manufacture of wood spirit, $\mathrm{CH}_{4} \mathrm{O}$, and acetic acid, $\mathrm{C}_{2} \mathrm{H}_{4} \mathrm{O}_{2}$. In such cases, the dry distillation is carried on in stills. The stills are nothing more than horizontal or vertical cylindrical retorts, made of boiler plate, heated with fuel and having apertures at the top and sometimes also at the bottom for the, exit of the light and heary products of distillation. The dry distillation of wood in stoves is carried on in two ways, either by burning a portion of the wood inside the stove in order to submit the remainder to dry distillation by means of the heat obtained in this manner, or by placing the wood in a stove the thin sides of which are surrounded with a flue leading from the fuel, placed in a space below.

The first niethod does not give such a large amount of liquid products of the dry distillation as the latter. In the latter process there is generally an outlet below for emptying out the charcoal at the close of the operation. For the dry distillation of 100 parts of wood from forty to twenty parts of fuel are used.

In the north of Russia wood is so plentiful and cheap that this locality is admirably fitted to become the centre of a general trade in the products of its dry distillation. Coal (Note 6), sea-weed, turf, animal substances (Chapter VI.), \&c., are also submitted to the process of dry distillation.

2 The result of imperfect combustion is not only the loss of a part of the fuel and the production of smoke, which in some respects is inconvenient and injurious to health, but also a low flame temperature, which means that a less amount of heat is transmitted to the object heated. Imperfect combustion is not only always accompanied by the fornan tion of soot or unburnt particles of charcoal, but also by that of carbonic oxide, $\mathrm{CO}$, in the smoke (Chapter IX.) which burns, emitting much heat. In works and factories where large quantities of fuel are consumed, many appliances are adopted to ensure perfect com.: bustion, and to combat against such a ruinous practice as the imperfect combustion of fuel. The most effective and radical means consists in employing combustible gases (producer and water gases), because by their aid perfect combustion can be easily vealised without a loss of heat-producing power and the highest temperature can be reached. When solid fuel is used (such as coal, wood, and turf), imperfect combustion. is most liable to occur when the furnace doors are opened for the introduction of fresh fuel. The step furnace may often prove a remedy for this defect. In the ordinary furnace fresh fuel is placed on the burning fuel, and the products of dry distillation of the fresh fuel have to burn at the expense of the oxygen remaining uncombined with the burnt fuel. Imperfect combustion is observed in this case also from the fact that the dry distillation and evaporation of the water of the fresh fuel lying on the top of that burnt, lowers the temperature of the flame, because part of the heat becomes latent. On this account a large amount of smoke (imperfect combustion) is observed when a fresh quantity of fuel is introduced into the furnace. This may be obviated by constructing the furnace (or managing the stoking) in such a way that the products of distillation pass. through the red-hot charcoal remaining from the burnt fuel. It is only necessary in order to ensure this to allow a sufficient quantity of air for perféct combustion. All this, 
The nature of the phenomenon, and the products arising from it, are the same as those produced by heating alone, since that part which is in a state of combustion serves to heat the remainder of the fuel. The decomposition which takes place on heating a compound composed of carbon, hydrogen, and oxygen is as follows :-A part of the hydrogen is separated in a gaseous state, another part in combination with oxygen, and a third part separates in combination with carbon, and sometimes in combination with carbon and oxygen in the form of gaseous or volatile products, or, as they are also called, the products of dry distillation. If the vapours of these products are passed through a strongly heated tube, they are changed again in a similar manner and finally resolve themselves into hydrogen and charcoal. Altogether these various products of decomposition contain a smaller amount of carbon than the original organic matter; part of the carbon remains in a free state, forming charcoal. ${ }^{3}$ It remains in that space where the decomposition took place, in the shape of the black, infusible, non-volatile charcoal familiar to all. The earthy matter and all non-

may be easily attained by the use of step fire-bars. The fuel is fed into a hopper and falls on to the fire-bars, which are arranged in the form of a staircase. The burning charcoal is below, and hence the flame formed by the fresh fuel is heated by the contact of the red-hot burning charcoal. An air supply through the fire grate, an equal dis. tribution of the fuel on the fire-bars (otherwise the air will blow through empty spaces.and lower the temperature), a proper proportion between the supply of air and the chimney draught, and a perfect admixture of air with the flame (without an undue excess of air), are the means by which we can contend against the imperfect combustion of such kinds of fuel as wood, peat, and ordinary (smoky) coal. Coke, charcoal, anthracite, burn without smoke, because they do not contain hydrogenous substances which furnish the products of dry distillation, but imperfect combustion may occur with them also; in that case the smoke contains carbonic oxide.

3 Under the action of air, organic substances are capable of oxidising to such an extent that all the carbon and all the hydrogen they contain will be transformed into carbonic anhy. dride and water. The refuse of plants and that of animals are subjected to such a change whether they slowly decompose and putrefy, or rapidly burn with direct access of air. But if the supply of air be limited, tirere can be no complete transformation into water and carbonic anhydride, there will be other volatile matters (rich in hydrogen), while charcoel must remain as a non-volatile substance. All organic substances are unstable, they do not resist heat, and change even at ordinary temperatures, particularly if water be present. It is therefore easy to understand that charcoal may in many cases be obtained through the transformation of substanoes entering into the composition of organisms, but that it is never found in a pure state.

However, water and carbonic anhydride are not the only products separated from organio substances. Carbon, hydrogen, and oxygen are capable of giving a multitude of compounds; some of these are volatile compounds, gaseous, soluble in water-they are carried off from organic matter, undergoing change without access of air. Others, on the oontrary, are non-volatile, rich in carbon, unaffected by heat and other agents. The latter remain in admixture with charcoal in the place where the decomposition takes place; such, for example, are tarry substances. The quantity of those bodies which are found mixed with the cliarcoal is very varied, and depends on the energy and duration of the decomposing agent. The annexed table shows, according to the data of Violette, those changes 
volatile substances (ash) forming a part of the organic matter, remain behind with the charcoal. The tar-like substances, which require a high temperature in order to decompose them, also remain mixed with charcoal. If a volatile organic substance, such as a gaseous compound containing oxygen and hydrogen, be taken, the carbon separates on passing the vapour through a trbe heated to a high temperature. Organic substances when burning with an insufficient supply of air give off soot-that is, charcoal-proceeding from carbon compounds in a state of vapour, the hydrogen of which has, by combustion, been converted into water; so, for instance, turpentine, naphthalene, and other hydrocarbons which are with difficulty decomposed by heat, easily yield carbon in the form of soot during combustion. Chlorine and other substances which, like oxygen, are capable of taking up hydro gen, and also substances which are capable of taking up wuter, can also separate carbon from (or char) most organic substances.

Wood charcoal is prepared in large quantities in a similar manner -that is, by the partial combustion of wood. ${ }^{4}$ In nature a similar

Which wood undergoes at various temperatures when submitted to dry distillation by' means of superheated steam:-

\begin{tabular}{|c|c|c|c|c|c|}
\hline Temperature & $\begin{array}{c}\text { Residue } \\
\text { from } 100 \text { parts } \\
\text { of alder wood }\end{array}$ & \multicolumn{4}{|c|}{ In 100 parts of the residual charcoal } \\
\hline $\begin{array}{r}150^{\circ} \\
350^{\circ} \\
1032^{\circ} \\
1500^{\circ}\end{array}$ & $\begin{array}{r}100 \cdot 0 \\
29 \cdot 7 \\
18 \cdot 7 \\
17 \cdot 3\end{array}$ & $\begin{array}{c}0 \\
47 \cdot 5 \\
76 \cdot 6 \\
81 \cdot 9 \\
95 \cdot 0\end{array}$ & $\begin{array}{l}\text { H } \\
6 \cdot 1 \\
4 \cdot 1 \\
2 \cdot 3 \\
0 \cdot 7\end{array}$ & $\begin{array}{c}O \text { and } N \\
46 \cdot 3 \\
18 \cdot 4 \\
14 \cdot 1 \\
3 \cdot 8\end{array}$ & $\begin{array}{l}\text { Ash } \\
0.1 \\
0.6 \\
1.6 \\
1.7\end{array}$ \\
\hline
\end{tabular}

4 The object of producing charcoal from wood has been explained in Note 1. Wood charcoal is obtained in so-called stacks by partially burning the wood, or by means of dry distillation (Note 1) without the access of air. It is principally manufactured for metallurgical processes, especially for smelting and forging iron. The preparation of charcoal in stacks has one advantage, and that is that it may be done on any spot in the forest. But in this way all the products of dry distillation are lost. For charcoal burning, a pile or stack is generally. built, in which the logs are placed close together, either horizontally, vertically, or inclined, forming a stack of from six to fifty feet in diameter and even larger. Under the stack are several horizontal air passages, and an opening in the middle to let out the smoke. The surface of the stack is covered with earth and sods to a considerable thickness, especially the upper part, in order to hinder the free passage of air and to concentrate the heat inside. When the stack is kindled, the pile-begins to settle down by degrees, and it is then necessary.to look after the turf casing and keep it in repair. As the combustion spreads throughout the whole pile, the temperature rises and real dry distillation commences. It is then necessary to stop the air holes, in order as much as possible to prevent unnecessary.combustion. The nature of the process is, that part of the fuel burns and develops the heat required for subjecting the remainder to dry distillation. The charring is stopped when the products of dry distillation, which are emitted, no longer burn with .2 brilliant flame, but the pale blue flame of carbonic oxide appears. Dry wood in stecks yields about oneSourth of its weight of charcoal. 
process of carbonisation of vegetable refuse takes place in its transformation under water, as shown by the marshy vegetation which forms peat. ${ }^{5}$ In this manner doubtless the enornous masses of coal were formed ${ }^{6}$ which, following the example set by England, are

8 When dead vegetable matter undergoes transformation in air, in the presence of moisture and lower organisms, there remains a substance much richer in carbon-namely, humus, black earth or mould. 100 parts of humus in a dry state contain about 70 p.c. of carbon. The roots, leaves, and stems of plants which wither and fall to the ground form a soil rich in humus. The non-vital vegetable substances (ligneous tissue) first form brown matter (ulmic compounds), and then black matter (humic substances), which are both insoluble in water; after this a brown acid is produced, which is soluble in water (apocrenic acid), and lastly a colourless acid also soluble in water (crenic acid). Alkali dis. solves a part of the original brown and black substances, forming solutions of a brown tint (ulmic and humic acids) which sometimes communicate their colour to springs and rivere. The proportion of humus in soil generally has a direct influence on its fertility; firstly, because putrefying plants develop carbonic anhydride and ammonia, and yield the substances forming the ashes of plants, which are necessary to vegetation; secondly, because humus is capable of attracting the moisture of the air and of absorbing water (twice its weight) and in this way keeps the soil in a damp condition, which is indispensable for nourishment; thirdly, humus renders the soil porous, and, fourthly, it renders it more capable of absorbing the heat of the sun's rays. On this account black earth is often most remarkable for its fertility. One object of manuring is to increase the quantity of humus in the soil, and any easily changeable vegetable or any animal matter (composts) may be used. The boundless tracts of black earth soil in Russia are capable of bestowing countless wealth on the country.

The origin and extent of black earth soil are treated in detail in Professor Dokou. chaeff's works.

If those substances which produce humus undergo decomposition nnder water, less ;carbonic anhydride is formed, a quantity of marsh gas, $\mathrm{CH}_{4}$, is evolved, and the solid residue forms an acid humus found in great quantities in marshy places and called peat. Peat is especially abundant in the lowlands of Holland, North Germany, Ireland, and Bavaria. In Russia it is likewise found in large quantities, especially in the North-West districts. The old hard forms of peat resemble in composition and pro. perties brown coal; the newest formations, as yet unhardened by pressure, form very porous masses which retain traces of the regetable matter from which they have been formed. Dried (and sometimes pressed) peat is used as fuel. The composition of peat varies considerably with the locality in which it is found. When dried in air it does not contain less than 15 p.c. of water and 8 p.c. of ash; the remainder consists of 45 p.o. of carbon, 4 p.c. of hydrogen, 1 p.c. of nitrogen, and 28 p.c. of oxygen. Its heating power is about equivalent to that of wood. The brown earthy varieties of coal were probably formed from peat. In other cases they have a marked woody structure, and are then known as lignites. The composition of the brown sorts of coal resembles in a marked degree that of peat-namely, in a dried state brown coal contains on an average 60 p.c. of carbon, 5 p.c. of hydrogen, 26 p.c. of oxygen and nitrogen, and 9 p.c. of ash. In Russia brown coal is met with in many districts near Moscow, in the Governments of Toula and Trer and the neighbonrhood; it is very usually used as fuel, particularly when found in thick seams. The brown coals usually burn with a flame like wood and poat, and are akin to them in heating power, which is half or a third that of the best coal.

- Grass and wood, the vegetation of primøval seas and similar refuse of all geological periods, must have been in many cases subjected to the same changes they now undergo-that is, under water they formed peat and lignites. Such substances, preeerved or a long time underground, subjected to the action of water, compressed by the new strata formed above them, transformed by the separation of their more volatile 


\section{now utilised everywhere as the principal material for heating steam}

component parts (peat and lignites, even in their last condition, still continue to evolve nitrogen, carbonio anhydride, and marsh gases) form coat. Coal is a dense homogeneous mass, black, with an oily or glassy lustre, or more rarely dull without any evident vegetable structure; this distinguishes it in sppearance from the majority of lignites. The density of coal (not counting the admixture of pyrites, \&c.) varies from 1.25 (dry bituminous coal) to 1.6 (anthracite, flameless), and even reaches 1.0 in the very dense variety of coal found in the Olonetzky government (termed thungite), which according to the investigations of Professor Inostrantzeff may be regarded as the extreme member of the various forms of coal.

In order to explain the formation of coal from vegetable matter, Cagniard de la Tour enclosed pieces of dried wood in a tube and heated them to the boiling point of meroury, when the wood was changed into a semi-liquid blaok mass from which a substance exceedingly like coal separated. In this manner some kinds of wood formed coal which on being heated left caking coke, others non-caking; precisely as we find with the natural varieties of coal. Violette repeated these experiments with wood dried at $150^{\circ}$, and showed that when wood is decomposed in this way, a gas, an aqueous liquor, and a residue are formed. The latter at a temperature of $200^{\circ}$ has the properties of wood charcoal incompletely burnt; at $800^{\circ}$ and higher a homogeneous mass like coal is formed which at $340^{\circ}$ is dense and without cavities. At $400^{\circ}$ the residue resembles anthracite. In nature probably the decomposition was in rare cases effected by heat alone; more generally it was effected by means of water and heat, but in either case the result ought to be almost the same.

The average composition of coal compiled from many analyses, disregarding the ash, is as follows: 84 parts of carbon, 5 parts of hydrogen, 1 part of nitrogen, 8 parts of oxygen, 2 of sulphur. The quantity of ash is on an average 5 p.c., but there are coals which contain a larger quantity, and naturally they are not so advantageons for use as fuel. The amount of water does not usually exceed more than 10 p.c. The anthracites form a remarkable variety of coals, they do not give any volatile products, or but a very small amount, as they contain but little hydrogen compared to oxygen. In the average com. position of coal we saw that for 5 parts of hydrogen there were 8 parts of oxygen; therefore 4 parts by weight of the hydrogen are capable of forming hydrocarbons, because 1 part of hydrogen is necessary in order to form water with the 8 parts of oxygen. These 4 parts by weight of hydrogen can convert 48 parts of carbon into volatile products, because 1 part of hydrogen by weight in these substances combines with 12 parts of carbon. The anthracites differ essentially from this: neglecting the ash, their average composition is as follows: 94 parts of carbon, 8 of hydrogen, and 8 of oxygen and nitrogen. According to the analyses of A. A. Voskresensky, the Grousheffsky anthracites (Don district) contains: $\mathrm{C}=93 \cdot 8 ; \mathrm{H}=1 \cdot 7, \mathrm{ash}=1 \cdot 5$. Therefore the anthracites contain but little hydrogen capable of combining with the carbon to form hydrocarbons which burn with a flame. Anthracites are the oldest forms of coal. The newest and least transformed cuals, which resemble some of the brown varieties, are the dry coals. They burn with a flame like wood, and leave a coke having the appearance of lumps of coal, half their component parts being absorbed by the flame (they contain much hydrogen and; oxygen). The remaining varieties of coal (gas coal, smithy coal, coking, and anthracite) according to. Griner in all respects form connecting links between the dry coals and the anthracites. These coals burn with a very smoky flame, and on being heated leave coke, which bears the same relation to coal that charcoal does to wood. The quantity and quality of colse vary considerably with the different sorts of coal from which it is formed. In practice coals are most often distinguished by the properties and quantity of the colke which they give. In this particular the so-called bituminous coals are especially valuable, as even the slack of this kind gives on dry distillation large spongy masses of coke. If large pieces of these kinds of coal are subjected to dry distillation, they, as it were, melt, flow together, and form caking masses of coke. The best coking coals give 65 p.c. of dense caking coke. Such copl is very valuable for metallurgical, 


\section{boilers, and in general for all purposes of heating and burning.? Russia possesses many very rich coalfields, amongst which the Donetz district is most worthy of remark. ${ }^{8}$ \\ During the imperfect combustion of volatile substances containing}

purposes (see Note 8). Besides coke, the dry distillation of coal prodnces gas (see further, illuminating gas, p. 861), coal-tar (which gires benzene, carbolic acid, naphthalene, tar for artificial asphalt, \&c.) and also an aqueous alkaline liquor (with wood and lignites the liquid is acid from acetic acid) which contains ammonium carbonate (see Note 6).

7 In England in 1850 the output of coal was as much as 48 million tons, and in latter years it has risen to about 190 millions. Besides this other countries contribute 300 millions-Russia about 6 millions. The United States of America come next to England with an output of 160 million tons, then Germany 90 millions; France produces but little (25 millions), and takes about 5 million tons from England. Thus the world consumes about 500 million tons of coal yearly. Besides household purposes, coal is chiefly used as fuel for steam-engines. As every horse-power ( $=75$ kilogrammetres per second) of a steam-engine expends on the average more than 25 kilograms in 24 hours, or in a year (counting stoppages) not less than 5 tons per horse-power, and there are not less than 40 million horse-power at work in the world, the consumption of coal for motive-power is at least equal to half the whole production. For this reason coal serves as a criterion of the industrial development of a country. About 15 p.c. of coul is used for the manufacture of cast iron, wrought iron, steel, and articles made of them.

8 The principal coal beds of Russia under exploitation are: The Don basin (150 million poods per annum, 62 poods $=1$ ton), the Polish basin (Dombrovo and others 120 million poods per annum), the Toula and Riazan beds of the Moscow basin (up to 25 million poods), the Ural basin (10 million poods), the Caucasian (Kviboul, near Kutais), the Khirjhis steppes, the smithy coal basin (Gov. of Tomsk), the Sahaline, \&c. The Polish and Moscow basins do not give any coking coals. The presence of every variety of coal (from the dry coal near Lisichansk on the Donetz to the anthracites of the entire south-east basin), the great abundance of excellent metallurgical coal (coking, see Note 6) in the western part. of the basin, its vast extent (as much as $25,000 \mathrm{sq}$. versts), the proximity of the seams to the surface (the shafts are now from 20 to 100 fathoms deep, and in England and Belgium as deep as 500 fathoms), the fertility of the soil (black earth), the proximity of the sea (about 100 versts from the Sea of Azoff) and of the rivers Donetz, Don, and Dneiper, the most abundant seams of excellent iron ore (Korsan Mogila, Krivoy Rog, Soulin, \&c., \&c.), copper ore, mercury ore (neàr Nikitovka, in the Bakhmouth district of the Ekaterinoslav Gov.), and other ores, the richest probably in the whole world, the beds of rock-salt (near the stations of the Stoupka and Brianzovka) the excellent clay of all kinds (china, fire-clay), gypsum, slate, sandstone, and other wealth of the Don coal basin, give complete assurance of the fact that with the growth of industrial activity in Russia this bountiful land of the Cossacks and New Russia will become the centre of the most extensive productive enterprise, not for the requirements of Russia alone," but of the whole world, because in no otber place can be found such a concentration of farourable conditions. The growth of enterprise and knowledge, together with the extinction of the forests which compels Russia to foster the production of coal, will help to bring about this desired result. Englaud with a whole fleet of merchant vessels exports annually about 25 million tons of coal, the price of which is higher than on the Donetz (where a pood of worked coal costs less than 5 'copecks on the average), where anthracites and semi-anthracites (like Cardiff or steam coal, which burns without smoke) and coking and metallurgical coals are able both in quantity and quality to satisfy the most fastidious requirements of the industry already existing and rapidly increasing everywhere. The coal mines of England and Belgium , are approaching a state of exhaustion, whilst in those of the Don basin, only at a depth of 100 fathoms, $1,200,000$ million poods of coal lie waiting to be worked. 
carbon and hydrogen, the hydrogen and part of the carbon first burn, and the remainder of the carbon forms soot. Tar, pitch, and similar substances for this reason burn with a smoky flame. Thus soot is finely-divided charcoal separated during the imperfect combustion of the vapours and gases of carbonaceous substances rich in carbon. Specially-prepared soot (lampblack) is very largely used as a black paint and a large quantity goes for the manufacture of printers' ink. It is prepared by burning tar, oil, natural gas, naphtha, \&c. The quantity of organic matter remaining undecomposed in the charcoal depends on the temperature to which it has been submitted. Charcoal prepared at the lowest temperature still contains a considerable quantity of hydrogen and oxygen-even as much as 4 p.c. of hydrogen and 20 p.c: of oxygen. Such charcoal still preservess the structure of the substance from which it was obtained. Ordinary charcoal, for instance, in which the structure of the tree is still visible, is of this kind. On submitting it to further heating, a fresh quantity of hydrogen with carbon and oxygen (in the form of gases or volatile matter) may be separated, and the purest charcoal will be obtained on submitting it to the greatest heat. ${ }^{9}$ If it be required to prepare pure charcoal from soot it is necessary first to wash it with alcohol and ether in order to remove the soluble tarry products, and then submit it to a powerful heat to drive off the impurities containing hydrogen and oxygen. Charcoal however when completely purified does not change in appearance. Its porosity, ${ }^{10}$ bad conducting power for heat,

$9 \mathrm{As}$ it is difficult to separate from the charcoal the admixture of ash-that is, the earthy matter contained in the vegetable substance used for producing charcual-in order to obtain it in its purest condition it is necessary to use such organic substances as do not contain any ash, for example completely refined or purified crystallised sugar, crystallised tartaric acid, \&c.

10 The cavities in charcoal are the passages through which those volatile products formed at the same time.as the charcoal have passed. The degree of porosity of charcoal varies considerably, and has a technical significance, in different kinds of charcoal. The most porous charcoal is very light; a cubio metre of wood charcoal weighs about 200 kilograms. Many of the properties of charcoal which depend exclusively on its porosity are shared by many other porous substances, and vary with the density of the charcoal and depend on the way it was prepared. The property which charcoal has of absorbing gases, liquids, and many substances in solution, is a case in point. The densest kind of charcoal is formed by the action of great heat on sugar and other fusible substances. The lustrous grey dense coke formed in gas retorts is also of this character. This dense coke collects on the internal walls of the retorts subjected to great heat, and is produced by the vapours and gases 'separated from the heated coal in the retorts. In virtue of its density such coke becomes a good conductor of the galvanic current and approaches graphite. It is principally used in galvanic batteries. Coke, or the charcoal remaining from the imperfect combustion of coal and tarry substances, is also but slightly porous, brilliant, does not soil or mark paper, is dense, almost devoid of the faculty of retaining liquids and solids, and does not absorb gases. The light sorts of charcoal produced from charred wood, on the other hand, show this absorptive power in 
capability of absorbing the luminous rays (hence its blackness and opacity), and many other qualities, are familiar from everyday ex. perience. $^{11}$ The specific gravity of charcoal varies from 1.4 to 1.9 , and that it floats on water is due to the air contained in its pores. If charcoal is reduced to a powder and moistened with spirit, it imme. diately sinks in.water. It is infusible in the furnace and even at the temperature of the oxyhydrogen flame. In the heat generated by means of a powerful galvanic.current charcoal only softens but does not completely melt, and on cooling it is found to have undergone a com. plete change both in properties and appearance, and is more or less transformed into graphite. The physical stability of charcoal is without doubt allied to its chemical stability. It is evidently a substance devoid of energy, for it is insoluble in all known liquids,

a most marked degree. This property is particularly developed in that very fine and friable charcoal prepnred by heating animal substances such as hides and bones. The absorptive power of charcoal for gases is similar to the condensation of gases in spongy platinum. Here evidently there is a case of the adherence of gases to a solid, precisely as liquids have the property of adhering to various solids. One volume of charcoal will absorb the following volumes of gases (charcoal is capable of absorbing an immense amount of chlorine, almost equal to its orwn weight) :-

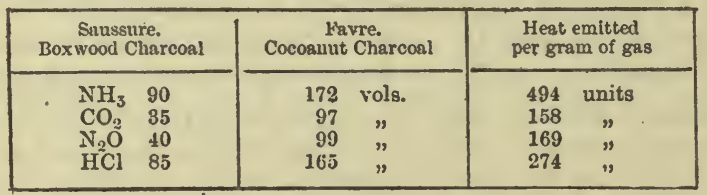

The quantity of gas absorbed by the charcoal increases with the pressure, and 10 approximately. proportional to it. The quantity of heat given out by the absorption nearly approaches that set free on dissolving, or passing into a liquid condition.

Charcoal absorbs not only gases, but a number of other substances. For instance, alcohol which contains disagreeably smelling fusel oil, on being mixed with charcoal or filtered through it, loses most of the fusel oil. The practice of filtering substances through charcoal in order to get rid of foreign matters is often applied in chemieal and manufacturing processes. Oils, spirits, various extracts, and vegetable and other solu. tions are filtered through charcoal in order to purify them. The bleaching power of charcoal may be tested by using various coloured solutions-such as aniline dyes, litmus, \&c. Charcoal, which has absorbed one substance to saturation is still capable of absorbing certain other substances. Animal charcoal, produced in a very. finely-divided state, especially by henting bones, makes the best sort for the purposes of absorption. Bone charcoal is used in large quantities in sugar works for filtering syrups and all saccharine solutions, in order to purify them, not only from colouring and odorous matter, but also from the lime which is mixed with the syrups in order to render them less unstable during boiling. The absorption of lime by animal charcoal depends, in all probability, in a great degree on the mineral component parts of bone charcoal.

11 Charcoal is a very bad conductor of heat, and therefore forms an excellent insulator or packing to prevent the transmission of heat. A charcoal lining is often used in crucibles for heating many substances, as it does not melt and resists a far greater beat than many other substances. 
and at an ordinary temperature does not combine with anytling; it is an inactive substance, like nitrogen. ${ }^{12}$ But these properties of charcoal change with a rise of temperature; thus, unlike nitrogen, charcoal, at a high temperature, combines directly with oxygen. This is well known, as charcoal burns in air. Indeed, not only does oxygen combine with charcoal at a red heat, but sulphur, hydrogen, silicon, and also iron and some other metals ${ }^{12}$ bis do so at $\{$ very high temperature-that is, when the molecules of the charcoal havereached a state of great instability - whilst at ordinary temperatures. neither oxygen, sulphur, nor metals act on charcoal in any way. When burning in oxygen, charcoal forms carbonic anhydride, $\mathrm{CO}_{2}$, whilst in the vapours of sulphur, carbon bisulphide, $\mathrm{CS}_{2}$, is formed, and wrought iron, when acted on by carbon, becomes cast iron. At the great heat obtained by passing the galvanic current through. carlon electrodes, charcoal combines with hydrogen, forming acetylene, $\mathrm{C}_{2} \mathrm{H}_{2}$. Charcoal does not combine directly with nitrogen, but in the presence of metals and alkaline oxides, nitrogen is absorbed, forming a metallic cyanide, as, for instance, potassium cyanide, KCN. From these few direct combinations which charcoal is capable of entering into, may be derived those numerous carbonaceous compounds which enter into the composition of plants and animals, and can be thus obtained artificially. Certain substances containing oxygen give up a

12 The unalterability of charcoal under the action of atmospheric agencies, which produce changes in the majority of stony and metallic substances, is often made use of in practice. For example, charcoal is frequently strewn in boundary ditches. The surface of wood is often charred to render it durable in those places where the soil is damp and wood itself would soon rot. The chambers (or in some works towers) through which acids pass (for example, sulphuric and hydrochloric) in order to bring them into contact with gases or liquids, are filled with charcoal or coke, because s.t ordinary tem. peratures it resists the action of even the strongest acids.

18 bls Maquenne (1892) discovered that carbon is capable of combining with the alkali metals. A 20 p.c. amalgam of the metals was heated to a.red heat with charcoal powder in a stream of hydrogen. The compounds so obtained possessed; after the mercury had been driven off, the compositions $\mathrm{BaC}_{2}, \mathrm{SrC}_{2}, \mathrm{CaC}_{2}$. All these compounds react with water forming acetylene, for example:

$$
\mathrm{BaC}_{2}+2 \mathrm{H}_{2} \mathrm{O}=\mathrm{C}_{2} \mathrm{H}_{2}+\mathrm{Ba}(\mathrm{OH})_{2} \text {. }
$$

Maquenne proposes the barium carbide as a source of acetylene. He obtained this compound by heating carbonate of barium, magnesium powder, and retort carbon in a Perreau furnace $\left(\mathrm{BaCO}_{3}+3 \mathrm{Mg}+\mathrm{C}=3 \mathrm{MgO}+\mathrm{BaC}_{2}\right)$. One hundred grams of $\mathrm{BaC}_{2}$ evolve 5,200 to 5,400 c.c. of acetylene, mixed with about 2-3 p.c. of hydrogen.

The relation of acetylene, $\mathrm{C}_{2} \mathrm{H}_{2}$, to these metallic carbides is evident from the fact that these metals $\left(\mathrm{Ca}, \mathrm{Sr}, \mathrm{Ba}\right.$ ) replace 2 atoms of hydrogen, and therefore $\mathrm{C}_{2} \mathrm{Ba}$ corre. sponds to $\mathrm{C}_{2} \mathrm{H}_{2}$, so that they may be regarded as metallic derivatives of acetylene. Moissan (1893) obtained similar cribides directly from the oxides by subjecting them to the action of the voltaic are, in the presence of carbon, for instance, $\mathrm{BaO}+3 \mathrm{C}=\mathrm{CO}+\mathrm{C}_{2} \mathrm{Ba}$, althongh at a furnace heat carbon has no action. on the oxides $\mathrm{CaO}, \mathrm{BaO}, \mathrm{SrO}$. $\mathrm{Con}$ cerning $\mathrm{Al}_{4} \mathrm{C}_{3}$, see Chapter XVII. Note 38. 
part of it to charcoal at a relatively low temperature. For instance, nitric acid when boiled with charcoal gives carbonic anhydride and nitric peroxide. Sulphuric acid is reduced to sulphurous anhydride when heated with carbon. When heated to redness charcoal ab. sorbs oxygen from a large number of the oxides. Even such oxides as those of sodium and potassium, when heated to redness, yield their oxygen to charcoal although they do not part with it to hydrogen. Only a few of the oxides, like silica (oxide of silicon) and lime (calcium oxide) resist the reducing action of charcoal. Charcoal is capable of changing its physical condition without undergoing any alteration in its essential chemical properties - that is, it passes into isomeric or allotropic forms. The two other particular forms in which carbon appears are the diamond and graphite. The identity of composition of these with charcoal is proved by burning an equal quantity of all three separately in oxygen (at a very high temperature), when each gives the same quantity of carbonic anhydride-namely, 12 parts of charcioal, diamond, or graphite in a pure state, yield on burning 44 parts by weight of carbonic anhydride. The physical properties present a marked contrast ; the densest sorts of charcoal have a density of only $1 \cdot 9$, whilst the density of graphite is about $2 \cdot 3$, and that of the diamond $3 \cdot 5$. A great many other properties depend on the density, for instance combustibility. The lighter charcoal is, the more easily it burns ; graphite burns with considerable difficulty even in oxygen, and the diamond burns only in oxygen and at a very high temperature. On burning, charcoal, the diamond, and graphite develop different quantities of heat, One part by weight of wood charcoal converted by burning into carbonic anhydride develops 8,080 heat units ; dense charcoal separated in gas retorts develops 8,050 heat units; natural graphite, 7,800 heat units; and the diamond 7,770. The greater the density the less the heat evolved by the combustion of the carbon. ${ }^{13}$

By means of intense heat charcoal may be transformed into graphite. If a charcoal rod $4 \mathrm{~mm}$. in cliameter and $5 \mathrm{~mm}$. long be enclosed in an exhausted receiver and the current from 600 Bunsen's elements, placed in parallel series of 100 , be passed through it, the charcoal

13 When subjected to pressure, charcoal loses heat, hence the densest form stands to the less dense as a solid to a liquid, or as a compound to an element. From this the conclusion may be drawn that the molecules of graphite are more complex than those of charcoal, and those of the diamond still more so. The specific heat shows the same variation, and as we shall see further on, the increased complexity of a molecule leads to a diminution of the specific heat. At ordinary temperatures the specific heat of charcoal is 0.24, graphite $0 \cdot 20$, the diamond $0 \cdot 147$. For retort carbon Le Chatelier (1893) found that the product of the sp. heat and atomic weight varies, between $0^{\circ}$ and $250^{\circ}$, according to the formula: $=1.92+0.0077 t$, and between $250^{\circ}$ and $1000^{\circ},=3.54+0.00246 t$ (seo Chapter XIV. Note 4). 
becomes strongly incandescent, partially volatilises, and is deposited in the form of graphite. If sugar be placed in a charcoal crucible and a powerful galvanic current passed through.$t$, it is baked into a mass similar to graphite. If charcoal be mixed with wrought iron and heated, cast iron is formed, which contains as much as five per cent. of charcoal. If molten cast iron be suddenly chilled, the carbon remains in combination with the iron, forming so called white cast iron; but if the cooling proceeds slowly, the greater part of the carbon separates in the form of graphite, and if such cast iron (so called grey cast iron) be dissolved in acid, the carbon remains in the form of graphite. Graphite is met with in nature, sometimes in the form of large compact masses, sometimes permeating rocky formations like the schists or slates, and in fact is met with in those places which, in all proba. bility, have been subjected to the action of subterranean heat. ${ }^{14}$ The graphite in cast iron, and sometimes also natural graphite, occasionally appears in a crystalline form in the shape of six-sided plates, but more often it occurs as a compact amorphous mass having the characteristic properties of the familiar black-lead pencil. ${ }^{15}$

The diamond is a crystalline and transparent form of carbon. It is

16 There are places where anthracite gradually changes into graphite as the strata sink. I myself had the opportunity of observing this gradual transformation in the valley of Aosta.

15 Pencils are made of graphite worked up into a homogeneous mass by disintegrating, powdering, and cleansing it from earthy impurities; the best kinds are made of completely homogeneous graphite sawn up into the requisite sticks. Graphite is found in many places. In Russia the so-called Aliberoffsky graphite is particularly renowned; it is found in the Altai mountains near the Chinese frontier; in many places in Finland and likewise on the banks of the Little Tungouska, Sidoroff also found a considerable quantity of graphite. When mixed with clay, graphite is used for making crucibles and pots for melting metals.

Graphite, like most forms of charcoal, still contains a certain quantity of hydrogen, oxygen, and ash, so that in its natural state it does not contain more than 98 p.c. of carbon.

In practice, graphite is purified simply by washing it when in a finely-ground state, by which means the bulk of the earthy matter may be separated. The following process, proposed by Brodie, consists in mixing the powdered graphite with $\frac{2}{14}$ part of its weight of potassium chlorate. The mixture is then heated with twice its weight of strong sulphuric acid until no more odoriferous gases are emitted; on cooling, the mixture is thrown into water and washed; the graphite is then dried and heated to a red heat; after this it shrinks considerably in volume and forms a very fine powder, which is then washed. By acting on graphite several times with a mixture of potassium chlorate and nitric acid heated up to $60^{\circ}$, Brodie transformed it into a yellow insoluble acid substance which he called graphitic acid, $\mathrm{C}_{11} \mathrm{H}_{4} \mathrm{O}_{5}$. The diamond remains unchanged when subjected to this treatment, whilst amorphous charcoal is completely oxidised. Availing himself of this possibility of distinguishing graphite from the diamond or amorphous charcoal, Berthelot showed that when compounds of carbon and hydrogen are decomposed by heat, amorphous charcoal is mainly formed, whilst when compounds of carbon with chlorine, sulphur, and boron are decomposed, graphite is principally deposited. 
bf rare occurrence in nature, and is found in the alluvial deposits of the diamond mines of Brazil, India, South Africa, de. It has also been found in meteorites. ${ }^{15}$ bis It crystallises in octahedra, dodecahedra, cabes, and other forms of the regular system. ${ }^{16}$ The efforts which have been made to produce diamonds artiticially, although they have not been fruitless, have not as yet led to the production of large-sized crystals, because those means by which crystals are generally formed are inapplicable to carbon. Indeed, carbon in all its forms being insoluble and infusible does not pass into a liquid condition by means of which crystallisation could take place. Diamonds have several times been successfully produced in the shape of minute crystals having the appearance of a black powder, but when viewed under the microscope appearing transparent, and possessing that hardness which is the peculiar characteristic of the diamond. This diamond powder is deposited on the negative electrode, when a weak galvanic current is passed through liquid chloride of carbon. ${ }^{16}$ bis

Moissan (Paris, 1893) produced diamonds artificially by means of the high temperature attained in the electrical farnace ${ }^{17}$ by dissolving

15 bis Diamonds are found in a particular dense rock, known by the name of itacolumnite, and are dug out of the debris produced by the destruction of the itacolumnite by water. When the debris is washed the diamonds remain behind; they are principally found in Brazil, in the provinces of Rio and Bahia, and at the Cape of Good Hope. The débris gives the black or amerphous diamond, carbonado, and the ordinary colourless or yellow translucent diamond. As the diamond possesses a very marked cleavage, the first operation consists in splitting it, and then roughly and finely polishing it with diamond powder. It is very remarkable that Professors P. A. Latchinoff and Eroféeff found (1887) diamond powder in a meteoric stone which fell in the Govern. ment of Penza, in the district of Krasnoslobodsk, near the settlement of Novo Urei (Sept. 10, 1886). Up to that time charcoal and graphite (a special variety, cliftonite) had been found in meteorites and the diamond only conjectured to occur therein. The Novo Urei meteorite was composed of siliceous matter and metallic iron (with nickel) like many other meteorites.

16 Diamonds are sometimes found in the shape of small balls, and in that case it is Impossible to cut them because directly the surface is ground or broken they fall into minute pieces. Sometimes minute diamond crystals form a dense mass like sugar, and this is generally reduced to diamond powder and used for grinding. Some known varieties of the diamond are almost opaque and of a black colour. Such diamonds are as hard as the ordinary ones, and are used for polishing diamonds and other precious stones, and also for rock boring and tunnelling.

16 bls Hannay, in 1880, obtained diamonds by heating a mixture of heavy liquid bydrocarbons (paraffin oils) with magnesium in a thick iron tube. This investigation, however, was not repeated.

17 The electrical furnace is an invention of recent times, and gives the possibility of obtaining a temperature of $3,500^{\circ}$, which is not only not obtainable in ordinary furnaces, but even in the oxyhydrogen flame, whose temperature does not exceed $2,000^{\circ}$. The electrical furnace consists of two pieces of lime, laid one on the other. A cavity is made in the lower piece for the reception of the substance to be melted between two thick electrodes of dense carbon. On passing a current of 70 volts and. 450 amperres a tem. perature of $8,000^{\circ}$ is easily obtained. At a temperaţure of $2,509^{\circ}$ (100 amperres and 40 
carbon in molten cast iron, and allowing the solution with an excess of carbon, to cool under the powerful pressure exerted by rapidly cooling the metal. ${ }^{17}$ bis $K$. Chroustchoff attained the same end by means of silver, which dissolves carbon to the extent of 6 p.c.

volts) not only do all metals melt, but even lime and magnesia (when placed in the space between the carbon electrodes, i.e. in the voltaio arc) become soft and crystallise on cooling. At $3,000^{\circ}$ lime becomes very fluid, metallio calcium partially separates out and a carbon compound, which remains liquid for a long time. At this tem. perature oxide of uranium is reduced to the snboxide and metal, zirconia and rock crystal fuse and partially volatilise, as also does alumina; platinum, gold, and even carbon distinctly volatilise; the majority of the metals form carbides. At such a tem. perature also cast iron and carbon give graphite, while according to Rousseau, between $2,000^{\circ}$ and $8,000^{\circ}$ the diamond passes into graphite and conversely graphite into the diamond, so that this is a kind of reversible reaction.

$17 \mathrm{bls}$ Moissan first investigated the solution of carbon in molten metals (and the formation of the carbides) such as magnesium, aluminium, iron, manganese, chromium, uranium, silver, platinum, and silicon. At the same time Friedel, owing to the discovery of the diamond in meteoric iron, admitted that the formation of the diamond is dependent upon the influence of iron and sulphur. With this object, that is to obtain the diamond, Friedel caused sulphur to react upon samples of cast iron rich in carbon, in a closed vessel at a maximum temperature of $500^{\circ}$, and after dissolving the sulphide of iron formed, he obtained a small quantity of a black powder which scratched corundum, i.e. diamond. Moissan's experiments (1893) were more successful, probably owing to hishaving employed the electrical furnace. If iron be saturated with carbon at a temperature between $1,100^{\circ}$ and $3,000^{\circ}$, then at $1,100^{\circ}-1,200^{\circ}$ a mixture of amorphous. carbon and graphite is formed, while at $3,000^{\circ}$ graphite alone is obtained in very. beautiful crystals. Thus under these conditions the diamond is not formed, and it can: only be obtained if the high temperature be aided by powerful pressures. For this purpose Moissan took advantage of the pressure produced in the passage of a mass of molten cast iron from a liquid into a solid state. He first melted 150-200 grams of iron in the electrical furnnce, and quickly introduced a cylinder of carbon into the molten iron. He then removed the crucible with the molten iron from the furnace and plunged it into $\Omega$ reservoir containing water. After treating with boiling hydrochloric acid, three varieties of carbon were obtained: (1) a small amount of graphite (if the cooling be rapid); (2) carbon of a chestnut colour in very flne twisted threads, showing that it had been subjected to a very high pressure 'a similar variety was met with in various samples of the Canon Diabolo), and lastly (3) an inconsiderable quantity of an exceeding dense mass which was freed from the admixture of the lighter modifications by treatment with aqua regia, sulphuric and hydrofluoric acids, and from which Moissan, by means of liquid bromoform (sp. gr. 2.900), succeeded in separating some small pieces, having a greater density than bromoform, which scratched the ruby and had the properties of the diamond. Some of these pieces were black, others were transparent and refracted light strongly. The dark grey tint of the former resembled that of the black diamonds (carbonado). Their density was between 3 and $3 \cdot 5$. The transparent specimens had a greasy appearance and seemed to be, as it were, surrounded by an envelope of carbon. At $1,050^{\circ}$ they did not burn entirely in a current of air, so that the imperfectly burnt particles, and a peculiar form of grains of a light ochre colour, which retained their crystalline form, could be examined under the microscope. Similar grains also remain after the imperfect combustion of the ordinary diamond. Moissan obtained the same results by rapidly cooling in a stream of coal gas a piece of cast iron, saturated with carbon obtained from sugar and first heated to $2,000^{\circ}$ In this instance he obtained small crystals of diamonds. K. Chroustchoff showed that at its boiling point silver dissolves 6 p.c. of carbon. This silver was rapidly cooled, so that a crust 
at a high temperature. Rousseau, for the same purpose, heated carbide of calcium in the electric furnace. There is no doubt that all these investigators obtained the diamond as a transparent body, which burnt into $\mathrm{CO}_{2}$, and possessed an exceptional hardness, but ouly in the form of a fine powder

Judging from the fact that carbon forms a number of gaseous bodies (carbonic oxide, carbonic anhydride, methane, ethylene, acetylene, \&c.) and volatile substances (for example; many hydrocarbons and their most simple derivatives), and considering that the atomic weight of carbon, $\mathrm{C}=12$, approaches that of nitrogen, $\mathrm{N}=14$, and that of oxygen, $\mathrm{O}=16$, and that the compounds $\mathrm{CO}$ (carbonic oxide) and $\mathrm{N}_{2} \mathrm{O}_{2}$ (cyanogen) are gases, it may be argued that if carbon formed the molecule $\mathrm{C}_{2}$, like $\mathrm{N}_{2}$ and $\mathrm{O}_{2}$, it would be a gas. And asithrough polymerism or the combination of like molecules (as $\mathrm{O}_{2}$ passes into $\mathrm{O}_{3}$ or $\mathrm{NO}_{2}$ into $\mathrm{N}_{2} \mathrm{O}_{4}$ ) the temperatures of ebullition and fusion rise (which is particularly clearly proved with the hydrocarbons of the $\mathrm{C}_{n} \mathrm{H}_{2 n}$ series), it ought to be con. sidered that the molecules of charcoal, graphite, and the diamond are very complex, seeing that they are insoluble, non-volatile, and infusible. The aptitude which the atoms of carbon show for combining together and forming complex molecules appears in all carbon compounds. Among the volatile compounds of carbon many are well known the molecules of which contain $\mathrm{C}_{5} \ldots \mathrm{C}_{10} \ldots \mathrm{C}_{20} \ldots \mathrm{C}_{30}$, \&c., in general $\mathrm{C}_{n}$, where $n$ may be very large, and in none of the other elements is this faculty of complexity so developed as in carhon. ${ }^{18} \mathrm{Up}$ to the present time there are no grounds for determining the degree of polymerism of the charcoal, graphite, or diamond molecules, and it can only be supposed that they contain $\mathrm{C}_{n}$ where $n$ is a large quantity. Charcoal and those complex non-volatile organic substances which represent the gradual transitions to charcoal ${ }^{19}$ and form the principal

formed on the surface and prevented the metal expanding, and so produced a powerful pressure. A portion of the carbon which separates ont under these. conditions exhibits the properties of the diamond.

18 The existence of a molecule $\mathrm{S}_{6}$ is known (up to $600^{\circ}$ ), and it must be held that this accounts for the formation of hydrogen persulphide, $\mathrm{H}_{2} \mathrm{~S}_{5}$. Phosphorus appears in the molecule $\mathrm{P}_{4}$ and gives $\mathrm{P}_{4} \mathrm{H}_{2}$. When expounding the data on specific heat we shall have occasion to return to the question of the complexity of the carbon molecule.

19 The hydrocarbons poor "in hydrogen and contrining many atoms of carbon, like chrysene and carbopetrocene, \&c., $\mathrm{C}_{n} \mathrm{H}_{2(n-m)}$, are solids, and less fusible as $n$ and $m$ increase. They present a marked approach to the properties of the diamond. And in proportion to the diminution of the water in the carbohydrates $\dot{\mathrm{C}}_{n} \mathrm{H}_{2 m} \mathrm{O}_{m}$-for example in the humic compounds (Note 5)-the transition of complex organic substances to charcoal is very evident. That residue resembling charcoal and graphite which is obtained by the separation (by means of copper sulphate and sodium chloride) of iron from white cast-iron containing carbon chemically combined with the iron, also seems, especially after the rescarches of G. A. Zaboudsky, to be a complex substance containing 
solid substances of organisms, contain a store or accumulation of internal power in the form of the energy binding the atoms into complex molecules. When charcoal or complex compounds of carbon burn, the energy of the carbon and oxygen is turned into heat, and this fact is taken advantage of at every turn for the generation of heat from fuel. ${ }^{20}$

No other two elements are capable of combining together in such variety as carbon and hydrogen. The hydrocarbons of the $\mathrm{C}_{n} \mathrm{H}_{2 m}$ series in many cases differ widely from each other, although they have some properties in common. All hydrocarbons, whether gaseous; liquid or solid, are combustible substances sparingly soluble or insoluble in water. The liquefied gaseous hydrocarbons, as well as those which are liquid at ordinary temperatures, and those solid hydrocarbons which have been liquefied by fusion, have the appearance and property of oily liquors, more or less viscid, or fluid. ${ }^{21}$ The solid hydrocarbons more or less resemble wax in their properties, although ordinary oils

$\mathrm{C}_{12} \mathrm{H}_{6} \mathrm{O}_{3}$. The endeavours which have been directed towards determining the measure of complexity of the molecules of charcoal, graphite, and the diamond will probably at some period lead to the solution of this problem and will most likely prove that the various forms of charcoal, graphite, and the diamond contain molecules of different and very considerable cornplexity. The constancy of the grouping of benzene, $\mathrm{C}_{6} \mathrm{H}_{6}$, and the wide diffusion and facility of formation of the carbohydrates containing $\mathrm{C}_{6}$ (for example, cellulose, $\mathrm{C}_{6} \mathrm{H}_{10} \mathrm{O}_{5}$, glucose, $\mathrm{C}_{6} \dot{\mathrm{H}}_{12} \mathrm{O}_{6}$ ) give reason for thinking that the group $\mathrm{C}_{6}$ is the first and simplest of those possible to free carbon, and it may be hoped that some time or other it may be possible to get carbon in this form. Perhaps in the diamond there may be found such a relation between the atoms as in the benzene group, and in charcoal such as in carbohydrates.

20 When charcoal burns, the complex molecule $\mathrm{C}_{n}$ is resolved into the simple molecules $n \mathrm{CO}_{2}$, and therefore part of the heat-probably no small amount-is expended in the destruction of the complex molecule $\mathbf{C}_{n}$. Perhaps by burning the most complex substances, which are the poorest as regards hydrogen, it may be possible to form an idea of the work required to split up $\mathrm{C}_{n}$ into separate atoms.

21 The viscosity, or degree of mobility, of liquids is determined by their internal friction. It is estimated by passing the liquids through narrow (capillary) tubes, the mobile liquids passing through with greater facility and speed than the viscid ones. The viscosity varies with the temperature and nature of the liquids, and in the case of solu. tions changes with the amount of the substance dissolved, but is not proportional to it. So that, for example, with alcohol at $20^{\circ}$ the viscosity will be 69 , and for a $5 v$ p.c. solution 160, the viscosity of water being taken as 100. The volume of the liquid which passes through by experiment (Poiseuille) and theory (Stokes) is proportional to the time, the pressure, and the fourth power of the diameter of the (capillary) tube, and inversely proportional to the length of the tube; this renders it possible to form com. parative estimates of the coefficients of internal friction and viscosity.

As the complexity of the molecules of hydrocarbons and their derivatives increases by the addition of carbon (or $\mathrm{CH}_{2}$ ), so does the degree of viscosity also rise. The exten. sive series of investigations referring to this subject still await the necessary generalisa tion. That connection which (already partly observed) ought to exist between the viscosity and the other physical and chemical properties, forces us to conclude that the magnitude of internal friction plays an important part in molecular mechanics. In investigating organic compounds and solutions, similar researches ought to stand foremost. Many observations have already been made, but not much has yet been done 
and wax generally contain oxygen in addition to carbon and hydrogen, but in relatively small proportion. There are also many hydrocarbons which have the appearance of tar-as, for instance; metacinnamene and gulta-percha. Those liquid hydrocarbons which boil at a high temperature are like oils, and those which have a low boiling point resemble ether, whilst the gaseous hydrocarbons in many of their properties are akin to hydrogen. All this tends to show that in hydrocarbons physically considered the properties of solid non-volatile charcoal are strongly modified and hidden, whilst those of the hydrogen predominate. All hydrocarbons are neutral substances (neither basic nor acid), but under certain conditions they enter into peculiar reactions. It has been seen in those hydrogen compounds which have been already considered (water, nitric acid, ammonia) that the hydrogen in almost all cases enters into reaction, being displaced by metals. The hydrogen of the hydrocarbons, it may be said, has no metallic character-that is to say, it is not directly ${ }^{22}$ displaced by metals, even by such as sodium and potassium. On the application of more or less heat all hydrocarbons decompose ${ }^{23}$ forming charcoal and hydrogen. The majority of hydrocarbons do not combine with the oxygen of the air or oxidise at ordinary temperatures, but under the action of nitric acid and many other oxidising substances most of them undergo oxidation, in which either a portion of the hydrogen and carbon is separated, or the oxygen enters into combination, or else the elements of hydrogen peroxide enter into combination with the hydrocarbon. ${ }^{24}$ When heated in air, hydro-

with them; the bare facts and some mechanical data exist, but their relation to molecular mechanics has not been cleared up in the requisite degree. It has already been seen from existing data that the viscosity at the temperature of the absolute boiling point becomes as small as in gases.

${ }^{23}$ In a number of hydrocarbons and their derivatives such a substitution of metals for the hydrogen may be attained by indirect means. The property shown by acetylene, $\mathrm{C}_{2} \mathrm{H}_{2}$, and its analogues, of forming metallic derivatives is in this respect particularly characteristic. Judging from the fact that carbon is an acid element (that is, gives an acid anhydride with oxygen), though comparatively slightly acid (for carbonic acid is not at all a strong acid and compounds of chlorine and carbon, even $\mathrm{CCl}_{4}$, are not decom. posed by water as is the case with phosphorus chloride and even silicic chloride and boric chloride, although they correspond with acids of but little energy), one might expect to find in the hydrogen of hydrocarbons this faculty for being substituted by metals. The metallic compounds which correspond with hydrocarbons are known under the name of organo-metallic compounds. Such, for instance, is zino ethyl, $\mathrm{Zn}\left(\mathrm{C}_{2} \mathrm{H}_{5}\right)_{2}$, which corresponds with ethyl hydride or ethane, $\mathrm{C}_{2} \mathrm{H}_{6}$, in which two atoms of hydrogen have been exchanged for one of zinc.

${ }_{23}$ Gaseous and volatile hydrocarbons decompose when passed through a heated tube. When hydrocarbons are decomposed by heating, the primary products are generally other more stable hydrocarbons, among which are acetylene, $\mathrm{C}_{2} \mathrm{H}_{2}$, benzene, $\mathrm{C}_{6} \mathrm{H}_{6}$, naphthalene, $\mathrm{C}_{10} \mathrm{H}_{8}$, \&c.

24 Wagner (1888) showed that when unsaturated bydrocarbons are shaken with a 
carbons burn, and, according to the amount of carbon they contain, their combustion is attended more or less with a separation of sootthat is, finely divided charcoal-which imparts great brilliancy to the flame, and on this account many of them are used for the purposes of illumination-as, for instance, kerosene, coal gas, oil of turpentine. As hydrocarbons contain reducing elements (that is, those capable of combining with oxygen), they often act as reducing agents-as, for instance, when heated with oxide of copper, they burn, forming carbonic anhydride and water, and leave metallic copper. Gerhardt proved that all hydrocarbons contain an even number of hydrogen atoms. Therefore, the general formula for all hydrocarbons is $\mathbf{C}_{n} \mathbf{H}_{2 m}$ where $n$ and $m$ are whole numbers. This fact is known as the law of even numbers. Hence, the simplest possible hydrocarbons ought to be: $\mathrm{CH}_{2}, \mathrm{CH}_{4}, \mathrm{CH}_{6} \ldots \mathrm{C}_{2} \mathrm{H}_{2}, \mathrm{C}_{2} \mathrm{H}_{4}, \mathrm{C}_{2} \mathrm{H}_{6}, \mathrm{C}_{2} \mathrm{H}_{8} \ldots$ but they do not all exist, since the quantity of $\mathrm{H}$ which can combine with a certain amount of carbon is limited, as we shall learn directly.

Some of the hydrocarbons are capable of combination, whilst others do not show that power. Those which contain less hydrogen belong to the former category, and those which, for a given quantity of carbon, contain the maximum amount of hydrogen, belong to the latter. The composition of those last mentioned is expressed by the general formula $\mathrm{C}_{n} \mathrm{H}_{2 n+2}$. These so-called saturated hydrocarbons are incapable of combination. ${ }^{25}$ The hydrocarbons $\mathrm{CH}_{6}, \mathrm{C}_{2} \mathrm{H}_{8}, \mathrm{C}_{3} \mathrm{H}_{10}$, \&c. . . do not exist. Those containing the maximum amount of hydrogen will be represented by $\mathrm{CH}_{4}(n=1,2 n+2=4), \mathrm{C}_{2} \mathrm{H}_{6}(u=2), \mathrm{C}_{3} \mathrm{H}_{8}(n=3)$, $\mathrm{C}_{4} \mathrm{H}_{10}$, \&c. This may be termed the law of limits. Placing this in juxtaposition with the law of even numbers, it is easy to perceive that the possible hydrocarbons can be ranged in series, the terms of which may be expressed by the general formulæ $\mathrm{C}_{n} \mathrm{H}_{2 n+2}, \mathrm{C}_{n} \mathrm{H}_{2 n}, \mathrm{C}_{n} \mathrm{H}_{2 n-2}$, \&c. . . Those hydrocarbons which belong to any one of the series

weak (1 p.c.) solution of potassium permanganate, $\mathrm{KMnO}_{4}$, at ordinary temperatures, they form glycols-for example, $\mathrm{C}_{2} \mathrm{H}_{4}$ yields $\mathrm{C}_{2} \mathrm{H}_{6} \mathrm{O}_{2}$.

${ }_{25} \mathrm{My}$ article on this subject appeared in the Jaurnal of the St. Petersburg Academy of Sciences in 1861. Up to that time, although many additive combinations with hydro. carbons and their derivatives were known, they had not been generalised, and were even. continually quoted as cases of substitution. Thus the combination of ethylene, $\mathrm{C}_{2} \mathrm{H}_{4}$, with chlorine, $\mathrm{Cl}_{2}$, was often regarded as a formation of the products of the substitution of $\mathrm{C}_{2} \mathrm{H}_{3} \mathrm{Cl}$ and $\mathrm{HCl}$, which it was supposed were held together as the water of crystallisation is in salts. Even esrlier than this (1857, Journal of the Petroffsky Academy) I considered similar cases as true compounds. In general, according to the law of limits, an unsaturated hydrocarbon, or its derivative, on combining with $r \mathrm{X}_{2}$, gives a substance which is saturated or else approaching the limit. The investigations of Frankland with many organo-metallic compounds clearly showed the limit in the case of metallio compounds, which we shall constantly refer to later on. 
expressible by a general formula are said to be homologous with one another. Thus, the hydrocarbons $\mathrm{CH}_{4}, \mathrm{C}_{2} \mathrm{H}_{6}, \mathrm{C}_{3} \mathrm{H}_{8}, \mathrm{C}_{4} \mathrm{H}_{10}$, drc. . . are members of the limiting (saturated) homologous series $\mathrm{C}_{n} \mathrm{H}_{2 n+2}$. That is, the difference between the members of the series is $\mathrm{CH}_{2}{ }^{26}$ Not only the composition but also the properties of the members of a. series tend to classification in one group. For instance, the members of the series $\mathrm{C}_{k} \mathrm{H}_{2 n+2}$ are not capable of forming additive compounds, whilst those of the series $\mathrm{C}_{n} \mathrm{H}_{2 n}$ are capable of combining with chlorine, sulphuric anhydride, \&c. ; and the members of the $\mathrm{C}_{n} \mathrm{H}_{2 n-6}$ group, belonging to the coal tar series, are easily nitrated (give nitro-compounds, Chapter VI.), and have other properties in common. The physical properties of the members of a given homologous series vary in some such manner as this; the boiling point generally rises and the internal friction increases as $n$ increases ${ }^{27}$-that is, with an increase in the relative amount of carbon and the atomic weight; the specific gravity also regularly changes as $n$ becomes greater. ${ }^{28}$

Many of the hydrocarbons met with in nature are the products of organisms, and do not belong to the mineral kingdom. A still greater number are produced artificially. These are formed by what is termed

26 The conception of homology has been applied by Gerhardt to all organic compounds in his classical work, 'Traité de Chimie Organique,' finished in 1855 (4 vols.), in which he divided all organic compounds into fatty and aromatic, which is in principle still adhered to at the present time, although the latter are more often called benzene derivatives, on account of the fact that Kelsule, in his beautiful investigations on the structure of aromatic compounds, showed the presence in them all of the 'benzene nucleus,' $\mathrm{C}_{6} \mathrm{H}_{6}$.

27 This is always true for hydrocarbons, but for derivatives of thelower homologues the law is sometimes different; for instance, in the series of saturated alcohols, $\mathrm{C}_{n} \mathrm{H}_{2 n+1}(\mathrm{OH})$, when $n=0$, we obtain water, $\mathrm{H}(\mathrm{OH})$, which boils at $100^{\circ}$, and whose specific gravity ut $15^{\circ}=0.9992$; when $n=1$, wood spirit $\mathrm{CH}_{3}(\mathrm{OH})$, which boils at $66^{\circ}$, and at $15^{\circ}$ has a specific gravity $=0.7964$; when $n=2$, ordinary alcohol, $\mathrm{C}_{2} \mathrm{H}_{5}(\mathrm{OH})$, boiling at $78^{\circ}$, specific gravity at $15^{\circ}=0.7936$, and with further increase of $\mathrm{CH}_{2}$ the specific gravity increases. For the glycols $\mathrm{C}_{n} \mathrm{H}_{2 n}(\mathrm{OH})_{2}$ the phenomenon of a similar kind 'is still more striking: at first the temperature of the boiling point and the density increase, and then for higher (more complex) members of the series diminish. The reason for this phenomenon, it is evident, must be sought for in the influence and properties of water, and that strong affinity which, acting between hydrogen and oxygen, determines many of the exceptional properties of water (Chapter I.).

${ }_{28}$ As. for example, in the saturated series of hydrocarbons $\mathrm{C}_{n} \mathrm{H}_{2 n+2}$, the lowest member $(n=0)$ must be taken as hydrogen $\mathrm{H}_{2}$, a gas which (t.c. below $-190^{\circ}$ ) is liquefied with great difficulty, and when in a liquid state has doubtless a very small density. Where $n=1,2,3$, the hydrocarbons $\mathrm{CH}_{4}, \mathrm{C}_{2} \mathrm{H}_{6}, \mathrm{C}_{3} \mathrm{H}_{8}$ are gases, more and more readily liquefiable. The temperature of the absolute boiling point for $\mathrm{CH}_{4}=-100^{\circ}$, and for ethane $\mathrm{C}_{2} \mathrm{H}_{6}$, and in the higher members it rises. The hydrocarbon $\mathrm{C}_{4} \mathrm{H}_{10}$, liquefies at about $0^{\circ} . \mathrm{C}_{5} \mathrm{H}_{12}$ (there are several isomers) boils at from $+9^{\circ}$ (Lvoff) to $37^{\circ}, \mathrm{C}_{6} \mathrm{H}_{14}$ from $58^{\circ}$ to $78^{\circ}, \& \mathrm{c}$. The specifio gravities in a liquid state at $15^{\circ}$ are :-

$$
\begin{array}{ccccc}
\mathrm{C}_{5} \mathrm{H}_{12} & \mathrm{C}_{6} \mathrm{H}_{14} & \mathrm{C}_{7} \mathrm{H}_{16} & \mathrm{C}_{10} \mathrm{H}_{22} & \mathrm{C}_{16} \mathrm{H}_{54} \\
0.63 & 0.66 & 0.70 & 0.75 & 0.85
\end{array}
$$


the combination of residues. For instance, if a mixture of the vapours of hydrogen sulphide and carbon bisulphide be passed through a tube in which copper is heated, this latter absorbs the sulphur from both the compounds, and the liberated carbon and hydrogen combine to form a hydrocarbon, methane. If carbon be combined with any metal and this compound $\mathrm{MC}_{n}$ be treated, with an acid $\mathrm{HX}$, then the haloid $\mathrm{X}$ will give a salt with the metal and the residual carbon and hydrogen will give a hydrocarbon. Thus cast iron which contains a compound of iron and carbon gives liquid hydrocarbons like naphtha under the action of acids. If a mixture of bromo-benzene, $\mathrm{C}_{6} \mathrm{H}_{5} \mathrm{Br}$, and ethyl bromide, $\mathrm{C}_{2} \mathrm{H}_{5} \mathrm{Br}$, be heated with metallic sodium, the sodium combines with the bromine of both compounds, forming sodium bromide, $\mathrm{NaBr}$. From the first combination the group $\mathrm{C}_{6} \mathrm{H}_{5}$ remains, and from the second $\mathrm{C}_{2} \mathrm{H}_{5}$. Having an odd number of hydrogen atoms, they, in virtue of the law of even numbers, cannot exist alone, and therefore combine together forming the compound $\mathrm{C}_{6} \mathrm{H}_{5} \cdot \mathrm{C}_{2} \mathrm{H}_{5}$ or $\mathrm{C}_{8} \mathrm{H}_{10}$ (ethylbenzene). Hydrocarbons are also produced by the breaking up of more complex organic or hydrocarbon compounds, especially by heating-that is, by dry distillation. For instance, gum-benzoin contains an acid called benzoic acid, $\mathrm{C}_{7} \mathrm{H}_{6} \mathrm{O}_{2}$, the vapours of which, when passed through a heated tube, split up into carbonic anhydride, $\mathrm{CO}_{2}$, and benzene, $\mathrm{C}_{6} \mathrm{H}_{6}$. Carbon and hydrogen only unite directly in one ratio of combination-namely, to form acetylene, having the composition $\mathrm{C}_{2} \mathrm{H}_{2}$, which, as compared with other hydrocarbons, exhibits a very great stability at a somewhat high temperature. ${ }^{29}$

29. If, at the ordinary temperature (assuming therefore that the water formed will be in a liquid state) a gram molecule ( $26 \mathrm{grams}$ ) of acetylene, $\mathrm{C}_{2} \mathrm{H}_{2}$, be burnt, 810 thousand calories will be emitted (Thomsen), and as 12 grams of charcoal produce 97 thousand calories, and 2 grams of hydrogen 69 thousand calories, it follows that, if the hydrogen and carbon of the acetylene were burnt there would be only $2 \times 97+69$, or 263 thousand calories produced. It is evident, then, that acetylene in its formation absorbs $310-263$, or 47 thousand calories.

For considerations relative to the combustion of carbon compounds, we will first enumerate the quantity of heat separated by the combustion of definite chemical carbon compounds, and then give a few figures bearing on the kinds of fuel used in practice.

For molecular quantities in perfect combustion the following amounts of heat are given out (when gaseous carbonic anhydride and liquid water are formed), according to Thomsen's data (1) for gaseous $\mathrm{C}_{n} \mathrm{H}_{2 n+2}: 52 \cdot 8+158 \cdot 8 n$ thousand calories; (2) for $\mathrm{C}_{n} \mathrm{H}_{2 n}: 17 \cdot 7+158 \cdot 1 n$ thousand calories; (3) according to Stohmann (1888) for liquid saturated alcohols, $\mathrm{C}_{n} \mathrm{H}_{2 n+2} \mathrm{O}: 11 \cdot 8+156 \cdot 3 n$, and as the latent heat of evaporation $=$ about $8 \cdot 2+0.6 n$, in a gaseous state, $20.0+156.9 n$; (4) for monobasic saturated liquid acids, $\mathrm{C}_{n} \mathrm{H}_{2 n} \mathrm{O}_{2}:-95 \cdot 3+154 \cdot 3 n$, and as their latent heat of evaporation is about $5 \cdot 0+1 \cdot 2 n$, in a gaseous form, about-90+155n; (5) for solid saturated bibasic acids, $\mathrm{C}_{n} \mathrm{H}_{2 n-2} \mathrm{O}_{4}$ : $-253.8+152 \cdot 6 n$, if theyrare expressed as $\mathrm{C}_{n} \mathrm{H}_{2 n} \mathrm{C}_{2} \mathrm{H}_{2} \mathrm{O}_{4}$, then $51.4+152.6 n$; (6) for benzene and its liquid homologues (still according to Stohmann) $\mathrm{C}_{n} \mathrm{H}_{2 n-6}:-158 \cdot 6+156 \cdot 3 n_{2}$ and in a gaseous form about-155+157n; $(7)$ for the gaseous homologues of acetylene, $\mathrm{C}_{n} \mathrm{H}_{2 n-2}$ (áccording to Thom sen) $-5+157 n$. It is evident from the preceding figures 
There is one substance known among the saturated hydrocarbons composed of 1 atom of carbon and 4 atoms of hydrogen; this is a compound containing the highest percentage of hydrogen $\left(\mathrm{CH}_{4}\right.$ contains 25 per cent. of hydrogen), and at the same time it is the only hydro. carbon whose molecule contains but a single atom of carbon. This saturated hydrocarbon, $\mathrm{CH}_{4}$, is called marsh gas or methane. If vegetable or animal refuse suffers decomposition in a space where the air has not free access, or no access at all, then the decomposition is accompanied with the formation of marsh gas, and this either at the ordinary temperature, or at a comparatively much higher one. On this account plants, when decomposing under water in marshes, give out this gas. ${ }^{29}$ bis It is well known that if the mud in bogs be stirred up, the act is accompanied with the evolution of a large quantity of gas bubbles, these may, although slowly, also separate of their own

that the group $\mathrm{CH}_{2}$, or $\mathrm{CH}_{3}$ substituted for $\mathrm{H}$, on burning gives out from 152 to 159 thousand calories. This is less than that given out by $\mathrm{C}+\mathrm{H}_{2}$, which is $97+69$ or 166 thousand; the reason for this difference (it would be still greater if carbon were gaseous) is the amount of heat-separated during the formation of $\mathrm{CH}_{2}$. According to Stohmann, for dextroglucose, $\mathrm{C}_{6} \mathrm{H}_{12} \mathrm{O}_{e}$, it is 673.7 ; for common sugar, $\mathrm{C}_{12} \mathrm{H}_{22} \mathrm{O}_{11}, 1325.7$; for cellulose, $\mathrm{C}_{6} \mathrm{H}_{10} \mathrm{O}_{3}, 678.0$; starch, $677 \cdot 5$; dextrin, $666 \cdot 2$; glycol, $\mathrm{C}_{2} \mathrm{H}_{6} \mathrm{O}_{2}, 281 \cdot 7$; glycerine, $397 \cdot 2$, \&c. The heat of combustion of the following solids (determined by Stohmann) is expressed per unit of weight : naphthalene, $\mathrm{C}_{10} \mathrm{H}_{8}, 9,621$; urea, $\mathrm{CN}_{2} \mathrm{H}_{4} \mathrm{O}, 2,465$; white of egg, 5,579; dry rye bread, 4,421; wheaten bread, 4,302; tallow, 9,365; butter, 9,192 ; linseed oil, 9,823. The most complete collection of arithmetical data for the heats of combustion will be found in V. F. Longinin's work, 'Description of the Various Methods of Determining the Heats of Combustion of Organic Compounds' (Moscow, 1894).

The number of units of heat given out by unit weight during the complete combustion and cooling of the following ordinary kinds of fuel in their usual state of dryness and purity are:-(1) for wood charcoal, anthracite, semi-anthracite, bituminous coal and coke, from 7,200 to 8,200 ; (2) dry, long flaming coals, and the best brown coals, from 6,200 to 6,800 ; (3) perfectly dry wood, 3,500 ; hardly dry, 2,500 ; (4) perfectly dry peat, best kind, 4,500 ; compressed and dried, 8,000 ; (5) petroleum refuse and similar liquid hydrocarbons, about 11,000 ; (6) illuminating gas of the ordinary composition (about 45 vols. $\mathrm{H}, 40$ vols. $\mathrm{CH}_{4}, 5$ vols. $\mathrm{CO}$, and 5 vols. N), about 12,000 ; (7) producer gas (see next Chapter), containing 2 vols. carbonio anhydride, 30 vols. carbonic oxide, and 68 vols. nitrogen for one part by weight of the whole carbon burnt, 5,300 , and for one part by weight of the gas, 910 , units of heat; and (8) water gas (see next chapter) containing 4 vols. carbonic anhydride, 8 vols. $\mathrm{N}_{2}, 24$ vols. carbonio oxide, and 46 vols. $\mathrm{H}_{2}$, for one part by weight of the carbon consumed in the generator 10,900 , and for one part by weight of the gas, 3,600 units of heat. In these figures, as in all calorimetric observations, the water produced by the combustion of the fuel is supposed to be liquid. As regards the temperature reached by the fuel, it is important to remark that for solid fuel it is indispensable to admit (to ensure complete combustion) twice the amount of air required, but liquid, or pulverised fuel, and especially gaseous fuel, does not require an excess of air; therefore, a kilogram of charcoal, giving 8,000 units of heat, requires about 24 kilograms of air (3 kilograms of air per thousand calories) and a kilogram of producer gas requires only 0.77 kilogram of air (0.85 kilo. of air per 1,000 calories), 1 kilogram of water gas about $4 \cdot 5$ of air (1.25 kilo. of air per 1,000 calories). CH.

99 bis Manure which decomposes under the action of bacteria gives off $\mathrm{CO}_{2}$ and 
accord. The gas which is evolved consists principally of marsh gas. ${ }^{30}$ If wood, coal, or many other vegetable or animal substances are decom. posed by the action of heat without access of air-that is, are subjected to dry distillation-they, in addition to many other gaseous products of decomposition (carbonic anhydride, hydrogen, and various other substances), evolve a great deal of methane. Generally the gas which is used for lighting purposes is obtained by this means and therefore always contains marsh gas, mixed with dry hydrogen and other vapours and gases, although it is subsequently purified from many of them. ${ }^{31}$

30 It is easy to collect the gas which is erolved in marshy places if a glass bottle be inverted in the water and a funnel put into it (both filled with water); if the mud of the bottom be now agitated, the bubbles which rise may be easily caught by the inverted funnel.

31 Illuminating gas is generally prepared by heating gas coal (see Note 6) in oval cylindrical horizontal cast-iron or clay retorts. Several such retorts $B B$ (fig. 58) are disposed in the furnace $A$, and heated together. When the retorts are heated to a red heat, lumps of coal are thrown into them, and they are then closed with a closely fitting cover. The illustration shows the furnace, with five retorts. Coke (see Note 1, dry distillation) remains in the retorts, and the volatile products in the form of vapours and gases travel along the pipe $d$, rising from each retort. These pipes branch above the stove, and communicate with the receiver $f$ (hydraulic main) placed above the fumace. Those products of the dry distillation which most easily pass from the gaseous into the liquid and solid states collect in the hydraulic main. From the hydraulic main the vapours and gases travel along the pipe $g$ and the series of vertical pipes $j$ (which are sometimes cooled by water trickling over the surface), where the vapours and gases cool from the contact of the colder surface, and a fresh quantity of vapour condenses. The condensed liquids pass from the pipes $g$ and $j$ and into the troughs $H$. These troughs always contain liquid at a constant level (the excess flowing away) so that the gas cannot escape, and thus they form, as it is termed, a hydraulic joint. In the state in which it leaves the condensers the gas consists principally of the following vapours and gases: (1) vapour of water, (2) ammonium carbonate, (3) liquid hydrocarbons, (4) hydrogen sulphide, $\mathrm{H}_{2} \mathrm{~S}$, (5) carbonic anhydride, $\mathrm{CO}_{2},(6)$ carbonic oxide, $\mathrm{CO}$, (7) sulphurous anhydride, $\mathrm{SO}_{2}$, but a great part of the illuminating gas consists of (8) hydrogen, (9) marsh gas, (10) olefiant.gas, $\mathrm{C}_{2} \mathrm{H}_{4}$, and other gaseous hydrocarbons. The hydrocarbons $(8,9$, and 10), the hydrogen, and carbonic oxide are capable of combustion, and are useful component parts, but the carbonio anhydride, the hydrogen sulphide, and sulphurous anhydride, as well as the vapours of ammonium carbonate, form an injurious admixture, because they do not burn $\left(\mathrm{CO}_{2}, \mathrm{SO}_{2}\right)$ and lower the temperature and brilliancy of the flame, or else, although capable of burning (for example, $\mathrm{H}_{2} \mathrm{~S}, \mathrm{CS}_{2}$, and others), they give out during combustion sulphurous anhydride which has a disagrceable smell, is injurions when inhaled, and spoils many surrounding objects. In order to separate the injurious products, the gas is washed with water, a cylinder (not shown in the illustration) filled with coke continually moistened with water serving for this purpose. The water coming into contact with the gas dissolves the ammonium carbonate; hydrogen sulphide, carbonic anhydride, and sulphurous anhydride, bcing only partly soluble in water, have to be got rid of by a special means. For this purpose the gas is passed through moist lime or other alkaline liquid, as the above-mentioned gases have acid properties and are therefore retained by the alkali. In the case of lime, calcium carbonate, sulphite and sulphide, all solid substances, are formed. It is necessary to renew the purifying material as its absorbing power decreases. A mixture of lime and sulphate of iron, $\mathrm{FeSO}_{4}$, acts still better, because the latter, with lime, $\mathrm{Ca}(\mathrm{HO})_{2}$, forms ferrous hydroxide, $\mathrm{Fe}(\mathrm{HO})_{2}$ and gypsum, $\mathrm{CaSO}_{4}$. The suboxide (partly turning into oxide) of iron absorbs 


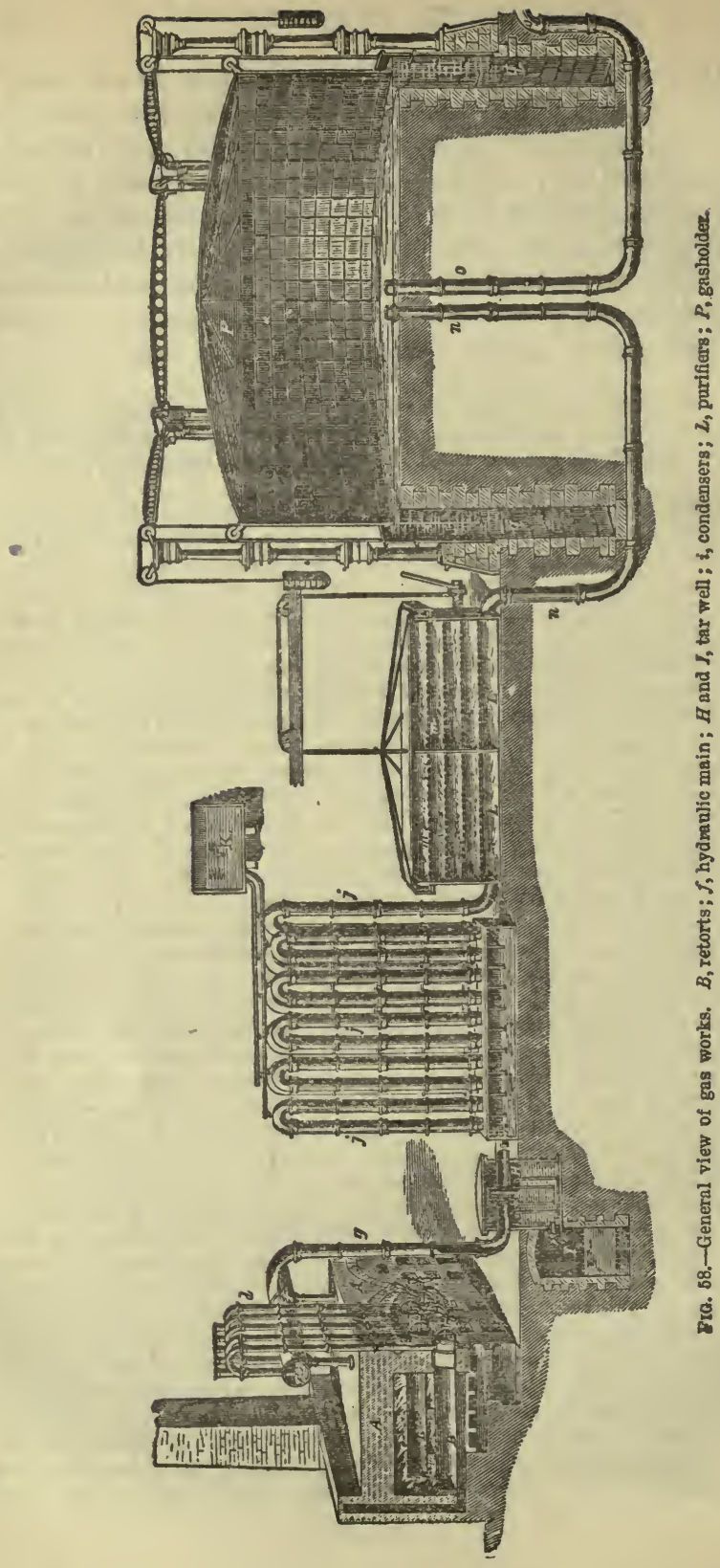




\section{As the decomposition of the organic matter which forms coal, is still} going on underground, the evolution of large quantities of marsh gas

$\mathrm{H}_{2} \mathrm{~S}$, forming $\mathrm{FeS}$ and $\mathrm{H}_{2} \mathrm{O}$, and the gypsum retains the remainder of the amimonia, the excess of lime absorbing carbonic anhydride and sulphuric anlyydride. [In English works a native hydrated ferric hydroxide is used for removing hydrogen sulphide.] This purification of the gas takes place in the apparatus $L$, where the gas passes through perforated trays $m$, covered with sawdust mixed with lime and sulphate of iron. It is necessary to remark that in the manufacture of gas it is indispensable to draw off the vapours from the retorts, so that they should not remain there long (otherwise the hydrocarbons would in a considerable degree be resolved into charcoal and hydrogen), and also to avoid a great pressure of gas in the apparatus, otherwise a quantity of gas would escape at all cracks such as must inevitably exist in such á complicated arrangement. For this purpose there are special pumps (exhausters) so regulated that they only pump off the quantity of gas formed (the pump is not shown in the illustration). The purified gas passes through the pipe $n$ into the gasometer (gasholder) $P$, a dome made of iron plate. The edges of the dome dip into water poured into a ring-shaped channel $g$, in which the sides of the dome rise and fall. The gas is collected in this holder, and distributed to its destination by pipes communicating with the pipe 0 , issuing from the dome. The pressure of the dome on the gas enables it, on issuing from a long pipe, to penetrate. through the small aperture of the burner. A hundred kilograms of coal give about 20 to 30 cubio metres of gaa, having a density from four to nine times greater than that of hydrogen. A cubic metre (1,000 litres) of hydrogen weighs about 87 grams; therefore 100 kilograms of coal give about 18 kilograms of gas, or about one-sixth of its weight. Illuminating gas is generally lighter than marsh gas, as it contains a considerable amount of hydrogen, and is only heavier than marsh gas when it contains much of the heavier hydrocarbons. Thus olefiant gas, $\mathrm{C}_{2} \mathrm{H}_{4}$, is fourteen times, and the vapours of benzene thirty-nine times; heavier than hydrogev, and illuminating gas sornetimes contains 15 p.c. of its volume of them. The brilliancy of the flame of the gas increases with the quantity of olefiant gas and similar heavy hydrocarbons, as it then contains more carbon for a given volume and a greater number of carbon particles are separated. Gas usually contains from 35 to 60 p.c. of its volume of marsh gas, from 30 to 50 p.c. of hydrogen, from 3 to 5 p.c. of carbonic oxide, from 2 to 10 p.c. heavy hydrocarbons, and from 3 to 10 p.c. of nitrogen. Wood gives almost the same sort of gas as coal and almost the same quantity, but the wood gas contains a great deal of carbonic anhydride, although on the other hand there is an almost complete absence of sulphur compounds. Tar, oils, naphtha, and such materials furnish a large quantity of good illuminating gas. An ordinary burner of 8 to 12 candle-power burns 5 to 6 cubic feet of coal gas per hour, but only 1 cubic foot of naphtha gas. One pood ( 36 lbs. Eng.) of naphtha gives 500 cubio feet of gas - that is, one kilogram of naphtha produces about one cubic metre of gas. The formation of combustible gas by heating coal was discovered in the beginning of the last

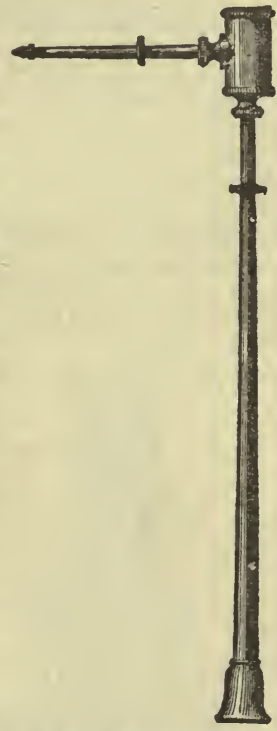

Fto. 59. - Blowplpe. Air is blown in at the trumpetsliaped mouthpiece, and escapes in a fine stream from the platinum jet placed at the extremity of the side tube.

century, but only put into practice towards the end by Le-Bon in France and Murdoch in England. In England, Murdoch, together with the renowned Watt, built the first gas works in 1805. 
frequently occurs in coal-mines. ${ }^{32}$ When mixed with air it forms an explosive mixture, which forms one of the great dangers of coal mining, as subterranean work has always to be carried on by lamp-light. This danger is, however, overcome by the use of Humphiry Davy's safety lamp. ${ }^{33}$ Sir Humphry Davy observed that on introducing a piece of wire gauze into a flame, it absorbs so much heat that combustion does not proceed beyond it (the unburnt gases which pass through it may be ignited on the other side). In accordance with this, the flame of the Davy lamp is surrounded with a thick glass (as shown in the drawing), and has no communication whatever with the explosive mixture except through a wire gauze which prevents it igniting the mixture of the marsh-gas issuing from the coal with air. In some districts, particularly in those where petroleum is found-as, for instance, near Baku, where a temple of the Indian fre-worshippers was built, and in Pennsylvania, and other places-marsh gas in abundance issues from the earth, and it is used, like coal gas, for the purposes of lighting and warming. ${ }^{34}$

In practice illuminating gas is not only used for lighting (electricity and kerosene are cheaper in Russia), but also as the motive power for gas engines (see p. 175), which consume about half a cubic metre per horse-power per hour; gas is also used in laboratories for heating purposes. When it is necessary to concentrate the heat, either the ordinary blowpipe (fig. 59) is applied, placing the end in the flame and blowing through the mouth. piece; or, in other forms, gas is passed through the blowpipe; when a large, hot, smokeless flame is required for heating crucibles or glass-blowing, a foot-hlower is used. High temperatures, which are often required for laboratory and manufacturing purposes, are most easily attained by the use of gaseous fuel (illuminating gas, producer gas, and water gas, which will be treated of in the following chapter), because complete combus-

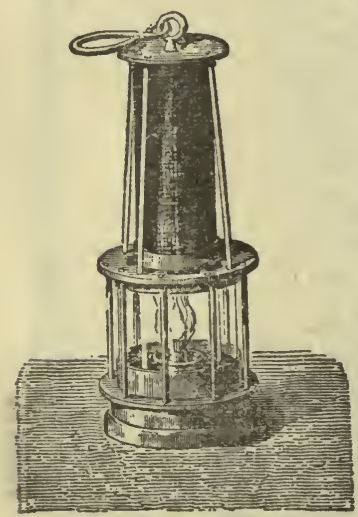

Fig. 60.-Davy safety-lamp. [Modern form.] tion may be effected without an access of air. It is evident that in order to obtain high temperatures means must be taken to diminish the loss of heat by radiation, and to ensure perfect combustion.

53 The gas which is set free in coal mines contains a good deal of nitrogen, some carbonio anhydride, and a large quantity of marsh gas. The best means of avoiding an explosion consists in efficient ventilation. It is best to light coal mines with electric lamps.

5s The Davy lamp, of which an improved form is represented in the accompanying figure, is used for lighting coal and other mines where combus. tible gas is found. The wick of the lamp is en. closed in a thick glass cylinder which is firmly held in a metallic holder. Over this a metallio cylinder and the wire gauze are placed. The pro. ducts of combnstion pass through the ganze, and the air enters throngh the space between the eylinder and the wire gauze. To ensure greater safety the lamp cannot be opened without extin. guishing the flame.

34 In Pennsylvania (beyond the Alleghany mountains) many of the shafts sunk for petroleum only emitted gas, but many useful spplications for it were found and it wase 
Tolerably pure marsh gas ${ }^{35}$ may be obtained by heating a mixture of an acetate with an alkali. Acetic acid, $\mathrm{C}_{2} \mathrm{H}_{4} \mathrm{O}_{2}$, on being heated is decomposed into marsh gas and carbonic anhydride, $\mathrm{C}_{2} \mathrm{H}_{4} \mathrm{O}_{2}=\mathrm{CH}_{4}+\mathrm{CO}_{2}$.

An alkali-for instance, $\mathrm{NaHO}$-gives with acetic acid a salt, $\mathrm{C}_{2} \mathrm{H}_{3} \mathrm{NaO}_{2}$, which on decomposition retains carbonic anhydride, forming a carbonate, $\mathrm{Na}_{2} \mathrm{CO}_{3}$, and marsh gas is given off :

$$
\mathrm{C}_{2} \mathrm{H}_{3} \mathrm{NaO}_{2}+\mathrm{NaHO}=\mathrm{Na}_{2} \mathrm{CO}_{3}+\mathrm{CH}_{4}
$$

Marsh gas is difficult to liquefy ; it is almost insoluble in water, and is without taste or smell. The most important point in connection with its chemical reactions is that it does not combine directly with anything, whilst the other hydrocarbons which contain less hydrogen than expressed by the formula $\mathrm{C}_{n} \mathrm{H}_{2 n+2}$ are capable of combining with hydrogen, chlorine, certain acids, \&c.

If the law of substitution gives a very simple explanation of the formation of hydrogen peroxide as a compound containing two aqueous residues $(\mathrm{OH})(\mathrm{OH})$, then on the basis of this law all hydro; carbons ought to be derived from methane, $\mathrm{CH}_{4}$, as being the simplest hydrocarbon. ${ }^{36}$ The increase in complexity of a molecule of methan $\theta$ is brought about by the faculty of mutual combination which exists in the atoms of carbon, and, as a consequence of the most detailed study of the subject, much that might have been foreseen and conjectured from the law of substitution has been actually brought about in such a manner as might have been predicted, and although this subject. on account of its magnitude really belongs, as has been already stated, to the sphere of organic chemistry, it has been alluded to here in order to show, although only in part, the best investigated example of the application of the law of substitution. According to this law, a molecule of methane, $\mathrm{CH}_{4}$, is capable of undergoing substitution in the four following ways :-(1) Methyl substitution, when the radicle, equivalent to hydrogen, called methyl $\mathrm{CH}_{3}$, replaces hydrogen. In $\mathrm{CH}_{4}$ this radicle is combined with $H$ and therefore can replace it, as $(\mathrm{OH})$ replaces $\mathrm{H}$ because with it it gives water ; (2) methylene substitution, or the exchange between $\mathrm{H}_{2}$ and $\mathrm{CH}_{2}$ (this radicle is called methylene), is founded on a similar division of the molecule $\mathrm{CH}_{4}$ into two equiva,

conducted in metallic pipes to works hundreds of miles distant, principally for metal. lurgical purposes.

${ }_{35}$ The purest gas is prepared by mixing the liquid substance called zino methyl, $\mathrm{Zn}\left(\mathrm{CH}_{3}\right)_{2}$, with water, when the following reaction occurs :

$$
\mathrm{Zn}\left(\mathrm{CH}_{3}\right)_{2}+2 \mathrm{HOH}=\mathrm{Zn}\left(\mathrm{HO}_{2}+2 \mathrm{CH}_{5} \mathrm{H}\right. \text {. }
$$

30 Methylene, $\mathrm{CH}_{2}$, does not exist. When attempts are made to obtain it (for example, by removing $\mathrm{X}_{2}$ from $\mathrm{CH}_{2} \mathrm{X}_{2}$ ), $\mathrm{C}_{2} \mathrm{H}_{4}$ or $\mathrm{C}_{3} \mathrm{H}_{6}$ are produced-that is to say, it undergoes polymerisation. 
lent parts, $\mathrm{H}_{2}$ and $\mathrm{CH}_{2}$; (3) acetylene substitution, or the exchange between $\mathrm{CH}$ on the one hand and $\mathrm{H}_{3}$ on the other; and (4) carbon substitution-that is, the substitution of $\mathrm{H}_{4}$ by an atom of carbon $\mathrm{C}$, which is founded on the law of substitution just as is the methyl substitution. These four cases of substitution render it possible to understand the principal relations of the hydrocarbons. For instance, the law of even numbers is seen from the fact that in all the cases of substitution mentioned the hydrogen atoms increase or decrease by an even number; but as in $\mathrm{CH}_{4}$ they are likewise even, it follows that no matter how many substitutions are effected there will always be obtained an even number of hydrogen atoms. When $H$ is replaced by $\mathrm{CH}_{3}$ there is an increase of $\mathrm{CH}_{2}$; when $\mathrm{H}_{2}$ is replaced by $\mathrm{CHI}_{2}$ there is no increase of hydrogen; in the acetylene substitution $\mathrm{CH}$ replaces $\mathrm{H}_{3}$, therefore there is an increase of $\mathrm{C}$ and a decrease of $\mathrm{H}_{2}$; in the carbon substitution there is a decrease of $\mathrm{H}_{4}$. In a similar way the law of limit may be deduced as a corollary of the law of substitution. For the largest possible quantity of hydrogen is intraduced by the methyl substitution, since it leads to the addition of $\mathrm{CH}_{2}$; starting from $\mathrm{CH}_{4}$ we obtain $\mathrm{C}_{2} \mathrm{H}_{6}, \mathrm{C}_{3} \mathrm{H}_{8}$, and in general, $\mathrm{C}_{n} \mathrm{H}_{2 n+2}$, and these contáin the greatest possible amount of hydrogen. Unsaturated hydrocarbons, containing less hydrogen, are evidently only formed when the increase of the new molecule derived from methane proceeds from one of the other forms of substitution. When the methyl substitution alone takes place in methane, $\mathbf{C H}_{4}$, it is evident that the saturated hydrocarbon formed is $\mathrm{C}_{2} \mathrm{H}_{6}$ or $\left(\mathrm{CH}_{3}\right)\left(\mathrm{CH}_{3}\right)^{37}$ This is called ethane. By means of the methylene substitution alone, ethylene, $\mathrm{C}_{2} \mathrm{H}_{4}$, or $\left(\mathrm{CH}_{2}\right)\left(\mathrm{CH}_{2}\right)$ may be directly obtained from $\mathrm{CH}_{4}$, and by the acetylene substitution $\mathrm{C}_{2} \mathrm{H}_{2}$ or

37 Although the methods of formation and the reactions connected with hydrocarbons are not described in this work, because they are dealt with in organic chemistry, yet in order to clearly show the mechanism of those.transformations by which the carbon atoms are built up into the molecules of the carbon compounds, we here give a general example of reactions of this kind. From marsh gas, $\mathrm{CH}_{4}$, on the one hand the substitution of chlorine or iodine, $\mathrm{CH}_{3} \mathrm{Cl}, \mathrm{CH}_{3} \mathrm{I}$, for the hydrogen may be effected, and on the other hand such metals as sodium may be substituted for the hydrogen, e.g. $\mathrm{CH}_{3} \mathrm{Na}$. These and similar products of substitution serve as a means of obtrining other more complex substances from given carbon compounds. If we place the two above-named products of substitution of marsh gas (metallic and haloid) in mutual contact, the metal combines with the halogen, forming a very stable compound-namely, common salt, $\mathrm{NaCl}$, and the carbon groups which were in combination with them separate in mutual combination, as shown by the equation:

$$
\mathrm{CH}_{3} \mathrm{Cl}+\mathrm{CH}_{3} \mathrm{Na}=\mathrm{NaCl}+\mathrm{C}_{2} \mathrm{H}_{6} \text {. }
$$

This is the most simple exumple of the formation of a complex hydrocarbon from these radicles. The cause of the reaction must be sought for in the property which the haloid (ohlorine) and sodium have of entering into mutual combination. 
(CH) (CH), or acetylene, both the latter being unsaturated hydrocarbons. Thus we have all the possible hydrocarbons with two atoms of carbon in the molecule, $\mathrm{C}_{2} \mathrm{H}_{6}$, ethane, $\mathrm{C}_{2} \mathrm{H}_{4}$, ethylene, and $\mathrm{C}_{2} \mathrm{H}_{2}$, acetylene. But in them, according to the law of substitution, the same forms of substitution may be repeated-that is, the methyl, methylene, acetylene, and even carbon substitutions (because $\mathrm{C}_{2} \mathrm{H}_{6}$ will still contain hydrogen when $\mathrm{C}$ replaces $\mathrm{H}_{4}$ ) and therefore further substitutions will serve as a source for the production of a fresh series of saturated and unsaturated hydrocarbons, containing more and more carbon in the molecule and, in the case of the acetylene substitution and carbon substitution, containing less and less hydrogen. Thus by means of the law of substitution we can foresee not only the limit $\mathrm{C}_{n} \mathrm{H}_{2 n+2}$, but an unlimited nurnber of unsaturated hydrocarbons, $\mathrm{C}_{n} \mathrm{H}_{2 n}^{\prime \prime}$ $\mathrm{C}_{n} \mathrm{H}_{2 n-2} \ldots, \mathrm{C}_{n} \mathrm{H}_{2}(n-m)$, where $m$ varies from 0 to $n-1,{ }^{38}$ and where $n$ increases indefinitely. From these facts not only does the existence of a multitude of polymeric hydrocarbons, differing in molecular weight, become intelligible, but it is also seen that there is a possibility of cases of isomerism with the same molecular weight. This polymerism, so common to hydrocarbon compounds is already apparent in the first unsaturated series $\mathrm{C}_{n} \mathrm{H}_{2 n}$, because all the terms of this series $\mathrm{C}_{2} \mathrm{H}_{4}, \mathrm{C}_{3} \mathrm{H}_{6}, \mathrm{C}_{4} \mathrm{H}_{8} \ldots \dot{\mathrm{C}}_{30} \mathrm{H}_{60} \ldots$. have one and the same composition $\mathrm{CH}_{2}$, but different molecular weights, as has been already explained in Chapter VII. The differences in the vapour density, boiling points, and melting points, of the quantities entering into reactions, ${ }^{39}$ and the methods of preparation 40 also so clearly tally with the conception of polymerism, that this example will always be the clearest and most conclusive for the illustration of polymerism and molecular weight. Such a case is also met with among other hydrocarbons. Thus benzene, $\mathrm{C}_{6} \mathrm{H}_{6}$, and cinnamene, $\mathrm{C}_{8} \mathrm{H}_{8}$, correspond with the composition of acetylcne or to a compound of the composition $\mathrm{CH} .{ }^{41}$ The first boils at $81^{\circ}$, the second at $144^{\circ}$;

ss When $m=n-1$, we have the series $\mathrm{C}_{n} \mathrm{H}_{2}$. The lowest member is acetylene, $\mathrm{C}_{2} \mathrm{H}_{2}$. These are hydrocarbons containing a minimum amount of hydrogen.

59 For instance, ethylene, $\mathrm{C}_{2} \mathrm{H}_{4}$, combines with $\mathrm{Br}_{2}, \mathrm{HI}, \mathrm{H}_{2} \mathrm{SO}_{4}$, as a whole molecule, as also does amylene, $\mathrm{C}_{5} \mathrm{H}_{10}$, and, in general, $\mathrm{C}_{n} \mathrm{H}_{2 n}$.

10 For instance, ethylene is obtained by removing the water from ethyl alcohol, $\mathrm{C}_{2} \mathrm{H}_{5}(\mathrm{OH})$, and amylene, $\mathrm{C}_{5} \mathrm{H}_{10}$, from amyl alcohol, $\mathrm{C}_{3} \mathrm{H}_{11}(\mathrm{OH})$, or in general $\mathrm{C}_{n} \mathrm{H}_{2 n}$, from $\mathrm{C}_{n} \mathrm{H}_{2 n+1}(\mathrm{OH})$.

$\$ 1$ Acetylene and its polymerides have an empirical composition $\mathrm{CH}$, ethylene and its homologues (and polymerides) $\mathrm{CH}_{2}$, ethane $\mathrm{CH}_{3}$, methane $\mathrm{CH}_{4}$. This series presents a good example of the law of multiple proportions, but such diverse proportions are met with between the number of atoms of the carbon and hydrogen in the hydrocarbons already known that the accuracy of Dalton's law might be doubted. Thus the substances $\mathrm{C}_{50} \mathrm{H}_{62}$ and $\mathrm{C}_{50} \mathrm{H}_{60}$ differ so slightly in their composition by weight as to be within the limits of experimental error, but their reactions and properties are so distinct that they 
the specific gravity of the first is 0.899 ; that of the second, 0.925 , at $0^{\circ}$-that is, here also the boiling point rises with the increase of molecular weight, and so also, as might be expected, does the density.

Cases of isomerism in the restricted sense of the word-that is, when with an identity of composition and of molecular weight, the properties of the substances are different-are very numerous among. the hydrocarbons and their derivatives. Such cases are particularly important for the comprehension of molecular structure and they also, like the polymerides, may be predicted from the above-mentioned conceptions, expressing the principles of the structure of the carbon compounds ${ }^{42}$ based on the law of substitution. According to it, for example, it is evident that there can be no isomerism in the cases of the saturated hydrocarbons $\mathrm{C}_{2} \mathrm{H}_{6}$ and $\mathrm{C}_{3} \mathrm{H}_{8}$, because the former is $\mathrm{CH}_{4}$, in which methyl has taken the place of. $\mathrm{H}$, and as all the hydrogen atoms of methane must be supposed to have the same relation to the carbon, it is all the same which of them be subjected to the methyl substitution--the resulting product can only be ethane, $\mathrm{CH}_{3} \mathrm{CH}_{3} ;{ }^{43}$ the same argument also applies in the case of propane, $\mathrm{CH}_{3} \mathrm{CH}_{2} \mathrm{CH}_{3}$, where one compound only can be imagined. It

can be distinguished beyond a doubt. Without Dalton's law chemistry could not have been brought to its present condition, but it cannot alone express ll those grada. tions which are quite clearly understood and predicted by the law of AvogadroGerhardt.

42 The conception of the structure of carbon compounds-that is, the expression of those unions and correlations which their atoms have in the molecules-was for a long time limited to the representation that organic substances contained complex radicles (for instance, ethyl $\mathrm{C}_{2} \mathrm{H}_{5}$, methyl $\mathrm{CH}_{3}$, phenyl $\mathrm{C}_{6} \mathrm{H}_{5}$, \&c.); then about the year 1840 the phenomena of substitution and the correspondence of the products of substitution with the primary bodies (nuclei and types) were observed, but it was not until about the year 1860 and later when on the one hand the teaching of Gerhardt about molecules was spreading, and on the other hand the materials had accumulated for discussing the transformations of the simplest hydrocarbon componnds, that conjectures began to appear as to the mutual connection of the atoms of carbon in the molecules of the com. plex hydrocarbon compounds. Then Kekule and A. M. Butleroff began to formulate the connection between the separate atoms of carbon, regarding it as a quadrivalent element. Although in their methods of expression and in some of their views they differ from each other and also from the way in which the subject is treated in this work, yet the essence of the matter-namely, the comprehension of the causes of isomerism and of the union between the separate atoms of carbon-remains the same. In addition to this, starting from the year 1870, there appears a tendency which from year to year increases to discover the actual spacial distribution of the atoms in the molecules. Thanks to the endeavours of Le-Bel (1874), Van 't Hoff (1874), and Wislicenus (1887) in observing cases of isomerism-such as the effect of different isomerides on the direction of the rotation of the plane of polarisation of light-this tendency promises much for chemical mechanics, but the details of the still imperfect knowledge in relation to this matter must be sought for in special works devoted to organic chemistry.

43 Direct experiment shows that however $\mathrm{CH}_{3} \mathrm{X}$ is prepared (where $\mathrm{X}=$ for instance $\mathrm{Cl}$, \&c.) it is always one and the same substanco. If, for example, in $\mathbf{C X}_{4}, \mathbf{X}$ is gradually replaced by hydrogen until $\mathrm{CH}_{3} \mathrm{X}$ is produced, or in $\mathrm{CH}_{4}$, the hydrogen by various 
is to be expected, however, that there should be two butanes, $\mathrm{C}_{4} \mathrm{H}_{10}$, and this is actually the case. In one, methyl may be considered as replacing the hydrogen of one of the methyls, $\mathrm{CH}_{3} \mathrm{CH}_{2} \mathrm{CH}_{2} \mathrm{CH}_{3}$; and in the other $\mathrm{CH}_{3}$ may be considered as substituted for $\mathrm{H}$ in $\mathrm{CH}_{2}$, and there it will consist of $\mathrm{CH}_{3} \mathrm{CH}_{\mathrm{CH}_{3}}^{\mathrm{CH}_{3}}$. The latter may also be regarded as methane in which three of hydrogen are exchanged for three of methyl. On going further in the series it is evident that the number of possible isomerides will be still greater, but we have limited ourselves to the simplest examples, showing the possibility and actual existence of isomerides. $\mathrm{C}_{2} \mathrm{H}_{4}$ and $\mathrm{CH}_{2} \mathrm{CH}_{2}$ are, it is evident, identical; but there ought to be, and are, two hydrocarbons of the composition $\mathrm{C}_{3} \mathrm{H}_{6}$, propylene and trimethylene; the first is ethylene, $\mathrm{CH}_{2} \mathrm{CH}_{2}$, in which one atom of hydrogen is exchanged for methyl, $\mathrm{CH}_{2} \mathrm{CHCH}_{3}$, and trimethylene is ethane, $\mathrm{CH}_{3} \mathrm{CH}_{3}$, with the substitution of methylene for two hydrogen atoms from two methyl groupsthat is, $\mathrm{CH}_{2} \mathrm{CH}_{2}$, where the methylene introduced is united to both the atoms of carbon in $\mathrm{CH}_{3} \mathrm{CH}_{3}$. It is evident that the cause of isomerism here is, on the one hand, the difference of the amount of hydrogen in union with the particular atoms of carbon, and, on the other, the different connection between the sereral atoms of carbon. In the first case they may be said to be chained together (more usually to form an open chain '), and in the second case, to be locked together (to form a 'closed chain' or 'ring'). Here also it is easily understood that on increasing the quantity of carbon atoms the number of possible and existing isomerides will greatly increase. If, at the same time, in addition to the substitution of one of the radicles of methane for hydrogen a further exchange of part of the hydrogen for some of the other groups of elements $\mathrm{X}, \mathrm{Y} \ldots$. . occurs, the quantity of possible isomerides still further increases in a considerable degree. For instance, there are even two possible isomerides for the derivatives of ethane, $\mathrm{C}_{2} \mathrm{H}_{6}$ : if two atoms of the hydrogen be exchanged for $\mathrm{X}_{2}$, means is replaced by $\mathrm{X}$, or else, for instance, if $\mathrm{CH}_{5} \mathrm{X}$ be obtrined by the decomposition of more complex compounds, the same product is always obtained.

This was shown in the year 1860 , or thereabout, by many methods, and is the fundamental conception of the structure of hydrocarbon compounds. If the atoms of hydrogen in methyl were not absolutely identical in value and position (as they are not, for instance, in $\mathrm{CH}_{5} \mathrm{CH}_{2} \mathrm{CH}_{3}$ or $\mathrm{CH}_{3} \mathrm{CH}_{2} \mathrm{X}$ ), then there would be as many different forms of $\mathrm{CH}_{5} \mathrm{X}$ as there were diversities in the atoms of hydrogen in $\mathrm{CH}_{4}$. The scope of this work does not permit of a more detailed account of this matter. It 18 given in works on organio chemistry.

44 The union of carbon atoms in closed chains or rings was first suggested by Kekule : as an explanation of the structure and isomerism of the derivatives of benzene, $\mathrm{C}_{6} \mathrm{H}_{60}$. forming aromatio compounds (Note 26). 
one will have the ethylene structure, $\mathrm{CH}_{2} \mathrm{XCH}_{2} \mathrm{X}$, and the other an ethylidene structure, $\mathrm{CH}_{3} \mathrm{CHX}_{2}$; such are, for instance, ethylene chloride, $\mathrm{CH}_{2} \mathrm{ClCH}_{2} \mathrm{Cl}$, and ethyliaiene chloride, $\mathrm{CH}_{3} \mathrm{CHCl}_{2}$. And as in the place of the first atom of hydrogen not only metals may be substi. tuted, but $\mathrm{Cl}, \mathrm{Br}, \mathrm{I}, \mathrm{OH}$ (the water radicle), $\mathrm{NH}_{2}$ (the arnmonia radicle), $\mathrm{NO}_{2}$ (the radicle of nitric acid), dcc, so also in exchange for two atoms of hydrogen $\mathrm{O}, \mathrm{NH}, \mathrm{S}$, \&c., may be substituted; hence it will be understood that the quantity of isomerides is sometimes very great. It is impossible here to describe how the isomerides are distinguished from each other, in what reactions they occur, how and when one changes into another, \&c. ; for this, taken together with the description of the hydrocarbons already known, and their derivatives, forms a very extensive and very thoroughly investigated branch of chemistry, called organic chemistry. Enriched with a mass of closely observed phenomena and strictly deduced generalisations, this branch of chemistry has been treated separately for the reason that in it the hydrocarbon groups are subjected to transformations which are not met with in such quantity in dealing with any of the other elements or their hydrogen compounds. It was important for us to show that notwithstanding the great variety of the hydrocarbons and their products, ${ }^{45}$ they are all of them governed by the law of substitution, and referring our readers for detailed information to works on organic chemistry, we will limit ourselves to a short exposition of the properties of the two simplest unsaturated hydrocarbons : ethylene, $\mathrm{CH}_{2} \mathrm{CH}_{2}$, and acetylene, $\mathrm{CHCH}$, and a short acquaintance with petroleum as the natural source of a mass of hydrocarbons. Ethylene, or olefiant gas, $\mathrm{C}_{2} \mathrm{H}_{4}$,

45 The following are the most generally known of the oxygenised but non-nitrogenous hydocarbon derivatives. (1) The alcohols. These are hydrocarbons in which hydrogen is exchanged for hydroxyl $(\mathrm{OH})$. The simplest of these is methyl alcolhol, $\mathrm{CH}_{3}(\mathrm{OH})$, or wood spirit obtained by the dry distillation of wood. The common spirits of wine or ethyl alcohol, $\mathrm{C}_{2} \mathrm{H}_{3}(\mathrm{OH})$, and glycol, $\mathrm{C}_{2} \mathrm{H}_{4}(\mathrm{OH})_{2}$, correspond with ethane. Normal propyl alcohol, $\mathrm{CH}_{3} \mathrm{CH}_{2} \mathrm{CH}_{2}(\mathrm{OH})$, and isopropyl alcohol, $\mathrm{CH}_{3} \mathrm{CH}(\mathrm{OH}) \mathrm{CH}_{3}$, propylene-glycol, $\mathrm{C}_{3} \mathrm{H}_{6}(\mathrm{OH})_{2}$, and glycerol, $\mathrm{C}_{3} \mathrm{H}_{5}(\mathrm{OH})_{3}$ (which, with stearic and other acids, forms fatty substances), correspond with propane, $\mathrm{C}_{3} \mathrm{H}_{5}$. All alcohols are capable of forming water and ethereal salts with acids, just as alkalis form ordinary salts. (2) Aldehydes are alcohols minus hydrogen; for instance, acetaldehyde, $\mathrm{C}_{2} \mathrm{H}_{4} \mathrm{O}$, corresponds with ethyl alcohol. (3) It is simplest to regard organic acids as hydrocarbons in which hydrogen has been exchanged for carboxyl $\left(\mathrm{CO}_{2} \mathrm{H}\right)$, as will be explained in the following chapter. There are a number of intermediate compounds; for example, the aldehyde-alcohols, alcohol-acids (or hydroxy-acids), \&c. Thus the hydroxy-acids are hydrocarbons in which some of the hydrogen has been replaced by hydroxyl, and some by carboxyl; for instance, lactic acid corresponds with $\mathrm{C}_{2} \mathrm{H}_{6}$, and has the constitution $\mathrm{C}_{2} \mathrm{H}_{4}(\mathrm{OH})\left(\mathrm{CO}_{2} \mathrm{H}\right)$. If to these products we add the haloid salts (where $\mathrm{H}$ is replaced by $\mathrm{Cl}, \mathrm{Br}, \mathrm{I}$ ), the nitrocompounds containing $\mathrm{NO}_{2}$ in place of $\mathrm{H}$, the amides, cyanides, ketones, and other compounds, it will be readily seen what an immense number of organic compounds there are and what a variety of properties these substances have; this we see also from the composition of plants and animals. 
is the lowest known member of the unsaturated hydrocarbon series of the composition $\mathrm{C}_{n} \mathrm{H}_{2 n}$. As in composition it is equal to two molecules of marsh gas deprived of two molecules of hydrogen, it is evident that it might be, and it actually can be, produced, although but in small quantities, together with hydrogen, by heating marsh gas. On being heated, however, oleñant gas splits up, first into acetylene and methane $\left(3 \mathrm{C}_{2} \mathrm{H}_{4}=2 \mathrm{C}_{2} \mathrm{H}_{2}+2 \mathrm{CH}_{4}\right.$, Lewes, 1894), and at a higher temperature into carbon and hydrogen; and therefore in those cases where marsh gas is produced by heating, olefiant gas, hydrogen, and charcoal will also be formed, although only in small quantities. The lower the temperature at which complex organic substances are heated, the greater the quantity of olefiant gas found in the gases given off ; at a white heat it is entirely decomposed into charcoal and marsh gas. If coal, wood, and more particularly petroleum, tars, and fatty substances, are subjected to dry distillation, they give off illuminating gas, which contains more or less olefiant gas.

Olefiant gas, almost free from other gases, ${ }^{46}$ may be obtained from ordinary alcohol (if possible, free from water) if it be mixed with five parts of strong sulphuric acid and the mixture heated to slightly above $100^{\circ}$. Under these conditions; the sulphuric acid removes the elements of water from the alcohol, $\mathrm{C}_{2} \mathrm{H}_{5}(\mathrm{OH})$, and gives olefiant gas ; $\mathrm{C}_{2} \mathrm{H}_{6} \mathrm{O}=\mathrm{H}_{2} \mathrm{O}+\mathrm{C}_{2} \mathrm{H}_{4}$. The greater molecular weight of olefiant gas compared with marsh gas.indicates that it may be comparatively easily converted into a liquid by means of pressure or great cold; this may be effected, for example, by the evaporation of liquid nitrous oxide. Its absolute boiling point is $+10^{\circ}$, it boils at $-103^{\circ}$ ( 1 atmosphere), liquefies at $0^{\circ}$, at a pressure of 43 atmospheres, and solidifies at $-160^{\circ}$. Ethylene is colourless, has a slight ethereal smell, is slightly soluble in water, and somewhat more soluble in alcohol and in ether (in five volumes of spirit and six volumes of ether). ${ }^{47}$

40 Ethylene bromide, $\mathrm{C}_{2} \mathrm{H}_{4} \mathrm{Br}_{2}$, when gently heated in alcoholic solution with finely divided zinc, yields pure ethylene, the zinc merely taking up the bromine (Sabaneyeff).

47 Ethylene decomposes somewhat easily under the influence of the electric spark, or a high temperature. In this case the volume of the gas formed may remain the same when olefiant gas is decomposed into carbon and marsh gas, or may increase to double its volume when hydrogen and carbon are formed, $\mathrm{C}_{2} \mathrm{H}_{4}=\mathrm{CH}_{4}+\mathrm{C}=2 \mathrm{C}+2 \mathrm{H}_{2}$. A mixture of olefiant gas and oxygen is highly explosive; two volumes of this gas require six volumes of oxygen for its perfect combustion. The eight volumes thus taken then resolve themselves into eight volumes of the products of combustion, a mixture of water and carbonic anhydride, $\mathrm{C}_{2} \mathrm{H}_{4}+3 \mathrm{O}_{2}=2 \mathrm{CO}_{2}+2 \mathrm{H}_{2} \mathrm{O}$. On cooling after the explosion dininution of volume occurs because the water becomes liquid. For two volumes of the olcfiant gas taken, the diminution will be equal to four volumes, and the same for marsh gas. The quantity of carbonic anlhydride formed by both gases is not the same. Two volumes of marsh gas give only two volumes of carbonic anhydride, and two, volumes of ethyleue give four volumes of carbonic anlydride. 
Like other unsaturated hydrocarbons, olefiant gas readily enters into combination with certain substances, such as chlorine, bromine, iodine, fuming sulphuric acid, or sulphuric anhydride, \&c. If olefiant gas be sealed up with a small quantity of sulphuric acid in a glass vessel, and constantly agitated (as, for instance, by attaching it to the moving part of a machine), the prolonged contact and repeated mixing causes the olefiant gas, little by little, to combine with the sulphuric acid, forming $\mathrm{C}_{2} \mathrm{H}_{4} \mathrm{H}_{2} \mathrm{SO}_{4}$. If, after this absorption, the sulphuric acid be diluted with water and distilled, alcohol separates, which is produced in this case by the olefiant gas combining with the elements of water, $\mathrm{C}_{2} \mathrm{H}_{4}+\mathrm{H}_{2} \mathrm{O}=\mathrm{C}_{2} \mathrm{H}_{6} \mathrm{O}$. In this reaction (Berthelot) we see an excellent example of the fact that if a given substance, like olefiant gas, is produced by the decomposition of another, then in the reverse way this substance, entering into combination, is capabie of forming the original substance -in our-example, alcohol. In combination with various molecules, $\mathrm{X}_{2}$, ethylene gives saturated compounds, $\mathrm{C}_{2} \mathrm{H}_{4} \mathrm{X}_{2}$ or $\mathrm{CH}_{2} \mathrm{XCH}_{2} \mathrm{X}$ (for example, $\mathrm{C}_{2} \mathrm{H}_{4} \mathrm{Cl}_{2}$ ), which correspond with ethane, $\mathrm{CH}_{3} \mathrm{CH}_{3}$ or $\mathrm{C}_{2} \mathrm{H}_{6} \cdot{ }^{48}$

Acetylene, $\mathrm{C}_{2} \mathrm{H}_{2}=\mathrm{CHCH}$, is a gas ; it was first prepared by Berthelot (1857). It has a very pungent smell, is characterised by its great stability under the action of heat, and is obtained as the only product of the direct combination of carbon with hydrogen when a luminous arc (voltaic) is formed between carbon electrodes. This arc contains particles of carbon passing from one pole to the other. If the carbons be surrounded with an atmosphere of hydrogen, the carbon in part combines with the hydrogen, forming $\mathrm{C}_{2} \mathrm{H}_{2} \cdot{ }^{48}$ bis Acetylene may be formed from olefiant gas if two atoms of hydrogen be taken from it. This may be effected in the following way : the olefiant gas is first made to combine with bromine, giving $\mathrm{C}_{2} \mathrm{H}_{4} \mathrm{Br}_{2}$; from this the hydrobromic acid is removed hy means of an alcoholic solution of caustic potash, leaving tire volatile product $\mathrm{C}_{2} \mathrm{H}_{3} \mathrm{Br}$; and from this yet another part of hydrobromic acid is withdrawn by passing it through anlydrous alcohol in which metallic sodium has been dissolved, or by heating it with a strong alcoholic solution of caustic potash. Under these circumstances (Berthelot, Sawitsch, Miasnikoff) the alkali takes up the hydrobromic acid from $\mathrm{C}_{n} \mathrm{H}_{2 n-1} \mathrm{Br}$, forming $\mathrm{C}_{n} \mathrm{H}_{2 n-2}$.

48 The homologues of ethylene, $\mathrm{C}_{n} \mathrm{H}_{2 n}$, are also capable of direct combination with halogens, \&c., but with various degrees of facility. The composition of these homologues can be expressed thus: $\left(\mathrm{CH}_{3}\right)_{x}\left(\mathrm{CH}_{2}\right)_{y}(\mathrm{CH})_{z} \mathrm{C}_{r}$, where the sum of $x+z$ is always an even number, and the sum of $x+z+r$ is equal to half the sum of $3 x+z$, whence $z+2 r=x$; by this means the possible isomerides are determined. For example, for butylenes, $\mathrm{C}_{4} \mathrm{H}_{8}$, $\left(\mathrm{CH}_{3}\right)_{2}(\mathrm{CH})_{2},\left(\mathrm{CH}_{3}\right)_{2}\left(\mathrm{CH}_{2}\right) \mathrm{C},\left(\mathrm{CH}_{2}\right)\left(\mathrm{CH}_{2}\right)_{2} \mathrm{CH}$, and $\left(\mathrm{CH}_{2}\right)_{4}$ are possible.

48 bis See also method of preparing $\mathrm{C}_{2} \mathrm{H}_{2}$ in Note 12 bis. 
Acetylene is also produced in all those cases where organic substances are decomposed by the action of a high temperature-for example, by dry distillation. On this account a certain quantity is always found in coal gas, and gives to it, at all events in part, its peculiar smell, but the quantity of acetylene in coal gas is very sinall. If the vapour of alcohol be passed through a heated tube a certain quantity of acetylene is formed. It is also produced by the imperfect combustion of olefiant and marsh gas-for example, if the flame of coal gas has not free access to air. ${ }^{49}$ The inner part. of every flame contains gases in imperfect combustion, and in them some amount of acetylene.

Acetylene, being further removed than ethylene from the limit $\mathrm{C}_{n} \mathrm{H}_{2 n+2}$ of hydrocarbon compounds, has a still greater faculty of combination than is shown by olefiant gas, and therefore can be more readily separated from any mixture containing it. Actually, acetylene not. only combines with one and two moleculcs of $\mathrm{I}_{2}, \mathrm{HI}, \mathrm{H}_{2} \mathrm{SO}_{4}, \mathrm{Cl}_{2}, \mathrm{Br}_{2}$, \&c. . . (many other unsaturated hydrocarbons combine with them), but also with cuprous chloride, $\mathrm{CuCl}$, forming a red precipitate. If a gaseous mixture containing acetylene be passed through an ammoniacal solution of cuprous chloride (or silver nitrate), the other gases do not combine, but the acetylene gives a red precipitate (or grey with silver), which detonates when struck with a hammer. This red precipitate gives off acetylene under the action of acids. Iu this manner pure acetylene may be obtained. Acetylene and its homologues also readily react with corrosive sublimate, $\mathrm{HgCl}_{2}$ (Koucheroff, Favorsky). Acetylene burns with a very brilliant flame, which is accounted for by the comparatively large amount of carbon it contains. ${ }^{50}$

The formation and existence in nature of large masses of petroleum or a mixture of liquid hydrocarbons, principally of the series $\mathrm{C}_{n} \mathrm{H}_{2 n+2}$ and $\mathrm{C}_{n} \mathrm{H}_{2 n}$ is in many respects remarkable. ${ }^{51}$ In some mountainous

19 This is easily accomplished with those gas burners which are used in laboratories and mentioned in the Introduction. In these burners the gas is first mixed with air in a. loug tube, above which it is kindled. But if it be lighted inside the pipe it does not burn completely, but forms acetylene, on account of the cooling effect of the walls of the metallic tube; this is detected by the sinell, and may be shown by passing the issuing gas (by aid of an aspirator) into an ammoniacal solution of cuprous chloride.

so Amongst the homologues of acetylene $\mathrm{C}_{n} \mathrm{H}_{2 n-2}$, the lowest is $\mathrm{C}_{3} \mathrm{H}_{4}$; allylene, $\mathrm{CH}_{5} \mathrm{CCH}$, and allene, $\mathrm{CH}_{2} \mathrm{CCH}_{2}$, are known, but the closed structure, $\mathrm{CH}_{2}(\mathrm{CH})_{2}$, is little investigated.

s1 The saturated hydrocarbons predominate in American petroleum, especially in its more volatile parts; in Baku naphtha the hydrocarbons of the composition $\mathrm{C}_{n} \mathrm{H}_{2 n}$ form the main part (Lisenko, Markovnikoff, Beilstein) but doubtless (Mendeléeff) it also contains saturated ones, $\mathrm{C}_{n} \mathrm{H}_{2 n+2}$. The structure of the naphtha hydrocarbons is only known for the lower homologues, but doubtless the distinction between the hydrocarbons of the Pennsylvanian and Baku naphthas, boiling at the same temperature (after the requisite. 
districts-as, for instance, by the slopes of the Caucasian chain, on inclines lying in a direction parallel to the range-an oily liquid issues from the earth together with salt water and hot gases (methane and others); it has a tarry smell and dark brown colour, and is lighter than water. This liquid is called naphtha or rock oil (petroleum) and is obtained in large quantities by sinking wells and deep bore-holes in those places where traces of naphtha are observed, the naphtha being sometimes thrown up from the wells in fountains of considerable height. ${ }^{52}$ The evolution of naphtha is always accompanied by salt water and marsh gas. Naphtha has from ancient times been worked in Russia in the Apsheron peninsula near Baku, and is also now worked in Burmah (India). in Galicia near the Carpathians, and in America, especially in Pennsylvania and Canada, \&c. Naphtha does not consist of one definite hydrocarbon, but of a mixture of several, and its density, external appearance, and other qualities vary with the amount of the different hydrocarbons of which it is composed. The light kinds of naphtha have a specific gravity about 0.8 and the heavy kinds up to 0.98 . The former are very mobile liquids, and more volatile; the latter contain less of the volatile hydrocarbons and are less mobile. When the light kinds of naphtha are distilled, the boiling. point taken in the vapours constantly changes, beginning at $0^{\circ}$ and going up to above $350^{\circ}$. That which passes over tirst is a very mobile, colourless ethereal liquid (forming gazolene, ligroin, benzoline, \&c.), from which the hydrocarbons whose boiling points start from $0^{\circ}$ may: be extracted-namely, the hydrocarbons $\mathrm{C}_{4} \mathrm{H}_{10}, \mathrm{C}_{5} \mathrm{H}_{12}$ (which boils at $\left.30^{\circ}\right), \mathrm{C}_{6} \mathrm{H}_{14}\left(\right.$ boils at $\left.62^{\circ}\right), \mathrm{C}_{7} \mathrm{H}_{16}$ (boils about $90^{\circ}$ ), dc. Those fractions of the naphtha distillate which boil above $130^{\circ}$, and contain hydrocarbons with $\mathrm{C}_{9}, \mathrm{C}_{10}, \mathrm{C}_{\mathrm{i}}$, drc., enter into the composition of the refining by repeated fractional distillation, which can be very conveniently done by means of steam rectification - that is, by passing the steam through the dense mass), depends not only on the predominance of saturated hydrocarbons in the former, and naphthenes, $\mathrm{C}_{n} \mathrm{H}_{2 n}$, in the latter, but also on the diversity of composition and structure of the corresponding portions of the distillation. The products of the Baku naphtha are richer in carbon (therefore in a suitably constructed lamp they ought to give a brighter light), they are of greater specific gravity, and have greater internal friction (and are therefore more suitable for lubricating machinery) than the American products collected at the same temperature.

32 The formation of naphtha fountains (which burst forth after the higher clay strata covering the layers of sands impregnated with naphtha have been bored through) is without doubt caused by the pressure or tension of the combustible hydrocarbon gases which accompany the naphtha, and are soluble in it under pressure. Sometimes these naphtha fountains reach a height of 100 metres-for instance, the fountain of 1887 near Baku. Naphtha fountains generally act periodically and their force dininishes with the lapse of time, which might be expected, because the gases which cause the fountains find an outlet, as the naphtha issuing from the bore-hole carries away the sand which was partially choking it up. 
oily suhstance, universally used for lighting, called kerosene or photogen or photonaphthalene, and by other names. The specific gravity of kerosene is from 0.78 to 0.84 , and it smells like naphtha. Those products of the distillation of naphtha which pass off below $130^{\circ}$ and have a specific gravity below 0.75 , enter into the composition of light petroleum (benzoline, ligroin, petroleum spirit, \&c.) ; which is used as a solvent for india-rubber, for remoring grease spots, \&c. Those portions of naphtha (which can only be distilled without change by means of superheated steam, otherwise they are largely decomposed) which boil above $275^{\circ}$ and up to $300^{\circ}$ and have a specific gravity higher than 0.85 , form an excellent oil, ${ }^{53}$ safe as regards inflammability (which is very important as diminishing the risks of tire), and may be used in lamps as an effective substitute for kerosene. ${ }^{54}$ Those portions of naphtha which pass over at a still higher temperature and have a higher specific gravity than 0.9 , which are found in abundance (about 30 p.c.) in the Baku naphtha, make excellent lubricating or machine oils. Naphtha has many important applications, and the naphtha industry is now of great commercial importance, especially as naphtha

33 This is a so-called intermediate oil (between kerosene and lubricating oils), solar oil, or pyronaphtha. Lamps are already being manufactured for burning it but still require improvement. Above all, however, it requires a more extended market, and this at present is wanting, owing to the two following reasons : (1) Those products of the American petroleum which are the most widely spread and almost universally consumed contain but little of this intermediate oil, and what there is is divided between the kerosene and the lubricating oils; (2) the Baku naphtha, which is capable of yielding a great deal (up to 30 p.c) of intermediste oil, is produced in enormous quantities, about 300 million poods, but has no regular markets abroad, and for the consumption in Russia (about 25 million poods of kerosene per annum) and for the limited export (60 million poods per annum) into Western Europe (by the Trans-Caucasian Railway) those volatile and more dangerous parts of the naphtha which enter into the composition of the American petroleum are sufficient, although Baku naphtha yields about 25 p.c. of such kerosene. For this reason pyronaphtha is not manufactured in sufficient quantities, and the whole world is consuming the unsafe kerosene. When a pipe line has been laid from Baku to the Black Sea (in America there are many which carry the raw naphtha to the sea-shore, where it is made into kerosene and other products) then the whole mass of the Baku naphtha will furnish safe illuminating oils, which without doubt will find an immense application. A mixture of the intermediate oil with kerosene or Saku oil (specific gravity 0.84 to 0.85 ) may be considered (on removing the benzoline) to be the best illuminating oil, because it is safe (flashing point from $40^{\circ}$ to $60^{\circ}$ ), cheaper (Baku naphtha gives as much as 60 p.c. of Baku oil), and burns perfectly well in lamps differing but little from those made for burning American kerosene (unsafe, flashing point $20^{\circ}$ to $30^{\circ}$ ).

54 The substitution of Baku pyronaphtha, or intermediate oil, or Baku oil (see Note 58), would not only be a great advantage as regards safety from fire, but would also be bighly economical. A ton ( 62 poods) of American crude petroleum costs at the coast considerably more than 24s. (12 roubles), and yields two-thirds of a ton of kerosene suitable for ordinary lamps. A ton of raw naphtha in Baku costs less than 4s. (1 rouble 80 copecks), and with a pipe line to the shore of the Black Sea would not cust more than 8 roubles, or 16s. Moreover, a ton of Baku naphtha will yield as much as two-thirds of a ton of kerosene, Baku oil, and pyronaphtha suitable or illuminating purposes. 


\section{and its refuse may be used as fuel. ${ }^{55}$ Whether naphtha was formed} from organic matter is very doubtful, as it is found in the most ancient Silurian strata which correspond with epochs of the earth's existence when there was little organic matter; it could not penetrate from the higher to the lower (more ancient) strata as it floats on water (and water penetrates through all strata). It therefore tends to rise to the surface of the earth, and it is always found in highlands parallel to the direction of the mountains. ${ }^{56}$ Much more probably its formation may be attributed to the action of water penetrating through the crevasses formed on the mountain slopes and reaching to the heart of the earth, to that kernel of heated metallic matter which must be accepted as existing in the interior of the earth. And as meteoric iron often contains carbon (like cast iron), so, accepting the existence of such carburetted iron at unattainable depths in the interior of the earth, it may be supposed that naphtha was produced by the action of water penetrating through the crevices of the strata during the upheaval of

35 Naphtha has been applied for heating purposes on a large scale in Russia, not only on account of the low cost of naphtha itself and of the residue from the preparation of kerosene, but also because the products of all the Baku naphtha do not find an outlet for general consumption. Naphtba itself and its various residues form excellent fuel, burning without smoke and giving a high temperature (steel and iron may be easily melted in the flame). A hundred poods of good cosl (for instance, Don coal) used as fuel for heating boilers are equivalent to 36 cubio feet (about 250 poods) of dry wood, while only 70 poods of naphtha will be required; and moreover there is no need for stoking, as the liquid can be readily and evenly supplied in the required quantity. The economic and other questions relating to American and Baku petroleums have been discussed more in detail in some separaté works of mine (D. Mendeléeff): (1) 'The Naphtha Industry of Pennsylvania and the Caucasus,' 1870; (2) 'Where to Build Naphtlia Works,' 1880; (3) 'On the Naphtha Question,' 1883; (4) 'The Baku Naphtha Question.' 1886 ; (5) the article on the naphtha industry in the account of the Russian industries printed for the Chicago Exhibition.

so As during the process of the dry distillation of wood, seaweed, and similar vegetable débris, and also when fats ure decomposed by the action of heat (in closed vessels), lydrocarbons similar to those of naphtha are formed, it was natural that this fact should lave been turned to account to explain the formation of the latter. But the hypothesis of the formation of naphtha from vegetable délris inevitably assumes coal to be the chief element of decomposition, and naphtha is met with in Pennsylvania and Canada, in the Silurian and Deronian strata, which do not contain coal, and correspond to an epoch not abounding in organic matter. Coal was formed from the vegetable débris of the Carboniferous, Jurassic, and other recent strata, but judging more from its composition and structure, it has been subjected to the same kind of decomposition as peat; nor could liquid hydrocarbons have been thus formed to such an extent as we see in naphtha. If we ascribe the derivation of naphtha to the decomposition of fat (adipose, animal fat) we encounter three almost insuperable difficulties: (1) Animal remains would furnish a great deal of nitrogenous matter, whilst there is but very little in naphtha; (2) the enormous quaritity of naphtha already discovered as compared with the insignificant amount of fat in the animal carcase; (3) the sources of naphtha always running parallel to mountrin chains is completely inexplicable. Being struck with this last-mentioned circumstance in Pennsylvania, and finding that the sources in the Caucasus surround the whole Caucasian range (Baku, Tiflis, Gouria, Kouban, Taman, Grozṇoe, Dagestan), I developed in 1876 the hypothesis of the mineral origin of naphtha expounded further on. 
mountain chains, ${ }^{57}$ because water with iron carbide ought to give iron oxide and hydrocarbons. ${ }^{58}$ Direct experiment proves that the so-called spiegeleisen (manganiferous iron, rich in chemically combined carbon) when treated with acids gives liquid hydrocarbons 59 which in com-

37 During the upheaval of mountain ranges crevasses would be formed at the peaks with openings upwards, and at the foot of the mountains with openings downwards. These cracks in cour'se of time fill up, but the younger the mountains the fresher the cracks (the Alleglany mountains are, without doubt, more ancient than the Caucasian, which wele formed during the tertiary epoch); through them water must gain access deep into the recesses of the earth to an extent that could not occur on the level (on plains). The situation of naphtha at the foot of mountain chains is the principal argument in my hypothesis.

Another fundamental reason is the consideration of the mean density of the earth. Cavendish, Airy, Comu, Boys, and many others who have investigated the subject by various methods, found that, taking water $=1$, the mean density of the earth is nearly 5.5. As at the surface water and all rocks (sand, clay, limestone, granite, \&c.) have a density less than 8 , it is evident (as solid substances are but slightly compressible even under the greatest pressure) that inside the earth there are substances of a greater density -indeed, not less than 7 or 8 . What conclusion, then, can be arrived at? Anything heavy contrined in the bosom of the earth must be distributed not only on its surface, but throughout the whole solar system, for everything tends to show that the sun and planets are formed from the same material, and according to the hypothesis of Laplace and Kant it is most probable, and indeed must necessarily be held, that the earth and planets are but fragments of the solar atmosphere, which have had time to cool con. siderably and become masses semi-liquid inside and solid outside, forming both planets and satellites. The sun amongst other heavy elements contains a great deal of iron, as shown by spectrum analysis. There is also much of it in an oxidised condition on the surface of the earth. Meteoric stones, carried as fragmentary planets in the solar system and sometimes falling upon the earth, consisting of siliceous rocks similar to terrestrial ones, often contain either dense masses of iron (for example, the Pallosovo iron preserved in the St. Petersburg Academy of Sciences) or granular masses (for instance, the Okhansk meteorite of 1886). It is therefore possible that the interior of the earth contains much iron in a metallic state. This might be anticipated from the hypothesis of Laplace, for the iron must have been compressed into a liquid at that period when the other component parts of the earth were still strengly heated, and oxides of iron could not then have been formed. The iron was covered with slags (mixtures of silicates like glass fused with rocky matter) which did not allow it to burn at the expense of the oxygen of the atmosphere or of water, just at that time when the temperature of the earth was very high. Carbon was in the same state; its oxides were also capable of dissociation (Deville); it is also but slightly volatile, and has an affinity for iron, and iron carbide is found in meteoric stones (as well as carbon and even the diamond). Thus the supposition of the existence of iron carbides in the interior of the earth was derived by me from many indications, which are to some extent confirmed by the fact that granular. pieces of iron have been found in some basalts (ancient lava) as well as in meteoric stones. The occurrence of iron in contact with carbon during the formation of the earth is all the more probable because those elements predominate in nature which have small atomio weights, and among them the most widely diffused, the most difficultly fusible, and therefore the most easily condensed (Chapter XV.) are carbon and iron. They passed into the liquid state when all compounds were at a temperature of dissociation.

38 The following is the typical equation for this formation:

$8 \mathrm{Fe}_{n} \mathrm{C}_{n}+44_{m} \mathrm{H}_{2} \mathrm{O}=m \mathrm{Fe}_{5} \mathrm{O}_{4}$ (magnetic oxide) $+\mathrm{C}_{5 n} \mathrm{H}_{8 m}$ (see Chapter XVII., Note 38).

59 Cloez investigated the bydrocarbons formed when cast-iron is dissolved in hydrochloric acid, and found $\mathrm{C}_{n} \mathrm{H}_{2 n}$ and others. I treated crystalline manganiferous cast-iron with the same acid, and obtained a liquid mixture of hydrocaxbons exactly similar to natural naphtha in taste, smell, and reaction. 


\section{position, appearance, and properties are completely identical with naphtha. ${ }^{60}$}

60 Probably naphtha was produced during the upheaval of all mountain chains, but only in some cases were the conditions favourable to its being preserved underground. The water penetrating below formed there a mixture of naphtha and watery vapours, and this mixture issued through fissures to the cold parts of the earth's crust. The raphtha vapours, on condensing, formed naphtha, which, if there were no obstacles, appeared on the surface of land and water. Here part of it soaked through formations (possibly the bituminous slates, schist3, dolomites, \&c., were thus formed), another part was carried away on the water, became oxidised, evaporated and was driven to the shores (the Caucasian naphtha probably in this way, during the existence of the Aralo. Caspian sea, was carried as far us the Sirran banks of the Volga, where many strata are impregnated with naphtha and products of its oxidation resembling asphalt and pitch); a great.part of it was burnt in one way or another-that is, gave carbonic anhydride and water. If the mixture of vapours, water, and naphtha formed inside the earth had no free outlet to the surface, it nevertheless would find its way through fissures to the superior and colder strata, and there become condensed. Some of the formations (clays) which do not absorb naphtha were only washed away by the warm water, and formed mud, which we also now observe issuing from the earth in the form of mud volcanoes. The neighbourhood of Baku and the whole of the Caucasus near the naphtha districts are full of such volcanoes, which from time to time are in a state of eruption. In old naphtha beds (such as the Pennsylvanian) even these blow-holes are closed, and. the mud rolcanoes have had time to be washed away. The naphtha and the gaseous: hydrocarbons formed with it under the pressure of the overlying earth and water im. pregnated the layers of sand, which are capable of absorbing a great quantity of such liquid, and if above this there were strata impermeable to naphtha (dense, clayey, damp strata) the naphtha would accumulate in them. It is thus preserved from remote geological periods up to the present day, compressed and dissolved under the pressure of the gases which burst out in places forming naphtha fountains. If this be granted, it may be thought that in" the comparatively new (geologically speaking) mountain chains such as the Caucasian, naphtha is even now being formed. Such a supposition may explain the remarkable fact that, in Pennsylvania, localities where naphtha had been rapidly worked for five years have become exhausted, and it becomes necessary to constantly have recourse to sinking new wells in fresh places. Thus, from the year 1859 , the workings were gradually transferred along a line running parallel to the Alleghany mountains for a distance of more than 200 miles, whilst in Baku the industry dates from, time immemorial (the Persians worked near the village of Ballaghana) and up to the present tims keeps to one and the same place. The amounts of the Pennsylvanian and Baku annual outputs are at present equal-namely, about 250 million poods (4 million tons). It may be that the Baku beds, as being of more recent geological formation, are not so exhausted by nature as those of Pennsylvaniu, and perhaps in the neighbourhood of Baku,naphtha, is still being formed, which is partially indicated by the continued activity of the mud volcanoes. As many varieties of naphtha contain in solution solid slightly volatile hydrocarbons like paraffin and mineral wax, the production of ozocerite, or mountain wax, is accounted for in conjunction with the formation of naphtha. Ozocerite is found in Galicia, also in the neighbourhood of Novorossisk, in the Caucrsus, and on the islands of the Caspian Sea (particularly in the Chileken and Holy Islands); it is met with in large masses, and is used for the production of paraffin and ceresene, for the manufacture of candles, and similar purposes.

As the naphtha treasures of the Caucasus have hardly been exploited (near Baku and near Kouban and Grosnyi), and as naphtha finds numerous uses, the subject presents most interesting features to chemists and geologists, and is worthy of the close attention cf practical men. 


\section{CHAPTER IX}

\section{COMPOUNDS OF CARBON WITH OXYGEN AND NITROGEA}

Carbonic anhydride (or carbonic acid or carbon dioxide, $\mathrm{CO}_{2}$ ) was tho first of all gases distinguished from atmospheric air. Paracelsus and Van Helmont, in the sixteenth century, knew that on heating limestone a particular gas separated, which is also formed during the alcoholic fermentation of saccharine solutions (for instance, in the manufacture of wine); they knew that it was identical with the gas which is produced by the combustion of charcoal, and that in some cases it is found

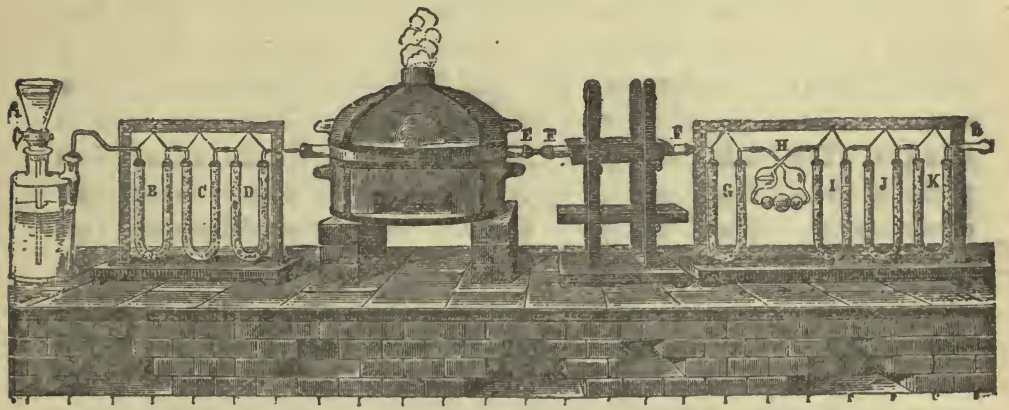

FrG. 61.-Dumas and Stas' apparatus for Aetermining the composition of carbonic anbydride. Carbon, graphite, or a diamond is placed in the tube $\mathrm{E}$ in the furnace, and heated in a stream of oxygen displaced from the bottle by water flowing from A. The oxygen is purified from carbonic anhydride and water in the tubes B, C, D. Carbonic anhydride, together with a certain amount of carbon monoxide, is formed in $\mathrm{E}$. The latter is converted into carbonic acid by passing the products of oombustion through a tube $\mathrm{F}$, containing cupric oxide heated in a furnace. The cuprio oxide oxidises this $\mathrm{CO}$ into $\mathrm{CO}_{2}$, forming metallio copper. The potash bulbs $\mathrm{H}$ and tubes I, J, $\mathbf{K}$ retain the carbonic anhydride. Thus, knowing the weight of carbon taken and the weiglit of the resultant carbonic anhydride (by weighing H, I, J, K before and after the experiment), the composition of carbonio anhydride and the equivalent of carbon may be determined.

in nature. In course of time it was found that this gas is absorbed by alkali, forming a salt which, under the action of acid, again yields this same gas. Priestley found that this gas exists in air, and Lavoisier determined its formation during respiration, combustion, putrefaction, and during the reduction of the oxides of metals by charcoal; he determined its composition, and showed that it only contains oxygen and carbon. Berzelius, Dumas with Stas, and Roscoe, determined its com. 
position, showing that it contains twelve parts of carbon to thirty-two of oxygen. The composition by volume of this gas is determined from the fact that during the combustion of charcoal in oxygen, the volumeremains unchanged; that is to say, carbonic anhydride occupies the same volume as the oxygen which it contains-that is, the atoms of the carbon are, so to speak, squeezed in between the atoms of the oxygen. $\mathrm{O}_{2}$ occupies two volumes and is a molecule of ordinary oxygen; $\mathrm{CO}_{2}$ likewise occupies two volumes, and expresses the composition and molecular weight of the gas. Carbonic anhydride exists in nature, both in a free state and in the most varied compounds. In a free state it is always contained (Chapter V.) in the air, and in solution is in all kinds of water. It is evolved from volcanoes, from mountain fissures, and in some caves. The well-known Dog grotto, near Agnano on the bay of Baiæ, near Naples, furnishes the best known example of such an evolution. Similar sources of carbonic anhydride are also found in other places. In France, for instance, there is a well-known poisonous fountain in Auvergne. It is a round hole, surrounded with luxurious vegetation and constantly evolving carbonic ashyddride. In the woods surrounding the Lacher See near the Rhine, in the neighbourhood of extinct volcanoes, there is a depression constantly filled with this same gas. The insects which fly to this place perish, animals being unable to breathe this gas. The birds chasing the insects also die, and this is turned to profit by the local peasantry. Many mineral springs carry into the air enormous quantities of this gas. Vichy in France, Sprüdel in Germany, and Narzan in Russia (in Kislovodsk near Piatigorsk) are known for their carbonated gaseous waters. Much of this gas is also evolved in mines, cellars, diggings, and wells. People descending into such places are suffocated. The combustion, putrefaction, and fermentation of organic substances give rise to the formation of carbonic anhydride. It is also introduced into the atmosphere during the respiration of animals at all times and during the respiration of plants in darkness and also during their growth. Very simple experiments prove the formation of carbonic anhydride under these circumstances; thus, for example, if the air expelled from the lungs be passed through a glass tube into a transparent solution of lime (or baryta) in water a white precipitate will soon be formed consisting of an insoluble compound of lime and carbonic anhydride. By allowing the seeds of plants to grow under a bell jar, or in a closed vessel, the formation of carbonic anhydride may be similarly confirmed. By confining an animal, a mouse, for instance, under a bell jar, the quantity of carbonic acid which it evolves may be exactly determined, and it will be found to be many grams per day for a mouse. Such experiments on the respiration of animals have 
been also made with great exactitude with large animals, such as men, bulls, sheep, \&c. By means of enormous hermetically closed bell receivers and the analysis of the gases evolved during respiration it was found that a man expels about 900 grams (more than two pounds) of : carbonic anhydride per diem, and absorbs during this time 700 grams of oxygen.' It must be remarked that the carbonic anhydride of the air constitutes the fundamental food of plants (Chapters III., V., and VIII.) Carbonic anhydride in a state of combination with a variety of other substances is perhaps even more widely distributed in nature than in a free state. Some of these substances are very stable and form a large portion of the earth's crust. For instance, limestones, calcium carbonate, $\mathrm{CaCO}_{3}$, were formed as precipitates in the seas existing preriously on the earth; this is proved by their stratified structure and the number of remains of sea animals which they frequently contain. Chalk, lithographic stone, limestone, marls (a mixture of limestone and clay), and many other rocks are examples of such sedimentary formations.

1 The quantity of carbonic acid gas exhaled by a man during the twenty-four hours is not evenly produced; during the night more oxygen is taken in than during the day (by night, in twelve hours, about 450 grams), and more carbonic anhydride is sepa. rated by day than during night-time and repose; thus, of the 900 grams produced during the tiwenty-four hours about 375 are given out during the night and 525 by day. This depends on the formation of carbonic anhydride during the work performed by the man in the day. Every movement is the result of some change of matter, for force cannot be self-created (in accordance with the law of the conservation of energy). Proportionally to the amount of carbon consumed an amount of energy is stored up in the organism and is consumed in the various movements performed by animals. This is proved by the fact that during work a man exhales 525 grams of carbonic anhydride in twelve hours instead of 375 , absorbing the same amount of oxygen as before. After a working day a man exhales by night almost the same anount of carbonic anhydride as after a day of rest, so that during a total twenty-four hours a man exhales about 900 grams of carbonic anhydride and absorbs about 980 grams of oxygen. Therefore during work the change of matter increases. The carbon expended on the work is obtained from the food; on this account the food of animals ought certainly to contain carbonaceous substances capable of dissolving under the action of the digestive fluids, and of passing into the blood, or, in other words, capable of being digested. Such food for man and all other animals is formed of vegetable matter, or of parts of other animals. The latter in every case obtain their carbonaceous matter from plants, in which it is formed by the separation of the carbon from the carbonic anhydride taken up during the day by the respiration of the plants. The volume of the oxygen exhaled by plants is almost equal to the volume of the carbonic anhydride absorbed; that is to say, nearly all the oxygen entering into the plant in the form of carbonic anhydride is libe. rated in a free state, whilst the carbon from the carbonic anhydride remains in the plant. At the same time the plant absorbs moisture by its leaves and roots. By a process which is unknown to us, this absorbed moisture and the carbon obtained from the carbonic an. hydride enter into the composition of the plants in the form of so-called carbohydrates, composing the greater part of the vegetable tissues, starch and cellulose of the com. position $\mathrm{C}_{6} \mathrm{H}_{10} \mathrm{O}_{5}$ being representatives of them. They may be considered like all carbo. hydrates as compounds of carbon and water, $6 \mathrm{C}+5 \mathrm{H}_{2} \mathrm{O}$. In this way a circulation of the carbon goes on in nature by means of vegetable and animal organisms, in which changes the principal factor is the carbonic anhydride of the air. 
Carbonates with various other bases - such as, for instance, magnesia, ferrous oxide, zinc oxide, \&c. - - are often found in nature. The shells of molluses also have the composition $\mathrm{CaCO}_{3}$, and many limestones were exclusively formed from the shells of minute organisms. As carbonic anhydride (together with water) is produced during the combustion of all organic compounds in a stream of oxygen or by heating them with substances which readily part with their oxygen-for instance, with copper oxide - this method is employed for estimating the amount of carbon in organic compounds, more especially as the $\mathrm{CO}_{2}$ can be easily collected and the amount of carbon calculated from its weight. For this purpose a hard glass tube, closed at one end, is filled with a mixture of the organic substance (about 0.2 gram) and copper oxide. The open end of the tube is fitted with a cork and tube containing

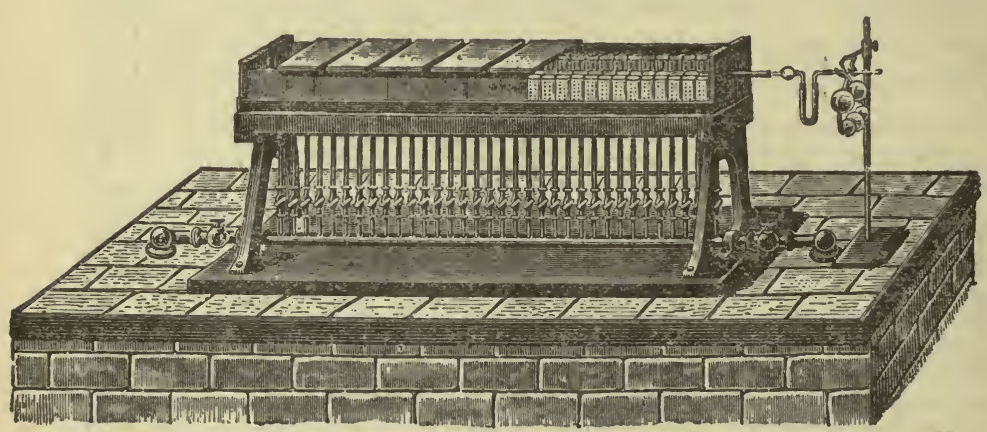

Frg. 62,-Apparatus for the combustion of organic substances by igniting them with oxide of copper.

calcium chloride for absorbing the water formed by the oxidation of the substance. This tube is hermetically connected (by a caoutchouc tube) with potash bulbs or other weighing apparatus (Chapter V.) containing alkali destined to absorb the carbonic anhydride. The increase in weight of this apparatus shows the amounts of carbonic anhydride formed during the combustion of the given substance, and the quantity of carbon may be determined from this, because three parts of carbon give eleven parts of carbonic anhydride.

For the preparation of carbonic anhydride in laboratories and often. in manufactories, various kinds of calcium carbonate are used, being treated with some acid; it is, however, most usual to employ the so. called muriatic acid-that is, an aquenus solution of hydrochloric acid, $\mathrm{HCl}$--because, in the first place, the substance formed, calcium chloride. $\mathrm{CaCl}_{2}$, is soluble in water and does not hinder the further 
action of the acid on the calcium carbonate, and secondly because, as we shall see further on, muriatic acid is a common product of chemical, works and one of the cheapest. For calcium carbonate, either limestone, chalk, or marble is used. ${ }^{2}$

$$
\mathrm{CaCO}_{3}+2 \mathrm{HCl}=\mathrm{CaCl}_{2}+\mathrm{H}_{2} \mathrm{O}+\mathrm{CO}_{2} \text {. }
$$

The nature of the reaction in this case is the same as in the decom. position of nitre by sulphuric acid ; only in the latter case a hydrate is formed, and in the former an anhydride of the acid, because the hydrate, carbonic acid, $\mathrm{H}_{2} \mathrm{CO}_{3}$, is unstable and as soon as it separates decomposes into water and its own anhydride. It is evident from the explanation of the cause of the action of sulphuric acid on nitre that not every acid can be employed for obtaining carbonic anhydride; namely, those will not set it free which chernically are but slightly energetic, or those which are insoluble in water, or are themselves as volatile as carbonic anhydricie. ${ }^{3}$ But as many acids are soluble in water and are less volatile than carbonic anhydride, the latter is evolved by the action of most acids on its salts, and this reaction takes place at ordinary temperatures. ${ }^{4}$

2 Other acids may be used instead of hydrochloric; for instance, ncetic, or even sulphuric, although this latter is not suitable, because it forms as a product insoluble calcium sulphate (gypsum) which surrounds the untouched calcium carbonate, and thus prevents a further evolution of gas. But if porous limestone-for instance, chalk-be treated with sulphuric acid diluted with an equal volume of water, the liquid is absorbed and acting on the mass of the salt, the evolution of carbonic anhydride continues evenly for a long time. Instead of calcium carbonale other carbonates may of course be used; for instance, washing-soda, $\mathrm{Na}_{2} \mathrm{CO}_{3}$, which is often chosen when it is required to produce a rapid stream of carbonic anhydride (for example, for liquefying it). But natural crystalline magnesium carbonate and similar salts are with difficulty decomposed by hydrochloric and sulphuric acids. When for manufacturing purposes-for instance, in precipitating lime in sugar-works-a large quantity of carbonic acid gas is required, it is generally obtained by burning charcoal, and the products of combustion, rich in carbonic anhydride, are pumped into the liquid containing the lime, and the carbonio anhydride is thus absorbed. Another method is also practised, which consists in using the carbonic anhydride separated during fermentation, or that evolved from limekilns. During the fermentation of sweet-wort, grape-juice, and other similar saccharine solutions, the glucose $\mathrm{C}_{6} \mathrm{H}_{12} \mathrm{O}_{6}$ changes under the intluence of the yeast organism, forming alcohol $\left(2 \mathrm{C}_{2} \mathrm{H}_{6} \mathrm{O}\right)$, and carbonic anhydride $\left(2 \mathrm{CO}_{2}\right)$ which separates in the form of gas; if the fermentation proceeds in closed bottles sparkling wine is obtained. When carbonic acid gas is prepared for saturating water and other beverages it is necessary to use it in a pure. state. Whilst in the state in which it is evolved from ordinary limestones by the aid of acids it contains, besides a certain quantity of acid, the organic matters of the limestone; in order to diminish the quantity of these substances the densest kinds of dolomites are used, which contain less organic matter, and the gas formed is passed through various washing apparatus, and then throngh a solution of potassium permanganate, which absorbs organio matter and does not take up carbonic anhydride.

5 Hypochlorous acid, $\mathrm{HClO}$, and its anhydride, $\mathrm{Cl}_{2} \mathrm{O}$, do not displace carbonic acid, and lyydrogen sulphide has the same relation to carbonic acid as nitric acid to hydrochlorioan excess of either one displaces the other.

4 Thus, in preparing the ordinary effervescing powders, sodium bicarbonate (or acid 
For the preparation of carbonic anhydride in laboratories, marble is generally used. It is placed in a Woulfe's bottle and treated with hydro. chloric acid in an apparatus similar to the one used for the production of hydrogen. The gas evolved carries away through the tube part of the volatile hydrochloric acid, and it is therefore necessary to wash the gas by passing it through another Woulfe's bottle containing water. If it be necessary to obtain dry carbonic anhydride, it must be passed through chloride of calcium. ${ }^{5}$

Carbonic anhydride may also be prepared by heating many of the salts of carbonic acid ; for instance, by heating magnesium carbonate, $\mathrm{MgCO}_{3}$ (e.g., in the form of dolomite), the separation is easily effected, particularly in the presence of the vapours of water. The acid salts of carbonic acid (for instance, $\mathrm{NaHCO}_{3}$, see further on) readily and abundantly give carbonic anhydride when heated.

Carbonic anhydride is colourless, has a slight smell and a faint acid taste; its density in a gaseous state is twenty-two times as great as that of hydrogen, because its molecular weight is forty - four. ${ }^{6}$

carbonate of soda) is used, and mixed with powdered citrio or tartaric acid. In a dry state these powders do not evolve carbonic anhydride, but when mixed with water the evolution takes place briskly, which is due to the substances passing into solution. The salts of carbonic acid may be recognised from the fact that they ovolve carbonic acid with a hissing noise when treated with acids. If vinegar, which contains acetic acid, be poured upon limestone, marble, malachite (containing copper carbonate), \&c., carbonic anhydride is evolved with a hissing noise. It is noteworthy that, neither hydrochlorio acid, nor even sulphuric acid nor acetic acid, acts on limestone except in presence of water. We shall refer to this later on.

s The direct observations made (1876) by Messrs. Bogouski and Kayander lead to the conclusion that the quantity of carbonic anhydride evolved by the action of acids on. marble (as homogeneous as possible) is direstly proportional to the time of action, the extent of surface, and the degree of concentration of the acid, and inversely proportional to the molecular weight of the acid. If the surface of a piece of Carrara marble be equal to one decimetre, the time of action one minute, and one cubio decimetre or litre contains one gram of hydrochlorio acid, then about 0.02 gram of carbonio anhydride will be evolved. If the litre contains $n$ grams of hydrochloric acid, then by experiment the amount will be $n \times 0.02$ of carbonic anhydride. Therefore, if the litre contains 36.5 . $(=\mathrm{HCl})$ grams, about $0.73 \mathrm{gram}$ of carbonic anhydride (about half a litre) would be: evolved per minute. If nitric acid or hydrobromic acid be used instead of hydrochloric, then, with a combining proportion of the acid, the same quantity of carbonio anhydride will be evolved; thus, if the litre contains $63\left(=\mathrm{HNO}_{3}\right)$ grams of nitric acid, or 81 $(=\mathrm{HBr})$ grams of hydrobromic acid, the quantity of carbonic anhydride evolved will still. be 0.73 gram. Spring, in 1890 , made a series of similar determinations.

- As carbonic anhydride is one and a half times heavier than air, it diffuses with difficulty, and therefore does not easily mix with air; but sinks in it. This may be shown in various ways; for instance, the gas may be carefully poured from one vessel into another containing air. If a lighted taper be plunged into the vessel containing carbonic anhy. dride it is extinguished, and then, after pouring the gas into the other cylinder, if will burn in the former and be extinguished in the latter. If a certain quantity of carbonic anhydride be potred into a vessel containing air, and soap-bubbles be introduced, they will only sink as far as the stratum where the atmosphere of carbonic anhydride com. mences, as this latter is heavier than the soap-bubbles filled with air. Naturally, after a 
It is an example of those gaseous substances which have been long ago transformed into all the three states. In order to obtain liquid carbonic anhydride, the gas must be submitted to a pressure of thirty-six atmospheres at $0^{0.7}$ Its absolute boiling point $=+32^{\circ} .8$ Liquid carbonic anhydride is colourless, does not mix with water, but is soluble in alcohol, ether, and oils ; at $0^{\circ}$ its specific gravity 15. $0.83 . .^{8}$ bis The boiling point of this liquid lies at $-80^{\circ}$-that is to say, the pressure of carbonic acid gas at that temperature does not exceed that of the atmosphere. At the ordinary temperature the liquid remains as such for some time under ordinary pressure, on account of its requiring a considerable amount of heat for its evaporation. If the evaporation takes place rapidly, especially if the liquid issues in a stream, such a decrease of temperature occurs that a part of the carbonic anhydride is transformed into a solid snowy mass. Water, mercury, and many other liquids freeze on coming into contact with snow-like carbonic anhydride. ${ }^{9}$ In this form carbonic anhydride may be preserved for a long time in the open air, because it requires still more heat to turn it into a gas than when in a liquid state. 9 bls

The capacity which carbonic anhydride has of being liquefied stands

certain lapse of time, the carbonic anhydride will be diffused throughout the vessel, and form a uniform mixture with the air, just as salt in water.

7 This liquefaction was first observed by Faraday, who sealed up in a tube a mixture of a carbonate and sulphuric acid. Afterwards this method was very considerably improved by Thilorier and Natterer, whose apparatus is given in Chapter VI. in describ. ing $\mathrm{N}_{2} \mathrm{O}$. It is, however, necessary to remark that the preparation of liquid carbonio anhydride requires good liquefying apparatus, constant cooling, and a rapid preparation of large masses of carbonic anhydride.

8 Carbonic anbydride, having the same molecular weight as nitrous oxide, very much resembles it when in a liquid state.

8 bis When poured into a tube, which is then sealed un, liquefied carboinic anluydride can be easily preserved, because a thick tube easily supports the pressure (about 50 atmospheres) exerted by the liquid at the ordinary temperature.

$\$$ When a fine stream of liquid carbonic anhydride is discharged into a closed metallio vessel, about one-third of its mass solidifies and the remainder evaporates. In employing solid carbonic anlydride for making experiments at low teniperatures, it is best to use it mixed with ether, otherwise there will be few points of contact. If a stream of air be blown through a mixture of liquid carbonic anhydride and ether, the evaporation proceeds rapidly, and great cold is obtained. At present in some special manufactories (and for making artificial mineral waters) carbonic anhydride is liquefied on the large scale, filled into wrought-iron cylinders provided with a valve, and in this manner it can be trans. ported and preserved safely for a long time. It is used, for instance, in breweries.

9 bls Solid carbonic anhydride, notwithstanding its very low tempernture, can be safely placed on the hand, because it continually evolves gas which prevents its coming into actual contact with the skin, but if a piece be squeezed between the fingers, it pro. duces a severe frost bite similar to a burn. If the snowlike solid be mixed with ether, a semi-liquid mass is obtained, which is employed for artificial refrigeration. This mixture may be used for liquefying many other gases - such as chlorine, nitrous oxide, hydrogen sulphicle, and others. The evaporation of such a mixture proceeds with far greater 
in connection with its considerable solubility in water, alcohol, and other liquids. Its solubility in water has been already spoken of in the first chapter. Carbonic anbydride is still more soluble in alcohol than in water, nameiy at $0^{\circ}$ one volume of alçohol dissolves 4.3 volumes of this gas, and at $20^{\circ} 2.9$ volumes.

Aqueous solutions of carbonic anhydride, under a pressure of several atmospheres, are now prepared artificially, because water saturated with this gas promotes digestion and quenches thirst. For this purpose the carbonic anhydride is pumped by means of a force-pump into a closed vessel containing the liquid, and then bottled off, taking special means to ensure rapid and air-tight corking. Various effervescing drinks and artificially effervescing wines are thus prepared. The presence of carbonic anhydride has an important significance in nature, because by its means water acquires the property of decomposing and dissolving many substances which are not acted on by pure water; for instance, calcium phosphates and carbonates are soluble in water containing carbonic acid. If the water in the interior of the earth is saturated with carbonic acid under pressure, the quantity of calcium carbonate in solution may reach three grams per litre, and on issuing at the surface, as the carbonic anhydride escapes, the calcium carbonate will be deposited.10 Water charged with carbonic anhydride brings about the destruction of many rocky formations by removing the lime, alkali, \&rc., from them. This process has been going on and continues on an enormous scale. Rocks

rapidity under the receiver of an air-pump, and consequently the refrigeration is more intense. By this means many gases may be liquefied which resist other methodsnamiely, olefiant gas, hydrochloric acid gas, and others. Liquid earbonic anhydride in this case congeals in the tube into a glassy transparent mass. Pictet availed himself of this method for liquefying many permanent gases (see Chapter II.)

Bleekrode, by compressing solid $\mathrm{CO}_{2}$ in a cylinder by means of a piston, obtained a semi-transparent stick, which contained as much as 1.3 and even 1.6 gram of $\mathrm{CO}_{2}$ per cubic centimetre. In this form the $\mathrm{CO}_{2}$ slowly evaporated, and could be kept for a long time.

20 If such water trickles through crevices and enters a cavern, the evaporation will be slow, and therefore in those places from which the water drips growths of calcium carbonate will be formed, just like the icicles formed on the roof-gutters in winter-time. Similar conical and cylindrical stony growths form the so-called stalactites or pendants hanging from above and stalagmites formed on the bottom of caves. Sometimes these two kinds meet together, forming entire columns filling the cave. Many of these caves are remarkable for their picturesqueness; for instance, the cave of Antiparos, in the Grecian Archipelago. This same cause also forms spongy masses of calcium curbonate in.those places where the springs come to the surface of the earth. It is therefore very evident that a calcareous solution is sometimes capable of penetrating plants and filling the whole of their mass with calcium carbonate. This is one of the forms of petrified plants. Calcium phosphate in solution in water containing carbonic acid plays an im. portant part in the nourishment of plants, because all plants contain both lime and phosphoric acid. 
contain silica and the oxides of various metals; amongst others. the oxides of aluminium, calcium, and sodium. Water charged with carbonic acid dissolves both the latter, transforming them into carbonates. The waters of the ocean ought, as the evolution of the carbonic anhydride proceeds, to precipitate salts of lime; these are actually found everywhere on the surface of the ground in those places which previously formed the bed of the ocean. The presence of carbonic anhydride in solution in water is essential to the nourishment and growth of water plants.

Although carbonic anhydride is soluble in water, yet no definite nydrate is formed; $"$ nevertheless an idea of the composition of this hydrate may be formed from that of the salts of carbonic acid, because a hydrate is nothing but a salt in which the metal is replaced by: hydrogen. As carbonic anhydride forms salts of the composition $\mathrm{K}_{2} \mathrm{CO}_{3}, \mathrm{Na}_{2} \mathrm{CO}_{3}, \mathrm{HNaCO}_{3}$, \&c., therefore carbonic acid ought to have the composition $\mathrm{H}_{2} \mathrm{CO}_{3}$-that is, it ought to contain $\mathrm{CO}_{2}+\mathrm{H}_{2} \mathrm{O}$. Whenever this substance is formed, it decomposes into its component: parts--that is, into water and carbonic anhydride. The acid properties of carbonic anhydride ${ }^{11}$ bis are demonstrated by its being directly absorbed by alkaline solutions and forming salts with them. In distinction from nitric, $\mathrm{HNO}_{3}$, and similar monobasic acids which with univalent metals (exchanging one atom for one atom of hydrogen) give salts such as those of potassium, sodium, and silver containing only one atom of the metal $\left(\mathrm{NaNO}_{3}, \mathrm{AgNO}_{3}\right)$, and with bivalent ${ }^{12}$ metals (such as calcium, barium, lead) salts containing two acid groups - for example, $\mathrm{Ca}\left(\mathrm{NO}_{3}\right)_{2}$, $\mathrm{Pb}\left(\mathrm{NO}_{3}\right)_{2}$-carbonic acid, $\mathrm{H}_{2} \mathrm{CO}_{3}$, is bibasic, that is contains two atoms of hydrogen in the hydrate or two atoms of univalent metals in their salts : for example. $\mathrm{Na}_{2} \mathrm{CO}_{3}$ is washing soda, a normal salt ; $\mathrm{NaHCO}_{3}$ is the bicarbonate, an acid salt. Therefore, if $\mathrm{M}^{\prime}$ be a univalent metal, its carbonates in general are the normal carbonate $\mathrm{M}_{2}^{\prime} \mathrm{CO}_{3}$ and the

11 The crystalluhydrate, $\mathrm{CO}_{2}, 8 \mathrm{H}_{2} \mathrm{O}$ of Wroblewski (Chapter I., Note 67), in the first place, is only formed under special conditions; in the second place, its existence still requires confirmation; and in the third place, it does not correspond with that hydrate. $\mathrm{H}_{2} \mathrm{CO}_{3}$,which should occur, judging from the composition of the salts.

11 bis It is easy to demonstrate the acid properties of carbonic anhydride by taking a long tube, closed at one end, and filling it with this gas; a test-tube is then filled with a solution of an alkali (for instance, sodium hydroxide), which is then poured into the long tube and the open end is corked. The solution is then well shaken in the tube, and the corked end plunged into water. If the cork be now withdrawn under water, the water will fill the tube. The vacuum obtained by the absorption of the carbonio anhydride by an alkali is so complete that even an electric discharge will not pass through it. This method is often applied to produce a vacurum.

12 The reasons for distinguishing the uni-, bi-, tri-, and quadri-valent metals will be explained hereafter on passing from the univalent metals $(\mathrm{Na}, \mathrm{K}, \mathrm{Li})$ to the bivalent (Mg. Ca, Ba), Chapter XIV 
acid carbonate, $\mathrm{M}^{\prime} \mathrm{HCO}_{3}$; or if $\mathrm{M}^{\prime \prime}$. be a bivalent metal (replacing $\mathrm{H}_{2}$ ) its normal carbonate will be $\mathrm{M}^{\prime \prime} \mathrm{CO}_{3}$; these metals do not usually form acid salts, as we shall see further on. The bibasic character of carbonic acid is akin to that of sulphuric acid, $\mathrm{H}_{2} \mathrm{SO}_{4}{ }^{13}$ but the latter, in distinction from the former, is an example of the energetic or strong acids (such as nitric or hydrochloric), whilst in carbonic acid we observe but feeble development of the acid properties; hence carbonic acid must be con. sidered a vieak acid. This conception must, however, be taken as only comparative, as up to this time theze is no definitely established rule for measuring the encrgy ${ }^{14}$ of acids. The feeble acid properties of carbonic

is Up to the year 1840 , or thereabout, acids were not distinguished by their basicity. Graham, while studying phosphoric acid, $\mathrm{H}_{3} \mathrm{PO}_{4}$, and Liebig, while studying many organic acids, distinguished mono-, bi-, and tri-basic acids. Gerhardt and Laurent generalised these relations, showing that this distinction extends over many reactions (for instance, to the faculty of bibasic acids of forming acid salts with alkalis, $\mathrm{KHO}$ or $\mathrm{NaHO}$, or with alcohols, RHO, \&c.) ; but now, since a definite:conception as to atoms and molecules has been arrived at, the basicity of an acid is determined by the number of hydrogen atoms, contained in a molecule of the acid, which can be exchanged for metals. If carbonic acid forms acid salts, $\mathrm{NaHCO}_{3}$, and normal salts, $\mathrm{Na}_{2} \mathrm{CO}_{3}$, it is evident that the hydrate is $\mathrm{H}_{2} \mathrm{CO}_{3}$, a bibasic acid. Otherwise it is at present impossible to account for the composition of these salts. But when $\mathrm{C}=6$ and $\mathrm{O}=8$ were taken, then the formula $\mathrm{CO}_{2}$ expressed the composition, but not the molecular weight, of carbonic anhydride ; and the composition of the normal salt would be $\mathrm{Na}_{2} \mathrm{C}_{2} \mathrm{O}_{6}$ or $\mathrm{NaCO}_{3}$, therefore carbonic acid imight have been considered as a monobasic acid. Then the acid salt would have been represented by $\mathrm{NaCO}_{3}, \mathrm{HCO}_{3}$. Such questions were the cause of much argument and difference of opinion among chemists about forty years ago. At present there cannot be two opinions on the subject if the law of Avogadro-Gerhardt and its consequences be strictly adhered to. It may, however, be observed here that the monobasic acids $\mathrm{R}(\mathrm{OH})$ were for a long time considered to be incapable of being decomposed into water and anhydride, and this property was ascribed to the bibasic acids $\mathrm{R}(\mathrm{OH})_{2}$ as containing the elements necessary for the separation of the molecule of water, $\mathrm{H}_{2} \mathrm{O}$. Thus $\mathrm{H}_{2} \mathrm{SO}_{4}$ or $\mathrm{SO}_{2}(\mathrm{OH})_{2}$, $\mathrm{H}_{2} \mathrm{CO}_{3}$, or $\mathrm{CO}(\mathrm{OH})_{2}$, and other bibasic acids decompose into an anhydride, $\mathrm{RO}$, and water, $\mathrm{H}_{2} \mathrm{O}$. But as nitrous, $\mathrm{HNO}_{2}$, iodic, $\mathrm{HIO}_{3}$, hypochlorous, $\mathrm{HClO}$, and other monobasio acids easily give their anhydrides $\mathrm{N}_{2} \mathrm{O}_{3}, \mathrm{I}_{2} \mathrm{O}_{5}, \mathrm{Cl}_{2} \mathrm{O}$, \&c., that method of distinguishing the basieity of acids, although it fairly well satisfies the requirements of organic chemistry, cannot. be considered correct. It may also be remarked that up to the present time not one of the bibasic acids has been found to have the faculty of being distilled without being decomposed into anhydride and water (even $\mathrm{H}_{2} \mathrm{SO}_{k}$, on being evpaporated and distilled, gives $\mathrm{SO}_{3}+\mathrm{H}_{2} \mathrm{O}$ ), and the decomposition of acids into water and anhydride proceeds particularly easily in dealing with feebly energetic acids, such as carbonic, nitrous, boric, and hypochlorons. Let us add that carbonic acid, as a hydrate corresponding to marsh gas, $\mathrm{C}(\mathrm{HO})_{4}=\mathrm{CO}_{2}+2 \mathrm{H}_{2} \mathrm{O}$, ought to be tetrabasic. But in general it does not form such salts. Basic salts, however, such as $\mathrm{CuCO}_{5} \mathrm{CuO}$, may be regarded in this sense, for $\mathrm{CCu}_{2} \mathrm{O}_{4}$ corresponds with $\mathrm{CH}_{4} \mathrm{O}_{4}$, as $\mathrm{Cu}$ corresponds with $\mathrm{H}_{2}$. Amongst the ethereal salts (alcoholic derivatives) of carbonic acid corresponding cases are, however, observed; for instance, ethylic orthocarbonate, $\mathrm{C}\left(\mathrm{C}_{2} \mathrm{H}_{5} \mathrm{O}\right)_{4}$ (obtained by the action of chloropicrin, $\mathrm{C}\left(\mathrm{NO}_{2}\right) \mathrm{Cl}_{3}$, on sodium ethoside, $\mathrm{C}_{2} \mathrm{H}_{5} \mathrm{ONa}$; boiling point $158^{\circ}$; specifio gravity, 0.92). The name orthocarbonic acid for $\mathrm{CH}_{4} \mathrm{O}_{4}$ is taken from orthophosphoric acid, $\mathrm{PH}_{3} \mathrm{O}_{4}$, which corresponds with $\mathrm{PH}_{3}$ (sec Chapter on Phosphorus).

14 Long ago endeavours were made to find a measure of affinity of acids and bases, beceuse some of the ucids, such as sulphuric or nitric, form comparatively stable salts, de: 
acid may, however, be judged from the joint evidence of many properties. With such energetic alkalis as soda and potash, carbonic acid forms normal salts, soluble in water, but having an alkaline reaction and in

composed with difficulty by heat and water, whilst others, like carbonic and hypochlorous acids, do not combine with feeble bases, and with most of the other bases form salts which sre easily decomposed. The same may be said with regard to bases, among which those of potassium, $\mathrm{K}_{2} \mathrm{O}$, sodium, $\mathrm{Na}_{2} \mathrm{O}$, and barium, $\mathrm{BaO}$, may serve as examples of the most powerful, because they combine with the most feeble acids and form a mass of alts of great stability, whilst as examples of the feeblest bases alumina, $\mathrm{Al}_{2} \mathrm{O}_{3}$, or bismuth oxide, $\mathrm{Bi}_{2} \mathrm{O}_{3}$, may be taken, becruse they form salts easily decomposed by water and by leat if the acid be volatile. Such a division of acids and bases into the feeblest and most. powerful is justified by all evidence concerning them, and is quoted in this work. But the teaching of this subject in certain circles has acquired quite a new tone, which, in my opinion, cannot be accepted withaut certain reservations and criticisms, although it comprises many interesting features. The fact is that Thomsen, Ostwald, and others proposed to express the measure of aftinity of acids to bases by figures drawn from data of the measure of displacement of acids in aqueous solutions, judging (1) from the amount of lieat developed by mixing a solution of the salt with a solution of another acid (the nvidity of acids, according to Thomsen); (2) from the change of the volumes accompranying such a mutaal action of solutions (Ostwald); (3) from the change of the index of refraction of solutions (Ostwald), \&c. Besides this there are many other methods which allow us to form an opinion about the distribution of bases among various acids in aqueous solutions. Some of these methods will be described lereafter. It ought, however, to be remarked that in making investigations in aqueous oolutions the affinity to water is generally left out of sight. If a base $\mathrm{N}$, combining with acids $X$ and $Y$ in presence of them both, divides in such a way that onethird of it combines with $\mathrm{X}$ and two-thirds with $\mathrm{Y}$, a conclusion is formed that the affinity, or power of forming salts, of the acid $\mathrm{Y}$ is twice as great as that of $\mathrm{X}$. But the presence of the water is not taken into account. If the acid $X$ has an affinity for water and for $N$. it will be distributed between them; and if $X$ has a greater affinity for water than $Y$, then less of $\mathrm{X}$ will combine with $\mathrm{N}$ than of $\mathrm{Y}$. If, in addition to this, the acid $\mathrm{X}$ is capable of forming an acid salt $\mathrm{NX}_{2}$, and $\mathrm{Y}$ is npt, the conclusion of the relative strength of $X$ and $Y$ will be still more erroneous, because the $X$ set free will form such a salt on the addition of $\mathrm{Y}$ to $\mathrm{NX}$. We shall see in Chapter $\mathrm{X}$. that when sulphuric and nitric acids in weak aqueous solution act on sodium, they are distributed exactly in this way: namely, one-third of the sodium combines with the sulphuric and two-thirds with the nitric acid; but, in my opinion, this does not show that sulphuric acid, compared witl nitric acid, possesses but half the degree of affinity for bases like soda, and only demonstrates the greater affinity of sulphurio acid for water compared with that of nitric acid. In this way the methods' of studying the distribution in aqueous solutions probably only shows the difference of the relation of the acid to a base.and to water.

In view of these considerations, although the teaching of the distribution of saltforming elements in aqueous solutions is an object of great and independent interest, it can hardly serve to determine the measure of affinity between bases and acids. Șimilar considerations ought to bo kept in view when determining the energy of acids by means of the electrical conductivity of their weak solutions. This method, proposed by Arrhenius (1884), and applied on an extensive scale by Ostwald (who developed it in great detail in his Lehrbuch $d$. allgemeinen Chemie, v. ii., 1887), is founded on the fact that the re: lation of the so-called molecular electrical-conductivity of weak solutions of various acids (I) coincides with the relation in which the same acids stand according to the distribution, (II) found by one of the above-mentioned methods, and with the relation deduced for therr from observations upon the velocity of reaction; (III) for instance, according to the rate of the splitting up of an ethereal salt (into alcohol and acid), or from the rate of the so. called inversion of sugar-that is, its transformation into glucose-as is seen by comparing 


\section{many cases themselves acting as alkalis. ${ }^{13}$ The acid salts of these alkalis,} $\mathrm{NaHCO}_{3}$ and $\mathrm{KHCO}_{3}$, have a neutral reaction on litmus, although they, like acids, contain hydrogen, which may be exchanged for metals. The acid salts of such acids-as, for instance, of sulphuric acid, $\mathrm{NaHSO}_{4}$ have a c'early defined acid reaction, and therefore carbonic acid is un. able to neutralise the powerful basic properties of such alkalis as potash or soda. Carbonic acid does not even combine at all with feeble bases, such as alumina, $\mathrm{Al}_{2} \mathrm{O}_{3}$, and therefore if a strong solution of sodium carbonate, $\mathrm{Na}_{2} \mathrm{CO}_{3}$, be added to a strong solution of aluminium sulphate, $\mathrm{Al}_{2}\left(\mathrm{SO}_{4}\right)_{3}$, although according to double saline decompositions aluminium carbonate, $\mathrm{Al}_{2}\left(\mathrm{CO}_{3}\right)_{3}$, ought to be formed, the carbonic acid separates, for this salt splits up in the presence of water into aluminium hydroxide and carbonic anhydride: $\mathrm{Al}_{2}\left(\mathrm{CO}_{3}\right)_{3}+3 \mathrm{H}_{2} \mathrm{O}=\mathrm{Al}_{2}(\mathrm{OH})_{6}$ $+3 \mathrm{CO}_{2}$. Thus feeble hases are unable to retain carbonic acid even at ordinary temperatures. For the same reason, in the case of bases of medium energy, although they form carbonates, the latter are compara. tively easily decomposed by heating, as is shown by the decomposition of copper carbonate, $\mathrm{CuCO}_{3}$ (see Introduction), and even of calcium carbo-

the annexed figures, in which the energy of hydrochloric acid is taken as equal to 100 :-

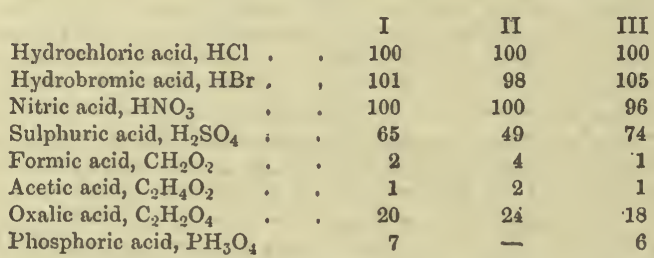

The coincidence of these figures, obtained by so many various methods, presents a most important and instructive relation between phenomena of different kinds, but in my opinion it does not permit us to assert that the degree of affinity existing between bases and various acids is determined by all these various methods, because the influence of the water must be taken into consideration. On this account, until the theory of solntion is more thoroughly worked out, this subject (which for the present ought to be treated of in special treatises on chemical mechanics) must be treated with great caution. But now we may hope to decide this question guided by a study of the rare of reaction, the influence of acids and bases upon indicators, \&c, all of which are treated fully in works on physical and theoretical chemistry.

15 Thus, for instance, in the washing of fabrics the caustic alkalis, such as sodium hydroxide, in weak solutions, act in removing the fatty matter just in the same way as carbonate solutions; for instance, a solution of soda crystals, $\mathrm{Na}_{2} \mathrm{CO}_{3}$. Soap acts in the same way, being composed of feeble acids, either fatty or resinous, combined with alkali. On this account all such substances are applied in manufacturing processes, and answer equally well in practice for bleaching and washing fabrics. Soda crystals or soap are preferred to caustic alkali, because an excess of the latter may have a destructive effect on the fabrics. It may be supposed that in aqueous solutions of soap or soda crystals, part of the base will form caustic alkali ; that is to say, the water will compete with the weak acids, and the alkali will be distributed between them and the water. 
nate, $\mathrm{CaCO}_{3}$. Only the normal (not the acid) salts of such powerful bases as potassium and sodium are capable of standing a red heat without decomposition. The acid salts-for instance, $\mathrm{NaHCO}_{3}$-decompose even on heating their solutions $\left(2 \mathrm{NaHCO}_{3}=\mathrm{Na}_{2} \mathrm{CO}_{3}+\mathrm{H}_{2} \mathrm{O}+\mathrm{CO}_{2}\right)$, evolving carbonic anhydride. The amount of heat given out by the combination of carbonic acid with bases also shows its feeble acid properties, being considerably less than with energetic acids. Thus if a weak solution of forty grams of sodium hydroxide be saturated (up to the formation of a normal salt) with sulphuric or nitric acid or another powerful acid, from thirteen to fifteen thousand calories are given out, but with carbonic acid only about ten thousand calories. $^{16}$ The majority of carbonates are insoluble in water, and therefore such solutions as sodium, potassium, or ammonium carbonates form in solutions of most other salts, $\mathrm{MX}$ or $\mathbf{M}^{\prime \prime} \mathrm{X}_{2}$, insoluble precipitates of carbonates, $\mathrm{M}_{2} \mathrm{CO}_{3}$ or $\mathrm{M}^{\prime \prime} \mathrm{CO}_{3}$. Thus a solution of barium chloride gives with sodium carbonate a precipitate of barium carbonate, $\mathrm{BaCO}_{3}$. For this reason rocks, especially those of aqueous origin, very often contain carbonates ; for example, calcium, ferrous, or magnesium carbonates, \&c.

Carbonic anhydride - which, like water, is formed with the development of a large amount of heat-is very stable. Only very few sub. stances are capable of depriving it of its oxygen. However, certain metals, such as magnesium, potassium and the like, on being heated, burn in it, depositing carbon and forming oxides. If a mixture of carbonio anhydride and hydrogen be passed through a heated tube, the formation of water and carbonic oxide will be observed; $\mathrm{CO}_{2}+\mathrm{H}_{2}=\mathrm{CO}+\mathrm{H}_{2} \mathrm{O}$.

16 Although carbonic acid is reckoned among the feeble acids, yet there are evidently many others still feebler-for instance, prussic acid, hypochlorous acid, many organio acids, \&c. Bases like alumina, or such feeble acids as silica, when in combination with alkalis, are decomposed in aqueous solutions by carbonsc acid, but on fusion-that is, without the presence of water-they displace it, which clearly shows in phenomena of this kind how much depends upon the conditions of reaction and the properties of the substances formed. These relations, which at first sight appear complex, may be best understood if we represent that two salts, $\mathrm{MX}$ and $\mathrm{NY}$, in general always give more or less of two other salts, MY and NX, and then examine the properties of the derived substances. Thus, in solution, sodium silicate, $\mathrm{Na}_{2} \mathrm{SiO}_{3}$, with carbonio anhydride will to some extent form sodium carbonate and silica, $\mathrm{SiO}_{2}$; but the latter, being colloid, separates, and the remaining mass of sodium silicate is again decomposed by, carbonic anhydride, so that finally silica separates and sodium carbonate is formed. In a fused state the case is different; sodium carbonate will react with silica to form carbonic anhydride and sodium silicate, but the carbonio anhydride will be separated as a gas, and therefore in the residue the same reaction will again take place, and ultimately the carbonic anhydride is entirely eliminated and sodium silicate remains. If, on the other hand, nothing is removed from the sphere of the reaction, distribution takes place. Therefore, although carbonic anhydride is a feeble acid, still not for this reason, but only in virtue of its gaseous. form, do all soluble acids displace it in saline solutions (see Chapter X.) 
But only a portion of the carbonic acid gas undergoes this change, and therefore the result will be a mixture of carbonic anhydride, carbonic oxide, hydrogen, and water, which does not suffer further change under the action of heat. ${ }^{17}$ Although, like water, carbonic anhydride is ex. ceedingly stable, still on being heated it partially decomposes into car. bonic oxide and oxygen. Deville showed that such is the case if car. bonic anhydride be passed through a long tube containing picces of porcelain and heated to $1,300^{\circ}$. If the products of decompositionnamely, the carbonic oxide and oxygen - be suddenly cooled, they can be collected separately, although they partly reunite together. A similar decomposition of carbonic anhydride into carbonic oxide and oxygen takes place on passing a series of electric sparks through it (for instance, in the eudiometer). Under these conditions an increase of volume occurs, because two volumes of $\mathrm{CO}_{2}$ give two volumes of $\mathrm{CO}$ and one volume of $O$. The decomposition reaches a certain limit (less than one-third.) and does not proceed further, so that the result is a mixture of carbonic anhydride, carbonic oxide, and oxygen, which is not altered in composition by the continued action of the sparks. This is readily understood, as it is a reversible reaction. If the carbonic anhydride be removed, then the mixture explodes when 2 spark is passed and forms carbonic anhydride ${ }^{17}$ bis If from an identical

27 Hydrogen and carbon are near akin to oxygen as regards affinity, but it ought to be considered that the affinity of hydrogen is slightly greater than that of carbon, be. cause during the combustion of hydrocarbons the hydrogen burns first. Some' idea of this similarity of affinity may be formed by the quantity of heat evolved. Gaseouse hydrogen, $\mathrm{H}_{2}$, on combining with an atom of oxygen, $\mathrm{O}=16$, derelops 69,000 heat-units if the water formed be condensed to a liquid state. If the water remains in the form of * gas (steam) the latent heat of evaporation must be subtracted, and then 58,000 calories will be developed. Carbon, $\mathrm{C}$, as a solid, on combining with $\mathrm{O}_{2}=32$ develops about 97,000 calories, forming gaseons $\mathrm{CO}_{2}$. If it were gaseous like hydrogen, and only contained $\mathrm{C}_{3}$ in its molecule, much more heat would be developed, and judging by other substances, whose molecules on passing from the solid to the gaseous state absorb about 10,000 to 15,000 calories, it must be held that gaseous carbon on forming gaseous carbonic anhydride would derelop not less than 110,000 calories-that is, approximately twice as much as is developed in the formation of water. And since there is twice as much oxygen in a molecule of carbonic anhydride as in a molecule of water, the oxygen develops approximately the same quantity of heat on combining with hydrogen as with carbon. That is to say, that here we find the same close affinity (see Chapter II., Note 7) determined by the quantity of heat as between hydrogen, zinc, and iron. For this reason here also, as in the case of hydrogen and iron, we ought to expect an equal distribution of oxygen between hydrogen and carbon, if they are both in excess compared with the amount of oxygen; but if there be an excess of carbon, it will decompose water, whilst an excess of hydrogen will decompose carbonic anhydride. Even if these phenomena and similar ones have been explained in isolated cases, a complete theory of the whole subject is still wanting in the present condition of chemical knowledge.

17 ble The degree or relative magnitude of the dissociation of $\mathrm{CO}_{2}$ varies with the temperature and pressure-that is, it increases with the temperature and as the pressure 
mixture the oxygen (and not the carbonic anhydride) be removed, and a series of sparks be again passed, the decomposition is renewed, and terminates with the complete dissociation of the carbonic anhydride. Phosphorus is used in order to effect the complete absorption of the oxygen. In these examples we see that a definite mixture of changeable substances is capable of arriving at a state of stable equilibrium, destroyed, however, by the removal of one of the substances composing the mixture. This is one of the instunces of the influence of mass.

Although carbonic anhydride is decomposed on heating, yielding oxygen, it is nevertheless, like water, an unchangeable substance at ordinary temperatures. Its decomposition, as effected by plants, is on this account all the more remarkable; in this case the whole of the oxygen of the carbonic anhydride is separated in the free state. The mechanism of this change is that the heat and light absorbed by the plants are expended in the decomposition of the carbonic anhydride. This accounts for the enormous influence of temperature and light on the growth of plants. But it is at present not clearly understood how this takes place, or by what separate intermediate reactions the whole process of decomposition of carbonic anhydride in plants into oxygen and the carbohydrates (Note 1) remaining in them, takes place. It is known that sulphurous anhydride (in many ways resembling carbonic anhydride) under the action of light (and also of heat) forms sulphur and sulphuric anhydride, $\mathrm{SO}_{3}$, and in the presence of water, sulphuric acid. But no similar decompo: sition has been obtained directly with carbonic anhydride, although it forms an exceedingly easily decomposable higher oxide-percarbonic

decreases. Deville found that at a pressure of 1 atmosphere in the flame of carbonic oxide burning in oxygen, about 40 per cent. of the $\mathrm{CO}_{3}$ is decomposed when the temperature is about $3,000^{\circ}$, and at $1,500^{\circ}$ less than 1 per cent. (Krafts); whilst under a pressure of 10 atmospheres about 34 per cent. is decomposed at $3,800^{\circ}$ (Mallard and Le Chatelier). It follows therefore that, under very smiall pressures, the dissociation of $\mathrm{CO}_{2}$ will be considerable even at comparatively moderate temperatures, but at the temperature of ordinary furnaces (about $1,000^{\circ}$ ) even under the small partial pressure of the carbonic acid, there are only small traces of decomposition which may be neglected in a practical estimation of the combustion of fuels. We may here cite the molecular specifio heat of $\mathrm{CO}_{2}$ (i.e. the amount of heat required to raise 44 units of weight of $\mathrm{CO}_{2} 1^{\circ}$ ), according to the determinations and calculations of Mallard and Le Chatelier, for a constant volume $C_{v}=6.26+0.0037 t$; for a constant pressure $C_{p}=C_{v}+2$ (see Chapter XIV., Note 7), i.e. the specific heat of $\mathrm{CO}_{2}$ increases rapidly with a rise of temperature : for example, at $0^{\circ}$ (per 1 part by weight), it is, at a constant pressure $=0.188$, at $1,000^{\circ}=0.272$, at $2,000^{\circ}$, about 0.856 . A perfectly distinct rise of the specific heat (for example, at $2,000^{\circ}, 0.409$ ), is given by a comparison of observations made by the above-mentioned investigators and by Berthelot and Vieille (Kournakoff). The cause of this must be looked for in dissociation. T. M. Cheltzoff, however, considers upon the basis of his researches upon explosives that it must be admitted that a maximum is reached at a certain temperature (about $2,500^{\circ} \mathrm{H}$ beyond which the specific heat begins to fall. 
acid; ${ }^{18}$ and perhaps that is the reason the oxygen separates. On the other hand, it is known that plants always form and contain organic acids, and these must be regarded as derivatives of carbonic acid, as is seen by all their reactions, of which we will shortly treat. For this reason it might be thought that the carbonic acid absorbed by the plants first forms (according to Baeyer) formic aldehyde, $\mathrm{CH}_{2} \mathrm{O}$, and from it organic acids, and that these latter in their tinal transformation form all the other complex organic substances of the plants. Many organic acids are found in plants in considerable quantity; for instance, tartaric acid, $\mathrm{C}_{4} \mathrm{H}_{6} \mathrm{O}_{6}$, found in grape-juice and in the acid juice of many plants; malic acid, $\mathrm{C}_{4} \mathrm{H}_{6} \mathrm{O}_{5}$, found not only in unripe apples but in still larger quantities in mountain ash berries; citric acid, $\mathrm{C}_{6} \mathrm{H}_{8} \mathrm{O}_{7}$, found in the acid juice of lemons, in gooseberries, cranberries, drc.; oxalic acid, $\mathrm{C}_{2} \mathrm{H}_{2} \mathrm{O}_{4}$, found in wood-sorrel and many other plants. Sometimes these acids exist in a free state in the plants, and sometimes in the form of salts ; for instance, tartaric acid is met with in grapes as the salt known as cream of tart3r, but in the impure state called argol, or tartar, $\mathrm{C}_{4} \mathrm{H}_{5} \mathrm{KO}_{6}$. In sorrel we find the so-called salts of sorrel, or acid potassium oxalate, $\mathrm{C}_{2} \mathrm{HKO}_{4}$. There is a very clear connection between carbonic anhydride and the abovementioned organic acids-namely, they all, under one condition or another, yield carbonic anhydride, and can all be formed by means of it from substances destitute of acid properties. The following exanples afford the best.demonstration of this fact : if acetic acid, $\mathrm{C}_{2} \mathrm{H}_{4} \mathrm{O}_{2}$, the acid of vinegar, be passed in the form of vapour through a heated tube,

18 Percarbonic acid, $\mathrm{H}_{2} \mathrm{CO}_{4}\left(=\mathrm{H}_{2} \mathrm{CO}_{3}+\mathrm{O}\right)$ is supposed by $\mathrm{A}$. Bach (1893) to be formed from carbonic acid in the action of light upon plants, (in the same manner as, according to the above scheme, sulphuric acid from sulphurous) with the formation of carbon, which remains in the form of hydrates of carbon: $3 \mathrm{H}_{2} \mathrm{CO}_{5}=2 \mathrm{H}_{2} \mathrm{CO}_{4}+\mathrm{CH}_{2} \mathrm{O}$. This substance $\mathrm{CH}_{2} \mathrm{O}$ expresses the composition of formic aldehyde which, according to Baeyer, by polymerisation and further changes, gives other hydrates of carbon and forms the first product which is formed in plants from $\mathrm{CO}_{2}$. And Berthelot (1872) had already, at the time of the discovery of persulphuric (Chapter XX.) and pernitric (Chapter VI., Note 26 ) acids pointed out the formation of the unstable percarbonic anhydride, $\mathrm{CO}_{3}$. Thus, notwithstanding the hypothetical nature of the above equation, it may be admitted all the more as it explains the comparative abundance of peroxide of hydrogen (Schöne, Chapter IV.) in the air, and this also at the period of the most energetic growth of plants (in July), because percarbonic acid should like all peroxides easily give $\mathrm{H}_{2} \mathrm{O}_{2}$. Besides which Bach (1894) showed that, in the first place, traces of formic aldehyde and oxidising agents $\left(\mathrm{CO}_{3}\right.$ or $\left.\mathrm{H}_{2} \mathrm{O}_{2}\right)$ are formed under the simultaneous action of $\mathrm{CO}_{2}$ and sunlight upon a solution containing a salt of uranium (which is oxidised), and diethylaniline (which reacts with $\mathrm{CH}_{2} \mathrm{O}$ ), and secondly, that by subjecting $\mathrm{BaO}_{2}$, shaken ap in water, to the action of a stream of $\mathrm{CO}_{2}$ in the cold, extracting (also in the cold) with ether, and then adding an alcoholic solution of $\mathrm{NaHO}$, crystalline plates of a sodium salt may be obtained, which with water evolve oxygen and leave sodium carbonate, they are therefore probably the per-salt. All these facts are of great interest and deserve further verification and elaboration: 
it splits up into carbonic anhydride and marsh gas $=\mathrm{CO}_{2}+\mathrm{CH}_{4}$. But conversely it can also be obtained from those components into which it decomposes. If one equivalent of hydrogen in marsh gas be replaced (by indirect means) by sodium, and the compound $\mathrm{CH}_{3} \mathrm{Na}$ is obtained, this directly absorbs carbonic anhydride, forming a salt of acetic acid, $\mathrm{CH}_{3} \mathrm{Na}+\mathrm{CO}_{2}=\mathrm{C}_{2} \mathrm{H}_{3} \mathrm{NaO}_{2}$; from this acetic acid itself may be easily obtained. Thus acetic acid decomposes into marsh gas and carbonic anhydride, and conversely is obtainable from them. The hydrogen of marsh gas does not, like that in acids, show the property of being directly replaced by metals; i.e. $\mathrm{CH}_{4}$ does not show any acid character whatever, but on combining with the elements of carbonic anhydride it acquires the properties of an acid. The investigation of all other organic acids shows similarly that their acid character depends on their containing the elements of carbonic anhydride. For this reason there is no organic acid containing less oxygen in its molecule than there is in carbonic anhydride; every organic acid contains in its molecule at least two atoms of oxygen. In order to express the rela. tion between carbonic acid, $\mathrm{H}_{2} \mathrm{CO}_{3}$, and organic acids, and in order to understand the reason of the acidity of these latter, it is simplest to turn to that law of substitution which shows (Chapter VI.) the relation between the hydrogen and oxygen compounds of nitrogen, and permits us (Chapter VIII.) to regard all hydrocarbons as derived from methane. If we have a given organic compound, $A$, which has not the properties of an acid, but contains hydrogen connected to carbon, as in hydrocarbons, then $\mathrm{ACO}_{2}$ will be a monobasic organic acid, $\mathrm{A} 2 \mathrm{CO}_{2}$ a bibasic, $\mathrm{A} 3 \mathrm{CO}_{2}$ a tribasic, and so on-that is, each molecule of $\mathrm{CO}_{2}$ transforms one atom of hydrogen into that state in which it may be replaced by metals, as in acids. This furnishes a direct proof that in organic acids it is necessary to recognise the group $\mathrm{HCO}_{2}$, or carboxyl. If the addition of $\mathrm{CO}_{2}$ raises the basicity, the removal of $\mathrm{CO}_{2}$ lowers it. Thus from the bibasic oxalic acid, $\mathrm{C}_{2} \mathrm{H}_{2} \mathrm{O}_{4}$, or phthalic acid, $\mathrm{C}_{8} \mathrm{H}_{6} \mathrm{O}_{4}$, by eliminating $\mathrm{CO}_{2}$ (easily effected experimentally) we obtain the monobasic formic acid, $\mathrm{CH}_{2} \mathrm{O}_{2}$, or benzoic acid, $\mathrm{C}_{7} \mathrm{H}_{6} \mathrm{O}_{2}$, respectively. The nature of carboxyl is directly explained by the law of substitution. Julging from what has been stated in Chapters VI. and VIII. concerning this law, it, is evident that $\mathrm{CO}_{2}$ is $\mathrm{CH}_{4}$ with the exchange of $\mathrm{H}_{4}$ for $\mathrm{O}_{2}$, and that the hydrate of carbonic anhydride, $\mathrm{H}_{2} \mathrm{CO}_{3}$, is $\mathrm{CO}(\mathrm{OH})_{2}$, that is, methane, in which two parts of hydrogen are replaced by two parts of the water radicle $(\mathrm{OH}$, hydroxyl) and the other two by oxygen. Therefore the group $\mathrm{CO}(\mathrm{OH})$, or carboxyl, $\mathrm{HCO}_{2}$, is a part of carbonic Rcid, and is equivalent to $(\mathrm{OH})$, and therefore also to $H$. That is, it 
is a univalent residue of carbonic acid capable of replacing one atom of hydrogen. Carbonic acid itself is a bibasic acid, both hydrogen atoms in it being replaceable by metals, therefore carboxyl, which contains one of the hydrogen atoms of carbonic acid, represents a group in which the hydrogen is, exchangeable for metals. And therefore if $1,2 \ldots n$ atoms of non-metallic hydrogen are exchanged $1,2 \ldots$ $n$ times for carboxyl, we ought to obtain $1,2 \ldots n$-basic acids. Organic acids are the products of the carboxyl substitution in hydrocarbons. 18 bis If in the saturated hydrocarbons, $\mathrm{C}_{\mathrm{n}} \mathrm{H}_{2 \mathrm{n}+2}$, one part of hydrogen is replaced by carboxyl, the monobasic saturated (or fatty) acids, $\mathrm{C}_{n} \mathrm{H}_{2 \mathrm{n}+1}\left(\mathrm{CO}_{2} \mathrm{H}\right)$, will be obtained, as, for instance, formic acid, $\mathrm{HCO}_{2} \mathrm{H}$, acetic acid, $\mathrm{CH}_{2} \mathrm{CO}_{2} \mathrm{H}, \ldots$. stearic acid, $\mathrm{C}_{17} \mathrm{H}_{35} \mathrm{CO}_{2} \mathrm{H}$, \&c. The double substitution will give bibasic acids, $\mathrm{C}_{n} \mathrm{H}_{2 \mathrm{n}}\left(\mathrm{CO}_{2} \mathrm{H}\right)\left(\mathrm{CO}_{2} \mathrm{H}\right)$; for instance, oxalic acid $n=0$, malonic acid $n=1$, succinic acid $n=2$, \&c. To benzene, $\mathrm{C}_{6} \mathrm{H}_{6}$, correspond benzoic acid, $\mathrm{C}_{6} \mathrm{H}_{5}\left(\mathrm{CO}_{2} \mathrm{H}\right)$, phthalic acid (and its isomerides), $\mathrm{C}_{6} \mathrm{H}_{4}\left(\mathrm{CO}_{2} \mathrm{H}\right)_{2}$, up to inellitic acid, $\mathrm{C}_{6}\left(\mathrm{CO}_{2} \mathrm{H}\right)_{6}$, in all of which the basicity is equal to the number of carboxyl groups. As many isomerides exist in hydrocarbons, it is readily understood not only that such can exist also in organic acids, but that their number and structure may be foreseen. This complex and most interesting branch of chemistry is treated separately in organic chemistry.

Carbonic Oxide.-This gas is formed whenever the combustion of organic substances takes place in the presence of a large excess of

18 bis If $\mathrm{CO}_{2}$ is the anhydride of a bibasic acid, and carboxyl corresponds with it, replacing the bydrogen of hydrocarbons, and giving thein the character of comparatively feeble acids, then $\mathrm{SO}_{3}$ is the anhydride of an energetic bibasic acid, and sulphoxyl, $\mathrm{SO}_{2}(\mathrm{OH})$, corresponds with it, being capable of replacing the hydrogen of hydrocarbons, and forming comparatively energetic sulphur oxyacids (sulphonic acids); for instance, $\mathrm{C}_{6} \mathrm{H}_{5}(\mathrm{COOH})$, benzoic acid, and $\mathrm{C}_{6} \mathrm{H}_{3}\left(\mathrm{SO}_{2} \mathrm{OH}\right)$, benzenesulphonic acid, are derived from $\mathrm{C}_{6} \mathrm{H}_{6}$. As the exchange of $\mathrm{H}$ for methyl, $\mathrm{CH}_{3}$, is equivalent to the addition of $\mathrm{CH}_{2}$, the exchange of carboxyl, $\mathrm{COOH}$, is equivalent to the addition of $\mathrm{CO}_{2}$; so the exchange of $\mathrm{H}$ for sulphoxyl is equivalent to the addition of $\mathrm{SO}_{3}$. The latter proceeds directly, for instance: $\mathrm{C}_{6} \mathrm{H}_{8}+\mathrm{SO}_{5}=\mathrm{C}_{6} \mathrm{H}_{5}\left(\mathrm{SO}_{2} \mathrm{OH}\right)$.

As, according to the determinations of Thomsen, the heat of combustion of the vapours of acids $\mathrm{RCO}_{2}$ is known where $\mathrm{R}$ is a hydrocarbon, and the heat of combustion of the hydrocarbons $\mathrm{R}$ themselves, it may be szen that the formation of acids, $\mathrm{RCO}_{2}$, from $\mathrm{R}+\mathrm{CO}_{2}$ is always accompanied by a small absorption or development of heut. We give the heats of combustion in thousands of calories, referred to the molecular weights of t'ie substances:-

$\begin{array}{lcccc}\mathrm{R}= & \mathrm{H}_{2} & \mathrm{CH}_{4} & \mathrm{C}_{2} \mathrm{H}_{6} & \mathrm{C}_{6} \mathrm{H}_{6} \\ & 68 \cdot 4 & 212 & 370 & 777 \\ \mathrm{RCO}_{2}= & 69 \cdot 4 & 225 & 387 & 766\end{array}$

Thus $\mathrm{H}_{2}$ corresponds with formic acid, $\mathrm{CH}_{2} \mathrm{O}_{2}$; benzene, $\mathrm{C}_{6} \mathrm{H}_{6}$, with benzoic acid, $\mathrm{C}_{7} \mathrm{H}_{6} \mathrm{O}_{2}$. The data for the latter are taken from Stohmann, and refer to the solid condition. For formio acid Stohmann gives the heat of combustion as 59,000 calories in a liquid state, but in a state of vapour, 64.6 thousand units, which is much less than according to Thomsen. 
incandescent charcoal, the air first burns the carbon into carbonic anhydricle, but this in penetrating through the red-hot charcoal is transformed into carbonic oxide, $\mathrm{CO}_{2}+\mathrm{C}=2 \mathrm{CO}$. By this reaction carbonic oxide is prepared by passing carbonic anhydride through charcoal at a red heat. It may be separated from the excess of carbonic anhydride by passing it through a solution of alkali, which does not absorb carbonic oxide. This reduction of carbonic anhydride explains why carbonic oxidé is formed in ordinary clear fires, where the incoming air passes over a large surface of heated coal. A blue flame is then observed burning above the coal ; this is the burning carbonic oxide. When charcoal is burnt in stacks, or when a thick layer of coal is burning in a brazier, and under many similar circumstances, carbonic oxide is also formed. In metallurgical processes, for instance when iron is smelted from the ore, very often the same process of conversion of carbonic anhydride into carbonic oxide occurs, especially if the combustion of the coal be effected in high, so-called blast, furnaces and ovens, wherc the air enters at the lower part and is compelled to pass through a thick layer of incandescent coal. In this way, also, com bustion with flame may be obtained from those kinds of fuel which under ordinary conditions burn without flame : for instance, anthracite, coke, charcoal. Heating by means of a gas-producer-that is, an apparatus producing combustible curbonic oxide from fuel-is carried on in the same manner. ${ }^{19}$ In transforming one part of char.

19 In gas-producers all carbonaceous fuels are transformed into inflammable gas. In those which (on account of their slight density and large amount of water, or incombustible admixtures which ab. sorb heat) are not as capable of giving a high temperature in ordinary furnacesfor instance, fir cones, peat, the lower kinds of coal, \&c.-the same gas is ob. tained as with the best kinds of coal, because the water condenses on cooling, and the ashes and earthy matter remain in the gas-producer. The construction of a gas-producer is seen from the ac companying drawing. The fuel lies on the fire-bars $O$, the air enters through them and the ash-hole (drawn by the draught of the chimney of the stove where

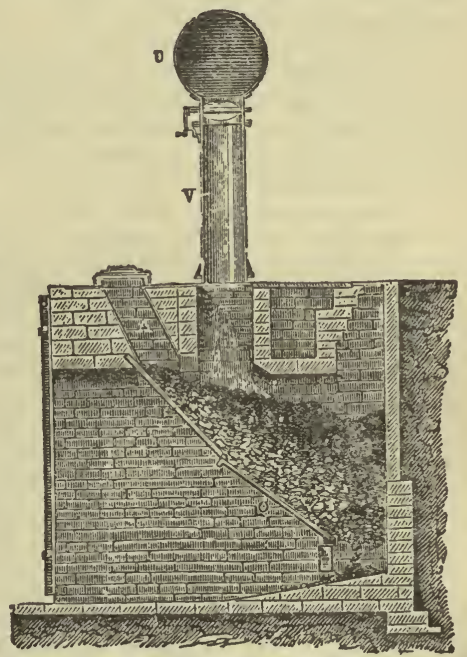

Fic. 63.-Gas-producer for the formation of carbon monoxide for lieating purposes.

the gas burns, or else forced by a blowing apparatus), the quantity of ax being exactly regulated by means of valves. The gases formed are then led by the tube $\mathrm{V}$; provided 
coal into carbonic oxide 2,420 heat units are given out, and on burning to carbonic anhydride 8,080 heat units. It is evident that on transforming the charcoal first into carbonic oxide we obtain a gas which in burning is capable of giving out 5,660 heat units for one part of charcoal. This preparatory transformation of fuel into carbonic oxide, or producer gas containing a mixture of carbonic oxide (about $\frac{1}{3}$ by volume) and nitrogen $\left(\frac{2}{3}\right.$ volume), in many cases presents most important advantages, as it is easy to completely burn gaseous fuel without an excess of air, which would lower the temperature. ${ }^{20}$ In stoves where solid fuel is burnt it is impossible to effect the complete combustion of the various kinds of fuel without admitting an excess of air. Gaseous fuel, such as carbonic oxide, is easily completely mixed with air and burnt without excess of it. If, in addition to this, the air and gas required for the combustion be previously heated by means of the heat which would otherwise be uselessly carried off in the products of combustion (smoke) ${ }^{21}$ it is easy to reach a high temperature, so high (about $1,800^{\circ}$ ) that platinum may be melted. Such an arrangement is known as a regene. rative furnace. ${ }^{22}$ By means of this process not only may the high temperatures indispensable in many industries be obtained (for instance,

with a valve, into the gas main U. The addition of fuel ought to proceed in such a way as to prevent the generated gas escaping; hence the space $A$ is kept filled with the combustible material and covered-with a lid.

20 An excess of air lowers the temperature of combustion, because it becomes heated itself, as explained in Chapter III. In ordinary furnaces the excess of air is three or four times greater than the quantity required for perfect combustion. In the best furnaces (with fire-bars, regulated air supply, and corresponding chimney draught) it is necessary to introdnce twice as much air as is necessary, otherwise the smoke contains much carbonic oxide.

21 If in manufactories it is necessary, for instance, to maintain the temperature in a furnace at $1,000^{\circ}$, the flame passes out at this or a higher temperature, and therefore much fuel is lost in the smoke. For the draught of the chimney a temperature of $100^{\circ}$ to $150^{\circ}$ is sufficient, and therefore the remaining heat ought to be utilised. For this purpose the flues are carried under boilers or other heating apparatus. The preparatory heating of the air is the best means of utilisation when a high temperature is desired (see Note 22).

22 Regenerative furnaces were introduced by the Brothers Siemens about the year 1860 in many industries, and mark a most important progress in the use of fuel, especially in obtaining high temperatures. The principle is as follows: The products of combustion from the furnace are led into a chamber, $I$, and heat up the bricks in it, and then pass into the outlet flue; when the bricks are at a red heat the products of com. bustion are passed (by altering the valves) into another adjoining chamber, II, and air requisite for the combustion of the generator gases is passed through $I$. In passing round about the incandescent bricks the air is heated, and the bricks are cooled-that is, the heat of the smoke is returned into the furnace. The air is then passed through II, and the smoke through I. The regenerative burners for illuminating gas are founded on this eame principle, the products of combustion heat the incoming air and gas, the tempera. ture is higher, the light brighter, and an economy of gas is effected. Absolute perfection in these appliances has, of course, not yet been attained; further improvement is still possible, but dissociation imposes a limit because at a certain high temperature 
glass-working, steel-melting, \&c.), but great adrantage also ${ }^{23}$ is gained as regards the quantity of fuel, because the transmission of heat to the object to be heated, other conditions being equal, is determined by the difference of temperatures.

The transformation of carbonic anhydride, by means of charcoal, into carbonic oxide $\left(\mathrm{C}+\mathrm{CO}_{2}=\mathrm{CO}+\mathrm{CO}\right)$ is considered a reversible reaction, because at a high temperature the carbonic oxide splits up into carbon and carbonic anhydride, as Sainte-Claire Deville showed by using the method of the 'cold and hot tule.' Inside a tube heated in a furnace another thin metallic (silvered copper) tube is fitted, through which a constant stream of cold water flows. The carbonic oxide coming into contact with the heated walls of the exterior tube forms charcoal, and its minute particles settle in the form of lampblack on the lower side of the cold tube, and, since they are cooled, do not act further on the oxygen or carbonic anhydride formed. ${ }^{24}$ A series

combinations do not ensue, possible temperatures being limited by reverse reactions. Here, as in a number of other cases, the further investigation of the matter must prove of direct value from a practical point of view

25 At first sight it appears absurd, useless, and paradoxical to lose nearly one-third of the heat which fuel can develop, by turning it into gas. Actually the advantage is enormous, especially for producing high temperatures, as is already seen from the fact that fuels rich in oxygen (for instance, wood) when damp are unable, with any kind of hearth whatever, to give the temperature required for glass-melting or steel-casting, whilst in the gas-producer they furnish exactly the same gas as the driest and most carbonaceous fuet. In order to understand the principle which is here involved, it is sufficient to remember that a large amount of heat, but having a low temperature, is in many cases of no use whatever. We are unable here to enter into all the details of the complicated matter of the application of fuel, and further particulars must be sought for in special technical treatises. The following footnotes, however, contain certain fundamental figures for calculations con. cerning combustion.

24 The first product of combustion of charcoal is always carbonic anhydride, and not carbonic oxide. This is seen from the fact that with a shallow layer of charcoal (less than a decimetre if the charcoal be closely packed) carbonic oxide is not formed at all. It is not even produced with a deep layer of charcoal if the temperature is not above $500^{\circ}$, and the current of air or oxygen is very slow. With a rapid current of air the charcoal becomes red-hot, and the temperature rises, and then carbonic oxide appears (Lang 1888). Ernst (1891) found that below $995^{\circ}$ carbonic oxide is always accompanied by $\mathrm{CO}_{2}$, and that the formation of $\mathrm{CO}_{2}$ begins about $400^{\circ}$. Naumann and Pistor determined that the reaction of carbonic anhydride with carbon commences at about $550^{\circ}$, and that between water and carbon at about $500^{\circ}$. At the latter temperature carbonic anhydride is formed, and only with a rise of temperature is carbonic oxide formed (Lang) from the action of the carbonic anhydride on the carbon, and from the reaction $\mathrm{CO}_{2}+\mathrm{H}_{2}=\mathrm{CO}+\mathrm{H}_{2} \mathrm{O}$. Rathke (1881) showed that at no temperature whatever is the reaction as expressed by the equation $\mathrm{CO}_{2}+\mathrm{C}=2 \mathrm{CO}_{2}$, complete; a part of the carbonic anhydride remains, and Lang determined that at about $1,000^{\circ}$ not less than 3 p.c. of the carbonic anhydride remains untransformed into carbonic oxide, even after the action has been continued for several hours. The endothermal reactions, $\mathrm{C}+2 \mathrm{H}_{2} \mathrm{O}=\mathrm{CO}_{2}+2 \mathrm{H}_{2}$, and $\mathrm{CO}+\mathrm{H}_{2} \mathrm{O}=\mathrm{CO}_{2}+\mathrm{H}_{2}$, are just as incomplete. This is made clear if we note that on the one hand the above-mentioned reactions are all reversible, and therefore bounded by a limit; and, on the other hand, that at about $500^{\circ}$ oxygen begins to combine with hydrogen and carbon, 
of electric sparks also decomposes carbonic oxide into carbonic anhydride and carbon, and if the carbonic anhydride be removed by alkali com. plete decomposition may be obtained (Deville). ${ }^{24}$ bis Aqueous vapour, which is so similar to carbonic anhydride in many respects, acts, at a high temperature, on charcoal in an exactly similar way, $\mathrm{C}+\mathrm{H}_{2} \mathrm{O}$ $=\mathrm{H}_{2}+\mathrm{CO}$. Froin 2 volumes of carbonic anhydride with charcoal 4 volumes of carbonic oxide (2 molecules) are obtained, and precisely the same from 2 volumes of water vapour with charcoal 4 volumes of a gas consisting of hydrogen and carbonic oxide $\left(\mathrm{H}_{2}+\mathrm{CO}\right)$ are formed. This mixture of combustible gases is called water gas. ${ }^{25}$

and also that the lower limits of dissociation of water, carbonic anhydride, and carbonic oxide lie near one anotlier between $500^{\circ}$ and $1,200^{\circ}$. For water and carbonic oxide the lower limit of the commencement of dissociation is unknown, but judging from the pub. lished data (according to Le Chatelier, 1888) that of carbonic anhydride may be taken as about $1,050^{\circ}$. Even at about $200^{\circ}$ half the carbonic anhydride dissociates if the pressure be small, about 0.001 atmosphere. At the atmosplieric pressure, not more than 0.05 p.c. of the carbonic anliydride decomposes. The reason of the influence of pressure is here evidently that the splitting np of carbonic anhydride into carbonic oxide and oxygen is accompanied by an increase in volume (as in the case of the dissociation of nitric peroxide. See Chapter VI., Note 46). As in stoves and lamps, and also with explosive snbstances, the temperature is not higher than $2,000^{\circ}$ to $2,500^{\circ}$, it is evident that although the partial pressure of carbonic anhydride is small, still its dissociation cannot here be considerable, and probably does not exceed 5 p.c.

24 bis Besides which L. Mond (1890) showed that the powder of freshly reduced metallic nickel (obtained by heating the oxide to redness in a stream of hydrogen) is able, when heated even to $350^{\circ}$, to completely decompose carbonic oxide into $\mathrm{CO}_{2}$ and carbon, which remains with the nickel and is easily removed from it by heating in a stream of air. Here $2 \mathrm{CO}=\mathrm{CO}_{2}+\mathrm{C}$. It should be remarked that heat is evolved in this reaction (Note 25), and therefore that the influence of 'contact' may here play a part. Indeed, this reaction inust be classed among the most remarkable instances.of the influence of contact, especially as metals analogous to $\mathrm{Ni}$ ( $\mathrm{Fe}$ and $\mathrm{Co}$ ) do not effect this reaction (see Chapter II., Note 17).

25 A molecular weight of this gas, or 2 volumes CO (28 grams), on combustion (forming $\mathrm{CO}_{2}$ ) gives out 68,000 heat units (Thomsen 67,960 calories). A molecular weight of hydrogen, $\mathrm{H}_{2}$ (or 2 volnmes), develops on burning into liquid water 69,000 heat units (according to Thomsen 68,300 ), but if it forms aqueous vapour 58,000 heat units. Charcoal, resolving itself by combustion irto the molecular quantity of $\mathrm{CO}_{2}$ (2 volumes), develops 97,000 leat units. From the data furnished by these exothermal reactions it follows: (1) that the oxidation of charcoal into carbonic oxide develops 29,000 heat units : (2) that the reaction $\mathrm{C}+\mathrm{CO}_{2}=2 \mathrm{CO}$ absorbs 39,000 heat units; (3) $\mathrm{C}+\mathrm{H}_{2} \mathrm{O}=\mathrm{H}_{2}+\mathrm{CO}$ absorbs (if the water be in a state of vapour) 29,000 calories, but if the water be liquid 40,000 calories (almost as much as $\mathrm{C}+\mathrm{CO}_{2}$ ); (4) $\mathrm{C}+\mathrm{H}_{2} \mathrm{O}=\mathrm{CO}_{2}+2 \mathrm{H}_{2}$ absorbs (if the water be in a state of vapour) 19,000 heat units; (5) the reaction $\mathrm{CO}+\mathrm{H}_{2} \mathrm{O}=\mathrm{CO}_{2}+\mathrm{H}_{2}$ levelops 10,000 heat units if the water be in the state of vapour; and (6) the decomposition expressed by the equation $2 \mathrm{CO}=\mathrm{C}+\mathrm{CO}_{2}$ (Note $24 \mathrm{bis}$ ) is accompanied by the evol $/$ tion of 39,000 units of heat.

Hence it follows that 2 volumes of $\mathrm{CO}$ or $\mathrm{H}_{2}$ burning into $\mathrm{CO}_{2}$ or $\mathrm{H}_{2} \mathrm{O}$ derelop almost the samne amount of hent, just as also the heat effects corresponding with the equations

are nearly equal.

$$
\begin{aligned}
& \mathrm{C}+\mathrm{H}_{2} \mathrm{O}=\mathrm{CO}+\mathrm{H}_{2} \\
& \mathrm{C}+\mathrm{CO}_{2}=\mathrm{CO}+\mathrm{CO}
\end{aligned}
$$


But aqueous vapour (and only when strongly superheated, otherwise it cools the charcoal) only acts on charcoal to form a large amount of carbonic oxide at a very high temperature (at which carbonic anhydride dissociates); it begins to react at about $500^{\circ}$, forming carbonic anhydride according to the equation $\mathrm{C}+2 \mathrm{H}_{2} \mathrm{O}=\mathrm{CO}_{2}+2 \mathrm{H}_{2}$. Besides this, carbonic oxide on splitting up forms carbonic anhydride, and therefore water gas always contains a mixture ${ }^{26}$ in which hydrogen predominates, the volume of carbonic oxide being comparatively less,

20 Water gas, obtained from steam and charcoal at a white heat, contains about 50 p.c. of hydrogen, about 40 p.c. of carbonic oxide, about 5 p:c. of carbonic anhydride, the remainder being nitrogen from the charcoal and air. Compared with producer gas, which contains much nitrogen, this is a gas much richer in combustible matter, and therefore capable of giving high temperatures, and is for this reason of the greatest utility. If carbonic anhydride could be as readily obtained in as pure a state as water, then $\mathrm{CO}$ might be prepared directly from $\mathrm{CO}_{2}+\mathrm{C}$, and in that case the utilisation of the heat of the carbon would be the same as in water gas, because $\mathrm{CO}$ evolves as much heat as $\mathrm{H}_{2}$, and even more if the temperature of the smoke be over $100^{\prime}$, and the water remains in the form of vapour (Note 25). But producer gas contains a large proportion of nitrogen, so that its effective temperature is below that given by water gas; therefore in places where a particularly high temperature is required (for instance, for lighting by means of incandescent lime or magnesia, or for steel melting, \&c.), and where the gas can be easily distributed through pipes, water gas is at present held in high estimation, but when (in ordinary furnaces, re-heating, glass-melting, and other furnaces) a very high temperature is not required, and there is no need to convey the gas in pipes, producer gas is generally preferred on account of the simplicity of its preparation, especially as for water gas such a high temperature is required that the plant soon becomes damaged.

There are numerons systems for making water gas, but the American patent. of T. Lowe ts generally used. The gas is prepared in a cylindrical generator, into which hot air is introduced, in order to raise the coke in it to a white heat. The products of combustion containing carbonic oxide are utilised for superheating steam, which is then passed over the white hot coke. Water gas, or a mixture of hydrogen and carbonic oxide, is thus obtained.

Water gas is sometimes called 'the fuel of the future,' because it is applicable to all purposes, develops a high temperature, and is therefore available, not only for domestic and industrial uses, but also for gas-motors and for lighting. For the latter purpose platinum, lime, magnesia, zirconia, and similar substances (as in the Drummond light, Chapter III.), are rendered incandescent in the flame, or else the gas is carburettedthat is, mixed with the vapours of volatile hydrocarbons (generally benzene or naphtha, naphthalene, or simply naphtha gas), which communicate to the pale flame of carbonic oxide and hydrogen a great brilliancy, owing to the high temperature developed by the combustion of the non-luminous gases. As water gas, possessing these properties, may be prepared at central works and conveyed in pipes to the consumers, and as it may be produced from any kind of fuel, and ought to be much cheaper than ordinary gas, it may as a matter of fact be expected that in course of time (when experience shall have deter. mined the cheapest and best way to prepare it) it will not only supplant ordinary gas, but will with advantage everywhere replace the ordinary forms of fuel, which in many respects are inconvenient. At present its consumption-spreads principally for lighting purposes, and for use in gas-engines instead of ordinary illuminating gas. In some cases Dowson gas is prepared in producers. This is a mixture of water and producer gases obtained by passing steam into an ordinary producer (Note 19), when the temperature of the carbon has become sufficiently high for the reaction $\mathrm{C}+\mathrm{H}_{2} \mathrm{O}=\mathrm{CO}+\mathrm{H}_{2}$. 
whilst the amount of carbonic anhydride increases as the temperature of the reaction decreases (generally it is more than 3 per cent.)

Metals like iron and zinc which at a red heat are capable of decomposing water with the formation of hydrogen, also decompose carbonic anhydride with the formation of carbonic oxide; so both the ordinary products of complete combustion, water and carbonic anhydride, are very similar in their reactions, and we shall therefore presently compare hydrogen and carbonic oxide. The metallic oxides of the above-mentioned metals, when reduced by charcoal, also give carbonic oxide. Priestley obtained it by heating charcoal with zinc oxide. As free carbonic anhydride may be transformed into carbonic oxide, so, in precisely the same way, may that carbonic acid which is in a state of combination; hence, if magnesium or barium carbo. nates $\left(\mathrm{MgCO}_{3}\right.$ or $\left.\mathrm{BaCO}_{3}\right)$ be heated to rerlness with charcoal, or iron or zinc, carbonic oxide will be produced-for instance, it is obtained by heating an intimate mixture of 9 parts of chalk and 1 part of charcoal in a clay retort.

Many organic substances ${ }^{27}$ on being heated, or under the action of various agents, yield carbonic oxide ; amongst these are many organic or carboxylic acids. The simplest are formic and oxalic acids. Formic acid, $\mathrm{CH}_{2} \mathrm{O}_{2}$, on being heated to $200^{\circ}$, easily decomposes into carbonic oxide and water, $\mathrm{CH}_{2} \mathrm{O}_{2}=\mathrm{CO}+\mathrm{H}_{2} \mathrm{O} .{ }^{27}$ bis Usually, however, carbonic oxide is prepared in laboratories, not from formic but from oxalic acid, $\mathrm{C}_{2} \mathrm{H}_{2} \mathrm{O}_{4}$, the more so as formic acid is itself prepared from oxalic acid. The latter acid is easily obtained by the action of nitric acid on starch, sugar, \&c.; it is also found in nature. Oxalic acid is easily decomposed by heat, its crystals first lose water, then partly volatilise, but the greater part is decomposed. The decomposition is of the following nature it splits up into water, carbonic oxide, and carbonic anhydride, ${ }^{28} \quad \mathrm{C}_{2} \mathrm{H}_{2} \mathrm{O}_{4}=\mathrm{H}_{2} \mathrm{O}+\mathrm{CO}_{2}+\mathrm{CO}$. This decomposition is generally practically effected by mixing oxalic acid with strong sul-

27 The so-called yellow prussiate, $\mathrm{K}_{4} \mathrm{FeC}_{6} \mathrm{~N}_{6}$, on being heated with ten parts of strong sulphuric acid forms a considerable quantity of very pure carbonic oxide quite free from carbonic anhydride..

27 bis To perform this reaction, the formic acid is mixed with glycerine, because when heated alone it volatilises much below its temperature of decomposition. When heated with sulphuric acid the salts of formic acid yield carbonic oxide.

29 The decomposition of formic and oxralicacids, with the formation of carbonic oxide, considering these acids as carboxyl derivatives, may be explained as follows:-The first is $\mathrm{H}(\mathrm{COOH})$ and the second $(\mathrm{COOH})_{2}$, or $\mathrm{H}_{2}$ in which one or both halves of the hydrogen are exchanged for carboxyl ; therefore they are equal to $\mathrm{H}_{2}+\mathrm{CO}_{2}$ and $\mathrm{H}_{2}+2 \mathrm{CO}_{2}$; but $\mathrm{H}_{2}$ reacts with $\mathrm{CO}_{2}$, as has been stated abøve, forming $\mathrm{CO}$ and $\mathrm{H}_{2} \mathrm{O}$. From this it is also evident that oxalic acid on losing $\mathrm{CO}_{2}$ forms formic acid, and also that the latter may proceed from $\mathrm{CO}+\mathrm{H}_{2} \mathrm{O}$, as we shall see further on. 
phuric acid, because the latter assists the decomposition by taking up the water. On heating a mixture of oxalic and sulphuric acids a mixture of carbonic oxide and carbonic anhydride is evolved. This mixture is passed through a solution of an alkali in order to absorb the carbonic anhydride, whilst the carbonic oxide passes on. ${ }^{28}$ bis

In its physical properties carbonic oxide resembles nitrogen; this is explained by the equality of their molecular weights. The absence of colour and smell, the low temperature of the absolute boiling point, $-140^{\circ}$ (nitrogen, $-146^{\circ}$ ), the property of solidifying at $-200^{\circ}$ (nitrogen, $-202^{\circ}$ ), the boiling point of $-190^{\circ}$ (nitrogen, $-203^{\circ}$ ), and the slight solubility (Chapter I., Note 30), of carbonic oxide are almost the same as in those of nitrogen. The chemical properties of both gases are, however, very different, and in these carbonic oxide resembles hydrogen. Carbonic oxide burns with a blue flame, giving 2 volumes of carbonic anhydride from 2 volumes of carbonic oxide, just as 2 volumes of hydrogen give 2 volumes of aqueous vapour. It explodes with oxygen, in the eudiometer, like hydrogen. ${ }^{29}$ When breathed it acts as a strong poison, being absorbed by the blood; ${ }^{30}$ this explains the action of charcoal fumes, the products of the

28 vis Greshoff (1888) showed that with a solution of nitrate of silver, iodoform, $\mathrm{CHI}_{3}$, forms $\mathrm{CO}$ according to the equation $\mathrm{CHI}_{3}+3 \mathrm{AgNO}_{3}+\mathrm{H}_{2} \mathrm{O}=3 \mathrm{AgI}+3 \mathrm{HNO}_{3}+\mathrm{CO}$. The reaction is immediate and is complete.

29 It is remarkable that, according to the investigations of Dixon, perfectly dry carbonic oxide does not explode with oxygen when a spark of low intensity is used, but an explosion takes place if there is the slightest admixture of moisture. L. Meyer, however, showed that sparks of an electric discharge of considerable intensity produce an explosion. N. N. Beketoff demonstrated that combustion proceeds and spreads slowly unless there be perfect dryness. I think that this may be explained by the fact that water with carbonic oxide gives carbonic anhydride and hydrogen, but hydrogen with oxygen gives hydrogen peroxide (Chapter VII.), which with carbonic oxide forms carbonic anhydride and water. The water, therefore, is renewed, and again serves the same purpose. But it may be that here it is necessary to acknowledge a simple contact influence. After Dixon had shown the inflience of traces of moisture upon the reaction $\mathrm{CO}+\mathrm{O}$, many researches were made of a similar nature. The fullest investigation into the influence of moisture upon the course of many chemical reactions was made by Baker in 1894. He showed that with perfect dryness, many chemical transformations (for example, the formaticn of ozone from oxygen, the decomposition of $\mathrm{AgO}, \mathrm{KClO}_{3}$ under the action of heat, \&c.) proceeds in exactly the same manner as in the presence of moisture; but that in many cases traces of moisture have an evident influence. We may mention the following instances: (1) $\mathrm{Dry} \mathrm{SO}_{3}$ does not act upan dry $\mathrm{CaO}$ or $\mathrm{CaO}$; (2) perfectly dry sal-ammoniac does not give $\mathrm{NH}_{3}$ with dry $\mathrm{CaO}$, but simply volatilises; (3) dry $\mathrm{NO}$ and $\mathrm{O}$ do not react ; (4) perfectly dry $\mathrm{NH}_{3}$ and $\mathrm{HCl}$ do not combine; (5) perfectly dry sal-ammoniac does not dissociate at $350^{\circ}$ (Chapter VII., Note 15 bis) ; and (6) perfectly dry chlorine does not act upon metals, \&c.

so Carbonic oxide is very rapid in its action, because it is absorbed by the blood in the same way as oxygen. In addition to this, the absorption spectrum of the blood changes so that by the help of blood it is easy to detect the slightest traces of carbonio oxide in the air. M. A. Kapoustin found that linseed oil and therefore oil painte, are capable of giving off carbonic oxide while drying (absorbing oxygen). 
incomplete combustion of charcoal and other carbonaceous fuels. Owing to its faculty of combining with oxygen, carbonic oxide acts as a powerful reducing agent, taking up the oxygen from many compounds at a red heat, and being itself transformed into carbonic anhydride. The reducing action of carbónic oxide, however, is (like that of hydrogen, Chapter II.) naturally confined to those oxides which easily part with their oxygen-as, for instance, copper oxide-whilst the oxides of magnesium or potassium are not reduced. Metallic iron itself is capable of reducing carbonic anhydride to carbonic oxide, just as it liberates the hydrogen from water. Copper, which does not decompase water, does not decompose carbonic oxide. If a platinum wire heated to $300^{\circ}$, or spongy platinum at the ordinary temperature, be plunged into a mixture of carbonic oxide and oxygen, or of hydrogen and oxygen, the mixture explodes. These reactions are very similar to those peculiar to hydrogen. The following important distinction, however, exists between them-namely : the molecule of hydrogen is composed of $\mathrm{H}_{2}$, a group of elements divisible into two like parts, whilst, as the molecule of carbonic oxide, $\mathrm{CO}$, contains unlike atoms of carbon and oxygen, in none of its reactions of combination can it give two molecules of matter containing its elements. This is particularly noticeable in the action of chlorine on hydrogen and on carbonic oxide respectively; with the former chlorine forms hydrogen chloride, and with the latter it produces the so-called carbonyl chloride, $\mathrm{COCl}_{2}$. that is to say, the molecule of hydrogen, $\mathrm{H}_{2}$, under the action of chlorine divides. forming two molecules of hydrochloric acid, whilst the molecule of carbonic oxide enters in its entirety inta the molecule of carbonyl chloride. This characterises the so-called diatomic or bivalent reactions of radicles or residues. $H$ is a monatomic residue or radicle, like $\mathrm{K}, \mathrm{Cl}$, and others, whilst carbonic oxide, $\mathrm{CO}$, is an indivisible (undecomposable) bivalent radicle, equivalent to $\mathrm{H}_{2}$ and not to $\mathrm{H}$, and therefore combining with $\mathrm{X}_{2}$ and interchangeable with $\mathrm{H}_{2}$. This distinction is evident from the annexed comparison .

$\begin{array}{ll}\mathrm{HH} \text {, hydrogen. } & \mathrm{CO} \text {, carbonic oxide. } \\ \mathrm{HCl} \text {, hydrochloric acid. } & \mathrm{COCl} \text {, carbonyl chloride. } \\ \mathrm{HKO} \text {, potash. } & \mathrm{CO}\left(\mathrm{KO}_{2} \text {, potassium carbonate. }\right. \\ \mathrm{HNH}_{2} \text {, ammonia. } & \mathrm{CO}\left(\mathrm{NH}_{2}\right)_{2} \text {, urea. } \\ \mathrm{HCH}_{3} \text {, methane. } & \mathrm{CO}\left(\mathrm{CH}_{3}\right)_{2} \text {, acetone. } \\ \mathrm{HHO} \text {, water. } & \mathrm{CO}(\mathrm{HO})_{2} \text {, carbonic acid. }\end{array}$

Such monatomic (univalent) residues, $\mathrm{X}$, as $\mathrm{H}, \mathrm{Cl}, \mathrm{Na}, \mathrm{NO}_{2}, \mathrm{NH}_{4}$, $\mathrm{CH}_{3}, \mathrm{CO}_{2} \mathrm{H}$ (carboxyl), $\mathrm{OH}$, and others, in accordance with the law of substitution, combine together, forming compounds, $\mathrm{XX}$, and with 
oxýgen, or in general with diatomic (bivalent) residues, $\mathrm{Y}$ - for instance, $\mathrm{O}, \mathrm{CO}, \mathrm{CH}_{2}, \mathrm{~S}, \mathrm{Ca}$, \&c. forming. compounds $\mathrm{XX}^{\prime} \mathrm{Y}$; but diatomio residues, $\mathrm{Y}$, sometímes capable of existing separately may combine together, forming $\mathrm{YY}^{\prime}$ and with $\mathrm{X}_{2}$ or $\mathrm{XX}^{\prime}$, as we see from the transition of $\mathrm{CO}$ into $\mathrm{CO}_{2}$ and $\mathrm{COCl}_{2}$. This combining power of carbonic oxide appears in many of its reactions. Thus it is very easily ab. 'sorbed by cuprous chloride, $\mathrm{CuCl}$, dissolvèd in fuming hydrochlorio acid, forming a crystalline compound, $\mathrm{COCu}_{2} \mathrm{Cl}_{2}, 2 \mathrm{H}_{2} \mathrm{O}$, decomposable by water; it combines directly with potassium (at $90^{\circ}$ ), forming $(\mathrm{KCO})_{n}{ }^{31}$ with platinum dichloride, $\mathrm{PtCl}_{2}$, with chlorine, $\mathrm{Cl}_{2}$, \&c.

But the most remarkable compounds are (1) the compound of $\mathrm{CO}$ with metallic nickel, a colourless volatile liquid, $\mathrm{Ni}(\mathrm{CO})_{4}$, obtained by L. Mond (described in Chapter XXII.) and (2) the compounds of car= bonic oxide with the alkalis, for instance with potassium or barium hydroxide, \&c.- -although it is not directly absorbed by them, as it has no acid properties. Berthelot (1861) showed that potash in the presence of water is capable of absorbing carbonic oxide, but the absorption takes place slowly, little by little, and it is only after being heated for many hours that the whole of the carbonic oxide is absorbed by the potash. The salt $\mathrm{CHKO}_{2}$ is obtained by this absorption; it corresponds with an acid found in nature-namely, the simplest organio (carboxylic) acid, formic acid, $\mathrm{CH}_{2} \mathrm{O}_{2}$. It can be extracted from the potassium salt by means of distillation with dilute sulphuric acid, just as nitric acid is prepared from sodium nitrate. The same acid is found in ants and in nettles (when the stings of the nettles puncture the skin they break, and the corrosive formic acid enters into the body) ; it is also obtained during the action of oxidising agents on many organic substances; it is formed from oxalic acid, and under many conditions splits up into carbonic oxide and water. In the formation of formic acid from carbonic oxide we olserve an example of the synthesis of organic compounds, such as are now very numerous, and are treated of in detail in works on organic chemistry.

Formic acid, $\mathrm{H}\left(\mathrm{CHO}_{2}\right)$, carbonic acid, $\mathrm{HO}\left(\mathrm{CHO}_{2}\right)$, and oxalic acid, $\left(\mathrm{CHO}_{2}\right)_{2}$, are the simple organic or carboxylic acids, $\mathrm{R}\left(\mathrm{CHO}_{2}\right)$ cor-

\$1 The molecule of metallic potassium (Scott, 1887), like that of mercury, contains only one atom, and it is probably in virtue of this that the molecules $\mathrm{CO}$ and $\mathrm{K}$ combine together. But as in the majority of cases potassium acts as a univalent radicle, the polymeride $\mathrm{K}_{2} \mathrm{C}_{2} \mathrm{O}_{2}$ is formed, and probably $\mathrm{K}_{10} \mathrm{C}_{10} \mathrm{O}_{10}$, -because products containing $\mathrm{C}_{10}$ are formed by the action of hydrochloric acid. The black mass formed by the combination of carbonic oxide with potassium explodes with great ease, and oxidises in the air. Although Brodie, Lerch, and Joannis (who obtained it in 1873 in a colourless form by means of $\mathrm{NH}_{3} \mathrm{~K}$, described in Chapter VI., Note 14) have greatly extended our knowledge of this compound, much still remains unexplained. It probably exists in various polymeric and isomeric forms, having the composition $(\mathrm{KCO})_{n}$ and $(\mathrm{NaCO})_{M}$. 
responding with $\mathrm{HH}$ and $\mathrm{HOH}$. Commencing with carbonic oxide, $\mathrm{CO}$, the formation of carboxylic acids is clearly seen from the fact that $\mathrm{CO}$ is capable of combining with $\mathrm{X}_{2}$, that is of forming $\mathrm{COX}_{2}$. If, for instance, one $\mathrm{X}$ is an aqueous residue, $\mathrm{OH}$ (hydroxyl), and the other $\mathrm{X}$ is hydrogen, then the simplest organic acid-formic acid, $\mathrm{H}(\mathrm{COOH})$-is obtained. As all hydrocarbons (Chapter VIII.) correspond with the simplest, $\mathrm{CH}_{4}$, so all organic acids may be considered to proceed from formic acid.

In a similar way it is easy to explain the relation to other com. pounds of carbon of those compounds which contain nitrogen. By way of an example, we will take one of the carboxyl acids, $\mathrm{R}\left(\mathrm{CO}_{2} \mathrm{H}\right)$, where $R$ is a hydrocarbon radicle (residue). Such an acid, like all others, will give by combination with $\mathrm{NH}_{3}$ an ammoniacal salt, $\mathrm{R}\left(\mathrm{CO}_{2} \mathrm{NH}_{4}\right)$. This salt contains the elements for the formation of two molecules of water, and under suitable conditions by the action of bodies capable of taking it up, water may in fact be separated from $\mathrm{R}\left(\mathrm{CO}_{2} \mathrm{NH}_{4}\right)$, forming by the loss of one molecule of water, amides, $\mathrm{RCONH}_{2}$, and by the loss of two molecules of water, nitriles, RCN, otherwise known as cyanogen compounds or cyanides. ${ }^{32}$ If all the carboxyl acids are united not only by many common reactions but also by a mutual conversion into each other (an instance of which we saw above in the conversion of oxalic acid into formaic and carbonic acids) one would expect the same for all the cyanogen compounds also. The common character of their reactions, and the reciprocity of their transformation, were long ago observed by Gay-Lussac, who recognised a common group or radicle (residue) cyanogen, $\mathrm{CN}$, in all of them. The simplest compounds are hydrocyanic or prussic acid, HCN, cyanic acid, $\mathrm{OHCN}$, and free cyanogen, $(\mathrm{CN})_{2}$, which correspond to the three simplest carboxyl acids : formic, $\mathrm{HCO}_{2} \mathrm{H}$, carbonic, $\mathrm{OHCO}_{2} \mathrm{H}$, and oxalic, $\left(\mathrm{CO}_{2} \mathrm{H}\right)_{2}$. Cyanogen, like carboxyl, is evidently a monatomic residue and acid, similar to chlorine. As regards the amides $\mathrm{RCONH}_{2}$, corresponding to the carboxyl acids, they contain the ammoniacal residue $\mathrm{NH}_{2}$, and form a numerous class of organic compounds met with in nature and obtained in many ways, ${ }^{33}$ but not

32 The connection of the cyanogen compounds with the rest of the hydrocarbons by means of carboxyl was enunciated by me, about the year 1860, at the first Annual Meeting of the Russian Naturalists.

33 Thus, for instance, oxamide, or the amide of oxalic acid, $\left(\mathrm{CNH}_{2} \mathrm{O}\right)_{2}$, is obtained in the form of an insoluble precipitate on adding a solution of ammonia to an alcoholic solution of ethyl oxalate, $\left(\mathrm{CO}_{2} \mathrm{C}_{2} \mathrm{H}_{5}\right)_{2}$, which is formed by the action of oxalic acid on alcohol: $\left(\mathrm{CHO}_{2}\right)_{2}+2\left(\mathrm{C}_{2} \mathrm{H}_{5}\right) \mathrm{OH}=2 \mathrm{HOH}+\left(\mathrm{CO}_{2} \mathrm{C}_{2} \mathrm{H}_{5}\right)_{2}$. As the nearest derivatives of ammonia, the amides treated with alkalis vield ammonia and form the salt of the acid. The nitriles âo not, however, give similar reactions so readily. The majority of amides corresponding to acids have a composition $\mathrm{RNH}_{2}$, and therefore recombine with water with great ease even when simply boiled with it, and with still greater facility in presence of 
distinguished by such characteristic peculiarities as the cyanogen com. pounds.

The reactiors and properties of the amides and nitriles of the organic acids are described in detail in books on organic chemistry; we will here only touch upon the simplest of them, and to clearly explain the derivative compounds will first consider the ammoniacal salts and amides of carbonic acid.

As carbonic acid is bibasic, its ammonium salts ought to have the following composition : acid carbonate of ammonium, $\mathrm{H}\left(\mathrm{NH}_{4}\right) \mathrm{CO}_{3}$, and normal carbonate, $\left(\mathrm{NH}_{4}\right)_{2} \mathrm{CO}_{3}$; they represent compounds of one ar two molecules of ammonia with carbonic acid. The acid salt appears in the form of a non-odoriferous and (when tested with litmus) neutral substance, soluble at the ordinary ternperature in six parts of water, insoluble in alcohol, and obtainable in a crystalline form either without water of crystallisation or with various proportions of it. If an aqueous solution of ammonia be saturated with an excess of carbonic anhyciride, and then evaporated over sulphuric acid in the bell jar of an air-pump, crystals of this salt are separated. Solutions of all other ammonium carbonates, when evaporated under the air-pump, yield crystals of this salt. A solution of this salt, even at the ordinary temperature, gives off carbonic anhydride, as do all the acid salts of carbonic acid (for instance, $\mathrm{NaHCO}_{3}$ ), and at $38^{\circ}$ the separation of carbonic anhydride takes place with great rapidity. On losing carbonic anhydride and water, the acid salt is converted into the normal salt, $2\left(\mathrm{NH}_{4}\right) \mathrm{HCO}_{3}$ $=\mathrm{H}_{2} \mathrm{O}+\mathrm{CO}_{2}+\left(\mathrm{NH}_{4}\right)_{2} \mathrm{CO}_{3}$; the latter, however, decomposes in solution, and can therefore only be obtained in crystals, $\left(\mathrm{NH}_{4}\right)_{2} \mathrm{CO}_{3}, \mathrm{H}_{2} \mathrm{O}$, at low temperatures, and from solutions containing an excess of ammonia as the product of dissociation of this salt: $\left(\mathrm{NH}_{4}\right)_{2} \mathrm{CO}_{3}=\mathrm{NH}_{3}$ $+\left(\mathrm{NH}_{4}\right) \mathrm{HCO}_{3}$. But the normal salt, ${ }^{34}$ according to the general type, is

acids or alkalis. Under the action of alkalis the amides naturally give off ammonia, through the combination of water with the amide, when a salt of the acid from which the amide was derived is formed: $\mathrm{RNH}_{2}+\mathrm{KHO}=\mathrm{RKO}+\mathrm{NH}_{3}$.

The same reaction takes place with acids, only an ammoniacal salt of the acid is of course formed whilst the acid held in the amide is liberated: $\mathrm{RNH}_{2}+\mathrm{HCl}+\mathrm{H}_{2} \mathrm{O}$ $=\mathrm{RHO}+\mathrm{NH}_{4} \mathrm{Cl}$.

Thus in the majority of cases amides easily pass into ammoniacal salts; but they differ essentially from them. No ammoniacal salt sublimes or volatilises unchanged, and generally when heated it gives off water and yields an amide, whilst many amides volatilise without alteration and frequently are volatile crystalline substances which may be easily sublimed. Such, for instance, are the amides of benzoic, formic, and many. other organic acids.

34 The acid salt, $\left(\mathrm{NH}_{4}\right) \mathrm{HCO}_{3}$, on losing water ought to form the carbamic acid, $\mathrm{OH}\left(\mathrm{CNH}_{2} \mathrm{O}\right)$; but it is not formed, which is accounted for by the instability of the acid salt itself. Carbonic anhydride is given off and ammonia is produced, which gives ammonium carbamate. 
capable of decomposing with separation of water, and forming ammonium carbamate, $\mathrm{NH}_{4} \mathrm{O}\left(\mathrm{CONH}_{2}\right)=\left(\mathrm{NH}_{4}\right)_{2} \mathrm{CO}_{3}-\mathrm{H}_{2} \mathrm{O}$; this still further complicates the chemical transformations of the carbonates of am. monium. It is in fact evident that, by changing the ratios of water, ammonia, and carbonic acid, various intermediate salts will be formed containing mixtures or combinations of those mentioned above. Thus the ordinary commercial carbonate of ammonia is oltained by heating a mixture of chalk and sulphate of ammonia (Chapter VI.), or sal-ammoniac, $2 \mathrm{NH}_{4} \mathrm{Cl}+\mathrm{CaCO}_{3}=\mathrm{CaCl}_{2}+\left(\mathrm{NH}_{4}\right)_{2} \mathrm{CO}_{3}$. The normal salt, however, through loss of part of the ammonia, partly forms the acid salt, and, partly through loss of water, forms carbamate, and most frequently presents the composition $\mathrm{NH}_{4} \mathrm{O}\left(\mathrm{CONH}_{2}\right)+2 \mathrm{OH}\left(\mathrm{CO}_{2} \mathrm{NH}_{4}\right)$ $=4 \mathrm{NH}_{3}+3 \mathrm{CO}_{2}+2 \mathrm{H}_{2} \mathrm{O}$. This salt, in parting under various conditions with ammonia, carbonic anhydride, and water, does not present a constant composition, and ought rather to be regarded as a mixture of acid salt and amide salt. The latter must be recognised as entering into the composition of the ordinary carbonate of ammonia, because it contains less water than is required for the normal or acid salt $;^{35}$ but on being dissolved in water this salt gives a mixture of acid and normal salts.

Each of the two ammoniacal salts of carbonic acid has its corresponding amide. That of the acid salt should be acid, if the water given off takes up the hydrogen of the ammonia, as it should acccrding to the common type of formation of the amides, so that $\mathrm{OHCONH}_{2}$, or carbamic acid, is formed from $\mathrm{OHCO}_{3} \mathrm{NH}_{4}$. This acid is not known in a free state, but its corresponding ammoniacal salt or ammonium car. bamate is known. The latter is easily and immediately formed by mixing 2 rolumes of $d r y$ ammonia with 1 volume of dry carbonic anhydride, $2 \mathrm{NH}_{3}+\mathrm{CO}_{2}=\mathrm{NH}_{4} \mathrm{O}\left(\mathrm{CONH}_{2}\right)$; it is a solid substance, smells strongly of ammonia, attracts moisture from the air, and decomposes completely at $60^{\circ}$. The fact of this decomposition may be proved ${ }^{36}$ by the density of its vapour, which $=13(\mathrm{H}=1)$; this exactly oorresponds with the density of a mixture of 2 volumes of ammonia and 1 volume

55 In the normal salt, $2 \mathrm{NH}_{3}+\mathrm{CO}_{2}+\mathrm{H}_{2} \mathrm{O}$, in the acid salt, $\mathrm{NH}_{3}+\mathrm{CO}_{2}+\mathrm{H}_{2} \mathrm{O}$, but in the commercial salt only $2 \mathrm{H}_{2} \mathrm{O}$ to $3 \mathrm{CO}_{2}$.

36 Naumann determined the following dissociation tensions of the vapour of ammonium carbamate (in millimetres of mercury) :-

$\begin{array}{cccccccc}-10^{\circ} & 0^{\circ} & +10^{\circ} & 20^{\circ} & 30^{\circ} & 40^{\circ} & 50^{\circ} & 60^{\circ} \\ 5 & 12 & 30 & 62 & 124 & 248 & 470 & 770\end{array}$

Horstmann and Isambert studied the tensions corresponding to excess of $\mathrm{NH}_{3}$ or $\mathrm{CO}_{2}$ and found, as might have been expected, that with such excess the mass of the salt formed (in a solid state) increases and the decomposition (transition into vapour) decreases. 
of carbonic anhydride. It is easily understood that such a combination will take place with any ammonium carbonate under the action of salts which take up the water-for instance, sociium or potassium carbonate ${ }^{37}$-as in an anhydrous state ammonia and carbonic anbydride only form one compound, $\mathrm{CO}_{2} 2 \mathrm{NH}_{3}{ }^{38}$ As the normal ammonium car bonate contains two ammonias, and as the amides are formed with the separation of water at the expense of the hydrogen of the ammonias, so this salt has its symmetrical amide, $\mathrm{CO}\left(\mathrm{NH}_{2}\right)_{2}$. This must be termed carbamide. It is identical with urea, $\mathrm{CN}_{2} \mathrm{H}_{4} \mathrm{O}$, which, contained in the urine (about 2 per cent. in human urine), is for the higher animals (especially the carnivorous) the ordinary product of excretion ${ }^{39}$ and oxidation of the nitrogenous substançes found in the organism, If ammonium carbamate be heated to $140^{\circ}$ (in a sealed tube, Bazaroff), or if carbonys chloride, $\mathrm{COCl}_{2}$, be treated with ammonia (Natanson), urea will be obtained, which shows its direct connection with carbonic acid-that is, the presence of carbonic acid and ammonia in it. From this it will be understoor how urea during the putrefaction of urine is converted into ammonium carbonate, $\mathrm{CN}_{2} \mathrm{H}_{4} \mathrm{O}+\mathrm{H}_{2} \mathrm{O}=\mathrm{CO}_{2}+2 \mathrm{NH}_{3}$.

Thus urea, both by its origin and decomposition, is an amide of oarbonic acid. Representing as it does ammonia (two molecules) in which hydrogen (two atoms) is replaced by the bivalent radicle of carbonic acid, urea retains the property of ammonia of entering into combination, with acids (thus nitric acid forms $\mathrm{CN}_{2} \mathrm{H}_{4} \mathrm{O}, \mathrm{HNO}_{3}$ ), with bases (for instance, with mercury oxide), and with salts (such as sodium chloride, ammonium chloride), but containing an acid residue it has no alkaline properties. It is soluble in water without change, but at a red heat loses ammonia and forms cyanic acid, $\mathrm{CNHO},{ }^{39}$ bis which is a nitrile of carbonic acid-that is to say, is a

5.7 Calcium chloride enters into double decomposition with ammonuum carbamate. Acids (for instance, sulphuric) take up ammonia, and set free carbonic anhydride, whilst alkalis (such as potash) take up carbonic anhydride and set free ammonia, and therefore, in this cașe for removing water only sodium or potassium carbonate cau be taken. An aqueous solution of ammonium carbamate does not entirely precipitate a solution of $\mathrm{CaCl}_{2}$, probably because calcium carbamate is soluble in water, and all the $\left(\mathrm{NH}_{3}\right)_{2} \mathrm{CO}_{2}$. is not converted by dissolving into the normal salt, $\left(\mathrm{NH}_{4} \mathrm{O}\right)_{2} \mathrm{CO}_{3}$.

38 It must be imagined that the reaction takes, place at first between equal volumes (Chapter VII.); but then carbamic acid, $\mathrm{HO}\left(\mathrm{CNH}_{2} \mathrm{O}\right)$, is produccd, wbich, as an acid, immediately combines with the ammonia, forming $\mathrm{NH}_{4} \mathrm{O}\left(\mathrm{CNH}_{2} \mathrm{O}\right)$.

59 Urea is undoubtedly.a product of the oxidation of complex nitrogenous matters (albumin) of the animal body. It is found in the blood. It is absorbed from the blood by the kidneys. A man excretes about 80 grams of urea per day. As a derivative of carbonic anhydride, into. which it is readily converted, urea is in a sense a product of oxidation.

39. is Its polymer, $\mathrm{C}_{3} \mathrm{~N}_{3} \mathrm{H}_{3} \mathrm{O}_{3}$, is formed together with it. Cyanic acld is. ai very unstable, easily changeable liquid, while cyanuric acid is a crystalline solid which is very stable at the ordinary temperature. 
cyanogen compound, corresponding to the acid ammonium carbonate, $\mathrm{OH}\left(\mathrm{CNH}_{4} \mathrm{O}_{2}\right)$, which on parting with $2 \mathrm{H}_{2} \mathrm{O}$ ought to form cyanic acid, $\mathrm{CNOH}$. Liquid cyanic acid, exceedingly unstable at the ordinary temperatures, gives its stable solid polymer cyanuric acid, $\mathrm{O}_{3} \mathrm{H}_{3} \mathrm{C}_{3} \mathrm{~N}_{3}$. Both have the same composition, and they pass one into another at different temperatures. If crystals of cyanuric acid be heated to a tem. perature, $t^{\circ}$, then the vapour tension, $p$, in millimetres of mercury (Troost and Hautefeuille) will be :

$$
\begin{aligned}
& \text { t. } 160^{\circ}, 170^{\circ}, 200^{\circ}, 250^{\circ}, 300^{\circ}, 350^{\circ} \\
& \text { p. 56, 68, 130, 220, 430, 1,200 }
\end{aligned}
$$

'The vapour contains cyanic acid, and, if it be rapidly cooled, it condenses into a mobile volatile liquid (specific gravity at $0^{\circ}=1 \cdot 14$ ). If the liquid cyanic acid be gradually heated, it passes into a new amorphous polymeride (cyamelide), which, on being heated, like cyanuric acid, forms vapours-of cyanic acid. If these fumes are heated above $150^{\circ}$ they pass directly into cyanuric acid. Thus at a temperature of $350^{\circ}$, the pressure does not rise above $1,200 \mathrm{~mm}$. on the addition of vapours of cyanic acid, because the whole excess is transformed into cyanuric acid. . Hence, the above-mentioned figures give the tension of dissociation of cyanuric acid, or the greatest pressure which the vapours of HOCN are able to attain at a given temperature, whilst at a greater pressure, or by the introduction of a larger mass of the substance into a given volume, the whole of the excess is converted into.cyanuric acid. The properties of cyanic acid which we have described were principally observed by Wöhler, and clearly show the faculty of polymerisation of cyanogen compounds. This is observed in many other cyanogen derivatives, and is to be regarded as the consequence of the above-mentioned explanation of their nature. All cyanogen compounds are ammonium salts, $\mathrm{R}\left(\mathrm{CNH}_{4} \mathrm{O}_{2}\right)$, deprived of water, $2 \mathrm{H}_{2} \mathrm{O}$; therefore the molecules, $\mathrm{RCN}$, ought to possess the faculty of combining with two molecules of water or with other molecules in exchange for it (for instance, with $\mathrm{H}_{2} \mathrm{~S}$, or $\mathrm{HCl}$, or $2 \mathrm{H}_{2}, \& \mathrm{c}$.), and are therefore capable of combining together. The combination of molecules of the same kind to form more complex ones is what is meant by polymerisation. ${ }^{40}$

40 Just as the aldehydes (such as $\mathrm{C}_{2} \mathrm{H}_{4} \mathrm{O}$ ) are alcohols (like $\mathrm{C}_{2} \mathrm{H}_{6} \mathrm{O}$ ) which have lost hydrogen and are also capable of entering into combination with many substances, and of polymerising, forming slightly volatile polymerides, which depolymerise on heating. Although there are also many similar phenomena (for instance, the transformation of yellow into red phosphorts, the transition of cinnamene into metacinnamene, \&c.) of polymerisation, in no other case are they so clearly and simply expressed as in cyanic acid. The details relating to this must be sought for in treatises on organic and theoretical chemistry. If we touch on certain sides of this question it is principally with the view of showing the phenomenon of polymerisation by typical examples, for it is of more frequent occurrence than was formerly supposed among compounds of several elements. 
Besides being a substance very prone to form polymerides, cyanic acid presents many other features of interest, expounded in greater detail in organic chemistry. However we may mention here the production of the cyanates by the oxidation of the metallic cyanides. Potassium cyanate, $\mathrm{KCNO}$, is most often obtained in this way. Solutions of oyanates by the addition of sulphuric acid yield cyanic acid, which, however, immediately decomposes : $\mathrm{CNHO}+\mathrm{H}_{2} \mathrm{O}=\mathrm{CO}_{2}+\mathrm{NH}_{3}$. A solution of ammonium cyanate, $\mathrm{CN}\left(\mathrm{NH}_{4}\right) \mathrm{O}$, behaves in the same manner, but only in 'the cold. On being heated it completely changes because it is transformed into urea. The composition of both substances is identical, $\mathrm{CN}_{2} \mathrm{H}_{4} \mathrm{O}$, but the structure, or disposition of, and connection between, the elements is different: in the ammonium cyanate one atom of nitrogen exists in the form of cyanogen, $\mathrm{CN}$ that is, united with carbon-and the other as ammonium, $\mathrm{NH}_{4}$, but, as cyanic acid contains the hydroxyl radicle of carbonic acid, $\mathrm{OH}(\mathrm{CN})$, the ammonium in this salt is united with oxygen. The composition of this salt is best expressed by supposing one atom of the hydrogen in water to be replaced by ammonium and the other by cyanogen-i.e. that its composition is not.symmetrical-whilst in urea both the nitrogen atoms are symmetrically and uniformly disposed as regards the radicle $\mathrm{CO}$ of carbonic acid: $\mathrm{CO}\left(\mathrm{NH}_{2}\right)_{2}$. For this reason, urea is much more stable than ammonium cyanate, and therefore the latter, on being slightly heated in solution, is converted into urea. This remarkable isomeric transformation was discovered by Wöhler in 1828.41 Formamide, $\mathrm{HCONH}_{2}$, and hydrocyanic acid, HCN, as a nitrile, correspond with formic acid, $\mathrm{HCOOH}$, and therefore ammonium formate, $\mathrm{HCOONH}_{4}$, and formamide, when acted on by heat and by substances which take up water (phosphoric anhydride)form hydrocyanic. acid, HCN, whilst, under many conditions (for instance, on combining with hydrochloric acid in presence of water), this hydrocyanic.acid forms formic acid and ammonia. Although coutaining hydrogen in the presence of two acid-forming elements-namely, carbon and nitrogen 42

41 It has an important historical interest, more especially as at that time such an easy preparation of substances occurring in organisms without the aid of organic life was quite unexpected, for thoy were supposed to be formed under the influence of the forces acting in organisms, and without the latter their formation was considered impossible. And in addition to destroying this illusion, the easy transition of $\mathrm{NH}_{4} \mathrm{OCN}$ into $\mathrm{CO}\left(\mathrm{NH}_{2}\right)_{2}$ is the best example of the passage of one system of equilibrium of atoms into another. more stable system.

42 If ammonia and methane (marah gas) do not show any acid properties, that is in all probability due to the presence of a large amount of hydrogen in both; but in hydrocyanic acid one atom of hydrogen is under the influence of two acid-forming elements. Acetylene, $\mathrm{C}_{2} \mathrm{H}_{2}$, which contains but little hydrogen, presents acid properties in certain respects, for its hydrogen is easily replaced by metals. Hydronitrous acid, $\mathrm{HN}_{3}$, which contains little hydrogen, also has the properties of an acid. 
-hydrocyanic acid does not give an acid reaction with litmus (cyanic acid' has very marked acid properties); but it forms salts, MCN, thus presenting the properties of a feeble acid, and for this reason is called an acid. The small amount of energy which it has is shown by the fact that the cyanides of the alkali metals - for instance, potas. sium cyanide $\left(\mathrm{KHO}+\mathrm{HCN}=\mathrm{H}_{2} \mathrm{O}+\mathrm{KCN}\right.$ ) in solution-have a strongly alkaline reaction. ${ }^{43}$ If ammonia be passed over charcoal at a red heat, especially in the presence of an alkali,.or if gaseous nitrogen be passed through a mixture of charcoal and an alkali (especially potash, $\mathrm{KHO}$ ), and also if a mixture of nitrogenous organic substances and alkali be heated to a red heat, in all these cases the alkali metal combines with the carbon and nitrogen, forming a metallic cyanide, $\mathrm{MCN}$ - for example, $\mathrm{KCN}_{0}{ }^{43}$ bis - Potassium cyanide is much used in the arts, and is obtained, as above stated, under many circumstances-as, for instance, in iron smelting, especially with the assistance of wood charcoal, the ash of which contains much potash. The nitrogen of the air, the alkali of the ash, and the charcoal are brought into contact at a high temperature during iron smelting, and therefore; under these conditions, a considerable quantity of potassium cyanide is formed. In practice it is not usual to prepare potassium cyanido directly, but a peculiar. compound of it containing potassium, iron, and cyanogen. This compound is potassium ferrocyanide, and is also known as yellow prussiate of potash. This saline substance (see Chapter XXII) has the composition $\mathrm{K}_{4} \mathrm{FeC}_{6} \mathrm{~N}_{6}+2 \mathrm{H}_{2} \mathrm{O}$. The name of cyanogen (кúavos) is derived from the property which this yellow prussiate possesses of forming, with a solution of a ferric salt, $\mathrm{FeX}_{3}$, the familiar pigment Prussian blue. The yellow prussiate is manu-

43 Solutions of cyanides-for-anstance, those of potassium or barium-are decomposed by carbonic acid. Even the carbonic anhydride of the air acts in a similar way, and for this reason these solutions do not keep, because, in the first place, free hydrocyanic acid itself decomposes and polymerises, and, in the second place, with alkaline liquids it forms ammonia and formic acid. Hydrocyanic acid does not liberate carbonic anhydride from solutions of sodium or potassium carbonates. But a mixture of solutions of potassium carbonate and hydrocyanic acid yields carbonic anhydride on the addition of oxides like zinc oxide, mercuric oxide, \&c. This is due to the great inclination which the cyanides exhibit of forming double salts. For instance, $\mathrm{ZnK}_{2}(\mathrm{CN})_{4}$ is formed, which is a soluble double salt.

43 bis The conyersion of the atmospheric nitrogen into cyanogen compounds, although possible, has not yet been carried out on a large scale, and one of the problems for future research should be the discovery of a practical and economical means of converting the atmospheric nitrogen into metallic cyanides, not only because potassium cyanide has found a vast and important use for the extraction of gold from even the poorest ores, but more especially because the cyanides furnish the means for effecting the synthesis of many complex carbon compounds, and the nitrogen contained in cywnogen easily passes into other forms of combination such as ammonia, which is of great importance in agriculture. 
factured on a large scale, and is generally used as the source of the other cyanogen compounds.

If four parts of yellow prussiate be mixed with eight parts of water and three parts of sulphuric acid, and the mixture be heated, it decomposes, volatile hydrocyanic acid separating. This was obtained for the first time by Scheele in 1782, but it was only known to bim in solution. In 1809 Ittner prepared anhydrous prussic acid, and in 1815 GayLussac finally settled its properties and showed that it contains only hydrogen, carbon, and nitrogen, $\mathrm{CNH}$. If the distillate (a weak solution of $\mathrm{HCN}$ ) be redistilled, and the first part collected, the anhy. drous acid may be prepared from this stronger solution. In order to do this, pieces of calcium chloride are added to the concentrated solution, when the anhydrous acid floats as a separate layer, because it is not soluble in an aqueous solution of calcium chloride. If this layer-be then distilled over a new portion of calcium chloride at-the lowest temperature possible, the prussic acid may be obtained completely free from water. It is, however, necessary to use the greatest caution in work of this kind, because prussic acid, besides being extremely poisonous, is exceedingly volatile. ${ }^{44}$

Anhydrous prussic acid is a very mobile and volatile liquid; its specific gravity is 0.697 at $18^{\circ}$; at lower temperatures, especially when mixed with a small quantity of water, it easily congeals ; it boils at $26^{b}$, and therefore very easily evaporates, and at ordinary temperatures may be regarded as a gas. An insignificant amount, when inhaled or brought into contact with the skin, oauses death. It is soluble in all

44 The mixture of the vapoirs of water and hydrocyanic acid, evolved on heating yellow prussiate with sulphuric acid, may be passed directly through vessels or tubes filled with calcium chloride. These tubes must be cooled, because, in the first place, hydrocyanic acid easily changes on being heated, and, in the second place, the calcium chloride when warm would absorb less water. The mixture of hydrocyanic acid and aqueous vapour on passing over a long layer of calcium chloridg gives up water, and hydrocyanic acid alone remains in the vapour. It onght to be coolea as carefully as possible in order to bring it into a liquid condition. The method which Gay-Lussac employed for obtaining pure hydrocyanic acid consisted in the action of hydrochloric acid gas on mercuric cyanide. The latter may be obtained in a pare state if a solution of yellow prussiate be boiled with a solution of mercuric nitrate, filtered, and crystallised by cooling; the mercuric cyanide is then obtained in the form of colourless crystals, $\mathrm{Hg}(\mathrm{CN})_{2}$.

If a strong solution of hydrochloric acid be poured upon these crystals, and the mixture of vapours evolved, consisting of aqueous vapour, hydrochloric acid, and hydrocyanic acid, be passed through a tube containing, first, marble (for absorbing the hydrochloric acid), and then lumps of calcium chloride, on cooling the hydrocyaric acid will be condensed. In order to obtain the latter in an anhydrous form, the decomposition of heated mercury cyanide by hydrogen sulphide may be made use of. Here the sulphur and cyanogen change ptaces, and hydrocyanic acid and mercury sulphide are formed $\mathrm{Hg}(\mathrm{CN})_{2}+\mathrm{H}_{2} \mathrm{~S}=2 \mathrm{HCN}+\mathrm{HgS}$. 
proportions in water, alcohol, and ether weak aqueous solutions are used in medicine. ${ }^{45}$

The salts MCN - for instance, potassium, sodium, ammonium-as well as the salts $\mathrm{M}^{\prime \prime}(\mathrm{CN})_{2}$ - for example, barium, calcium, mercury-are soluble in water, but the cyanides of manganese, zinc, lead, and many others are insoluble in water. They form double salts with potassium cyanide and similar metallic cyanides, an example of which we will con. sider in a further description of the yellow prussiate. Not only are some of the double salts remarkable for their constancy and comparative stability, but so also are the soluble salt $\mathrm{HgC}_{2} \mathrm{~N}_{2}$, the insoluble silver cyanide $\mathrm{AgCN}$, and even potassium cyanide in the absence of water. The last salt, ${ }^{46}$ when fused, acts as a reducing agent with its elements $\mathbf{K}$ and $\mathbf{C}$, and oxidises when fused with lead oxide, forming potassium. cyanate, KOCN, which establishes the connection between HCN and $\mathrm{OHCN}$ - that is, between the nitriles of formic and carbonic acids-and this connection is the same as that between the acids themselves, since formic acid, on oxidation, yields carbonic acid. Free cyanogen, $(\mathrm{CN})_{2}$ or CNCN, corresponds to hydrocyanic acid in the same manner as free chlorine, $\mathrm{Cl}_{2}$ or $\mathrm{ClCl}$, corresponds to hydrochloric acid. This composition, judging from what has been already stated, exactly expresses that of the nitrile of oxalic acid, and, as a matter of fact, oxalate of ammonia and the amide corresponding with it (oxamide, Note 33), on being beated with phosphoric anhydride, which takes up the water, yield cyanogen, $(\mathrm{CN})_{2}$. This substance is also produced by simply heating some of the

45 A weak (up to 2 p.c.) aqueous solution of hydrocyanic acid is obtained by the dis. tillation of certain vegetable substances. The so-called laurel water in particular enjoys considerable votoriety from its containing hydrocyanic acid. It is obtained by the steeping and distillation of laurel leaves. A similar kind of water is formed by the infusion and distillation of bitter almonds. It is well known that bitter almonds are poisonous, and have a peculiar characteristic taste. This bitter taste is due to the presence of a certain substance called amygdalin, which can be extracted by alcohol, This anygdalin decomposes in an infusion of bruised almonds, forming the so-called bitter almond oil, glucose, and hydrocyanic acid :

$$
\underset{\substack{\text { Amygdalin in } \\
\text { bitter almonds }}}{\mathrm{C}_{10} \mathrm{H}_{27} \mathrm{NO}_{11}}+\underset{\text { Water }}{\mathrm{H}_{2} \mathrm{O}}=\underset{\begin{array}{c}
\text { Bitter } \\
\text { almond oil }
\end{array}}{\mathrm{C}_{7} \mathrm{H}_{6} \mathrm{O}}+\underset{\begin{array}{c}
\text { Hydrocyanic } \\
\text { acid }
\end{array}}{\mathrm{CNH}} \quad \begin{gathered}
2_{6} \mathrm{C}_{12} \mathrm{O}_{6} \\
\text { Glucose }
\end{gathered}
$$

If after this the infusion of bitter almonds be distilled with water, the hydrocyanic acid and the volatile bitter almond oil are carried over with the aqueous vapour. The oil is insoluble in water, or only sparingly soluble, while the hydrocyanic acid remains as an aqueous solution. Bitter almond water is similar to laurel water, and is used like the former in medicine, naturally only in small quantitıes because any considerable amount has poisonous effects. Perfectly pure anhydrous hydrocyanio acid keeps without change, just like the weak solutions, but the strong solutions only keep in the presence of other acids. In the presence of many admixtures these solutions easily give a brown polymeric cubstance, which is also formed in a solution of potassium cyanide.

46 This salt will be described in Chapter XIII. 
metallic cyanides. Mercuric cyanide is particularly adapted for this purpose, because it is easily obtained in a pure state and is then very stable. If mercuric cyanido be heated, it decomposes, in like manner to mercury oxide, into metallic mercury and cyanogen : $\mathrm{HgC}_{2} \mathrm{~N}_{2}=\mathrm{Hg}$ $+\mathrm{C}_{2} \mathrm{~N}_{2} \cdot{ }^{47}$ When cyanogen is formed, part of it always polymerises into a dark brown insoluble substance called paracyanogen, capable of forming cyanogen when heated to redness. ${ }^{48}$ Cyanogen is a colourless, poisonous gas, with a peculiar smell and easily condensed by cooling into a colourless liquid, insoluble in water and having a specitic gravity of 0.86 . It boils at about $-21^{\circ}$, and therefore cyanogen may be easily condensed into a liquid by a strong freezing mixture. At $-35^{\circ}$ liquid cyanogen solidifies. The gas is soluble in water and in alcohol to a considerable extent-namely, 1 volume of water absorbs as much as $4 \frac{1}{2}$ volumes, and alcohol 23 volumes. Cyanogen resists the action of a tolerably high temperature without decomposing, but under the action of the electric spark the carbon is separated, leaving a volume of nitrogen equal to the volume of the gas taken. As it contains carbon it burns, and the colour of the flame is reddish-violet, which is due to the presence of nitrogen, all compounds of which impart more or less of this reddish-violet hue to the flame. During the combustion of

4: For the preparation it is necessary to taike completely dry mercuric cyanide, because when heated in the presence of moisture it gives ammonia, carbonic anhydride, and hydrocyanic acid. Instead of mércurio cyanide, a mixture of perfectly dry yellow prus. siate and mercuric chloride may be used, then double decomposition and the formation of mercuric cyanide take place in the retort. Silver oyanide also disengages cyanogen, on being heated.

48 Paracyanogen is a brown substance (having the composition of cyanogen) which is formed during the preparation of cyanogen by all methods, and remains as a residue. Silver cyanide, on being slightly heated, fuses, and on being further heated evolves a gas : a considerable quantity of paracyanogen remains in the residue. Here it is remarkable that exactly half the cyanogen becomes gaseous, and the other half is transformed into paracyanogen. Metallic silver will be found in the residue with the paracyanogen; it may be extracted with mercury or nitric acid, which does not act on paracyanogen. If paracyanogen be heated in a vacuum it decomposes, forming cyanogen; but here the pressure $p$ for a given temperature $t$ cannot exceed a certain limit, so that the pheno. menon presents all the external appearance of a physical transformation into vapour; but, nevertheless, it is a complete change in the nature of the substance, though limited by the pressure of dissociation, as we saw before in the transformation of cyanuric into hydrocyanic acid, and as would be expected from the fundamental principles of dissociation. Troost and Hautefeuille (1868) found that for paracyanogen,

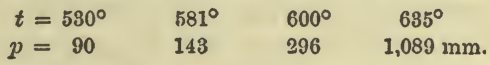

However, even at $550^{\circ}$ part of the cyanogen decomposes into carbon and nitrogen. The reverse transition of cyanogen into paracyanogen commences at $350^{\circ}$, and at $600^{\circ}$ proceeds rapidly. And if the transition of the first kind is likened to evaporation, then the reverse transition; or polymerisation, presents a likeness to the transition of vapours into the solid state. 
cyanogen, carbonic anhydride and nitrogen are formed. The same products are obtained in the eudiometer with oxygen or by. the action of cyanogen on many oxides at a red heat.

The relation of cyanogen to the metallic cyanides is seen not only in the fact that it is formed from mercuric cyanide, but also by its forming cyanide of sodium or potassium on being heated with either of those metals, the sodium or potassium taking fire in the cyanogen. On heating a mixture of hydrogen and cyanogen to $500^{\circ}$ (Berthelot), ${ }^{49}$ or under the action of the silent discharge (Boilleau), hydrocyanic acid is formed, so that the reciprocity. of the transitions does not leave any doubt in the matter that all the nitriles of the organic acids contain cyanogen, just as all the organic acids contain carboxyl and in it the elements of carbonic anhydride. Besides the amides, ${ }^{50}$ the nitriles (or cyanogen compounds, RCN), and nitro-compounds (containing the radicle of nitric acid, $\mathrm{RNO}_{2}$ ), there are a great number of other substances containing at the same time carbon and nitrogen, particulars of which must be sought for in special works on organic chemistry.

49 Cyanogen (like chlorine) is absorbed by a solution of sodium hydroxide, sodium cyanide and cyanate being produced: $\mathrm{C}_{2} \mathrm{~N}_{2}+2 \mathrm{NaHO}=\mathrm{NaCN}+\mathrm{CNNaO}+\mathrm{H}_{2} \mathrm{O}$. But the latter salt decomposes relatively easily, and moreover part of the cyanogen liberated by heat from its compounds undergoes a more complex transformation.

so If, in general, compounds containing the radicle $\mathrm{NH}_{2}$ are called amides, some of the amines ought to be ranked with them; namely, the hydrociarbons $\mathrm{C}_{n} \mathrm{H}_{2 m}$, in which part of the hydrogen is replaced by $\mathrm{NH}_{2}$; for instance, methylamine, $\mathrm{CH}_{3} \mathrm{NH}_{2}$, aniline, $\mathrm{C}_{6} \mathrm{H}_{5} \mathrm{NH}_{2}$, sc. In general the amines may be represented as ammonia in which part or all of the hydrogen is replaced by hydrocarbon radicles-as, for example, trimethylamine, $\mathrm{N}\left(\mathrm{CH}_{3}\right)_{3}$. They, like ammonia, combine with acids and form crystalline salts. Analogous substances are sometimes met with in nature, and bear the general name of alkaloids; such are, for instance, quinine in cinchona bark, nicotine in tobacco, \&c. 


\section{CHAPTER X}

\section{SODIUM CHLORIDE-BERTHOLLET'S LAWS-HYDROCHLORIC ACID}

IN the preceding chapters we have become acquainted with the most important properties of the four elements, hydrogen, oxygen, nitrogen, and carbon. They are sometimes termed the organogens, because they enter into the composition of organic substances. Their mutual com. binations may serve as types for all other chemical compounds-that is, they present the same atomic relations (types, forms, or grades of combinations) as those in which the other elements also combine together.

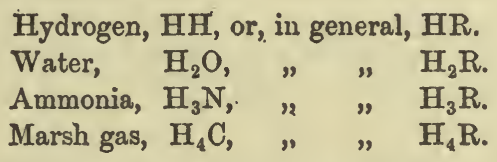

One, two, three, and four atoms of hydrogen enter into these molesules for one atom of another element. No compounds of one atom of oxygen with three or four atoms of hydrogen are known; hence the atom of oxygen does not possess certain properties which are found in the atoms of carbon and nitrogen.

The faculty of an element to form a compound of definite composition with hydrogen (or an element analogous to it) gives the possibility of foretelling the composition of many other of its compounds. Thus, if we know that an element, $M$, combines with hydrogen, forming, by preference, a gaseous substance such as HM, but not forming $\mathrm{H}_{2} \mathrm{M}, \mathrm{H}_{3} \mathrm{M}, \mathrm{H}_{\mathrm{n}} \mathrm{M}_{\mathrm{m}}$, then we must conclude, on the basis of the law of substitution, that this element will give compounds $\mathrm{M}_{2} \mathrm{O}, \mathrm{M}_{3} \mathrm{~N}, \mathrm{MHO}$, $\mathrm{MH}_{3} \mathrm{C}$, \&c. Chlorine is an example of this kind. If we know that another element, $\mathrm{R}$, like oxygen, gives with hydrogen a molecule $\mathrm{H}_{2} \mathrm{R}$, then we may expect that it will form compounds similar to hydrogen peroxide, the metallic oxides, carbonic anhydride, or carbonic oxide, and others. Sulphur is an instance of this kind. Hence the elements may be classified according to their resemblance to hydrogen, oxygen, nitrogen, and carbon, and in conformity with this analogy it is possible 
to foretell, if not the properties (for example, the acidity or basicity), at any rate the composition, ${ }^{3}$ of some of their compounds. This forms the substance of the conception of the valency or atomicity of the elements. Hydrogen is taken as the representative of the univalent elements, giving compounds, $\mathrm{RH}, \mathrm{R}(\mathrm{OH}), \mathrm{R}_{2} \mathrm{O}, \mathrm{RCl}, \mathrm{R}_{3} \mathrm{~N}, \mathrm{R}_{4} \mathrm{C}$, \&c. Oxygen, in that form in which it gives water, is the representative of the

1 But it is impossible to foretell all the compounds formed by an element from its atomicity or valency, because the atomicity of the elements is variable, and furthermore this variability is not identical for different elements. In $\mathrm{CO}_{2}, \mathrm{COX}_{2}, \mathrm{CH}_{4}$, and the multitude of carbon compounds corresponding with them, the $\mathrm{C}$ is quadrivalent, but in CO either the carbon must be taken as bivalent or the atomicity of oxygen be accounted as variable. Moreover, carbon is an example of an element which preserves its atomicity to a greater degree than most of the other elements. Nitrogen in $\mathrm{NH}_{5}, \mathrm{NH}_{2}(\mathrm{OH})$, $\mathrm{N}_{2} \mathrm{O}_{3}$, and even in $\mathrm{CNH}$, must be considered as trivalent, but in $\mathrm{NH}_{4} \mathrm{Cl}, \mathrm{NO}_{2}(\mathrm{OH})$, and in all their corresponding compounds it is necessarily pentavalent. In $\mathrm{N}_{2} \mathrm{O}$, if the atomicity of oxygen $=2$, nitrogen has an uneven atomicity $(1,3,5)$, whilst in $N O$ it is bivalent. If sulphur be bivalent, like oxygen, in many of its campounds (for example, $\mathrm{H}_{2} \mathrm{~S}, \mathrm{SCl}_{2}, \mathrm{KHS}$, \&c.), then it could not be foreseen from this that it would form $\mathrm{SO}_{2}$, $\mathrm{SO}_{3}, \mathrm{SCl}_{4}, \mathrm{SOCl}_{2}$, and a series of similar compounds in which its atomicity must be acknowledged as greater than 2. Thus $\mathrm{SO}_{2}$, dulphurous anhydride, has many points in common with $\mathrm{CO}_{2}$, and if carbon . be quadrivalent then the $\mathrm{S}$ in $\mathrm{SO}_{2}$ is quadrivalent. Therefore the principle of atomicity (valency) of the elements cannot be considered established as the basis for the study of the elements, although it gives an easy method of grasping many analogies. I consider the four following as the chief obstacles to acknowledging the atomicity of the elements as a primary conception for the consideration of the properties of the elements: 1 . Such univalent elements as $\mathrm{H}, \mathrm{Cl}$, \&c., appear in a free state as molecules $\mathrm{H}_{2}, \mathrm{Cl}_{2}$, \&c., and are consequently like the univalent radicles $\mathrm{CH}_{3}, \mathrm{OH}, \mathrm{CO}_{2} \mathrm{H}$, \&c., which, as might be expeeted, appear as $\mathrm{C}_{2} \mathrm{H}_{6}, \mathrm{O}_{2} \mathrm{H}_{2}$, $\mathrm{C}_{2} \mathrm{O}_{4} \mathrm{H}_{2}$ (ethane; hydrogen peroxide, oxalic acid), whilst on the other.hand, potassium and sodium (perhaps also iodine at a high temperature) contain only one atom, $\mathrm{K}, \mathrm{Na}$, in the molecule in a free state. Hence it follows that free affinities may exist. Granting this, nothing prevents the assumption that free affinities exist in all unsaturated compounds; for example, two free affinities in $\mathrm{NH}_{3}$. If such instances of free aftinities be admitted, then all the possible advantages to be gained by the application of the doctrine of atomicity (valency) are lost. 2. There are instances-for example, $\mathrm{Na}_{2} \mathrm{H}$-where univalent elements are combined in molecules which are more complex than $R_{2}$, and form molecules, $\mathbf{R}_{3}, \mathbf{R}_{4}$, \&c.; this may again be either taken as evidence of the existence of free affinities, or else necessitates such primary univalent elements as sodium and hydrogen being considered as variable in their atomicity. 3. The periodio system of the elements, with which we shall afterwards become acquainted, shows that there is a law or rule for the variation of the forms of oxygen and hydrogen compounds; chlorine is univalent with respect to hydrogen, and septavalent with respect to oxygen; sulphur is bivalent to hydrogen, and sexavalent to oxygen; phosphorus is triralent to hydrngen and pentavalent in respect to oxygen-the sum is in every case equal to 8. Only carbon and its analogues (for example, silicon) are quadrivalent to both hydrogen and oxygen, Hence the power of the elements to change their atomicity is an essential part of their nature, and therefore constant valency cannot be considered as a fundamental property. 4. Crystallo-hydrates (for instance, $\mathrm{NaCl}_{2} \mathrm{H}_{2} \mathrm{O}$, or $\mathrm{NaBr}, 2 \mathrm{H}_{2} \mathrm{O}$ ), double salts (such as $\mathrm{PtCl}_{4}, 2 \mathrm{KCl}, \mathrm{H}_{2} \mathrm{SiF}_{6}$, \&c.), and similar complex compounds (and, according to Chap. I., solutions also) demonstrate the capacity not only of the elements themselves, but also of their saturated and limiting compounds, of entering into further combination. Therefore, the admission of a definite limited atomicity of the elements includes in itself an admission of limitation which is not in accordance with the nature of chemical reactions. 
bivalent elements, forming $\mathrm{RH}_{2}, \mathrm{RO}, \mathrm{RCl}_{2}, \mathrm{RHCl}, \mathrm{R}(\mathrm{OH}) \mathrm{Cl}, \mathrm{R}(\mathrm{OH})_{2}$, $\mathrm{R}_{2} \mathrm{C}, \mathrm{RCN}$, \&c. Nitrogen in ammonia is the representative of the trivalent elements, giving compounds $\mathrm{RH}_{3}, \mathrm{R}_{2} \mathrm{O}_{3}, \mathrm{R}(\mathrm{OH})_{3}, \mathrm{RCl}_{3}, \mathrm{RN}$, $\mathrm{RHC}$, dc. In carbon are exemplified the properties of the quadrivalent elements, forming $\mathrm{RH}_{4}, \mathrm{RO}_{2}, \mathrm{RO}(\mathrm{OH})_{2}, \mathrm{R}(\mathrm{OH})_{4}, \mathrm{RHN}, \mathrm{RCl}_{4}, \mathrm{RHCl}_{3}$, ic. We meet with these forms of combination, or degrees of union of atoms, in all other elements, some being analogous to hydrogen, others to. oxygen, and others to nitrogen or to carbon. But besides these quan. titative analogies or resemblances, which are foretold by the law of substitution (Chapter VI.), there exist among the elements qualitative analogies and relations which are not fully seen in the compounds of the elements which have been considered, but are most distinctly exhibited in the formation of bases, acids, and salts of different types and properties. Therefore, for a complete study of the nature of the elements and their compounds it is especially important to become acquainted, with the salts, as substances of a peculiar character, and with the corresponding acids and-bases. Common table salt, or sodium chloride, $\mathrm{NaCl}$, may in every respect be taken as a type of salts in general, and we will therefore pass to the consideration of this substance, and of hydrochloric acid, and of the base sodium hydroxide, formed by the non-metal chlorine and the metal sódium, which correspond with it.

Sodium chloride, $\mathrm{NaCl}$, the familiar table salt, occurs, although in very small quantities, in all the primary formations of the earth's crust, ${ }^{2}$ from which it is washed away by the atmospheric waters ; it is contained in small quantities in all waters flowing through these formations, and is in this manner conreyed to the oceans and seas. The immense mass of salt in the oceans has been accumulated by this process from the remote ages of the earth's creation, because the water has evaporated from them while the salt has remained in solution. The salt of sea water serves as the source not only for its direct extraction, but

2 The primary formations are those which do not bear any distinct traces of having been deposited from water (have not a stratified formation and contain no remains of animal or vegetable life), occur under the sedimentary formations of the earth, and are everywhere uniform in composition and structure, the latter being generally distinctly crystalline. If it be assumed that the earth was originally in a molten condition, the first primary formations are those which formed the first solid crust of the earth. But even with this hypothesis of the earth's origin, it is necessary to admit that the first aqueous deposits must have caused a change in the original crust of the earth, and therefore under the head of primary formations must be understood the most ancient of the products of decomposition (mostly by atmospheric, aqueous, and organic agency, \&c.), from which all the rocks and substances of the earth's surface have arisen. In speaking of the origin of one or another substance, we can only, on the basis of facts, descend to the primary formations, of which granite, gneiss, and trachyte may be taken as examples. 
also for the formation of other masses of workable salt, such as rock salt, and of saline springs and lakes. ${ }^{2}$ bis

The extraction of salt from sea water is carried on in several ways. In southern climes, especially on the shores of the Atlantic Ocean and the Mediterranean and Black Seas, the summer heats are taken advantage of. A convenient low-lying sea shore is chosen, and a whole series of hasins, communicating with each other, are constructed along it. The upper of these basins are filled with sea water by pumping, or else adrantage is taken of high tides. These basins are sometimes separated from the sea by natural sand-banks (limans) or by artificial means, and in spring the water already begins to eraporate considerably. As the solution becomes more concentrated, it is run into the succeeding basins, and the upper ones are supplied with a fresh quantity of sea water, or else an arrangement is made enabling the salt water to flow by degrees through the series of basins. It is evident that the beds of the basins should be as far as possible impervious to water, and for this purpose they are made of beaten clay. The crystals of salt begin to separate out when the concentration attains 28 p.c. of salt (which corresponds to $28^{\circ}$ of Baumés hydrometer). They are raked off, and employed for all those purposes to which table salt is applicable. In the majority of cases only the first half of the sodium chloride which can be separated from the sea water is extracted, because the second half has a bitter taste from the presence of magnesium salts which separate out together with the sodium salt. But in certain localities-as, for instance, in the estuary of the Rhone, on the island of Camarga ${ }^{3}$-the evaporation is carried on to the very end, in order to obtain those magnesium and potassium salts which separate out at the end of the evaporation of sea water. Various salts are separated from sea water in its evaporation. From 100 parts of sea water there separates out, by natural and artificial evaporation, about one part of tolerably pure table salt at tho very commencement of the operation; the total amount held in solution being about $2 \frac{1}{2}$ p.c. The remaining portion separates out inter-

2 bls Chloride of sodium has been found to occur in the atmosphere in the form of a fine dust; in the lower strata it is present in larger quantities than in the upper, so that the rain water falling on mountuins contains less $\mathrm{NaCl}$ than that falling in valleys. Mitintz (1891) found that a litre of rain water collected on the summit of the Pic du Midi (2,877 metres above the sea level) contained 0.34 milligram of chlorid 3 ,of sodium. while a litre of rain collected from the valley contained $2 \cdot 5-7 \cdot 6$ milligrams.

s The extraction of the potassium salts (or so-called summer salts) was carried on at the Isle of Camargr about 1870, when I had occasion to visit that spot. At the present time the deposits of Stassfurt provide a much cheaper salt, owing to the evaporation and separation of the salt being carried on there by natural means and only requiring a treatment and refining, which is also vecessary in addition for the 'summer salt' obtained from sea-water. 
mixed with the bitter salts of magnesium which, owing to their solubility and the small amount in which they are present (less than 1 p.c.), only separate out, in the first crystallisations, in traces. Gypsum, or calcium sulphate, $\mathrm{CaSO}_{4} 2 \mathrm{H}_{2} \mathrm{O}$, because of its sparing solubility, separates together with or even before the table salt. When about half of the latter has separated, then a mixture of table salt and magnesium sulphate separates out, and on still further evaporation the chlorides of potassium and magnesium begin to separate in a state of combination, forming the double salt $\mathrm{KMgCl}_{3}, 6 \mathrm{H}_{2} \mathrm{O}$, which occurs in nature as carnallite. ${ }^{4}$ After the separation of this salt from sea water, there remains a mother liquor containing a large amount of magnesium chloride in admixture with various other salts. ${ }^{5}$ The extraction of sea. salt is usually carried on for the purpose of procuring table salt, and therefore directly it begins to separate mixed with a considerable proportion ${ }^{6}$ of magnesium salts (when it acquires a bitter taste) the remaining liquor is run back into the sea.

The same process which is employed for artificially obtaining salt in a crystalline form from sea water has been repeatedly accomplished during the geological evolution of the earth on a gigantic scale; up heavals of the earth have cut off portions of the sea from the remainder (as the Dead Sea was formerly a part of the Mediterranean, and the Sea of Aral of the Caspian), and their water has evaporated and formed (if the mass of the inflowing fresh water were less than that of the mass evaporated) deposits of rock salt. It is always accompanied by gypsum, because the latter is separated from sea water with or before the sodiun chloride. For this reason rock salt may always be looked for

4 The double salt $\mathrm{KCl}, \mathrm{MgCl}_{2}$ is a crystallohydrate of $\mathrm{KCl}$ and $\mathrm{MgCl}_{2}$, and is only formed from solutions containing an excess of magnesium chloride, because water decomposes this double salt, extracting the more soluble maguesium chloride from it.

5 Owing to the fundamental property of salts of interchanging their metals, it cannot be said that sea water contains this or that salt, but only that it contains certain amounts of certain metals $\mathrm{M}$ (univalent like $\mathrm{Na}$ and $\mathrm{K}$, and bivalent like $\mathrm{Mg}$ and $\mathrm{Ca}$ ), and haloids $\mathrm{X}$ (univalent like $\mathrm{Cl}, \mathrm{Br}$, and bivalent like $\mathrm{SO}_{4}, \mathrm{CO}_{3}$ ), which are disposed in every possible kind of grouping; for instance, $\mathrm{K}$ as $\mathrm{KCl}, \mathrm{KBr}, \mathrm{K}_{2} \mathrm{SO}_{4}, \mathrm{Mg}$ as $\mathrm{MgCl}_{2}$, $\mathrm{MgBr}_{2}, \mathrm{MgSO}_{4}$, and so on for all the other metals. In evaporation different salts separate out consecutively only because they reach saturation. A proof of this may be seen in the fact that a solution of a mixture of sodium chloride and magnesium sulphate (both of which salts are obtained from sea water, as was mentioned above), when evaporated, deposits crystals of these salts, but when refrigerated (if the solution be sufficiently saturated) the salt $\mathrm{Na}_{2} \mathrm{SO}_{4}, 10 \mathrm{H}_{2} \mathrm{O}$ is first deposited because it is the first to arrive at saturation at low temperatures. Consequently this solution contains $\mathrm{MgCl}_{2}$ and $\mathrm{Na}_{2} \mathrm{SO}_{4}$, besides $\mathrm{MgSO}_{4}$ and $\mathrm{NaCl}$. So it is with sea water.

6 The salt extracted from water is piled up in heaps and left exposed to the action of rain water, which purifies it, owing to the water becoming saturated with sodium chloride and then no longer dissolving it, but washing out the impurities. 
in those localities where there are deposits of gypsum. But inasmuch as the gypsum remains on the spot where it has been deposited (as it is a sparingly soluble salt), whilst the rock salt (as one which is very soluble) may be washed away by rain or fresh running water, it may sometimes happen that although gypsum is still found there may be no salt; but, on the other hand, where there is rock salt there will always be gypsun. As the geological changes of the earth's surface are still proceeding at the present day, so in the midst of the dry land salt lakes are net with, which are sometimes scattered over vast districts formerly covered by seas now dried up. Such is the origin of many of the salt lakes about the lower portions of the Volga and in the Kirghiz steppes, where at a geological epoch preceding the present the Aralo-Caspian Sea extended. Such are the Baskunchaksky (in the Government of Astrakhan, 112 square kilometres superficial area), the Eltonsky (140 versts from the left bank of the Volga, and 200 square kilometres in superficial area), and upward of 700 other salt lakes lying about the lower portions of the Volga. In those in which the inflow of fresh water is less than that yearly evaporated, and in which the concentration of the solution has reached saturation, the selfcleposited salt is found already deposited on their beds, or is being yearly deposited during the summer months. Certain limans, or sea-side lakes, of the Azoff Sea are essentially of the same character-as, for instance, those in the neighbourhood of Henichesk and Berdiansk. The saline soils of certain Central Asian steppes, which suffer from a want of atmospheric fresh water, are of the same origin. Their salt originally proceeded from the salt of seas which previously covered these localities, and has not yet been washed away by fresh water. The main result of the above-described process of nature is the formation of masses of rock salt, which are, however, being gradually washed away by the subsoil waters flowing in their neighbourhood, and afterwards rising to the surface in certain places as saline springs, which indicate the presence of masses of deposited rock salt in the depths of the earth. If the subsoil water flows along a stratum of salt for a sufficient length of time it becomes saturated; but in flowing in its further course along an impervious stratum (clay) it becomes diluted by the fresh water leaking through the upper soil, and therefore the greater the distance of a saline spring from the deposit of rock salt, the poorer will it be in salt. A-perfectly saturated brine, however, may be procured from the depths of the earth by means of bore-holes. The deposits of rock salt themselves, which are sometimes hidden at great depths below the earth's strata, may be discovered by the guidance of bore-holes and the direction of the strata of the district. Deposits of rock salt, about 
35 metres thick and 20 metres below the surface, were discovered in this manner in the neighbourhood of Brianstcheffky and Dekonoftky, in the Bakhmut district of the Government of Ekaterinoslav Large quantities of most excellent rock salt are now (since 1880) obtained from these deposits, whose presence was indicated by the neighbouring salt springs (near Slaviansk and Bakhmut) and by bore-holes which had been sunk in these localities for procuring strong (saturated) brines. But the Stassfurt deposits of rock salt near Magdeburg in Germany are celebrated as being the first discovered in this manner, and for their many remarkable peculiarities. ${ }^{7}$ The plentiful distribution of saline springs in this and the neighbouring districts suggested the presence of deposits of rock salt in the vicinity. Deep bore-holes sunk in this locality did in fact give a richer brine-even quite saturated with salt. On sinking to a still greater depth, the deposits of salt themselves were at last arrived at. But the first deposit which was met with consisted of a bitter salt unfit for consumption, and was therefore called refuse salt (Abraumsalz). On sinking still deeper vast beds of real rock salt were struck. In this instance the presence of these upper strata containing salts of potassium, magnesium, and sodium is an excellent proof of the formation of rock salt from sea water. It is very evident that not only a case of evaporation to the end-as far, for instance, as the separation of carnallite-but also the preservation of such soluble salts as separate out from sea water after the sodium chloride, must be a very exceptional phenomenon, which is not repeated in all deposits of rosk salt. The Stassfurt deposits therefore are of particular interest, not only from a scientific point of view, but also because they form a rich source of potassium salts which have many practical uses. ${ }^{\text {lis }}$

3 When the German savants pointed out the exact locality of the Stassfurt salt. beds and their depth below the surface, on the basis of information collected from various quarters respecting bore-holcs and the direction of the strata, and when the borings, conducted by the Government, struck a salt-bed which was bitter and unfit for use, there was a great outcry against science, and the doubtful result even caused the cessation of the further work of deepening the shafts It required a great effort to persuade the Government to continue the work. Now, when the pure salt encountered below forms one of the important riches of Germany, and when those 'refuse salts' have proved to be most valuable (as a source of potassium and magnesium), we should see in the utilisation of the Stassfurt deposits one of the conquests of science for the common welfare.

7 bis In Western Europe, deposits of rock salt have long been known at Wieliczkr, near Cracow, and at Cardona in Spain. In Russia the following deposits are.known: (a) the vast masses of rock salt (3 square kilometres area and up to 140 metres thick) lying directly on the surface of the earth at Iletzky Zastchit, on the left bank of the river Ural, in the Government of Orenburg; $(b)$ the Chingaksky deposit, 90 versts from the river Volga, in the Enotaeffsky district of the Government of Astrakhan; $(c)$ the Kulepinsky (and other) deposits (whose thickness attains 150 metres), on the Araks, in 
A saturated brine, formed by the continued contact of subsoil water with rock salt, is extracted by means of bore-holes, as, for instance, in the Governments of Perm, Kharkoff, and Ekaterinoslav. Sometimes, as at Berchtesgaden (and at Hallein) in Austria, spring water is run on to underground beds of rock salt containing much clay.

If a saline spring, or the salt water pumped from bore-holes, contains but little salt, then the first concentration of the natural solution

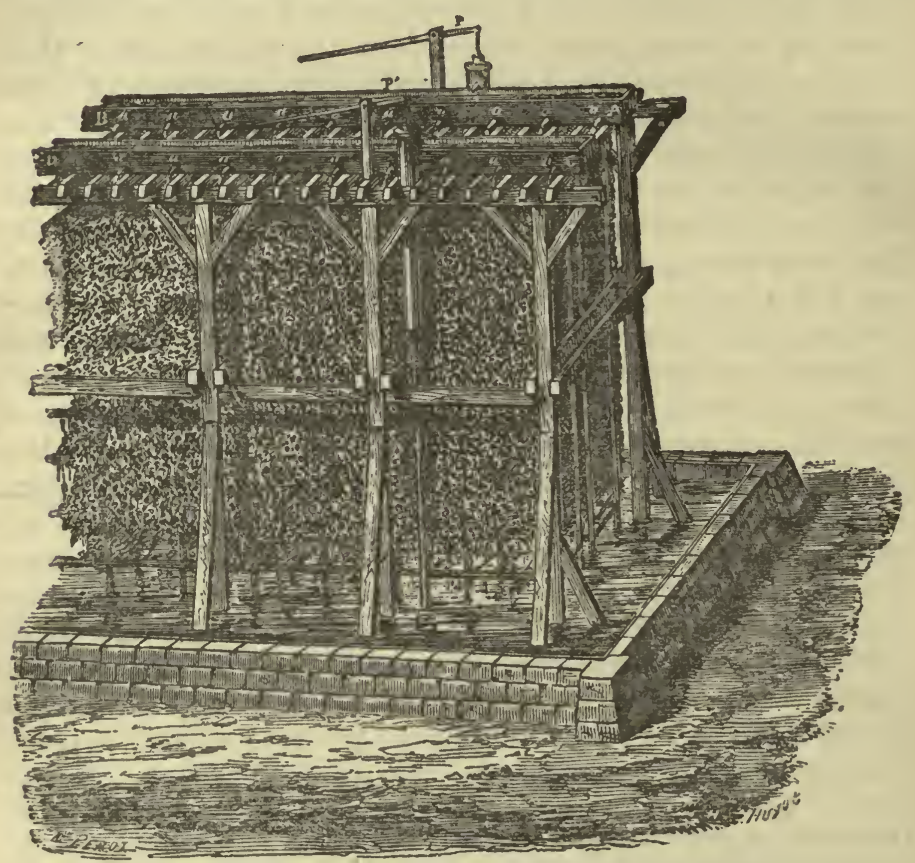

FI. 64.-Graduator for the evaporation of the water of saline springs.

is not carried on by the costly consumption of fuel, but by the cheaper method of evaporation by means of the wind For this purpose so-called graduators are constructed : they consist of long and lofty sheds, which are sometimes several versts long, and generalls extend in \& direction at right angles to that of the usual course of the wind in the district. These sheds are open at the sides, and are filled with brushwood as shown in fig. 64 . Troughs, $A B, C D$, into which the salt the Government of Erivan in the Caucasus; $(d)$ the-Katchiezmansky deposit in the province of Kars ; $(e)$ the Krasnovodsky deposit in the Trans-Caspian province; and $(f)$ the Bardymkulsky salt mines in Kokhand. 
water is pumped, run along the top. On flowing from these troughs, through the openings, $a$, the water spreads over the brushwood and distributes itself in a thin layer over it, so that it presents a very large surface for evaporation, in consequence of which it rapidly becomes concentrated in warm or windy weather. After trickling over the brushwood, the solution collects in a reservoir under the graduator, whence it is usually punped up by the pumps $\mathrm{P} \mathrm{P}^{\prime}$, and again run a second and third time through the graduator, until the solution reaches a degree of concentration at which it becomes profitable to extract the salt by direct heating. Generally the evaporation in the graduator is not carried beyond a concentration of 12 to 15 parts of salt in 100 parts of solution. Strong natural solutions of salt, and also the graduated solutions, are evaporated in large shallow metallic vessels, which are either heated by the direct action of the flame from below or from above. These vessels are made of boiler plate, and are called salt-pans. Various means are employed for accelerating the evaporation and for economising fuel, which are mainly based on an artificial draught to carry off the steam as it is formed, and on subjecting the saline solution to a preliminary heating by the waste heat of the steam and furnace gases. Furthermore, the first portions of the salt which crystallise out in the salt-pans are invariably contaminated with gypsum, since the waters of saline springs always contain this substance. It is only the portions of the salt which separate later that are distinguished by their great purity. The salt is ladled out as it is deposited, left to drain on inclined tables and then dried, and in this manner the socalled bay salt is obtained. Since it has become possible to discover the saline deposits themselves, the extraction of table salt from the water of saline springs by evaporation, which previously was in general use, has begun to be disused, and is only able to hold its ground in places where fuel is cheap.

In order to understand the full importance of the extraction of salt, it need only be mentioned that on the average $20 \mathrm{lbs}$. of table salt are consumed yearly per head of population, directly in food or for cattle. In those countries where common salt is employed in technical processes, and especially in England, almost an equal quantity is consumed in the production of substances containing chlorine and sodium, and especially in the manufacture of washing soda, \&c., and of chlorine compounds (bleaching powder and hyrdochloric acid). The yearly pro. duction of salt in Europe amounts to as much as $7 \frac{1}{2}$ million tons.

Although certain lumps of rock salt and crystals of bay salt sometimes consist of almost pure sodium chloride, still the ordinary com. mercial salt contains various impurities, the most common of which are 
maghesium salts. If the salt be pure, its solution gives -no precipitate with sodium carbonate, $\mathrm{Na}_{2} \mathrm{CO}_{3}$, showing the absence of magnesiun salts, because magnesium carbonate, $\mathrm{MgCO}_{3}$, is insoluble in water: Rock salt, which is ground for use, generally contains also a considerable admixture of clay and other insoluble impurities. ${ }^{8}$ For ordinary use the bulk of the salt obtained .can be employed directly without further purification; but some salts are purified by solution and crystallisation of the solution after standing, in which case the evaporation is not carried on to dryness, and the impurities remain in the mother liquor or in the sediment, When perfectly pure salt is required for chemical purposes it is best obtained as follows: a saturated solution of table salt is prepared; and hydrochloric acid gas is passed through it; this precipitates the sodium chloride (which is not soluble in a strong solution of hydrochloric acid), while the impurities remain in solution. By repeating the operation and fusing the salt (when adhering hydrochloric acid is volatilised) a pure salt is obtained, which is again crystallised from its solution by evaporation. ${ }^{9}$

Pure sodium chloride, in well-defined crystals (slowly deposited at the bottom of the liquid) or in compact masses (in which form rock salt is sometimes met with), is a colourless and transparent substance resembling, but more brittle and less hard than, glass. ${ }^{10}$ Common salt always crystallises in the cubic system, most frequently in cubes, and more rarely in octahedra. Large transparent cubes of common salt, having edges up to 10 . centimetres long, are sometimes found in inasses of rock salt. ${ }^{11}$ When evaporated in the open the salt often-

8 The fracture of rock salt generally shows the presence of interlayers of impurities which are sometimes very small in weight, but visible owing to their refraction. In the excellently laid out salt mines of Briansk I counted (1888), if my memory does not deceive $m e$, on an average ten interlayers per metre of thickness, between which the salt was in general very pure, and in places quite transparent. If this be the case, then there would be 850 interlayers for the whole thickness (about 35 metres) of the bed. They probably correspond with the yearly deposition of the salt. In this case the deposition would have extended over more than 300 years. This should be observable at the present day in lakes where the salt is saturated and in course of deposition.

9 My own investigations have shown that not only the sulphates, but also the potassium salts, are entirely removed by this method.

10 According to the determinations of Klodt, the Briansk rock salt withstands a pressure of 340 kilograms per square centimetre, whilst glass withstands 1,700 kilos. In this respect salt is twice as secure as bricks, and therefore immense masses may be extracted from underground workings with perfect safety, withont having recourse to brickwork supports, merely taking advantage of the properties of the salt itself.

11 To obtain well-formed crystals, a saturated solution is mixed with ferric chloride, several small crystals of sodium chloride are placed at the bottom, and the solution is allowed to evaporate slowly in a vessel with a loose-fitting cover. Octahedral crystals are obtained by the addition of borax, urea, \&c., to the solution. Very fine crystals are formed in a mass of gelatinous silica. 
(separates out on the surface ${ }^{12}$.as cubes, which grow on to each other in the form of pyramidal square funnels. In still weather, these clusters are able to support themselves on the surface of the water for a long time, and sometimes go on increasing to a considerable extent, but they sink directly the water penetrates inside them. Salt fuses to a colourless liquid (sp. gr. 1.602, according to Quincke) at $851^{\circ}$ (V.Meyer); if pure it solidifies to a non-crystalline mass, and if impure to an opaque mass whose surface is not smooth In fusing, sodium chloride commences to volatilise (its weight decreases) and at a white heat it volatilises with.great ease and completely; but at the ordinary temperature it may, like all ordinary salts, be considered as non-volatile, although as yet no exact experiments have been made in this direction.

A saturated ${ }^{13}$ solution of table salt (containing 26.4 p.c.) has at the ordinary temperature a specific gravity of about $1 \cdot 2$. The specific gravity of the crystals is $2.167\left(17^{\circ}\right)$. The salt which separates out at the ordinary and higher temperatures contains no water of crystallisation $;^{14}$ but if the crystals are formed at a low temperature,

12 If a solution of sodium chloride be slowly heated from above, where the evaporation takes place, then the upper layer will become saturated before the lower and cooler layers, and therefore crystallisation will begin on the surface, and the crystals first formed will float, having also dried from above, on the surface until they become quite soaked. Being heavier.than the solution the crystals are partially immersed under it, and the following crystallisation, also proceeding on the surface, will only form crystals along the side of the original crystals. A funnel is formed in this manner. It will be borne on the surface like a boat (if the liquid be quiescent), because it will grow more from the npper edges. We can thus understand this at first sight strange funnel form of crystallisation of salt. In explanation why the crystallisation under the above conditions begins at the surface and not at the lower layers, it must be mentioned that the specific gravity of a crystal of sodium chloride $=2 \cdot 16$, and that of a solution saturated at $25^{\circ}$ contains 26.7 p.c. of salt and has a specific gravity at $25^{\circ} / 4^{\circ}$ of 1.2004 ; at $15^{\circ}$ a saturated solution contains $26^{\circ} 5$ p.o. of salt and has a sp. gr. 1.208 at $15^{\circ} / 4^{\circ}$. Hence a solution saturated at a higher temperature is specifically lighter, notwithstanding the greater amount of salt it contains. With many substances surface crystallisation cannot take place because their solubility increases more rapidly with the temperature than their specific gravity decreases. In this case the saturated solution will always be in the lower layers, where also the crystallisation will take place. Besides which it may be added that as a consequence of the properties of water and solutions, when they are heated from above (for instance, by the sun's rays), the warmer layers being the lightest remain above, whilst when heated from below they rise to the top. For this reason the water at great depths below the surface is always cold, which has long been known. These circumstances, as well as those observed by Soret (Chapter I., Note 19), explain the great differences of density and temperature, and in the amount of salts held in the oceans at different latitudes (in polar and tropical climes) and at various depths.

$13 \mathrm{By}$ combining the results of Poggiale, Muiller, and Karsten (they are evidently more accurate than those of Gay-Lussac and others) I found that a saturated solution at $t^{\circ}$, from $0^{\circ}$ to $108^{\circ}$, contains $35 \cdot 7+0.024 t+0.0002 t^{2}$ grams of salt per 100 grams of water. This formula gives a solubility at $0^{\circ}=85^{\circ} 7 \mathrm{grams}\left(=26{ }^{\circ} 8\right.$ p.c. $)$, whilst according to Kar. sten it is $86 \cdot 09$, Poggiale $85 \cdot 5$, and Müller $35 \cdot 6$ grams.

14 Perfectly puro fused salt is not hygroscopic, uccording to Karsten, whilst the crystallised salt, even when quite pure, attracts as much $a s 0.6$ p.o. of water from moist 
especially from a saturated solution cooled to $-12^{\circ}$, then they present a prismatic form, and contain two equivalents of water, $\mathrm{NaCl}, 2 \mathrm{H}_{2} \mathrm{O}$. At the ordinary temperature these crystals split up into sodium chloride and its solution. ${ }^{15}$ Unsaturated solutions of table salt when cooled below $0^{\circ}$ give ${ }^{16}$ crystals of ice, but when the solution has a composition $\mathrm{NaCl}, 10 \mathrm{H}_{2} \mathrm{O}$ it solidifies completely at a temperature of $-23^{\circ}$ A solution of table salt saturated at its boiling point boils at about $109^{\circ}$, and contains about 42 parts of salt per 100 parts of water.

Of all its physical properties the specific gravity of solutions of sodium chloride is the one which has been the most fully investigated. A comparison of all the existing determinations of the specific gravity

air, according to Stas. (In the Briansk mines, where the temperature throughout the whole year is about $+10^{\circ}$, it may be observed, as Baron Klodt informed me, that in the summer during damp weather the walls become moist, while in winter they are dry).

If the salt contain impurities - such as magnesium sulphate, \&c.-it is more hygroscopic. If it contain any magnesium chloride, it partially deliquesces in a damp atmosphere. The crystallised and not perfectly pure salt decrepitates when heated, owing to its containing water. The pure salt, and also the transparent rock salt, or that which has been once fused, does not decrepitate. Fused sodium chloride shows a faint alkaline reaction to litmus, which has been noticed by many observers, and is due to the presence of sodium oxide (probably by the action of the oxygen of the atmosphere). According to A. Stcherbakoff very sensitive litmus (washed in alcohol and neutralised with oxalic acid) shows an alkaline reaction even with the crystallised salt.

It may be observed that rock salt sometimes contains cavities filled with a colourless liquid. Certain kinde of rock salt emit an odour like that of hydrocarbons. These pheuomena have as yet received very little attention.

is By cooling a solution of table salt saturated at the ordinary temperature to $-15^{\circ}$, I obtained first of all well-formed tabular (six-sided) crystals, which when warmed to the ordinary temperature disintegrated (with the separation of anhydrous sodium chloride), and then prismatic needles up to $20 \mathrm{~mm}$. long were formed from the same solution. I have not yet investigated the reason of the difference in crystalline form. It is known (Mitscherlich) that $\mathrm{NaI}, 2 \mathrm{H}_{2} \mathrm{O}$ also crystallises either in plates or prisms. Sodium bromide also crystallises with $2 \mathrm{H}_{2} \mathrm{O}$ at the codinary temperature.

18 Notwithstanding the great simplicity (Chapter I., Note 49) of the observations on the formation of ice from solution, still even for sodium chloride they cannot yet be considered as sufficiently harmonious. According to Blagden and Raoult, the temperature of the formation of ice from a solution containing $c$ grams of salt per 100 grams of water $=-0.6 c$ to $c=10$, according to Rosetti $=-0.649 c$ to $c=8.7$, according to De Coppet (to $c=10)=-0.55 c-0.006 c^{2}$, according to Karsten (to $\left.c=10\right)-0.762 c+0.0084 c^{2}$, and according to Guthrie a much lower figure. By taking Rosetti's figure and applying the rule given in Chapter I., Note 49 we obtain-

$$
i=0.649 \times \frac{58 \cdot 5}{18 \cdot 5}=2 \cdot 05 \text {. }
$$

Pickering (1893) gives for $c=1-0.608$, for $c=2-1 \cdot 220$; that is ( $c$ up to $2 \cdot 7$ ) about $-(0.600+0.005 c) c$.

The data for strong eolutions are not less contradictory. Thus with 20 p.c. of salt, ice is formed at $-144^{\circ}$ according to Karsten, $-17^{\circ}$ according to Guthrie, $-17^{\circ} 6^{\circ}$ according to De Coppet. Riidorfi states that for strong solutions the temperature of the formation of ice descends in proportion to the ccntents of the compound, $\mathrm{NaCl}, 2 \mathrm{H}_{2} \mathrm{O}$ (per 100 grams of water) by $0^{\circ} .842$ per 1 gram of salt, and $D_{\theta}$ Coppet shows that there is no proportios. ality, in a strict sense, for either a percentage of $\mathrm{NaCl}$ or of $\mathrm{NaCl}_{2} 2 \mathrm{H}_{2} \mathrm{O}$. 
of solutions of $\mathrm{NaCl}^{17}$ at $15^{\circ}$ (in vacuo, taking water at $4^{\circ}$ as 10,000 ), with regard to $p$ (the percentage amount of the salt in solution), show that it is expressed by the equation $\mathrm{S}_{1.5}=9991 \cdot 6+71 \cdot 17 p+0.2140 p^{2}$. For instance, for a solution $200 \mathrm{H}_{2} \mathrm{O}+\mathrm{NaCl}$, in which case $p=1 \cdot 6$, $S_{15}=1 \cdot 0106$. It is seen from the formula that the addition of water produces a contraction. ${ }^{18}$ The specific gravity ${ }^{19}$ at certain temperatures and concentrations in vacuo referred to water at $4^{\circ}=10,000^{20}$ is here given for

\begin{tabular}{|c|c|c|c|c|}
\hline & $0^{\circ}$ & $15^{\circ}$ & $30^{\circ}$ & $100^{\circ}$ \\
\hline$p=5$ & 10372 & 10353 & 10307 & 9922 \\
\hline 10 & 10768 & 10728 & 10669 & 10278 \\
\hline 15 & 11164 & 11107 & 11043 & 10652 \\
\hline 20 & 11568 & 11501 & 11429 & 11043 \\
\hline
\end{tabular}

It should be remarked that Baume's hydrometer is graduated by taking a 10 p.c. solution of sodium chloride as $10^{\circ}$ on the scale, and therefore it gives approximately the percentage amount of the salt in a

17 A collection of observations on the specific gravity of solutions of sodium chloride is given in my work cited in Chapter I., Note 50.

Solutions of common salt have also been frequently investigated as regards rate of diffusion (Chapter I.), but as yet there are no complete data in this respect. It may be mentioned that Graham and De Vries demonstrated that diffusion in gelatinous masses (for instance, gelatin jelly, or gelatinous silica) proceeds in the same manner as in water, which may probably lead to a convenient and accurate method for the investigation of the phenomena of diffusion. N. Umoff (Odessa, 1888) investigated the diffusion of common salt by means of glass globules of definite density. Having poured water into a cylinder over a layer of a solution of sodium chloride, he observed during a period of several months the position (height) of the globules, which floated up higher and higher as the salt permeated upwards. Umoff found that at a constant temperature the distances of the globules (that is, the length of a column limited by layers of definite concentration) remain constant; that at a given moment of time the concentration, $q$, of different layers siturted at a depth $z$ is expressed by the equation $\mathrm{B}-\mathrm{K} z=\log \cdot(\mathrm{A}-q)$, where $A, B$, and $K$ are constants; that at a given moment the rate of diffusion of tho different layers is proportional to their depth, \&c.

18 If $S_{a}$ be the specific gravity of water, and $S$ the specific gravity of a solution containing $p$ p.c. of salt, then by mixing equal weights of water and the solution, we shall obtain a solution containing $\frac{1}{2} p$ of the salt, and if it be formed without contraction, then its specific gravity $x$ will be determined by the equation $\frac{2}{x}=\frac{1}{S_{0}}+\frac{1}{S}$, , because the volume is equal to the weight divided by the density. In reality, the specific gravity is always found to be greater than that calculated on the supposition of an absence of contraction.

19 Generally the specific gravity is observed by weighing in air and dividing the weight in grams by the volume in cubic centimetres, the latter being found from the weight of water displaced, divided by its density at the temperature at which the experiment is carried out. If we call this specific gravity $S_{1}$, then as a cubic centimetre of air under the usual conditions weighs about 0.0012 gram, the sp. gr. in a vacuum $S=S_{1}+0.0012\left(S_{1}-1\right)$, if the density of water $=1$.

20 If the sp. gr. $S_{2}$ be found directly by dividing the weight of a solution by the weight of water at the same temperature and in the same volume, then the true sp. gx. $S$ referred to water at $4^{\circ}$ is found by multiplying $S_{2}$ by the sp. gr. of water at the temperature of observation. 
solution. Common salt is somewhat soluble in alcohol, ${ }^{21}$ but it is insoluble in ether and in oils.

Common salt gives very few compounds ${ }^{22}$ (double salts) and these are very readily decomposed : it is also decomposed with great difficulty and its dissociation is unknown. ${ }^{23}$ But it is easily decomposed, both when fused and in solution, by the action of a galvanic current. If the dry salt, be fused in a crucible and an electric current be passed through it by immersing carbon or platinum electrodes in it (the positive electrode is made of carbon and the negative of platinum or mercury), it is decomposed: the suffocating gas, chlorine, is liberated at the positive pole and metallic sodium at the negative pole. Both of them act on the excess of water at the moment of their evolution; the sodium evolves hydrogen and forms caustic soda, and the chlorine evolves oxygen and forms hydrochloric acid, and therefore on passing a current through a solution of common salt metallic sodium will not be obtained-but oxygen, chlorine, and hydrochloric acid will appear at the positive pole, and hydrogen and caustic soda at the negative pole. ${ }^{23 \mathrm{hls}}$ Thus salt, like other salts, is decomposed by the action of an electric current into a metal and a haloid (Chapter III.) Naturally, like all other salts, it may be formed from the corresponding base and acid with the separation of water. In fact if we mix caustic soda (base) with hydrochloric acid (acid), table salt is formed, $\mathrm{NaHO}+\mathrm{HCl}$ $=\mathrm{NaCl}+\mathrm{H}_{2} \mathrm{O}$.

21 According to Schiff 100 grams of alcohol, containing $p$ p.c. by weight of $\mathrm{C}_{2} \mathrm{H}_{0} \mathrm{O}$, dissolves at $15^{\circ}-$

$$
\begin{array}{ccccc}
p=10 & 20 & 40 & 60 & 80 \\
28 \cdot 5 & 22 \cdot 6 & 18 \cdot 2 & 5.9 & 1 \cdot 2 \text { grams NaCl. }
\end{array}
$$

${ }^{23}$ Amongst the double salts formed by sodium chloride that obtained by Ditte (1870) by the evaporation of the solution remaining after heating sodium iodate with hydrochloric acid until chlorine ceases to be liberated, is a remarkable one. Its composition is $\mathrm{NaIO}_{3}, \mathrm{NaCl}, 14 \mathrm{H}_{2} \mathrm{O}$. Rammelsberg obtained a similar (perhaps the same) salt in well. formed crystals by the direct reaction of both salts.

23 But it gives sodium in the flame of a Bunsen's burner (see Spectrum Analysis), doubtless under the reducing action of the elements carbon and hydrogen. In the presence of an excess of hydrochloric acid in the flame (when the sodium would form sodium chloride), no sodium is formed in the flame and the salt does not communicate its usual coloration.

25 bis There is no doubt, however, but that chloride of sodium is also decomposed in its aqueous solutions with the separation of sodium, and that it does not simply enter into double decomposition with the water $\left(\mathrm{NaCl}+\mathrm{H}_{2} \mathrm{O}=\mathrm{NaHO}+\mathrm{HCl}\right)$. This is seen from the fact that when a saturated solution of $\mathrm{NaCl}$ is rapidly decomposed by an electric current, a large amount of chlorine appears at the anodo and a sodium amalgam forms at the mercury cathode, which acts but slowly upon the strong solvtion of salt. Castner's process for the electrolysis of brine into chlorine and caustio soda is an application of this method which has been already worked in England on andustrial scale. 
With respect to the double decompositions of sodium chloride it should be observed that they are most varied, and serve as means of obtaining nearly all the other compounds of sodium and chlorine.

The double decompositions of sodium chloride are almost exclusively based on the possibility of the metal sodium being exchanged for hydrogen and other metals: But neither hydrogen nor any other metal can directly displace the sodium from sodium chloride. This would result in the separation of metallio sodium, which itself displaces hydrogen and the majority of other metals from their compounds, and is not, so far as is known, ever separated by them. The replacement of the sodium in sodium chloride by hydrogen and various metals can only take place by the transference of the sodium into some other combination. If hydrogen or a metal, $\mathrm{M}$, be combined with an element $\mathrm{X}$, then the double decomposition $\mathrm{NaCl}+\mathrm{MX}=\mathrm{NaX}+\mathrm{MCl}$ takes place. Such double decompositions take place under special conditions, sometimes completely and sometimes only partially, as we shall endeavour to explain. In order to acquaint ourselves with the double decompositions of sodiun chloride, we will follow the methods actually employed in practice to procure compounds of sodium and of chlorine from common silt. For this purpose we will first describe the treatment of sodium chloride with sulphuric acid for the preparation of hydrochloric acid and sodium sulphate. We will then describe the substances obtained from hydrochloric acid and sodium sulphate. Chlorine itself, and nearly all the compounds of this element, may be procured from hydrochloric acid, whilst sodium carbonate, caustic soda, metallic sodium itself and all its compounds, may be obtained from sodium sulphate.

Even in the animal organism salt undergoes similar changes, furnishing the sodium, alkali, and hydrochloric acid which take part in the processes of animal life.

Its necessity as a constituent in the food both of human beings and of animals becomes evident when we consider that both hydrochloric acid and salts of sodium are found in the substances which are separated out from the blood into the stomach and intestines.- Sodium salts are found in the blood and in the bile which is elaborated in the liver and acts on the food in the alimentary cunal, whilst hydro. chloric acid is found in the acid juices of the stomach. Chlorides of the metals are always found in considerable quantities in the urine, and if they are excreted they must be replenished in the organism; and for the replenishment of the loss, substances containing chlorine compounds must be taken in food. Not only do animals consume those small amounts of sodium chloride which are found in drinking water orin plants 
or other animals, but experience has shown that many wild animals travel long distances in search of salt springs, and that domestic animals which in their natural condition do not require salt, willingly take it, and that the functions of their organisms become much more regular from their doing so.

The action of sulphuric acid on sodium chloride.-If sulphuric acid be poured over common salt, then even at the ordinary temperature, as Glauber observed, an odorous gas, hydrochloric acid, is evolved. The reaction which takes place consists in the sodium of the. salt and the hydrogen of the sulphuric acid changing places.

$$
\underset{\text { Sodium chloride }}{\mathrm{NaCl}}+\underset{\text { Sulphuric acid }}{\mathrm{H}_{2} \mathrm{SO}_{4}}=\underset{\text { Hydrochloric acid }}{\mathrm{HCl}}+\underset{\text { Acid sodium sulphate }}{\mathrm{NaHSO}_{4}}
$$

At the ordinary temperature this reaction is not complete, but soon ceases. When the mixture is heated, the decomposition proceeds until, if there be sufficient salt present, all the sulphuric acid taken is converted into acid. sodium sulphate. Any excess of acid will remain unaltered. If 2 molecules of sodium chloride (117 parts) be taken per molecule of sulphuric acid (98 parts), then on heating the mixture to a moderate temperature only one-half $(58.5)$ of the salt will suffer change. Complete decomposition, 'after which neither hydrogen nor chlorine is left in the residue, proceeds (when 117 parts of table salt are taken per 98 parts of sulphuric acid) at a red leat only. Then-

$$
\underset{\text { Table salt }}{2 \mathrm{NaCl}}+\underset{\text { Sulphuric acid }}{\mathrm{H}_{2} \mathrm{SO}_{4}}=\underset{\text { Hydrochloric acid }}{2 \mathrm{HCl}}+\underset{\text { Sodium sulphate }}{\mathrm{Na}_{2} \mathrm{SO}_{4}}
$$

This double decomposition is the result of the action of the acid salt; $\mathrm{NaHSO}_{4}$, first formed, on sodium chloride, for the acid salt, since it contains hydrogen, itself acts like an acid, $\mathrm{NaCl}+\mathrm{NaHSO}_{4}$ $=\mathrm{HCl}+\mathrm{Na}_{2} \mathrm{SO}_{4}$. $\quad$ By adding this equation to the first we obtain the second, which expresses the ultimate reaction. Hence in the above reaction, non-volatile or sparingly volatile table salt and sparingly volatile sulphuric acid are taken, and as the result of their reaction, after the hydrogen and sodium have exchanged places, there is obtained non-volatile sodium sulphate and gaseous hydrochloric acid. The fact of the latter being a gaseous substance forms the main reason for the reaction proceeding to the very end. The mechanism of this kind of double decomposition, and the cause of the course of the reaction, are exactly the same as those we saw in the decomposition of nitre (Chapter VI.) by the action of sulphuric acid. The sulphuric acid in each case displaces the other, volatile, acid. 
Not only in these two instances, but in every instance, if a volatile acid can be formed by the substitution of the hydrogen of sulphuric acid for a metal, then this volatile acid. will be formed. From this it may be concluded that the volatility of the acid should be considered as the: cause of the progiress of the reaction; and indeed if the acid be soluble but not volatile, or if the reaction take place in an enclosed space where the resulting acid cannot volatilise, or at the ordinary temperature when it does not pass into the state of elastic vapour-then the decomposition does not proceed to the end, but only up to a certain limit. In this respect the explanations given at the beginning of this century by the French chemist Berthollet in his work ' Essai de Statique Chimique' are very important. The doctrine of Berthollet starts from the supposition that the chemical reaction of substances is determined not only by the degrees of affinity between the different parts, but also by the relative masses of the reacting substances and by those physical conditions under which the reaction takes place. Two substances containing the elements $\mathrm{MX}$ and $\mathrm{NY}$, being brought into contact with each other, form by double decomposition the compounds MY and $\mathrm{NX}$; but the formation of these two new compounds will not proceed to the end unless one of them is removed from the sphere of action. But it can only be removed if it possesses different physical properties from those of the other substances which are present with it. Either it must be a gas while the others are liquid or solid, or an inscluble solid while the others are liquid or soluble. The relative amounts of the resultant substances, if nothing separates out from their intermixture, depend only on the relative quantities of the substances MX and NY, and upon the degrees of attraction existing between the elements $M$, $\mathrm{N}, \mathrm{X}$, and $\mathrm{Y}$; but however great their mass may be, and however considerable the attractions, still in any case if nothing separates out from the sphere of action the decomposition will presently cease, a state of. equilibrium will be established, and instead of two there will remain four substances in the mass : namely, a portion of the original bodies $\mathrm{MX}$ and $\mathrm{NY}$, and a certain quantity of the newly formed substances $M Y$ and $N X$, if it be assurned that neither MN or XY nor any other substances are produced, and this may for the present ${ }^{24}$ be admitted in the case of

24 If MX and NY represent the molecules of two salts, and if there be. no third substance present (such as water in a solution), the formation of XY would also be possible; for instance, cyanogen, iodine, \&c. are capable of combining with simple haloids, as well as with the complex groups which in certain salts play the part of haloids. Besides. which the salts MX and NY or MY with NX may forn double salts. If the number of molecules be unequal, or if the valency of the elements or groups contained in them be different, as in $\mathrm{NaCl}+\mathrm{H}_{2} \mathrm{SO}_{4}$, where $\mathrm{Cl}$ is a univalent haloid and $\mathrm{SO}_{4}$ is bivalent, then the matter may be complicated by the formation of other compounds besides MY and NX, and 
the double decomposition of salts in which $\mathrm{M}$ and $\mathrm{N}$ are metals and $\mathrm{X}$ and $\mathrm{Y}$ haloids. As the ordinary double decomposition here consists merely in the exchange of metals, the above simplification is applicable. The sum total of existing data concerning the double decomposition of salts leads to the conclusion that from salts $\mathrm{MX}+\mathrm{NY}$ there always arises a certain quantity of $N X$ and $M Y$, as should be the case according to Berthollet's doctrine. A portion of the historical 'data concerning this subject will be afterwards mentioned, but we will at once proceed to point out the observations made by Spring (1888) which show that even in a solid state salts are subject to a similar interchange of metals if in a condition of sufficiently close contact (it requires time, a finely divided state, and intimate mixture). Spring took two non-hygroscopic salts, potassium nitrate, $\mathrm{KNO}_{3}$, and well-dried sodium acetate, $\mathrm{C}_{2} \mathrm{H}_{3} \mathrm{NaO}_{2}$, and left a mixture of their powders for several months in a desiccator. An interchange of metals took place, as was seen from the fact that the resultant mass rapidly attracted the moisture of the air, owing to the formation of sodium nitrate, $\mathrm{NaNO}_{3}$, and potassium acetate, $\mathrm{C}_{2} \mathrm{H}_{3} \mathrm{KO}_{2}$, both of which are highly hygroscopic. ${ }^{24}$ bis

When Berthollet enunciated his doctrine the present views of atoms and molecules had yet to be developed, and it is now necessary to submit the matter to examination in the light of these conceptions ; we will therefore consider the reaction of salts, taking $M$ and $N, X$ and $Y$ as equivalent to each other-that is, as capable of replacing each other 'in toto,' as $\mathrm{Na}$ or $\mathrm{K}, \frac{1}{2} \mathrm{Ca}$ or $\frac{1}{2} \mathrm{Mg}$ (bivalent elements) replace hydrogen.

And since, according to Berthollet's doctrine, when $m \mathrm{MX}$ of one salt comes into contact with $n \mathrm{NY}$ of unother salt, a certain quantity $x \mathrm{MY}$ and $x \mathrm{NX}$ is formed, there remains $m-x$ of the salt $\mathrm{MX}$, and $n-x$ of the salt NY. If $m$ be greater than $n$, then the maximum interchange could lead to $x=n$, whilst from the salts taken there would be formed $n \mathrm{MY}+n \mathrm{NX}+(m-n) \mathrm{MX}$-that is, a portion of one only of the salts taken would remain unchanged because the reaction could only proceed between $n \mathrm{MX}$ and $n \mathrm{NY}$ If $x$ were actually equal

when a solvent participates in the action, and especially if present in large proportion, the phenomena must evidently become still more complex; and this is actually the case in nature. Hence while placing before the reader a certain portion of the existing store of knowledge concerning the phenomena of double saline decompositions, I cannot consider the theory of the subject as complete, and have therefore limited myself to a few data, the completion of which must be sought in more detailed works on the subject of theoretical chemistry, without losing sight of what has been said above.

21 bis When the mixture of potassium nitrate and sodium acetate was heated by Spring to $100^{\circ}$, it was completely fused into one mass, although potassium nitrate fuses at about $340^{\circ}$ and sodium nitrate at about $820^{\circ}$ 
to $n$, the mass of the salt MX would not have any influence on the modus operandi of the reaction, which is equally in accordance with the teaching of Bergmann, who supposed double reactions to be independent of the mass and determined by affinity only. If $M$ had more affinity for $\mathrm{X}$ than for $\mathrm{Y}$, and $\mathrm{N}$ more affinity for $\mathrm{Y}$ than for $\mathrm{X}$, then according to Bergmann there would be no decomposition whatever, and $x$ would equal 0 . If the affinity of $\mathrm{M}$ for $\mathrm{Y}$ and of $\mathrm{N}$ for $\mathrm{X}$ were greater than those in the original grouping, then the affinity of $\mathrm{M}$ for $\mathrm{X}$ and of $\mathrm{N}$ for $\mathrm{Y}$ would beovercome, and, according to Bergmann's doctrine, complete interchange would take place-i.e. $x$ would equal $n$. According to Berthollet's teaching, a distribution of $\mathrm{M}$ and $\mathrm{N}$ between $\mathrm{X}$ and $\mathrm{Y}$ will take place in everry case, not only in proportion to the degrees of affinity, but also in proportion to the masses, so that with a small affinity and a large mass the same action can be produced as with a large affinity and a small mass. Therefore, (1) $x$ will always be less than $n$ and their ratio $\frac{x}{n}$ less than unity-that is, the decomposition will be expressed by the equation, $m \mathrm{MX}+n \mathrm{NY}=(m-x) \mathrm{MX}+(n-x) \mathrm{NY}$ $+x \mathrm{MY}^{\prime}+x \mathrm{NX}$; (2) by increasing the mass $m$ we increase the de. composition-that is, we increase $x$ and the ratio $\frac{x}{(n-x)}$, until with an infinitely large quaritity $m$ the fraction $\frac{x}{n}$ will equal 1 , and the de. composition will be complete, however small the affinities uniting MY and $\mathrm{NX}$ may.be ; and (3) if $m=n$, by taking $\mathrm{MX}+\mathrm{NY}$ or MY $+\mathrm{NX}$ we arrive at one and the same system in either case : $(n-x) \mathrm{MX}$ $+(n-x) \mathrm{NY}+x \mathrm{MY}+x \mathrm{NX}$. These direct consequęnces of Berthollet's teaching are verified by experience. Thus, for example, a mixture of solutions of sodium nitrate and potassium chloride in all cases has entirely the same properties as a mixture of solutions of potassium nitrate and sodium chloride, of course on condition that the mixed solutions are of identical elementary composition. But this identity of properties might either proceed from one system of salts passing entirely into the other (Bergmann's hypothesis) in conformity with the predominating affinities (for instance, from $\mathrm{KCl}+\mathrm{NaNO}_{3}$ there might arise $\mathrm{KNO}_{3}+\mathrm{NaCl}$, if it be admitted that the affinities of the elements as combined in the latter system are greater than in the former); or, on the other hand, it might be because both systems by the interchange of a portion of their elements give one and the same state of equilibrium, as according to Berthollet's teaching. Experiment proves the latter hypothesis to be the true one. But before citing the most historically important experiments verifying Berthollet's 
doctrine, we must stop to consider the conception of the mass-of the reacting substances. Berthollet understood by mass the actual relative quantity of a substance; but now it is impossible to understand this term otherwise than as the number of molecules, for they act as chemical units, and in the special case of double saline decompositions it is better to take it as the number of equivalents. Thus in the reaction $\mathrm{NaCl}+\mathrm{H}_{2} \mathrm{SO}_{4}$ the salt is taken in one equivalent and the acid in two. If $2 \mathrm{NaCl}+\mathrm{H}_{2} \mathrm{SO}_{4}$ act, then the number of equivalents are equal, and so on. The influence of mass on the amount of decomposition $\frac{x}{n}$ forms the root of Berthollet's doctrine, and therefore we will first of all turn our attention to the establishment of this principle in relation to the double decomposition of salts.

About $1840 \mathrm{H}$. Rose ${ }^{2.5}$ showed that water decomposes metallic sul. phides like calcium sulphide, CaS, forming hydrogen sulphide, $\mathrm{H}_{2} \mathrm{~S}$, notwithstanding the fact that the affinity of hydrogen sulphide, as an acid, for lime, $\mathrm{CaH}_{2} \mathrm{O}_{2}$, as a base, causes them to react on each other, forming calcium sulphide and water, $\mathrm{CaS}+2 \mathrm{H}_{2} \mathrm{O}$. Furthermore, Rose showed that the greater the amount of water acting on the calcium sulphide, the more complete is the decomposition. The results of this reaction are evident from the fact that the hydrogen sulphide formed may be expelled from the solution by heating, and that the resulting lime is sparingly soluble in water. Rose clearly saw from this that such feeble agents, in a chemical sense, as carbonic anhydride and water, by acting in a mass and for long periods of time in nature on the durable rocks, which resist the action of the most powerful acids, are able to bring about chemical change-to extract, for example, from rocks the bases, lime, soda, potash. The influence of the mass of water on antimonious chloride, bismuth nitrate, \&c., is essentially of the same character. These substances give up to the water a quantity of acid which is greater in proportion as the mass of the water acting on them is greater. ${ }^{25}$ bis

$25 \mathrm{H}$. Rose is more especially known for his having carefully studied and perfected several methods for the exact chemical analysis of many mineral substances. His predecessor in this branch of researclı was Berzelins, and his successor Fresenius.

25 bis Historically the influence of the mass of water was the first well-observed phenomenon in support of Berthollet's teaching, and it should not now be forgotten. In double decompositions taking place in dilute solutions where the mass of water is large, its influence, notwithstanding the weakness of affinities, must be great, according to the very essence of Berthollet's doctrine.

As explaining the action of the mass of water, the experiments of Pattison Muir (1879) are very instructive. These experiments demonstrate that the decomposition of bismuth chloride is the more complete the greater the relative quantity of water, and the leas the mass of hydrochloric acid forming one of the products of the reaction. 
Barium sulphate, $\mathrm{BaSO}_{4}$, which is insoluble in water, when fused with sodium carbonate, $\mathrm{Na}_{2} \mathrm{CO}_{3}$, gives, but not completely, barium carbonate, $\mathrm{BaCO}_{3}$, (also insoluble), and sodium sulphate, $\mathrm{Na}_{2} \mathrm{SO}_{4}$. If a solution of sodium carbonate acts on precipitated barium suIphate, the same decomposition is also effected (Dulong, Rose), but it is restricted by a limit and requires time. A mixture of sodium carbonate and sulphate is obtained in the solution and a mixture of barium carbonate and sulphate in the precipitate. If the solution be decanted off and a fresh solution of sodium carbonate be poured over the precipitate, then a fresh portion of the barium sulphate passes into barium carbonate, and so by increasing the mass of sodium carbonate it is possible to entirely convert the barium sulphate into barium carbonate. If a definite quantity of sodium sulphate be added to the solution of sodium carbonate, then the latter will have no action whatever on the barium sulphate, because then a system in equilibrium determined by 'the reverse action of the sodium sulphate on the barium carbonate and by the presence of both sodium carbonate and sulphate in the solution, is at once arrived at. On the other hand, if the mass of the sodium sulphate in the solution be great, then the barium carbonate is reconverted into sulphate until a definite state of equilibrium is attained between the two opposite reactions, producing barium carbonate by the action of the sodium carbonate and barium sulphate by the action of the sodium sulphate.

Another most important principle of Berthollet's teaching is the existence of a limit of exchange decomposition, or the attain. ment of a state of equilibrium. In this respect the determinations of Malaguti (1857) are historically the most important. He took a mixture of solutions of equivalent quantities of two salts, MX and $\mathrm{NY}$, and judged the amount of the resulting exchange from the composition of the precipitate produced by the addition of alcohol. When, for example, zinc sulphate and sodium chloride $\left(\mathrm{ZnSO}_{4}\right.$ and $2 \mathrm{NaCl}$ ) were taken, there were produced by exchange sodium sulphate and zinc chloride. A mixture of zinc sulphate and sodium. sulphate was precipitated by an excess of alcohol, and it appeared from the composition of the precipitate that 72 per cent. of the salts laken had been decomposed. When, however, a mixture of . solutions of sodium sulphate and zinc chloride was taken, the precipitate presented the same composition as before-that. is, about 28 per cent. of the salts taken had been subjected to decomposition. In a similar experiment with a mixture of sodium chloride and unagnesium sulphate, $2 \mathrm{NaCl}+\mathrm{MgSO}_{4}$ or $\mathrm{MgCl}_{2}+\mathrm{Na}_{2} \mathrm{SO}_{4}$, about half of the metals under . went the decomposition, which may be expressed by the equation 
$4 \mathrm{NaCl}+2 \mathrm{MgSO}_{4}=2 \mathrm{Na} \cdot \mathrm{Cl}+\mathrm{MgSO}_{4}+\mathrm{Na}_{2} \mathrm{SO}_{4}+\mathrm{MgCl}_{2}=2 \mathrm{Na}_{2} \mathrm{SO}_{4}+$ $2 \mathrm{MgCl}_{2}$. A no less clear limit expressed itself in another of Malaguti's researches when he investigated the above-mentioned reversible reactions of the insoluble salts of barium. When, for example, bariun carbonate. and sodium sulphate. $\left(\mathrm{BaCO}_{3}+\mathrm{Na}_{2} \mathrm{SO}_{4}\right)$. were taken, then about 72 per cent. of the salts were decomposed, that is, were converted into barium sulphate and sodium carbonate. But when the two latter salts were taken, then about 19 per cent. of them passed into barium carbonate and sodium sulphate. Probably the end of the reaction was not reached in either case, because this would require a considerable time and a uniformity of conditions attainable with difficulty.

Gladstone (1855) took advantage of the colour of solutions of different ferric salts for determining the measure of exchange between metals. Thus a solution of ferric thiocyanate has.a most intenso red colour, and by making a comparison between the colour of the resulting solutions and the colour of solutions of known strength it was possible to judge to a certain degree the quantity of the thiocyanate formed. This colorimetric method of determination has an important signiticance as being the first in which a method was applied for determining the composition of a solution withuut the removal of any of its component parts. When Gladstone took equivalent quantities of ferric nitrate and potassium thiocyanate- $\mathrm{Fe}\left(\mathrm{NO}_{3}\right)_{3}+3 \mathrm{KCNS}$ -only 13 per cent. of the salts underwent decomposition. On increasing the mass of the latter salt the quantity of ferric thiocyanate formed increased, but even when more than 300 equivalents of potassium thiocyanate were taken a portion of the iron still remained as nitrate. It is evident that the affinity acting between $\mathrm{Fe}$ and $\mathrm{NO}_{3}$ and between $\mathrm{K}$ and CNS on the one hand, is greater than the affinity acting between $\mathrm{Fe}$ and CNS, together with the affinity of $\mathrm{K}$ for $\mathrm{NO}_{3}$, on the other hand. The investigation of the variation of the fluorescence of quinine sulphate, as well as the variation of the rotation of the plane of polarisation of nicotine, gave in the hands of Gladstone many proofs of the entire applicability of Berthollet's doctrine, and in particular demonstrated the influence of mass which forms the chief distinctive feature of the teaching of Berthollet, teaching little appreciated in his own time.

At the beginning of the year 1860, the doctrine of the limit of reaction and of the influence of mass on the process of chemical transformations received a very important support in the researches of Berthelot and P. de Saint-Gilles on the formation of the ethereal salts $\mathrm{RX}$ from the alcohols $\mathrm{ROH}$ and acids $\mathrm{HX}$, when water is also formed. This conversion is essentially very similar to the formation of salts, but 
differs in that it proceeds slowly at the ordinary temperature, extend. ing over whole years, and is not complete-that is, it has a distinct limit determined by a reverse reaction; thus an ethereal salt $\mathrm{RX}$ with water gives an alcohol $\mathrm{ROH}$ and an acid HX-up to that limit generally corresponding with two-thirds of the alcohol taken, if the action proceed between molecular quantities of alcohol and acid. Thus common alcohol, $\mathrm{C}_{2} \mathrm{H}_{5} \mathrm{OH}$, with acetic acid, $\mathrm{HC}_{2} \mathrm{H}_{3} \mathrm{O}_{2}$, gives the following system rapidly when heated, or slowly at the ordinary temperature, $\mathrm{ROH}+\mathrm{HX}+2 \mathrm{RX}+2 \mathrm{H}_{2} \mathrm{O}$, whether we start from $3 \mathrm{RHO}+3 \mathrm{HX}$ or from $3 \mathrm{RX}+3 \mathrm{H}_{2} \mathrm{O}$. The process and completion of the reaction in this instance are very easily observed, because the quantity of free acid is easily determined from the amount of alkali requisite for its saturation, as neither alcohol nor ethereal salt acts on litmus or other reagent for acids. Under the influence of an increased mass of alcohol the reaction proceeds further. If two molecules of alcohol, RHO, be taken for every one molecule of acetic acid, HX, then instead of 66 p.c., 83 p.c. of the acid passes into ethereal salt, and with fifty molecules of RHO nearly all the acid is etherised. The researches of Menschutkin in their details touched on many important aspects of the same subject, such as the influence of the composition of the alcohol and acid on the limit and rate of exchange-but these, as well as other details, must be looked for in special treatises on organic and theoretical chemistry. In any case the study of etheritication has supplied chemical mechanics with clear and valuable data, which directly confirm the two fundamental propositions of Berthollet; the influence of mass, and the limit of reaction-that is, the equilibrium between opposite reactions. The study of numerous instances of dissociation which we have already touched on, and shall again meet with on several occasions, gave the same results. With respect to double saline decompositions, it is also necessary to mention the researches of Wiedemann on the decomposing action of a mass of water on the ferric salts, which could be determined by measuring the magnetism of the solutions, because the ferric oxide (soluble colloid) set free by the water is less magnetic than the ferric salts.

A very important epoch in the history of Berthollet's doctrine was attained when, in 1867, the Norwegian chemists, Guldberg and Waage, expressed it as an algebraical formula They defined the antive mass as the number of molecules contained in a given volume, and assumed, as follows from the spirit of Berthollet's teaching, that the action between the substances was equal to the product of the masses of the reacting substances. Hence if the salts $\mathrm{MX}$ and $\mathrm{NY}$ be taken in equivalent quantities $(m=1$ and $n=1)$ and the salts $\mathrm{MY}$ and $\mathrm{NX}$ are 
not added to the mixture but proceed from it, then if $k$ represent the coefficient of the rate of the action of MX on NY and if $k^{\prime}$ represent the same coefficient for the pair MY and NX, then we shall have at the mument when the decomposition equals $x$ a measure of action for the first pair : $k(1-x)(1-x)$ and for the second pair $k^{\prime} x x$, and $a$ state of equilibrium or limit will be reached when $k(1-x)^{2}=k^{\prime} x^{2}$, whence the ratio $k / k^{\prime}=[x /(1-x)]^{2}$. Therefore in the case of the uction of alcohol on an acid, when $x=\frac{2}{3}$, the magnitude $k / k^{\prime}=4$, that is, the reaction of the alcohol on the acid is four times as fast as thai of the ethereal salt on water. If the ratio $k / k^{\prime}$ be known, then the influence of mass may be easily determined from it. Thus if instead of one molecule of alcohol two be taken, then the equation will be $k(2-x)(1-x)=k^{\prime} x x$, whence $x=0.85$ or 85 per cent., which is close to the result of experiment. If 300 molecules of alcohol be taken, then $x$ prores to be approximately 100 per cent., which is also found to be the case by experiment. ${ }^{26}$

But it is impossible to subject the formation of salts to any process directly analogous to that which is so conveniently effected in etherification. Many efforts have, however, been made to solve the problem of the measure of reaction in this case also. Thus, for example, Khichinsky (1866), Petrieff (1885), and many others investigated the distribution of metals and haloid groups in the case of one metal and several haloids taken in excess, as acids; or conversely with an excess of bases, the distribution of these bases with relation to an acid; in cases where a portion of the substances forms a precipitate and a portion remains in solution. But such complex cases, although they in general confirm Berthollet's teaching (for instance, a solution of silver nitrate gives some silver oxide with lead oxide, and a solution of nitrate of lead precipitates some lead oxide under the action of silver oxide, as Petrieff demonstrated), still, owing to the complexity of the phenomena (for instance, the formation of basic and double salts), they cannot gire simple results. But much more instructive and complete are researches like those made by Pattison Muir (1876), who took the simple case of the precipitation of calcium carbonate, $\mathrm{CaCO}_{3}$, from the mixture of solutions of calcium chloride and sodium or potassium carbonate, and found in this case that not only was the

26. From the above it follows that an excess of acid should infiuence the reaction like an excess of -alcohol. It is in fact shown by experiment that if two molecules of acetic acid be taken to one molecule of alcohol, 84 p.c. of alcohol is etherified. If with a large preponderance of acid or of alcohol certain discrepancies are observed, their cause must be looked for in the incomplete correspondence of the conditions and exterial influences. 
rate of action (for example, in the case of $\mathrm{CaCl}_{2}+\mathrm{Na}_{2} \mathrm{CO}_{3}, 75$ per' cent. of $\mathrm{CaCO}_{3}$ was precipitated in five minutes, 85 per cent. in thirty minutes, and 94 per cent. in two days) determined by the temperature, relative mass, and amount of water (a large mass of water decreases the rate), but that the limit of decomposition was also dependent on these influences. However, even in researches of this kind the conditions of reaction are counplicated by the non-uniformity of the media, inasmuch as a portion of the substance is obtained or remains in the form of a precipitate, so that the system is heterogeneous. The investigation of double saline decompositions offers many difficulties which cannot be considered as yet entirely overcome. Ailthough many efforts have long since been made, the majority of the researches were carried on in aqueous solutions, and as water is itself a saline compound and able to combine with salts and enter into double decomposition with them, such reactions taking place in solutions in reality present very complex cases. ${ }^{27}$ In this sense ther reaction between alcohols and acids

27 As an example two methods may be mentioned, Thomsen's and Ostwald's. Thomsen (1869) applied a thermo-chemical method to exceedingly dilute solutions without taking the water into further consideration. He took solutions of caustic soda containing $100 \mathrm{H}_{2} \mathrm{O}$ per $\mathrm{NaHO}$, and sulphuric acid containing $\frac{1}{2} \mathrm{H}_{2} \mathrm{SO}_{4}+100 \mathrm{H}_{2} \mathrm{O}$. In order that these solutions may be mixed in such quantities that atomic proportious of acid and alkali would act, for forty grams of caustic soda (which answers to its equivalent) there. should be employed 49 grams of sulphuric acid, and then $+15,689$ heat units would be evolved. If the normal sodium sulphate so formed be mixed with $n$ equivalents of sulphuric acid, a certain amount of heat is absorbed, namely a quantity equal to $\frac{n .1650}{(n+0.8)}$ heat units. An equivalent of caustic soda, in combining with an equivalent of nitric acid, evolves $+18,617$ units of heat, and the augmentation of the amount of pitric acid entails an absorption of heat for each equivalent equal to -27 units; so also in combining with hydrochloric acids $+13,740$ heat units are absorbed, and for each equivalent of hydrochloric acid beyond this amount there are absorbed -32 heat units. Thomsen nixed each one of three neutral salts, sodium sulphate, sodium chloride and sodium nitrate, with an acid which is not contained in it; for instance, he mixed a solution of sodiun sulphate with a solution of nitric acid and determined the number of heat units then absorbed. An absorption of heat ensued because a normal salt was taken in the first instance, and the mixture of all the above normal salts with acid produces an absorption of heat. The amount of heat absorbed enabled him to obtain an insight into the process taking place in this mixture, for sulphuric acid added to sodium sulphate absorbs a considerable quantity of heat, whilst hydrochloric and nitric acids absorb a very small amount of heat in this case. By mixing an equivalent of sodium sulphate. with various numbers of equivalents of nitric acid, Thomsen observed that the amount of heat absorbed increased more and more as the amount of nitric acid was increased; thus when $\mathrm{HNO}_{3}$ was taken per $\frac{1}{2} \mathrm{Na}_{2} \mathrm{SO}_{4}, 1,752$ heat units were absorbed per equivalent of soda contained in the sodium sulphate. When twice as much nitric acid was taken, 2,026. heat units, and when three times as much, 2,050 heat units were absorbed. Had the double decomposition been complete in the case where one equivalent of nitric acid was. taken per equivalent of $\mathrm{Na}_{2} \mathrm{SO}_{4}$, then according to calculation from similar data there should hrve been absorbed $-2,989$ units of heat, while in reality only $-1,752$ units were absorbed. Hence Thomsen concluded that a displacement of only about two-thirds of the sulphuric acid had taken place-that is, the ratio $k: k^{\prime}$ for the reaction $\frac{1}{8} \mathrm{Na}_{2} \mathrm{SO}_{4}+\mathrm{HNO}_{3}$. 
is much more simple, and therefore its significance in confirmation of Berthollet's doctrine is of particular importance. The only cases

and $\mathrm{NaNO}_{5}+\frac{1}{2} \mathrm{H}_{2} \mathrm{SO}_{4}$ is equal, as for ethereal salts, to 4. By taking this figure and ad. mitting the above supposition, Thomsen found that for all mixtures of soda with nitrio acid, and of sodium nitrate with sulphuric acid, the amounts of heat followed Guldberg and Waage's law; that is, the limit of decomposition reached was greater the greater the mass of acid added. The relation of hydrochloric to sulphuric acid gave the same results. Therefore the researches of Thomsen fully confirm the hypotheses of Guldberg and Warge and the doctrine of Berthollet.

Thomsen concludes his investigation with the words: (a) 'When equivalent quantities of $\mathrm{NaHO}, \mathrm{HNO}_{3}$ (or $\mathrm{HCl}$ ) and $\frac{1}{2} \mathrm{H}_{2} \mathrm{SO}_{4}$ react on one another in an aqueous solution, then two-thirds of the soda combines with the nitric and one-third with the sulphuric acid; (b) this subdivision repeats itself, whether the soda be taken combined with nitric or with sulphuric acid; $(c)$ and therefore nitric acid has double the tendency to combine with the base that sulphuric acid has, and hence in an aqueous solution it is a stronger acid than the latter.'

'It is therefore necessary,' Thomsen afterwards remarks, 'to have an expression indicating the tendency of an acid for the saturation of bases. This idea cannot be expressed by the word affinity, because by this term is most often understood that force which it is necessary to overcome in order to decompose a substance into its component parts. This force should therefore be measured by the amount of work or heat employed for the decomposition of the substance. The above-mentioned phenomenon is of an entirely different nature,' and Thomsen introduces the term avidity, by which he desig. nates the tendency of acids for neutralisation. 'Therefore the avidity of nitric acid with respect to soda is twice as great as the avidity of sulphuric acid. An exactly similar result is obtained with hydrochloric acid, so that its avidity with respect to soda is also double the avidity of sulphuric acid. Experiments conducted with other acids showed that not one of the acids investigated had so great an avidity as nitric acid; some had a greater avidity than sulphuric acid, others less, and in some instances the avidity $=0$.' The reader will naturally see clearly that the path chosen by Thomsen deserves to be. worked out, for his results concern important questions of chemistry, but great faith cannot be placed in the deductions he has already. arrived at, because great complexity of relations is to be seen in the very method of his investigation. It is especially important to turn attention to the fact that all the reactions investigated are reactions of double decomposition. In them $\mathrm{A}$ and $\mathrm{B}$ do not combine with $\mathrm{C}$ and distribute them. selves according to their affinity or avidity for combination, but reversible reactions are induced. MX and NY give MY and NX, and conversely; therefore the affinity or avidity for combination is not here directly determined, but only the difference or relation of the affinities or avidities. The affinity of nitric acid not only for the water of constitution, but also for that serving for solution, is much less than that of sulphuric acid. This is seen from thermal data. The reaction $\mathrm{N}_{2} \mathrm{O}_{5}+\mathrm{H}_{2} \mathrm{O}$ gives $+3,600$ heat units, and the solution of the resultant hydrate, $2 \mathrm{NHO}_{3}$, in a large excess of water evolves $+14,986$ heat units. The formation of $\mathrm{SO}_{3}+\mathrm{H}_{2} \mathrm{O}$ evolves $+21,308$ heat units, and the solution of $\mathrm{H}_{2} \mathrm{SO}_{4}$ in an excess of water 17,860 - that is, sulphuric acid gives more heat in both cases. The interchange between $\mathrm{Na}_{2} \mathrm{SO}_{4}$ and $2 \mathrm{HNO}_{3}$ is not only accomplished at the expense of the production of $\mathrm{NaNO}_{3}$, but also at the expense of the formation of $\mathrm{H}_{2} \mathrm{SO}_{4}$, hence the affinity of sulphuric acid for water plays its part in the phenomena of displacement Therefore in determinations like those made by Thomsen the water does not form a medium which is present without participating in the process; it also takes part in the reaction. (Compare Chapter IX., Note 14.)

Whilst retaining essentially the methods of Thomsen, Ostwald (1876) determined the variation of the sp. gr. (and afterwards of volume), proceeding in the same dilute solutions, on the saturation of acids by bases, and in the decomposition of the salts of one acid by the other, and arrired at conclusions of just the same nature as Thomsen's. Ostwald's 
which can be compared with these reactions for simplicity are those exchange decompositions investigated by G. G. Gustavson, which

method will be clearly understood from an example. A solution of caustic soda containing an almost molecular ( 40 grams) weight per litre had a specific gravity of 1.04051. The specific gravities of solutions of equal volume and equivalent composition of sulphuric and nitric acids were 1.02970 and 1.03084 respectively. On mixing the solutions of $\mathrm{NaHO}$ and $\mathrm{H}_{2} \mathrm{SO}_{4}$ there was formed a solution of $\mathrm{Na}_{2} \mathrm{SO}_{4}$ of sp. gi. 1.02959; hence there ensued a decrease of specific gravity which we will term Q, equal to 1.04051 $+1 \cdot 02970-2(1 \cdot 02959)=0.01103$. So also the specific gravity after mixture of the solutions of $\mathrm{NaHO}$ and $\mathrm{HNO}_{3}$ was 1.02633 , and therefore $Q=0.01869$. When one volume of the solution of nitric acid was added to two volumes of the solution of sodium sulphate, a solution of sp. gr. 1.02781 was obtained, and therefore the resultant decrease of $\mathrm{sp}$. gr.

$$
\mathrm{Q}_{1}=2(1 \cdot 02959)+1 \cdot 03084-3(1: 02781)=0.00659 \text {. }
$$

Had there been no chemical reaction between the salts, then according to Ostwald's reasoning the specific gravity of the solutions would not have changed, and if the nitric acid had entirely displaced the sulphuric acid $Q_{2}$ would be $=0.01869-0.01103=0.00766$. It is evident that a portion of the sulphuric acid was displaced by the nitric acid. But the measure of displacement is not equal to the ratio between $Q_{1}$ and $Q_{2}$, because a decrease of sp. gr. also occurs on mixing the solution of sodium sulphate with sulphuric acid, whilst the mixing of the solutions of sodium nitrate and nitric acid only produces a slight variation of sp. gr. which falls within the limits of experimental error. Ostwald deduces from similar data the same conclusions as Thomsen, and thus reconfirms the formula deduced by Guldberg and Waage, and the teaching of Berthollet.

The participation of water is seen still more clearly in the methods adopted by Ostwald than in those of Thomsen, because in the saturation of solutions of acids by alkalis (which Kremers, Reinhold, and others had previously studied) there is observed, not a contraction, as might have been expected from the quantity of heat which is then evolved, but an expansion, of volume (a decrease of specific gravity, if we calculate as Ostwald did in his first investigations). Thus by mixing 1,880 grams of a solution of sulphuric acid of the composition $\mathrm{SO}_{3}+100 \mathrm{H}_{2} \mathrm{O}$, occupying a volume of 1,815 c.c., with a corresponding quantity of a solution $2\left(\mathrm{NaHO}+5 \mathrm{H}_{2} \mathrm{O}\right)$, whose volume $=1,793$ c.c., we obtain not 3,608 but 3,633 c.c., an expansion of 25 c.c. per gram molecule of the resulting salt, $\mathrm{Na}_{2} \mathrm{SO}_{4}$. It is the same in other cases. Nitric and hydrochloric acids give a still greater expansion than sulphuric acid, and potassium hydroxide than sodium hydróxide, whilst a solution of ammonia gives a contraction. The relation to water must be considered as the cause of these phenomena. When sodium hydroxide and sulphuric acid dissolve in water they develop heat and give a vigorous contraction; the water is sepa. rated from such solutions with great difficulty. After mutual saturation they form the salt $\mathrm{Na}_{2} \mathrm{SO}_{4}$, which retains the water but feebly and evolves but little heat with it, i.e., in other words, has little affinity for water. In the saturation of sulphuric acid by soda the water is; so to say, displaced from a stable combination and passes into an unstable conbination; hence an expansion (decrease of sp. gr.) takes place. It is not the reaction of the acid on the alkali, but the reaction of water, that produces the phenomenon by which Ostwald desires to measure the degree of salt formation. The water, which escaped attention, itself has affinity, and influences those phenomena which are being investigated. Furthermore, in the given instance its influence is very great because its mass is lnrge. When it is not present, or only present in small quantities, the attraction of the base to the acid leads to contraction, and not expansion. $\mathrm{Na}_{2} \mathrm{O}$ has a sp. gr. 2.8, hence its molecular volume $=22$; the sp. gr. of $\mathrm{SO}_{3}$ is 1.9 and volume 41 , hence the sum of their volumes is 63 ; for $\mathrm{Na}_{2} \mathrm{SO}_{4}$ the sp. gr. is 2.65 and volume 53.6 , consequently there is a contraction of 10 c.c. per gram-molecule of salt. The volume of $\mathrm{H}_{2} \mathrm{SO}_{4}=53 \cdot 3$, that of $2 \mathrm{NaHO}=37 \cdot 4$; there is produced $2 \mathrm{H}_{2} \mathrm{O}$, volume $=36,+\mathrm{Na}_{2} \mathrm{SO}_{4}$, volume $=53.6$. There react $90 \cdot 7$ c.c., and on satu. ration there result $89 \cdot 6$ c.c.; consequently contraction again ensues, although less, and 
take place between $\mathrm{CCl}_{4}$ and $\mathrm{RBr}_{n}$ on the one liand, and $\mathrm{CBr}_{4}$ and $\mathrm{RCl}_{n}$ on the other. This case is convenient for investigation inas-, much as the $\mathrm{RCl}_{n}$ and $\mathrm{RBr}_{n}$ taken (such as $\mathrm{BCl}_{3}, \mathrm{SiCl}_{4}, \mathrm{TiCl}_{4}, \mathrm{POCl}_{3}$, and $\mathrm{SnCl}_{4}$ ) belong to those substances which are decomposed by water, whilst $\mathrm{CCl}_{4}$ and $\mathrm{CBr}_{4}$ are not decomposed by water; and therefore, by heating, for instance, a mixture of $\mathrm{CCl}_{4}+\mathrm{SiBr}_{4}$ it is possible to arrive at a conclusion as to the amount of interchange by treating the product with water, which decomposes the $\mathrm{SiBr}_{4}$ left unchanged and the $\mathrm{SiCl}_{4}$ formed by the exchange, and therefore by determining the composition of the product acted on by the water it is possible to form a conclusion as to the amount of decomposition. The mixture was always formed with equivalent quantities-for in. stance, $4 \mathrm{BCl}_{3}+3 \mathrm{CBr}_{4}$. It appeared that there was no exchange whatever on simple intermixture, but that it proceeded slowly, when the mixture was heated (for example, with the mixture above mentioned at $123^{\circ} 4.86$ per cent. of Cl was replaced by Brafter 14 days' heating, and 6.83 per cent. after 28 days, and 10.12 per cent. when heated at $150^{\circ}$ for 60 days). A limit was always reached which corresponded with that of the complemental system; in the given instance the system $4 \mathrm{BBr}_{3}+3 \mathrm{CCl}_{4}$. In this last 89.97 per cent. of bromine in the $\mathrm{BBr}_{3}$ was replaced by chlorine; that is, there were obtained 89.97 molecules of $\mathrm{BCl}_{3}$ and there remained 10.02 molecules of $\mathrm{BBr}_{3}$, and therefore the same state of equilibrium was reached as that given by the system $4 \mathrm{BCl}_{3}+3 \mathrm{CBr}_{4}$. Both systems gave one and the same state of equilibrium at the limit, which is in agreement with Berthollet's doctrine. ${ }^{28}$

although this reaction is one of substitution and not of combination. Consequently the phenomens studied by Ostwald depend but little on the measure of the reaction of the salts, and more on the relations of the dissolved substances to water. In substitutions, for instance $2 \mathrm{NaNO}_{3}+\mathrm{H}_{2} \mathrm{SO}_{4}=2 \mathrm{HNO}_{3}+\mathrm{Na}_{2} \mathrm{SO}_{4}$; the volumes vary but slightly: in the above example they are $2(38 \cdot 8)+58 \cdot 3$ and $2(41 \cdot 2)+53 \cdot 6$; hence 181 volumes act, and 186 volumes are produced. It may be concluded, therefore, on the basis of what has been said, that on taking water into consideration the phenomena, studied by Thomsen and Ostwald are much more complex than they at first appear, and that this method can scarcely lead to a correct interpretation as to the distribution of acids between bases. We may add that P. D. Chroustcheff (1890) introduced a new method for this class of research, by investigating the electro.conductivity of solutions and their mixtures, and obtained remarkable results (for example, that hydrochloric acid almost entirely displace fornic acid and only $\frac{2}{3}$ of sulphuric acid), but details of these methods must be looked, for in text-books of theoretical chemistry.

28 G. G. Gustavson's researches, which were conducted in the laboratory of the St. Petersburg University in 1871-72, are among the first in which the measure of the affinity of the elements for the halogens is recognised with perfect clearness in the limit of substitution and in the rate of reaction. The researches conducted by A. L. Potilitzin (of which mention will be made in Chapter XI., Note 66) in the same laboratory touch on another aspect of the same problem which bas not yet made much progress, nọtwith. 
Thus we now find ample confirmation from various quarters for the following rules of Berthollet, applying them to double saline decom. positions - 1. From two salts MX and NY containing different haloids and metals there result from their reaction two others, MY and NX, but such a substitution will not proceed to the end unless one product passes from the sphere of action. 2. This reaction is limited by the existence of an equilibrium between $\mathrm{MX}, \mathrm{NY}, \mathrm{MY}$, and $\mathrm{NX}$, because a reverse reaction is quite as possible as the direct reaction. 3. This limit is determined both by the measure of the active affinities and by the rela. tive masses of the substances as measured by the number of the reacting molecules. 4. Other conditions being constant, the chemical action is proportional to the product of the chemical masses in action. ${ }^{29}$

standing its importance and the fact that the theoretioal side of the subject (thanks especially to Guldberg and Van't Hoff) has since been rapidly pushed forward. If the re. searches of Gustarson took account of the influence of mass, and were more fully supplied with data concerningivelocities and temperatures, they would be very important, because of the great significance which the case considered has for the understanding of double saline decompositions in the absence of water.

Furthermore, Gustavson showed that the greater the atomic weight of the element $\left(\mathrm{B}, \mathrm{Si}, \mathrm{Ti}, \mathrm{As}_{\mathrm{B}}, \mathrm{Sn}\right)$ combined with chlorine the greater the amount of chlorine replaced by bromine by the action of $\mathrm{CBr}_{4}$, and consequently the less the amount of bromine replaced by chlorine by the action of $\mathrm{CCl}_{4}$ on bromine compounds. For instanoe, for chlorine compounds the percentage of substitution (at the limit) is -

$\begin{array}{ccccc}\mathrm{BCl}_{3} & \mathrm{SiCl}_{4} & \mathrm{TiCl}_{4} & \mathrm{AsCl}_{3} & \mathrm{SnCl}_{4} \\ 10 \cdot 1 & 12.5 & 43.6 & 71 \cdot 8 & 77 \cdot 5\end{array}$

It should be observed, however, that Thorpe, on the basis of his experiments, denies the universality of this conclusion. I may mention one conclusion which it appears to me may be drawn from the above-cited figures of Gustavson, if they are subsequently verified even within narrow limits. If $\mathrm{CBr}_{4}$ be heated with $\mathrm{RCl}_{4}$, then an exchange of the bromine for chlorine takes place. But what would be the result if it were mixed with $\mathrm{CCl}_{4}$ ? Judging by the magnitnde of the atomic weights, $B=11, C=12, \mathrm{Si}=28$, about 11 p.c. of the chlorine would be replaced by bromine. But to what does this point? I think that this shows the existence of a motion of the atoms in the molecule. The mixture of $\mathrm{CCl}_{4}$ and $\mathrm{CBr}_{4}$ does not remain in a condition of static equilibrium; not only are the molecules contained in it in a state of motion, but also the atoms in the molecules, and the above figures show the measure of their translation under these conditions. The bromine in the $\mathrm{CBr}_{4}$ is, within the limit, substituted by the chlorine of the $\mathrm{CCl}_{4}$ in a quantity of about 11 out of 100 : that is,'a portion of the atoms of bromine previously to this moment in combination with one atom of carbon pass over to the other atom of carbon, and the chlorine passes over from this second atom of carbon to replace it. Therefore, also, in the homogeneous mass $\mathrm{CCl}_{4}$ all the atoms of $\mathrm{Cl}$ do not remain constantly combined with the same atoms of carbon, and there is an exchange of atoms between different mole. cules in a homogeneous medium also. This hypothesis may in my opinion explain cer tain phenomena of dissociation, but though mentioning it I do not consider it worth while to dwell upon it. I will only observe that a similar hypóthesis suggested itself to $m e$ in my researches on solutions, and that Pfaundler enunciated an essentially similar liypothesis, and in recent times a like view is beginning to find favour with respect to the electrolysis of saline solutions.

29 Berthollet's doctring is hardly at all affected in principle by showing that there are cases in which there is no decomposition between salts, because the affinity may be so 
Thus if the salts MX and NY after reaction partly formed salts MY and NX, then a state of equilibrium is reached and the reaction ceases ; but if one of the resultant compounds, in virtue of its physical properties, passes from the sphere of action of the remaining substances, then the reaction will continue. This exit from the sphere of action depends on the physical properties of the substance and on the conditions under which the reaction takes place. Thus, for instance, the salt NX may, in the case of reaction between solutions, separate as is precipitate, an insoluble substance, while the other three substances remain in solution, or it may pass into vapour, and in this manner also pass away from the sphere of action of the remaining substances. Let us now suppose that it passes away in some form or other from the sphere of action of the remaining substances-for instance, that it is transformed into a precipitate or vapour-then a fresh reaction will set in and a re-formation of the salt NX. If this be removed, then, although the quantity of the elements $\mathrm{N}$ and $\mathrm{X}$ in the mass will be diminished, still, according to Berthollet's law, a certain amount of NX should be again formed. When this substance is again formed, then, owing to its physical properties, it will again pass away; hence the reaction, in consequence of the physical properties of the resultant substances, is able to proceed to completion notwithstanding the possible weakness of the attraction existing between the elements entering into the composition of the resultant substance NX. Naturally, if the resultant substance is formed of elements having a considerable degree of affinity, then the complete decomposition is considerably facilitated.

Such a representation of the modus operandi of chemical transformations is applicable with great clearness to a number of reactions studied in chemistry, and, what is especially important, $\cdot$ the application of this aspect of Berthollet's teaching does not in any way require the determination of the measure of affinity acting between the substances present. For instance, the action of anmonia on solutions

small that even a large mass would still give no observablo displacements. The fundamental condition for the application of Berthollet's doctrine, as vell as Deville's doctrine of dissociation, lies in the reversibility of reactions. There are practically irreversible reactions (for instance, $\mathrm{CCl}_{4}+2 \mathrm{H}_{2} \mathrm{O}=\mathrm{CO}_{2}+4 \mathrm{HCl}$ ), just as there are non-volatile substances. But while accepting the doctrine of reversible reactions and retaining the theory of the evaporation of liquids, it is possible to admit the existence of non-volatile substances, and in just the same way of reactions, without any visible conformity to Berthollet's doctrine. This doctrine evidently comes nearer than the opposite doctrine of Bergmann to solving the complex problems of chemical mechanics for the successful solution of which at the present time the most valuable help is to be expected from the working out of data concerning dissociation, the influence of mass, and the equilibrium and velocity of reactions. But it is evident that from this point of view we must not regard a solvent as a non-participant space, but must take into consideration the chemical reactions accompanying solution, or else bring about reactions without solution. 
of salts ; the displacement, by its means, of basic hydrates insoluble in water; the separation of volatile nitric acid by the aid of non-volatile sulphuric acid, as well as the decomposition of common salt by means of sulphuric acid, when gaseous hydrochloric acid is formed-may be taken as examples of reactions which proceed to the end, inasmuch as one of the resultant substances is entirely removed from the sphere of action, but they in no way indicate the measure of affinity. ${ }^{30}$

As a proof that double decompositions like the above are actually accomplished in the sense of Berthollet's doctrine, the fact may be cited that common salt may be entirely decomposed by nitric acid, and nitre may be completely decomposed by hydrochloric acid, just as they are decomposed by sulphuric acid ; but this only takes place when, in the first instance, an excess of nitric acid is taken, and in the second instance, an excess of hydrochloric acid, for a given quantity of the sodium salt, and when the resultant acid passes off. If sodium chloride be put into a porcelain evaporating basin, nitric acid added to it, and the mixture heated, then both hydrochloric and nitric acids are expelled by the heat. Thus the nitric acid partially acts on the sodium chloride, but on heating, as both acids are volatile, they are both converted into

so Conmon salt not only enters into double decomposition with acids but also with every salt. However, as clearly follows from Berthollet's doctrine, this form of decomposition will only in a few cases render it possible for new metallic chlorides to be obtained, because the decomposition will not be carried on to the end unless the metallic chloride formed separates from the mass of the active substances. Thus, for example, if a solution of common salt be mixed with $\approx$ solution of magnesium sulphate, double decomposition ensues, but not completely, because all the substances remain in the solution. In this case the decomposition must result in the formation of sodium sulphate and magnesium chloride, substances which are soluble in water; nothing is disengaged, and therefore the decomposition $2 \mathrm{NaCl}+\mathrm{MgSO}_{4}=\mathrm{MgCl}_{2}+\mathrm{Na}_{2} \mathrm{SO}_{4}$ cannot proceed to the end. However, the sodium sulphate formed in this manner may be separated by freezing the mixture. The complete separation of the sodium sulphate will naturally not take place, owing to a portion of the salt remaining in the solution. Nevertheless, this kind of decomposition is made use of for the preparation of sodium sulphate from the residues left $\mathrm{nfter}$ the evaporation of sea-water, which contain a mixture of magnesium sulphate and common salt. Such a mixture is found at Stassfurt in a natural form. It might be said that this form of double decomposition is only accomplished wlth a change of tem. perature; but this would not be true, as may be concluded from other analogous cases. Thus, for instance, a solution of copper sulphate is of a blue colour, while a solution of copper chloride is green. If we mix the two salts together the green tint is distinctly visible, so that by this means the presence of the copper chloride in the solution of copper sulphate is clearly seen. If now we add a solution of common salt to a solution of copper sulphate, a green coloration is obtained, which indicates the formation of copper chloride. In this instance it is not separated, but it is immediately formed on the addition of common salt, as it should be according to Berthollet's doctrine.

The complete formation of a metallic chloride from common salt can only occur, judging from the above, when it separates from the sphere of action. The salts of silver are instances in point, because the silver chloride is insoluble in water; and therefore if we add a solution of sodium chloride to a solution of a silver salt, silver chloride and the sodium salt of that acid which was in the silver salt are formed. 
vapour, and therefore the residue will contain a mixture of a certain quantity of the sodium chloride taken and of the sodium nitrate formed. If a fresh quantity of nitric acid be then added, reaction will again set in, a certain portion of hydrochloric acid is again evolved, and on heat. ing is expelled together with nitric acid. If this be repeated several times, it is possible to expel all the hydrochloric acid, and to obtain sodium nitrate only in the residue. If, on the contrary, we take sodium nitrate and add hydrochloric acid to it in an aqueous solution, a certain quantity of the hydrochloric acid displaces a portion of the nitric acid, and on heating the excess of hydrochloric acid passes away with the nitric acid formed. On repeating this process, it is possible to displace the nitric acid with an excess of hydrochloric acid, just as it was possible to displace the hydrochloric acid by an excess of nitric acid. The influence of the mass of the substance in action and the influence of volatility are here very distinctly seen. Hence it may be affirmed that sulphuric acid does not displace hydrochloric acid because of an especially high degree of affinity, but that this reaction is only carried on to the end because the sulphuric acid is not volatile, whilst the hydrochloric acid which is formed is volatile.

The preparation of hydrochloric acid in the laboratory and on a large scale is based upon these data. In the first instance, an excess of sulphuric acid is employed in order that the reaction may proceed easily at a low temperature, whilst on a large scale, when it is necessary to economise every material, equivalent quantities are taken in order to obtain the normal salt $\mathrm{Na}_{2} \mathrm{SO}_{4}$ and not the acid salt, which would require twice as much acid. The hydrochloric acid evolved is a gas which is very soluble in water. It is most frequently used in practice in this state of solution under the name of muriatic acid. ${ }^{31}$

51 The apparatus shown in fig. 46 (Chapter VI., Note 12) is generally employed for the preparation of small quentities of hydrochloric acid. Common salt is placed in the retort; the salt is generally previously fused, as it otherwise froths and boils over in the apperatus. When the apparatus is placed in order sulphuric acid mixed with water is poured down the thistle funnel into the retort. Strong sulphuric acid (about balf as much again as the weight of the salt) is usually taken, and it is diluted with a small quantity of water (half) if it be desired to retard the action, as in using strong sulphuric acid the action immediately begins with great vigour. The mixture, at first without the aid of heat and then at a moderate temperature (in a water-bath), evolves hydrochloric acid. Commercial hydrochloric acid contains many impurities; it is usually purified by distillation, the middle portions being collected. It is purified from arsenic by adding $\mathrm{FeCl}_{2}$, distilling, and rejecting the first third of the distillate. If free hydrochloric acid gas be required, it is passed through a vessel containing strong sulphuric acid to dry it, and is collected over a mercury bath.

Phosphoric anhydride absorbs hydrogen chloride (Bailey and Fowler, 18s8; $2 \mathrm{P}_{2} \mathrm{O}_{3}+3 \mathrm{HCl}=\mathrm{POCl}_{3}+3 \mathrm{HPO}_{3}$ ) at the ordinary temperature, and therefore the gas cannot be dried by this substance. 
In chemical works the decomposition of sodium chloride by means of sulphuric acid is carried on on a very large scale, chiefly with a view to the preparation of normal sodium sulphate; the hydrochloric acid being a bye-product. ${ }^{31}$ bis The furnace employed is termed a salt cake furnace. It is represented in fig. 65 , and consists of the following two parts $\cdot$ the pan $B$ and the roaster $C$, or enclosed space built up of large bricks $a$ and enveloped on all sides by the smoke and flames from the fire grate, $F$ The ultimate decomposition 'of the salt by the sulphuric acid is accomplished in the roaster. But the first decomposition of sodium chloride by sulphuric acid does not require so high a temperature as the ultimate decomposition, and is therefore carried on in the front and cooler portion, B, whose bottom is heated by gas flues. When the reaction in this portion ceases and the evolution

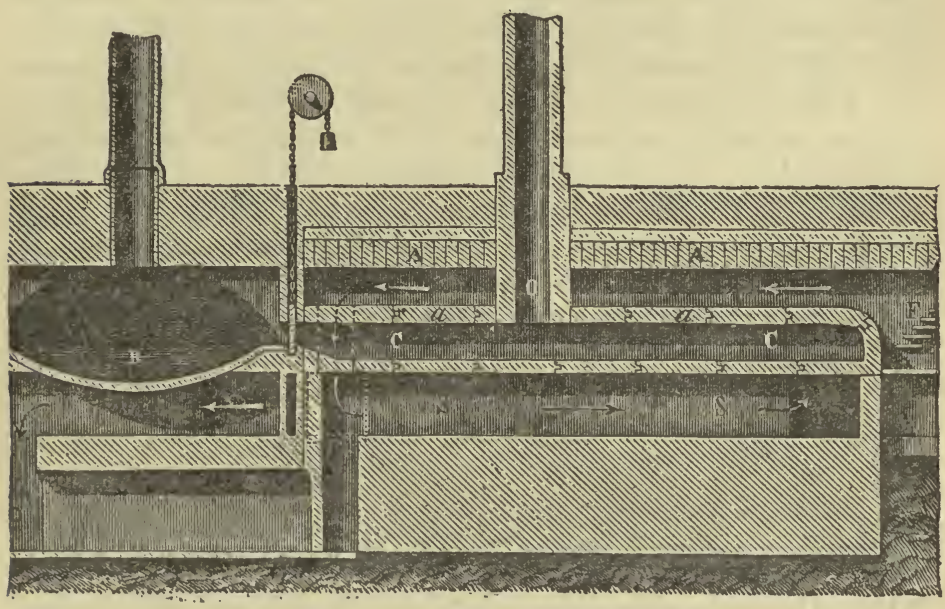

Fra. 65.-Section of a salt-cake furnace, B, pan in which the sodium chloride and sulphuric acid are first mixed and heated. $\mathrm{C}$, muffle for the ultimate decomposition.

of hydrochloric acid stops, then the mass, which contains about half of the sodium chloride still undecomposed, and the sulphuric acid in the form of acid sodium sulphate, is removed from $\mathrm{B}$ and thrown into the roaster $\mathrm{C}$, where the action is completed. Normal sodium sulphate, which we shall afterwards describe, remains in the roaster. It is employed both directly in the manufacture of glass, and in the preparation of other sodium compounds-for instance, in the

81 bla In chemical works where sulphuric acid of $60^{\circ}$ Baume (22 p.c. of water) is employed. 117. parts of sodium chloride are taken to about 125 parts of sulphurio acia 
preparation of soda ash, as will afterwards be described. For the present we will only turn our attention to the hydrochloric acid evolved in B and $\mathrm{C}$.

The hydrochloric acid gas evolved is subjected to condensation by dissolving it in water. ${ }^{32}$ If the apparatus in which the decomposition is accomplished were hermetically closed, and only presented one outlet, then the escape of the hydrochloric acid would only proceed through the escape pipe intended for this purpose. But as it is impossible to construct a perfectly hermetically closed furnace of this kind, it is necessary to increase the draught by artificial means, or to oblige the hydrochloric acid gas to pass through those arrangements in which it is to. be condensed. This is done by connecting the ends of the tubes through which the hydrochloric acid gas escapes from the furnace with high chimneys, where a strong draught is set up from the combustion of the fuel. This causes a current of hydrochloric acid' gas to pass through the absorbing apparatus in a definite direction. Here it encounters a current of water flowing in the opposite direction, by which it is absorbed. It is not customary to cause the acid to pass through the water, but only to bring it into contact with the surface of the water. The absorption apparatus consists of large earthenware vessels having four orifices, two above and two lateral ones in the wide central portion of each vessel. The upper orifices serve for connecting the vessels together, and the hydrochloric acid gas escaping from the furnace passes through these tubes. The water for absorbing the acid enters at the upper, and

$53 \mathrm{As}$ in works which treat common salt in order to obtain sodium sulphate, the hydrochloric acid is sometimes held to be of no value, it might be allowed to escape with the waste furnace gases into the atmosphere, which would greatly injure the air of the neighbourhood and destroy all vegetation. In all countries, therefore, there are laws forbidding the factories to proceed in this manner, and requiring the absorption of the hydrochloric acid by water at the works themselves, and not permitting the solution to be run into rivers and streams, whose waters it would spoil. It may be remarked that the absorption of hydrochloric acid presents no particular difficulties (the absorption of sulphurous acid is much more difficult) because hydrochloric acid has a great affinity for water and gives a hydrate which boils abore $100^{\circ}$. Hence, even steam and hot water, as well as weaker solutions, can be used for absorbing the acid. However, Warder (1888) showed that weak solutions of composition $\mathrm{H}_{2} \mathrm{O}+n \mathrm{HCl}$ when boiled (the residue will be almost $\mathrm{HCl}, 8 \mathrm{H}_{2} \mathrm{O}$ ) evolve (not water but) a solution of the composition $\mathrm{H}_{2} \mathrm{O}+445 n^{4} \mathrm{HCl}$; for example, on distilling $\mathrm{HCl}, 10 \mathrm{H}_{2} \mathrm{O}, \mathrm{HCl}, 23 \mathrm{H}_{2} \mathrm{O}$ is first obtained in the distillate. As the strength of the residue becomes greater, so also does that of the distillate, and therefore in order to completely absorb hydrochloric acid it is necessary in the end to have recourse to water.

As in Russia the manufacture of sodium sulphate from sodium chloride has not yet been sufficiently developed, and as hydrochloric acid is required for many technical purposes (for instance, for the preparation of zinc chloride, which is employed for soaking cailway sleepers), therefore salt is often treated mainly for the manufacture of hydro. chloric acid. 
flows out from the lower, vessel, passing through the lateral orifices in the vessels. The water flows from the chimney towards the furnace and it is therefore evident that the outflowing water will be the most saturated with acid, of which it actually contains about 20 per cent. The absorption in these vessels is not complete. The ultimate absorption of the hydrochloric acid is carried on in the so-called coke towers, which usually consist of two adjacent chimneys. A lattice-work of bricks is laid on the bottom of these towers, on which coke is piled up to the top of the tower. Water, distributing itself over the coke, trickles down to the bottom of the tower, and in so doing absorba the hydrochloric acid gas rising upwards.

It will be readily understood that hydrochloric acid may be obtained from all other metallic chlorides. ${ }^{33}$ It is frequently formed in other reactions, many of which we shall meet with in the further course of this work. It is, for instance, formed by the action of water on sulphur chloride, phosphorus chloride, antimony chloride, \&c.

Hydrochloric acid is a colourless gas having a pungent suffocating odour and an acid taste. This gas fumes in air and attracts moisture, because it forms vapour containing a compound of hydrochloric acid and water. Hydrochloric acid is liquefied by cold, and under a pressure of 40 atmospheres, into a colourless liquid of sp. gr. 0.908 at $0^{\circ}, 34$ boiling point $-35^{\circ}$ and absolute boiling point $+52^{\circ}$. We have already seen (Chapter I:) that hydrochloric acid combines very energetically with water, and in so doing evolves a considerable amount of heat. The solution saturated in the cold attains a density 1-23. On heating such, a solution containing about 45 parts of acid per 100 parts, the hydro-

35 Thus the metallic chlorides, which are decomposed to a greater or less degree by water, correspond with feeble bases. Such are, for example, $\mathrm{MgCl}_{2}, \mathrm{AlCl}_{3}, \mathrm{SbCl}_{3}, \mathrm{BiCl}_{3}$. The decomposition of magnesium chloride (and also carnallite) by sulphuric acid proceeds at the ordinary temperature; water decomposes $\mathrm{MgCl}_{2}$ to the extent of 50 p.c. when aided by heat, and may be employed as a convenient method for the production of hydrochloric acid. Hydrochlorio acid is also produced by the ignition of certain metallic chlorides in a stream of hydrogen, especially of those metals which are easily reduced and diffichltly oxidised-for instance, silver chloride. Lead chloride, when heated to redness in a current of steam, gives hydrochloric acid and lead oxide. The multitude of the cases of formation of hydrochloric acid are understood from the fact that it is a substance which is comparatively very stable, resembling water in this respect, and even most probably more stable than water, because, at a high temperature and even under the action of light, chlorine decomposes $\cdot$ water, with the formation of hydrochloric acid. The combination of chlorine and hydrogen also proceeds by their direct action, as we shall afterwards describe.

5t According to Ansdell (1880) the sp. gr. of liquid hydrochloric acid at $0^{\circ}=0.908$, so $11.67^{\circ}=0.854$, at $22 \cdot 7^{\circ}=0.808$, at $83^{\circ}=0.748$. Hence it is seen that the expansion of this liquid is greater than that of gases (Chapter II., Note 34). 
chloric acid gas is expelled with only a slight admixture of aqueous vapour. But it is impossible to entirely separate the whole of the hydrochloric acid from the water by this means, as could be done in the case of an ammoniacal solution. The temperature required for the evolution of the gas rises and reaches $110^{\circ}-111^{\circ}$, and after this remains constant - that is, a solution having a constant boiling point is obtained (as with $\mathrm{HNO}_{3}$ ), which, however, does not (Roscoe and Dittmar) present a constant composition under different pressures, because the hydrate is decomposed in distillation, as is seen from the determinations of its vapour density (Bineau). Judging from the facts (1) that with. decrease of the pressure under which the distillation proceeds the solution of constant boiling point approaches to a composition of 25 p.c. of hydrochloric acid, ${ }^{35}$ (2) that by passing a stream of dry air through a solution of hydrochloric acid there is obtained in the residue a solution which also approaches to 25 p.c. of acid, and more nearly as the tempera. ture falls, ${ }^{36}(3)$ that many of the properties of solutions of hydrochloric acid vary distinctly according as they contain more or less than 25 p.c. of hydrochloric acid (for instance, antimonious sulphide gives hydro. gen sulphide with a stronger acid, but is not acted on by a weaker solution, also a stronger solution fumes in the air, \&c.), and (4) that the composition $\mathrm{HCl}, 6 \mathrm{H}_{2} \mathrm{O}$ corresponds with $25 \cdot 26$ p.c. $\mathrm{HCl}$-judging from all these data, and also from the loss of tension which occurs in the combination of hydrochloric acid with water, it may be said that they form a definite hydrate of the composition $\mathrm{HCl}, 6 \mathrm{H}_{2} \mathrm{O}$. Besides this hydrate there exists also a crystallo-hydrate, $\mathrm{HCl}, 2 \mathrm{H}_{2} \mathrm{O},{ }^{37}$ which is formed by the absorption of hydrochloric acid by a saturated solution at a temperature of $-23^{\circ}$. - It crystallises and melts at $-18^{\circ} .38$

The mean specific gravities at $15^{\circ}$, taking water at its maximum

35 According to Roscoe and Dittmar at a pressure of three atmospheres the solution of corstant boiling point contains 18 p.c. of hydrogen chloride, and at a pressure of one. tenth atmosphere 28 p.c. The percentage is intermediate at medium pressures.

s6 At $0^{\circ} 25$ p.c., at $100^{\circ} 20.7$ p.c. ; Roscoe and Dittmar.

37 This crystallo-hydrate (obtained by Pierre and Puchot, and investigated by Rooze. boom) is analogous to $\mathrm{NaCl}, 2 \mathrm{H}_{2} \mathrm{O}$. The crystals $\mathrm{HCl}, 2 \mathrm{H}_{2} \mathrm{O}$ at $-22^{\circ}$ have a specifio gravity 1.46; the vapour tension (under dissociation) of the solution having a composition $\mathrm{HCl}, 2 \mathrm{H}_{2} \mathrm{O}$ at $-24^{\circ}=760$, at $-19^{\circ}=1,010$, at $-18^{\circ}=1,057$, at $-17^{\circ}=1,112 \mathrm{~mm}$. of mer. cury. In a solid state the crystallo-hydrato at $-17.7^{\circ}$ has the same tension, whilst at lower temperatures it is much less: at $-24^{\circ}$ about 150 , at $-19^{\circ}$ about $580 \mathrm{~mm}$. A mixture of fuming hydrochloric acid with snow reduces the temperature to $-88^{\circ}$. If another equivalent of water be added to the hydrate $\mathrm{HCl}, 2 \mathrm{H}_{2} \mathrm{O}$ at $-18^{\circ}$, the temperature of solidification falls to $-25^{\circ}$, and the hydrate $\mathrm{HCl}, 3 \mathrm{H}_{2} \mathrm{O}$ is formed (Pickering, 1893).

33 According to Roscoe at $0^{\circ}$ one hundred grams of water a a pressure $p$ (in millimetres of mercury) dissolves-

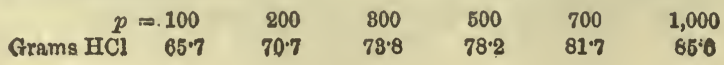


density $\left(4^{\circ}\right)$ as 10,000 , for solutions containing $p$ per cent. of hydrogen chloride are-

$\begin{array}{rccc}p & S^{\circ} & p & S \\ 5 & 10,242 & 25 & 11,266 \\ 10 & 10,490 & 30 & 11,522 \\ 15 & 10,744 & 35 \cdot & 11,773 \\ 20 & 11,001 & 40 & 11,997\end{array}$

The formula $S=9,991 \cdot 6 \cdot 49 \cdot 43 p+0 \cdot 0571 p^{2}$, up to $p=25 \cdot 26$, which answers. to the hydrate $\mathrm{HCl}, 6 \mathrm{H}_{2} \mathrm{O}$ mentioned above, gives the specific gravity. Above this percentage $S \doteq 9,785 \cdot 1+65 \cdot 10 p-0 \cdot 240 p^{2}$. The

At a pressure of 760 millimetres and temperature $t$, one hundred grams of water dissolves

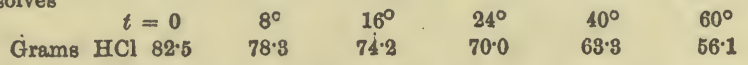

Roozeboom (1886) showed that at $t^{\circ}$ solutions containing 0 grams of hydrogen chlorive per 100 grams of water may (with the variation of the pressure $p$ ) be formed together with the crystallo-hydrate $\mathrm{HCl}, 2 \mathrm{H}_{2} \mathrm{O}$ :

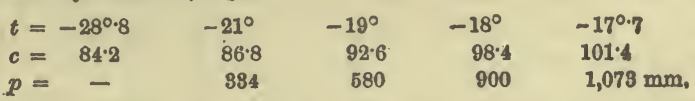

The last combination answers to the melted crystallo-hydrate $\mathrm{HCl}, 2 \mathrm{H}_{2} \mathrm{O}$, which splits up at temperatures above $-17^{\circ} \cdot 7$, and at a constant atmospheric pressure when there are no crystals-

$$
\begin{array}{lrrrr}
t=-24^{\circ} & -21^{\circ} & -18^{\circ} & -10^{\circ} & 0^{\circ} \\
c=101^{\circ} 2 & 98^{\circ} 3 & 95^{\circ} \cdot 7 & 89^{\circ} \cdot 8 & 84^{\circ} \cdot 2
\end{array}
$$

From these data it is seen that the hydrate $\mathrm{HCl}, 2 \mathrm{H}_{2} \mathrm{O}$ can exist in a liquid state, which is not the case for the hydrates of carbonic and sulphurous anhydrides, chlorine, \&c.

According to Marignac, the specific heat $c$ of a solution $\mathrm{HCl}+m \mathrm{H}_{2} \mathrm{O}$ (at about $30^{\circ}$, taking the speciflo heat of water $=1$ ) is given by the expression-

$$
\mathrm{C}(36 \cdot 5+m 18)=18 m-28 \cdot 39+140 / m-268 / m^{2}
$$

if $m$ be not less than 6.25. For example, for $\mathrm{HCl}+25 \mathrm{H}_{2} \mathrm{O}, \mathrm{C}=0.877$.

According to Thomsen's data, the amount of heat $Q$, expressed in thousands of calories, evolved in the solution of 36.5 grams of gaseous hydrochloric acid in $m \mathrm{H}_{2} \mathrm{O}$ or $18 \mathrm{~m}$ grams of water is equal to-

$$
\begin{aligned}
& m=2 \quad 4 \quad 10 \quad 50 \quad 400 \\
& Q=11 \cdot 4 \quad 14.8 \quad 16.2 \quad 17 \cdot 1 \quad 17 \cdot 8
\end{aligned}
$$

In these quantities the latent heat of liquefaction is included, which must be talken as 5-9 thousand calories per molecular quantity of hydrogen chloride.

The researches of Scheffer (1888) on the rate of diffusion (in water) of solutions of bydrochloric acid show that the coefficient of diffusion $\dot{k}$ decreases with the amount of water $n$, if the composition of the solution is $\mathrm{HCl} n \mathrm{H}_{3} \mathrm{O}$ at $0^{\circ}:-$

$\begin{array}{llllcc}n=5 & 6.9 & 9.8 & 14 & 27.1 & 129.5 \\ k=2.81 & 2.08 & 1.86 & 1.67 & 1.62 & 1.39\end{array}$

It also appears that strong solutions diffuse more rapidly into dilute solutions than into water. 
rise of specific gravity with an increase of percentage (or the differential $\left.\begin{array}{c}d s \\ d \dot{p}\end{array}\right)$ reaches a maximum at about 25 p.c. $^{39}$ The intermediate solution, $\mathrm{HCl}, 6 \mathrm{H}_{2} \mathrm{O}$, is further distinguished by the fact that the variation of the specific gravity with the variation of temperature is a constant quantity, so that the specific gravity of this solution is equal to $11,352.7(1-0.000447 t)$, where 0.000447 is the coefficient of expansion of the solution..$^{40}$ In the case of more dilute solutions, as with water, the specific gravity per $1^{\circ}$ (or the differential $\frac{d s}{d t}$ ) rises with a rise of tem. perature. ${ }^{41}$

$\begin{array}{rccccc}p & =0 & 5 & 10^{\circ} & 15 & 20 \\ S_{0}-S_{15}=7 \cdot 2 & 23 & 38 & 52 & 64 \\ S_{15}-S_{30} & =34 \cdot 1 & 42 & 50 & 59 & 67\end{array}$

Whilst for solutions which contain a greater proportion of hydrogen chloride than $\mathrm{HCl}, 6 \mathrm{H}_{2} \mathrm{O}$, these coefficients decrease with a rise of temperature; for instance, for 30 p.c. of hydrogen chloride $S_{0}-S_{15}$ $=88$ and $S_{15}-S_{30}=87$. (according to Marignac's data). In the cas of $\mathrm{HCl}, 6 \mathrm{H}_{2} \mathrm{O}$ these differences are constant, and equal 76 .

Thus the formation of two definite hydrates, $\mathrm{HCl}, 2 \mathrm{H}_{2} \mathrm{O}$ and $\mathrm{HCl}, 6 \mathrm{H}_{2} \mathrm{O}$, between hydrochloric acid and water may be accepted upon the basis of many facts. But both of them, if they occur in a liquid state, dissociate with great facility into hydrogen chloride and water, and are completely decomposed when distilled.

All solutions of hydrochloric acid present the properties of an energetic acid. They not only transform blue vegetable colouring matter into red, and disengage carbonic acid gas from carbonates, \&c., but they also entirely saturate bases, even such energetic ones as potash, lime, \&c. In a dry state, however, hydrochloric acid does not alter

39 If it be admitted that the maximum of the differential corresponds with $\mathrm{HCl}, 6 \mathrm{H}_{2} \mathrm{O}$, then it might be thought that the specific gravity is expressed by a parabola of the third order; but such an admission does not give expressions in accordance with fact. This is all more fully considered in my work mentioned in Chapter I., Note 19.

$40 \mathrm{As}$ in water, the coefficient of expansion (or the quantity $k$ in the expression $\mathrm{S}_{t}=\mathrm{S}_{o}-k \mathrm{~S}_{o} t$, or $\mathrm{V}_{t}=1 /(1-k t)$ attains a magnitude 0.000447 at about $48^{\circ}$, it might be thought that at $48^{\circ}$ all solutions of hydrochloric acid would have the same coefficient of expansion, but in reality this is not the case. At low and at the ordinary temperatures the coefficient of expansion of aqueous solutions is greater than that of water, and increases with the amount of substance dissolved.

41 The figures cited above may serve for the direct determination of the variation of the specific gravity of solutions of hydrochloric acid with the temperature. Thus, knowing that at $15^{\circ}$ the specific gravity of a 10 p.c. solution of hydrochloric acid $=10,492$, we find that at $t^{\top}$ it $=10,530-t(2 \cdot 13+0 \cdot 027 t)$. Whence also may be found the coefficient of expansion (Note 40). 
vegetable dyes, and does not effect many double decompositions which easily take place in the presence of water. This is explained by the fact that the gaso-elastic state of the hydrochloric acid prevents its entering into reaction. However, incandescent iron, zinc, sodium, \&c., act on gaseous hydrochloric acid, displacing the hydrogen and leaving half a volume of hydrogen for each volume of hydrochloric acid gas ; this reaction may serve for determining the composition of hydrochloric acid. Combined. with water hydrochloric acid acts as an acid much resembling nitric acid 42 in its energy and in many of its reactions ; however, the latter contains oxygen, which is disengaged with great ease, and so very frequently acts as an oxidiser, which hydrochloric acid is not capable of doing. The majority of metals (even those which do not displace the $\mathrm{H}$ from $\mathrm{H}_{2} \mathrm{SO}_{4}$, but which, like copper, decompose it to the limit of $\mathrm{SO}_{2}$ ) displace the hydrogen from hydrochloric acid Thus hydrogen is disengaged by the action of zinc, and even of copper and tin. ${ }^{42}$ bis Only a few metals withstand its action; for example, gold and platinum. Lead in compact masses is only acted on feebly, because the lead chloride formed is insoluble and prevents the further action of the acid on the metal. The same is to be remarked with respect to the feeble action of hydrochloric acid on mercury and silver, because the compounds of these metals, $\mathrm{AgCl}$ and $\mathrm{HgCl}$, are insoluble in water. Metallic chlorides are not only formed by the action of hydrochloric acid on the metals, but also by many other methods ; for instance, by the action of hydrochloric acid on the carbonates, oxides, and hydroxides, and also by the action of chlorine on metals and certain of their compounds. Metallic chlorides have a composition $\mathrm{MCl}$; for example, $\mathrm{NaCl}, \mathrm{KCl}, \mathrm{AgCl}, \mathrm{HgCl}$, if the metal replaces hydrogen equivalent for equivalent, or, as it is said, if it be monatomic or univalent. In the case of bivalent metals, they have a composition $\mathrm{MCl}_{2}$; for example, $\mathrm{CaCl}_{2}, \mathrm{CuCl}_{2}, \mathrm{PbCl}_{2}, \mathrm{HgCl}_{2}, \mathrm{FeCl}_{2}, \mathrm{MnCl}_{2}$. The composition of the haloid salts of other metals presents a further variation ; for example, $\mathrm{AlCl}_{3}, \mathrm{PtCl}_{4}$, \&c. Many metals, for instance $\mathrm{Fe}$, give several degrees of combination with chlorine $\left(\mathrm{FeCl}_{2}, \mathrm{FeCl}_{3}\right)$ is with hydrogen. In their composition the metallic chlorides differ from the corresponding oxides, in that the $\mathrm{O}$ is replaced by $\mathrm{Cl}_{2}$, as should follow from the law of substitution, because oxygen gives $\mathrm{OH}_{2}$, and is

'42 Thus, for instance, with feable bases they evolve in dilute solutions (Chapter III., Note 58) almost equal amounts of heat; their relation to sulphuric acid is quite identical. They both form fuming solutions as well as hydrates; they both form solutions of con. siant boiling point.

42 bis Pybalkin (1891) found that copper begins to disengage hydrogen at $100^{\circ}$, and that chloride of copper begins to give up its chlorine to hydrogen gas at $230^{\circ}$; for silver these temperatures are $117^{\circ}$ and $260^{\circ}$-that is, there is less difference between them. 
consequently bivalent, whilst chlorine forms $\mathrm{HCl}$, and is therefore univalent. So, for instance, ferrous oxide, $\mathrm{FeO}$, corresponds with ferrous chloride, $\mathrm{FeCl}_{2}$, and the oxide $\mathrm{Fe}_{2} \mathrm{O}_{3}$ with ferric chloride, which is also seen from the origin of these compounds, for $\mathrm{FeCl}_{2}$ is obtained by the action of hydrochloric acid on ferrous oxide or carbonate and $\mathrm{FeCl}_{3}$ by its action on ferric oxide. In a word, all the typical properties of acids are shown by hydrochloric acid, and all the typical properties of salts in the metallic chlorides derived from it. Acids and salts composed like $\mathrm{HCl}$ and $\mathrm{M}_{n} \mathrm{Cl}_{2 m}$ without any oxygen bear the name of haloid salts ; for instance, $\mathrm{HCl}$ is a haloid acid, $\mathrm{NaCl}$ a haloid salt, chlorine a halogen. The capacity of hydrochloric acid to give, by its action on bases, $\mathrm{MO}$, a metallic chloride, $\mathrm{MCl}_{2}$, and water, is limited at high temperatures by the reverse reaction $\mathrm{MCl}_{2}+\mathrm{H}_{2} \mathrm{O}=\mathrm{MO}+2 \mathrm{HCl}$, and the more pronounced are the basic properties of MO the feebler is the reverse action, while for feebler bases such as $\mathrm{Al}_{2} \mathrm{O}_{3}, \mathrm{MgO}$, \&c., this reverse reaction proceeds with ease. Metallic chlorides corresponding with the peroxides either do not exist, or are easily decomposed with the disengagement of chlorine. Thus there is no compound $\mathrm{BaCl}_{4}$ corresponding with the peroxide $\mathrm{BaO}_{2}$. Metallic chlorides having the general aspect of salts, like their representative sodium chloride, are, as a rule, easily fusible, more so than the oxides (for instance, $\mathrm{CaO}$ is infusible at a furnace heat, whilst $\mathrm{CaCl}_{2}$ is easily fused) and many other salts. Under the action of heat many chlorides are more stable than the oxides, some can even be converted into vapour; thus corro. sive sublimate, $\mathrm{HgCl}_{2}$, is particularly volatile, whilst the oxide $\mathrm{HgO}$ decomposes at a red heat. Silver chloride, $\mathrm{AgCl}$, is fusible and is decomposed with difficulty, whilst $\mathrm{Ag}_{2} \mathrm{O}$ is easily decomposed. The majority of the metallic chlorides are soluble in water, but silver chloride, cuprous chloride, mercurous chloride, and lead chloride are sparingly soluble in water, and are therefore easily obtained as precipitates when a solution of the salts of these metals is mixed with a solution of any chloride or even with hydrochloric acid. The metal contained in a haloid salt may often be replaced by another metal, or even by hydrogen, just as is the case with a metal in an oxide. Thus copper displaces mercury from a solution of mercuric chloride, $\mathrm{HgC}_{2}+\mathrm{Cu}=\mathrm{CuCl}_{2}+\mathrm{Hg}$, and hydrogen at a red heat displaces silver from silver chloride, $2 \mathrm{AgCl}+\mathrm{H}_{2}=\mathrm{Ag}_{2}+2 \mathrm{HCl}$. These, and a whole series of similar reactions, form the typical methods of double saline decompositions. The measure of decomposition and the conditions under which reactions of double saline decompositions proceed in one or in the other direction are determined by the properties of the compounds which take part in the reaction, and of those capable of formation at the 
temperature, \&c., as was shown in the preceding portions of this chapter, and as will be frequently found hereafter.

If hydrochloric acid enters into double decomposition with basic oxides and their hydrates, this is only.due to its acid properties; and for the same reason it rarely enters into double decomposition with acids and acid anhydrides. Sometimes, however, it combines with the latter, as, for instance, with the anhydride of sulphuric acid, forming the compound $\mathrm{SO}_{3} \mathrm{HCl}$; and in other cases it acts on acids, giving up its hydrogen to their oxygen and forming chlorine, as will be seen in the following chapter.

Hydrochloric acid, as may already be concluded from the composition of its molecule, belongs to the monobasic acids, and does not, therefore, give true acid salts (like $\mathrm{HNaSO}_{4}$ or $\mathrm{HNaCO}_{3}$ ) ; nevertheless many metallic chlorides, formed from powerful bases, are capable of combining with hydrochloric acid, just as they combine with water, ôr with ammonia, or as they give double salts. Compounds have long been known of hydrochloric acid with auric, platinic, and antimonious chlorides, and other similar metallic chlorides corresponding with very feeble bases. But Berthelot, Engel, and others have shown that the capacity of $\mathrm{HCl}$ for combining with $\mathrm{M}_{n} \mathrm{Cl}_{m}$ is much more frequently encountered than was previously supposed. Thus, for instance, dry hydrochloric acid when passed into a solution of zinc.chloride (containing an excess of the salt) gives in the cold $\left(0^{\circ}\right)$ a compound $\mathrm{HCl}, \mathrm{ZnCl}_{2}, 2 \mathrm{H}_{2} \mathrm{O}$, and at the ordinary temperature $\mathrm{HCl}, 2 \mathrm{ZnCl}_{2}, 2 \mathrm{H}_{2} \mathrm{O}$; just as it is able at low temperatures to form the crystallo-hydrate $\mathrm{ZnCl}_{2}, 3 \mathrm{H}_{2} \mathrm{O}$ (Engel, 1886). Similar compoundsare obtained with $\mathrm{CdCl}_{2}, \mathrm{CuCl}_{2}, \mathrm{HgCl}_{2}, \mathrm{Fe}_{2} \mathrm{Cl}_{6}$, \&c. (Berthelot, Ditte, Cheltzoff, Lachiroff, and others). These compounds with hydrochloric acid are generally more soluble in water than the metallic chlorides themselres, so that whilst hydrochloric acid decreases the solubility of $\mathrm{M}_{n} \mathrm{Cl}_{m}$, corresponding with energetic hases (for instance, sodium or barium chlorides), it increases the solubility of the metallic chlorides corresponding with feeble bases (cadmium chloride, ferric chloride, \&c.) Silver chloride, which is insoluble in water, is soluble in hydrochloric acid. Hydrochloric acid also combines with certain unsaturated hydrocarbons (for instance, with turpentine, $\mathrm{C}_{10} \mathrm{H}_{16}, 2 \mathrm{HCl}$ ) and their derivatives. Sal-ammoniac, or ammonia hydrochloride, $\mathrm{NH}_{4} \mathrm{Cl}$ $=\mathrm{NH}_{3}, \mathrm{HCl}$, also belongs to this class of ccmpounds. ${ }^{43}$ If hydrogen chloride gas be mixed with ammonia gas a solid compound consisting

43 When an unsaturated hydrocarbon, or, in general, an unsaturated compound, assimilates to itself the molecules $\mathrm{Cl}_{2}, \mathrm{HCl}, \mathrm{SO}_{3}, \mathrm{H}_{2} \mathrm{SO}_{4}$, \&c., the cause of the reaction" is most simple. As nitrogen, besides the type $\mathrm{NX}_{3}$ to which $\mathrm{NH}_{3}$, belongs, gives com. pounds of the type $\mathrm{NX}_{5}$ for example, $\mathrm{NO}_{2}(\mathrm{OH})$ - the formation of the salts of 
of equal volumes of each is immediately formed. The same com. pound is obtained on mixing solutions of the two gases. It is also produced by the action of hydrochloric acid on ammonium carbonate. Sal-ammoniac is usually prepared, in practice, by the last method.48 The specific gravity of sal-ammoniac is 1.55 . We have already seen (Chapter VI.) that sal-ammoniac, like all other ammonium salts, easily decomposes; for instance, by volatilisation with alkalis, and even partially when its solution is boiled. The other properties and reactions of sal-ammoniac, especially in solution, fully recall those already mentioned in speaking of sodium chloride. Thus, for instance, with silver nitrate it gives a precipitate of silver chloride : with sulphuric acid it gives hydrochloric acid and ammonium sulphate, and it forms double salts with certain metallic chlorides and other salts. ${ }^{45}$

ammonium should be understood in this way $\mathrm{NH}_{3}$ gives $\mathrm{NH}_{4} \mathrm{Cl}$ because $\mathrm{NX}_{3}$ is capable of giving $\mathrm{NX}_{5}$. But as saturated compounds-for instance, $\mathrm{SO}_{3} \mathrm{H}_{2} \mathrm{O}, \mathrm{NaCl}$, \&c.-are also capable of combination even between themselves, it is impossible to deny the capacity of $\mathrm{HCl}$ also for combination. $\mathrm{SO}_{3}$ combines with $\mathrm{H}_{2} \mathrm{O}$, and also with $\mathrm{HCl}$ and the unsaturated hydrocarbons. It is impossible to recognise the distinction formerly sought to be established between atomic and molecular compounds, and regarding, for instance, $\mathrm{PCl}_{3}$ as an atomic compound and $\mathrm{PCl}_{5}$ as a molecular one, only because it ensily splits up into molecules $\mathrm{PCl}_{3}$ and $\mathrm{Cl}_{2}$.

44 Sal-ammoniac is prepared from ammonium carbonate, obtained in the dry distillation of nitrogenous substances (Chapter VI.), by saturating the resultant solution with hydrocbloric acid. A solution of sal-ammoniao is thus produced, which is evaporated, and in the residue a mass is obtained containing a mixture of various other, especially terry, products of dry distillation. The sal-ammoniac is generally purified by sublima. tion. For this purpose iron vessels covered with hemispherical metallic covers are employed, or else simply clay crucibles covered by other crucibles. The upper portion, or head, of the apparatus of this kind will have a lower temperature than the lower portion, which is under the direct action of the flame. The sal-ammonia volatilises when heated, and settles on the cooler portion of the apparatus. It is thus freed from many impurities, and is obtained as a crystalline crust, generally several centimetres thick, in which form it is commonly sold. The solubility of sal-ammoniac rises rapidly with the temperature: at $0^{\circ}, 100$ parts of water dissolve about 28 parts of $\mathrm{NH}_{4} \mathrm{Cl}$, at $50^{\circ}$ about 50 parts, and at the ordinary temperature about 85 parts. This is sometimes takeb advantage of for separating $\mathrm{NH}_{4} \mathrm{Cl}$ from solutions of other salts.

45 The solubility of sal-ammoniac in 100 parts of water (according to Alluard) is-

$\begin{array}{ccccccccc}0^{\circ} & 10^{\circ} & 20^{\circ} & 30^{\circ} & 40^{\circ} & 60^{\circ} & 80^{\circ} & 100^{\circ} & 110^{\circ} \\ 28^{\circ} 40 & 32.48 & 87.28 & 41.72 & 46 & 55 & 64 & 73 & 77\end{array}$

A saturated solution boils at $115^{\circ} .8$. The specific gravity at $15^{\circ} / 4^{\circ}$ of solutions of sal. ammoniac (water $\left.4^{\circ}=10,000\right)=9,991 \cdot 6+31 \cdot 26 p-0.085 p^{2}$, where $p$ is the amount by weight of ammonium chloride in 100 parts of solution. With the majority of salts the differential $d s / d p$ increases, but here it decreases with the increase of $p$. For (unlike the sodium and potassium salts) a solution of the alkali plus a solution of acid occupy a greater volume than that of the resultant ammonium salt. In the solution of solid ammonium chloride a contraction, and not expansion, generally takes place. It may further be remarked that solutions of sal-ammoniac have an acid reaction even when prepared from the salt remaining after prolonged washing of the sublimed salt witb water (A. Stcherbakofi). 


\section{CHAPTER XI}

THE HALOGENS CHLORINE, BROMINE, IODINE, AND FLUORINE

Although hydrochloric acid. like water, is one of the most stable substances, it is nevertheless decomposed not only by the action of a galvanic current, ' but also by a high temperature. Sainte-Claire Deville showed that decomposition already occurs at $1,300^{\circ}$, because a cold tube (as with $\mathrm{CO}$, Chapter IX.) covered with an amalgam of silver absorbs chlorine from hydrochloric acid in a red-hot tube, and the escaping gas contains hydrogen. V Meyer and Langer (1885) observed the decomposition of hydrochloric acid at $1,690^{\circ}$ in a platinum vessel , the decomposition in this instance was proved not only from the fact that hydrogen diffused through the platinum (p. 142), owing to which the volume was diminished, but also from chlorine being obtained in the residue (the hydrogen chloride was mixed with nitrogen), which liberated iodine from potassium iodide. ${ }^{2}$ The usual method for the preparation of chlorine consists in the abstraction of the hydrogen by oxidising agents. ${ }^{2}$ is

The decomposition of fused sodium chloride by an electric current has been proposed in America and Russia (N. N. Beketoff) as a means for the preparation of chlorine and sodium, A strong solution of hydrochloric acid is decomposed into equal volumes of chlorine and hydrogen by the action of an electrio current. If sodium chloride and lead be melted in a crucible, the former being connected with the cathode and a carbon anode immersed in the lead, then the lead dissolves sodium and chlorine is disengaged as gas. This electrolytic method has not yet been practised on a large scale, probably because gaseous chlorine has not many applications, and because of the difficulty there is in dealing with it.

To obtain so high a temperature (at which the best kinds of porcelain soften) Langer and Meyer employed the dense graphitoidal carbon from gas retorts, and a powerful blast. They determined the temperature by the alteration of the volume of nitrogen in the platinum vessel, for this gas does not permeate through platinum, and is unaltered by heat.

- us The acid properties of hydrochlorio acld were known when Lavoisier pointed out the formation of acids by the combination of water with the oxides of the non-metals, and therefore there was reason. for thinking that hydrochlorio acid was formed by the combination of water with the oxide of some element. Hence when Scheele obtained chlorine by the action of hydrochlorio acid on manganese peroxide he considered it as the acid contained in common salt. When it became known that chlorine gives hydro. chlorio acid with hydrogen, Liavoisier and Berthollet supposed it to be a compound with oxygen of an enhydride contained in hydrochlorio acid. They suppossd that hydro. 
An aqueous solution of hydrochloric acid is generally employed for the evolution of chlorine. The hydrogen has to be abstracted from the hydrochloric acid. This is accomplished by nearly all oxidising substances, and especially by those which are able to evolve oxygen at a red heat (besides bases, such as mercury and silver oxides, which are able to give salts with hydrogen chloride); for example, manganese peroxide, potassium chlorate, chromic acid, \&c The decomposition essentially consists in the oxygen of the oxidising substance displacing the chlorine from $2 \mathrm{HCl}$. forming water, $\mathrm{H}_{2} \mathrm{O}$, and setting the chlorine free. $2 \mathrm{HCl}+\mathrm{O}$ (disengaged by the oxidising substances) $=\mathrm{H}_{2} \mathrm{O}+\mathrm{Cl}_{2}$. Even nitric acid partially produces a like reaction ; but as we shall afterwards see its action is more complicated, and it is therefore not suitable for the preparation of pure chlorine. ${ }^{3}$ But other oxidising substances which do not give any other volatile products with hydrochloric acid may be employed for the preparation of chlorine. Among these may be mentioned potassium chlorate, acid potassium chromate, sodium manganate, manganese peroxide, \&c. Manganese peroxide is commonly employed in the laboratory, and on a large scale, for the preparation of chlorine. The chemical process in this case may be represented as follows an exchange takes place between $4 \mathrm{HCl}$ and $\mathrm{MnO}_{2}$, in which the manganese takes the place of the four atoms of hydrogen, or the chlorine and oxygen exchange places-that is, $\mathrm{MnCl}_{4}$ and $2 \mathrm{H}_{2} \mathrm{O}$ are produced. The chlorine com. pound, $\mathrm{MnCl}_{4}$, obtained is very unstable, it splits up into chlorine, which as a gas passes from the sphere of action, and a lower compound containing less chlorine than the substance first formed, which remains in the apparatus in which the mixture is heated, $\mathrm{MnCl}_{4}=\mathrm{MnCl}_{2}$ $+\mathrm{Cl}_{2} 3$ bis The action of hydrochloric acid requires a temperature of

chloric acid contained water and the oxide of a particular radicle, and that chlorine was a higher degree of oxidation of this radicle murias (from the Latin name of hydrochlorio acid, acidum muriaticum). It was only in 1811 that Gay-Lussac and Thenard in France and Davy in England arrived at the conclusion that the sulstance obtained by Scheele does not contain oxygen, nor under any conditions give water with hydrogen, and that there is no water in hydronliloric acid gas, and therefore concluded that chlorine is an elementary substance. They named it 'chlorine' from the Greek word $\chi \lambda \omega \rho$ ss, signifying a green colour, because of the peculiar colour by which this gas is chartice terised.

3 However, nitric acid has been proposed as a means for obtaining chlorine, but by methods which have the drawback of being very complicated.

sole This representation of the process of the reaction is the most natural. However, this decomposition is generally represented as if chlorine gave only one degree of combination with manganese, $\mathrm{MnCl}_{2}$, and therefore directly reacts in the following manner$\mathrm{MnO}_{2}+4 \mathrm{HCl}=\mathrm{MnCl}_{2}+2 \mathrm{H}_{2} \mathrm{O}+\mathrm{Cl}_{2}$, in which case it is supposed that manganese peroxide, $\mathrm{MnO}_{2}$, breaks up, as it were, into manganous oxide, $\mathrm{MnO}$, and oxygen, both of which react with hydrochloric acid, the manganous oxide acting upon $\mathrm{HCl}$ as a base, giving $\mathrm{MnCl}_{2}$ and at the same time $2 \mathrm{HCl}+\mathrm{O}=\mathrm{H}_{2} \mathrm{O}+\mathrm{Cl}_{2}$. In reality, a mixture of oxygen 
about $100^{\circ}$ In the laboratory the preparation of chlorme is carried on in flasks, heated over a water-bath, by acting on manganese peroxide

and hydrochloric acid does give chlorine at a red heat, and this reaction may also take place at the moment of its evolution in this case.

All the oxides of manganese $\left(\mathrm{Mn}_{2} \mathrm{O}_{3}, \mathrm{MnO}_{2}, \mathrm{MnO}_{3}, \mathrm{Mn}_{2} \mathrm{O}_{7}\right)$, with the exception of man. gənous oxide, $\mathrm{MnO}$, disengage chlorine from hydrochloric acid, because manganous chloride, $\mathrm{MnCl}_{2}$, is the only compound of chlorine and manganese which exists as a stable compound, all the higher chlorides of manganese being unstable and evolving chlorine. - Hence we here take note of two separatechanges: (1) an exchange between oxygen and chlorine, and (2) the instability of the higher chlorine conipounds. As (according to the law of substitution) in the substitution of oxyger by chlorine, $\mathrm{Cl}_{2}$ takes the place of $\mathrm{O}$, the cllorine compoinds will contain more atoms than the corresponding oxygen compounds. It is not surprising, therefore, that certain of the chlorine compounds corresponding with oxygen compounds do not exist, or if they are formed are very unstable. And furthermore, an atom of chlorine is heavier than an atom of oxygen, and therefore a given element would have to retain a large mass of chlorine if in the higher oxides the oxygen were replaced by chlorine. For this reason equivalent com. pounds of chlorine do not exist for all oxygen compounds. Many of the former are immediately decomposed, when formed, with the evolution of ollorine. From this it is evident that there should exist such chlorine compounds as would evolve chlorine as peroxides evolve oxygen, and indeed a large-number of such compounds are known. Amongst them may be mentioned antimony pentachloride, $\mathrm{SbCl}_{5}$, which splits up into chlorine and antimony trichloride when heated. Cupric chloride, corresponding with copper oxide, and having a composition $\mathrm{CuCl}_{2}$, similar to $\mathrm{CuO}$, when heated parts with half its chlorine, just as bariurn peroxide evolves half its oxygen. This method may even be taken advantage of for the preparation of chlorine and cuprous chloride, $\mathrm{CnCl}$. The latter attracts oxygen from the atmosphere, and in so doing is converted from a colourless substance into a green "compound whose composition is $\mathrm{Cu}_{2} \mathrm{Cl}_{2} \mathrm{O}$. With hydrochloric acid this substarice gives cupric chloride $\left(\mathrm{Cu}_{2} \mathrm{Cl}_{2} \mathrm{O}+2 \mathrm{HCl}=\mathrm{H}_{2} \mathrm{O}+2 \mathrm{CuCl}_{2}\right)$. which has only to be dried and heated in order again to obtain chlorine. Thus, in solution, and at the ordinary temperature, the compound $\mathrm{CuCl}_{2}$ is stable, but when heated it splits up. On this property is founded Deacon's process for the preparation of chlorine from hydrochloric acid with the aid of air and copper salts, by passing a mixture of air and hydrochloric acid at about $440^{\circ}$ over bricks saturated with a solution of a copper salt (a mixture of solutions of $\mathrm{CuSO}_{4}$ and $\mathrm{Na}_{2} \mathrm{SO}_{4}$ ). $\mathrm{CuCl}_{2}$ is then formed by the double decomposition of the salt of copper and the hydrochloric acid; the $\mathrm{CuCl}_{2}$ liberates chlorine, and the $\mathrm{CuCl}$ forms $\mathrm{Cu}_{2} \mathrm{Cl}_{2} \mathrm{O}$ with the oxygen of the air, which again gives $\mathrm{CuCl}_{2}$ with $2 \mathrm{HCl}$, and so on.

Magnesium chloride, which is obtained from sea-water, carnallite, \&c., may serve not. only as a means for the preparation of hydrochloric acid, but also of chlorine, because its basic salt (magriesium oxychloride) when heated in the air gives magnesium oxide and chlorine (Weldon-Pechiney's process, 1888). Chlorine is now prepared on a large scale by this method. Several new methods based upon this reaction have been proposed for procuring chlorine from the bye-products of other chemical processes. Thus, Lyte and Tattars (1891) obtained up to 67 p.c. of chlorine from $\mathrm{CaCl}_{2}$ in this manner. A solu. tion of $\mathrm{CaCl}_{2}$, containing a certain amount of common salt, is evaporated and oxide of magnesium added to it. When the solution attains a density of 1.2445 (at $15^{\circ}$ ), it is treated with carbonio acid, which precipitates carbonate of calcium, while chloride of magnesium remains in solution. After adding ammonium chloride, the solution is evaporated to dryness and the double chloride of magnesium and ammonirm formed is ignited, which drives off the chloride of ammonium. The chloride of magnesium which remains behind is used in the Weldon-Pechiney process. The De Wilde-Reychler (1892) process for the manufacture of chlorine consists in passing alternate currents of hot air and hydrochlorio acid gas through a cylinder containing a mixture of the chlorides of magnesium and manganese. A certain amount of sulphate of magnesium which does 
with hydrochlorio acid or a mixture of common salt and sulphuric acid 4 and washing the gas with water to remove hydrochloric acid." Chlorine cannot be collected over mercury, because it combines with it as with many other metals, and it is soluble in water; however, it is but slightly soluble in hot water or brine. Owing to its great weight, chlorine may be directly collected in a dry vessel by carrying the gas-conducting tube down to the bottom of the vessel. The chlorine will lie in a heavy layer at the bottom of the vessel, displace the air, and the extent to which it fills the vessel may be followed by its colour.

not participate in any way in the reaction, is added to the mixture to prevent its fusing. The reactions may be expressed by the following equations: (1) $8 \mathrm{MgCl}_{2}+8 \mathrm{MnCl}_{2}+80$ $=\mathrm{Mg}_{5} \mathrm{Mn}_{3} \mathrm{O}_{8}+12 \mathrm{Cl}$; (2) $\mathrm{Mg}_{3} \mathrm{Mn}_{5} \mathrm{O}_{3}+16 \mathrm{HCl}=3 \mathrm{MgCl}_{2}+3 \mathrm{MnCl}_{2}+8 \mathrm{H}_{2} \mathrm{O}+4 \mathrm{Cl}$. As nitric acid is able to take up the hydrogen from hydrochlorio acid, a heated mixture of these acids is also employed for the preparation of chlorine. The resultant mixture of chlorine and lower oxides of nitrogen is mixed with air and steam which regenerates the $\mathrm{HNO}_{5}$, while the chlorine remains as a gas together with nitrogen, in which form it is quite capable of bleaching, forming chloride of lime, \&c Besides these, Solvay and Mond's methods of preparing chlorine must be mentioned. The first is based upon the reaction $\mathrm{CaCl}_{2}+\mathrm{SiO}_{2}+\mathrm{O}$ (air) $=\mathrm{CaOSiO}_{2}+\mathrm{Cl}_{2}$, the second on the action of the oxygen of the air (heated) upon $\mathrm{MgCl}_{2}$ (and certain similar chlorides) $\mathrm{MgCl}_{2}+\mathrm{O}=\mathrm{MgO}+\mathrm{Cl}_{2}$. The remaining $\mathrm{MgO}$ is treated with sal-ammoniac to re-form $\mathrm{MgCl}_{2}\left(\mathrm{MgO}+2 \mathrm{NH}_{4} \mathrm{Cl}=\mathrm{MgCl}_{2}+\mathrm{H}_{2} \mathrm{O}+2 \mathrm{NH}_{3}\right)$ and the resultant $\mathrm{NH}_{5}$ again converted into sal-ammoniac, so that hydrochloric acid is the only substance cousumed The latter processes have not yet found much application.

- The following proportions are accordingly taken by weight: 5 parte of powdered man. ganese peroxide, 11 parts of salt (best fused, to prevent its frothing), and 14 parts of sul. phuric acid previously mixed with an equal volume of water The mixture is heated in a salt bath, so as to obtain a temperature above $100^{\circ}$ The corks in the apparatus must be soaked in paraffin (otherwise they are corroded by the chlorine), and black india-rubber tubing smeared with vaseline must be used, and not vulcanised rubber (which contains sulphur, and becomes brittle under the action of the chlorine).

The reaction which proceeds may be expressed thus. $\mathrm{MnO}_{2}+2 \mathrm{NaCl}+2 \mathrm{H}_{2} \mathrm{SO}_{4}$ $=\mathrm{MnSO}_{4}+\mathrm{Na}_{2} \mathrm{SO}_{4}+2 \mathrm{H}_{2} \mathrm{O}+\mathrm{Cl}_{2}$. The method of preparation of $\mathrm{Cl}_{2}$ from manganese peroxide and hydrochloric acid was discovered by Scheele, and from sodium chloride by Berthollet.

3 The reaction of hydrochlorio acid upon bleaching powder gives chlorine without the aid of heat, $\mathrm{CaCl}_{2} \mathrm{O}_{2}+4 \mathrm{HCl}=\mathrm{CaCl}_{2}+2 \mathrm{H}_{2} \mathrm{O}+2 \mathrm{Cl}_{2}$, and is therefore also used for the preparation of chlorine. This reaction is very violent if all the acid be added at once; it should be poured in drop by drop (Mermé, Kämmerer). C. Winkler proposed to mix bleaching powder with one quarter of burnt and powdered gypsum, and having damped the mixture with water, to press and cut it up into cubes and dry at the ordinary temperature. These cubes can be used for the preparation of chlorine in the $\operatorname{sam} \theta$ apparatus as that used for the evolution of hydrogen and carbonio anhydride-the disengagement of the chlorine proceeds uniformly.

A misture of potassium dichromate and hydrochloric acid evolves chlorine perfectly tree from oxygen ( $V$. Meyer and Langer).

6 Chlorine is manufactnred on a large scale from manganese peroxide and hydrochlorio acid. It is most conveniently prepared in the apparatus shown in fig. 66, which con. eists of a three-necked earthenware vèssel whose central orifice is the largest. A clay or lead funnel, furnished with a number of orifices, is placed in the central wide neck of the vessel. Roughly-ground lumps of natural mangnnese peroxide are placed in the funnel, which is then closed by the cover $\mathrm{N}$, and luted with clay. One orifice is closed 
Chlorine is a gas of a yellowish green colour, and has a very suffocating and characteristic odour. On lowering the temperature to $-50^{\circ}$ or increasing the pressure to six atmospheres (at $0^{\circ}$ ) chlorine condenses ${ }^{7}$ into a liquid which has a yellowish-green colour, a density of $1 \cdot 3$, and boils at $-34^{\circ}$ The density and atomic weight of chlorine is 35.5 times greater than that of hydrogen, hence the molecule contains $\mathrm{Cl}_{2}{ }^{8}$ At $0^{\circ}$ one volume of water dissolves about $1 \frac{1}{2}$ volume of chlorine, at $10^{\circ}$ about 3 volumes, at $50^{\circ}$ again $1 \frac{1}{2}$ volume. ${ }^{9}$ Such

by a clay stopper, and is used for the introduction of the hydrochloric acid and withdrawal of the residues. The chlorine disengaged passes along a leaden gase conducting tube placed in the other orifice. A row of these vessels is surrounded by a water-bath to ensure their being uniformly heated. Manganese chloride is found in the residue. In Weldon's process lime is added to the acid solution of manganese chloride. A double decomposition takes place, resulting in the formation of manganous hydroxide and calcium chloride. When the insoluble manganous hydroxide has settled, a further excess of milk of lime is added (to make a mixture $2 \mathrm{Mn}(\mathrm{OH})_{2}+\mathrm{CaO}+x \mathrm{CaCl}_{2}$, which is found to be the best propor. tion, judging from experiment), and then air is forced through the mixtare. The hydroxide is thus converted from a colourless to a brown substance, containing peroxide, $\mathrm{MnO}_{2}$, and oxide of manganese, $\mathrm{Mn}_{2} \mathrm{O}_{3}$. This is due to the manganous oxide absorb-

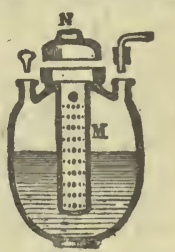

Eia. 66.-Clay retort for the preparation of chlorine on a large scsle.

ing oxygen from the air. Under the action of hydrochloric acid this mixture evolves chlorine, because of all the compounds of chlorine and manganese the chloride $\mathrm{MnCl}_{2}$ is the only one which is stable (see Note 8). Thus one and the same mass of manganese may be repeatedly used for the preparation of chlorine. The same result is attained in other ways If manganous oxide be subjected to the action of oxides of nitrogen and air (Coleman's process), then manganese nitrate is formed, which at a red heat gives oxides of nitrogen (which are again used in the process) and manganese peroxide, which is thus renewed for the fresh evolntion of chlorivio.

Davy and Faraday liquefied chlorine in 1823 by heating the crystallo-hydrate $\mathrm{Cl}_{2} 8 \mathrm{H}_{2} \mathrm{O}$ in a bent tube (as with $\mathrm{NH}_{3}$ ), surrounded by warm water, while the other end of the tube was immersed in a freezing mixture. Meselan condensed chlorine in freshly-burnt charcoal (placed in a glass tube), which when cold absorbs an equal weight of chlorine. The tube was then fused up, the bent end cooled, and the charcoal heated, by which means the chlorine was expelled from the charcoal, and the pressure increased.

8 Judging from Ludwig's observations (1868), and from the fact that the coefficient of expansion of gases increases with their molecular weight (Chapter II., Note 26, for hydrogen $=0.867$, carbonic anhydride $=0.378$, hydrogen bromide $=0.886$ ), it might be expected that the expansion of chlorine would be greater than that of air or of the gases composing it. V. Meyer and Langer (1885) having remarked that at $1,400^{\circ}$ the density of chlorine (taking its expansion as equal to that of nitrogen) $=29$, consider that the molecules of chlorine oplit up and partially give molecules $\mathrm{Cl}$, but it might be maintained that the decrease in density observed only depends on the increase of the coefficient of expansion.

9 Investigations on the solubility of chlorine in water (the solutions evolve all their chlorine on boiling and passing air through them) show many different peculiarities. First Gay-Lussac, and subsequently Pelouze, determined that the solubility increases between $0^{\circ}$ and $8^{\circ}-10^{\circ}$ (from $1 \frac{1}{8}$ to 2 vols. of chlorine per 100 vols. of water at $0^{\circ}$ up to 8 to $2 \frac{3}{4}$ at $10^{\circ}$ ). In the following note we shall 'see that this is not due to the breaking-up of the hydrate at abont $8^{\circ}$ to $10^{\circ}$, but to its formation below $9^{\circ}$. Roscoe observed an increase in the solu. bility of chlorine in the presence of hydrogen-even in the dark. Berthelot determined 
a solution of chlorine is termed "chlorine water ;" and is employed in a diluted form in medicine and as a laboratory reagent. It is prepared by passing chlorine through a series of Woulfe's bottles or into an inverted retort filled with water. Under the action of light, chlorine water gives oxygen and hydrochloric acid. At $0^{\circ}$ a saturated solution of chlorine yields a crystallo-hydrate, $\mathrm{Cl}_{2}, 8 \mathrm{H}_{2} \mathrm{O}$, which easily splits up into chlorine and water when heated, so that if it be sealed up in a tube and heated to $35^{\circ}$, two layers of liquid are formed-a lower stratum of chlorine containing a small quantity of water, and an upper stratum of water containing a small quantity of chlorine..$^{10}$

Chlorine explodes with hydrogen, if a mixture of equal volumes be exposed to the direct action of the sun's rays " or brought into contact

an increase of solubility with the progress of time. Schönbein and others suppose thao chlorine acts on water, forming hypochlorous and hypochloric acids, $(\mathrm{HClO}+\mathrm{HCl})$

The equilibrium between chlorine and steam as gases and between water, liquid chlorine, ice, and the solid crystallo-hydrate of chlorine is evidently very complex. Gibbs, Guldberg (1870) and others gave a theory for similar states of equilibrium, which was afteswards developed by Roozeboon (1887), but it would be inopportune here to enter into its details. It will be sufficient in the first place to mention that there is now no doubt (according to the theory of heat, and the direct observations of Ramsay and Young) that the vapour tensions at one and the same temperature are different for the liquid and solid states of substances; secondly, to call attention to the following note; and, thirdly, to state that, in the presence of the crystallo-hydrate, water between $0^{\circ} .24$ and $+28^{\circ} \cdot 7$ (when the hydrate and a solution may occur simultaneously) dissolves a different amount of chlorine than it does in the absence of the crystallo-hydrate.

10 According to Faraday's data the hydrate of chlorine contains $\mathrm{Cl}_{2}, \mathrm{10H}_{2} \mathrm{O}$, but Rooze. boom (1885) showed that it is poorer in water and $=\mathrm{Cl}_{2}, 8 \mathrm{H}_{2} \mathrm{O}$. At first small, almost colourless, crystals are obtained, but they gradually form (if the temperature be below their critical point $28^{\circ} \cdot 7$, ibbove which they do not exist) large yellow crystals, like those of potassium chromate. The specific gravity is $1 \cdot 23$. The hydrate is formed if there be more chlorine in a solution than it is able to dissolve under the dissociation pressure corresponding with a given temperature. In the presence of the hydrate the percentage amount of chlorine at $0^{\circ}=0.5$, at $9^{\circ}=0.9$, and at $20^{\circ}=1.82$. At temperatures below $9^{\circ}$ the solubility (determined by Gry-Lussac and Pelouze, see Note 9 ) is dependent on the formation of the hydrate; whilst at higher temperatures under the ordinary pressure the hydrate cannot be formed, and the solubility of chlorine falls, as it does for all gases (Chapter I.). If the crystallo-hydrate is not formed, then below $9^{\circ}$ the solubility follows the same rule $\left(6^{\circ} 1.07\right.$ p.c. $\mathrm{Cl}, 9^{\circ} 0.95$ p.c.). According to Roozeboom, the chlorine evolved by the hydrate presents the following tensions of dissociation: at $0^{\circ}=249 \mathrm{~mm}$., at $4^{\circ}=398$, at $8^{\circ}=620$, at $10^{\circ}=797$, at $14^{\circ}=1,400 \mathrm{~mm}$. In this case a portion of the crystallo-hydrate remains solid. At $9^{\circ} \cdot 6$ the tension of dissociation is equal to the atmospheric pressure. At a higher pressure the crystallo-hydrate may form at temperatures above $9^{\circ}$ up to $28^{\circ} .7$, when the vapour tension of the hydrate equals the tension of the chlorine. It is evident that the equilibrium which is established is on the one hand a case of a complex heterogeneous system, and on the other hand a case of the solution of solid and gaseous substances in water.

The crystallo-hydrate or chlorine water must be kept in the dark, or the access of light be prevented by coloured glass, otherwise oxygen is evolved and hydrochlorio acid formed.

11 The chemical action of light on a mixture of chlorine and hydrogen was discovered by Gay-Iussac and Thénard (1809). It has been investigated by many savants, and especially 
with spongy platinum, or a strongly heated substance, or when subjected to the action of an electric spark. The explosion in this case takes place for exactly the same reasons-i.e. the evolution of heat and expansion of the resultant product-as in the case of detonating gas (Chapter III:) Diffused light acts in the same way, but slowly, whilst direct sunlight causes an explosion. ${ }^{12}$ The hydrochloric acid gas produced by the

by Draper, Bunsen, and Roscoe. Electric or magnesium light, or the light emitted by the combustion of carbon bisulphide in nitric oxide, and actinic light in general, acts in the same manner as sunlight, in proportion to its intensity. At temperatures below $-12{ }^{\circ}$ light no longer brings about reaction, or at all events does not give an explosion. It was long supposed that chlorine that had been subjected to the aotion of light was afterwards able to act on hydrogen in the dark, but it was shown that this only takes place with moist chlorine, and depends on the formation of oxides of chlorine. The presence of foreign gases, and even of excess of chlorine or of hydrogen, very much enfeebles the explosion, and therefore the experiment is conducted with a detonating mixture prepared by the action of an electric current on a strong solution (sp. gr. 1.15) of hydro. chloric acid, in which case the water is not decomposed-that is, no oxygen becomes mixed with the chlorine.

12 The quantity of chlorine and hydrogen which combine is proportional to the intensity of the light-not of all the rays, but only those so-termed chemical (actinic) rays which produce chemical action. Hence a mixture of chlorine and hydrogen, when exposed to the action of light in vessels of known capacity and surface, may be employed as an actino. meter-that is, as a means for estimating the intensity of the chemical rays, the infuence of the heat rays being previously destroyed, which may be done by passing the rays through water. Investigations of this kind (photo-chemical) showed that chemical action is chiefly limited to the violet ond of the spectrum, and that even the invisible pltra-violet rays produce this action. A colourless gas flame contains no chemically active rays; the flame coloured green by a salt of copper evinces more chemical action than the colourless flame, but the flame brightly coloured yellow by salts of sodium has no more chemical action then that of the colourless flame.

As the chemical action of light becomes evident in plants, photography, the bleaching of tissues, and the fading of colours in the sunlight, and as a means for studying the phenomenon is given in the reaction of chlorine on hydrogen, this subject has been the most fully investigated in photo-chemistry. The researches of Bunsen and Roscoe in the fifties and sixties are the most complete in this respect. Their actinometer contains hydrogen and chlorine, and is surrounded by a solution of chlorine in water. The hydro. chloric acid is absorbed as it forms, and therefore the variation in volume indicates the progress of the combination. AB was to be expected, the action of light proved to be proportional to the time of exposure and intensity of the light, so that it was possible to conduct.detailed photometrical investigations respecting the time of day and season of the year, various sources of light, its absorption, \&c. This subject is considered in detail in special works, and we only stop to mention one circumstance, that a small quantity of a foreign gas decreases the action of light; for example, $\frac{3}{380}$ of hydrogen by 38 p.c., होo of oxygen by 10 p.c., rfo of chlorine by 60 p.c., \&c. According to the researches of Klimenko and Pekatoros (1889), the photo-chemical alteration of chlorine water is retarded by the presence of traces of metallic chlorides, and this influence varies with different metals.

As much heat is evolved in the reaction of chlorine on hydrogen, and as this reaction, being exothermal, mas proceed by itself, the action of light is essentially the came as that of heat-that is, it brings the chlorine and hydrogen into the condition necessary for the reaction-it, es we may say, disturbs the original equilibrium; this is the work done by the luminous energy. It seems to me that the action of light on the mired gases should bo understood in this sense, as Pringsheim (1877) pointed out. 
reaction of chlorine on hydrogen oćcupies (at the original temperature and pressure) a volume equal to the sum of the original volumes, that is, a reaction of substitution here takes place $\mathrm{H}_{2}+\mathrm{Cl}_{2}=\mathrm{HCl}+\mathrm{HCl}$ In this reaction twenty-two thousand heat units are evolved for one part by weight [ 1 gram] of hydrogen. ${ }^{13}$

These relations show that the affinity of chlorine for hydrogen is very great and analogous to the affinity between hydrogen and oxygen. Thus ${ }^{14}$ on the one hand by passing a mixture of steam and chlorine through a red-hot tube, or by exposing water and chlorine to the sunlight, oxygen is disengaged, whilst on the other hand, as we saw above, oxygen in many cases displaces chlorine from its compound with hydrogen, and therefore the reaction $\mathrm{H}_{2} \mathrm{O}+\mathrm{Cl}_{2}=2 \mathrm{HCl}+\mathrm{O}$ belongs to the number of reversible reactions, and hydrogen will distribute itself between oxygen and chlorine. This determines the relation of $\mathrm{Cl}$ to substances containing hydrogen and its reactions in the presence of water, to which we shall turn our attention after having pointed out the relation of chlorine to other elements.

Many metals when brought into contact with chlorine immediately combine with it, and form those metallic chlorides which correspond with hydrogen chloride and with the oxide of the metal taken. This combination may proceed rapidly with the evolution of heat and light; that is, metals are able to burn in chlorine. Thus, for example, sodium ${ }^{15}$ burns in chlorine, synthesising common salt. Metals in the form of powders burn without the aid of heat, and become highly incandescent in the process ; for instance, antimony, which is a metal easily converted into a powder. ${ }^{16}$ Even such metals as gold and

13 In the formation of steam (from one part by weight [ 1 gram] of hydrogen) 29,000 heat units are evolved. The following are the quantities of heat (thousands of units) evolved in the formation of various other corresponding compounds of oxygen and of chlorine (from Thomsen's, and, for $\mathrm{Na}_{2} \mathrm{O}$, Beketoff's results)

$\left\{\begin{array}{rrrl}2 \mathrm{NaCl}, 195 ; & \mathrm{CrCl}_{3}, 170 ; & \mathrm{HgCl}_{2}, 68 ; & 2 \mathrm{AgCl}, 59 . \\ \mathrm{Na}_{2} \mathrm{O}, 100 ; & \mathrm{CaO}, 131 ; & \mathrm{HgO}_{4}, 42 ; & \mathrm{Ag}_{2} \mathrm{O}, 6 . \\ 2 \mathrm{CaCl}_{3}, 143 ; & 2 \mathrm{PCl}_{3}, 210 ; & \mathrm{CCl}_{4}, 21 ; & 2 \mathrm{HCl}, 44 \text { (gas). } \\ \mathrm{As}_{2} \mathrm{O}_{5}, 155 ; & \mathrm{P}_{3} \mathrm{O}_{3}, 870 ; & \mathrm{CO}_{2}, 97 ; & \mathrm{H}_{2} \mathrm{O}, 58 \text { (g\&). }\end{array}\right.$

With the first four elements the formation of the chlorine compound gives the most heat, and with the four following the formation of the oxygen compound evolves the greater amount of heat. The first four chlorides are true salts formed from $\mathrm{HCl}$ and the oxide, whilst the remainder have other properties, as is seen from the fact that they are not formed from hydrochloric acid and the oxide, but give hydrochloric acid with water.

14 This has been already pointed out in Clapter III., Note 5.

1s Sodium remains unaltered in perfectly dry chlorine at the ordinary temperature, and even when slightly warmed; but the combination is exceedingly violent at a red heat

16 An instructive experiment on combustion in chlorine may be conducted as follows: leaves of Dutch metal (used instead of gold for gilding) are placed in a glass globe, and a 
platinum ${ }_{2}^{17}$ which do not combine directly with oxygen and give very. unstable compounds with it, unite directly with chlorine to form metallic chlorides. Either chlorine water or aqua regia may be employed for this purpose instead of gaseous chlorine. These dissolve gold and platinum, converting them into metallic chlorides. Aqua regia is a mixture of 1 part of nitric acid with 2 to 3 parts of hydrochloric acid. This mixture converts into soluble chlorides not only those metals which are acted on by hydrochloric and nitric acids, but also gold and platinum, which are insoluble in either acid separately. This action of aqua regia depends on the fact that nitric acid in acting on hydrochloric acid evolves chlorine. If the chlorine evolved be transferred to a metal, then a fresh quantity is formed from the remaining acids and also combines with the metal. ${ }^{18}$ Thus the aqua regia acts by virtue of the chlorine which it contains and disengages.

The majority of non-metals also react directly on chlorine; hot sulphur and phosphorus burn in it and combine with it at the ordinary temperature. Only nitrogen, carbon, and oxygen do not combine directly with it. The chlorine compounds formed by the non-metalsfor instance, phosphorus trichloride, $\mathrm{PCl}_{3}$, and sulphurous chloride, \&c., do not have the properties of salts, and, as we shall afterwards see more fully, correspond to acid anhydrides and acids ; for examiple, $\mathrm{PCl}_{3}$ -to phosphorous acid, $\mathrm{P}(\mathrm{OH})_{3}$

$$
\begin{array}{lllll}
\mathrm{NaCl} & \mathrm{FeCl}_{2} & \mathrm{SnCl}_{4} & \mathrm{PCl}_{3} & \mathrm{HCl} \\
\mathrm{Na}(\mathrm{HO}) & \mathrm{Fe}(\mathrm{HO})_{2} & \mathrm{Sn}(\mathrm{HO})_{4} & \mathrm{P}(\mathrm{HO})_{3} & \mathrm{H}(\mathrm{HO})
\end{array}
$$

gas-conducting tube furnished with a glass cock is placed in the cork closing it, and the air is pumped out of the globe. The gas-conducting tube is then connected with a vessel containing chlorine, and the cock opened; the chlorine rushes in, and the metallic leaves are consumed.

17 The behaviour of flatinum to chlorine at a high temperature $\left(1,400^{\circ}\right)$ is very remarkable, because platinous chloride, $\mathrm{PtCl}_{2}$, is then formed, whilst this substance de. composes at a much lower temperature into chlorine and platinum. Hence, when chlorine comes into contact with platinum at such high temperatures, it forms fumes of platinous chloride, and they on cooling decompose, with the liberation of platinum, so that the phenomenon appears to be dependent on the volatility of platinum. Deville proved the formation of platinous chloride by inserting a cold tube inside a red-hot one (as in the experiment on carbonic oxide). However, V. Meyer was able to observe the density of chlorine in a platinum vessel at $1,690^{\circ}$, at which temperature chlorine does not exert this action on platinum, or at least only to an insignificant degree.

18 When left exposed to the air aqua regia disengages chlorine, and afterwards it no longer acts on gold. Gay-Lussac, in explaining the action of aqua regia, showed that when heated it evolves, besides chlorine, the vapours of two chloranhydrides-that of nitric acid, $\mathrm{NO}_{2} \mathrm{Cl}$ (nitric acid, $\mathrm{NO}_{2} \mathrm{OH}$, in which $\mathrm{HO}$ is replaced by chlorine; see Chapter on Phosphorus), and that of nitrous acid, $\mathrm{NOCl}$-but these do not aot on gold. The formation of aqua regia may therefore be expressed by $4 \mathrm{NHO}_{3}+8 \mathrm{HCl}=2 \mathrm{NO}_{2} \mathrm{Cl}+2 \mathrm{NOCl}$ $+6 \mathrm{H}_{2} \mathrm{O}+2 \mathrm{Cl}_{2}$. The formation of the chlorides $\mathrm{NO}_{2} \mathrm{Cl}$ and $\mathrm{NOCl}$ is explained by the fact that the nitrio acid is deoxidised, gires the oxides $\mathrm{NO}$ and $\mathrm{NO}_{2}$, and they directly combink with chlorine to form the sbove anhydrides. 
As the above-mentioned relation in composition-i.e. substitution of $\mathrm{Cl}$ by the aqueous residue-exists between many chlorine compounds and their corresponding hydrates, and as furthermore some (acid) hydrates are obtained from chlorine compounds by the action of water, for instance,

$$
\underset{\substack{\text { Phosphorus } \\
\text { trichloride }}}{\mathrm{PCl}_{3}}+\underset{\text { Wates }}{3 \mathrm{H}_{2} \mathrm{O}}=\underset{\begin{array}{c}
\text { Phosphorous } \\
\text { acid }
\end{array}}{\mathrm{P}(\mathrm{HO})_{3}}+\underset{\begin{array}{c}
\text { Hydrochlorio } \\
\text { acid }
\end{array}}{3 \mathrm{HCl}}
$$

whilst other chlorine compounds are formed from hydroxides and hydrochloric acid, with the liberation of water, for example,

$$
\mathrm{NaHO}+\mathrm{HCl}=\mathrm{NaCl}+\mathrm{H}_{2} \mathrm{O}
$$

we endeavour to express this intimate connection between the hydrates and chlorine compounds by calling the latter chloranhydrides. In general terms, if the hydrate be basic, then,

$\underset{\text { hydrate }}{\mathrm{M}(\mathrm{HO})}+\underset{\text { hydrochloric acid }}{\mathrm{HCl}}=\underset{\text { chloranhydride }}{\mathrm{MCl}}+\underset{\text { water }}{\mathrm{H}_{2} \mathrm{O}}$

and if the hydrate $\mathrm{ROH}$ be acid. then,

$$
\begin{array}{cl}
\mathrm{RCl}+\mathrm{H}_{2} \mathrm{O} & =\mathrm{R}(\mathrm{HO})+\underset{\mathrm{HCl}}{\text { hydrate }}+\text { hydrochloric acid }
\end{array}
$$

The chloranhydrides $\mathrm{MCl}$ corresponding to the bases are evidently metallic chlorides or salts corresponding to $\mathrm{HCl}$. In this manner a distinct equivalency is marked between the compounds of chlorine and the so-called hydroxyl radicle $(\mathrm{HO})$, which is also expressed in the analogy existing between chlorine, $\mathrm{Cl}_{2}$, and hydrogen peroxide, $(\mathrm{HO})_{2}$.

As regards the chloranhydrides corresponding to acids and nonmetals, they bear but little resemblance to metallic salts. They are nearly all volatile, and have a powerful suffocating smell which irritates the eyes and respiratory organs. They react on water like many anhydrides of the acids, with the evolution of heat and liberation of. hydrochloric acid, forming acid hydrates. For this reason they cannot usually be obtained from hydrates-that is, acids-by the action of hydrochloric acid, as in that case water would be formed together with them, and water decomposes them, converting them into hydrates. There are many intermediate chlorine compounds between true saline metallio chlorides like sodium chloride and true acid chloranhydrides, just as there are all kinds of transitions between bases and acids. Acid chloranhydrides are not only obtained from chlorine and non-metals, but also from many lower oxides, by the aid of chlorine. Thus, for example, $\mathrm{CO}, \mathrm{NO}, \mathrm{NO}_{2}, \mathrm{SO}_{2}$, and other lower oxides which are capable of combining with oxygen may also combine with a corre. 
sponding quantity of chlorine. Thus $\mathrm{COCl}_{2}, \mathrm{NOCl}, \mathrm{NO}_{2} \mathrm{Cl}, \mathrm{SO}_{2} \mathrm{Cl}_{2}$, ic., are obtained. They correspond with the hydrates $\mathrm{CO}(\mathrm{OH})_{2}$, $\mathrm{NO}(\mathrm{OH}), \mathrm{NO}_{2}(\mathrm{OH}), \mathrm{SO}_{2}(\mathrm{OH})_{2}$, \&c., and to the anhyhrides $\mathrm{CO}_{2}$, $\mathrm{N}_{2} \mathrm{O}_{3}, \mathrm{~N}_{2} \mathrm{O}_{5}, \mathrm{SO}_{3}$, \&c. Here we should notice two aspects of the matter : (1) chlorine combines with that with which oxygen is able to combine, because it is in many respects equally if not more energetic than oxygen and replaces it in the proportion $\mathrm{Cl}^{2}: \mathrm{O}$; (2) that highest limit of possible combination which is proper to a given element or grouping of elements is very easily and often attained by combination with chlorine. If phosphorus gives $\mathrm{PCl}_{3}$ and $\mathrm{PCl}_{5}$, it is evident that $\mathrm{PCl}_{5}$ is the higher form of combination compared with $\mathrm{PCl}_{3}$. To the form $\mathrm{PCl}_{5}$, or in general $\mathrm{PX}_{5}$, correspond $\mathrm{PH}_{4} \mathrm{I}, \mathrm{PO}(\mathrm{OH})_{3}, \mathrm{POCl}_{3}$, \&c. If chlorine does not always directly give compounds of the highest possible forms for a given element, then generally the lower forms combine with it in order to reach or approach the limit. This is particularly clear in hydrocarbons, where we see the limit $\mathrm{C}_{n} \mathrm{H}_{2 n+2}$ very distinctly. The unsaturated hydrocarbons are sometimes able to combine with chlorine with the greatest.ease and thus reach the limit. Thus ethylene, $\mathrm{C}_{2} \mathrm{H}_{4}$, combines with $\mathrm{Cl}_{2}$, forming the so-called Dutch liquid or ethylene chloride, $\mathrm{C}_{2} \mathrm{H}_{4} \mathrm{Cl}_{2}$, because it then reaches the limit $\mathrm{C}_{n} \mathrm{X}_{2 n+2^{2}}$. In this and all similar cases the combined chlorine is able by reactions of substitution to give a hydroxide and a whole series of other derivatives. Thus a hydroxide called glycol, $\mathrm{C}_{2} \mathrm{H}_{4}(\mathrm{OH})_{2}$, is obtained from $\mathrm{C}_{2} \mathrm{H}_{4} \mathrm{Cl}_{2}$.

Chlorine in the presence of water very often acts directly as an oxidising agent. A substance A combines with chlorine and gives, for example, $\mathrm{ACl}_{2}$, and this in turn a hydroxide, $\mathrm{A}(\mathrm{OH})_{2}$, which on losing water forms AO. Here the chlorine has oxidised the substance A. This frequently happens in the simultaneous action of water and chlorine : $\mathrm{A}+\mathrm{H}_{2} \mathrm{O}+\mathrm{Cl}_{2}=2 \mathrm{HCl}+\mathrm{AO}$. Examples of this oxidising action of chlorine may frequently be observed both in practical chemistry and technical processes. Thus, for instance, chlorine in the presence of water oxidises sulphur and metallic sulphides. In this case the sulphur is converted into sulphuric acid, and the chlorine into hydrochloric acid, or a metallic chloride if a metallic sulphide be taken. A mixture of carbonic oxide and chlorine passed into water gives carbonic anhydride and hydrochloric acid. Sulphurous anhydride is oxidised by chlorine in the presence of water into sulphuric acid, just as it is by the action of nitric acid: $\mathrm{SO}_{2}+2 \mathrm{H}_{2} \mathrm{O}+\mathrm{Cl}_{2}=\mathrm{H}_{2} \mathrm{SO}_{4}+2 \mathrm{HCl}$.

The oxidising action of chlorine in the presence of water is taken advantage of in practice for the rapid bleaching of tissues and fibres. The colouring matter of the fibres is altered by oxidation and con. 
verted into a colourless substance, but the chlorine afterwards acts on the tissue itself. Bleaching by means of chlorine therefore requires a certain amount of technical skill in order that the chlorine should not act on the fibres themselves, but that its action should be limited to the colouring matter only The fibre for making writing paper, for instance, is bleached in this manner. The bleaching property of chlorine was discovered by Berthollet, and forms an important acquisition to the arts, because it has in the majority of cases replaced that which before was the universal method of bleaching-namely, exposure to the sun of the fabrics damped with water, which is still employed for linens, \&c. Time and great trouble, and therefore money also, have been considerably saved by this change. ${ }^{19}$

The power of chlorine for combination is intimately connected with its capacity for substitution, because, according to the law of substitution, if chlorine combines with hydrogen, then it also replaces hydrogen, and furthermore the combination and substitution are accomplished in the same quantities. Therefore the atom of chlorine which combines with the atom of hydrogen is also able to replace the atom of hydrogen. We mention this property of chlorine not only because it illustrates the application of the law of substitution in clear and historically important examples, but more especially because reactions of this kind explain those indirect methods of the formation of many substances which we have often mentioned and to which recourse is had in many cases in chemistry. Thus chlorine does not act on carbon, ${ }^{20}$ oxygen, or nitrogen, but nevertheless its compounds with these elements may be obtained by the indirect method of the substitution of hydrogen by chlorine.

As chlorine easily combines with hydrogen, and does not act on carbon, it decomposes hydrocarbons (and many of their derivatives) at a high temperature, depriving them of their hydrogen and liberating the carbon, as, for example, is clearly seen when a lighted candle is placed in a vessel containing chlorine. The flame becomes smaller, but

19 Ozone and peroxide of hydrogen also bleach tissues. As the action of peroxide of hydrogen is easily controlled by taking a weak solution, and as it has hardly any action upon the tissues themselves, it is replacing chlorine more and more as a bleaching agent. The oxidising property of chlorine is apparent in destroying the majority of organic tissues, and proves fatal to organisms. This action of chlorine is taken advantage of in quarantine stations. But the simple fumigation by chlorine must be "carried on with great care in dwelling places, because chlorine disengaged into the atmosphere renders it harmful to the health.

20 A certain propensity of carbon to attract chlorine is evidenced in the immense absorption of chlorine by charcoal (Note 7), but, so far as is at present known (if I am not mistaken, no one has tried the aid of light), no combination takes place between the chlorine and carbon. 
continues to burn for a certain time, a large amount of soot is obtained, and hydrochloric acid is formed. In this case the gaseous and incan. descent substances of the flame are decomposed by the chlorine, the hydrogen combines with it, and the carbon is disengaged as soot. ${ }^{21}$ This action of chlorine on hydrocarbons, \&c., proceeds otherwise at lower temperatures, as we will now consider.

A very important epoch in the history of chemistry was inaugurated by the discovery of Dumas and Laurent that chlorine is able to displace and replace hydrogen. This discovery is important from the fact that chlorine proved to be an element which combines with great ease simultaneously with both the hydrogen and the element with which the hydrogen was combined. This clearly proved that there is no' opposite polarity between elements forming stable compounds. Chlorine does not combine with hydrogen because it has opposite properties, as Dumas and Laurent stated previously, accounting hydrogen to be electro-positive and chlorine electro-negative ; this is not the reason of their combining together, for the same chlorine which combines with hydrogen is also able to replace it without altering many of the properties of the resultant substance. This substitution of hydrogen by chlorine is termed metalepsis. The mechanism of this substitution is very constant. If we take a hydrogen compound, preferably a hydrocarbon, and if chlorine acts directly on it, then there is produced on the one hand hydrochloric acid and on the other hand a compound containing chlorine in the place of the hydrogen-so that the chlorine divides itself into two equal portions, one portion is evolved as hydrochloric acid, and the other portion takes the place of the hydrogen thus liberated. Hence this metalepsis is always accompanied by the formation of hydrochloric acid. ${ }^{22}$ The scheme of the process is as follows :

$$
\underset{\text { Hydrocarbon }}{\mathrm{C}_{n} \mathrm{H}_{m} \mathrm{X}}+\underset{\text { Free chlorine }}{\mathrm{Cl}_{2}}=\underset{\text { Product of metalepsis }}{\mathrm{C}_{n} \mathrm{H}_{m-1} \mathrm{ClX}}+\underset{\text { Hydrochloric acid }}{\mathrm{HCl}}
$$

Or, in general terms-

$$
\mathrm{RH}+\mathrm{Cl}_{2},=\mathrm{RCl}+\mathrm{HCl} \text {. }
$$

The conditions under which metalepsis takes place are also very constant. In the dark chlorine does not usually act on hydrogen com-

21 The same reaction takes place under the action of oxygen, with the difference that it burns the carbon, which chlorine is not able to do. If chlorine and oxy gen compete together at a high temperature, the oxygen will unite with the carbon, and the chlorine with the hydrogen.

22 This division of chlorine into two portions may at the same time be taken as a clear confirmation of the conception of molecules. According to Avogadro-Gerhardt's law, the molecule of ohlorine (p. 310 ) contains two atoms of this substance; one atom replaces hydrogen, and the other combines with it. 
pounds, but the action commences under the influence of light. The divect action of the sun's rays is particularly propitious to metalepsis. It is also remarkable that the presence of traces of certain substances, ${ }^{23}$ especially of iodine, aluminium chloride, antimony chloride, \&c., promotes the action. A trace of iodine added to the substance subjected to metalepsis often produces the same effect as sunlight. ${ }^{24}$

If marsh gas be mixed with chlorine and the mixture ignited, then the hydrogen is entirely taken up from the marsh gas and hydrochlorio acid and carbon formed, but there is no metalepsis. ${ }^{25}$ But if a mixture of equal volumes of chlorine and marsh gas be exposed to the action of diffused light, then the greenish yellow mixture gradually becomes colourless, and hydrochloric acid and the first product of metalepsis-namely, methyl chloride-are formed

$\underset{\text { Marsh gaa }}{\mathrm{CH}_{4}}+\underset{\text { Chlorine }}{\mathrm{Cl}_{8}}=\underset{\text { Methyl chloride }}{\mathrm{CH}_{3} \mathrm{Cl}}+\underset{\text { Hydrochloric aoid }}{\mathrm{HCl}}$

The volume of the mixture reniains unaltered. The methyl chloride which is formed is a gas. If it be separated from the hydrochloric acid (it is soluble in acetic acid, in which hydrochloric acid is but sparingly soluble) and be again mixed with chlorine, then it may be

25 Such carriers or media for the transference of chlorine and the halogens in general were long known to exist in iodine and antimonious chloride, and have been most fully studied by Gustarson and Friedel, of the Petroffsky Academy - the former with respect to aluminium bromide, and the latter with respect to aluminium chloride. Gustarson showed that if a trace of metallic aluminium be dissolved in bromine (it floats on bromine, and when combination takes place much heat and light are evolved), the latter becomes endowed with the property of entering into metalepsis, which it is not able to do of its own accord. When pure, for instance, it acts very slowly on benzene, $\mathrm{C}_{6} \mathrm{H}_{6}$, but in the presence of a trace of aluminium bromide the reaction proceeds violently and easily, so that each drop of the hydrocarbon gives a mass of hydrobromio acid, and of the product of metalepsis. Gustarson showed that the modus operandi of this instructive reaction is based on the property of aluminium bromide to enter into combination with hydrocarbons and their derivatives. The details of this and all researches concerning the metalepsis of the hydrocarbons must be looked for in works on organic chemistry.

24 As small admixtures of iodine, aluminium bromide, \&c., aid the metalepsis of large quantities of a substance, just as nitric oxide aids the reaction of sulphurous anhydride on oxygen and water, so the principle is essentially the same in both cases. Effects of this kind (which should also be explained by a chemical reaction proceeding at the surfaces) only differ from true contact phenomens in that the latter are produced by solid bodies and are accomplished at their surfaces, whilst in the former all is in solution. Probably the action of iodine is founded on the formation of iodine chloride, which rescts mare easily than chlorine.

25 Motalepsis belongs to the number of delicate reactiono-if it may be saexpressedas compared with the energetic. reaction of combustion. Many cases of substitution are of this kind. Reactions of metalepsis are accompanied by an evolution of heat, but in a less quantity than that evolved in the formation of the resulting quantity of the halogen acids. Thus the, reaction $\mathrm{C}_{2} \mathrm{H}_{6}+\mathrm{Cl}_{2}=\mathrm{C}_{2} \mathrm{H}_{3} \mathrm{Cl}+\mathrm{HCl}$, according to the data given by Thomsen, evolves about 20,000 heat units, whilst the formation of hydrochloric acid evolves 22,000 units 
subjected to a further metalepsical substitution-the second atom of hydrogen may be substituted by chlorine, and a liquid substance, $\mathrm{CH}_{2} \mathrm{Cl}_{2}$, called methylene chloride, will be obtained. In the same manner the substitution may be carried on still further, and $\mathrm{CHCl}_{3}$, or chloroform, and lastly carbon tetrachloride, $\mathrm{CCl}_{4}$, will be produced. Of these substances the best known is chloroform, owing to its being formed from many organic substances (by the action of bleaching powder) and to its being used in medicine as an anæsthetic ; chloroform boils at $62^{\circ}$ and carbon tetrachloride at $78^{\circ}$. They are both colourless odoriferous liquids, heavier than water. The progressive substitution of hydrogen by chlorine is thus evident, and it can be clearly seen that the double decompositions are accomplished between molecular quanti. ties of the substance-that is, between equal volumes in a gaseous state.

Carbon tetrachloride, which is obtained by the metalepsis of marsh gas, cannot be obtained directly from chlorine and carbon, but it may be obtained from certain compounds of carbon-for instance, from carbon bisulphide-if its vapour mixed with chlorine be passed through a red-hot tube. Both the sulphur and carbon then combine with the chlorine. It is evident that by ultimate metalepsis a corresponding carbon chloride may be obtained from any hydrocarbon-indeed, the number of chlorides of carbon $\mathrm{C}_{n} \mathrm{Cl}_{2 m}$ already known is very large.

As a rule, the fundamental chemical characters of hydrocarbons are not changed by metalepsis ; that is, if a neutral substance be taken, then the product of metalepsis is also a neutral substance, or if an acid be taken the product of metalepsis also has acid properties. Even the crystalline form not unfrequently remains unaltered after metalepsis. The metalepsis of acetic acid, $\mathrm{CH}_{3} \cdot \mathrm{COOH}$, is historically the most important. It contains three of the atoms of the hydrogen of marsh gas, the fourth being replaced by carboxyl, and therefore by the action of chlorine it gives three products of metalepsis (according to the amount of the chlorine and conditions under which the reaction takes place), mono-, di-, and tri-chloracetic acids $-\mathrm{CH}_{2} \mathrm{Cl} \cdot \mathrm{COOH}, \mathrm{CHCl}_{2} \cdot \mathrm{COOH}$, and $\mathrm{CCl}_{3} \cdot \mathrm{COOH}$; they are all, like acetic acid, monobasic. The resulting products of metalepsis, in containing an element which so easily acts on metals as chlorine, possess the possibility of attaining a further com: plexity of molecules of which the original hydrocarbon is often in no way capable. Thus on treating with an alkali (or first with a salt and then with an alkali, or with a basic oxide and water, \&c.) the chlorine forms a salt with its metal, and the hydroxyl radicle takes the place of the chlorine-for example, $\mathrm{CH}_{3} \cdot \mathrm{OH}$ is obtained from $\mathrm{CH}_{3} \mathrm{Cl}$. By the action of metallic derivatives of hydrocarbons-for example, $\mathrm{CH}_{3} \mathrm{Na}-$ the chlorine also gives a salt, and the hydrocarbon radicle-for instance, 
$\mathrm{CH}_{3}$-takes the place of the chlorine. In this, or in a similar manner, $\mathrm{CH}_{3} \cdot \mathrm{CH}_{3}$, or $\mathrm{C}_{2} \mathrm{H}_{6}$ is obtained from $\mathrm{CH}_{3} \mathrm{Cl}$ and $\mathrm{C}_{6} \mathrm{H}_{5} \cdot \mathrm{CH}_{3}$ from $\mathrm{C}_{6} \mathrm{H}_{6}$. The products of metalepsis also often react on ammonia, forming hydrochloric acid (and thence $\mathrm{NH}_{4} \mathrm{Cl}$ ) and an amide ; that is, the product of metalepsis, with the ammonia radicle $\mathrm{NH}_{2}$, \&c. in the place of chlorine. Thus by means of metalepsical substitution methods were found in chemistry for an artificial and general means of the formation of com. plex carbon compounds from more simple compounds which are often totally incapable of direct reaction. Besides which, this key opened the doors of that secret edifice of complex organic compounds into which man had up to then feared to enter, supposing the hydrocarbon elements to be united only under the influence of those mystic forces acting in organisms. ${ }^{26}$

It is not only hydrocarbons which are subject to metalepsis. Certain other hydrogen compounds, under the action of chlorine, also give corresponding chlorine derivatives in exactly the same manner; for instance, ammonia, caustic potash, caustic lime, and a whole series of alkaline substances. ${ }^{27}$ In fact, just as the hydrogen in marsh gas can be replaced by chlorine and form methyl chloride, so the hydrogen in caustic potash, $\mathrm{KHO}$, ammonia, $\mathrm{NH}_{3}$, and calcium hydroxide,

8v. With the predominance of the representation of compound radicles (this doctrine dates from Lavoisier and Gay-Lussac) in organic chemistry, it was a very important moment in its history when it became possible to gain an insight into the structure of the radicles themselves. It was clear, for instance, that ethyl, $\mathrm{C}_{2} \mathrm{H}_{5}$, or the radicle of common alcohol, $\mathrm{C}_{2} \mathrm{H}_{5} \cdot \mathrm{OH}$, passes, without changing, into a number of ethyl 'derivatives, but its relation to the still simpler hydrocarbons was not clear, and occupied the attention of science in the 'forties' and 'fifties.' Huving obtained ethyl hydride, $\mathrm{C}_{2} \mathrm{H}_{5} \mathrm{H}=\mathrm{C}_{2} \mathrm{H}_{8}$, it was looked on as containing the same ethyl, just as methyl hydride, $\mathrm{CH}_{4}=\mathrm{CH}_{3} \mathrm{H}$, was considered as existing in methane. Having obtained free methyl, $\mathrm{CH}_{3} \mathrm{CH}_{3}=\mathrm{C}_{2} \mathrm{H}_{6}$, from it, it was considered as a derivative of methyl alcolıol, $\mathrm{CH}_{5} \mathrm{OH}$, and as only isomeric with ethyl hydride. By means of the products of metalepsis it was proved that this is not a case of isomerism but of strict identity, and it therefore became clear that ethyl is methylated methyl, $\mathrm{C}_{2} \mathrm{H}_{5}=\mathrm{CH}_{2} \mathrm{CH}_{3}$. In its time a still greater impetus was given by the study of the reactions of monochloracetic acid, $\mathrm{CH}_{2} \mathrm{Cl} \cdot \mathrm{COOH}$, or $\mathrm{CO}\left(\mathrm{CH}_{2} \mathrm{Cl}\right)(\mathrm{OH})$. It appeared that metalepsical chlorine, like the chlorine of chloranhydrides-for instance, of methyl chloride, $\mathrm{CH}_{3} \mathrm{Cl}$, or ethyl chloride, $\mathrm{C}_{2} \mathrm{H}_{3} \mathrm{Cl}$-is capable of substitution; for example, glycollic acid, $\mathrm{CH}_{2}(\mathrm{OH})\left(\mathrm{CO}_{2} \mathrm{H}\right)$, or $\mathrm{CO}\left(\mathrm{CH}_{2} \cdot \mathrm{OH}\right)(\mathrm{OH})$, was obtained from it, and it uppeared that the $\mathrm{OH}$ in the group $\mathrm{CH}_{2}(\mathrm{OH})$ reacted like that in alcohols, and it became clear, therefore, that it was necessary to examine the radicles themselves by analysing them trom the point of view of the bonds connecting the constituent atoms. Whence arose the present doctrine of the structure of the carbon compounds. (Sen Chapter VIII., Note 42.)

$n$ By including many instances of the action of chlomne under metalepsts we not only explain the indirect formation of $\mathrm{CCl}_{4}, \mathrm{NCl}_{5}$, and $\mathrm{Cl}_{2} \mathrm{O}$ by one method, but we also arrive at the fact that the reactions of the metalepsis of the hydrocarbons lose thus exclusiveness which was often ascribed to them Also by subjecting the chemical repre. sentations to the law of substitution we may foretell metalepsis as a particular case of a general law. 
$\mathrm{CaH}_{2} \mathrm{O}_{2}$ or $\mathrm{Ca}(\mathrm{OH})_{2}$, may be replaced by chlorine and give potassium hypochlorite, $\mathrm{KClO}$, calcium hypochlorite, $\mathrm{CaCl}_{2} \mathrm{O}_{2}$, and the so-called chloride of nitrogen, $\mathrm{NCl}_{3}$. For not only is thecorrelation in composition the same as in the substitution in marsh gas, but the whole mechanism of the reaction is the same. Here also two atoms of chlorine act : one takes the place of the hydrogen whilst the other is evolved as hydrochloric acid, only in the former case the hydrochloric acid evolved remained free, and in the latter, in presence of alkaline substances, it reacts on them. Thus, in the action of chlorine on caustic potash, the hydrochloric acid formed acts on another quantity of caustic potash and gives potassium chloride and water, and therefore not only $\mathrm{KHO}+\mathrm{Cl}_{2}=\mathrm{HCl}+\mathrm{KClO}$, but also $\mathrm{KHO}+\mathrm{HCl}=\mathrm{H}_{2} \mathrm{O}+\mathrm{KCl}$, and the result of both simultaneous phases will be $2 \mathrm{KHO}+\mathrm{Cl}_{2}$ $=\mathrm{H}_{2} \mathrm{O}+\mathrm{KCl}+\mathrm{KClO}$. We will here dis̄cuss certain special cases.

The action of chlorine on ammonia may either result in the entire breaking up of the ammonia, with the evolution of gaseous nitrogen, or in a product of metalepsis (as with $\mathrm{CH}_{4}$ ). With an excess of chlorine and the aid of heat the ammonia is decomposed, with the disengagement of free nitrogen. ${ }^{28}$ This reaction evidently results in the formation of sal-ammoniac, $8 \mathrm{NH}_{3}+3 \mathrm{Cl}_{2}=6 \mathrm{NH}_{4} \mathrm{Cl}+\mathrm{N}_{2}$. But if the ammonium salt be in excess, then the reaction takes the direction of the replacement of the hydrogen in the ammonia by chlorine. The principal result is that $\mathrm{NH}_{3}+3 \mathrm{Cl}_{2}$ forms $\mathrm{NCl}_{3}+3 \mathrm{HCl} .{ }^{29}$

28 This may be taken advantage of in the preparation of nitrogen. If a large excess of chlorine water be poured into a beaker, and a small quantity of a solution of ammonia be added, then, after shaking, nitrogen is evolved. If chlorine act on a dilute solution of ammonia, the volume of nitrogen does not correspond with the volume of the chlorine taken, because anmonium hypochlorite is formed. If ammonia gas be passed through a fine orifice into a vessel contrining chlorine, the reaction of the formation of nitrogen is accompanied by the emission of light and the appearance of a cloud of sal. ammoniac. In all these instances an excess of chlorine must be present.

29 The hydrochloric acid formed combines with ammonia, and therefore the final result is $4 \mathrm{NH}_{5}+3 \mathrm{Cl}_{2}=\mathrm{NCl}_{3}+3 \mathrm{NH}_{4} \mathrm{Cl}$. For this reason, more ammonia must enter into the reaction,-but the metalepsical reaction in reality only takes place with an excess of ammonia or its salt. If bubbles of chlorine be passed through a fine tube into a vessel containing ammonia gas, each bubble gives rise to an explosion. If, however, chlorine be passed into a solution of ammonia, the reaction at first brings abont the formation of nitrogen, because chloride of nitrogen acts on ammonia like chlorine. But when sal-ammoniac has begun to form, then the reaction directs itself towards the formation of chloride of nitrogen. The first action of chlorine on a solution of sal-ammoniac always causes the formation of chloride of nitrogen, which then reacts on ammonia thus : $\mathrm{NCl}_{3}+4 \mathrm{NH}_{5}=\mathrm{N}_{2}+3 \mathrm{NH}_{4} \mathrm{Cl}$. Therefore, so long as the liquid is alkaline from the presence of ammonia the chief product will be nitrogen. The reaction $\mathrm{NH}_{4} \mathrm{Cl}+8 \mathrm{Cl}_{2}=\mathrm{NCl}_{3}+4 \mathrm{HCl}$ is reversible; with a dilute solution it proceeds in the above. described direction (perhaps owing to the affinity of the hydrochloric acid for the excess of water), but with a strong solution of hydrochloric acid it takes the opposite direction 


\section{The resulting product of metalepsis, or chloride of nitrogen, $\mathrm{NCl}_{3}$,} discovered by Dulong, is a liquid having the property of decomposing with excessive ease not only when heated, but even under the action of mechanical influences, as by a blow or by contact with certain solid substances. The explosion which accompanies the decomposition is due to the fact that the liquid chloride of nitrogen gives gaseous products, nitrogen and chlorine. ${ }^{29}$ bis

(probably by virtue of the affinity of hyddrochlorio acid for ammonia). Therefore there must exist a very interesting case of equilibrium between ammónia, hydrochloric acid, chlorine, water, and chloride of nitrogen which has not yet been investigated. The reaction $\mathrm{NCl}_{5}+4 \mathrm{HCl}=\mathrm{NH}_{4} \mathrm{Cl}+3 \mathrm{Cl}_{2}$ enabled Deville and Hautefeuille to determine the composition of chloride of nitrogen, When slowly decomposed by water, chloride of nitrogen gives, like of chloranhydride, nitrous acid or its anhydride, $2 \mathrm{NCl}_{3}+3 \mathrm{H}_{2} \mathrm{O}$ $=\mathrm{N}_{2} \mathrm{O}_{3}+6 \mathrm{HCl}$. From these observations it is evident that chloride of nitrogen presents. great chemical interest, which is strengthened by its analogy with trichloride of phosphorus. The researches of $\mathrm{F}$. F. Selivanoff $(1891-94)$ prove that $\mathrm{NCl}_{3}$ may be regarded as an ammonium derivative of hypochlorous acid. Chloride of nitrogen is decomposed by dilute sulphuric acid in the following manner: $\mathrm{NCl}_{3}+3 \mathrm{H}_{2} \mathrm{O}+\mathrm{H}_{2} \mathrm{SO}_{4}=\mathrm{NH}_{4} \mathrm{HSO}_{4}+3 \mathrm{HClO}$. This reaction is reversible and is only complete when some substance, combining with HClO (for instance, succinimide) or decomposing it, is added to the liquid. This is easily understood from the fact that hypochlorous acid itself, $\mathrm{HClO}$, may, according to the view held in this book, be regarded as the product of the metalepsis of water, and consequently bears the same relation to $\mathrm{NCl}_{3}$ as $\mathrm{H}_{2} \mathrm{O}$ does to $\mathrm{NH}_{3}$, or as $\mathrm{RHO}$ to $\mathrm{RNH}_{2}, \mathrm{R}_{2} \mathrm{NH}$, and $\mathrm{R}_{3} \mathrm{~N}$ - that is to say, $\mathrm{NCl}_{3}$ corresponds as an ammonium derivative to $\mathrm{ClOH}$ and $\mathrm{Cl}_{2}$ in exactly the same manner as $\mathrm{NR}_{3}$ corresponds to $\mathrm{ROH}$ and $\mathrm{R}_{2}$. The connection of $\mathrm{NCl}_{3}$ and other similar explosive chloro-nitrogen compounds (called chloryl compounds by Selivanoff; for example, the $\mathrm{C}_{2} \mathrm{H}_{5} \mathrm{NCl}_{2}$ of Wurtz is chloryl ethylamine), such as $\mathrm{NRCl}_{2}$ (as $\mathrm{NC}_{2} \mathrm{H}_{5} \mathrm{Cl}_{2}$ ), and $\mathrm{NR}_{2} \mathrm{Cl}$ (for instance, $\mathrm{N}\left(\mathrm{CH}_{3} \mathrm{CO}\right) \mathrm{HCl}$, chloryl. acetamide, and $\mathrm{N}\left(\mathrm{C}_{2} \mathrm{H}_{5}\right)_{2} \mathrm{Cl}$, chloryl diethylamine) with $\mathrm{HClO}$ is evident from the fact that under certain circumstances these compounds give hypochlorous acid, with water, for instance, $\mathrm{NR}_{2} \mathrm{Cl}+\mathrm{H}_{2} \mathrm{O}=\mathrm{NR}_{2} \mathrm{H}+\mathrm{HClO}$, and frequently act (like $\mathrm{NCl}_{3}$ and $\mathrm{HClO}$, or $\mathrm{Cl}_{2}$ ) in an oxidising and chloridising manner. We may take chloryl succinimide, $\mathrm{C}_{2} \mathrm{H}_{4}(\mathrm{CO})_{2} \mathrm{NCl}$ for example. It was obtained by Bender by the action of $\mathrm{HClO}$ upon succinimide, $\mathrm{C}_{2} \mathrm{H}_{4}(\mathrm{CO})_{2} \mathrm{NH}$, and is decomposed by water with the re-formation of amide and $\mathrm{HClO}$ (the reaction is reversible). Selivanoff obtained, investigated, and classified many of the compounds $\mathrm{NR}_{2} \mathrm{Cl}$ and $\mathrm{NRCl}_{2}$, where $\mathrm{R}$ is a residue of organio acids or alcohols, and showed their distinction from the chloranhydrides, and thus supplemented the history of chloride of nitrogen, which is the simplest of the amides containing chlorine, $\mathrm{NR}_{3}$, where $\mathbf{R}$ is fully substituted by chlorine.

29 bis In preparing $\mathrm{NCl}_{5}$ every precaution must be used to guard against an explosion, and care should be taken that the $\mathrm{Nel}_{3}$ remains under a layer of water. Whenever an ammoniacal substance comes into contact with chlorine great care must be taken, because it may be a case of the formation of such products and a very dangerous explosion may ensue. The liquid product of the metalepsis of ammonia may be most safely prepared in the form of small drops by theaction of a galvanic current on a slightly warm solution of sal-ammoniac; chlorine is then evolved at the positive pole, and this chlorine acting on the ammonia gradually forms the product of metalepsis which floats on the surface of the liquid (being carried ap by the gas), and if a layer of turpentine be poured on to it these small drops, on coming into contact with the turpentine, give feeble explosions, which are in no way dangerous owing to the small mass of the substance formed. Drops of chloride of nitrogen may with great caution be collected for investigation in the following manner. The neck of a funnel is immersed in a basin containing mercury, and first a saturated solutiop of common salt is poured into the funnel, 
Chloride of nitrogen is a yellow oily liquid of sp. gr. 1.65, which boils at $71^{\circ}$, and breaks up into $\mathrm{N}+\mathrm{Cl}_{3}$ at $97^{\circ}$. The contact of phosphorus, turpentine, india-rubber, \&c. causes an explosion, which is sometimes. so violent that a small drop will pierce through a thick board. The great ease with which chloride of nitrogen decomposes is dependent upon the fact that it is formed with an absorption of heat, which it evolves when decomposed, to the amount of about 38.000 heat units for $\mathrm{NCl}_{3}$, as Deville and Hautefeuille determined.

Chlorine, when absorbed by a solution of caustio soda (and also of other alkalis) at the ordinary temperature, causes the replacement of the hydrogen in the caustic soda by the chlorine, with the formation of sodium chloride by the hydrochloric acid, so that the reaction may be represented in two phases, as described above. In this manner, sodium hypochlorite, $\mathrm{NaClO}$, and sodium chloride are simultaneously formed: $2 \mathrm{NaHO}+\mathrm{Cl}_{2}=\mathrm{NaCl}+\mathrm{NaClO}+\mathrm{H}_{2} \mathrm{O}$. The resultant solution contains $\mathrm{NaClO}$ and is termed 'eau de Javelle. An exactly similar reaction takes place when chlorine is passed over dry hydrate of lime at the ordinary temperature: $2 \mathrm{Ca}(\mathrm{HO})_{2}+2 \mathrm{Cl}_{2}$ $=\mathrm{CaCl}_{2} \mathrm{O}_{2}+\mathrm{CaCl}_{2}+2 \mathrm{H}_{2} \mathrm{O}$. A mixture of the product of metalepsis with calcium chloride is obtained. This mixture is employed in practice on a large scale, and is termed 'bleaching powder, owing to its acting, especially when mixed with acids, as a bleaching agent on tissues, so that it resembles chlorine in this respect. It is however preferable to chlorine, because the destructive action of the chlorine can be moderated in this case, and because it is much more convenient to deal with a solid substance than with gaseous chlorine. Bleaching powder is also called chloride of lime, because it is obtained from chlorine and hydrate of lime, and contains ${ }^{30}$ both these substances. It

and above it a solution of sal-ammoniac in 9 parts of water. Chlorine is then slowly passed through the solutions. when drops of chloride of nitrogen fall into the salt water.

60 Quicklime, $\mathrm{CaO}$ (or calcium carbonate, $\mathrm{CaCO}_{3}$ ), does not absorb chlorine when cold, but as a red heat, in a current of chlorine, it forms calcium chloride, with the evolution of oxygen. (This was confirmed in 1898 by Wells, at Oxford.) This reaction corresponds with the decomposing action of chlorine on methane, ammonia, and water. Slaked lime (calcium hydroxide, $\mathrm{CaH}_{2} \mathrm{O}_{2}$ ) also, when ary, does not absorb chlorine at $100^{\circ}$. The absorption proceeds at the ordinary temperature (below $40^{\circ}$ ). The dry mass thus ob. tained contains not less than three equivalents of caloitum hydroxide to four equivalents of chlorine, so that its composition is $\left[\mathrm{Ca}\left(\mathrm{HO}_{2}\right]_{3} \mathrm{Cl}_{4}\right.$. In all probability a simple absorp. tion of chlorine by the lime at first takes place in this case, as may be seen from the fact that even carbonic anhydride, when acting on the dry mass obtained as above, disengages all the chlorine from it, leaving only calcium carbonate. But if the bleaching powder be obtained by a wet method, or if it be dissolved in water (in which it is very soluble), and carbonic anhydride be passed into it, then chlorine is no longer disengaged, but chlorine oxide, $\mathrm{Cl}_{2} \mathrm{O}$, and only half of the chlorine is converted into this oxide, while the other hall remains in the liquid as calcium chloride. From this it may be inferred that calcium 
may be prepared in the laboratory by passing a current of chlorine through a cold mixture of water and lime (milk of lime). The mixture must be kept cold, as otherwise $3 \mathrm{Ca}(\mathrm{ClO})_{2}$ passes into $2 \mathrm{CaCl}_{2}$ $+\mathrm{Ca}\left(\mathrm{ClO}_{3}\right)_{2}$. In the manufacture of bleaching powder in large quantities at chemical works, the purest possible slaked lime is taken and laid in a thin layer in large flat chambers, $\mathbf{M}$ (whose walls are made of Yorkshire flags or tarred wood, on which chlorine has no action), and into which chlorine gas is introduced by lead tubes. The distribution of the plant is shown in the annexed drawing (fig. 67).

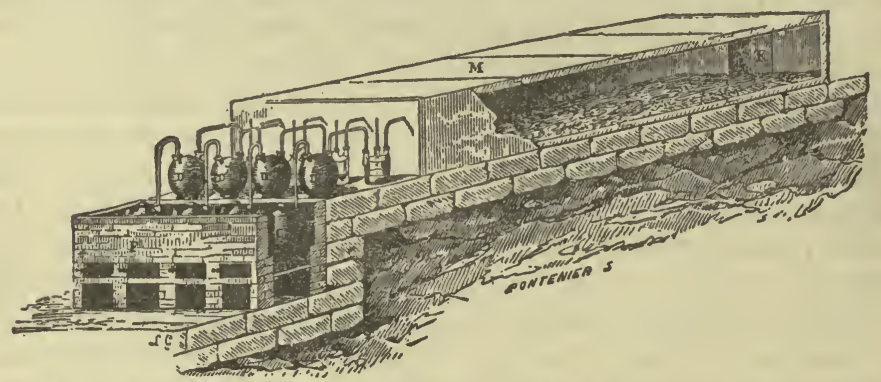

FiG. 67.-A pparatus for the manufacture of bleaching powder (on a small scale) by the action of chlorine, which is generated in the vessels $\mathrm{C}$, on lime; which is charged tnto $M$.

The products of the metalepsis of alkaline hydrates, $\mathrm{NaClO}$ and $\mathrm{Ca}(\mathrm{ClO})_{2}$, which are present in solutions of 'Javelle salt' and bleaching

chloride is formed by the action of water on bleaching powder, and this is proved to be the case by the fact that small quantities of water extract a considerable amount of calcium chloride from bleaching powder. If a large quantity of water act on bleaching powder an excess of calcium hydroxide remains, a portion of which is not subjected to change. The action of the water may be expressed by the following formulø: From the dry mass $\mathrm{Ca}_{3}(\mathrm{HO})_{6} \mathrm{Cl}_{4}$ there is formed lime, $\mathrm{Ca}(\mathrm{HO})_{2}$, calcium chloride, $\mathrm{CaCl}_{2}$, and a saline substance, $\mathrm{Ca}(\mathrm{ClO})_{2}$. $\mathrm{Ca}_{3} \mathrm{H}_{6} \mathrm{O}_{6} \mathrm{Cl}_{4}=\mathrm{CaH}_{2} \mathrm{O}_{2}+\mathrm{CaCl}_{2} \mathrm{O}_{2}+\mathrm{CaCl}_{2}+2 \mathrm{H}_{2} \mathrm{O}$. The resulting substances are not equally soluble; water first extracts the calcium chloride, which is the most soluble, then the compound $\mathrm{Ca}(\mathrm{ClO})_{2}$ and ultimately calcium hydroxide is left. A mixture of calcium chloride and hypochlorite passes into solution. On evaporation there remains $\mathrm{Ca}_{2} \mathrm{O}_{2} \mathrm{Cl}_{4} 3 \mathrm{H}_{2} \mathrm{O}$. The dry bleaching powder does not absorb more chlorine, but the solution is able to absorb it in considerable quantity. If the liquid be boiled, a considerable amount of chlorine monoxide is evolved. After this calcium chloride alone remains in solution, and the decomposition may be expressed as follows: $\mathrm{CaCl}_{2}+\mathrm{CaCl}_{2} \mathrm{O}_{2}+2 \mathrm{Cl}_{2}=2 \mathrm{CaCl}_{2}+2 \mathrm{Cl}_{2} \mathrm{O}$. Chlorine monoxide may be prepared in this manner.

It is sometimes said that bleaching powder contains a substance, $\mathrm{Ca}(\mathrm{OH})_{2} \mathrm{Cl}_{2}$, that is calcium peroxide, $\mathrm{CaO}_{2}$, in which one atom of oxygen is replaced by $(\mathrm{OH})_{2}$, and the other by $\mathrm{Cl}_{2}$; but, judging from what has been said above, this can only be the case in the dry state, and not in solutions.

On being kept for some time, bleaching powder sometimes decomposes, with the evolution of oxygen (because $\mathrm{CaCl}_{2} \mathrm{O}_{2}=\mathrm{CaCl}_{2}+\mathrm{O}_{2}$, see p. 168); the samo takes place when it is heated. 
powder (they are not obtained free from metallic chlorides), must be counted as salts, because their metals are capable of substitution. But the hydrate $\mathrm{HClO}$ corresponding with these salts, or hypochlorous acid, is not obtained in a free or pure state, for two reasons : in the first place, because this hydrate, as a very feeble acid, splits up (like $\mathrm{H}_{2} \mathrm{CO}_{3}$ or $\mathrm{HNO}_{3}$ ) into water and the anhydride, or chlorine monoxide. $\mathrm{Cl}_{2} \mathrm{O}=2 \mathrm{HClO}-\mathrm{H}_{2} \mathrm{O}$; and, in the second place, because, in a number of instances, it evolves oxygen with great facility, forming hydrochloric acid : $\mathrm{HClO}=\mathrm{HCl}+\mathrm{O}$. Both hypochlorous acid and chlorine monoxide may be regarded as products of the metalepsis of water, because $\mathrm{HOH}$ corresponds with $\mathrm{ClOH}$ and. $\mathrm{ClOCl}$. Hence in many instances bleaching salts (a mixture of hypochlorites and chlorides) break up, with the evolution of (1) chlorine, under the action of an excess of a powerful acid capable of evolving hydrochloric acid from sodium or calcium chlorides, and this takes place most simply under the action of hydrochloric acid itself, because (p. 462) $\mathrm{NaCl}+\mathrm{NaClO}$ $+3 \mathrm{HCl}=2 \mathrm{NaCl}+\mathrm{HCl}+\mathrm{Cl}_{2}+\mathrm{H}_{2} \mathrm{O}$; (2) oxygen, as we saw in Chapter III.-The bleaching properties and, in general, oxidising action of bleaching. salts is based on this evolution of oxygen (or chlorine) ; oxygen is also disengaged on heating the dry salts-for instance, $\mathrm{NaCl}+\mathrm{NaClO}=2 \mathrm{NaCl}+\mathrm{O} ;$ (3) and, lastly, chlorine monoxide, which contains both chlorine and oxygen. Thus, if a little sulphuric, nitric, or similar acid (not enough to liberate hydrochloric acid from the $\mathrm{CaCl}_{2}$ ) be added to a solution of a bleaching salt (which has an alkaline reaction, owing either to an excess of alkali or to the feeble acid properties of $\mathrm{HClO}$ ), then the hypochlorous acid set free gives water and chlorine monoxide. If carbonic anhydride (or boracic or a similar very feeble acid) act on the solution of a bleaching salt, then hydrochloric acid is not evolved from the sodium or calcium chlorides, but the hypochlorous acid is displaced and gives chlorine monoxide, ${ }^{31}$ because hypochlorous acid is one of the most feeble acids. Another method for the preparation of chlorine monoxide is based on these feeble acid properties of hypochlorous acid. Zinc oxide and mercury oxide, under the action of chlorine in the presence of water, do not give a salt of hypochlorous acid, but form a chloride and hypochlorous acid, which fact shows the incapacity of this acid to

31 For this reason it is necessary that in the preparation of bleaching powder the chlorine should be free from hydrochloric acid, and even the lime from calcium chloride: An excess of chlorine, in acting on a solution of bleaching powder, may also give chlorine monoxide, because calcium carbonate also gives chlorine monoxide under the acticr of chlorine. This reaction may be brought about by treating freshly precipitated calcium carbonate with a stream of chlorine in water: $2 \mathrm{Cl}_{2}+\mathrm{CaCO}_{3}=\mathrm{CO}_{2}+\mathrm{CaCl}_{2}+\mathrm{Cl}_{2} \mathrm{O}$. From this we may conclude that, altbough carbonic anhydride displaces hypochlorous anhy dride, it may be itself displaced by an excess of the latter. 
combine with the bases mentioned. Therefore, if such oxides as those of zinc or mercury be shaken up in water, and chlorine be passed through the turbid liquid, ${ }^{32}$ a reaction occurs which may be expressed in the following manner $2 \mathrm{HgO}+2 \mathrm{Cl}_{2}=\mathrm{Hg}_{2} \mathrm{OCl}_{2}+\mathrm{Cl}_{2} \mathrm{O}$. In this case, a compound of mercury oxide with mercury chloride, or the socalled mercury oxychloride, is obtained: $\mathrm{Hg}_{2} \mathrm{OCl}_{2}=\mathrm{HgO}+\mathrm{HgCl}_{2}$. This is insoluble in water, and is not affected by hypochlorous anhydride, so that the solution will contain hypochlorous acid only, but the greater part of it splits up into the anhydride and water. ${ }^{32}$ bis

Chlorine monoxide, which corresponds to bleaching and hypochlorous salts, containing as it does the two elements oxygen and chlorine, forms a characteristic example of a compound of elements which, in the majority of cases, act chemically in an analogous manner. Chlorine monoxide, as prepared from an aqueous solution by the abstraction of water or by the action of dry chlorine on cold mercury oxide, is, at the ordinary temperature, a gas or vapour which condenses into a red liquid boiling at $+20^{\circ}$ and giving a vapour whose density (43 referred to hydrogen) shows that 2 vols. of chlorine and 1 vol. of oxygen give 2 vols. of chlorine monoxide. In an anhydrous form the gas or liquid easily explodes, splitting up into chlorine and oxygen. This explosiveness is determined by the fact that heat is evolved in the decomposition to the amount of about 15,000 heat units for $\mathrm{Cl}_{2} \mathrm{O}^{33}$ The explosion may even take place spontaneously, and also

52 Dry red mercury oxide acts on chlorine, forming dry hypochlorous anhydride (chlorine monoxide) (Balard); when mixed with water, red mercury oxide acts feebly on chlorine, and when freshly precipitated it evolves oxygen and chlorine. An oxide of mercury which easily and abundantly evolves chlorine monoxide under the action of chlorine in the presence of water may be prepared as follows: the oxide of mercury, precipitated from a mercuric salt by an alkali, is heated to $300^{\circ}$ and cooled (Pelouze). If a salt, $\mathrm{MClO}$, be added to a solution of mercuric salt, $\mathrm{HgX}_{2}$, mercuric oxide is liberated, because the hypochlorite is decomposed.

32 bis A solution of hypochlorous anhydride is also obtained by the action of chlorine on many salts ; for example, in the action of chlorine on a solution of sodium sulphate the following reaction takes place: $\mathrm{Na}_{2} \mathrm{SO}_{4}+\mathrm{H}_{2} \mathrm{O}+\mathrm{Cl}_{2}=\mathrm{NaCl}+\mathrm{HClO}+\mathrm{NaHSO}_{4}$. Here the hypochlorous acid is formed, together with $\mathrm{HCl}$, at the expense of chlorine and water, for $\mathrm{Cl}_{2}+\mathrm{H}_{2} \mathrm{O}=\mathrm{HCl}+\mathrm{HClO}$. If the crystallo-hydrate of chlorine be mixed with mercury oxide, the hydrochloric acid formed in the reaction gives mercury chloride, and hypochlorous acid remains in solution. A dilute solution of hypochlorous acid or cllorine monoxide may be concentrated by distillation, and if a substance which takes up water (without destroying the acid)-for instance, calcium nitrate-be added to the stronger solution, then the anhydride of hypochlorous acid-i.e. chlorine monoxide-is disengaged.

53 All explosive substances are of this kind-ozone, hydrogen peroxide, chloride of nitrogen, nitro-compounds, \&c. Hence they cannot be formed directly from the elements or their simplest compounds, but, on the contrary, decompose into them. In a liquid state chlorine monoxide explodes even on contact with powdery substances, or when rapidly agitated - for instance, if a file be rasped over the vessel in which it is contained. 
in the presence of many oxidisable substances (for instance, sulphur, organic compounds, \&r..), but the solution, although unstable and showing a strong oxidising tendency, does not explode. ${ }^{34}$ It is evident that the presence of hypochlorous acid, $\mathrm{HClO}$, may be assumed in an aqueous solution of $\mathrm{Cl}_{2} \mathrm{O}$, since $\mathrm{Cl}_{2} \mathrm{O}+\mathrm{H}_{2} \mathrm{O}=2 \mathrm{HClO}$.

Hypochlorous acid, its salts, and chlorine monoxide serve as a transition between hydrochloric acid, chlorides, and chlorine, and a whole series of compounds containing the same elements combined with a still greater quantity of oxygen. The higher oxides of chlorine, as their origin indicates, are closely connected with hypochlorous acid and its salts

$\begin{array}{llll}\mathrm{Cl}_{2}, & \mathrm{NaCl}, & \mathrm{HCl}, & \text { hydrochloric acid, } \\ \mathrm{Cl}_{2} \mathrm{O}, & \mathrm{NaClO}, & \mathrm{HClO}, & \text { hypochlorous acid } \\ \mathrm{Cl}_{2} \mathrm{O}_{3}, & \mathrm{NaClO}_{2}, & \mathrm{HClO} & \text { chlorous acid. }{ }^{35} \\ \mathrm{Cl}_{2} \mathrm{O}_{5}, & \mathrm{NaClO}_{3}, & \mathrm{HClO}_{3}, & \text { chloric acid. } \\ \mathrm{Cl}_{2} \mathrm{O}_{7}, & \mathrm{NaClO}_{4}, & \mathrm{HClO}_{4}, & \text { perchloric acid. }\end{array}$

When heated, solutions of hypochlorites undergo a remarkable change. Themselves so unstable, they, without any further addition, yield two fresh salts which are both much more 'stable; one contains more oxygen than $\mathrm{MClO}$, the other contains none at all.

$$
\underset{\text { hypochlorite }}{3 \mathrm{MClO}}=\underset{\text { chlorate }}{\mathrm{MClO}_{3}}+\underset{\text { chloride }}{2 \mathrm{MCl}}
$$

34 A solution of chlorine monoxide, or hypochlorous acid, does not explode, owing to the presence of the mass of water. In dissolving, chlorine monoxide evolves about 9,000 heat units, so that its store of heat becomes less.

The capacity of hypochlorous acid (studied by Carius and others) for entering into com. bination with the unsaturated hydrocarbons is very often taken advantage of in organio chemistry. Thus its solution absorbs ethylene, forming the chlorhydrin $\mathrm{C}_{2} \mathrm{H}_{4} \mathrm{ClOH}$.

The oxidising action of hypochlorous acid and its salts is not only applied to bleaching but also to many reactions of oxidation. Thus it converts the lower oxides of manganese into the peroxide.

35 Chlorous acid, $\mathrm{HClO}_{2}$ (according to the data given by Millon, Brandau, and others) in many.respects resembles hypochlorous acid, $\mathrm{HClO}$, whilst they both differ from chlorio and perchloric acids in their degree of stability, which is expressed, for instance, in their bleaching properties; the two higher acids do not bleach, but both the lower ones do so (oxidise at the ordinary temperature). On the other hand, chlorous acid is analogous to nitrous acid, $\mathrm{HNO}_{2}$ The anhydride of chlorous acid, $\mathrm{Cl}_{2} \mathrm{O}_{3}$, is not known in a pure state, but it probably occurs in admixture with chlorine dioxide, $\mathrm{ClO}_{2}$, which is obtained by the action of nitric and sulphuric acids on a mixture of potassium chlorate with such reducing substances as nitric oxide, arsenious oxide, sugar, \&c. All that is at present known is that pure chlorine dioxide $\mathrm{ClO}_{2}$ (see Notes 39-43) is gradually converted into a mixture of hypochlorous and chlorous acids under the action of water (and alkalis); that is, it acts like nitric peroxide, $\mathrm{NO}_{2}$ (giving $\mathrm{HNO}_{3}$ and $\mathrm{HNO}_{2}$ ), or as a mired anhy. dricle, $2 \mathrm{ClO}_{2}+\mathrm{H}_{2} \mathrm{O}=\mathrm{HClO}_{3}+\mathrm{HClO}_{3}$. The silver salt, $\mathrm{AgClO}_{2}$, is sparingly soluble in water. The investigations of Garzarolli-Thurnlackh and others seem to show that the anhydride $\mathrm{Cl}_{2} \mathrm{O}_{3}$ does not exist in a free state. 
Part of the salt-namely, two-thirds of it-parts with its oxygen in order to oxidise the remaining third. ${ }^{36}$ From an intermediate sub. stance, $\mathrm{RX}$, two extremes, $\mathrm{R}$ and $\mathrm{RX}_{3}$ are formed, just as nitrous anhydride splits up into nitric oxide and nitric anhydride (or nitric acid). The resulting salt, $\mathrm{MClO}_{3}$, corresponds with chloric acid and potassium chlorate, $\mathrm{KClO}_{3}$. It is evident that a similar salt may be obtained directly by the action of chlorine on an alkali if its solution be heated, because $\mathrm{RClO}$ will be first formed, and then $\mathrm{RClO}_{3}$; for example, $6 \mathrm{KHO}+3 \mathrm{Cl}_{2}=\mathrm{KClO}_{3}+5 \mathrm{KCl}+3 \mathrm{H}_{2} \mathrm{O}$. Chlorates are so prepared; for instance, potassium chlorate, which is easily separated from potassium chloride, being sparingly soluble in cold water. ${ }^{37}$

S6 Hydrochlorio acid, which is an example of compounds of this kind, is a satu. rated substance which does not combine directly with oxygen, but in which, nevertheless, a considerable quantity of oxygen may be inserted between the elements forming it The same may be observed in a number of other cases. Thus oxygen may be added or inserted between the elements, sometimes in considerable quantities, in the saturated hydrocarbons; for instance, in $\mathrm{C}_{3} \mathrm{H}_{8}$, three atoms of oxygen produce an alcohol, glycerin or gitycerol, $\mathrm{C}_{3} \mathrm{H}_{5}(\mathrm{OH})_{3}$. We shall meet with similar examples hereafter. This is generally explained by regarding oxygen as a bivalent element-that is, as capable of combining with two different elements, such as chlorine, hydrogen, \&c. On the basis of this view, it may be inserted between each pair of combined elements; the oxygen will then be combined with one of the elements by one of its affinities and with the other element by its other affinity. This view does not, however, express the entire truth of the matter, even when applied to the compounds of chlorine, Hypochlorous acid, $\mathrm{HOCl}$-that is, hydrochloric acid in which one atom of oxygen is inserted-is, as we have already seen, a substance of small stability; it might therefore be expected that on the addition of a fresh quantity of oxygen, a still less stable substance would be obtained, because, according to the above view, the chlorine and hydrogen, which form such a stable compound together, are then still further removed from each other. But it appears that chlorio and perchloric acid, $\mathrm{HClO}_{3}$ and $\mathrm{HClO}_{4}$, are much more stable substances. Furthermore, the addition of oxygen hes also its limit, it can only be added to a certain extent. If the above representation were true and not merely hypothetical, there would be no limit to the combination of oxygen, and the more it entered into one continuons chain the more unstable would be the resultant compound. Bnt not more than four atoms of oxygen can be added to hydrogen sulphide, nor to hydrochloric acid, nor to hydrogen phosphide. This peculiarity must lie in the properties of oxygen itself; four atoms of oxygen seem to have the power of forming a kind of radicle which retains two or several atoms of various other substances-for example, chlorine and hydrogen, hydrogen and sulphur, sodium and manganese, phosphorus and metals, \&c., forming comparatively stable compounds, $\mathrm{NaClO}_{4}, \mathrm{Na}_{2} \mathrm{SO}_{4}, \mathrm{NaMnO}_{4}, \mathrm{Na}_{3} \mathrm{PO}_{4}, \&$ c. See Chapter $\mathrm{X}$. Note 1 and Chapter XV.

37 If chlorine be passed through a cold solution of potash, a bleaching compound, potassium chloride and hypochlorite, $\mathrm{KCl}+\mathrm{KClO}$, is formed, but if it be passed through a hot solution potassium chlorate is formed. As this is sparingly soluble in water, it chokes the gas-conducting tube, which should therefore be widened out at the end.

Potassium chlorate is usually obtained on a large scale from calcium chlorate, which is prepared by passing chlorine (as long as it is absorbed) into water containing.lime, the mixture being kept warm. A mixture of calcium chlorate and chloride is thus formed in the solution. Potassium chloride is then added to the warm solution, and on cooling a precipitate of potassium chlorate is formed as a substance which is sparingly soluble in 
If dilute sulphuric acid be added to a solution of potassium chlorate, chloric acid is liberated, but it cannot be separated by distillation, as it is decomposed in the process. To obtain the free acid, sulphuric acid must be added to a solution of barium chlorate. ${ }^{38}$ The sulphuric acid gives a precipitate of barium sulphate, and free chloric acid remains in solution. The solution may be evaporated under the receiver of an air-pump. This solution is colourless, has no smell, and acts as a powerful acid (it neutralises sodium hydroxide, decomposes sodium carbonate, gives hydrogen with zinc, \&c.); when heated above $40^{\circ}$, however, it decomposes, forming chlorine, oxygen, and perchloric acid: $4 \mathrm{HClO}_{3}=2 \mathrm{HClO}_{4}+\mathrm{H}_{2} \mathrm{O}+\mathrm{Cl}_{2}+\mathrm{O}_{3}$. In a concentrated condition the acid acts as an exceedingly energetic oxidiser, so that organic substances brought into contact with it burst into flame. Iodine, sulphurous acid, and similar oxidisable substances form higher oxidation products and reduce the chloric acid to hydrochloric acid. Hydrochloric acid gas gives chlorine with chloric acid

cold water, especially in the presence of other salts. The double decomposition taking place is $\mathrm{Ca}\left(\mathrm{ClO}_{3}\right)_{2}+2 \mathrm{KCl}=\mathrm{CaCl}_{2}+2 \mathrm{KClO}_{3}$. On a small scale in the laboratory potassium chlorate is best prepared from a strong solution of bleaching powder by passing chlorine through it and then adding potassium chloride. $\mathrm{KClO}_{3}$ is always formed by the action of an electric current on a solution of $\mathrm{KCl}$, especially at $80^{\circ}$ (Häussermann and Naschold, 1894), so that this method is now used on a large scale.

Potassium chlorate crystallises easily in large colourless tabular crystals. Its solu. bility in 100 parts of water at $0^{\circ}=3$ parts, $20^{\circ}=8$ parts, $40^{\circ}=14$ parts, $60^{\circ}=25$ parts, $80^{\circ}=40$ parts. For comparison we will cite the following figures showing the solubility of potassium chloride and perchlorate in 100 parts of water : potassium chloride at $0^{\circ}=28$ parts, $20^{\circ}=\varepsilon 5$ parts, $40^{\circ}=40$ parts, $100^{\circ}=57$ parts ; potassium perchlorate at $0^{\circ}$ about 1. part, $20^{\circ}$ about $1 \frac{3}{4}$ part, $100^{\circ}$ about 18 parts. When heated, potassium chlorate melts (the melting point has been given as from $335^{\circ}-376^{\circ}$; according to the latest determination by Carnelley, $359^{\circ}$ ) and decomposes with the evolution of oxygen, potassium perchlorate being at first formed, as will afterwards be described (see Note 47). A mixture of potassium chlorate and nitric and hydrochloric acids effects oxidation and chlorination in solutions. It deflagrates when thrown upon incandescent carbon, and when mixed with sulphur ( $\frac{1}{3}$ by weight) it ignites it on being struck, in which case an explosion takes place. The same occurs with many metallic sulphides and organic substandefs: Such mixtures are also ignited by a drop of sulphuric acid. All these effects are due to the large amount of oxygen contained in potassium chlorate, and to the ease with which it is evolved. A mixture of two parts of potassium chlorate, one part of sugar, and one part of yellow prussiate of potash acts like gunpowder, but burns too rapidly, and therefore bursts the guns, and it also has a very strong oxidising-action on their metal. The sodinm salt, $\mathrm{NaClO}_{3}$, is much more soluble than the potassium salt, and it is therefore more difficult to free it from sodium chloride, \&c. The barium salt is also more soluble than the potassium salt; $0^{\circ}=24$ parts, $20^{\circ}=37$ parts, $80^{\circ}=98$ parts of salt per 100 of water.

38 Barium chlorate, $\mathrm{Ba}\left(\mathrm{ClO}_{3}\right)_{2}, \mathrm{H}_{2} \mathrm{O}$, is prepared in the following way : impure chloric acid is first prepared and saturated with baryta, and the barium salt purified by crystallisation. The impure free chloric acid is obtained by converting the potassium in potas. sium chlorate into an insoluble salt. This is done by adding tartaric or hydrofluosilicio acid to a solution of potassium chlorate, because potassium tartrate and potassium silico: fluoride are very sparingly soluble in water. Chloric acid is easily soluble in water. 
(and consequently with $\mathrm{KClO}_{3}$ also) acting in the same manner as it acts on the lower acids: $\mathrm{HClO}_{3}+5 \mathrm{HCl}=3 \mathrm{H}_{2} \mathrm{O}+3 \mathrm{Cl}_{2}$.

By cautiously acting on potassium chlorate with sulphuric acid, the dioxide (chloric peroxiide), $\mathrm{ClO}_{2}{ }^{39}$ is obtained (Davy, Millon). This gas is easily liquefied in a freezing mixture, and boils at $+10^{\circ}$. The vapour density (about 35 if $\mathrm{H}=1$ ) shows that the molecule of this substance is $\mathrm{ClO}_{2} .^{40}$ In a gaseous or liquid state it very easily explodes (for instance, at $60^{\circ}$, or by contact with organic compounds or finely divided substances, \&c.), forming $\mathrm{Cl}$ and $\mathrm{O}_{2}$, and in many instances ${ }^{41}$ therefore it acts as an oxidising agent, although (like nitric peroxide) it may itself be further oxidised. ${ }^{42}$ In dissolving in water or alkalis chloric peroxide gives chlorous and hypochlorous acids $-2 \mathrm{ClO}_{2}+2 \mathrm{KHO}$ $=\mathrm{KClO}_{3}+\mathrm{KClO}_{2}+\mathrm{H}_{2} \mathrm{O}$-and therefore, like nitric peroxide, the dioxide may be regarded as an intermediate oxide between the (unknown) anhydrides of chlorous and chloric acids : $4 \mathrm{ClO}_{2}=\mathrm{Cl}_{2} \mathrm{O}_{3}$ $+\mathrm{Cl}_{2} \mathrm{O}_{6} \cdot{ }^{43}$

As the salts of chloric acid, $\mathrm{HClO}_{3}$, are produced by the splitting up of the salts of hypochlorous acid, so in the same way the salts of

30 To prepare $\mathrm{ClO}_{2} 100$ grams of sulphuric acid are cooled in a mixture of ice and salt, and 15 grams of powdered potassium chlorate are gradually added to the acid, which is then carefully distilled at $20^{\circ}$ to $40^{\circ}$, the vapour given off being condensed in 8 freezing mixture. Potassium perchlorate is then formed: $8 \mathrm{KClO}_{3}+2 \mathrm{H}_{2} \mathrm{SO}_{4}=2 \mathrm{KHSO}_{4}$ $+\mathrm{KClO}_{4}+2 \mathrm{ClO}_{2}+\mathrm{H}_{2} \mathrm{O}$. The reaction may result in an explosion. Calvert and Davies obtained chloric peroxide, without the least danger by heating a mixture of oxalio acid and potassium chlorate in a test tube in a water-bath. In this case $2 \mathrm{KClO}_{3}$ $+3 \mathrm{C}_{2} \mathrm{H}_{2} \mathrm{O}_{4} 2 \mathrm{H}_{2} \mathrm{O}=2 \mathrm{C}_{2} \mathrm{HKO}_{4}+2 \mathrm{CO}_{2}+2 \mathrm{ClO}_{2}+8 \mathrm{H}_{2} \mathrm{O}$. The reaction is still further facilitated by the addition of a small quantity of sulphuric acid. If a solution of $\mathrm{HCl}$ acts upon $\mathrm{KClO}_{3}$ at the ordinary temperature, a mixture of $\mathrm{Cl}_{2}$ and $\mathrm{ClO}_{2}$ is formed, but if the temperature be raised to $80^{\circ}$ the greater part of the $\mathrm{ClO}_{2}$ decomposes, and when passed: through a hot solution of $\mathrm{MnCl}_{2}$ it oxidises it. Gooch and Kreider proposed (1894) to employ this method for preparing small quantities of chlorine in the laboratory.

40 By analogy with nitric peroxide it might be expected that at low temperatures a doubling of the molecule into $\mathrm{Cl}_{2} \mathrm{O}_{4}$ would take place, as the reactions of $\mathrm{ClO}_{3}$ point to its being a mixed anhydride of $\mathrm{HClO}_{2}$ and $\mathrm{HClO}_{3}$.

41 Owing to the formation of this chlorine dioxide, a mixture of potassium chlorate and sugar is ignited by a drop of sulphuric acid. This property was formerly made use of for making matches, and is now sometimes employed for setting fire to explosive charges by means of an arrangement in which the acid is caused to fall on the mixture at the moment required. An interesting experiment on the combustion of phosphorus under water may be conduoted with chlorine dioxide. Pieces of phosphorus and of potassium chlorate are placed under water, and sulphuric acid is poured on to them (through a long funnel); the phosphorus then burns at the expense of the chlorine dioxide.

43 Potassium permanganate oxidises chlorine dioxide into chloric acid (Fürst).

45. The euchlorine obtained by Davy by gently heating potassiun chlorate with hydro. chloric acid is (Pebal) a mixture of chlorine dioxide and free chlorine. The liquid and gaseous chlorine oxide (Note 35), which Millon considered to be $\mathrm{Cl}_{2} \mathrm{O}_{3}$, probably contains a mirture of $\mathrm{ClO}_{2}$ (vapour density 35 ), $\mathrm{Cl}_{2} \mathrm{O}_{3}$ (whose vapour density should be 59), and chlorine (vapour density $85^{\circ} 5$ ), since its vapour density was determined to be about 40 . 
perchloric acid, $\mathrm{HClO}_{4}$, are produced from the salts of chloric acid, $\mathrm{HClO}_{3}$. But this is the highest form of the oxidation of $\mathrm{HCl}$. Perchloric acid, $\mathrm{HClO}_{4}$, is the most stable of all the acids of chlorine. When fused potassium chlorate begins to swell up and solidify, after having parted with one-third of its oxygen, potassium chloride and potassium perchlorate have been formed according to the equation $2 \mathrm{KClO}_{3}$ $=\mathrm{KClO}_{4}+\mathrm{KCl}+\mathrm{O}_{2}$.

The formation of this salt is easily observed in the preparation of oxygen from potassium chlorate, owing to the fact that the potassium perchlorate fuses with greater difficulty than the chlorate, and therefore appears in the molten salt as solid grains (see Chapter III. Note 12). Under the action of certain acids-for instance, sulphuric and nitric-potassium chlorate also gives potassium perchlorate. This latter may be easily purified, because it is but sparingly soluble in water, although all the other salts of perchloric acid are very soluble and even deliquesce in the air. The perchlorates, although they contain more oxygen than the chlorates, are decomposed with greater difficulty, and even when thrown on ignited charcoal give a much feebler deflagration than the chlorates. Sulphuric acid (at a temperature not below $100^{\circ}$ ) evolves volatile and to a certain extent stable perchloric acid from potassium perchlorate. Neither sulphuric nor any other acid will further decompose perchloric acid as it decomposes chloric acid. Of all the acids of chlorine, perchloric acid alone can be distilled.44 The pure hydrate $\mathrm{HClO}_{4}{ }^{45}$ is a colourless and exceedingly caustic substance

44 If a solution of chloric acíd, $\mathrm{HClO}_{3}$, be first concentrated over sulphuric acid under the receiver of an air-pump and afterwards distilled, chlorine and oxygen are evolved and perchloric acid is formed: $4 \mathrm{HClO}_{3}=2 \mathrm{HClO}_{4}+\mathrm{Cl}_{2}+8 \mathrm{O}+\mathrm{H}_{2} \mathrm{O}$. Roscoe accordingly decomposed directly a solution of potassium chlorate by hydrofluosilicic acid, decanted it from the precipitate of potassium silicofluoride, $\mathrm{K}_{2} \mathrm{SiF}_{6}$, concentrated the solution of chloric acid, and then distilled it, perchloric acid being then obtained (see following footnote). That chloric acid is capable of passing into perchloric acid is alsa seen from the fact that potassium permanganate is decolorised, although slowly, by the action of a solution of chloric acid. On decomposing a solution of potassium chlorate by the action of an electric current, potassium perchlorate is obtained at the positive electrode (where the oxygen is evolved). Perchloric acid is also formed by the action of an electric current on solutions of chlorine and chlorine monoxide. Perchloric acid was obtained by Count Stadion and afterwards by Serullas, and was studied by Roscoe and others.

45 Perchloric acid, which is obtained in a free state by the action of sulphuric acid on its salts, may be separated from a solution very easily by distillation, being volatile, although it is partially decomposed by distillation. The solution obtained after distillation may be concentrated by evaporation in open vessels. In the distillation the solution reaches a temperature of $200^{\circ}$, and then a very constant liquid hydrate of the composition $\mathrm{HClO}_{4}, 2 \mathrm{H}_{2} \mathrm{O}$ is obtained in the distillate. If this hydrate be mixed with sulphuric acid, it begins to decompose at $100^{\circ}$, but nevertheless a portion of the acid passes over into the receiver without decomposing, forming a crystalline hydrate $\mathrm{HClO}_{4}, \mathrm{H}_{2} \mathrm{O}$ which melts at $50^{\circ}$. On carefully heating this hydrate it breaks up into perchloric acid, which distils over below $100^{\circ}$, and into the liquid hydrate $\mathrm{HClO}_{4}, 2 \mathrm{H}_{2} \mathrm{O}$. The acid $\mathrm{HClO}_{4}$ may 
which fumes in the air and has a specific gravity $1 ; 78$ at $15^{\circ}$ (sometimes, after being kept for some time, it decomposes with a violent ex. plosion). It explodes violently when brought into contact with charcoal, paper, wood, and other organic substances. If a small quantity of water be added to this hydrate, and it be cooled, a .crystallo-hydrate, $\mathrm{ClHO}_{4}, \mathrm{H}_{2} \mathrm{O}$, separates out. This is much more stable, but the liquid hydrate $\mathrm{HClO}_{4}, 2 \mathrm{H}_{2} \mathrm{O}$ is still more so. The acid dissolves in water in all proportions, and its solutions are distinguished for their stability. ${ }^{46}$ When ignited both. the acid and its salts are decomposed, with the evolution of oxygen. ${ }^{47}$

also be obtained by adding one-fourth part of strong sulphuric acid to potassium chlorate, carefully distilling and subjecting the crystals of the hydrate $\mathrm{HClO}_{4}, \mathrm{H}_{2} \mathrm{O}$ obtained in the distillate to a fresh distillation. Perchlorio acid, $\mathrm{HClO}_{4}$, itself does not distil, and is decomposed on distillation until the more stable hydrate $\mathrm{HClO}_{4}, \mathrm{H}_{2} \mathrm{O}$ is formed; this decomposes into $\mathrm{HClO}_{4}$ and $\mathrm{HClO}_{4}, 2 \mathrm{H}_{2} \mathrm{O}$; which latter hydrate distils withont decom. position. This forms an excellent example of the influence of water on stability, and of the property of chlorine of giving compounds of the type $\mathrm{ClX}_{7}$, of which all the above hydrates, $\mathrm{ClO}_{3}(\mathrm{OH}), \mathrm{ClO}_{2}(\mathrm{OH})_{3}$, and $\mathrm{ClO}(\mathrm{OH})_{5}$, are members. Probably further research will lead to the discovery of a hydrate $\mathrm{Cl}(\mathrm{OH})_{7}$.

46 According to Roscoe the specific gravity of perchloric acid $=1.782$ and of the bydrate $\mathrm{HClO}_{4}, \mathrm{H}_{2} \mathrm{O}$ in a liquid state $\left(50^{\circ}\right) 1.811$; hence a considerable contraction takes place in the combination of $\mathrm{HClO}_{4}$ with $\mathrm{H}_{2} \mathrm{O}$.

47 The decomposition of salts analogous to potassium chlorate has been more fully studied in recent years by Potilitzin and P. Frankland. Professor Potilitzin, by decomposing, for example, lithium chlorate $\mathrm{LiClO}_{3}$, found (from the quantity of lithium chloride and oxygen) that at first the decomposition of the fused salt $\left(368^{\circ}\right)$ takes place according to the equation, $3 \mathrm{LiClO}_{3}=2 \mathrm{LiCl}+\mathrm{LiClO}_{4}+50$, and that towards the end the remaining salt is decomposed thus: $5 \mathrm{LiClO}_{3}=4 \mathrm{LiCl}+\mathrm{LiClO}_{4}+100$. The phenomens observed by Potilitzin obliged him to admit that lithium perchlorate is capable of decom. posing simultaneously with lithium chlorate, with the formation of the latter salt and oxygen; and this was confirmed by direct experiment, which showed that lithium chlorate is always formed in the decomposition of the perchlorate. Potilitzin drew particular attention to the fact that the decomposition of potassinm chlorate and of salts analogous to it, although exothermal (Chapter III., Note 12), not only does not proceed spontaneously, but requires time and a rise of temperature in order to attain completion, which again shows that chemical equilibria are not determined by the heat effects of ro. ections only.

P. Frankland and J. Dingwall (1887) showed that at $448^{\circ}$ (in the vapour of sulphur) \& mixture of potassium chlorate and powdered glass is decomposed almost in accordanci $\theta$ with the equation $2 \mathrm{KClO}_{3}=\mathrm{KClO}_{4}+\mathrm{KCl}+\mathrm{O}_{2}$, whilst the salt by itself evolves about half as much oxygen, in accordance with the equation, $8 \mathrm{KClO}_{3}=5 \mathrm{KClO}_{4}+8 \mathrm{KCl}+2 \mathrm{O}_{2}$. The decomposition of potassium perchlorate in admixture with manganese peroxide proceeds to completion, $\mathrm{KClO}_{4}=\mathrm{KCl}+2 \mathrm{O}_{2}$. But in decomposing by itself the salt at first gives potassium chlorate, approximately according to the equation $7 \mathrm{KClO}_{4}=2 \mathrm{KClO}_{3}+5 \mathrm{KC}$ i $+110_{2}$. Thus there is now no doubt that when potassium chlorate is heated, the perchlorate is formed, and that this salt, in decomposing with evolution of oxygen, again gives the former salt.

In the decomposition of barium hypochlorite, 50 per cent. of the whole amount pasees into chlorate, in the decomposition of strontium hypochlorite (Potilitzin, 1890) 12.5 per cent., and of calcium hypochlorite about 2.5 per cent. Besides which Potilitzin showed that the decomposition of the hypochlorites and also of the chlorates is always accom. panied by the formation of a certain quantity of the oxides and by the evolution of 
On comparing chlorine as an element not only with nitrogen and carbon but with all the other non-metallic elements (chlorine has so little analogy with the metals that a comparison with them would be superfluous), we find in it the following fundamental properties of the halogens or salt-producers. With metals chlorine gives selts (such as sodium chloride, \&c.); with hydrogen a very energetic and monobasic acid $\mathrm{HCl}$, and the same quantity of chlorine is able by metalepsis to replace the hydrogen; with oxygen it forms unstable oxides of an acid character. These properties of chlorine are possessed by three other elements, bromine, iodine, and fluorine. They are members of one natural family. Each representative has its peculiarities, its individual properties and points of distinction, in combination and in the free state-otherwise they would not be independent elements; but the repetition in all of them of the same chief characteristics of the family enables one more quickly to grasp all their various properties and to classify the elements themselves.

In order to have a guiding thread in forming comparisons between the elements, attention must however be turned not only to their points of resemblance but also to those of their properties and characters in which they differ most from each other. And the atomic weights of the elements must be considered as their most elementary property, since this is a quantity which is most firmly established, and must be taken account of in all the reactions of the element. The halogens have the following atomic weights-

$$
\mathrm{F}=19, \quad \mathrm{Cl}=35 \cdot 5, \quad \mathrm{Br}=80, . \quad \mathrm{I}=127 .
$$

All the properties, physical and chemical, of the elements and their corresponding compounds must evidently be in a certain dependence

chlorine, the chlorine being displaced by the oxygen disengaged. Spring and Prost (1889) represent the evolution of oxygen from $\mathrm{KClO}_{3}$ as due to the salt first splitting up into base and anhydride, thus (1) $2 \mathrm{MClO}_{5}=\mathrm{M}_{2} \mathrm{O}+\mathrm{Cl}_{2} \mathrm{O}_{5}$; (2) $\mathrm{Cl}_{2} \mathrm{O}_{5}=\mathrm{Cl}_{2}+\mathrm{O}_{5}$; and (8) $\mathrm{M}_{2} \mathrm{O}+\mathrm{Cl}=2 \mathrm{MCl}+\mathrm{O}$.

I may further remark that the decomposition of potassium chlorate as a reaction evolving heat easily lends itself for this very reason to the contect action of manganese peroxide and other similar admixtures; for such very feeble influences as those of contact may become evident either in those cases (for instance, detonating gus, hydrogen peroxide, \&c.), when the reaction is accompanied by the evolution of heat, or when (for instance, $\mathrm{H}_{2}+\mathrm{I}_{2}$, \&c.) little heat is absorbed or evolved. In these cases it is evident that the existing equilibrium is not very stable, and that a small alteration in the conditions at the surfaces of contact may suffice to npset it. In. order to conceive the modus operandi of contact phenomena, it is enough to imagine, for instance, that a the surface of contact the movement of the atoms in the molecules changes from a circular to an elliptical path. Momentary and transitory compounds may be formed, bat their formation cannot affect the explanation of the phenomena. 
on this fundamental point, if the grouping in one family be natural.47 bis And we find in reality that, for instance, the properties of bromine, whose atomic weight is almost the mean between those of iodine and chlorine, occupy a mean position between those of these two elements. The second measurable property of the elements is their equivalence or their capacity for forming compounds of definite forms. Thus carbon or nitrogen in this respect differs widely from the halogens. Although the form $\mathrm{ClO}_{2}$ corresponds with $\mathrm{NO}_{2}$ and $\mathrm{CO}_{2}$, yet the last is the highest oxide of carbon, whilst that of nitrogen is $\mathrm{N}_{2} \mathrm{O}_{5}$, and for chlorine, if there were an anhydride of perchloric acid, its composition would be $\mathrm{Cl}_{2} \mathrm{O}_{7}$, which is quite different from that of carbon. In respect to the forms of their compounds the halogens, like all elements of one family or group, are perfectly analogous to each other, as is seen from their hydrogen compounds :

\section{$\mathrm{HF}, \mathrm{HCl}, \mathrm{HBr}, \mathrm{HI}$.}

Their oxygen compounds exhibit a similar analogy. Only fluorine does not give any oxygen compounds. The iodine and bromine com. pounds corresponding with $\mathrm{HClO}_{3}$ and $\mathrm{HClO}_{4}$ are $\mathrm{HBrO}_{3}$ and $\mathrm{HBrO}_{4}$, $\mathrm{HIO}_{3}$ and $\mathrm{HIO}_{4}$. On comparing the properties of these acids we can even predict that fluorine will not form any oxygen compound. For iodine is easily oxidised-for instance, by nitric acid-whilst chlorine is not directly oxidised. The oxygen acids of iodine are com. paratively more stable than those of chlorine ; and, generally speaking, the affinity of iodine for oxygen is much greater than that of chlorine. Here also bromine occupies an intermediate position. In fluorine we may therefore expect a still smaller affinity for oxygen than in chlorine-and up to now it has not been combined with oxygen. If any oxygen compounds of fluorine should be obtained; they will naturally be exceedingly unstable. The relation of these elements to hydrogen is the reverse of the above. Fluorine has so great an affinity for hydrogen that it decomposes water at the ordinary temperature; whilst iodine

47 bs See, for example the melting point of $\mathrm{NaCl}, \mathrm{NaBr}, \mathrm{NaI}$ in Chapter II. Note 27. According to F. Freyer and V. Meyer (1892), the following are the boiling points of some of the corresponding compounds of chlorine and bromine:

$\begin{array}{rrlr}\mathrm{BCl}_{3} & 17^{\circ} & \mathrm{BBr}_{5} & 90^{\circ} \\ \mathrm{SiCl}_{3} & 59^{\circ} & \mathrm{SiBr}_{4} & 153^{\circ} \\ \mathrm{PCl}_{5} & 76^{\circ} & \mathrm{PBr}_{3} & 175^{\circ} \\ \mathrm{SbCl}_{3} & 223^{\circ} & \mathrm{SbBr}_{3} & 275^{\circ} \\ \mathrm{BiCl}_{3} & 447^{\circ} & \mathrm{BiBr}_{5} & 453^{\circ} \\ \mathrm{SnCl}_{4} & 606^{\circ} & \mathrm{SnBr}_{4} & 619^{\circ} \\ \mathrm{ZnCl}_{2} & 730^{\circ} & \mathrm{ZnBr}_{2} & 650^{\circ}\end{array}$

Thus for all the more volatile compounds the replacement of chlorine by bromine raises the boiling point, but in the case of $\mathrm{ZnX}$, it lowers it (Chapter XV. Note 19). 
has so little aftinity for hydrogen that hydriodic acid, HI, is formed with difficulty, is easily decomposed, and acts as a reducing agent in a number of cases.

From the form of their compounds the halogens are univalent elements with respect to hydrogen and septivalent with respect to oxygen, $\mathrm{N}$ being trivalent to hydrogen (it gives $\mathrm{NH}_{3}$ ) and quinquivalent to oxygen (it gives $\mathrm{N}_{2} \mathrm{O}_{5}$ ), and $\mathrm{C}$ being quadrivalent to both $\mathrm{H}$ and $\mathrm{O}$ as it forms $\mathrm{CH}_{4}$ and $\mathrm{CO}_{2}$. And as not only their oxygen compounds, but also their hydrogen compounds, have acid properties, the halogens are elements of an exclusively acid character. Such metals as sodium, potassium, barium only give basic oxides. In the case of nitrogen, although it forms acid oxides, still in ammonia we find that capacity to give an alkali with hydrogen which indicates a less distinctly acid character than in the halogens. In no other elements is the acidgiving property so strongly developed as in the halogens.

In describing certain peculiarities characterising the halogens, we shall at every step encounter a confirmation of the above-mentioned general relations.

As fuorine decomposes water with the evolution of oxygen, $\mathrm{F}_{2}+\mathrm{H}_{2} \mathrm{O}=2 \mathrm{HF}+\mathrm{O}$, for a long time all efforts to obtain it in free state by means of methods similar to those for the preparation of chlorine proved fruitless. ${ }^{48}$ Thus by the action of hydrofluoric acid on manganese peroxide, or by decomposing a solution of hydrofluoric acid by an electric current, either oxygen or a mixture of oxygen and fluorine were obtained instead of fluorine. Probably a certain quantity of fluorine 48 bis was set free by the action of oxygen or an electric current on incandescent and fused calcium fluoride, but at a high temperature fluorine acts even on platinum, and therefore it was not obtained When chlorine acted on silver fuoride, $\mathrm{AgF}$, in a vessel of natural fluor spar, $\mathrm{CaF}_{2}$, fluorine was also liberated; but it was mixed

48 Even before free fluorine was obtained (1886) it was evident from experience gained in the efforts made to obtain it, and from analogy, that it would decompose water (see first Russian edition of the Principles of Chemistry).

$48 \mathrm{sto}$ It is most likely that in this experiment of Fremy's, which corresponds with the action of oxygen on calcium chloride, fluorine was set free, but that a converse reaction also proceeded, $\mathrm{CaO}+\mathrm{F}_{2}=\mathrm{CaF}_{2}+\mathrm{O}$-that is, the calcium distributed itself between the oxygen and fluorine. $\mathrm{MnF}_{4}$, which is capable of splitting up into $\mathrm{MnF}_{2}$ and $\mathrm{F}_{2}$, is without doubt formed by the action of a strong solution of hydrofluoric acid on manganese peroxide, but under the action of vater the fluorine gives hydrofluorio acid, and probably this is aided by the affinity of the manganese fluoride and hydrofluoric acid. In all the attempts made (by Davy, Knox, Louget, Fremy, Gore, and others) to decompose fluorides (those of lead, silver, calcium, and others) by chlorine, there were doubtless also cases of distribution, a portion of the metal combined with chlorine and a portion of the fluorine was evolved; but it is improbable that any decisive results were obtained. Fremy probably obtained fluorine, bùt nót in a pure state. 
with chlorine, and it was impossible to study the properties of the resultant gas. Brauner (1881) also obtained fluorine by igniting cerium fluoride, $2 \mathrm{CeF}_{4}=2 \mathrm{CeF}_{3}+\mathrm{F}_{2}$; but this, like all preceding efforts, only showed fluorine to be a gas which decomposes water, and is capable of acting in a nuppber of instances like chlorine, but gave no possibility of testing its properties. It was evident that it was necessary to avoid as far as possible the presence of water and a rise of temperature; this Moissan succeeded in doing in 1886. He decomposed anhydrous hydrofluoric acid, liquefied at a temperature of $-23^{\circ}$ and contained in a $U$-shaped tube (to which a small quantity of potassium fluoride had been added to make it a better conductor), by the action of a powerful electric current (twenty Bunsen's elements in series). Hydrogen was then evolved at the negative pole, and fluorine appeared at the positive pole (of iridium platinum) as a pale green gas which decomposed water with the formation of ozone and hydrofluoric acid, and combined directly with silicon (forming silicon fluoride, $\mathrm{SiF}_{4}$ ), boron (forming $\mathrm{BF}_{3}$ ), sulphur, \&c. Its density $(\mathrm{H}=1)$ is 18 , so that its molecule is $\mathbf{F}_{2}$. But the action of fluorine on metals at the ordinary temperature is comparatively feeble, because the metallic fluoride formed coats the remaining mass of the metals; it is, however, completely absorbed by iron. Hydrocarbons (such as naphtha), alcohol, \&c., immediately absorb fluorine, with the formation of hydrofluoric acid. Fluorine when mixed with hydrogen can easily be made to explode violently, forming hydrofluoric acid. ${ }^{49}$

In 1894, Brauner obtained fluorine directly by igniting the easily

49 According to Moissan, fluorine is disengaged by the action of an electric current on fused hydrogen potassium fluoride, $\mathrm{KHF}_{2}$. The present state of chemical knowledge is such that the knowledge of the properties of an element is much more general than the knowledge of the free element itself. It is useful and satisfactory to learn that even fluorine in the free state has not succeeded in eluding experiment and research, that the efforts to isolate it have been crowned with success, but the sum total of chemical data concerning fluorine as an element gains but little by this achievement. The gain will, however, be augmented if it be now possible to subject fluorine to a comparative study in relation to oxygen and chlorine. There is particular interest in the phenomena of the distribution of fluorine and oxygen, or fluorine and chlorine, competing under different conditions and relations. We may add that Moissan (1892) found that free fluorine decomposes $\mathrm{H}_{2} \mathrm{~S}, \mathrm{HCl}, \mathrm{HBr}, \mathrm{CS}_{2}$, and $\mathrm{CNH}$ with a flash; it does not act upon $\mathrm{O}_{2}, \mathrm{~N}_{2}, \mathrm{CO}$, and $\mathrm{CO}_{2} ; \mathrm{Mg}, \mathrm{Al}, \mathrm{Ag}$, and $\mathrm{Ni}$, when heated, burn in it, as also do $\mathrm{S}, \mathrm{Se}, \mathrm{P}$ (forms $\mathrm{PF}_{5}$ ); it reacts upon $\mathrm{H}_{2}$ even in the dark, with the evolution of $366^{\circ} 00$ nnits of heat. At a temperature of $-95^{\circ}, \mathrm{F}_{2}$ still retains its gaseous statè. Soot and carbon in gẹneral (but not the diamond) when heated in gaseous fluorine form fiuoride of carbon, $\mathrm{CF}_{4}$ (Moissan, 1890); this compound is also formed at $800^{\circ}$ by the double decomposition of $\mathrm{CCl}_{4}$ and $\mathrm{AgF}$; it is a gas which liquefies at $10^{\circ}$ under a pressure of 5 atmospheres. With an alcoholic solution of $\mathrm{KHO}, \mathrm{CF}_{4}$ gives $\mathrm{K}_{2} \mathrm{CO}_{5}$, according to the equation $\mathrm{CF}_{4}+6 \mathrm{KHO}$ $=\mathrm{K}_{2} \mathrm{CO}_{3}+4 \mathrm{KF}+3 \mathrm{H}_{2} \mathrm{O}$. $\mathrm{CF}_{4}$ is not soluble in water, but it is easily soluble in $\mathrm{CCl}_{4}$ and alcohol 
formed 49 bis double lead salt $\mathrm{HF}, 3 \mathrm{KF}, \mathrm{PbF}_{4}$, which first, at $230^{\circ}$, decomposes with the evolution of $\mathrm{HF}$, and then splits up forming $3 \mathrm{KF}, \mathrm{PbF}_{2}$ and fluorine $\mathrm{F}_{2}$, which is recognised by the fact that it liberates iodine from KI and easily combines with silicon, forming $\mathrm{SiF}_{4}$. This method gives chemically pure fluorine, and is based upon the breaking up of the higher compound-tetrafluoride of lead, $\mathrm{PbF}_{4}$, corresponding to $\mathrm{PbO}_{2}$, into free fluorine, $\mathrm{F}_{2}$, and the lower more stable form-bifluoride of lead, $\mathrm{PbF}_{2}$, which corresponds to $\mathrm{PbO}$; that is, this method resembles the ordinary method of obtaining chlorine by means of $\mathrm{MnO}_{2}$, as $\mathrm{MnCl}_{4}$ here breaks up into $\mathrm{MnCl}_{2}$ and chlorine, just as $\mathrm{PbF}_{4}$ splits up into $\mathrm{PbF}_{2}$ and fluorine.

Among the compounds of fluorine, calcium fluoride, $\mathrm{CaF}_{2}$, is somewhat widely distributed in nature as fluor spar, $^{50}$ whilst cryolite, or aluminium sodium fluoride, $\mathrm{Na}_{\triangleleft} \mathrm{AlF}_{6}$, is found more rarely (in large masses in Greenland). Cryolite, like fluor spar, is also insoluble in water, and gives hydrotluoric acid with sulphuric acid. Small quantities of fluorine have also in a number of cases been found in the bodies of animals, in the blood, urine, and bones. If fluorides occur in the bodies of animals, they must have been introduced in food, and must occur in plants and in water. And as a matter of fact river, and especially sea, water alwuys contains a certain, although small, quantity of fluorine compounds.

Hydrofluoric acid, $\mathrm{HF}$, cannot be obtained from fluor spar in glass retorts, because glass is acted on by and destroys the acid. It is

49 bis T. Nikolukin (1885) and subsequently Friedrich and Classen obtained $\mathrm{PbCl}_{4}$ and a double ammonium salt of tetrachloride of lead (starting from the binoxide), $\mathrm{PbCl}_{4} 2 \mathrm{NH}_{4} \mathrm{Cl}$; Hutchinson and Pallard obtained a similar salt of acetic acid (1893) corresponding to $\mathrm{PbX}_{4}$ by treating red lead with strong acetic acid; the composition of this salt is $\mathrm{Pb}\left(\mathrm{C}_{2} \mathrm{H}_{3} \mathrm{O}_{2}\right)_{4}$; it melts (and decomposes) at about $175^{\circ}$. Branner (1894) pbtained a salt corresponding to tetrafluoride of lead, $\mathrm{PbF}_{4}$, and the acid corresponding to it, $\mathrm{H}_{4} \mathrm{PbF}_{8}$. For example, by treating potassium plumbate (Chapter XVIII. Note 55) with strong $\mathrm{HF}$, and also the above-mentioned tetra-acetate with a solution of $\mathrm{KHF}_{2}$, Brauner obtained crystalline $\mathrm{HK}_{5} \mathrm{PbF}_{8}$-i.e. the salt from which he obtained fluorine.

so It is called spar because it very frequently occurs as crystals of a clearly laminar structure, and is therefore easily split up into pieces bounded by planes. It is called fluor spar because when used as a flux it renders ores fusible, owing to its reacting with silica, $\mathrm{SiO}_{2}+2 \mathrm{CaF}_{2}=2 \mathrm{CaO}_{2}+\mathrm{SiF}_{4}$; the silicon fluoride escapes as a gas and the lime combines with a further quantity of silica, and gives a vitreous slag. Fluor spar occurs in mineral veins and rocks, sometimes in considerable quantities. It always crystallises in the cubic system, sometimes in very large semi-transparent cubic crystals, which are colourless or of different colours. It is insoluble in water. It melts under the action of heat, and crystallises on cooling. The specific gravity is 3.1. When steam is passed over incan. descent fluor spar, lime and hydrofluoric acid are formed: $\mathrm{CaF}_{2}+\mathrm{H}_{2} \mathrm{O}=\mathrm{CaO}+2 \mathrm{HF} \quad A$ double decomposition is also easily produced by fusing fluor spar with sodium or potassium hydroxides, or potash, or even with their carbonates; the fluorine then palsses over to the potassium or sodium, and the oxygen to the calcium. Iu solutions-for example, $\mathrm{Ca}\left(\mathrm{NO}_{3}\right)_{2}+2 \mathrm{KF}=\mathrm{CaF}_{2}$ (precipitate) $\div 2 \mathrm{KNO}_{3}$ (in solution)-the formation of caloium fluoride takes place, owing tó its very sparing solubility. 26,000 parts of water dissolve one part of fluor spar. 
prepared in lead vessels, and when it is required pure, in platinum vessels, because lead also acts on hydrofluoric acid, although only very feebly on the surface, and when once a coating of fluoride and sulphate of lead is formed no further action takes place. Powdered fluor spar and sulphuric acid evolve hydrofluoric acid (which fumes in the air) even at the ordinary temperature, $\mathrm{CaF}_{2}+\mathrm{H}_{2} \mathrm{SO}_{4}=\mathrm{CaSO}_{4}+2 \mathrm{HF}$. At $130^{\circ}$ fluor spar is completely decomposed by sulphuric acid. The acid is then evolved as vapour, which may be condensed by a freezing mixture into an anhydrous acid. The condensation is aided by pouring water into the receiver of the condenser, as the acid is easily soluble in cold water.

In the liquid anhydrous form hydrofluoric acid boils at $+19^{\circ}$, and its specific gravity at $12.8^{\circ} \doteq 0.9849 .51$ It dissolves in water with the evolution of a considerable amount of heat, and gives a solution of constant boiling point which distils over at $120^{\circ}$; showing that the acid is able to combine with water. The specific gravity of the compound is $1 \cdot 15$, and its composition $\mathrm{HF}, 2 \mathrm{H}_{2} \mathrm{O} .{ }^{52}$ With an excess of water a dilute solution distils over first. The aqueous solution and the acid itself must be kept in platinum vessels, but the dilute acid may be conveniently preserved in vessels made of various organic materials, such as guttapercha, or even in glass vessels having an interior coating of paraffin. Hydrofluoric acid does not act on hydrocarbons and many other substances, but it acts in a highly corrosive manner on metals, glass, porcelain, and the majority of rock substances. ${ }^{33}$ It also attacks the skin,

31 According to Gore. Fremy obtained anhydrous hydrofluoric acid by decomposing lead fluoride at a red heat, by hydrogen, or by heating the double salt $\mathrm{HKF}_{2}$, which easily crystallises (in cubes) from a solution of hydrofluoric acid, half of which has been saturated with potassium hydroxide. Its vapour density corresponds to the formula HF.

32 This composition corresponds to the crystallo-hydrate $\mathrm{HCl}, 2 \mathrm{H}_{2} \mathrm{O}$. All the properties of hydrofluorio acid recall those of hydrochloric acid, and therefore the com. parative ease with which hydrofluoric acid is liquefied (it boils at $+19^{\circ}$, hydrochloric acid at $-35^{\circ}$ ) must be explained by a polymerisation taking place at low temperatures, as will be afterwards explained, $\mathrm{H}_{2} \mathrm{~F}_{2}$, being formed, and therefore in a liquid state it differs from hydrochloric acid, in which a phenomenon of a similar kind has not yet been observed.

33 The corrosive action of hydrofluoric acid on glass and similar siliceous compounds is based upon the fact that it acts on silica, $\mathrm{SiO}_{2}$, as we shall consider more fully in describing that compound, forming gaseous silicon fluoride, $\mathrm{SiO}_{2}+4 \mathrm{HF}=\mathrm{SiF}_{4}+2 \mathrm{H}_{2} \mathrm{O}$. Silica, on the other hand, forms the binding (scid) element of glass and of the mass of mineral substances forming the salts of silica. When it is removed the cohesion is destroyed. This is made use of in the arts, and in the laboratory, for etching designs and scales, \&c., on glass. In engraving on glass the surface is covered with a varnish composed of four parts of wax and one part of turpentine. This varnish is not acted on by hydrofluoric acid, and it is soft enough to allow of designs being drawn upon it whose lines lay bare the glass. The drawing is made with a steel point, and the glass is afterwards laid in a lead trough in which a mixture of fiuor spar and sulphuric acid is placed. The sulphuric acid must be used in considerable excess, as otherwise transparent lines are 
and is distinguished by its poisonous properties, so that in working with the acid a strong draught must be kept up, to prevent the possibility of the fumes being inhaled. The non-metals do not act on hydrofluoric acid, but all metals-with the exception of mercury, silver, gold, and platinum, and, tó a certain degree, lead-decompose it with the evolution of hydrogen. With bases it gives directly metallic fluorides, and behaves in many respects like hydrochloric acid. There are, however, several distinct individual differences, which are furthermore much greater than those between hydrochloric, hydrobromic, and hydriodic acids. Thus the silver compounds of the latter are insoluble in water, whilst silver fluoride is soluble. Calcium fluoride, on the contrary, is insoluble in water, whilst calcium chloride, bromide, and iodide are not only soluble, but attract water with great energy. Neither hydrochloric, hydrobromic, nor hydriodic acid acts on sand and glass, whilst hydrofluoric acid corrodes them, forming gaseous silicon fluoride. The other halogen acids only form normal salts, $\mathrm{KCl}, \mathrm{NaCl}$, with $\mathrm{Na}$ or $\mathrm{K}$, whilst hydrofluoric acid gives acid salts, for instance $\mathrm{HKF}_{2}$ (and by dissolving $\mathrm{KF}$ in liquid $\mathrm{HF}, \mathrm{KHF}_{2} 2 \mathrm{HF}$ is obtained). This latter property is in close connection with the fact that at the ordinary temperature the vapour density of hydrofluoric acid is nearly 20 , which corresponds with a formula $\mathrm{H}_{2} \mathrm{~F}_{2}$, as Mallet (1881) showed; but a depolymerisation occurs with a rise of temperature, and the density approaches 10 , which answers to the formula HF. ${ }^{34}$

The analogy between chlorine and the other two halogens, bromine and iodine, is much more perfect. Not only have their hydrates or halogen acids much in common, but they themselves resemble chlorine in many respects, ${ }^{65}$ and even the properties of the corresponding

obtained (owing to the formation of hydrofluosilicic acid). After being exposed for some time, the, varnish is removed (melted) and the design drawn by the steel point is found reproduced in dull lines. The drawing may be also made by the direct application of a mixture of a silicofluoride and sulphurie acid, which forms hydrofluoric acid.

3. Mallet (1881) determined the density at $80^{\circ}$ and $100^{\circ}$, previous to which Gore (1869) had determined the vapour density at $100^{\circ}$, whilst Thorpe and Hambly (1888) made fourteen determinations between $26^{\circ}$ and $88^{\circ}$, and showed that within this limit of temperature the density gradually diminishes, just like the vapour of acetio acid, nitrogen dioxide, and others. The tendency of $\mathrm{HF}$ to polymerise into $\mathrm{H}_{2} \mathrm{~F}_{2}$ is probably connected with the property of many fluorides of forming acid salts-for example, $\mathrm{KHF}_{2}$ and $\mathrm{H}_{2} \mathrm{SiF}_{6}$. We saw above that $\mathrm{HCl}$ has the same property (forming, for instance, $\mathrm{H}_{2} \mathrm{PtCl}_{6}$, \&o., p. 457), and hence this property of hydrofluorio acid does not stand isolated from the properties of the other halogens.

5s For instance, the experiment with Dutch metal foil (Note 16) may be made with bromine just as well as with ohlorine. A very instructire experiment on the direot com. bination of the halogens with metals may be made by throwing a small piece (a shaving) of aluminium into a vessel containing liquid bromine; the aluminium, being lighter, floats on the bromine, and after a certain time reaction sets in accompanied by the evolution of heat, light, and fumes of bromine. The incondescent piece of metal moves trpidly 
metallic compounds of bromine and iodine are very much alikê. Thus, the chlorides, bronides, and iorlides of sodium and potassium crystallise in the cubic system, and are soluble. in water; the chlorides of calcium, aluminiur, magnesium, and barium are just as soluble in water as the bromides and iodides of these metals. The iodides and bromides of silver and lead are sparingly soluble in water, like the chlorides of these metals. The oxygen compounds of bromine and iodine also present.a very strong analugy to the corresponding compounds of chlorine: A hypobromous acid is known corresponding with hypo. chlorous acid. The salts of this acid have the same bleaching property as the salts of hypochlorous acid. Iodine was discovered in 1811 by Courtois in kelp, and was shortly afterwards investigated by Clement, Gay-Lussac, and Davy. Bromine was discovered in 1826 by Balard in the mother liquor of sea water.

Bromine and iodine, like chlorine, occur in sea water in combina. tion with metals. However, the amount of bromides, and especially of iodides, in sea water is so small that their presence can only be discovered by means of sensitive reactions. ${ }^{56}$ In the extraction of salt from sea water the bromides remain in the mother liquor. Iodine and bromine also occur combined with silver, in admixture with silver chloride, as a rare ore which is mainly found in America. Certain

over the surface of the bromine in which the resultant aluminium bromide dissolves. For the sake of comparison we will proceed to cite several thermochemical data (Thom. sen) for analogous actions of (1) chlorine, (2) bromine, and (3) iodine, with respect to metals; the halogen being expressed by the symbol $\mathbf{X}$, and the plus sign connecting the reacting substances. All the figures are given in thousands of celories, and refer to molecular quantities in grams and to the ordinary temperature :-

$\begin{array}{lrrr} & 1 & 2 & 3 \\ \mathrm{Z}_{2}+\mathrm{X}_{2} & 211 & 191 & 160 \\ \mathrm{Na}_{2}+\mathrm{X}_{2} & 105 & 172 & 188 \\ \mathrm{Ag}_{2}+\mathrm{X}_{2} & 59 & 45 & 28 \\ \mathrm{Hg}_{2}+\mathrm{X}_{2} & 83 & 68 & 18 \\ \mathrm{Hg}_{\mathrm{g}}+\mathrm{X}_{2} & 68 & 51 & 84 \\ \mathrm{C} \mathrm{Z}_{2}+\mathrm{X}_{2} & 170 & 141 & - \\ \mathrm{Ba}+\mathrm{X}_{2} & 195 & 170 & - \\ \mathrm{Zn}+\mathrm{X}_{2} & 97 & 76 & 49 \\ \mathrm{~Pb}+\mathrm{X}_{2} & 83 & 64 & 40 \\ \mathrm{Al}+\mathrm{X}_{2} & 161 & 120 & 70^{\circ}\end{array}$

We may remark that the latent heat of vaporisation of the molecular weight $\mathrm{Br}_{2}$ is about $7 \cdot 2$, and of iodine 6.0 thousand heat units, whilst the latent heat of fusion of $\mathrm{Br}_{2}$ is about 0.8 , and of $I_{2}$ about 8.0 thousand heat units. From this it is evident that the difference between the amounts of heat evolved does not depend on the difference in physical state. For instance, the vapour of iodine in combining with $\mathrm{Zn}$ to form $\mathrm{ZnI}_{2}$ would give $48+8+8$, or about sixty thousand heat units, or $1 \frac{1}{2}$ times less than $\mathrm{Zn}+\mathrm{Cl}_{2}$.

so One litre of sea-water contains about 20 grams of chlorine, and about $0.07 \mathrm{gram}$ of bromine. The Dead Sea contains about ten times as much bromine. 
mineral waters (those of Kreuznach and Staro-rossüsk) contain metallio bromides and iodides, always in admixture with an excess of sodium chloride. Those upper strata of the Stassfurt rock salt (Chapter X.) which are a source of potassium salts also contain metallic bromides, ${ }^{37}$ which collect in the mother liquors left after the crystallisation of the potassium salts; and this now forms the chief source (together with certain American springs) of the bromine in common use. Bromine may be easily liberated from a mixture of bromides and chlorides, owing to the fact that chlorine displaces bromine from its compounds with sodium, magnesium, calcium, \&c. A colourless solution of bromides and chlorides turns an orange colour after the passage of chlorine, owing to the disengagement of bromine. ${ }^{38}$ Bromine may be extracted on a large scale by a similar method, but it is simpler to add a small quantity of manganese peroxide and sulphuric acid to the mother liquid direct. This sets free a portion of the chlorine, and this chlorine liberates the bromine.

Bromine is a dark brown liquid, giving brown fumes, and having a poisonous suffocating smell, whence its name (from the Greek $\beta \rho \hat{\omega} \mu \circ$, signifying evil smelling). The vapour density of bromine shows that its molecule is $\mathrm{Br}_{2}$. In the cold bromine freezes into brown-grey scales like iodine. The melting point of pure bromine is $-7^{\circ} .05 .39$ The density of liquid bromide at $0^{\circ}$ is $3 \cdot 187$, and at $15^{\circ}$ about 3.0 . The boiling point of bromine is about $58^{\circ} .7$. Bromine, like chlorine, is soluble in water; 1 part of bromine at $5^{\circ}$ requires 27 parts of water, and at $15^{\circ} 29$ parts of water. The aqueous solution of bromine is of

37 But there is no iodine in Stessfurt carnallite.

58 The chlorine must not, however, be in large excess, as otherwise the bromine would contain chlorine. Commercial bromine not unfrequently contains chlorine, as bromine chloride; this is more soluble in water than bromine, from which it may thus be freed. To obtain pure bromine the commercial bromine is washed with water, dried by sulphuric acid, and distilled, the portion coming over at $58^{\circ}$ being collected; the greater part is then converted into potassium bromide and dissolved, and the remainder is added to the solution in order to separate iodine, which is removed by shaking with carbon bisulphide. By heating the potassium bromide thus obtained with manganese peroxide end sulphuric acid, bromine is obtained quite free from iodine, whioh, however, is not present in certain kinds of commercial bromine (the Stassfurt, for instance). By treatment with potash, the bromine is then converted into a mixture of potassium bromide and bromate, and the mixture (which is in the proportion given in the equation) is distilled with sulphurio acid, bromine being then evolved: $5 \mathrm{KBr}+\mathrm{KBrO}_{5}+6 \mathrm{H}_{2} \mathrm{SO}_{4}$ $=6 \mathrm{KHSO}_{4}+3 \mathrm{H}_{2} \mathrm{O}+8 \mathrm{Br}_{2}$. After dissolving the bromine in a strong solntion of calcium bromide and precipitating with an excess of water, it loses all the chlorine it eontained, because chlorine forms calcium chloride with $\mathrm{CaBr}_{2}$.

59 There has long existed a difference of opinion as to the melting point of pure bromine. By some investigators (Regnault, Pierre) it was given as between $-7^{\circ}$ and $-8^{\circ}$, and by others (Balard, Liebig, Quincke, Baumhnuer) as between $-20^{\circ}$ and $-25^{\circ}$. There is now no doubt, thanks more especially to the researches of Ramsay and Young (1885), that pure bromine melts at about $-7^{\circ}$. This figure is not only established by direct $e x$ - 
an orange colour, and when cooled to $-2^{\circ}$ yields crystals containing 10 molecules of water to 1 molecule of bromine. ${ }^{60}$ Álcohol dissolves a greater quantity of bromine, and ether a still greater amount. But after a certain time products of the action of the bromine on these organic substances are formed in the solutions. Aqueous solutions of the bromides also absorb a large amount of bromine.

With respect to iodine, it is almost exclusively extracted from the mother liquors after the crystallisation of natural sodium nitrate (Chili saltpetre) and from the ashes of the sea-weed cast upon the shores of France, Great Britain, and Spain, sometimes in considerable quantities, by the high tides. The majority of these sea-weeds are of the genera Irucus, Laminaria, \&c. The fused ashes of these sea-weeds are called 'kelp' in Scotland and 'varech' in Normandy. A somewhat considerable quantity of iodine is contained in these sea-weeds. After

periment (Van der Plaats confirmed it), but also by means of the determination of the vapour tensions. For solid bromine the vapour tension $p$ in $\mathrm{mm}$. at $t$ was found to be-

$$
\begin{array}{cccccc}
p=20 & 25 & 80 & 35 & 40 & 45 \mathrm{~mm} . \\
t=-16^{\circ} \cdot 6 & -14^{\circ} & -12^{\circ} & -10^{\circ} & -8 \cdot 5^{\circ} & -7^{\circ}
\end{array}
$$

For liquid bromino-

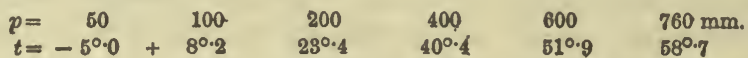

These curves intersect at $-7^{\circ} .05$. Besides which, in comparing the vapour tension of many liquids (for example, those given in Chapter II., Note 27), Ramsay and Young observed that the ratio of the absolute temperatures $(t+273)$ corresponding with equal tension varies for every pair of substances in rectilinear proportion in dependence npon $t$, and, therefore, for the above pressure $p$. Ramsay and Young determined the ratio of

\begin{tabular}{|c|c|c|c|c|c|c|}
\hline$p=$ & 20 & 25 & 80 & 85 & 40 & 45 \\
\hline $278+t=$ & $256 \cdot 4$ & 259 & 261 & 268 & $264 \cdot 6$ & 266 \\
\hline $278+t^{\prime}=$ & $295 \cdot 8$ & 299 & $802 \cdot 1$ & $804 \cdot 8$ & $307 \cdot 2$ & $809 \cdot 8$ \\
\hline$c=$ & $1 \cdot 152$ & $1 \cdot 154$ & $1 \cdot 157$ & 1159 & $1 \cdot 161$ & $1 \cdot 168$ \\
\hline
\end{tabular}
$t+278$ for water and bromine, and found that the straight lines expressing these ratios for liquid and solid bromine intersect also at $7^{\circ} .05$; thus, for example, for solid bromine-

where $t^{\prime}$ indicates the temperature of water corresponding with a rapour tension $p$, and where $c$ is the ratio of $278+t^{\prime}$ to $273+t$. The magnitude of $c$ is evidently expressed with great accuracy by the straight line $c=1 \cdot 1703+0.0011 t$. In exactly the same way we find the ratio for liquid bromine and water to be $c_{1}=1 \cdot 1585+0.00057 t$. The intersection of these straight lines in fact corresponds with $-7^{\circ} 06$, which again confirms the melting point given above for bromine. In this manner it is possible with the existing store of data to accurately establish and verify the melting point of substances. Ramsay and Young established the thermal constants of iodine by exactly the same method.

60 The observations made by Paterno and Nasini (by Rroult's method, Chapter I. Note 49) on the temperature of the formation of ice (-1. 1115 , with 1.391 gram of bromine in 100 grams of water) in an aqueous solution of bromine, showed that bromine is contrined in solutions as the molecule $\mathrm{Br}_{2}$. Similar experiments conducted on iodine (Kloboukofis 1889 and Beckmann 1890) show that in solution the molecnle is $I_{2}$.

B. Roozeboom investigated the hydrate of bromine as completely as the hydrate of chlorine (Notes 9, 10), The temperature of the complete decomposition of the hydrate is $+6^{\circ} \cdot 2$; the density of $\mathrm{Br}_{8} 10 \mathrm{H}_{2} \mathrm{O}=1 \cdot 49$. 
being burnt (or subjected to dry distillation) an ash is left which chiefly contains salts of potassium, sodium, and calcium. The metals occur in the sea-weed as salts of organic acids. On being burnt these organic salts are decomposed, forming carbonates of potassium and sodium. Hence, sodium carbonate is found in the ash of sea plants. The ash is dissolved in hot water, and on evaporation sodium carbonate and other salts separate, but a portion of the substances remains in solution. These mother liquors left after the separation of the sodium carbonate contain chlorine, bromine, and iodine in combination with metals, the chlorine and iodine being in excess of the bromine 13,000 kilos of kelp give about 1,000 kilos of sodium carbonate and 15 kilos of iodine.

The liberation of the iodine from the mother liquor is effected with comparative ease, because chlorine disengages iodine from potassium iodide and its other combinations with the metals. Not only chlorine, but also sulphuric acid, liberates iodine from sodium iodide. Sulphuric acid, in acting on an iodide, sets hydriodic acid free, but the latter easily decomposes, especially in the presence of substances capable of evolving oxygen, such as chromic acid, nitrous acid, and even ferric salts. ${ }^{61}$ Owing to its sparing solubility in water, the iodine liberated separates as a precipitate. To obtain pure iodine it is sufficient to distil it, and neglect the first and last portions of the distillate, the middle portion only being collected. Iodine passes directly from a state of vapour into a crystalline form, and settles on the cool portions of the

61 In general, $2 \mathrm{HI}+\mathrm{O}=\mathrm{I}_{2}+\mathrm{H}_{2} \mathrm{O}$, if the oxygen proceed from a substance from which it is easily evolved. For this reason compounds corresponding with the higher stages of oxidation or chlorination frequently give a lower stage when treated with hydriodic acid. Ferric oxide, $\mathrm{Fe}_{2} \mathrm{O}_{3}$, is a higher oxide, and ferrou's oxide, $\mathrm{FeO}$, a lower oxide; the former corresponds with $\mathrm{FeX}$, and the latter with $\mathrm{FeX}_{2}$, and this passage from the higher to the lower takes place under the action of hydriodic acid. Thus hydrogen peroxide and ozone (Chapter IV.) are able to liberate iodine from hydriodic acid. Compounds of copper oxide, $\mathrm{CnO}$ or $\mathrm{CuX}_{2}$, give compounds of the suboxide $\mathrm{Cu}_{2} \mathrm{O}$, or $\mathrm{CuX}$. Even sulphuric scid, which corresponds to the higher stage $\mathrm{SO}_{3}$, is able to act thus, forming the lower oxide $\mathrm{SO}_{2}$. The liberation of iodine from hydriodic acid proceeds with still greater ease under the action of substances capable of disengaging oxygen. In practice, many methods are employed for liberating iodine from acid liquids containing, for example, sulphuric acid and hydriodic acid. The higher oxides of nitrogen are most commonly used; they then pass into nitric oxide. Iodine may even be disengaged from hydriodic acid by the action of iodic acid, \&c. But there is a limit in these reactions of the oxidation of hydri. odic acid because, under certain conditions, especially in dilute solutions, the iodine set free is itselt able to act as an oxidising agent-that is, it exhibits the character of chlorine, and of the halogens in general, to which we shell again have occasion to refer. In Chili, where a large quantity of iodine is extracted in the manufacture of Chili nitre, which contains $\mathrm{NaIO}_{3}$, it is mixed with the acid and normal sulphites of sodium In solution; the iodine is then precipitated according to the equation $2 \mathrm{NaIO}_{3}+8 \mathrm{Na}_{2} \mathrm{SO}_{3}$ $+2 \mathrm{NaHSO}_{3}=5 \mathrm{Na}_{2} \mathrm{SO}_{4}+\mathrm{I}_{2}+\mathrm{H}_{2} \mathrm{O}$. The iodine thus obtained is purified by sublimation, 
apparatus in tabular crystals, having a black grey colour and metallic lustre. ${ }^{62}$

The specific gravity of the crystals of iodine is 4.95 . It melts at $114^{\circ}$ and boils at $184^{\circ}$. Its rapour is formed at a much lower tempera-

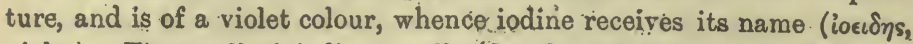
violet). The smell of iodine recalls the characteristic smell of hypochlorousacid; it has a sharp sour taste. It destroys the skin and organs of the body, and is therefore frequently employed for cauterising and as an irritant for the skin. In small quantities it turns the skin brown, but the coloration disappears after a certain time, partly owing to the. volatility of the iodine. Water dissolves only rodo part of iodine. A brown solution is thus obtained. which bleaches, but much more feebly than bromine and chlorine. Water which contains salts, and especially iodides, in solution dissolves iodine in considerable quantities, and the resultant solution is of a dark brown colour. Pure alcohol dissolves a small amount of iodine, and in so doing acquires a brown colour, but the solubility of iodine is considerably increased by the presence of a small quantity of an iodine compound-for instance, ethyl iodide-in the alcohol. ${ }^{63}$ Ether dissolves a larger amount of iodine than alcohol but iodine is particularly soluble in liquid hydrocarbons, in carbon bisulphide, and in chloroform. A small quantity of iodine dissolved in carbon bisulphide tints it rose-colour, but in a somewhat larger amount it gives a violet colour. Chloroform (quite free from alcohol) is also tinted rose colour by a small amount of iodine. This gives an casy means for detecting the presence of free iodine in small quantities. The blue coloration which free iodine gives with starch may also, as has already been frequently-mentioned (see Chapter IV.), serve for the detection of iodine.

If we compare the four elements, fluorine, chlorine, bromine, and iodine, we see in them an example of analogous substances which arrange themselves by their physical properties in the same order as

62 For the final purification of iodine, Stas dissolved it in a strong solution of potassium iodide, and precipitated it by the addition of water (see Note 58).

65 The solubility of iodine in solutions containing iodides, and compounds of iodine In general, may serve, on the one hand, as an indication that solution is due to a similarity between the solvent and dissolved substance, and, on the other hand, as an indirect proof of that view as to solutions which was cited in Chapter I., because in many instances un. stable highly iodised compounds, resembling crystallo-hydrates, have been obtained from such solutions. Thus iodide of tetramethylammonium, $\mathrm{N}\left(\mathrm{CH}_{5}\right)_{4} \mathrm{I}$, combines with $\mathrm{I}_{2}$ and $\mathrm{I}_{4}$. Even a solution of iodine in a saturated solution of potassium iodide presents indications of the formation of a definite compound $\mathrm{KI}_{3}$. Thus, an alcoholic solution of $\mathrm{KI}_{3}$ does not give up iodine to carbon bisulphide, although this solvent takes up iodine from an alcoholic solution of iodine itself (Girault Jörgensen, and others). The instrbility of these compounds resembles the instability of many crystallo-hydrates, for instance of $\mathrm{HCl}_{2} 2 \mathrm{H}_{2} \mathrm{O}$. 
they stand in respect to their atomic and molecular weights. If the weight of the molecule be large, the substance has a higher specific gravity, a higher melting and boiling point, and a whole serles of pro. perties depending on this difference in its fundamental properties. Chlorine in a free state boils at about $-35^{\circ}$, bromine boils at $60^{\circ}$, and iodine only above $180^{\circ}$. According to Avogadro-Gerhardt's law, the vapour densities of these elements in a gaseous state are proportional to their atomic weights, and here, at all events approximately, the densities in a liquid (or solid) state are also almost in the ratio of their atomic weights. Dividing the atomic weight of chlorine $(35.5)$ by its specific gravity in a liquid state $(1 \cdot 3)$, we obtain a volume $=27$, for bromine $(80 / 3 \cdot 1) 26$, and for iodine also (127/4:9) 26.64

The metallic bromides and iodides are in the majority of cases, in most respects analogous to the corresponding chlorides ${ }_{2}^{65}$ but chlorine displaces the bromine and iodine from them, and bromine liberates iodine from iodides, which is taken advantage of in the preparation of these halogens. However, the researches of Potilitzin showed that a reverse displacement of chlorine by bromine may occur both in. solutions and in ignited metallic chlorides in an atmosphere of bromine vapour-that is, a distribution of the metal (according to Berthollet's doctrine) takes place between the halogens, although however the larger portion. still unites with the chlorine, which shows its greater affinity for metals as compared with that of bromine and iodine. ${ }^{66}$ The latter, however,

64 The equality of the atomic volumes of the halogens themselves is all the more cemarkable because in all the halogen compounds the volume augments with the substi. tation of fluorine by chlorine, bromine, and iodine. Thus, for example, the volume of codiu'm fluoride (obtained by dividing the weight expressed by its formula by its specifo gravity) is abont 15, of sodium chloride 27 , of sodium bromide 32 , and of sodium iodide 11. The volume of silicon chloroform, $\mathrm{SiHCl}_{3}$, is 82 , and those of the corresponding bromine and iodine compounds are 108 and 122 respectively. The same difference also exists in solutions; for example, $\mathrm{NaCl}+200 \mathrm{H}_{2} \mathrm{O}$ has a sp. gr. (at $15^{\circ} / 4^{\circ}$ ) of 1.0106 , consequently the volume of the solution ' $3,658 \cdot 5 / 1: 0106=3,620$, hence the volume of sodium chloride in solntion $=3,620-8,603$ (this is the volume of $200 \mathrm{H}_{2} \mathrm{O}$ ) $=17$, and in similar solutions, $\mathrm{NaBr}=26$ and $\cdot \mathrm{NaI}=35$.

65 But the density (and also molecular volume, Note 64) of a bromine compound is always greater than that of a chlorine compoupd, whilst that of an iodine componnd is still greater. The order is the same in many other respects. For example, an iodine compound has a higher boiling point than a bromine compound, \&c.

*6 A. L. Potilitzin showed that in heating various metallic chlorides in es closed tube; with an equivalent quantity of bromine, a distribution of the metal between the halogen always occurs, and that the amounts of chlotine replaced by the bromine in the ultimate product are proportional to the atomic weights of the metals taken and inversely propor. tional to their equivalence. Thus, if $\mathrm{NaCl}+\mathrm{Br}$ be taken, then out of 100 partis of chlorine, 5.54 are replaced by the bromine, whilst with $\mathrm{AgCl}+\mathrm{Br} 27.28$ parts are replaced. These figures are in the ratio $1: 4 \cdot 9$, and the atomic weights $\mathrm{Na}: \mathrm{Ag}=1: 4 \cdot 7$. In general terms, if a chloride $\mathrm{MCl}_{n}$ be taken, it gives with $n \mathrm{Br}$ a percentage sub. etitution $=4 \mathrm{M} / n^{2}$, where $M$ is the atomic weight of the metal. This. law was deduced 
sometimes behave with respect to metallic oxides in exactly the same manner us chlorine. Gay-Lussac, by igniting potassium carbonate in iodine vapour, obtained (as with chlorine) an evolution of oxygen and carbonic anhydride, $\mathrm{K}_{2} \mathrm{CO}_{3}+\mathrm{I}_{2}=2 \mathrm{KI}+\mathrm{CO}_{2}+\mathrm{O}$, only the reactions between the halogens and oxygen are more easily reversible with bromine and iodine than with chlorine. Thus, at a red heat oxygen displaces iodine from barium iodide. Aluminium iodide burns in a current of oxygen (Deville and Troost), and a similar, although not so clearly marked, relation exists for aluminium chloride, and shows that the halogerns have a distinctly smaller affinity for those metals which only form feeble bases. This is still more the case with the non-metals, which form acids and evolve much more heat with oxygen than with the halogens (Note 13). But in all these instances the affinity (and amount of heat evolved) of iodine and bromine is less than that of chlorine, probably because the atomic weights are greater.

from observations on the chlorides of $\mathrm{Li}, \mathrm{K}, \mathrm{Na}, \mathrm{Ag}(n=1), \mathrm{Ca}, \mathrm{Sr}, \mathrm{Ba}, \mathrm{Co}, \mathrm{Ni}, \mathrm{Hg}, \mathrm{Pb}$ $(n=2), \mathrm{Bi}(n=8), \mathrm{Sn}(n=4)$, and $\mathrm{F}_{\mathrm{e}_{2}}(n=6)$.

In these determinations of Potilitzin we see not only a brilliant confirmation of Berthollet's doctrine, but also the first effort to directly determine the affinities of elementr by means of displacement. The chief object of these researches consisted in proving whether a displacement occurs in those cases where heat is absorbed, and in this instance it should be absorbed, because the formation of all metallic bromides is attended with the evolution of less heat than that of the chlorides, as is seen by the figures given in Note 55 .

If the mass of the bromine be increased, then the amount of chlorine displaced also increases. For example, if masses of bromine of 1 and 4 equivalents act on a molecule of sodium chloride, then the percentages of the chlorine displaced will be 6.08 p.o. and 12.46 p.c.; in the action of $1,4,25$, and 100 molecules of bromine on a molecule of barinm chloride, there will be displaced $7.8,17.6,85.0$, and 45.0 p.o. of chlorine. If an equivalent quantity of hydrochloric acid act on metallic bromides in closed tubes, and in the absence of water at a temperature of $300^{\circ}$, then the percentages of the substitution of the bromine by the chlorine in the double decomposition taking place between univalent metals are inversely proportional to their atomic weights. For example, $\mathrm{NaBr}+\mathrm{HCl}$ gives at the limit 21 p.c. of displacement, $\mathrm{KCl} 12$ p.o. and $\mathrm{AgCl} 4 \frac{1}{4}$ p.c. Essentially the same action takes place in an aqueous solution, although the phenomenon is complicated by the participation of the water. The reactions proceed spontaneously in one or the other direction at the ordinary temperature but at different rates. In the action of a dilute solution (1 equivalent per 5 litres) of sodium chloride on silver bromide at the ordinary temperature the amount of bromine replaced in six and a half days is 2.07 p.e., and with potassium chloride 1.5 p.c. With an excess of the chloride the mag. nitude of the substitution incresses. These conversions also proceed with the absorption of heat. The reverse reactions evolving heat proceed incomparably more rapidly, but also to a certain limit; for example, in the reaction $\mathrm{AgCl}+\mathrm{RBr}$ the following percentages of silver bromide are formed in different times:

$\begin{array}{lccccc}\text { hours } & 2 & 3 & 22 & 96 & 120 \\ \mathrm{~K} & 79.82 & 87 \cdot 4 & 88.22 & - & 94 \cdot 21 \\ \mathrm{Na} & 83.63 & 90.74 & 91.70 & 95.49 & -\end{array}$

That is, the conversions which are accompanied by an evolution of heat proceed with very much greater rapidity than the reverse conversions. 
The smaller store of energy in iodine and bromine is seen still more clearly in the relation of the halogens to hydrogen. In a gaseous state they all enter, with more or less ease, into direct combination with gaseous hydrogen-for example, in the presence of spongy platinum, forming halogen acids, $\mathrm{HX}$ - but the latter are far from being equally stable; hydrogen chloride is the most stable, hydrogen iodide the least so, and hydrogen bromide occupies an intermediate position A very high temperature is required to decompose hydrogen chloride even par. tially, whilst hydrogen iodide is decomposed by light even at the ordinary temperature and very easily by a red heat. Hence the reaction $\mathrm{I}_{2}+\mathrm{H}_{2}=\mathrm{HI}+\mathrm{HI}$ is very easily reversible, and consequently has a limit, and hydrogen iodide easily dissociates. ${ }^{67}$ Judging by the direct measurement of the heat evolved (22,000 heat units) in the formation of $\mathrm{HCl}$, the conversion of $2 \mathrm{HCl}$ into $\mathrm{H}_{2}+\mathrm{Cl}_{2}$ requires the expenditure

67 The dissociation of hydriodic acid has been studied in detail by Hautefenille and Lemoine, from whose researches we extract the following information. The decom. position of hydriodic acid is decided, but proceeds slowly at $180^{\circ}$; the rate and limit of decomposition increase with a rise of temperature. The reverse action-that is, $\mathrm{I}_{2}+\mathrm{H}_{2}$ $=2 \mathrm{HI}-$ proceeds not only under the influence of spongy platinum (Corenwinder), which also accelerates the decomposition of hydriodic acid, but also by itself, although slowly. The limit of the reverse reaction remains the same with or without spongy platinum. An increase of pressure has a very powerful accelerative effect on the rate of formation of hydriodic acid, and therefore spongy platinum by condensing gases has the same effect as increase of pressure. At the atmospheric pressure the decompesit.on of hydriodic acid reaches the limit at $250^{\circ}$ in several months, and at $440^{\circ}$ in several hours. The limit at $250^{\circ}$ is about 18 p.c. of decomposition-that is, out of 100 parts of hydrogen previously combined in hydriodic acid, about 18 p.c. may be disengaged at this temperature (this hydrogen may be easily measured, and the measure of dissociation determined), but not more; the limit at $440^{\circ}$ is about 26 p.o. If the pressure under which $2 \mathrm{HI}$ passes into $\mathrm{H}_{2}+\mathrm{I}_{2}$ be $4 \frac{1}{2}$ atmospheres, then the limit is 24 p.c.; under a pressure of $\frac{1}{3}$ atmosphere the limit is 29 p.c. The small influence of pressure on the dissociation of hydriodic acid (compared with $\mathrm{N}_{2} \mathrm{O}_{4}$, Chapter VI. Note 46) is due to the fact that the reaction $2 \mathrm{HI}=\mathrm{I}_{2}+\mathrm{H}_{2}$ is not accompanied by a change of volume. In order to show the influence of time, we will cite the following figures referring to $350^{\circ}$ : (1) Reaction $\mathrm{H}_{2}+\mathrm{I}_{2}$; after 8 hours, 88 p.c. of hydrogen remained free: 8 hours, 69 p.c.; 34 hours, 48 p.c.; 76 hours, 29 p.c.; and 327 hours, $18 \cdot 5$ p.c. (2) The reverse decomposition of $2 \mathrm{HI}$; after 9 hours, 3 p.c. of hydrogen was set free, and after 250 hours 18.6 p.c.-that is, the limit was reached. The addition of extraneous hydrogen diminishes the limit of the reaction of decomposition, or increases the formation of hydriodic acid from iodine and hydrogen, as would be expected from Berthollet's doctrine (Chapter $\mathrm{X}$.). Thus at $440^{\circ} 26$ p.c. of hydriodic acid is decomposed if there be no admixture of hydrogen, while if $\mathrm{H}_{2}$ be added, then at the limit only half as large a mass of $\mathrm{HI}$ is decomposed. Therefore, if an infinite mass of hydrogen be added there will be no decomposition of the hydriodic acid. Light aids the decomposition of hydriodic acid very powerfully. At the ordinary temperature 80 p.c. is decomposed under the influence of light, whilst under the infuence of heat alone this, limit corre. sponds with a very high temperature. The distinct action of light, spongy platinum, and of impurities in glass (especially of sodium sulphate, which decomposes hydriodic acid), not only render the investigations difficult, but also show that in reactions like $2 \mathrm{HI}=\mathrm{I}_{2}+\mathrm{H}_{23}$, which are accompanied by slight heat effects, all foreign and feeble influences may strongly affect the progress of the action (Note 47). 
of 44,000 heat units. The decomposition of $2 \mathrm{HBr}$ into $\mathrm{H}_{2}+\mathrm{Br}_{2}$ only requires, if the bromine be obtained in a gaseous state, a consumption of about 24,000 units, whilst in the decomposition of $2 \mathrm{HL}$ into $\mathrm{H}_{2}+\mathrm{I}_{2}$ as vapour about. 3,000 heat units are evolved; ${ }^{68}$ these facts, without doubt, stand in causal connection with the great stability of hydrogen chloride, the easy decomposability of hydrogen iodide, and the intermediate properties of hydrogen bromide. From this it would be expected that chlorine is capable of decomposing water with the evolution of oxygen, whilst iodine has not the energy to produce this disengagement, ${ }^{69}$ although it is able to liberate the oxygen from the oxides of potassium and sodium, the affinity of these metals for the halogens being very considerable. For this reason oxygen, especially in compounds from which it can be evolved readily (for instance, ClHO, $\mathrm{CrO}_{3}$, \&c.), easily decomposes hydrogen iodide. A mixture of hydrogen iodide and oxygen burns in the presence of an ignited substance, forming water and iodine. Drops of nitric acid in an atmosphere of hydrogen iodide cause the disengagement of violet fumes of iodine and brown fumes of nitric peroxide. In the presence of alkalis and an excess of water, however, iodine is able to effect oxidation like chlorine-that is,

68 The thermal determinations of Thomsen (at $18^{\circ}$ ) gave in thousands of calories, $\mathrm{Cl}+\mathrm{H}=+22, \mathrm{HCl}+\mathrm{Aq}$ (that is, on dissolving $\mathrm{HCl}$ in a large amount of water) $=+17 \cdot 3$, and therefore $\mathrm{H}+\mathrm{Cl}+\mathrm{Aq}=+39 \cdot 3$. In taking molecules, all these figures must be doubled. $\mathrm{Br}+\mathrm{H}=+8 \cdot 4 ; \mathrm{HBr}+\mathrm{Aq}=19 \cdot 9 ; \mathrm{H}+\mathrm{Br}+\mathrm{Aq}=+28 \cdot 3$. According to Ber. thelot 7.2 are required for the vaporisation of $\mathrm{Br}_{2}$, hence $\mathrm{Br}_{2}+\mathrm{H}_{2}=16 \cdot 8+7 \cdot 2=+24$, if $\mathrm{Br}_{2}$ be taken as vaponr for comparison with $\mathrm{Cl}_{2} . \mathrm{H}+\mathrm{I}=-6 \cdot 0, \mathrm{HI}+\mathrm{Aq}=19 \cdot 2$; $\mathrm{H}+\mathrm{I}+\mathrm{Aq}=+18 \cdot 2$, and, according to Berthelot, the heat of fusion of $\mathrm{I}_{2}=3.0$, and of vaporisation $6 \cdot 0$ thousand heat units, and therefore $I_{2}+H_{2}=-2(6 \cdot 0)+3+6=-3 \cdot 0$, if the iodine be taken as vapour. Berthelot, on the basis of his determinations, gives, however, +0.8 thousand heat units. Similar contradictory results are often met with in thermochemistry owing to the imperfection of the existing methods, and particularly the necessity of depending on indirect methods for obtaining the fundamental figures. Thus Thomsen decomposed a dilute solution of potassium iodide by gaseous chlorine; the reaction gave $+26^{\prime} 2$, whence, having first determined the heat effects of the reactions $\mathrm{KHO}+\mathrm{HCl}, \mathrm{KHO}+\mathrm{HI}$ and $\mathrm{Cl}+\mathrm{H}$ in aqueous solutions, it was possible to find $\mathrm{H}+\mathrm{I}+\mathrm{Aq}$; then, knowing $\mathrm{HI}+\mathrm{Aq}$, to find $\mathrm{I}+\mathrm{H}$. It is evident that unavoidable errors may accumulate.

69 One can believe, however, on the basis of Berthollet's doctrine, and the observations of Potilitzin (Note 66), that a certain slow decomposition of water by iodine. takes place. On this view the observations of Dossios and Weith on the fact that the solubility of iodine in water increases after the lapse of several months will be comprehen. sible. Hydriodic acid is then formed, and it increases the solubility. If the iodine be extracted from such a solution by carbon bisulphide, then, as the authors showed, after the action of nitrous anhydride iodine may be again detected in the solution by means of starch. It can easily be understood that a number of similar reactions, requiring much time and taking place in small quantities, have up to now eluded the attention of inves. tigators, who even still doubt the universal application of Berthollet's doctrine, or only see the thermochemical side of reactions, or else neglect to pay attention to the element of time and the influence of mass. 
it decomposes water; the action is here aided by the affinity of hydrogen iodide for the alkali and water, just as sulphuric acid helps zinc to decom. pose water. But the relative instability of hydriodic acid is best seen in comparing the acids in a gaseous state. If the 'halogen acids be dissolved in water, they evolve so much heat that they approach much nearer to each other in properties. This is seen from thermochemical data, for in the formation of $\mathrm{HX}$ in solution (in a large excess of water) from the gaseous elements there is evolved for $\mathrm{HCl} 39,000$, for $\mathrm{HBr} 32,000$, and for HI 18,000 heat units. ${ }^{70}$ But it is especially evident from the fact that solutions of hydrogen bromide and iodide in water have many points in common with solutions of hydrogen chloride, both in their capacity to form hydrates and fuming solutions of constant boiling point, and in their capacity to form haloid salts, \&c. by reacting on bases.

In consequence of what has been said above, it follows that hydrobromic and hydriodic acids, being substances which are but slightly stable, cannot be evolved in a gaseous state under many of those conditions under which hydrochloric acid is formed. Thus if sulphuric acid in solution acts on sodium iodide, all the same phenomena take place as with sodium chloride (a portion of the sodium iodide gives hydriodic acid, and all remains in solution), but if sodium iodide be mixed with strong sulphuric acid, then the oxygen of the latter decomposes the hydriodic acid set free, with liberation of iodine, $\mathrm{H}_{2} \mathrm{SO}_{4}+2 \mathrm{HI}$ $=2 \mathrm{H}_{2} \mathrm{O}+\mathrm{SO}_{2}+\mathrm{I}_{2}$. This reaction takes place in the reverse direction in the presence of a large quantity of water $(2,000$ parts of water per 1 part of $\mathrm{SO}_{2}$ ), in which case not only the affinity of hydriodic acid for water is brought to light but also the action of water in directing chemical reactions in which it participates. ${ }^{71}$ Therefore, with a halogen salt, it is easy to obtain gaseous hydrochloric acid by the action of sulphuric acid, but neither hydrobromic nor hydriodic acid can be so obtained in the free state (as gases). ${ }^{72}$ Other methods have to be resorted to for their preparation, and recourse must not be had to compounds of oxygen, which are so easily able to destroy these acids. Therefore hydrogen sulphide, phosphorus, \&c., which themselves easily take up oxygen, are introduced as means for the conversion of bromine and iodine into hydrobromio and hydriodic acids in the presence of water. For example, in the action of phosphorus the essence of the matter is that the oxygen of the water goes

70 On the basts of the data in Note 68.

71 A number of similar cases confirm what has been said in Chapter X.

72 This is prevented by the reducibility of sulphurio acid. If volatile acids be taken they pass over, together with the hydrobromic and hydriodic acids, when distilled; whilst many non-volatile acids which are not reduced by hydrobromic and hydriodio acids only act feebly (like phosphnric acid), or do not act at all (like boric acid). 
to the phosphorus, and the union of the remaining elements leads to the formation of hydrobromic or hydriodic acid ; but the matter is complicated by the reversibility of the reaction, the affinity for water, and other circumstances which are understood by following Berthollet's doctrine. Chlorine (and bromine also) directly decomposes hydrogen sulphide, forming hydrochloric acid and liberating sulphur, both in a gaseous form and in solutions, whilst iodine only decomposes hydrogen sulphide in weak solutions, when its affinity for hydrogen is'aided by the affinity of hydrogen iodide for water. In a gaseous state iodine does not act on hydrogen sulphide, ${ }^{73}$ whilst sulphur is able to decompose gaseous hydriodic acid, forming hydrogen sulphide and a compound of sulphur and iodine which with water forms hydriodic acid. ${ }^{74}$

If hydrogen sulphide be passed through water containing iodine, the reaction $\mathrm{H}_{2} \mathrm{~S}+\mathrm{I}_{2}=2 \mathrm{HI}+\mathrm{S}$ proceeds so long as the solution is dilute, but when the mass of free HI increases the reaction stops, because the iodine then passes into solution. A solution having a composition approximating to $2 \mathrm{HI}+4 \mathrm{I}_{2}+9 \mathrm{H}_{2} \mathrm{O}$ (according to Bineau) does not react with $\mathrm{H}_{2} \mathrm{~S}$, notwithstanding the quantity of free iodine. Therefore only weak solutions of hydriodic acid can be obtained by passing hydrogen sulphide into water with iodine. ${ }^{74}$ bls

To obtain ${ }^{75}$ gaseous hydrobromic and hydriodic acids it is most

73 This is in agreement with the thermochemical data, because if all the substances be taken in the gaseous state (for sulphur the heat of fusion is 0.3 , and the heat of vaporisation 2.3) we have $\mathrm{H}_{2}+\mathrm{S}=4 \cdot 7 ; \mathrm{H}_{2}+\mathrm{Cl}_{2}=44 ; \mathrm{H}_{2}+\mathrm{Br}_{2}=24$, and $\mathrm{H}_{2}+\mathrm{I}_{2}=$ -3 thousand heat units; hence the formation of $\mathrm{H}_{2} \mathrm{~S}$ gives less heat than that of $\mathrm{HCl}$ and $\mathrm{HBr}$, but more than that of $\mathrm{HI}$. In dilute solutions $\mathrm{H}_{2}+\mathrm{S}+\mathrm{Aq}=9 \cdot 3$, and consequently less than the formation of all the halogen acids, as $\mathrm{H}_{2} \mathrm{~S}$ evolves but little heat with water, and therefore in dilute solutions chlorine, bromine, and iodine decompose hydrogen sulphide.

74 Here there are three elements, hydrogen, sulphur, and iodine, each pair of which is able to form a compound, $\mathrm{HI}, \mathrm{H}_{2} \mathrm{~S}$, and $\mathrm{SI}$, besides which the latter may unite in various proportions. The complexity of chemical mechanics is seen in such examples as these. It is evident that only the study of the simplest cases can give the key to the more complex problems, and on the other hand it is evident from the examples cited in the last pages that, without penetrating into the conditions of chemical equilibria, it would be impossible to explain chemical phenomena. By following the footsteps of Berthollet the possibility of unravelling the problems will be reached; but work in this direction has only been begun during the last ten years, and much remains to be done in collecting experimental material, for which occasions present themselvès at every step. In speaking of the halogens I wished to turn the reader's attention to problems of this kind.

74 bis The same essentially takes place when sulphurous anhydride, in a dilute solution, gives hydriodic acid and sulphuric acid with iodine. On concentration a reverse reaction takes place. The equilibrated systems and the part played by water are everywhere distinctly seen.

75 Methods of formation and preparation are nothing more than particular cases of chemical reaction. If the knowledge of chemical mechanics were more exact and complete than it now is it would be possible to foretell all cases of preparation with every 
convenient to take advantage of the reactions between phosphorus, the halogens, and water, the latter being present in small quantity (otherwise the halogen acids formed are dissolved by it); the halogen is gradually added to the phosphorus moistened with water Thus if rèd phosphorus be placed in a flask and moistened with water, and bromine be added drop by drop (from a tap funnel), hydrobromic acid is abundantly and uniformly disengaged. ${ }^{76}$ Hydrogen

detail (of the quantity of water, temperature, pressure, mass, \&c.) The study of practical methods of preparation is therefore one of the paths for the study of chemical mechanics. The reaction of iodine on phosphorus and water is a case like that men. tioned in Note 74, and the matter is here further complicated by the possibility of the formation of the compound $\mathrm{PH}_{3}$ with $\mathrm{HI}$, as well as the production of $\mathrm{PI}_{2}, \mathrm{PI}_{3}$, and the affinity of hydriodic acid and the acids of phosphorus for water. The theoretical interest of equilibria in all their complexity is naturally very great, but it falls into the background in presence of the primary interest of discovering practical methods for the isolation of substances, and the means of employing them for the requirements of man. It is only after the satisfaction of these requirements that interests of the other order arise, which in their turn must exert an influence on the former. For these reasons, whilst considering it opportune to point ont the theoretical interest of chemical equilibria, the chief attention of the reader is directed in this work to questions of practical importance.

76 Hydrobromic acid is also obtained by the action of bromine ois paraffin heated to $180^{\circ}$. Gustarson proposed to prepare it by the action of bromine (best added in drops together with traces of aluminium bromide) on anthracene (a solid hydrocarbon from coal tar). Balard prepared it by passing bromine vapour over moist pieces of common phosphorus. The liquid tribromide of phosphorus, directly obtained from phosphorus and bromine, also gives hydrobromic acid when treated with water. Bromide of potassium or sodium, when treated with sulphuric acid in the presence of phosphorus, also gives hydrobromic acid, bat hydriodic acid is decomposed by this method. In order to free hydrobromic acid from bromine vapour it is passed over moist phosphorus and dried either by phosphoric anhydride or calcium bromide (calcium chloride cannot be used, as hydrochloric acid would be formed). Neither hydrobromic nor hydriodic acids can be collected over mercury, on which they act, but they may be directly collected in a dry vessel by leading the gas-conducting tube to the bottom of the vessel, both gases being much heavier than air. Merz and Holtzmann (1889) propose to prepare $\mathrm{HBr}$ directly from bromine and hydrogen. For this purpose pure dry hydrogen is passed through a flask containing boiling bromine. The mixture of gas and rapour then passes through a tube provided with one or two bulbs, which is heated moderately in the middle. Hydrobromic acid is formed with a series of flashes at the part heated. The resultant $\mathrm{HBr}$, together with traces of bromine, passes into a Woulfe's bottle into which hydrogen is also introduced, and the mixture is then carried through another heated tube, after which it is passed through water which dissolves the hydrobromic acid. According to the method proposed by Newth (1892) a mixture of bromine and hydrogen is led through a tube containing a platinum spiral, which is heated to redness after the air has been displaced from the tube. If the vessel contrining the bromine be kept at $60^{\circ}$, the hydrogen takes up almost the theoretical amount lof bromine required for the formation of $\mathrm{HBr}$. Although the flame which appears in the neighbourhood of the platinum spiral does not penetrate into the vessel containing the bromine, still, for safety, a tube filled with cotton wool may be interposed.

Hydriodic acid is obtained in the same manner as hydrobromic. The iodine is heated in a small fiask, and its vapour is carried over by hydrogen into a strongly heated tube. The gas passing from the tube is found to contain a considerable amount of $\mathrm{HI}$, together with some free iodine. At a low red heat about 17 p.c of the iodine vapour enters 
iodide is prepared by adding 1 part of common (yellow) dry phosphorus to 10 parts of dry iodine in a glass flask. On shaking the flask, union proceeds quietly between them (light and heat being evolved), and when the mass of iodide of phosphorus which is formed has cooled, water is added drop by drop (from a tap funnel) and hydrogen iodide is evolved directly without the aid of heat. These methods of preparation will be at once understood when it is remembered (p. 468) that phosphorus chloride gives hydrogen chloride with water. It is exactly the same here-the oxygen of the water passes over to the phosphorus, and the hydrogen to the iodine, thus, $\mathrm{PI}_{3}+3 \mathrm{H}_{2} \mathrm{O}$ $=\mathrm{PH}_{3} \mathrm{O}_{3}+3 \mathrm{HI} .{ }^{77}$

In a gaseous form hydrobromic and hydriodic acids are closely analogous to hydrochloric acid ; they are liquefied by pressure and cold, they fume in the air, form solutions and hydrates, of constant boiling point, and react on metals, oxides and salts, \&cc. ${ }^{78}$ Only the relatively

into combination; at \& higher temperature, 78 p.c. to 79 p.c., and at a strong heat about 82 p.c.

77 But generally more phosphorus is taken than is required for the formation of $\mathrm{PI}_{3}$, because otherwise a portion of the iodine distils over. If less than one-tenth part of iodine be taken, much phosphonium iodide, $\mathrm{PH}_{4} \mathrm{I}$, is formed. This proportion was established by Gay-Iussac and Kolbe. Hydriodic acid is also prepared in many other ways. Bannoff dissolves two parts of lodine in one part of a previously prepared strong (sp. gr. 1.67) solution of hydriodic acid, and pours it on to red phosphorus in a retort. Personne takes a mixture of fifteen parts of water, ten of iodine, and one of red phosphorus, which, when heated, disengages hydriodic acid mixed with iodine vapour; the latter is removed by passing it over moist phosphorus (Note 76). 'It must be remembered however that reverse reaction (Oppenheim) may take place between the hydriodic acid and phosphorus, in which the compounds $\mathrm{PH}_{4} \mathrm{I}$ and $\mathrm{PI}_{2}$ are formed.

It should be observed that the reaction between phosphorus, iodine and water must be carried out in the above proportions and with caution, as they may react with explosion. With red phosphorus the reaction proceeds quietly, but nevertheless requires care.

L. Meyer showed that with an excess of iodine the reaction proceeds without the formation of bye-products $\left(\mathrm{PH}_{4} \mathrm{I}\right)$, according to the equation $\mathrm{P}+5 \mathrm{I}+4 \mathrm{H}_{2} \mathrm{O}=\mathrm{PH}_{3} \mathrm{O}_{4}+5 \mathrm{HI}$. For this purpose 100 grams of iodine and 10 grams of water are placed in a retort, and a paste of 5 grams of red phosphoru and 10 grams of water is added little by little (at first with great care). The hydriodic acid may be obtained free from iodine by directing the neck of the retort upwards and causing the gas to pass through a shallow layer of water (respecting the formation of $\mathrm{HI}$, see also Note r5) $^{2}$

78 The specific gravities of their Eolutions as deduced by me on the basis of Topköe and Berthelot's determinations for $15^{\circ} / 4^{\circ}$ are as follows :-

$\begin{array}{lcccccc} & 10 & 20 & 30 & 40 & 50 & 60 \text { p.ck } \\ \text { HBr } & 1.071 & 1.156 & 1.258 & 1.874 & 1.505 & 1.650 \\ \text { HI } & 1.075 & 1.164 & 1.267 & 1.399 & 1.567 & 1.769\end{array}$

Hydrobromic acid forms two hydrates, $\mathrm{HBr}, 2 \mathrm{H}_{2} \mathrm{O}$ and $\mathrm{HBr}, \mathrm{H}_{2} \mathrm{O}$, which have been studied by Roozeboom with as much completeness as the hydrate of bydrochlorio acid (Chapter X. Note 37).

With metallic silver, solutions of hydriodic acid give hydrogen with great ease, forming silver iodide. Mercury, lead, and other metals act in a similar manner. 
easy decomposability of hydrobromic acid, and especially of hydriodic acid, clearly distinguish these acids from hydrochloric acid. For this reason, hydriodic acid acts in a number of cases as a deoxidiser or reducer, and frequently even serves as a means for the transference of hydrogen. Thús Berthelot, Baeyer, Wreden, and others, by heating unsaturated hydrocarbons in a solution of hydriodic acid, obtained their compounds with hydrogen nearer to the limit $\mathrm{C}_{n} \mathrm{H}_{2 n+2}$ or even the saturated compounds. For example, benzene, $\mathrm{C}_{6} \mathrm{H}_{6}$, when heated in a closed tube with a strong solution of hydriodic acid, gives hexylene, $\mathrm{C}_{6} \mathrm{H}_{12}$. The easy decomposability of hydriadic acid accounts for the fact that iodine does not act by metalepsis on hydrocarbons, for the hydrogen iodide liberated with the product of metalepsis, RI, formed, gives iodine and the hydrogen compound, $\mathrm{RH}$, back again. And therefore, to obtain the products of iodine substitution, either iodic acid, $\mathrm{HIO}_{3}$ (Kekulé), or mercury oxide, $\mathrm{HgO}$ (Weselsky), is added, as they immediately react on the hydrogen iodide, thus: $\mathrm{HIO}_{3}+5 \mathrm{HI}=3 \mathrm{H}_{2} \mathrm{O}+3 \mathrm{I}_{2}$, or, $\mathrm{HgO}+2 \mathrm{HI}=-\mathrm{HgI}_{2}+\cdot \mathrm{H}_{2} \mathrm{O}$. From these considerations it will be readily understood that iodine acts like chlorine (or bromine) on ammonia and sodium hydroxide, for in these cases the hydriodic acid produced forms $\mathrm{NH}_{4} \mathrm{I}$ and $\mathrm{NaI}$. With tincture of iodine or even the solid element, a solution of ammonia immediately forms a highly-explosive solid black product of metalepsis, $\mathrm{NHI}_{2}$, generally known as iodide of nitrogen, although it still contains hydrogen (this was proved beyond doubt by Szuhay 1893), which may be replaced by silver (with the formation of $\mathrm{NAgI}_{2}$ ): $3 \mathrm{NH}_{3}+2 \mathrm{I}_{2}$ $=2 \mathrm{NH}_{4} \mathrm{I}+\mathrm{NHI}_{2}$. However, the composition of ' the last product is variable, and with an excess of water $\mathrm{NI}_{3}$ seems to-be formed. Iodide of nitrogen is just as explosive as nitrogen chloride. ${ }^{78}$ bis In the

78 bis Iodide of nitrogen, $\mathrm{NHI}_{2}$ is obtained as a brown pulverulent precipitate on adding a solution of iodine (in alcohol, for instance) to a solution of ammonia. If it be collected on a filter-paper, it does not decompose so long as the precipitate is moist; but when dry it explodes.violently, so that it can only be experimented upon in small quantities. Usually the filter-paper is torn into bits while moist, and the pieces laid upon a brick; on drying an explosion proceeds not only from friction or a blow, but even spontaneously. The more dilute the solution of ammonia, the greater is the amount of iodine required for the formation of the precipitate of $\mathrm{NHI}_{2}$. A low temperature facilitates its formation. $\mathrm{NHI}_{2}$ dissolves in ammonia water, and when heated the solution forms $\mathrm{HIO}_{3}$ and iodine. With KI, iodide of nitrogen gives iodine, $\mathrm{NH}_{3}$ and $\mathrm{KHO}$. These reactions (Selivanoff) are explained by the formation of $\mathrm{HIO}$ from $\mathrm{NHI}_{2}+2 \mathrm{H}_{2} \mathrm{O}=\mathrm{NH}_{3} 42 \mathrm{HIO}$-and then $\mathrm{KI}+\mathrm{HIO}=\mathrm{I}_{2}+\mathrm{KHO}$. Selivanoff (see Note 29) usually observed a temporary formation of hypoiodous acid, HIO, in the reaction of ammonia upon iodine, so that here the formation of $\mathrm{NHI}_{2}$ is preceded by that of $\mathrm{HIO}-$ i.e. first $\mathrm{I}_{2}+\mathrm{H}_{2} \mathrm{O}=\mathrm{HIO}+\mathrm{HI}$, and then not only the HI combines with $\mathrm{NH}_{3}$, but also $2 \mathrm{HIO}+\mathrm{NH}_{3}=\mathrm{NHI}_{2}+2 \mathrm{H}_{2} \mathrm{O}$. With dilute sulphuric acid iodide of nitrogen (like $\mathrm{NCl}_{3}$ ) forms hypoiodous acid, but it immediately passes into iodic acid, as is expressed by the equation $5 \mathrm{HIO}=2 \mathrm{I}_{2}+\mathrm{HIO}_{3}$ $+2 \mathrm{H}_{2} \mathrm{O}$ (first $3 \mathrm{HIO}=\mathrm{HIO}_{3}+2 \mathrm{HI}$, and then $\mathrm{HI}+\mathrm{HIO}=\mathrm{I}_{2}+\mathrm{H}_{2} \mathrm{O}$ ). Moreover, Selivanoff 
action of iodine on sodium hydroxide no bleaching compound is formed (whilst bromine gives one), but a direct reaction is always accomplished with the formation of an iodate, $6 \mathrm{NaHO}+3 \mathrm{I}_{2}=5 \mathrm{NaI}+3 \mathrm{H}_{2} \mathrm{O}+\mathrm{NaIO}_{3}$ (Gay-Lussac). Solutions of other alkalis, and even a mixture of water and oxide of mercury, act in the same manner. ${ }^{79}$ This direct formation of iodic acid, $\mathrm{HIO}_{3}=\mathrm{IO}_{2}(\mathrm{OH})$, shows the propensity of iodine to give compounds of the type $\mathbf{I X}_{5}$. Indeed, this capacity of indine to form compnunds of a high type emphasises itself in many ways. But it is most important to turn attention to the fact that iodic acid is easily and directly formed by the action of oxidising substances on iodine. Thus, for instance, strong nitric acid directly converts iodine into iodic acid, whilst it has no oxidising action on chlorine. 79 bis This shows a greater affinity in iodine for oxygen than in chlorine, and this conclusion is confirmed by the fact that iodine displaces chlorine from

found that iodide of nitrogen, $\mathrm{NHI}_{2}$, dissolves in an excess of ammonia water, and that with potassium iodide the solution gives the reaction for hypoiodous acid (the evolution of iodine in an alkaline solution). This shows that HIO participates in the formation and decomposition of $\mathrm{NHI}_{2}$, and therefore the condition of the iodine (its metaleptio position) in them is analogous, and differs from the condition of the halogens in the haloid-anhydrides (for instance, $\mathrm{NO}_{2} \mathrm{Cl}$ ). The latter are tolerably stable, while (the haloid being designated by X) $\mathrm{NHX}_{2}, \mathrm{NX}_{3}, \mathrm{XOH}$, RXO (see Chapter XIII. Note 43), \&o., are unstable, easily-decomposed with the evolution of heat, and, under the action of water, the haloid is easily replaced by hydrogen (Selivanoff), as would be expected in true products of metalepsis.

79 Hypoiodous acid, HIO, is not known, but organio compounds, RIO, of this type are known. To illustrate the peculiarities of their properties we will mention one of these compounds, namely, iodosobenzol, $\mathrm{C}_{6} \mathrm{H}_{3} \mathrm{IO}$. This substance was obtained by Willgerodt (1892), and also by V. Meyer, Wachter, and Askenasy, by the action of caustic alkalis upon phenoldiiodochloride, $\mathrm{C}_{6} \mathrm{H}_{5} \mathrm{ICl}_{2}$ (according to the equation, $\mathrm{C}_{6} \mathrm{H}_{3} \mathrm{ICl}_{2}+2 \mathrm{MOH}=\mathrm{C}_{6} \mathrm{H}_{5} \mathrm{IO}+2 \mathrm{MCl}+\mathrm{H}_{2} \mathrm{O}$ ). Iodosobenzol is an amorphous yellow substance, whose melting point could not be determined because it explodes at $210^{\circ}$, decomposing with the evolution of iodine vapour. This substance dissolves in hot water and alcohol, but is not soluble in the majority of other neutral organio solvents. If acids do not oxidise $\mathrm{C}_{6} \mathrm{H}_{5} \mathrm{IO}$, they give saline compounds in which iodosobenzol appears as a basic oxide of a diatomic metal, $\mathrm{C}_{6} \mathrm{H}_{5} \mathrm{I}$. Thus, for instance, when an acetic acid solution of iodosobenzol is treated with a solution of nitric acid, it gives large monoclinic crystals of a nitric acid salt having the composition $\mathrm{C}_{6} \mathrm{H}_{5} \mathrm{I}\left(\mathrm{NO}_{3}\right)_{2}$ (like $\left.\mathrm{Ca}\left(\mathrm{NO}_{3}\right)_{2}\right)$ : In appearing as the analogue of basic oxides, iodosobenzol displaces iodine from potassium iodide (in a solution acidulated with acetic or hydrochlorio acid)-i.e. it acts with its oxygen like HClO. The action of peroxide of hydrogen, chromic acid, and other similar oxidising agents gives iodoxybenzol, $\mathrm{C}_{6} \mathrm{H}_{5} \mathrm{IO}_{2}$, which is a neutral substance-i.e. incapable of giving salts with acids (compare Chapter XIII. Note 48).

78 bis The oxidation of iodine by strong nitric acid was discovered by Connell; Millon howed that it is effected, although more slowly, by the action of the hydrates of nitric acid up to $\mathrm{HNO}_{3}, \mathrm{H}_{2} \mathrm{O}$, but that the solution $\mathrm{HNO}_{3}, 2 \mathrm{H}_{2} \mathrm{O}$, and weaker solutions, do not oxidise, but simply dissolve, iodine. The participation of water in reactions is seen in this instance. It is also seen, for example, in the fact that dry ammonia combines directly with iodine-for instance, at $0^{2}$ forming the compound $\mathrm{I}_{2}, 4 \mathrm{NH}_{5}$-whilst iodide of nitrogen is only formed in presence of water. 
its oxygen acids, ${ }^{80}$ and that in the presence of water chlorine oxidises iodine. ${ }^{81}$ Even ozone or a silent discharge passed through a mixture of oxygen and iodine vapour is able to directly oxidise iodine ${ }^{82}$ into iodic acid. It is disengaged from solutions as a hydrate, $\mathrm{HIO}_{3}$, which loses water at $170^{\circ}$, and gives an anhydride, $\mathrm{I}_{2} \mathrm{O}_{5}$. Both these substances are crystalline (sp. gr. $\mathrm{I}_{2} \mathrm{O}_{5} 5.037, \mathrm{HIO}_{3} 4.869$ at $0^{\circ}$ ), colourless and soluble in water $; 83$ both decompose at a red heat into iodine and oxygen, are in many cases powerfully oxidising - for instance, they oxidise sulphurous anhydride, hydrogen sulphide, carbonic oxide, \&c.-form chloride of iodine and water with hydrochloric acid, and with bases form salts, not only normal $\mathrm{MIO}_{3}$, but also acid; for example, $\mathrm{KIO}_{3} \mathrm{HIO}_{3}, \mathrm{KIO}_{3} 2 \mathrm{HIO}_{3}{ }^{83}$ bis With hydriodic acid iodic acid immediately reacts, disengaging iodine, $\mathrm{HIO}_{3}+5 \mathrm{HI}=3 \mathrm{H}_{2} \mathrm{O}+3 \mathrm{I}_{2}$.

80 Bromine also displaces chlorine-for instance, from chloric acid, directly forming bromic acid. If a solution of potassium chlorate be taken (75 parts per 400 parts of water), and iodine be added to it ( 80 parts), and then a small quantity of nitric acid, chlorine is disengaged on boiling, and potassium iodate is formed in the solution. In this instance the nitric acid first evolves a certain portion of the chloric acid, and the latter, with the iodine, evolves chlorine. The iodic acid thus formed acts on a further quantity of the potassium chlorate, sets a portion of the chloric acid free, and in this manner the action is kept up. Potilitzin (1887) remarked, however, that not only do bromine and iodine displace the chlorine from chloric acid and potassium chlorate, but also chlorine displaces bromine from sodium bromate, and, furthermore, the reaction does not proceed as a direct substitution of the halogens, but is accompanied by the formation of free acids; for example, $5 \mathrm{NaClO}_{3}+3 \mathrm{Br}_{2}+3 \mathrm{H}_{2} \mathrm{O}=5 \mathrm{NaBr}+5 \mathrm{HClO}_{3}+\mathrm{HBrO}_{3}$.

81 If iodine be stirred up in water, and chlorine passed through the mixture, the iodine is dissolved; the liquid becomes colourless, and contains, according to the relative amounts of water and chlorine, either $\mathrm{IHCl}_{2}$, or $\mathrm{ICl}_{3}$, or $\mathrm{HIO}_{3}$. If there be a small amount of water, then the iodic acid may separate out directly as crystals, but a complete con. version (Bornemann) only occurs when not less than ten parts of water are taken to one part of iodine- $\mathrm{ICl}+3 \mathrm{H}_{2} \mathrm{O}+2 \mathrm{Cl}_{2}=\mathrm{IHO}_{3}+5 \mathrm{HCl}$.

82 Schönebein and Ogier proved this. Ogier found that at $45^{\circ}$ ozone immediately oxidises iodine vapour, forming first of all the oxide $\mathrm{I}_{2} \mathrm{O}_{3}$, which is decomposed by water or on heating into iodic anhydride and iodine. Iodic acid is formed at the positive pole when a solution of hydriodio acid is decomposed by a galvanic current (Riche). It is also formed in the combustion of hydrogen mixed with a small quantity of hydriodic acid (Salet).

85 Kämmerer showed that a solution of sp. gr. $2 \cdot 127$ at $14^{\circ}$, containing $2 \mathrm{HIO}_{5}, 9 \mathrm{H}_{2} \mathrm{O}$, solidified completely in the cold. On comparing solutions $\mathrm{HI}+m \mathrm{H}_{2} \mathrm{O}$ with $\mathrm{HIO}_{3}+m \mathrm{H}_{2} \mathrm{O}$, we find that the specific gravity increases but the volume decreases, whilst in the passage of solutions $\mathrm{HCl}+m \mathrm{H}_{2} \mathrm{O}$ to $\mathrm{HClO}_{3}+m \mathrm{H}_{2} \mathrm{O}$ both the specific gravity and the volume increase, which is also observed in certain other cases (for example, $\mathrm{H}_{3} \mathrm{PO}_{3}$ and $\mathrm{H}_{3} \mathrm{PO}_{4}$ ).

83 bis Ditte (1890) obtained many iodates of great variety. A neutral salt, $2\left(\mathrm{LiIO}_{5}\right) \mathrm{H}_{2} \mathrm{O}_{\text {, }}$ is obtained by saturating a solution of lithia with iodic acid. There is an analogous ammonium salt, $2\left(\mathrm{NH}_{4} \mathrm{IO}_{3}\right) \mathrm{H}_{2} \mathrm{O}$. He also obtained hydrates of a more complex com. position, such as $6\left(\mathrm{NH}_{4} \mathrm{IO}_{3}\right) \mathrm{H}_{2} \mathrm{O}$ and $6\left(\mathrm{NH}_{4} \mathrm{IO}_{3}\right) 2 \mathrm{H}_{2} \mathrm{O}$. Salts of the alkaline earths, $\mathrm{Ba}\left(\mathrm{IO}_{3}\right)_{2} \mathrm{H}_{2} \mathrm{O}$ and $\mathrm{Sr}\left(\mathrm{IO}_{3}\right)_{2} \mathrm{H}_{2} \mathrm{O}$, may be obtained by a reaction of double decomposition from the normal salts of the type $2\left(\mathrm{MeIO}_{3}\right) \mathrm{H}_{2} \mathrm{O}$. When evaporated at $70^{\circ}$ to $80^{\circ}$ with nitric acid these salts lose water. A mixture of solutions of nitrate of zinc and an alkaline iodate precipitates $\mathrm{Zn}\left(\mathrm{IO}_{3}\right)_{2} 2 \mathrm{H}_{2} \mathrm{O}$. An anhydrous salt is thrown out if nitrio 
As with chlorine, so with iodine, a periodic acid, $\mathrm{HIO}_{4}$, is formed. This acid is produced in the form of its salts, by the action of chlorine on alkaline solutions of iodates, and also by the action of iodine on chloric acid. ${ }^{84}$ It crystallises from solutions as a hydrate containing $2 \mathrm{H}_{2} \mathrm{O}$ (corresponding with $\mathrm{HClO}_{4}, 2 \mathrm{H}_{2} \mathrm{O}$ ), but as it forms salts containing up to 5 atoms of metals, this water must be counted as water of constitution. Therefore $\mathrm{IO}(\mathrm{OH})_{5}=\mathrm{HIO}_{4}, 2 \mathrm{H}_{2} \mathrm{O}$ corresponds with the bighest form of halogen compounds, $\mathrm{IX}_{7}{ }^{85}$ In decomposing (at

acid be added to the solutions. Analogous salts of cadmium, silver, and copper give compounds of the type $2 \mathrm{Me}^{-} \mathrm{TO}_{5} 4 \mathrm{NH}_{5}$ and $\mathrm{Me}^{\prime \prime}\left(\mathrm{IO}_{3}\right)_{2} 4 \mathrm{NH}_{3}$, with gaseous ammonia ( $\mathrm{Me}^{\prime}$ and $\mathrm{Me}^{\prime \prime}$ being elements of the first $(\mathrm{Ag})$ and second $(\mathrm{Cd}, \mathrm{Zn}, \mathrm{Cu})$ groups). With an aqueous solution of ammonia the above salts give substances of a different composition, such as $\mathrm{Zn}\left(\mathrm{IO}_{3}\right)_{2}\left(\mathrm{NH}_{4}\right)_{2} \mathrm{O}, \mathrm{Cd}\left(\mathrm{IO}_{3}\right)_{2}\left(\mathrm{NH}_{4}\right)_{2} \mathrm{O}$. Copper gives $\mathrm{Cu}\left(\mathrm{IO}_{3}\right)_{2} 4\left(\mathrm{NH}_{4}\right)_{2} \mathrm{O}$ and $\mathrm{Cu}\left(\mathrm{IO}_{5}\right)_{2}\left(\mathrm{NH}_{4}\right)_{2} \mathrm{O}$. These salts may be regarded as compounds of $\mathrm{I}_{2} \mathrm{O}_{5}$, and $\mathrm{MeO}$ and $\left(\mathrm{NH}_{4}\right)_{2} \mathrm{O}$; for example, $\mathrm{Zn}\left(\mathrm{IO}_{3}\right)_{2}\left(\mathrm{NH}_{4}\right)_{2} \mathrm{O}$ may be regarded as $\mathrm{ZnO}\left(\mathrm{NH}_{4}\right)_{2} \mathrm{OI}_{2} \mathrm{O}_{5}$, or, as derived from the hydrate, $\mathrm{I}_{2} \mathrm{O}_{5} 2 \mathrm{H}_{2} \mathrm{O}=2\left(\mathrm{HIO}_{3}\right) \mathrm{H}_{2} \mathrm{O}$.

84 If sodium iodate be mired with a solution of sodium hydroxide, heated, and chlorine passed through the solution, a sparingly soluble salt separates out, which corresponds. with periodic acid, and has the composition $\mathrm{Na}_{4} \mathrm{I}_{2} \mathrm{O}_{3}, 3 \mathrm{H}_{2} \mathrm{O}$.

$$
6 \hat{\mathrm{N}} a \mathrm{HO}+2 \mathrm{NaIO}_{3}+4 \mathrm{Cl}=4 \mathrm{NaCl}+\mathrm{Na}_{4} \mathrm{I}_{2} \mathrm{O}_{9}+3 \mathrm{H}_{2} \mathrm{O} \text {. }
$$

This compound is sparingly soluble in water, but dissolves easily in a very dilnte solntion of nitric acid. If silver nitrate be added to this solution a precipitate is formed which contains the corresponding compound of silver, $\mathrm{Ag}_{4} \mathrm{I}_{2} \mathrm{O}_{3}, 8 \mathrm{H}_{2} \mathrm{O}$. If this sparingly soluble silver compound be dissolved in hot nitric acid, orange crystals of a salt having the composition $\mathrm{AgIO}_{4}$ separate on evaporation. This salt is formed from the preceding by the nitric acid taking up silver oxide- $\mathrm{Ag}_{4} \mathrm{I}_{2} \mathrm{O}_{9}+2 \mathrm{HNO}_{3}=2 \mathrm{AgNO}_{3}+2 \mathrm{AgIO}_{4}+\mathrm{H}_{2} \mathrm{O}$. The silver salt is decomposed by water, with the re-formation of the preceding salt, whilst iodic acid remams in solution-

$$
4 \mathrm{AgIO}_{4}+\mathrm{H}_{2} \mathrm{O}=\mathrm{Ag}_{4} \mathrm{I}_{2} \mathrm{O}_{9}+2 \mathrm{HIO}_{4} \text {. }
$$

The structure of the first of these salts, $\mathrm{Na}_{4} \mathrm{I}_{2} \mathrm{O}_{9}, 3 \mathrm{H}_{2} \mathrm{O}$, presents itself in a simpler form if the water of crystallisation is regarded as an integral portion of the sailt; the formula is then divided in two, and takes the form of $\mathrm{IO}(\mathrm{OH})_{3}(\mathrm{ONa})_{2}$-that is, it answers to the type $\mathrm{IOX}_{5}$, or IX 7 , like $\mathrm{AgIO}_{4}$ wwhich is $\mathrm{IO}_{3}(\mathrm{OAg})$. The composition of all the salts of periodic acids are expressed by this type $\mathrm{IX}_{7}$. Kimmins (1889) refers all the salts of periodio acid to four types-the meta-salts of $\mathrm{HIO}_{4}$ (salts of $\mathrm{Ag}, \mathrm{Cu}, \mathrm{Pb}$ ), the meso-salts of $\mathrm{H}_{3} \mathrm{IO}_{3}\left(\mathrm{PbH}, \mathrm{Ag}_{2} \mathrm{H}, \mathrm{CdH}\right)$, the para-salts of $\mathrm{H}_{3} \mathrm{IO}_{6}\left(\mathrm{Na}_{2} \mathrm{H}_{3}, \mathrm{Na}_{3} \mathrm{H}_{2}\right)$, and the di-salts of $\mathrm{H}_{4} \mathrm{I}_{2} \mathrm{O}_{9}\left(\mathrm{~K}_{4}, \mathrm{Ag}_{4}, \mathrm{Ni}_{2}\right)$. The three first are direct compounds of the type $\mathrm{IX}_{7}$, numely, $\mathrm{IO}_{3}(\mathrm{OH}), \mathrm{IO}_{2}(\mathrm{OH})_{3}$, and $\mathrm{IO}(\mathrm{OH})_{5}$, and the last are types of diperiodio salts, which correspond with the type of the meso-salts, as pyrophosphoric salts correspond with orthophosphoric saltg-i.e. $2 \mathrm{H}_{5} \mathrm{IO}_{3}-\mathrm{H}_{2} \mathrm{O}=\mathrm{H}_{4} \mathrm{I}_{2} \mathrm{O}_{9}$.

8s Periodic acid, discovered by Magnus and Ammermüller, and whose salts were afterwards studied by Langlois, Rammelsberg, and many others, presents an example of hydrates in which it is evident that there is not that distinction between the water of hydration and of crystallisation which was at first considered to be so clear. In $\mathrm{HClO}, 2 \mathrm{H}_{2} \mathrm{O}$ the water, $2 \mathrm{H}_{2} \mathrm{O}$, is not displaced by bases, and must be regarded as water of crystallisation, whilst in $\mathrm{HIO}_{4}, 2 \mathrm{H}_{2} \mathrm{O}$ it must be regarded as water of hydration. We shall afterwards see that the systen of the elements obliges us to consider the halogens as substances giving a highest saline type, $G X_{7}$, where $G$ signifies a halogen, and $X$ oxygen $\left(\mathrm{O}=\mathrm{X}_{2}\right), \mathrm{OH}$, and other like elements. The hydrate $\mathrm{IO}(\mathrm{OH})_{5}$ corresponding with many of the salts of periodic acid (for example, the salts of barium, strontium, mercury) does not exhaust all the possible forms. It is evident that various other pyro-, metar, \&c., forms are possible by the loss of water, as will be more fully explained in speaking of phosphorio acid, and as was pointed out in the preceding note. 
$200^{\circ}$ ) or acting as an oxidiser, periodic acid first gives iodic acid, but it may also be ultimately decomposed.

Compounds formed between chlorine and iodine must be classed among the most interesting halogen bodies. ${ }^{86}$ These elements combine together directly with evolution of heat, and form iodine monochloride; $\mathrm{ICl}$, or iodine trichloride, $\mathrm{ICl}_{3} \cdot{ }^{87} \mathrm{As}$ water reacts on these substances, forming iodic acid and iodine, they have to be prepared from dry iodine and chlorine. ${ }^{88}$ Both substances are formed in a number of reactions; for example, by the action of aqua regia on iodine, of chlorine on hydriodic acid, of hydrochloric acid on periodic acid, of iodine on potassium chlorate (with the aid of heat, \&c.) Trapp obtained iodine monochloride, in beautiful red crystals, by passing a rapid current of chlorine into molten iodine. The monochloride then distils over and solidifies, melting at $27^{\circ}$ By passing chlorine over the

se With respect to hydrogen, oxygen, chlorine, and other elements, bromine occupies an intermediate position between chlorine and iodine, and therefore there is no particular need for considering at length the compounds of bromine This is the great advantage of a natural grouping of the elements.

87 They were both obtained by Gry-Lussao and many others. Recent data respect. ing iodine munochloride, ICl, entirely confirm the numerous observations of Trapp (1854), and even confirm his statement as to the existence of two isomeric (liquid and crystalline) forms (Stortenbeker). With a small excess of iodine, iodine monochloride remains liquid, but in the presence of traces of iodine trichloride it easily crystallises. Tanatar (1893) showed that of the two modifications of ICl, one is stable, and melts at $27^{\circ}$; while the other, which easily passes into the first, and is formed in the absence of $\mathrm{ICl}_{3}$, melts at $14^{\circ}$. Schiitzenberger amplified the data concerning the action of water on the chlorides (Note 88), and Christomanos gave the fullest data regarding the trichloride.

After being kept for some time, the liquid monochloride of iodine yields red deliques. cent octahedra, having the composition $\mathrm{ICl}_{4}$, which are therefore formed from the mono. chloride with the liberation of free iodine, which dissolves in the remaining quantity of the monochloride. This substance, however, judging by certain observations, is impure iodine trichloride. If 1 part of indine be stirred up in 20 parts of water, and chlorine be passed through the liquid, then all the iodine is dissolved, and a colourless liquid is ultimately obtrined which contains a certain proportion of chlorine, because this compound gives a metallic chloride and iodate with alkalis without evolving any free iodine: $\mathrm{ICl}_{5}+6 \mathrm{KHO}$ $=5 \mathrm{KCl}+\mathrm{KIO}_{3}+8 \mathrm{H}_{2} \mathrm{O}$ The existence of a pentachloride $\mathrm{ICl}_{5}$ is, however, denied, because this substance has not been obtained in a free state.

Stortenbeker (1888) investigated the equilibrium of the system containing the molecules $\mathrm{I}_{2}, \mathrm{ICl}, \mathrm{ICl}_{3}$, and $\mathrm{Cl}_{2}$, in the same way that Roozeboom (Chapter X. Note 38) examined the equilibrium of the molecules $\mathrm{HCl}, \mathrm{HCl}, 2 \mathrm{H}_{2} \mathrm{O}$, and $\mathrm{H}_{2} \mathrm{O}$. $\mathrm{He}$ found that iodine monochloride appears in two states, one (the ordinary) is stable and melts at $27^{\circ} \cdot 2$, whilst the other is obtained by rapid cooling, and melts at $13^{\circ} .9$, and easily passes into the first form. Iodine trichloride melts at $101^{\circ}$ only in a closed tube under a pressure of 16 atmospheres.

$88 \mathrm{By}$ the action of water on iodine monochloride and trichloride a compound $\mathrm{IHCl}_{2}$ is obtained, which does not seem to be altered by water. Besides this compound, iodine and iodic acid are always formed, $10 \mathrm{ICl}+3 \mathrm{H}_{2} \mathrm{O}=\mathrm{HIO}_{3}+6 \mathrm{IHCl}_{2}+2 \mathrm{I}_{2}$; and in this respect iodine trichloride may be regarded as a mixture, $\mathrm{ICl}+\mathrm{ICl}_{5}=2 \mathrm{ICl}_{3}$, but $\mathrm{ICl}_{5}+3 \mathrm{H}_{2} \mathrm{O}$ $=\mathrm{IHO}_{3}+5 \mathrm{HCl}$; henoe iodic acid, iodine, the compound $\mathrm{IHCl}_{2}$, and hydrochlotic acid are also formed by the action of water. 
crystals of the monochloride, it is easy to obtain iodine trichloride in orange crystals, which melt at $34^{\circ}$ and volatilise at $47^{\circ}$, but in so doing decompose (into $\mathrm{Cl}_{2}$ and $\mathrm{ClI}$ ). The chemical properties of these chlorides entirely resemble those of chlorine and iodine, as would be expected, because, in this instance, a combination of similar substances has taken place as in the formation of solutions or alloys. Thus, for instance, the unsaturated hydrocarbons (for example, $\mathrm{C}_{2} \mathrm{H}_{4}$ ), which are capable of directly combining with chlorine and iodine, also directly combine with iodine monochlorido. 


\section{CHAPTER XII}

SODIUM

Tre neutral salt, sodium sulphate, $\mathrm{Na}_{2} \mathrm{SO}_{4}$, obtained when a mixture of sulphuric acid and common salt is strongly heated (Chapter X.), ${ }^{1}$ forms a colourless saline mass consisting of fine crystals, soluble in water. It is the product of many other double decompositions, sometimes carried out on a large scale; for example, when ammonium sulphate is heated with common salt, in which case the sal-ammoniac is volatilised, \&c. A similar decomposition also takes place when, for instance, a mixture of lead sulphate and common salt is heated; this mixture easily fuses, and if the temperature be further raised heavy vapours of lead chloride appear. When the disengagement of these vapours ceases, the remaining mass, on being treated with water, yields a solution of sodium sulphate mixed with a solution of undecomposed common salt. A considerable quantity, however, of the lead sulphate remains unchanged during this reaction, $\mathrm{PbSO}_{4}+2 \mathrm{NaCl}=\mathrm{PbCl}_{2}+\mathrm{Na}_{2} \mathrm{SO}_{4}$, the vapours will contain lead chloride, and the residue will contain the mixture of the three remaining salts. The cause and nature of the reaction are just the same as were pointed out when considering the action of sulphuric acid upon $\mathrm{NaCl}$. Here too it may be shown that the double decomposition is determined by the removal of $\mathrm{PbCl}_{2}$ from the sphere of the action of the remaining substances. This is seen from the fact that sodium sulphate, on being dissolved in water and mixed with a solution of any lead salt (and even with a solution of lead chloride, although this latter is but sparingly soluble in water), immediately gives a white precipitate of lead sulphate. In this case the lead takes up the elements of sulphuric acid from the sodium sulphate in the solutions.

1 Whilst describing in some detail the properties of sodium chloride, hydrochloric acid, and sodium sulphate, I wish to impart, by separate examples, an idea of the properties of saline substances, but the dimensions of this work and its purpose and aim do not permit of entering into particulars concerning every salt, acid, or other substance. The fundamental object of this work-an account of the characteristics of the elements and an acquaintance with the forces acting between atoms-has nothing to gain from the multiplication of the number of as yet ungeneralised properties and relations. 
On heating, the reverse phenomenon is observed. The reaction in the solution depends upon the insolubility of the lead sulphate, and the decomposition which takes place on heating is due to the volatility of the lead chloride. Silver sulphate, $\mathrm{Ag}_{2} \mathrm{SO}_{4}$, in solution with common salt, gives silver chloride, because the latter is insoluble in water, $\mathrm{Ag}_{2} \mathrm{SO}_{4}+2 \mathrm{NaCl}=\mathrm{Na}_{2} \mathrm{SO}_{4}+2 \mathrm{AgCl}$. Sodium carbonate, mixed in solution with the sulphates of iron, copper, manganese, magnesium, \&c., gives in solution sodium sulphate, and in the precipitate a carbonate of the corresponding metal, because these salts of carbonic acid are insoluble in water; for instance, $\mathrm{MgSO}_{4}+\mathrm{Na}_{2} \mathrm{CO}_{3}=\mathrm{Na}_{2} \mathrm{SO}_{4}$ $+\mathrm{MgCO}_{3}$. In precisely the same way sodium hydroxide acts on solutions of the majority of the salts of sulphuric acid containing metals, the hydroxides of which are insoluble in water-for instance, $\mathrm{CuSO}_{4}+2 \mathrm{NaHO}=\mathrm{Cu}(\mathrm{H} O)_{2}+\mathrm{Na}_{2} \mathrm{SO}_{4}$. Sulphate of magnesium, $\mathrm{MgSO}_{4}$, on being mixed in solution with common salt, forms, although not completely, chloride of magnesium, and sodium sulphate. On cooling the mixture of such (concentrated) solutions sodium sulphate is deposited, as was shown in Chapter $\mathbf{X}$. This is made use of for preparing it on the large scale in works where sea-water is treated. In this case, on cooling, the reaction $2 \mathrm{NaCl}+\mathrm{MgSO}_{4}=\mathrm{MgCl}_{2}+\mathrm{Na}_{2} \mathrm{SO}_{4}$ takes place.

Thus where sulphates and salts of sodium are in contact, it may be expected that sodium sulphate will be formed and separated if the conditions are favourable; for this reason it is not surprising that sodium sulphate is often found in the native state. Some of the springs and salt lakes in the steppes beyond the Volga, and in the Caucasus, contain a considerable quantity of sodium sulphate, and yield it by simple evaporation of the solutions. Beds of this salt are also met with; thus at a depth of only $5^{5}$ feet, about 38 versts to the east of Tifis, at the foot of the range of the 'Wolf's mane' (Voltchia griva) mountains, a deep stratum of very pure Glauber's salt, $\mathrm{Na}_{2} \mathrm{SO}_{4} ; 10 \mathrm{H}_{2} \mathrm{O}$, has been found, ${ }^{2}$ A layer two metres thick of the same salt lies at the bottom of several lakes (an area of about 10 square kilometres) in the Kouban district near Batalpaschinsk, and here its working has been commenced (1887). In Spain, near Arangoulz and in many parts of the Western States of North America, mineral sodium sulphate has likewise been found, and is already being worked.

The methods of obtaining salts by means of double decomposition

2 Anhydrous (ignited) sodium sulphate, $\mathrm{Na}_{2} \mathrm{SO}_{4}$, is known in trade as 'sulphate' or salt-cake, in mineralogy thenardite. Crystalline decahydrated salt is termed in mineralogy mirabilite, and in trade Glauber's salt. On fusing it, the monohydrate $\mathrm{Na}_{2} \mathrm{SO}_{4} \mathrm{H}_{2} \mathrm{O}$ is obtained, together with a supersaturated solution. 
from others already prepared are so general, that in describing a given salt there is no necessity to enumerate the cases hitherto observed of its being formed through various double decompositions. ${ }^{3}$ The possi. bility of this occurrence ought to be foreseen according to Berthollet's doctrine from the properties of the salt in question. On this account it is important to know the properties of salts ; all the more so because up to the present time those very properties (solubility, formation of crystallo-hydrates, volatility, \&c.) which may be made use of for separating them from other salts have not been generalised. ${ }^{4}$ These properties as yet remain subjects for investigation, and are rarely to be foreseen. The crystallo-hydrate of the normal sodium sulphate, $\mathrm{Na}_{2} \mathrm{SO}_{4}, 10 \mathrm{H}_{2} \mathrm{O}$, very easily parts with water, and may be obtained in an anhydrous state if it be carefully heated until the weight remains constant; but if heated further, it partly loses the elements of sulphuric anhydride. The normal salt fuses at $843^{\circ}$ (red heat), and volatilises to a slight extent when very strongly heated, in which case it naturally decomposes with the evolution of $\mathrm{SO}_{3}$. At $0^{\circ} 100$ parts of water dissolve 5 parts of the anhydrous salt, at $10^{\circ} 9$ parts, at $20^{\circ} 19.4$, at $30^{\circ} 40$, and at $34^{\circ} 55$ parts, the same being the case in the presence of an excess of crystals of $\mathrm{Na}_{2} \mathrm{SO}_{4}, 10 \mathrm{H}_{2} \mathrm{O} .^{5}$ At $34^{\circ}$ the latter fuses, and the solubility decreases at higher temperatures. ${ }^{6}$. A concentrated solution at $34^{\circ}$ has a composition nearly approacning to $\mathrm{Na}_{2} \mathrm{SO}_{4}+14 \mathrm{H}_{2} \mathrm{O}$,

5 The salts may be obtained not only by methods of substitution of various kinds, but also by many other combinations. Thus sodium sulphate may be formed from sodium oride and sulphuric anhydride, by oxidising sodiun sulphide, $\mathrm{Na} 2 \mathrm{~S}$, or sodium sulphite, $\mathrm{Na}_{2} \mathrm{SO}_{3}$, \&c. When sodium chloride is heared in a mixture of the vapours of water, air, and sulphurous anhydride, sodium sulphate is formed. According to this method (patented by Hargreaves and Robinson), sodium sulphate, $\mathrm{Na}_{2} \mathrm{SO}_{4}$, is obtained from $\mathrm{NaCl}$ without the preliminary manufacture of $\mathrm{H}_{2} \mathrm{SO}_{4}$. Lumps of $\mathrm{NaCl}$ pressed into bricks are loosely packed into a cylinder and subjected, at a red heat, to the action of steam, air and $\mathrm{SO}_{2}$. Under thess conditions, $\mathrm{HCl}$, sulphate, and a certain amount of unaltered $\mathrm{NaCl}$ are obtained. This mixture is converted into soda by Gossage's process (see Note 15) and may have some practical value.

4 Many observations have been made, but little general information has been obtained from particular cases. In addition to which, the properties of a given salt are changed by the presence of other salts. This takes place not only in virtue of mutual decomposition or formation of double salts capable of separate existence, but is determined by the influence which some saits exert on others, or by forces similar to those which act during solution. Here nothing has been generalised to that extent which would render it possible to predict without previous investigation, if there be no close analogy to help us. Let us state one of these numerous cases: 100 parts of water at $20^{\circ}$ dissolve 84 parts of potassium nitrate but on the addition of sodium nitrate the solubility of potassium nitrate increases to 48 parts in 10 of water (Camelley and Thomson). In general, in all cases of which there are accurate observations it appears that the presence of foreign salts changes the properties of any given salt.

s The information concerning solubility (Chapter I.) is given according to the determinations of Gay-Lussac, Lovell, and Mulder.

- In Chapter I, Note 24, we have already seen that with many other sulphates the 
and the decahydrated salt contains 78.9 of the anhydrous salt combined with 100 parts of water. From the above figures it is seen that the decahydrated salt cannot fuse without decomposing, ${ }^{7}$ like hydrate of chlorine, $\mathrm{Cl}_{2}, 8 \mathrm{H}_{2} \mathrm{O}$ (Chapter XI., Note 10). Not only the fused deca. hydrated salt, but also the concentrated solution at $34^{\circ}$ (not all at once, but gradually), yields the monohydrated salt, $\mathrm{Na}_{2} \mathrm{SO}_{4}, \mathrm{H}_{2} \mathrm{O}$. The heptahydrated salt, $\mathrm{Na}_{2} \mathrm{SO}_{4}, 7 \mathrm{H}_{2} \mathrm{O}$, also splits up, even at low temperatures, with the formation of this monohydrated salt, and therefore from $35^{\circ}$ the solubility can be given only for the latter. For 100 parts of water this is as follows : at $40^{\circ} 48 \cdot 8$, at $50^{\circ} 46.7$, at $80^{\circ} 43 \cdot 7$, at $100^{\circ} 42.5$ parts of the anhydrous. salt. If the decahydrated salt be fused, and the solution allowed to cool in the presence of the monohydrated salt, then at $30^{\circ} 50.4$ parts of anhydrous salt are retained in the solution, and at $20^{\circ} 52.8$ parts. Hence, with respect to the anhydrous and monohydrated salts, the solubility is identical, and falls with increasing temperature, whilst with respect to decahydrated salt, the solubility rises with increasing temperature. So that if in contact with a solution

solubility also decreases after a certain temperature is passed. Gypsum, $\mathrm{CaSO}_{4}, 2 \mathrm{H}_{2} \mathrm{O}$, lime, and many other compounds present such a phenomenon. An observation of Tilden's (1884) is most instructive; he showed that on raising the temperature (in closed vessels) above $140^{\circ}$ the solubility of sodium sulphate again begins to increase. At $100^{\circ} 100$ parts of water dissolve about 43 parts of anhydrous salt, at $140^{\circ} 42$ parts, at $160^{\circ} 43$ parts, at $180^{\circ} 44$ parts, at $230^{\circ} 46$ parts. According to Etard (1892) the solubility of 30 parts of $\mathrm{Na}_{2} \mathrm{SO}_{4}$ in 100 of solution (or 48 per 100 of water) corresponds to $80^{\circ}$, and above $240^{\circ}$ the solubility again falls, and very rapidly, so that at $320^{\circ}$ the solution contrins 12 per 100 of solution (about 14 per 100 of water) and a further rise of temperature is followed by a further deposition of the salt. It is evident that the phenomenon of saturation, deter. mined by the presence of an excess of the dissolved substance, is very complex, and therefore that for the theory of solutions considered as liquid indefinite chemical com. pounds, many useful statements can hardly be given.

7 Already referred to in Chapter I., Note 56.

The example of sodium sulphate is historically very important for the theory of solutions. Notwithstanding the number of investigations which have been made, it is still insufficiently studied, especially from the point of the vapour tension of solutions and crystallo-hydrates, so that those processes cannot be applied to it which Guldberg, Roozeboom, Van't Hoff, and others applied to solutions and crystallo-hydrates. It would also be most important to investigate the influence of pressure on the various phenomena corresponding with the combinations of water and sodium sulphate, because when crystals are separated-for instance, of the decahydrated salt-an increase of volume takes place, as can be seen from the following data:-the sp. gr. of the anhydrous salt is $2 \cdot 66$, that of the decahydrated salt $=1 \cdot 46$, but the sp. gr. of solutions at $15^{\circ} / 4^{\circ}=9,992+90 \cdot 2 p+0 \cdot 35 p^{2}$ where $p$ represents the percentage of anhydrous salt in the solution, and the sp. gr. of water at $4^{\circ}=10,000$. Hence for solutions containing 20 p.c. of anhydrous salt the sp. gr. $=1.1936$; therefore the volume of 100 grams of this solution $=88.8$ c.c., and the volume of anhydrous salt contained in it is equal to $20 / 2 \cdot 66$, or $=7.5$ c.c., and the volume of water $=80.1$ c.c. Therefore, the solution, on decomposing into anhydrous salt and water, increases in volume (from 83.8 to 87.6 ); but in the same way 83.8 c.c. of 20 p.c. solution are formed from $(45 \cdot 4 / 1 \cdot 46=) 31.1$ c.c. of the decahydrated salt, and 54.6 c.c. of waterthat is to say, that during the formation of a solution from $85 \cdot 7$ c.c., 83.8 c.c. are formed. 
of sodium sulphate there are only crystals of that heptahydrated salt (Chapter I., Note 54), $\mathrm{Na}_{2} \mathrm{SO}_{4}, 7 \mathrm{H}_{2} \mathrm{O}$, which is formed from saturated solutions, then saturation sets in when the solution has the following composition per 100 parts of salt : at $0^{\circ} 19 \cdot 6$, at $10^{\circ} 30.5$, at $20^{\prime}$ 44.7 , and at $25^{\circ} 52.9$ parts of anhydrous salt. Above $27^{\circ}$ the heptahydrated salt, like the decahydrated salt at $34^{\circ}$, splits up into the monohydrated salt and a saturated solution. Thus sodium sulphate has three curves of solubility : one for $\mathrm{Na}_{2} \mathrm{SO}_{4}, 7 \mathrm{H}_{2} \mathrm{O}$ (from $0^{\text {' }}$ to $26^{\circ}$ ), one for $\mathrm{Na}_{2} \mathrm{SO}_{4}, 10 \mathrm{H}_{2} \mathrm{O}$ (from $0^{\circ}$ to $34^{\circ}$ ), and one for $\mathrm{Na}_{2} \mathrm{SO}_{4}, \mathrm{H}_{2} \mathrm{O}$ (a descending curve beginning at $26^{\circ}$ ), because there are three of these crystallo-hydrates, and the solubility of a substance only depends upon the particular condition of that portion of it which has separated from the solution or is present in excess. ${ }^{8}$

Thus solutions of sodium sulphate may give crystallo-hydrates of three kinds on cooling the saturated solution : the unstable heptahydrated salt is obtained at temperatures below $26^{\circ}$, the decahydrated salt forms under ordinary conditions at temperatures below $34^{\circ}$, and the monohydrated salt at temperatures above $34^{\circ}$. Both the latter crystallo-hydrates present a stable state of equilibrium, and the hepta. hydrated salt decomposes into them, probably according to the equation $3 \mathrm{Na}_{2} \mathrm{SO}_{4}, 7 \mathrm{H}_{2} \mathrm{O}=2 \mathrm{Na}_{2} \mathrm{SO}_{4}, 10 \mathrm{H}_{2} \mathrm{O}+\mathrm{Na}_{2} \mathrm{SO}_{4}, \mathrm{H}_{2} \mathrm{O}$. The ordinary decahydrated salt is called Glauber's salt. All forms of these crystallohydrates lose their water entirely, and give the anhydrous salt when dried over sulphuric acid. ${ }^{9}$

Sodium sulphate, $\mathrm{Na}_{2} \mathrm{SO}_{4}$, only enters into, a few reactions of combination with other salts, and chiefly with salts of the same acid, forming double sulphates. Thus, for example, if a solution of sodium

8 From this example it is evident the solution remains unaltered until from the contact of a solid it becomes either saturated or supersaturated, crystallisation being determined by the attraction to a solid, as the phenomenon of supersaturation clearly demonstrates. This partially explains certain apparently contradictory determinations of solubility. The best investigated example of such complex relations is cited in Chapter XIV., Note 50 (for $\mathrm{CaCl}_{2}$ ).

9 According to Pickering's experiments (1886), the molecular weight in grams (that is, 142 grams) of anhydrous sodium sulphate, on being dissolved in a large mass of water, at $0^{\circ}$ absorbs (hence the $\left.-\operatorname{sign}\right)-1,100$ heat units, at $10^{\circ}-700$, at $15^{\circ}-275$, at $20^{\circ}$ gives out +25 , at $25^{\circ}+300$ calories. For the decahydrated salt, $\mathrm{Na}_{2} \mathrm{SO}_{4}, 10 \mathrm{H}_{2} \mathrm{O}$, $5^{\circ}-4,225,10^{\circ}-4,000,15^{\circ}-3,570,20^{\circ}-3,160,25^{\circ}-2,775$. Hence (just as in Chapter I., Note 56) the heat of the combination $\mathrm{Na}_{2} \mathrm{SO}_{4}, 10 \mathrm{H}_{2} \mathrm{O}$ at $5^{\circ}=+3,125,10^{\circ}=+3,250$, $20^{\circ}=+3,200$, and $25^{\circ}=+3,050$.

It is evident that the decahydrated salt dissolving in water gives a decrease of temperature. Solutions in hydrochloric acid give a. still greater decrease, because they contain the water of crystallisation in a solid state-that is, like ice-and this on melting absorbs heat. A mixture of 15 parts of $\mathrm{Na}_{2} \mathrm{SO}_{4}, 10 \mathrm{H}_{2} \mathrm{O}$ and 12 parts of 'strong hydrochloric acid' produces sufficient cold to freeze water. During the treatment with hydrochloric acid a certain quantity of sodium chloride is formed. 
sulphate be mixed with a solution of aluminium, magnesium, or ferrous sulphate, it gives crystals of a double salt when evaporated. Sulphuric acid itself forms a compound with sodium sulphate, which is exactly like these double salts. It is formed with great ease when sodium sulphate is dissolved in sulphuric acid and the solution evaporated. On evaporation, crystals of the acid salt separate, $\mathrm{Na}_{2} \mathrm{SO}_{4}+\mathrm{H}_{2} \mathrm{SO}_{4}$ $=2 \mathrm{NaHSO}_{4}$. This separates from hot solutions, whilst the crystallohydrate, $\mathrm{NaHSO}_{4}, \mathrm{H}_{2} \mathrm{O},{ }^{10}$ separates from cold solutions. The crystals when exposed to damp air decompose into $\mathrm{H}_{2} \mathrm{SO}_{4}$, which deliquesces, and $\mathrm{Na}_{2} \mathrm{SO}_{4}$ (Graham, Rose) ; alcohol also extracts sulphuric acid from the acid salt. This shows the feeble force which holds the sulphuric acid to the sodium sulphate." ${ }^{11}$ Both acid sodium sulphate and all mixtures of the normal salt and sulphuric acid lose water when heated, and are converted into sodium pyrosulphate, $\mathrm{Na}_{2} \mathrm{~S}_{2} \mathrm{O}_{7}$, at a low red heat." bis This anhydrous salt, at a bright red heat, parts with the elements of sulphuric anhydride, the normal sodium sulphate remaining behind $-\mathrm{Na}_{2} \mathrm{~S}_{2} \mathrm{O}_{7}=\mathrm{Na}_{2} \mathrm{SO}_{4}+\mathrm{SO}_{3}$. From this it is seen that the normal salt is able to combine with water, with other sulphates, and with sulphuric anhydride or acid, \&c.

Sodium sulphate may by double decomposition be converted into a sodium salt of any other acid, by means of heat and taking advantage of the volatility, or by means of solution and taking advantage of the different degree of solubility of the different salts. Thus, for instance, owing to the insolubility of barium sulphate, sodium hydroxide or caustic soda may be prepared from sodium sulphate, if barium hydroxide be added to its solution, $\mathrm{Na}_{2} \mathrm{SO}_{4}+\mathrm{Ba}(\mathrm{HO})_{2}=\mathrm{BaSO}_{4}+2 \mathrm{NaHO}$. And by taking any salt of barium, $\mathrm{BaX}_{2}$, the corresponding salt of sodium may be obtained, $\mathrm{Na}_{2} \mathrm{SO}_{4}+\mathrm{BaX}_{2}=\mathrm{BaSO}_{4}^{+}+2 \mathrm{NaX}$. Barium

10 The very large and well-formed crystals of this salt resemble the hydrate $\mathrm{H}_{2} \mathrm{SO}_{4}, \mathrm{H}_{2} \mathrm{O}$, or $\mathrm{SO}(\mathrm{OH})_{4}$. In general the replacement of hydrogen by sodium modifies many of the properties of acids less than its replacement by other metals. This most probably depends on the volumes being nearly equal.

11 In solution (Berthelot) the acid salt in all probability decomposes most in the greatest mass of water. The specific gravity (according to the determinations of Marignac) of solutions at $15^{\circ} / 4^{\circ}=9,992+77^{\circ} 92 p+0.231 p^{2}$ (see Note 7 ). From these figures, and from the specific gravities of sulphuric acid, it is evident that on mixing solutions of this acid and sodium sulphate expansion will always take place; for instance, $\mathrm{H}_{2} \mathrm{SO}_{4}+25 \mathrm{H}_{2} \mathrm{O}$ with $\mathrm{Na}_{2} \mathrm{SO}_{4}+25 \mathrm{H}_{2} \mathrm{O}$ increases from 483 volumes to 486 . In addition to which, in weak solutions heat is absorbed, as shown in Chapter X., Note 27. Nevertheless, even more acid salts may be formed and obtained in a crystalline form. For instance, on cooling a solution of 1 part of sodium sulphate in 7 parts of sulphurio acid, crystals of the composition $\mathrm{NaHSO}_{4}, \mathrm{H}_{2} \mathrm{SO}_{4}$ are separated (Schultz, 1868). This compound fuses at about $100^{\circ}$; the ordinary acid salt, $\mathrm{NaHSO}_{4}$, at $149^{\circ}$.

11 bis $\mathrm{On}$ decreasing the pressure, sodium hydrogen sulphate, $\mathrm{NaHSO}_{4}$, dissociates much more easily than at the ordinary pressure; it loses water and forms the pyrosulphate, $\mathrm{Na}_{2} \mathrm{~S}_{2} \mathrm{O}_{7}$; this reaction is atilised in chemical works. 
sulphate thus formed, being a very sparingly-soluble salt, is obtained as a precipitate, whilst the sodium hydroxide, or salt, $\mathrm{NaX}$, is obtained in solution, because all salts of sodium are soluble. Berthollet's doctrine permits all such cases to be foreseen

The reactions of decomposition of sodium sulphate are above all noticeable by the separation of oxygen. Sodium sulphate by itself is very stable, and it is only at a temperature sufficient to melt iron that it is possible to separate the elements $\mathrm{SO}_{3}$ from it, and then only partially. However, the oxygen may be separated from sodium sulphate, as from. all other sulphates, by means of many substances which are able to combine with oxygen, such as charcoal and sulphur, but hydrogen is not able to produce this action. If sodium sulphate be heated with charcoal, then carbonic oxide and anhydride are evolved, and there is produced, according to the circumstances, either the lower oxygen compound, sodium sulphite, $\mathrm{Na}_{2} \mathrm{SO}_{3}$ (for instance, in the formation of glass); or else the decomposition proceeds further, and sodium sulphide, $\mathrm{Na}_{2} \mathrm{~S}$, is formed, according to the equation $\mathrm{Na}_{2} \mathrm{SO}_{4}+2 \mathrm{O}$ $=2 \mathrm{CO}_{2}+\mathrm{Na}_{2} \mathrm{~S}$.

On the basis of this reaction the greater part of the sulphate of sodium prepared at chemical works is converted into soda ash-that is, sodium carbonate, $\mathrm{Na}_{2} \mathrm{CO}_{3}$, which is used for many purposes. In the form of carbonates, the metallic oxides behave in many cases just as they do in the state of oxides or hydroxides, owing to the feeble acid properties of carbonic acid. However, the majority of the salts of carbonic acid are insoluble, whilst sodium carbonate is one of the few soluble salts of this acid, and therefore reacts with facility. Hence sodium carbonate is employed for many purposes, in which its alkaline properties come into play. Thus, even under the action of feeble organic acids it immediately parts with its carbonic acid, and gives a sodium salt of the acid taken. Its solutions exhibit an alkaline reaction on litmus. It aids the passage of certain organic substances (tar, acids) into solution, and is therefore used, like caustic alkalis and soap (which latter also acts by virtue of the alkali it contains), for the removal of certain organic substances, especially in bleaching cotton and similar fabrics. Besides which a considerable quantity of sodium carbonate is used for the preparation of sodium hydroxide or caustic soda, which has also a very wide application. In large chemical works where sodium carbonate is manufactured from $\mathrm{Na}_{2} \mathrm{SO}_{4}$, it is usual first to manufacture sulphuric acid, and then by its aid to convert common salt into sodium sulphate, and lastly to convert the sodium sulphate thus obtained into carbonate and caustic soda. Hence these works prepare both alkaline substances (soda ash and caustic 
soda) and acid substances (sulphuric and hydrochloric acids), the two classes of chemical products which are distinguished for the greatest energy of their reactions and are therefore most frequently applied to technical purposes. Factories manufacturing soda are generally called alkali works.

The process of the conversion of sodium sulphate into sodium carbonate consists in strongly heating a mixture of the sulphate with charcoal and calcium carbonate. The following reactions then take place : the sodium sulphate is first deoxidised by the charcoal, forming sodium sulphide and carbonic anhyouride, $\mathrm{Na}_{2} \mathrm{SO}_{4}+2 \mathrm{C}=\mathrm{Na}_{2} \mathrm{~S}+2 \mathrm{CO}_{2}$. The sodium sulphide thus formed then enters into double decomposition with the calcium carbonate taken, and gives calcium sulphide and sodium carbonate, $\mathrm{Na}_{2} \mathrm{~S}+\mathrm{CaCO}_{3}=\mathrm{Na}_{2} \mathrm{CO}_{3}+\mathrm{CaS}$.

Besides which, under the action of the heat, a portion of the excess of calcium carbonate is decomposed into lime and carbonic anhydride, $\mathrm{CaCO}_{3}=\mathrm{CaO}+\mathrm{CO}_{2}$, and the carbonic anhydride with the excess of charcoal forms carbon monoxide, which towards the end of the operation shows itself by the appearance of a blue flame. Thus from a mass containing sodium sulphate we obtain a mass which includes sodium carbonate, calcium sulphide, and calcium oxide, but none of the sodium sulphide which was formed on first heating the mixture. The entire process, which proceeds at a high temperature, may be expressed by a combination of the three above-mentioned formulæ, if it be considered that the product contains one equivalent of calcium oxide to two equivalents of calcium sulphide. ${ }^{12}$ The sum of the reactions may then be expressed thus: $2 \mathrm{Na}_{2} \mathrm{SO}_{4}+3 \mathrm{CaCO}_{3}+9 \mathrm{C}=2 \mathrm{Na}_{2} \mathrm{CO}_{3}$ $+\mathrm{CaO}, 2 \mathrm{CaS}+10 \mathrm{CO}$ Indeed, the quantities in which the substances are mixed together at chemical works approaches to the proportion required by this equation. The entire process of decomposition is carried on in reverberatory furnaces, into which a mixture of 1,000 parts of sodium sulphate, 1,040 parts of calcium carbonate (as a somewhat porous limestone), and 500 parts of small coal is introduced from above. This mixture is first heated in the portion of the furnace which is

12 Calcium sulphide, $\mathrm{CaS}$, like many metallic sulphides which are soluble in water, is decomposed by it (Chapter $\mathrm{X}$.), $\mathrm{CaS}+\mathrm{H}_{2} \mathrm{O}=\mathrm{CaO}+\mathrm{H}_{2} \mathrm{~S}$, because hydrogen sulphide is a very feeble acid. If calcium sulphide be acted on by a large mass of water, lime may be precipitated, and a state of equilibrium will be reached, when the system $\mathrm{CaO}+2 \mathrm{CaS}$ remains unchanged. Lime, being a product of the action of water on $\mathrm{CaS}$, limits this action. Therefore, if in black ash the lime were not in excess, a part of the sulphide would be in solution (actually there is but very little). In this manner in the mannfacture of sodinm carbonate the conditions of equilibrium which ente: into double decompositions have been made use of (see above), and the aim is to form directly the unchangeable product $\mathrm{CaO}, 2 \mathrm{CaS}$. This was first regarded as a special insoluble compound, but there is no evidence of its independent existence. 
furthest removed from the fire-grate; it is then brought to the portion nearest to the fre-grate, when it is stirred during heating. The

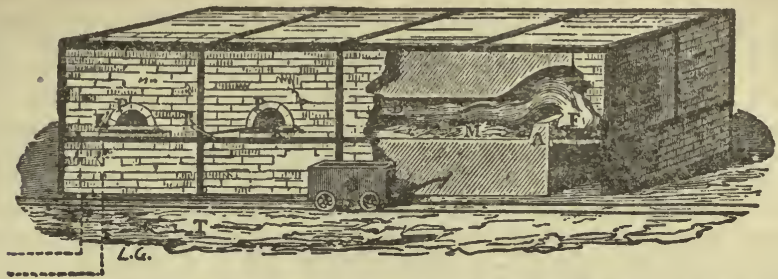

FTo. 68.- Reverberatory furnace for the manufacture of sollium carbonate. F, grate. A, bridge. M, hearth for the ultimate calcination of the mixture of sodium sulphate, coal, and calcium carbonate, which is charged from above into the part of the furnace furthest removed from the fire $F$. $\mathbf{P}, \mathbf{P}$, doors for stirring and bringing the mass towards the grate $\mathbf{F}$ by means of stirrers $\mathbf{R}$. At the end of the operstion the semifused mass is charged mto trucks $C$.

partially fused mass obtained at the end of the process is cooled, and then subjected to methodical lixiviation ${ }^{13}$ to extract the sodium car-

15 Methodical lixiviation is the extraction, by means of water, of a soluble substance from the mass containing it. It is carried on so as not to obtain wealk aqueous solutions, and in such \& way that the residue shall not contain any of the soluble substance. This problem is pract:cally of great importance in many industries. It is required to extract from the mass all that is soluble in water. This is easily effected if water be first poured

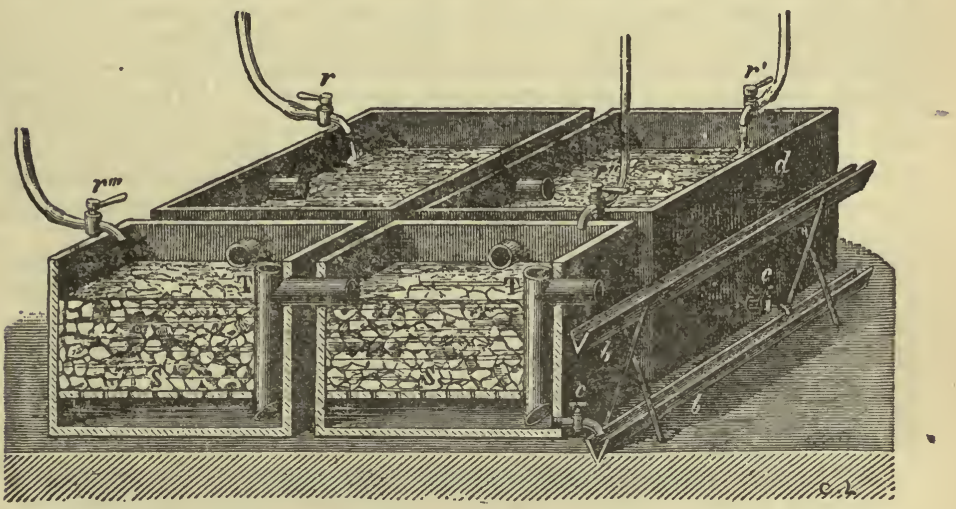

FiG. 69.-Apparatus for the methodical lixiviation of black ash, \&c. Water flows into the tanks from the pipes $r, r$, and the saturated liquid is drawn off from $c . c$.

on the mass, the strong solution thus obtained deoanted, then water again poured on, time being allowed for it to act, then again decanted, and so on until fresh water does not take up anything. But then finally such weak solutions are obtained that it would be very disadvantageous to evaporate them. This is avoided by pouring the fresb hot water destined for the lixiviation, not onto the fresh mass, but upon a mass 
bonate, the mixture of calcium oxide and sulphide forming the so.called 'soda waste ' or 'alkali waste.' 14

which has already been anbjected to a first lixiviation by weak solutions. In this way the fresh water gives a weak solution. The strong solution which goes to the evaporating pan flows from those parts of the apparatus which contain the fresh, as yet unlixiviated, mass, and thus in the latter parts the weak alkali formed in the other parts of the apparatus becomes saturated as far as possible with the soluble substance. Generally several intercommunicating vessels are constructed (standing at the same level) into which in turn the fresh mass is charged which is intended for lixiviation; the water is poured in, the alkali drawn off, and the lixiviated residue removed. The illustration represents such an apparatus, consisting of four communicating vessels. The water poured into one of them flows through the two nearest and issues from the third. The fresh mass being placed in one of these boxes or vessels, the stream of water passing through the apparatus is directed in such a manner as to finally issue from this vessel containing the fresh unlixiviated mass. The fresh water is added to the vessel containing the material which has been almost completely exhausted. Passing through this ressel it is conveyed by the pipe (syphon passing from the bottom of the first box to the top of the second) communicating with the second; it finally passes (also through a syphon pipe) into the box (the third) containing the fresh material. The water will extract all that is soluble in the first vessel, leaving only an insoluble residue. This vessel is then ready to be emptied, and refilled with fresh material. The levels of the liquids in the various vessels will naturally be different. in consequence of the various strengths of the solutions which they contain

It must not, however, be thought that sodium carbonate alone passes into the solution; there is also a good deal of caustic soda with it, formed by the action of lime on the carbonate of sodium, and there are also certain sodium sulphur compounds with which we shall partly become acquainted hereafter. The sodium carbonate, therefore, is not obtained in a very pure state. The solution is concentrated by evaporation. This is conducted by means of the waste heat from the soda furnaces, together with that of the gases given off. The process in the soda furnaces can only be carried on at a high temperature, and therefore the smoke and gases issuing from them are necessarily very hot. If the heat they contain was not made use of there would be a great waste of fuel; consequently in immediate proximity to these furnaces there is generally a series of pans or evaporating boilers, under which the gases pass, and into which the alkali solution is poured. On evaporating the solution, first of all the undecomposed sodium sulphate separates, then the sodium carbonate or soda crystals. These crystals as they separate are raked out and placed on planks, where the liquid drains away from them. Caustio soda remains in the residue, and also any sodium chloride which was not decomposed in the foregoing process.

Part of the sodium carbonate is recrystallised in order to purify it more thoroughly. In order to do this a saturated solution is left to crystallise at a temperature below $30^{\circ}$ in a current of air, in order to promote the separation of the water vapour. The large transparent crystals (efflorescent in air) of $\mathrm{Na}_{2} \mathrm{CO}_{3}, 10 \mathrm{H}_{2} \mathrm{O}$ are then formed which have already been spoken of (Chapter I.).

14 The whole of the sulphur used in the production of the sulphuric acid employed in decomposing the common salt is contained in this residue. This is the great burden and expense of the soda works which use Leblanc's method. As an instructive example from a chemical point of view, it is worth while mentioning here two of the various methods of recovering the sulphur from the soda waste. Chance's process is treated in Chapter XX., Note 6.

Kynaston (1885) treats the soda waste with a solution (sp. gr. 1.21) of magnesium chloride, which disengages sulphuretted hydrogen: $\mathrm{CaS}+\mathrm{MgCl}_{2}+2 \mathrm{H}_{2} \mathrm{O}=\mathrm{CaCl}_{2}$ $+\mathrm{Mg}(\mathrm{OH})_{2}+\mathrm{H}_{2} \mathrm{~S}$. Sulphurous anhydride is passed through the residue in order to form the insoluble celcium sulphite: $\mathrm{CaCl}_{2}+\mathrm{Mg}(\mathrm{OH})_{2}+\mathrm{SO}_{2}=\mathrm{CaSO}_{5}+\mathrm{IgCl}_{2}+\mathrm{H}_{2} \mathrm{O}$. The 
The above-mentioned process for making soda was discovered in the year 1808 by the French doctor Leblanc, and is known as the Leblanc process. The particulars of the discovery are somewhat remarkable. Sodium carbonate, having a considerable application in industry, was for a long time prepared exclusively from the ash of marine plants (Chapter XI., page 497). Even up to the present time this process is carried on in Normandy. In France, where for a long time the manufacture of large quantities of soap (so-called Marseilles soap) and various fabrics required a large amount of soda, the quantity prepared at the coast was insufficient to meet the demand. For this reason during the wars at the beginning of the century, when the import of foreign goods into France was interdicted, the want of sodium carbonate was felt. The French Academy offered a prize for the discovery of a profitable method of preparing it from common salt. Leblanc then proposed the above-mentioned process, which is remarkable for its great simplicity. ${ }^{15}$

solution of magnesium clloride obtained is agam used, and the washed calcium sulphite is brought into contact at a low temperature with hydrochloric acid (a weak aqueous solution) and hydrogen sulphide, the whole of the sulphur then separating:

$$
\mathrm{CaSO}_{3}+2 \mathrm{H}_{2} \mathrm{~S}+2 \mathrm{HCl}=\mathrm{CaCl}_{2}+3 \mathrm{H}_{2} \mathrm{O}+8 \mathrm{~S} \text {. }
$$

But most efforts have been directed towards avoiding the formation of soda waste.

15 Among the drawbacks of the Leblanc process are the accumulation of 'soda waste' (Note 14) owing to the impossibility at the comparatively low price of sulphur (especially in the form of pyrites) of finding employment for the sulphur and sulphur compounds for which this waste is sometimes treated, and also the insufficient purity of the sodium carbonate for many purposes. The advantages of the Leblanc process, besides its simplicity and cheapness, are that almost the whole of the acids obtained as bye-products have a commercial value; for chlorine and bleaching powder are produced from the large amount of hydrochloric acid which appears as a bye-product; caustic soda also is very easily made, and the demand for it increases every year. In those places where salt, pyrites, charcoal, and limestone (the materials required for alkali works) are found side by side-as, for instance, in the Ural or Don districts-conditions are favourable to the development of the manufacture of sodium carbonate on an enormous scale; and where, as in the Caucasus, sodium sulphate occurs naturally, the conditions are still more favourable. A large amount, however, of the latter salt, even from soda works, is used in making glass. The most important soda works, as regards the quantity of products obtained from them, are the English works.

As an example of the other numerous and varied methods of manufacturing soda from sodium chloride, the following may be mentioned: Sodium chloride is decom. posed by oxide of lead, $\mathrm{PbO}$, forming lead chloride and sodium oxide, which, with carbonio anhydride, yields sodium carbonate (Scheele's process). In Cornu's method sodium chloride is treated with lime, and then exposed to the air, when it yields a small quantity of sodium carbonate. In E. Kopp's process sodium sulphate (125 parts) is mixed with oxide of iron ( 80 parts) and charcoal ( 55 parts), and the mixture is heated in reverbe. ratory furnaces. Here a compound, $\mathrm{Na}_{6} \mathrm{Fe}_{4} \mathrm{~S}_{3}$, is formed, which is insoluble in water, absorbs oxygen and carbonic anhydride, and then forms sodium carbonate and ferrous sulphide; this when roasted gives sulphurous anhydride, the indispensable materia!! 
Of all other industrial processes for manufacturing sodium carbonate, the ammonia process is the most worthy of mention. ${ }^{16}$ In this the vapours of ammonia, and then an excess of carbonic anhydride, are directly introduced into a concentrated solution of sodium chloride in order to form the acid ammonium carbonate, $\mathrm{NH}_{4} \mathrm{HCO}_{3}$. Then, by means of the double saline decomposition of this salt, sodium chloride is decomposed, and in virtue of its slight solubility acid sodium carbonate, $\mathrm{NaHCO}_{3}$, is precipitated and ammonium chloride, $\mathrm{NH}_{4} \mathrm{Cl}$, is obtained in solution (with a portion of the sodium chloride and acid sodium carbonate). The reaction proceeds in the solution owing to the sparing solubility of the $\mathrm{NaHCO}_{3}$ according to the equation $\mathrm{NaCl}+\mathrm{NH}_{4} \mathrm{HCO}_{3}$ $=\mathrm{NH}_{4} \mathrm{Cl}+\mathrm{NaHCO}_{3}$. The ammonia is recovered from the solution by heating with lime or magnesia, ${ }^{16}$ bis and the precipitated acid sodium carbonate is converted into the normal salt by heating. It is thus obtained in a very pure state. ${ }^{17}$

for the manufacture of sulphuric acid, and ferric oxide which is again used in the process. In Grant's method sodium sulphate is transformed into sodium sulphide, and the latter is decomposed by a stream of carbonic anhydride and steam, when hydrogen sulphide is disengaged and sodium carbonate formed. Gossage prepares $\mathrm{Na}_{2} \mathrm{~S}$ from $\mathrm{Na}_{2} \mathrm{SO}_{4}$ (by heating it with carbon), dissolves it in water and subjects the solution to the action of an excess of $\mathrm{CO}_{2}$ in coke towers, thus obtuining $\mathrm{H}_{2} \mathrm{~S}$ (a gas which gives $\mathrm{SO}_{2}$ under perfect combustion, or sulphur when incompletely burnt, Chapter XX., Note 6) and bicarbonate of sodium; $\mathrm{Na}_{2} \mathrm{~S}+2 \mathrm{CO}_{2}+2 \mathrm{H}_{2} \mathrm{O}=\mathrm{H}_{2} \mathrm{~S}+2 \mathrm{HNaCO}_{3}$. The latter gives soda and $\mathrm{CO}_{2}$ when ignited. This process quite eliminates the formation of soda-waste (see Note 3 ) and should in my opinion be suitable for the treatment of native $\mathrm{Na}_{2} \mathrm{SO}_{4}$, like that which is found in the Cancasus, all the more since $\mathrm{H}_{2} \mathrm{~S}$ gives sulphur as a bye-product.

Repeated efforts have been made in recent times to obtain soda (and chlorine, see Chapter II., Note 1) from strong solutions of salt (Chapter X., Note 28 bis) by the action of an electric current, but until now these methods have not been worked out sufficiently for practical use, probably partly owing to the complicated apparatus needed, and the fact that the chlorine given off at the anode corrodes the electrodes and vessels and has but a limited industrial application. We may mention that according to Hempel (1890) soda in crystals is deposited when an electric current and a stream of carbonic acid gas are passed through a saturated solution of $\mathrm{NaCl}$.

Sodium carbonate may likewise be obtained from cryolite (Chapter XVII., Note 23) the method of treating this will be mentioned under Aluminium.

16 This process (Chapter XVII.) was first pointed out by Turck, worked out by Schloesing, and finally applied industrially by Solvay. The first (1883) large soda factories erected in Russia for working this process are on the banks of the Kama at Bererniak, near Ousolia, and belong to Lubimoff. But Russia, which still imports from abroad a large quantity of bleaching powder and exports a large amount of manganese ore, most of all requires works carrying on the Leblanc process. In 1890 a factory of this kind was erected by P. K. Oushkoff, on the Kama, near Elagoubi.

16 bis Mond (see Chapter XI., Note 3 bis) separates the $\mathrm{NH}_{4} \mathrm{Cl}$ from the residual solutions by cooling (Chapter X., Note 44); ignites the sal-ammoniac and passes the vapour over $\mathrm{MgO}$, and so re-obtains the $\mathrm{NH}_{3}$ and forms $\mathrm{MgCl}$ : the former goes back for the manufacture of soda, while the latter is employed either for making $\mathrm{HCl}$ or $\mathrm{Cl}_{2}$.

17 Commercial soda ash (calcined, anhydrous) is rarely pure; the crystallised soda is generally purer. In order to purify it further, it is best to boil a concentrated solution of soda ash until two-thirds of the liquid remain, collect the soda which settles, wash with 
Sodium carbonate, like sodium sulphate, loses all its water on being heated, and when anhydrous fuses at a bright-red heat $\left(1098^{\circ}\right)$. A small quantity of sodium carbonate placed in the loop of a platinum wire volatilises in the heat of a gas flame, and therefore in the furnaces of glass works part of the soda is always transformed into the condition of vapour. Sodium carbonate resembles sodium sulphate in its relation to water. ${ }^{18}$ Here also the greatest solubility is at the temperature of $37^{\circ}$, both salts, on crystallising at the ordinary temperature, combine with ten molecules of water, and such crystals of soda, like crystals of Glauber's salt, fuse at $34^{\circ}$ Sodium carbonate also forms a supersaturated solution, and, according to the conditions, gives various combinations with water of crystallisation (mentioned on page 108), \&c.

At a red heat superheated steam liberates carbonic anhydride from sodium carbonate and forms caustic soda, $\mathrm{Na}_{2} \mathrm{CO}_{3}+\mathrm{H}_{2} \mathrm{O}=2 \mathrm{NaHO}$ $+\mathrm{CO}_{2}$. Here the carbonic anhydride is replaced by water; this depends on the feebly acid character of carbonic anhydride. By direct heating, sodium carbonate is only slightly decomposed into sodium oxide and carbonic anhydride; thus, when sodium carbonate is fused, about 1 per cent. of carbonic anhydride is disengaged.19 The carbonates of many other metals-for instance, of calcium, copper, magnesium, iron, \&c.- on being heated lose all their carbonic anhydride. This shows cold water, and then shake up with a strong solution of ammonia, pour off the residue, end heat. The impurities will then remain in the mother liquors, \&c.

Some numerical data may be given for sodium carbonate. The specific gravity of the anhydrous salt is $2 \cdot 48$, that of the decshydrated salt $1 \cdot 46$. Two varieties are known of the heptahydrated salt (Löwel, Marignac, Rammelsberg), which are formed together by allowing a saturated solution to cool under a layer of alcohol; the one is less stable (like the corresponding sulphate) and at $0^{\circ}$ has a solubility of 82 parts (of anhydrous salt) in 100 water; the other is more stable, and its solubility 20 parts (of anhydrous salt) per 190 of water. The solubility of the decahydarated salt in 100 water $=a t 0^{\circ}, 7.0$; at $20^{\circ}, 21.7$; at $80^{\circ}, 87^{\circ} 2$ parts (of anhydrons salt). At $80^{\circ}$ the solubility is only $46 \cdot 1$, at $90^{\circ} 45 \cdot 7$, at $100^{\circ}$, 45.4 parts (of anhydrous salt). That is, it falls as the temperature rises, like $\mathrm{Na}_{2} \mathrm{SO}_{4}$. The specific gravity (Note 7) of the solutions of sodium carbonate, according to the data of Gerlach and Kohlrausch, at $15^{\circ} / 4^{\circ}$ is expressed by the formula, $s=9,992+104 \cdot 5 p$ $+0.165 p^{2}$. Weak solutions occupy a volume not only less than the sum of the volumes of the anhydrous salt and the water, but even less than the water contained in them. For instance, 1,000 grams of a 1 p.o. solution occupy (at $15^{\circ}$ ) a volume of $990 \cdot 4$ c.c. (sp. gr. 1.0097), but contain 990 grams of water, occupying at $15^{\circ}$ a volume of 990.8 o.c. A similar case, which is comparatively rare occurs also with sodium hydroxide, in those dilute solutions for which the factor $A$ is greater than 100 if the sp. gr. of water at $4^{\circ}=$ 100,000 , and if the sp. gr. of the solution be expressed by the formula $S=S_{0}$ o $A p+B p^{2}$, where $S_{0}$ is the specific gravity of the water. For 5 p.c. the sp. gr. $15^{\circ} / 4^{\circ}=1.0520$; for 10 p.c. 1.1057; for 15 p.c. 1·1603. The ohanges in the sp. gr. with the temperature are here almost the same as with solutions of sodium chloride with an equal value of $p$.

18 The resemblance is so great that, notwithstanding the difference in the molecular composition of $\mathrm{Na}_{2} \mathrm{SO}_{4}$ and $\mathrm{Ne}_{2} \mathrm{CO}_{3}$, they ought to be classed under the type $(\mathrm{NaO})_{2} \mathrm{R}$, where $\mathrm{R}=\mathrm{SO}_{2}$ or CO. Many other sodium salts also contain $10 \mathrm{~mol} . \mathrm{H}_{2} \mathrm{O}$.

19 According to the observations of Pickering. According to Rose, when solutions of codium carbonate are boiled accertain amount of carbonio anhydride is disengagod. 
the considerable basic energy which sodium possesses. With the soluble salts of most metals, sodium carbonate gives precipitates either of insoluble carbonates of the metals, or else of the hydroxides (in this latter case' carbonic anhydride is disengaged), for instance, with barium salts it precipitates an insoluble barium carbonate $\left(\mathrm{BaCl}_{2}+\mathrm{Na}_{2} \mathrm{CO}_{3}=2 \mathrm{NaCl}+\mathrm{BaCO}_{3}\right)$ and with the aluminium salts it precipitates aluminium hydroxide, carbonic anhydride being disengaged $3 \mathrm{Na}_{2} \mathrm{CO}_{3}+\mathrm{Al}_{2}\left(\mathrm{SO}_{4}\right)_{3}+3 \mathrm{H}_{2} \mathrm{O}=3 \mathrm{Na}_{2} \mathrm{SO}_{4}+2 \mathrm{Al}(\mathrm{OH})_{3}$ $+3 \mathrm{CO}_{2}$. Sodium carbonate, like all the salts of carbonic acid, evolves carbonic anhydride on treatment with all acids which are to any extent energetic. But if an acid diluted with water be gradually added to a solution of sodium carbonate, at first such an evolution does not take place, because the excess of the carbonic anhydride forms acid sodium carbonate (sodium bicarbonate), $\mathrm{NaHCO}_{3}{ }^{20}$ The acid sodium carbonate is an unstable salt. Not only when heated alone, but even on being slightly heated in solution, and also at the ordinary temperature in damp air, it loses carbonic anhydride and forms the normal salt. And at the same time it is easy to obtain it in a pure crystalline form, if a strong solution of sodium carbonate be cooled and a stream of carbonic anhydride gas passed through it. The acid salt is less soluble in water than the normal, ${ }^{21}$ and therefore a strong

20 The composition of this salt, however, may be alśo represented as a combination of carbonic acid, $\mathrm{H}_{2} \mathrm{CO}_{3}$, with the normal salt, $\mathrm{Na}_{2} \mathrm{CO}_{5}$, just as the latter also combines with water. Such a oombination is all the more likely because (1) there exists another salt, $\mathrm{Na}_{2} \mathrm{CO}_{3}, 2 \mathrm{NaHCO}_{3}, 2 \dot{\mathrm{H}}_{2} \mathrm{O}$ (sodium sesquicarbonate), obtained by cooling a boiling solution of sodium bicarbonate, or by mixing this salt with the normal salt; but the formula of this salt cannot be derived from that of normal carbonic acid, as the formula of the bicarbonate can. At the same time the sesqui-salt has all the properties of a definite compound; it crystallises in transparent orystals, has a constant composition, its solubility (at $0^{\circ}$ in 100 of water, 12.6 of anhydrous salt) differs from the solubility of the normal and acid salts; it is found in nature, and is known by the names of trona and urao. The observations of Watts and Richards showed (1886) that on pouring a strong solntion of the acid salt into a solntion of the normal salt saturated by heating, crystals of the salt $\mathrm{NaHCO}_{3}, \mathrm{Na}_{2} \mathrm{CO}_{3}, 2 \mathrm{H}_{2} \mathrm{O}$ may be easily obtained, as long as the temperature is above $35^{\circ}$. The natural urao (Boussingault) has, according to taurent, the same composition. This salt it very stable in air, and may be used for purifying sodium carbonate on the large scale. Such compounde have been little studied from a theoretical point of view, although particularly interesting, since in all probability they correspond with ortho-carbonic acid, $\mathrm{C}(\mathrm{OH})_{4}$, and at the same time correspond with double salts like astrachanite (Chapter XIV., Note 25). (2) Water of crystallisation does not enter into the composition of the crystals of the acid salt, so that on its formation (occurring only at low temperatures, as in the formation of crystalline com. pounds with water) the water of crystallisation of the normal salt separates and the water is, as it were, replaced by the elements of carbonic acid. If anhydrous sodium carbonate be mixed with the amount of water requisite for the formation of $\mathrm{N}_{2} \mathrm{CO}_{3} \mathrm{H}_{2} \mathrm{O}$, this salt will, when powdered, absorb $\mathrm{CO}_{2}$ as easily at the ardinary temperature as it does water.

${ }^{21} 100$ parts of water at $0^{\circ}$ dissolve 7 parts of the acid salt, which corresponds with 4.8 parts of the anhydrous normal salt, but at $0^{\circ} 100$ parts of water dissolve 7 parte of 
solution of the latter gives crystals of the acid salt if carbonic anhydride be passed through it. The acid salt may be yet more conveniently formed from effloresced crystals of sodium carbonate, which, on being considerably heated, very easily absorb carbonic anhydride. ${ }^{22}$ ' The acid salt crystallises well, but not, however, in such large crystals as the normal salt; it has a brackish and not an alkaline taste like that of the normal salt; its reaction is feebly alkaline, nearly neutral. At $70^{\circ}$ its solution begins to lose carbonic anhydride; and on boiling the evolution becomes very abundant. From the preceding remarks it is clear that in most reactions this salt, especially when heated, acts similarly to the normal salt, but has, naturally, some distinction from it. Thus, for example, if a solution of sodium carbonate be added to a normal magnesium salt, a turbidity (precipitate) is formed of magnesium carbonate, $\mathrm{MgCO}_{3}$. No such precipitate is formed by the acid salt, because magnesium carbonate is soluble in the presence of an excess of carbonic anhydride.

Sodium carbonate is used for the preparation of caustic soda ${ }^{23}$ that is, the hydrate of sodium oxide, or the alkali which corresponds to sodium. For this purpose the action of lime on a solution of sodium carbonate is generally made use of. The process is as follows : a weak, generally 10 per cent., solution of sodium carbonate is taken, ${ }^{24}$

the latter. The solubility of the bi- or acid salt varies with considerable regularity, 100 parts of water dissolves at $15^{\circ} 9$ parts of the salt, at $30^{\circ} 11$ parts.

The ammonium, and more especially the calcium, salt, is much more soluble in water. The ammonia process (see p. 524) is founded upon this. Ammonium bicarbonate (acid carbonate) at $0^{\circ}$ has a solubility of 12 parts in 100 water, at $30^{\circ}$ of 27 parts. The solu. bility therefore increases very rapidly with the temperature. And its saturated solntion is more stable than a solution of sodium bicarbonate. In fact, saturated solutions of these salts have a gaseous tension like that of a mixture of carbonic anhydride and waternamely, at $15^{\circ}$ and at $50^{\circ}$, for the sodium salt 120 and 750 millimetres, for the ammonium salt 120 and 563 millimetres. These data are of great importance in understanding the phenomena connected with the ammonia process. They indicate that with an increased pressure the formation of the sodium salt ought to increase if there be an excess of ammonium salt.

22 Crystalline sodium carbonate (broken into lumps) also absorbs carbonic anhydride, but the water contained in the crystals is then disengaged: $\mathrm{Na}_{2} \mathrm{CO}_{3}, 10 \mathrm{H}_{2} \mathrm{O}+\mathrm{CO}_{2}$ $=\mathrm{Na}_{2} \mathrm{CO}_{3}, \mathrm{H}_{2} \mathrm{CO}_{3}+9 \mathrm{H}_{2} \mathrm{O}$, and dissolves part of the carbonate; therefore part of the sodium carbonate passes into solution together with all the impurities. When it is required to avoid the formation of this solution, a mixture of ignited and crystalline sodium carbonate is taken. Sodium bicarbonate is prepared chiefly for medicinal use, and is then often termed carbonate of soda, also, for instance, in the so-called soda powders, for preparing certain artificial mineral waters, for the manufacture of digestive lozenges like those made at Essentuki, Vichy, \&c.

25 In chemistry, sodium oxide is termed ' soda, which word must be carefully distin guished from the word sodium, meaning the metal.

24 With a small quantity of water, the reaction either does not take place, or even proceeds in the reverse way-that is, sodium and potassium hydroxides remove carbonic enhydride from calcium carbonate (Liebig, Watson, Mitscherlich, and others). The in. 
and boiled in a cast-iron, wrought-iron, or silver boiler (sodium hydrox. ide does not act on these metals), and lime is added, little by little, during the boiling. This latter is soluble in water, although but very slightly. The clear solution becomes turbid on the addition of the lime because a precipitate is formed; this precipitate consists of calcium carbonate, almost insoluble in water, whilst caustic soda is formed and remains in solution. The decomposition is effected according to the equation $\mathrm{Na}_{2} \mathrm{CO}_{3}+\mathrm{Ca}(\mathrm{HO})_{2}=\mathrm{CaCO}_{3}+2 \mathrm{NaHO}$. On cooling the solution the calcium carbonate easily settles as a precipitate, and the clear solution or alkali above it contains the easily soluble sodium hydroxide formed in the reaction. ${ }^{25}$ After the necessary quantity of lime has been added, the solution is allowed to stand, and is then decanted off and evaporated in cast or wrought iron boilers, or in silver pans if a perfectly pure product is required. ${ }^{26}$ The evaporation cannot

fluence of the mass of water is evident. According to Gerberts, however, strong solutions of sodium carbonate are decomposed by lime, which is very interesting if confirmed by further investigation.

${ }^{25} \mathrm{As}$ long as any undecomposed sodium carbonate remains in solution, exoess of acid added to the solution disengages carbonic anhydride, and the solution after dilution gives a white precipitate with a barium salt soluble in acids, showing the presence of a carbonate in solution (if there be sulphate present, it also forms a white precipitate, but this is insoluble in acids). For the decomposition of sodium carbonate, milk of lime -that is, slaked slime suspended in water-is employed. Formerly pure sodium hydroxide was prepared (according to Berthollet's process) by dissolving the impure substance in alcohol (sodium carbonate and sulphate are not soluble), but now that metallio sodium has become cheap and is purified by distillation, pure caustic soda is prepared by acting on a small quantity of water with sodium. Perfectly pure sodium hydroxide may also be obtained by allowing strong solutions to crystallise (in the cold) (Note 27).

In alkali works where the Leblanc process is used, caustic soda is prepared directly from the alkali remaining in the mother liquors after the separation of the sodium carbonate by evaporation (Note 14). If excess of lime and charcoal have been used, much sodium hydroxide may.be obtained. After the removal as much as possible of the sodium carbonate, a red liquid (from iron oxide) is left, containing sodium hydroxide mixed with com. pounds of sulphur and of cyanogen (see Chapter IX.) and also containing iron. This red alkali is evaporated and air is blown through it, which oxidises the impurities (for this purpose sometimes sodium nitrate is added, or bleaching powder, \&c.) and leaves fused caustio soda. The fused mass is allowed to settle in order to separate the ferruginous precipitate, and ponred inta iron drums, where the sodium hydroxide solidifies. Such caustio soda contains about 10 p.c. of water in excess and some saline impurities, but when properly manufactured is almost free from carbonate and from iron. The greater part of the caustic soda, which forms so important an article of commerce, is manufactured in this manner.

${ }_{20}$ Löwig gave a method of preparng sodium hydroxide from sodium carbonate by heating it to a dull red heat with an excess of ferric oxide. Carbonic anhydride is given off, and warm water extracts the caustic soda from the remaining mass. This reaction, as experiment shows, proceeds very easily, and is an example of contact action similar to that of ferrio oxide on the decomposition of potassium chlorate. The reason of this may be that a small quantity of the sodium carbonate enters into double decom. position with the ferrie oxide, and the ferric carbonate produced is decomposed into carbonio anhydride and ferrio oxide, the action of which is renewed. Similar explana. 
be conducted in china, glass, or similar vessels, because caustic soda attacks these materials, although but slightly. The solution does not crystallise on evaporation, because the solubility of caustia soda when hot is very great, but crystals containing water of crystallisation may be obtained by cooling. If the evaporation of the alkali be conducted until the specifio gravity reaches $1 \cdot 38$, and the liquid is then cooled to $0^{\circ}$, transparent crystals appear containing $2 \mathrm{NaHO}, 7 \mathrm{H}_{2} \mathrm{O}$; they fuse at $+6^{\circ} .27$ If the evaporation be conducted solong as water is disengaged, which requires a considerable amount of heat, then, on cooling, the hydroxide, $\mathrm{NaHO}$, solidifies in a semi-transparent crystalline mass, ${ }^{28}$ which eagerly absorbs moisture and carbonic anhydride from the air. ${ }^{29}$ Its specific gravity is $2 \cdot 13,{ }^{30}$ it is easily soluble in water, with disengagement of a considerable quantity of heat. ${ }^{31}$ A saturated solution at the ordiuary temperature has a specific gravity of about 1.5 , contilins about 45 per cent. of sodium hydroxide, and boils at $130^{\circ}$; at $55^{\circ}$ water dissolves an equal weight of it. ${ }^{32}$ Caustic soda is not only soluble in

tions expressing the reason for a reaction really add but little to that elementary con. ception of contact which, according to my opinion, consists in the change of motion of the atoms in the molecules under the influence of the substance in contact. In order to represent this clearly it is sufficient, for instance, to imagine that in the sodium carbonate the elements $\mathrm{CO}_{2}$ move in a circle round the elements $\mathrm{Na}_{2} \mathrm{O}$, but at the points of contact with $\mathrm{Fe}_{2} \mathrm{O}_{3}$ the motion becomes elliptic with a long axis, and at some distance.from $\mathrm{Na}_{2} \mathrm{O}$ the elements of $\mathrm{CO}_{2}$ are parted, not having the faculty of attaching themselves to $\mathrm{Fe}_{2} \mathrm{O}_{3}$.

27 By allowing strong solutions of sodium hydroxide to crystallise in the cold, impurities-such as, for instance, sodium sulphate-may be separated from them. The fused crystallo-hydrate $2 \mathrm{NaHO}, 7 \mathrm{H}_{2} \mathrm{O}$ forms a solution having a specific gravity of 1.405 . (Hermes). The crystals on dissolving in water produce cold, while NaHO produces heat. Besides which Pickering obtained hydrates with $1,2,4,5$, and $7 \mathrm{H}_{2} \mathrm{O}$.

28 In solid caustic soda there is generally an excess of water beyond that required by the formula $\mathrm{NaHO}$. The caustic soda used in laboratories is generally cast in sticks, which are broken into pieces. It must be preserved in carefally closed vessels, because it absorbs water and carbonic anhydride from the air.

29 By the way it changes in air it is easy to distinguish caustic soda from caustic potash, which in general resembles it. Both alkalis absorb water and carbonic anhydride from the air, bat caustic potash forms a deliquescent mass of potassium carbonate, whilst caustio soda forms a dry powder of efflorescent salt.

30 As the molecular weight of $\mathrm{NaHO}=40$, the volume of its molecule $=40 / 2 \cdot 13=18 \cdot 5$, which very nearly approaches the volume of a molecule of water. The same rule applies to the compounds of sodium in general-for instance, its salts have a molecular volume approaching the volume of the acids from which they are derived.

81 The molecular quantity of sodium hydroxide ( 40 grams), on being dissolved in a large mass (200 gram molecules) of water, develops, according to Berthelot 9,780, and according to Thomsen 9,940, heat-units, but at $100^{\circ}$ about 18,000 (Bérthelot). Solutions of $\mathrm{NaHO}+n \mathrm{H}_{2} \mathrm{O}$, on being mixed with water, evolve heat if they contain less than $6 \mathrm{H}_{2} \mathrm{O}$, but if more they absorb heat:

32 The specific gravity of solutions of sodinm hydroxide at $15^{\circ} / 4^{\circ}$ is given in the short table below:-

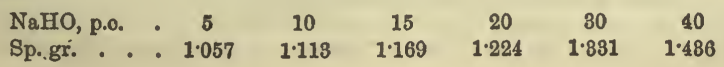


water but in alcohol, and even in ethèr. Dilute solutions of sodium hydroxide produce a soapy feeling on the skin because the active base of soap consists of caustic soda. ${ }^{33}$ Strong solutions have a corroding action.

The chemical reactions of sodium hydroxide serve as a type for those of a whole class of alkalis - that is, of soluble basic hydroxides, $\mathrm{MOH}$. The solution of sodium hydroxide is a very caustic liquid-that is to say, it acts in a destructive way on most substances, for instance on most organic tissues-hence caustic soda, like all soluble alkalis, is a poisonous substance; acids, for example hydrochloric, serve as antidotes. The action of caustic soda on bones, fat, starch, and similar vegetable and animal substances explains its action on organisms. Thus bones, when plunged into a weak solution of caustic soda, fall to powder, ${ }^{34}$

1,000 grams of a 5 p.c. solution occupies a volume of 946 c.c., that is, less than the water serving to make the solution (see Note 18).

53 Sodium hydroxide and some other alkalis are capable of hydrolysing-saponifying, as it is termed-the compounds of acids with alcohols. If $\mathrm{RHO}$ (or $\mathrm{R}(\mathrm{HO})$ ) represent the composition of an alcohol-that is, of the hydroxide of a hydrocarbon radicle-and $\mathrm{QHO}$ an acid, then the compound of the acid with the alcohol or ethereal salt of the given acid will have the composition RQO. Ethereal salts, therefore, present a likeness to metallic salts, just as aloohols resemble basic hydroxides. Sodium hydroxide acts on ethereal salts in the same way that it acts on the majority of metallic salts-namely, it liberates alcohol, and forms the sodium salt of that acid which was in the ethereal zalt. The reaction takes place in the following way :-

$\underset{\substack{\text { Ethereal } \\ \text { salt }}}{\mathrm{RQO}}+\underset{\substack{\text { Canstic } \\ \text { soda }}}{\mathrm{NaFO}}=\underset{\begin{array}{c}\text { Sodium } \\ \text { salt }\end{array}}{\mathrm{NaQO}}+\underset{\text { Alcohol }}{\mathrm{RHO}}$

Such a decomposition is termed saponification; similar reactions were known very long ago for the ethereal salts corresponding with gtyeerin, $\mathrm{C}_{3} \mathrm{H}_{5}(\mathrm{OH})_{3}$ (Chapter IX.), found in animals and plants, and composing what are called fats or oils. Caustic soda, acting on fat and oil, forms glycerin, and sodium salts of those acids which were in union with the glycerin in the fat, as Cherreul showed at the beginning of this century. The sodium salts of the fatty acids are commonly known as soaps. That is to say, soap is made from fat and caustic soda, glycerin being separated and a sodium salt or soap formed. As glycerin is usually found in union with certain acids, so also are the sodium salts of the same acids found in soap. The greater part of the acids found in conjunction with glycerin in fats are the solid palmitic and stearic acids, $\mathrm{C}_{16} \mathrm{H}_{32} \mathrm{O}_{2}$ and $\mathrm{C}_{18} \mathrm{H}_{38} \mathrm{O}_{2}$, and the liquid oleio acid, $\mathrm{C}_{18} \mathrm{H}_{34} \mathrm{O}_{2}$. In preparing soap the fatty substences are mixed with a solution of caustic soda until an emulsion is formed; the proper quantity of caustic soda is then added in order to produce saponification on heating, the soap being separated from the solution either by means of an excess of caustic soda or else by common salt, which displaces the soap from the aqueous solution (salt water does not dissolve soap, neither does it form a lather). Water acting on soap partly decomposes it (because the acids of the soap are feeble), and the alkali set free acts during the application of soap. Hence it may be replaced by a very feeble alkali. Strong solutions of alkali corrode the skin and tissues. They are not formed from snap, because the reaction is reversible, and the alkali is only set free by the excess of water Thus we see how the teaching of Berthollet renders it possible to understand many phenomena which occur in every-day experience (see Chapter IX., Note 15).

34 $\mathrm{Ob}$ this is founded the process of Henkoff and Engelhardt for treating bones 
and erolve a smell of ammonia, owing to the caustic soda changing the gelatinous organio substance of the bones (which contains carbon, hydrogen, nitrogen, oxygen, and sulphur, like albumin), dissolving it and in part destroying it, whence ammonia is disengaged. Fats, tallow, and oils become saponified by a solution of caustic soda-that is to say, they form with it soaps soluble in water, or sodium salts of the organic acids contained in the fats. ${ }^{35}$ The most characteristic reactions of sodium hydroxide are determined by the fact that it saturates all acids, forming salts with them, which are almost all soluble in water, and in this respect caustic soda is as characteristic amongst the bases as nitric acid is among the acids. It is impossible to detect sodium by means of the formation of precipitates of insoluble sodium salts, as may be done with other metals, many of whose salts are but slightly soluble. The powerful alkaline properties of caustic soda determine its capacity for combining with even the feeblest acids, its property of disengaging ammonia from ammonium salts, its faculty of forming precipitates from solutions of salts whose bases are insoluble in water, \&c. If a solution of the salt of almost any metal be mixed with caustic soda, then a soluble sodium salt will be formed, and an insoluble hydroxide of the metal will be separated-for instance, copper nitrate yields copper, hydroxide, $\mathrm{Cu}\left(\mathrm{NO}_{3}\right)_{2}+2 \mathrm{NaHO}=\mathrm{Cu}(\mathrm{HO})_{2}+2 \mathrm{NaNO}_{3}$. Even many basic oxides precipitated by caustic soda are capable of combining with it and forming soluble compounds, and therefore caustic soda in the presence of salts of such metals first forms a precipitate of hydroxide, and then, employed in excess, dissolves this precipitate. This pheno: menon occurs, for example, when caustic soda is added to the salts of aluminium. This shows the property of such an alkali as caustic soda of combining not only with acids, but also with feeble basic oxides. For this reason caustic soda acts on most elements which are capable of form. ing acids or oxides similar to them; thus the metal aluminium gives hydrogen with caustic soda in consequence of the formation of alumina, which combines with the caustic soda-that is, in this case, the caustic alkali acts on the metal just as sulphuric acid does on $\mathrm{Fe}$ or $\mathrm{Zn}$. If caustic soda acts in this manner on a metalloid capable of combining with the hy.drogen evolved (aluminium does not give a compound with hydrogen), then it forms such a hydrogen compound. Thus, for instance, phosphorus acts in this way on caustic soda, yielding hydrogen phos. phide. When the hydrogen compound disengaged is capable of combin.

The bones are mixed with ashes, lime, and water; it is true that in this case more potassium hydroxide than sodium hydroxide is formed, but their action is almost identical.

ss As explained in Note ss. 
ing with the alkali, then, naturally, a salt of the corresponding acid is formed. For example, chlorine and sulphur act in this way on caustio soda. Chlorine, with the hydrogen of the caustic soda, forms hydrochloric acid, and the latter forms common salt with the sodium hydroxide, whilst the other atom in the molecule of chlorine, $\mathrm{Cl}_{2}$, takes the place of the hydrogen, and forms the hypochlorite, $\mathrm{NaClO}$ In the same way, by the action of sodium hydroxide on sulphur, hydrogen sulphide is formed, which acts on the soda forming sodium sulphide, in addition to which sodium thiosulphate is formed (see Chapter XX.) By virtue of such reactions, sodium hydroxide acts on many metals and non-metals. Such action is often accelerated by the presence of the oxygen of the air, as by this means the formation of acids and oxides rich in oxygen is facilitated. Thus many metals and their lower oxides, in the presence of an alkali, absorb oxygen and form acids. Even manganese peroxide, when mixed with caustic soda, is capable of absorbing the oxygen of the air, and forming sodium manganate. Organic acids when heated with caustic soda give up to it the elements of carbonic anhydride, forming sodium carbonate, and separating that hydiocarbon group which exists, in combination with carbonic anhydride, in the organic acid.

Thus sodium hydroxide, like the soluble alkalis in general, ranks amongst the most active substances in the chemical sense of the term, and but few substances are capable of resisting it. Even siliceous rocks, as we shall see further on, are transformed by it, forming when fused with it vitreous slags. Sodium hydroxide (like ammonium and potassium hydroxides), as a typical example of the basic hydrates, in distinction. from many other basic oxides, easily forms acid salts. with acids (for instance, $\mathrm{NaHSO}_{4}, \mathrm{NaHCO}_{3}$ ), and does not form any basic salts at all; whilst many less energetic bases, such as the oxides of copper and lead, easily form basic salts, but acid salts only with difficulty. This capability of forming acid salts, particularly $w^{\text {ith }}$ polybasic acids, may be explained by the energetic basic properties of sodium hydroxide, contrasted with the small development of these properties in the bases which easily form basic salts. An energetic base is capable of retaining a considerable quantity of acid, which a slightly energetic base would not have the power of doing. Also, as will be shown in the subsequent chapters, sodium belongs to the univalent metals, being exchangeable for hydrogen atom for atom-that is, amongst metals sodium may, like chlorine amongst the non-metals, serve as the representative of the univalent properties. Most of the elements which are not capable of forming acid salts are bivalent. Whence it may be understood that in a bibasic acid-for instance, carbonic, 
$\mathrm{H}_{2} \mathrm{CO}_{3}$, or sulphuric, $\mathrm{H}_{2} \mathrm{SO}_{4}$-the hydrogen may be exchanged, atom for atom, for sodium, and yield an acid salt by means of the first substitution, and a normal salt by means of the second-for instance, $\mathrm{NaHSO}_{4}$, and $\mathrm{Na}_{2} \mathrm{SO}_{4}$, whilst such bivalent metals as calcium and barium do not form acid salts because one of their atoms at once takes the place of both hydrogen atoms, forming, for example, $\mathrm{CaCO}_{3}$ and $\mathrm{CaSO}_{4} \cdot{ }^{35 \mathrm{bis}}$

We have seen the transformation of common salt into sodium sulphate, of this latter into sodium carbonate, and of sodium carbonate into caustic soda. Lavoisier still regarded sodium hydroxide as an element, because he was unacquainted with its decomposition with the formation of metallic sodium, which separates the hydrogen from water, reforming caustic soda.

The preparation of metallic sodium was one of the greatest discoveries in chemistry, not only because through it the conception of elements became broader and more correct, but especially because in sodium, chemical properties were observed which were but feebly shown in the other metals more familiarly known. This discovery was made in 1807 .by the English chemist Davy by means of the galvanic current. By connecting with the positive pole (of copper or carbon) a piece of caustic soda (moistened in order to obtain electrical conductivity), and boring a hole in it filled with mercury connected with the negative pole of a strong Volta's pile, Davy observed that on passing the current a peculiar metal dissolved in the mercury, less volatile than mercury, and capable of decomposing water, again forming caustic soda. In this way (by analysis and synthesis) Davy demonstrated the compound nature of alkalis. On being decomposed by the galvanic current, caustic soda disengages hydrogen and sodium at the

35 bls It might be expected, from what has been mentioned above, that bivalent metals would easily form acid salts with acids containing more than two atoms of hydrogen-for instance, with tribasic acids, such as phosphoric acid, $\mathrm{H}_{3} \mathrm{PO}_{4}$-and actually such salts do exist; but all such relations are complicated by the fact that the character of the base very often changes and becomes weakened with the increase of valency and the change of atomic weight; the feebler bases (like silver oxide), although corresponding with univalent metals, do not form acid salts, while the feeblest bases ( $\mathrm{CuO}, \mathrm{PbO}$, \&c.) easily form basio salts, and notwithstanding their valency do not form acid salts which are in any degree stable-that is, which are undecomposable by water. Basic and acid salts ought to be regarded rather as compounds similar to crystallo-hydrates, because such acids as sulphurio form with sodium not only an acid and a normal salt, as might be expected from the valency of sodium, but also salts containing a greater quantity of acid. In sodium sesquicarbonate we saw an example of such compounds. Taking all this into consideration, we must say that the property of more or less easily forming acid salts depends more upon the energy of the base than upon its valency, and the best statement is that the capacity of a base for forming acid and basic salts is characteristic, just is the faculty of forming compounds with hydrogen is characteristic of elements. 
negative pole and oxygen at the positive pole. Dary showed that the metal formed volatilises at a red heat, and this is its most important physical property in relation to its extraction, all later methods being founded on it Besides this Davy observed that sodium easily oxidises, its vapour taking fire in air, and the latter circumstance was for a long time an obstacle to the easy preparation of this metal. The properties of sodium were subsequently more thoroughly investigated by Gay-Lussac and Thénard, who observed that metallic iron at a high temperature was capable of reducing caustic soda to sodium. ${ }^{36}$ Brunner latterly discovered that not only iron, but also charcoal, has this property, although hydrogen has not. ${ }^{37}$ But still the methods of ex. tracting sodium were very troublesome, and consequently it was a great rarity. The principal obstacle to its production was that an endeavour was made to condense the easily-oxidising vapours of sodium in vacuo in complicated apparatus. For this reason, when Donny and Maresca, having thoroughly studied the matter, constructed a specially simple condenser, the production of sodium was much facilitated. Furthermore, in practice the most important epoch in the history of the production of sodium is comprised in the investigation of Suinte-Claire Deville, who avoided the complex methods in vogue up to that time, and furnished those simple means by which the production of sodium is now rendered feasible in chemical works.

For the production of sodium according to Deville's method, a mixture of anhydrous sodium carbonate (7 parts), charcoal (two parts), and lime or chalk (7 parts) is heated. This latter ingredient is only added in order that the sodium carbonate, on fusing, shall not separate

36 Deville supposes that such a decomposition of sodium hydroxide by metallic iron depends solely' on the diesociation of the alkali at a white heat into sodium, hydrogen, and oxygen. Here the part played by the iron is only that it retains the oxygen formed, otherwise the decomposed elements would again reunite upon cobling, as in other cases of dissociation. If it be supposed that the temperature at the commencement of the dis. sociation of the iron oxides is higher than that of sodium oxide, then the decomposition may be explained by Deville's hypothesis. Deville demonstrates his views by the following experiment :-An iron bottle, filled with iron borings, was heated in such a way that the upper part became red hot, the lower part remaining cooler; sodium hydroxide was introduced into the upper part. The decomposition was then effected-that is, sodium vapours were produced (this experiment was really performed with potassium hydroxide). On opening the bottle it was found that the iron in the upper part was not oxidised, but only that in the lower part. This may be explained by the decomposition of the alkali into sodium, hydrogen, and oxygen taking place in the upper part, whilst the iron in the lower part absorbed the oxygen set free. If the whole bottle be subjected to the same moderate heat as the lower extremity, no metallic vapours are formed. In that case, according to the hypothesis, the temperature is insufficient for the dissociation of the sodium hydroxide.

37 It has been previously remarked (Chapter II. Note 9) that Beketoff showed the displacement of sodium by hydrogen, not from sodium hydroxide but from the oxide $\mathrm{Na}_{2} \mathrm{O}$; then, however, only one half is displaced, with the formation of $\mathrm{NaHO}$ 
from the charcoal. 38 The chalk on being heated loses carbonic anhy. dride, leäving infusible lime, which is permeated by the sodiun carbonate and forms a thick mass, in which the charcoal is intimately mixed with the sodiun carbonate. When the charcosl is heated with the sodium carbonate, at a white beat, carbonic oxide and vapours of sodium are disengaged, according to the equation :

$$
\mathrm{N}_{2} \mathrm{CO}_{3}+2 \mathrm{O}=\mathrm{Na} \mathrm{a}_{2}+8 \mathrm{CO}
$$

On cooling the vapours and gases disengaged, the vapours condense into molten metal (in this form sodium does not easily oxidise, whilst in vapour it burns) and the carbonic oxide remains as gas.

In sodium works an iron tube, about a metre long and a decimeter

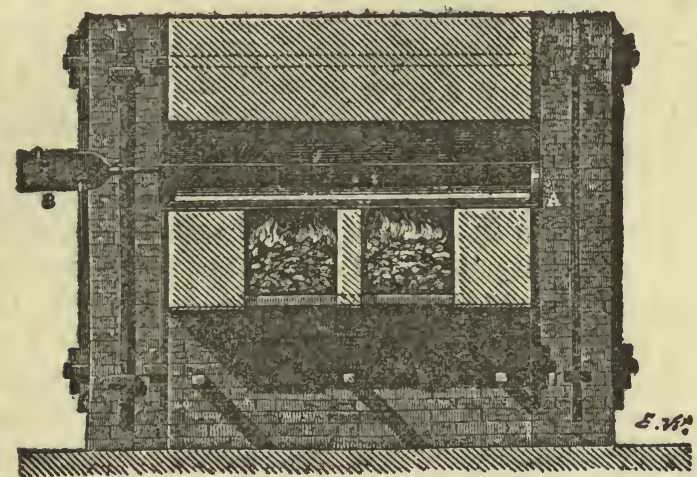

Fro. 70-Manufacture of sodium by Devillo's process A C, fron tube containing a mixtare of odas, charcoal, ada chalk. B, condenser.

in diameter, is made out of boiler plate. The pipe is luted into a furnace having a strong draught, capable of giving a high temperature, and the tube is charged with the mixture required for the preparation of sodium. One end of the tube is closed with a cast-iron stopper $\mathbf{A}$ with clay luting, and the other with the cast-iron stopper $\mathbf{C}$ provided

58 Since the close of the eighties in England, where the preparation of sodium is at present carried out on a lange oommencial sicale (from 1860 to 1870 it wes only manufactured in a few works in France), it has been the practice to add to Deville's mixture iron, or iron oxide which with the charcoal gives metallio and carburetted iron, which still further facilitates the decomposition. At present a kilogram of sodium may be purchased for about the same sum (2/-) as a gram cost thirty years aga. Castner, in England, greatly improved the manufacture of sodium in large quantities, and so cheapened it as a reducing agent in the preparation of metallic aluminium. He heated a mixture of 44 parts of $\mathrm{NaHO}$, and 7 parts of carbide of iron in large iron retorto at $1,000^{\circ}$ and obtained about $6 \frac{1}{2}$ parts of tmetallic sodium. The reaction proceeds more easily than with carbon or iron alone, and the decomposition o the $\mathrm{NaHO}$ proceede according to the equation: $8 \mathrm{NaHO}+\mathrm{C}=\mathrm{Na}_{2} \mathrm{CO}_{3}+3 \mathrm{H}+\mathrm{Na}$. Subsequently, in 1891, aluminium was prepared by electrolysis (see Chapter XVII.), and metallic sodium found 
with an aperture. On heating, first of all the moisture contained. in the various substances is given off, then carbonic anhydride and the products of the dry distillation of the charcoal, then the latter begins
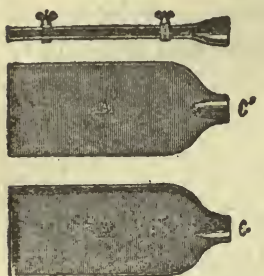

[ Jua.71.-Donny and Maresca's sodium condenser, consist. Ing of two cast-iron plates corewed together. to act on the sodium carbonate, and carbonio oxide.and vapours of sodium appear. It is easy to observe the appearance of the latter, because on issuing from the aperture in the stopper $O$ they take fire spontaneously and burn with a very bright yellow flame. A pipe is then in. troduced into the aperture $\mathrm{C}$, compelling the vapours arid gases formed to pass through the condenser $B$. This condenser consists of two square cast-iron trays, $A$ and $A^{\prime}$, fig. 71, with wide edges' firmly screwed together. Between these two trays there is a space in which the condensation of the vapours of sodium is effected, the thin metallic walls of the condenser being cooled by the air but remaining hot enough to preserve the sodium in a liquid state, so that it does not choke the apparatus, but continually flows from it. The vapours of sodium, condensing in the cooler, flow in the shape of liquid metal into a vessel containing some non-volatile naphtha or hydrocarbon. This is used in order to prevent the sodium oxidising as it issues from the condenser at a somewhat high temperature. In order to obtain sodium of a pure quality it is necessary to distil it ance more, which may-even be done in porcelain retorts, but the distillation must be conducted in a stream of some gas on which sodium does not act, for instance in a stream of nitrogen; carbonic anhydride is not applicable, because sodium partially de. composes it, absorbing oxygen from jit. Although the above described methods of preparing sodium by chemical means have proved very con. venient in practice, still it is now (since 1893) found profitable in England to obtain it (to the amount of several tons a week) by. Davy's classical method, i.e. by the action of an electric current at a moderately high temperature, because the means for producing an electric current (by motors and dynamos) now render this quite feasible. This may be regarded as a sign that in process of time many other technical methods for producing various substances by decomposition maj bo profitably carried on by elestrolysis.

Pure sodium is a lustrous metal, white as silver, soft as wax; it becomes brittle in the cold. In ordinary moist air it quickly tarnishes

two new uses ; (1) for the manufacture of peroxide of sodium (see later on) which is used in blewobing works, and (2) in the manufacture of potassium and oddium oyanide from rellow prussiate (Chepter XIII., Note 12). 
and becomes covered with a film of hydroxide, $\mathrm{NaHO}$, formed at the expense of the water in the air. In perfectly dry air sodium retains its lustre for an indefinite time. Its density at the ondinary tempera. ture is equal to 0.98 , so that it is lighter than water; it fuses very easily at a temperature of $95^{\circ}$, and distils at a bright red heat $\left(742^{\circ}\right.$ according to Perman, 1889). Scott (1887) determined the density of sodium vapour and found it to be nearly 12 (if $H=1$ ). This shows that its molecule contains one atom (like mercury and cadmium) $\mathrm{Na}_{0}{ }^{38}$ bis It forms alloys with most metals, combining with them, heat being sometimes evolved and sometimes absorbed. Thus, if sodium (having a clean surface) be thrown into mercury, especially when beated, there is a flash, and such a considerable amount of heat is evolved that part of the mercury is transformed into vapour ${ }^{39}$ Compounds or solutions of sodium in mercury, or amalgams of sodium, even when containing 2 parts of sodium to 100 parts of mercury, are solids. Only those amalgams which are the very poorest in sodium are liquid. Such alloys of sodium with mercury are often used instead of sodium in chemical investigations, because in combination with mercury sodium is not easily acted on by air, and is heavier than water, and therefore more convenient to bandle, whilst at the same time it retains the principal properties of sodium, 40 for instance it decomposes water, forming $\mathrm{NaHO}$.

It is easy to form an alloy of mercury and sodium having a crystal. line structure, and a definite atomic composition, $\mathrm{NaHg}_{5}$. The alloy of sodium with hydrogen or sodium hydride, $\mathrm{Ne}_{2} \mathrm{H}$, which has the external

88 bls This is also shown by the fall in the temperature of solidification of tin produced by the addition of sodium (and also $\mathrm{Al}$ and $\mathrm{Zn}$ ). Heycock and Neville (1889).

$59 \mathrm{By}$ dissolving sodium amsigams in water and acids, and deducting the heat of colution of the sodium, Berthelot found that for each atom of the sodium in amalgam. containing a larger amount of mercury than $\mathrm{NaHg}_{5}$, the amount of heat evolved increases, after which the heut of formation falls, and the heat evolved decreases. In the formation of $\mathrm{NaHg}_{5}$ about 18,500 calories are evolved; when $\mathrm{NaHg}_{3}$ is formed, about 14,000; and for $\mathrm{NaHg}$ about 10,000 calories. Kraft regarded the definite crystalline amalgam as having the composition of $\mathrm{NaHg}_{6}$, but at the present time, in accordance with Grimaldi's results, it is thought to be $\mathrm{NaHg}_{5}$. A similar amalgam is very easily obtained if e 8 p.c. amalgam be left several days in a solution of sodium hydroxide until a crystalline mass is formed, from which the mercury may be remored by strongly pressing in chamois leather. This amalgam with a solution of potassium hydroxide forms a potassium eimalgam, $\mathrm{KHg}_{10}$. It may be mentioned here that the latent heat of fusion (of atomio quantities) of $\mathrm{Hg}=860$ (Personne), $\mathrm{Na}=730$ (Joannis), and $\mathrm{K}=610$ calories (Joannis).

40 Alloys are so similar to solutions (exhibiting such complete parallelism in proper. ties) that they are included in the same class of so-called indefinite compounds. But in alloys, as substances passing from the liquid to the solid state, it is easier to discover the formation of definite chemical compounds. Besides the alloys of $\mathrm{Na}$ with $\mathrm{Hg}$, those with cin (Bailey 1892 found $\mathrm{Na}_{2} \mathrm{Sn}$ ), lead (NaPb), bismuth (NazBi), \&c. (Joannis 1892 and othero) have been investigated. 
appearance of a metal, 41 is a most instructive example of the characteristics of alloys. At the ordinary temperature sodium does not absorb hydrogen, but from $300^{\circ}$ to $421^{\circ}$ the absorption takes place at the ordinary pressure (and at an increased pressure even at higher tem. peratures), as shown by Troost and Hautefeuille (1874). One volume of sodium absorbs as much as 238 volumes of hydrogen. The inetal increases in volume, and when once formed the alloy can be preserved for some time without change at the ordinary temperature. The appearance of sodium hydride resembles that of sodium itself; it is as soft as this latter, when heated it becomes brittle, and decomposes above $300^{\circ}$, evolving hydrogen. In this decomposition all the phenomona of dissociation are very clearly shown-that is, the hydrogen gas evolved has a definite tension ${ }^{42}$ corresponding with each definite temperature. This cunfirms the fact that the formation of substances capable of dissociation can only be accomplished within the dissociation limits. Sodium hydride melts more easily than sodium itself, and then does not undergo decomposition if it is in an attosphere of hydrogen. It oxidises easily in air, but not so easily as potassium hydride. The chemical reactions of sodium are rettained in its hydride, and, if we may 80 express it, they are even increased by the addition of hydrogen. At all events, in the properties of sodium hydride ${ }^{43}$ we see other properties than in such hydrogen compounds as $\mathrm{HC}, \mathrm{H}_{2} \mathrm{O}, \mathrm{H}_{3} \mathrm{~N}, \mathrm{H}_{4} \mathrm{C}$, or even in the gaseous metallio hydrides $\mathrm{AsH}_{3}, \mathrm{TeH}_{2}$. Platinum, palladium, nickel, and iron, in absorbing bydrogen form compounds in which hydrogen is in a similar state. In them, as in sodium hydride, the hydrogen is compressed, absorbed, occluded (Chapter II.) 43 bls

41 Potessium forms a similas compound, but lithtum, under the same circumstancee, does not.

- The tension of dissociation of hydrogen $p$, in millimetres of metoury, is :-

$\begin{array}{cccccc} & t= & 880^{\circ} & 850^{\circ} & 400^{\circ} & 430^{\circ} \\ \text { for } \mathrm{Nr}_{2} \mathrm{H} & p= & 88 & 57 & 447 & 910 \\ \text { for } \mathrm{K}_{2} \mathrm{H} & & 40 & 78 & 548 & 1100\end{array}$

43 In general, during the formation of alloye the volumes change very alightly, and therefore from the volume of $\mathrm{Na}_{2} \mathrm{H}$ some idea may be formed of the volume of hydrogen in a solid or liquid state. Ever Archimedes concluded that there was gold In an alloy of copper and gold by reason of its volume and density. From the fact that the density of $\mathrm{Na}_{2} \mathrm{H}$ is equal to 0.959 , it may be seen that the volume of 47 grams (the gram molecule) of this compound $=49^{\circ} 0 \mathrm{coc}$. The volume of $46 \mathrm{grams}$ of sodium con. tained in the $\mathrm{Na}_{2} \mathrm{H}$ (the density under the same conditions being 0.97 ) is equal to $47^{\prime}$ 0.c. Therefore the rolume of $1 \mathrm{gram}$ of hydrogen in $\mathrm{Na}_{2} \mathrm{H}$ is equal. to 1.6 c.c., and conse. quently the density of metallio hydrogen, or the weight of $1 \mathrm{c.0}$, approaches $0.6 \mathrm{gram}$. This density is also proper to the hydrogen alloyed with potassium and palladium. Judging from the scanty information which is at present available, liquid hydrogen near its absolute boiling point (Chapter II) has a much lower density.

43 ble We may remark that at low temperatures $\mathrm{Na}$ absorbs $\mathrm{NH}_{8}$ and forms $\left(\mathrm{NH}_{3} \mathrm{Na}_{2}\right.$ (see Chapter VI, Note 14); this substance absorbs CO and gives (NaCO)n (Chapter IX., Noto 81), although by itsels $\mathrm{Na}$ does not combine directly with $\mathrm{CO}$ (bat $\mathrm{K}$ does). 
The most important chemical property of sodium is its power of easily decomposing water and evolving hydrogen from the majority of the hydrogen compounds, and especially from all acids, and hydrates in which hydroxyl must be recognised. This depends on its power of combining with the elements which are in combination with the hydrogen. We already know that sodium disengages hydrogen, not only from water, hydrochloric acid, ${ }^{44}$ and all other acids, but also from ammonia, ${ }^{44}$ bis with the formation of sodamide $\mathrm{NH}_{2} \mathrm{Na}$, although it does not displace hydrogen from the hydrocarbons. ${ }^{45}$ Sodium burns

44 H. A. Schmidt remarked that perfectly dry hydrogen chloride is decomposed with great difficulty by sodium, although the decomposition proceeds easily with potassium and with sodium in moist hydrogen chloride. Wanklyn also remarked that sodium aburns with great difficulty in dry chlorine. Probably these fscts are related to other phenomena observed by Dixon, who found that perfectly dry carbonio oxide does not explode with oxygen on passing an electric spark

44 bio Sodamide, $\mathrm{NH}_{2} \mathrm{Na}$, (Chapter IV., Noto 14), discovered by Gay.Lussac and Thenard, has formed the object of repeated research, but has been most fully investigated by A. W. Titherley (1894). Until recently the following was all that was known about this compound:-

By heating sodium in dry ammonia, Gay-Lussac and Thénard obtained an olivegreen, easily-fusible mass, sodamide, $\mathrm{NH}_{2} \mathrm{Na}$, hydrogen being separated. This substance with water forms sodium hydroxide and ammonia; with carbonic oxide, $\mathrm{CO}$, it forms sodium cyanide, $\mathrm{NaCN}$, and water, $\mathrm{H}_{2} \mathrm{O}$; and with dry hydrogen chloride it forms sodium and ammonium chlorides. These and other reactions of sodamide show that the metal in it prèserves its energetic properties in reaction, and that this compound of sodium is more stable than the corresponding chlorine amide. When heated, sodamide, $\mathrm{NH}_{2} \mathrm{Na}$, only partially decomposes, with evolution of hydrogen, the principal part of it giving ammonia and sodium nitride, $\mathrm{Na}_{3} \mathrm{~N}$, according to tho equátion $3 \mathrm{NH}_{2} \mathrm{Na}_{2}=2 \mathrm{NH}_{3}+\mathrm{NNa}_{3}$. The latter is an almost black powdery mass, decomposed by water into ammonia and sodium hydroxide.

Titherley's researches added the following data :-

Iron or silver vessels should be used in preparing this body, because glass and porcelain are corroded at $800^{\circ}-400^{\circ}$, at which temperature ammonia gas acts upon sodium and forms the amide with the evolution of hydrogen. The reaction proceeds slowly, but is complete if there be an excess of $\mathrm{NH}_{3}$. Pure $\mathrm{NH}_{2} \mathrm{Na}$ is colourless (its colouration is due to various impurities), semi-transparent, shows traces of crystallisation, has a conchoidal fracture, and melts at $145^{\circ}$. Judging from the increase in weight of the sodium and the quantity of hydrogen which is disengaged, the composition of the amide is exactly $\mathrm{NH}_{2} \mathrm{Na}$. It partially volatilises (sublimes) in vacuo at $200^{\circ}$, and breaks up into $2 \mathrm{Na}+\mathrm{N}_{2}+2 \mathrm{H}_{2}$ at $500^{\circ}$. The same aunide is formed when oxide of sodium is heated in $\mathrm{NH}_{3}: \mathrm{Na}_{2} \mathrm{Q}+2 \mathrm{NH}_{3}=2 \mathrm{NaH}_{2} \mathrm{~N}+\mathrm{H}_{2} \mathrm{O}$. NaHO is also formed to some extent by the resultant $\mathrm{H}_{2} \mathrm{O}$. Potassium and lithium form similar amides. With water, alcohol, and acids, $\mathrm{NH}_{2} \mathrm{Na}$ gives $\mathrm{NH}_{3}$ and $\mathrm{NaHO}$, which react further. Anhydrous $\mathrm{CaO}$ absorbs $\mathrm{NH}_{2} \mathrm{Na}$ when heated without decomposing it. When sodanide is heated with $\mathrm{SiO}_{2}, \mathrm{NH}_{3}$ is disengaged, and silicon nitride formed. It acts still more readily upon boric anhydride when heated with it: $2 \mathrm{NH}_{2} \mathrm{Na}+\mathrm{B}_{2} \mathrm{O}_{3}=2 \mathrm{BN}+2 \mathrm{NaHO}+\mathrm{H}_{2} \mathrm{O}$. When slightly heated, $\mathrm{NH}_{2} \mathrm{Na}+\mathrm{NOCl}=\mathrm{NaCl}+\mathrm{N}_{2}+\mathrm{H}_{2} \mathrm{O}\left(\mathrm{NHNa}_{2}\right.$ and $\mathrm{NNa} a_{3}$ are apparently not formed at a higher temperature). The halogen organic compounds react with the aid of heat, but with so much energy that the reaction frequently leads to the ultimate deotruction of the organic groups and production of carbon.

45 As sodium does not displace hydrogen from the hydrocarbons, it may be preserved in liquid hydrocarbons. Naphtha is generally used for this purpose, as it consists of a mixture of various liquid hydrocarbons. However, in naphtha sodium usually becomes coated with a crast composed of matter produced by the action of the sodium on certain 
both in chlorine and in oxygen, evolving much heat. These properties are closely connected with its power of taking up oxygen, chlorine, and similar elements from most of their compounds. Just as it removes the oxygen from the oxides of nitrogen and from carbonic anhydride, so also does it decompose the majority of oxides at definite temperatures. Here the action is essentially the same as in the decomposition of water. Thus, for instance, when acting on magnesium chloride the sodium displaces the magnesium, and when acting on aluminium chloride it displaces metallic aluminium. Sulphur, phosphorus, arsenic and a whole series of other elements, also combine with sodium. ${ }^{46}$

With oxygen sodium unites in three degrees of combination, forming a suboxide $\mathrm{Na}_{4} \mathrm{O}, 45$ bis an oxide, $\mathrm{Na}_{2} \mathrm{O}$, and a peroxide, $\mathrm{NaO}$. They are thus termed because $\mathrm{Na}_{2} \mathrm{O}$ is a stable basio oxide (with water it forms a basic hydroxide), whilst $\mathrm{Na}_{4} \mathrm{O}$ and $\mathrm{NaO}$ do not form corresponding saline hydrates and salts. The suboxide is a grey intlammable substance which easily decomposes water, disengaging hydrogen; it is formed by the slow oxidation of sodium at the ordinary temperature. The peroxide is a greenish yellow substance, fusing at a bright red heat; it is produced by burning sodium in an excess of oxygen, and it fields oxygen when treated with water

$$
\begin{aligned}
& \text { Suboxide : } \mathrm{Na}_{4} \mathrm{O}+3 \mathrm{H}_{2} \mathrm{O}=4 \mathrm{NaHO}+\mathrm{H}_{2}^{47} \\
& \text { Oxide: } \mathrm{Na}_{2} \mathrm{O}+\mathrm{H}_{2} \mathrm{O}=2 \mathrm{NaHO} 48 \\
& \text { Peroxide : } \mathrm{Na}_{2} \mathrm{O}_{2}+\mathrm{H}_{2} \mathrm{O}=2 \mathrm{NaHO}+\mathrm{O}^{49}
\end{aligned}
$$

of the substances contained in the mixture composing naphtha. In order that sodium may retain its lustre in naphtha, secondary octyl alcohol is added. (This alcohol is obtained by distilling castor oil with caustio potash.) Sodium keeps well in a mixture of pure benzene and paraffin.

40 If sodium does not directly displace the hydrogen in hydrocarbons, still by indirect means compounds may be obtained which contain sodium and hydrocarbon gronps. Some of these compounds have been produced, although not in a pure state. Thus, for : instance, zinc ethyl, $\mathrm{Zn}\left(\mathrm{C}_{2} \mathrm{H}_{5}\right)_{2}$, when treated with sodium, loses zino and forms sodium ethyl, $\mathrm{C}_{2} \mathrm{H}_{5} \mathrm{Na}$, but this decomposition is not complete, and the compound formed cannot: be separated by distillation from the remaining zinc ethyl. In this compound the energy of the sodium is clearly manifest, for it reacts with substances containing haloids, oxygen, \&c., and directly absorbs carbonia anbydride, forming a salt of a carboxylic acid (propionic).

46 bls It is even doubtful whether the suboxide exists (see Note 47 ).

47 A compound, $\mathrm{Na}_{2} \mathrm{Cl}_{2}$ which corresponds with the suboxide, is apparently formed when a galvanio current is passed through fused common.salt; the sodium liberated dissolves in the common salt, ind does not separate from the compound either on cooling or on treatment with mercury. It is therefore supposed to be $\mathrm{Na}_{2} \mathrm{Cl}$; the more so as the mass obtained gives hydrogen when treated with water: $\mathrm{Na}_{2} \mathrm{Cl}+\mathrm{H}_{2} \mathrm{O}$ $=\mathrm{H}+\mathrm{NaHO}+\mathrm{NaCl}$, that is, it acts like suboxide of sodium. If $\mathrm{Na}_{2} \mathrm{Cl}$ really exists as a salt, then the corresponding base $\mathrm{Nr}_{4} \mathrm{O}$, according to the rule with other bases of the composition $\mathrm{M}_{4} \mathrm{O}$, ought to be called a quaternary oxide. According to certain evidence, as suboxide is formed when thin sheets or fine drops of sodium slowly oxidise in moist air.

48 Aocording to observations easily made, sodium when fused in air exidises but does bot bur $\dot{n}_{2}$, the combustion only commencing with the formation of vapour-that is, when 
All three oxides form sodium hydroxide with water, but only the oxide $\mathrm{Na}_{2} \mathrm{O}$ is directly transformed into a hydrate. The other oxides liberate either hydrogen or oxygen; they also present a similar distinction yith reference to many other agents. Thus carbonic anhydride combines directly with the oxide $\mathrm{Na}_{2} \mathrm{O}$, which when heated in the gas burns, forming sodium carbonate, whilst the peroxide yields oxygen in addition. When treated with acids, sodium and all its oxides only form the salts corresponding with sodium oxide-that is of the formula or type $\mathrm{NaX}$. "Thus the oxide of sodium, $\mathrm{Na}_{2} \mathrm{O}$, is the only salt-forming

considerably heated. Davy and Karsten obtained the oxides of potassium, $\mathrm{K}_{2} \mathrm{O}$, and of sodium, $\mathrm{Na}_{2} \mathrm{O}$, by heating the metals with their hydroxides, whence $\mathrm{NeHO}+\mathrm{Na}=\mathrm{Na}_{2} \mathrm{O}+\mathrm{H}$, but N. N. Beketoff failed to obtain oxides by this means. He prepared them by directly igniting the metals in drI air, and afterwards heating with the metel in order to destroy any peroxide. The oxide produced; $\mathrm{N}_{2} \mathrm{O}$, when-hęatêd în an étmosphere of hydrogen, gave a mixture of sodium and its hydroxide: $\mathrm{Na}_{2} \mathrm{O}+\mathrm{H}=\mathrm{NaHO}+\mathrm{Na}$ (see Chapter II., Note 9). If both the observations mentioned are accuratel then the resction is reversible. Sodium oxide ought to be formed during the decomposition of sodium carbonate by oxide of iron (see Note 26), and during the decomposition of sodium nitrite. According to Karsten, its specifio gravity is $2: 8$, according to Beketoff $2 \cdot 8$. The difficulty in obtaining it is owing to an excess of sodium forming the suboxide, and an excess of oxygen the peroxide. The grey colour peculiar to the suboxide and oxide perhaps shows that they contain metallic sodium. In addition to this, in the presence of water it may contain sodium hydride and $\mathrm{NaHO}$.

40 Of the oxides of sodinm, that easiest to form is the peroxide, $\mathrm{NaO}_{2}$ or $\mathrm{Na}_{2} \mathrm{O}_{2}$; this is obtained when sodium is burnt in an excess of oxygen. If $\mathrm{NaNO}_{3}$ be melted, it gives $\mathrm{Na}_{2} \mathrm{O}_{2}$ with metallic $\mathrm{Na}$. In a fused state the peroxide is reddish yellow, but it becomes almost colourless when cold. When heated with iodine vapour, it loses oxygen: $\mathrm{Na}_{2} \mathrm{O}_{2}+\mathrm{I}_{2}$ $=\mathrm{Ne}_{2} \mathrm{OI}_{2}+\mathrm{O}$. The compound $\mathrm{Na}_{2} \mathrm{OI}_{2}$ is akin to the compound $\mathrm{Cu}_{2} \mathrm{OCl}_{2}$ obtained by oxidising $\mathrm{CuCl}$. This reaction is one of the few in which iodine directly displaces oxygen. The substance $\mathrm{Na}_{2} \mathrm{OI}_{2}$ is soluble in water, and when acidifled gives free iodine and a sodium salt. Carbonic oxide is absorbed by heated sodium peroxide with formation of sodium carbonate: $\mathrm{Na}_{2} \mathrm{CO}_{3}=\mathrm{Na}_{2} \mathrm{O}_{2}+\mathrm{CO}$, whilst carbonio anhydride liberates oxygen from it. With nitrous oxide it reacts thas: $\mathrm{Na}_{2} \mathrm{O}_{2}+2 \mathrm{~N}_{2} \mathrm{O}=2 \mathrm{NaNO}_{2}+\mathrm{N}_{2}$; with nitrio oxide it combines directly, forming sodium nitrite, $\mathrm{NaO}+\mathrm{NO}=\mathrm{NaNO}_{2}$. Sodium peroxide, when treated with water, does not give hydrogen peroxide, because the latter in the presence of the alkali formed $\left(\mathrm{Na}_{2} \mathrm{O}_{3}+2 \mathrm{H}_{2} \mathrm{O}=2 \mathrm{NaHO}+\mathrm{H}_{2} \mathrm{O}_{2}\right)$ decomposes into water and oxygen. In the presence of dilute sulphurio acid it forms $\mathrm{H}_{2} \mathrm{O}_{2}\left(\mathrm{Na}_{2} \mathrm{O}_{2}+\mathrm{H}_{2} \mathrm{SO}_{4}\right.$ $=\mathrm{Ne}_{2} \mathrm{SO}_{4}+\mathrm{H}_{2} \mathrm{O}_{2}$ ). Peroxide of sodium is now prepared on a large scale (by theaction of air upon $\mathrm{Na}$ at $800^{\circ}$ ) for bleaching wool, silk \&c. (when it acts in virtue of the $\mathrm{H}_{2} \mathrm{O}_{2}$ formed). The oxidising properties of $\mathrm{Na}_{2} \mathrm{O}_{2}$ under the action of heat are seen, for instance, in the fact that when heated with I it forms sodium iodate; with $\mathrm{PbO}, \mathrm{Na}_{2} \mathrm{PbO}_{5}$; with pyrites, eulphates, \&c. When peroxide of sodium comes into contact with water, it evolves much heat; forming $\mathrm{H}_{2} \mathrm{O}_{2}$, and decomposing with the disengagement of oxygen; but, as a rule, there is no explosion. But if $\mathrm{Na}_{2} \mathrm{O}_{2}$ be placed in contact with organic matter, such as sawdust, cotton, \&c., it gives a violent explosion when heated, ignited, or acted on by water. Peroxide of sodium forms an excellent oxidising agent for the preparation of the higher product of oxidation of $\mathrm{Mn}, \mathrm{Cr}, \mathrm{W}$, \&c., and also for oxidising the metallio sulphides. It should therefore find many applications in chemical analysis. To prepare $\mathrm{Na}_{2} \mathrm{O}_{2}$ on a large scale, Castner melts $\mathrm{Na}$ in an aluminium vessel, and at $800^{\circ}$ passes firet air deprived of a portion of its oxygen (having been already onco used), and then ordinary ding air over ib. 
oxide of this metal, as water is in the case of hydrogen. Although the peroxide $\mathrm{H}_{2} \mathrm{O}_{2}$ is derived from hydrogen, and $\mathrm{Na}_{2} \mathrm{O}_{2}$ from sodium, get there are no corresponding salts known, and if they are formed they are probably as unstable as hydrogen peroxide. Although carbon forms carbonic oxide, $\mathrm{CO}$, still it has only one salt-forming oxidecarbonio anhydride, $\mathrm{CO}_{2}$. Nitrogen and chlorine both give several salt.forming oxides and types of salts. But of the oxides of nitrogen, $\mathrm{NO}$ and $\mathrm{NO}_{2}$ do not form salts, as do $\mathrm{N}_{8} \mathrm{O}_{3}, \mathrm{~N}_{2} \mathrm{O}_{4}$, and $\mathrm{N}_{2} \mathrm{O}_{5}$, although $\mathrm{N}_{2} \mathrm{O}_{4}$ does not form special salts, and $\mathrm{N}_{2} \mathrm{O}_{5}$ corresponds with the highest form of the saline compounds of nitrogen. Such distinctions between the elements, according to their power of giving one or several saline forms, is a radical property of no less importance than the basio or acid properties of their oxides. Sodium as a typical metal does not form any acid oxides, whilst chlorine, as a typical non-metal, does not form bases with oxygen. Therefore sodium as an elenvent may bo thus characterised : it forms one very stable salt-forming oxide, $\mathrm{Na}_{2} \mathrm{O}$, having powerful basic properties, and its salts are of the general formula, $\mathrm{NaX}$, therefore in its compounds it is, like hydrogen, a basio and univalent element.

On comparing sodium and its analogues, which will be described later with other metallic elements, it will be seen that these properties, together with the relative lightness of the metal itself and its com. pounds, and the magnitude of its atomic weight comprise the most essential properties of this element, clearly distinguishing it from others, and enabling us easily to rocognise its analogues. 


\section{CHAPTER XIII}

POTASSIUM, ROBIDIUM, CESIUM, AND LITHIOM. BPECTROM ANATYSIS

JUST as the series of halogens, fluorine, bromine and iodine correspond with the chlorine contained in common salt, so also there exists a corresponding series of elements: lithium, $L i=7$, potassium, $K=39$, rubidium, $\mathrm{Rb}=85$, and cæsium, $\mathrm{Cs}=133$, which are analogous to the sodium in common salt. These elements bear as great a resemblance to sodium, $\mathrm{N} a=23$, as fluorine, $\mathrm{F}=19$, bromine, $\mathrm{Br}=80$, and iodine, $\mathrm{I}=127$, do to chlorine, $\mathrm{Cl}=35.5$. Indeed, in a free state, these elements, like sodium, are soft metals which rapidly oxidise in moist air and decompose water at the ordinary temperature, forming soluble hydroxides having clearly-defined basic properties and the composition $\mathrm{RHO}$, like that of caustic soda. The resemblance between these metals is sometimes seen with striking clearness, especially in compounds such as salts. 1 The corresponding salts of nitric, sulphuric, carbonic, and nearly all acids with these metals have many points in common. The motals which resemble sodium so much in their reactions are termed the metals of the alkalis.

1 Tutton's researches (1894) npon the anslogy of the crystalline forms of $\mathrm{K}_{2} \mathrm{SO}_{4}, \mathrm{Rb}_{2} \mathrm{SO}_{4}$ and $\mathrm{Cs}_{2} \mathrm{SO}_{4}$ may be taken as a typical example of the comparison of analogous compounds. We cite the following data from these excellent researches: the sp. gr. at $20^{\circ} / 4^{\circ}$ of $\mathrm{K}_{2} \mathrm{SO}$, is 2.6633 of $\mathrm{Rb}_{2} \mathrm{SO}_{4}, 8.6113$, and of $\mathrm{Cs}_{2} \mathrm{SO}_{4}, 4.2434$. The coefficient of cubical expansion (the mean between $20^{\circ}$ and $60^{\circ}$ ) for the $\mathrm{K}$ salt is 0.0053 , for the $\mathrm{Rb}$ salt 0.0052 , for the Cs salt 0.0051. The linear expansion (the maximum for the vertical axis) along the axis of crystallisation is the same for all three salts, within the limits of experimental error. The replacement of potassium by rubidium causes the distance between the oentres of the moleoules in the direction of the three ares of crystallisation to increase equally, and less than with the replacement of rubidium by crsium. The index of refraction for all rays and for every crystalline path (direction) is greater for the rubidium salt than for the potassium salt, and less than for the cosium ealt, and the differences are neaxly in the ratio $2: 5$. The lengths of the rhombio crystalline axes for $\mathrm{K}_{2} \mathrm{SO}_{4}$ are in the ratio $0.5727: 1$ : 0.7418 , for $\mathrm{Rb}_{2} \mathrm{SO}_{4}, 0.5728: 1: 0.7485$, and for $\mathrm{Cs}_{2} \mathrm{SO}_{4}, 0.5712: 1$ : 0.7521. The development of the basic and brachy-pinacoids gradually increases in passing from $\mathrm{K}$ to $\mathrm{Rb}$ and $\mathrm{Cs}$. The optical properties also follow the same order both at the ordinary and at a higher temperature. Tutton draws the general conolusion that the cxystallo, graphic properties of the isomorphic rhombic sulphates $\mathrm{R}_{2} \mathrm{SO}_{4}$ are a function of the atomic weight of the metals contained in them (see Chapter XV.) Suoh researches as Whese should do much towards hastening the establishmont of a true molecular mechanies of physico-chemioal phonomona. 
Among the metals of the alkalis, the most widely distributed in nature, after sodium, is potassium. Like sodium, it does not appear either in a free state or as oxide or hydroxide, but in the form of salts, which present much in common with the salts of sodium in the manner of their occurrence. The compounds of potassium and sodium in the earth's crust occur as mineral compounds of silica. With silica, $\mathrm{SiO}_{2}$, potassium oxide, like sodium oxide, forms saline mineral substances resembling glass. If other oxides, such as $\operatorname{lime}, \mathrm{CaO}$, and alunina, $\mathrm{Al}_{2} \mathrm{O}_{3}$, combine with these compounds, glass is formed, a vitreous stony mass, distinguished by its great stability, and its very slight wariation under the action of water. It is such complex silicious com: pounds as these which contain potash (potassium oxide), $\mathrm{K}_{2} \mathrm{O}$, or soda (sodium oxide), $\mathrm{Na}_{2} \mathrm{O}$, and sometimes both together, silica, $\mathrm{SiO}_{2}$, lime, $\mathrm{CaO}$, alumina, $\mathrm{Al}_{2} \mathrm{O}_{3}$, and other oxides, that form the chief mass of rocks, out of which, judging by the direction of the strata, the chief mass of the accessible crust (envelope) of the earth is made up. The primary rocks, like granite, porphyry, \&c., ${ }^{1}$ bis are formed of such crystalline silicious rocks as these. The oxides entering into the composition of these rocks do not form a homogeneous amorphous mass like glass, but are distributed in a series of peculiar, and in the majority of cases crystalline, compounds, into which the primary rocks may be divided. Thus a felspar (orthoclase) in granite contains from 8 to 15 per cent. of potassium, whilst another variety (plagioclase) which also occurs in granite contains 1.2 to 6 per cent. of potassium, and 6 to 12 per cent. of sodium. The mica in granite contains 3 to 10 per cent. of potassium. As already mentioned, and further explained in Chapter XVII., the friable, crumbling, and stratified formations which in our times cover a large part of the earth's surface have been formed' from these primary rocks by the action of the atmosphere and of water containing carbonic acid. It is evident that in the chemical alteration of the primary rocks by the action of water, the compounds of potassium, as well as the compounds of sodium, must have been dissolved by the water (as they are soluble in water), and that therefore the compounds of potassium must be accumulated together with those of sodium in sea water. And indeed compounds of potassium are always found in sea water, as we have already pointed out (Chapters I. and X.). This forms one of the sources from which they are extracted. After the evaporation of sea water, there remains a mother liquor, which contains potassium chloride and a large proportion of magnesium chloride. On cooling this solution crystals separate out which contain chlorides of magnesium and potassium. A double salt of this kind, called carnallite,

i vie The origin of the primary rocks has been mentioned in Chapter $\mathrm{X}$, Note 2. 
$\mathrm{KMgCl}_{3}, 6 \mathrm{H}_{2} \mathrm{O}$, occurs at Stassfurt. This carnallite ${ }^{2}$ is now employed as a material for the extraction of potassium chloride, and of all the compounds of this element. ${ }^{3}$ Besides which, potassium chloride itself is sometimes found at Stassfurt as sylvine. ${ }^{3 \text { bis }}$ By a method of

- Carnallite belongs to the number of double salts which are directly decomposed by water, and it only crystallises from solutions which contain an excess of magnesium chloride. It may be prepared artificially by mixing strong solutions of potassium and magnesium chlorides, when colourless crystals of sp.gr. 1.60 separate, whilst the Stassfurt salt is usually of a reddish tint, owing to traces of iron. At the ordinary temperature sixty-five parts of carnallite are soluble in one hundred parts of water in the presence of an excess of the salt. It deliquesces in the air, forming a solution of magnesium chloride and leaving potassium chloride. The quantity of carnallite produced at Stassfurt is now as much as 100,000 tons a year.

5 The method of separating sodium chloride from potassium chloride has been de. scribed in Chapter I. On evaporation of a mixture of the saturated solutions, sodium chloride separates; and then, on cooling, potassium chloride separates, owing to the difference of rate of variation of their solubilities with the temperature. The following are the most trustworthy figures for the solubility of potassium chloride in one hundred parts of water (for sodium chloride, seo Chapter X., Note 13) :-

$\begin{array}{lllll}10^{\circ} & 20^{\circ} & 40^{\circ} & 60^{\circ} & 100^{\circ} \\ 82 & 85 & 40 & 40 & 57\end{array}$

When mixed with solutions of other salts the solubility of potassium chloride, naturalls varies, but not to any great extent.

5 bin The specific gravity of the solid salt is 1.99-that is, less than that of sodium chloride. All the salts of sodium are specifically heavier than the corresponding salts of potassium, as are also their solutions for equal percentage compositions. If the specifip gravity of water at $4^{\circ}=10,000$, then at $15^{\circ}$ the specifio gravity of a solution of $p$ p.c. potassium chloride $=9,992+63 \cdot 29 p+0 \cdot 226 p^{2}$, and therefore for 10 p.c. $=1.0647,20$ p.c. - 1.1348, \& 0 .

Potassium chloride corribines with iodine trichloride to form a compound $\mathrm{KCl}+\mathrm{ICl}_{3}$ $=\mathrm{KICl}_{4}$, which has a yellow colour, is fusible, loses lodine trichloride at a red heat, and gives potassium lodate and hydrochlorio acid with water. It is not only formed by direct combination, but also by many other methods; for instance, by passing chlorine into a solution of potassium iodide so long as the gas is absorbed, $\mathrm{KI}+2 \mathrm{Cl}_{2}=\mathrm{KOl}, \mathrm{ICl}_{3}$. Potassium iodide, when treated with potassium: chlorate and strong hydrochlorio acid, alșo gives this compound; another method for its formation is given by the equation $\mathrm{KClO}_{3}+\mathrm{I}+6 \mathrm{HCl}=\mathrm{KCl}, \mathrm{ICl}_{5}+3 \mathrm{Cl}+8 \mathrm{H}_{2} \mathrm{O}$. This is a kind of salt corresponding with $\mathrm{KIO}_{2}$ (unknown) in which the oxygen .js replaced by chlorine. If valency be taken as the starting-point in the study of chemical compounds, and the elements considerel as having a constant atomicity (number of bonds)-that is, if $\mathrm{K}, \mathrm{Cl}$, and I be taken as univalent elements-then it is impossible to explain the formation of such a compound because, according to this view, tnivalent elements are only able to form dual com. pounds with each other: such as, KCl, ClI, KI, \&o., whilst here they are grouped together in the molecule $\mathrm{KICl}_{4}$. Wells, Wheeler, and Penfield (1892) obtained a large number of such poly-haloid salts. They mày all be divided into two large classes: the tri-haloid and the penta-haloid salts. They have been obtained not only for $\mathrm{K}$ but also for $\mathrm{Rb}$ and $\mathrm{Cs}$, and partially also for $\mathrm{Na}$ and $\mathrm{Li}$. The general method of their formation consists in dissolving the ordinary halogen salt of the metal in water, and treating it with the requisite amount of free halogen. The poly-haloid ralt separates out after evaporating the solution at a more or less low tempersture. In this manner, emong the tri-haloid salts, may be obtained : $\mathrm{KI}_{3}, \mathrm{KBr}_{2} \mathrm{I}, \mathrm{KCl}_{2} \mathrm{I}$, and the corresponding calts of rubidium and cesium, for instance, $\mathrm{CsI}_{3}, \mathrm{CsBrI}_{2}, \mathrm{CsBr}_{2} \mathrm{I}, \mathrm{CsClBrI} \mathrm{CsCl}_{2} \mathrm{I}$, $\mathrm{CsBr}_{5}, \mathrm{CsClBr}_{2}, \mathrm{Cs}_{8} \mathrm{Cl}_{2} \mathrm{Br}$, and in general $\mathrm{MA}_{3}$ where 8 is a halogen. The colinur of the 
double saline decomposition, the chloride of potassium may be converted into all the other potassium salts, ${ }^{4}$ some of which are of practical use. The potassium salts have, however, their greatest importance as an indispensable component of the food of plants."

The primary rocks contain an almost equal proportion of potassium and sodium. But in sea water the compounds of the latter metal pre. dominate. It may be asked, what became of the compounds of potas. sium in the disintegration of the primary rocks, if so small a quantity went to the sea water? They remained with the other products of the decomposition of the primary rocks. When granite or any other similar rock formation is disintegrated, there are formed, besides the soluble substances, also insoluble substances-sand and finely-divided clay, containing water, alumina, and silica. This.clay is carried away by the water, and is then deposited in strata. It, and especially its admixture with vegetable remains, retain compounds of potassium in a greater quantity than those of sodium. This has been proved with absolute certainty to be the case, and is due to the absorptive power of the soil. If a dilute solution of a potassium compound be filtered through common mould used for growing plants, containing clay and the remains of vegetable decomposition, this mould will be

crystals varies according to the halogen, thus $\mathrm{CsI}_{3}$ is black, $\mathrm{CrBr}_{3}$ yellowish red, $\mathrm{CsBrI}_{2}$ reddish brown, $\mathrm{CsBr}_{2} \mathrm{I}$ red, $\mathrm{CsCl}_{2} \mathrm{Br}$ yellow. The cæsium salts are the most stable, and those of potassium least so, as also those which contain $\mathrm{Br}$ and I separately or together; for cæsium no compounds containing $\mathrm{Cl}$ and I were obtained. The penta-haloid salts form a smaller class; among those selts potassium forms $\mathrm{KCl}_{4} \mathrm{I}$, rubidium $\mathrm{RbCl} \mathbf{I}$, cæsium $\mathrm{CsI}_{5}, \mathrm{CsBr}_{4} \mathrm{CsCl}_{4} \mathrm{I}$, lithium $\mathrm{LiCl}_{4} \mathrm{I}$ (with $4 \mathrm{H}_{2} \mathrm{O}$ ) and sodium $\mathrm{NaCl}_{4} \mathrm{I}$ (with $2 \mathrm{H}_{2} \mathrm{O}$ ). The most stable are those salts containing the metal with the greatest atomic weight-crosinm (see Chapter XI, Note 68).

4 It is possible to extract the compounds of potassium directly from the primary rocks which are so widely distributed over the earth's surface and so abundant in some localities. From a chemical point of view this problem presents no difficulty; for instance, by fusing powdered orthoclase with lime and fluor spar (Ward's method) and then extracting the alkali with water (on fusion the silica gives an insoluble compound with $\lim \theta$ ), or by treating the orthoclase with bydrofluoric acid (in which case silicon fluoride is evolved as a gas) it is possible to transfer the alkali of the orthoclase to an aqueous solution, and to separate it in this manner from the other insoluble oxides. However, as yet there is no profit in, nor necessity for, recourse to this treatment, as carnallite and potash form abundant materials for the extraction of potassium compounds by cheaper methods. Furthermore, the salts of potassium are now in the majority of chemical reactions replaced by salts of sodium, especially since the preparation of sodium carbonate has been facilitated by the Leblano process. The replacement of potassium compounds by sodium compounds not only has the advantage that the salts of sodium are in general cheaper than those of potassium, but also that a smaller quantity of a sodium salt is needed for a given reaction than of a potassium salt, because the combin. ing weight of sodium (23) is less than that of potassium (39).

5 It has been shown by direct experiment on the cultivation of plants in ertifioial soils and in solutions that under conditions (physical, chemical, and physiological) other. wise identical plants are able to thrive and become fully developed in the entire absence of sodium salts, but that their development is impossible without potassium salts. 
found to have retained a somewhat considerable percentage of the potassium compounds. If a salt of potassium be taken, then during the filtration an equivalent quantity of a salt of calcium - which is also found, as a rule, in soils-is set free. Such a process of filtration through finely divided earthy substances proceeds in nature, and the compounds of potassiun are everywhere retained by the friable earth in considerable quantity. This explains the presence of so small an amount of potassium salts in the water of rivers, lakes, streams, and oceans, where the lime and soda have accumulated. The compounds of potassium retained by the friable mass of the earth are absorbed as an aqueous solution by the roots of plants. Plants, as everyone knows, when burnt leave an ash, and this ash, besides various other substances; without exception contains compounds of potassium. Many land plants contain a very small amount of sodium compounds, ${ }^{6}$ whilst potassium and its compounds occur in all kinds of vegetable ash. Among the generally cultivated plants, grass, potatoes, the turnip. and buckwheat are particularly rich in potrssium compounds. The ash of plants, and especially of herbaceous plants, buckwheat straw, sunflower and potato leaves are used in practice for the extraction of potassium compounds. There is no doubt that potassium occurs in the plants themselves in the form of complex compounds, and often as salts of organio acids. In certain cases such salts of potassium are even extracted from the juice of plants. Thus, sorrel and oxalis, for example, contain in their juices the acid oxalate of potassium, $\mathrm{O}_{8} \mathrm{HKO}_{4}$, which is employed for removing ink stains. Grape juice contains the so-called cream of tartar, which is the acid tartrate of potassium, $\mathrm{O}_{4} \mathrm{H}_{5} \mathrm{KO}_{6}{ }^{\text {? }}$

6 If herbaceous plants contain much sodium salta, it is evident that these salts mainly come from the sodium compounds in the water absorbed by the plants.

- As plants always contain mineral substances and cannot thrive in a medium which does not contain them, more especially in one which is free from the salts of the four basio oxides, $\mathrm{K}_{2} \mathrm{O}$, $\mathrm{CaO}, \mathrm{MgO}$, and $\mathrm{Fe}_{2} \mathrm{O}_{5}$, and of the four acid oxides, $\mathrm{CO}_{2}, \mathrm{~N}_{2} \mathrm{O}_{3}$, $\mathrm{P}_{2} \mathrm{O}_{5}$, and $\mathrm{SO}_{3}$, and as the amount of ash-forming substances in plants is small, the question inevitably arises as to what part these play in the development of plants. With the existing chemical data only one answer is possible to this question, and it is still only a hypothesis. This answer was particularly clearly expressed by Professor Gustavson of the Petroffsky Agricultural Academy. Starting from the fact (Chapter XI. Note 55) that a small quantity of aluminium renders possible or facilitates the reaction of bromine on hydrocarbons at the ordinary temperatare, it is easy to arrive at the conclusion, which is very probable and in accordance with many data respecting the reactions of organic compounds, that the addition of mineral substances to organio com. pounds lowers the temperature of reaction and in general facilitates chemical reactions In plants, and this aids the conversion of the most simple nourishing substances into the complex component parts of the plant organism. The province of chemical reactions proceeding in organic substances in the presence of a small quantity of mineral substances has as yet been but little investigated, although there are already several disconnected data concerning reactions of this kind, and although a great deal is known with regard to such reactions among inorganio compounds. The essence of the matter may be ete. 
This salt also separates as a sediment from wine. When the plants, containing one or more of the salts of potassium, are burnt, the carbonaceous matter is oxidised, and in consequence the potassium is obtained in the ash as carbonate, $\mathrm{K}_{2} \mathrm{CO}_{3}$, which is generally known as potashes. Hence potashes occur ready prepared in the ash of plants, and therefore the ash of land plants is emplojed as a source for the extraction of potassium compounds. Potassium carbonate is extracted by lixiviating the ash with water. ${ }^{8}$ Potassium carbonato

pressed thus-two substances, A and B, do not react on each other of their own accord, but the addition of a small quantity of a third particularly active substance, C, produces the reaction of $A$ on $B$, because $A$ combires with $C$, forming $A C$, and $B$ reacts on this new compound, which has a different store of chemical energy, forming-the compound $A B$ or its products, and setting $C$ free again or retaining it.

It may here be remarked that all the mineral substances necessary for plants (those enumerated at the beginning of the note) are the highest saline compounds of their elements, that they enter into the plants as salts, that the lower forms of oxidation of the same elements (for instance, sulphites and phosphites) are harmful to plants (poisonous), and that strong solutions of the salts assimilated by plants (their osmotio pressure being great and contracting the cells, as Do Vries showed, (see Chapter I. Note 19) not only do not enter into the plants but kill them (poison them)

Besides which, it will be understood from the preceding paragraph, that the salts of potassium may become exhausted from the soil by long cultivation, and that there may therefore be cases when the direct fertilisation by salts of potasisium may be profitable. But manure and animal excrements, eshes, and, in general, nearly all refuse which may cerve for fertilising the soil, contain a considerable quantity of potassium salts, and therefore, as regards the natural salts of potassium (Stassfurt), and especially potassium sulphate, if they often improve the crops, it is in all probability due to their action on the properties of the soil. The agriculturist cannot therefore be advised to add potassium salts, without making special experiments showing the advantage of such o fertiliser on a given kind of soil and plant.

The animal body also contains potassium compounds, wilich is natural, since animals consume plants. For example, milk, and especially human milk, contains a somewhat con. siderable quantity of potasaium compounds. Cow's milk, however, does not contain much potassium salt. Sodium compounds generally predominate in the bodies of animals. The excrement of animals, and especially of herbivorous animals, on the contrary, often con. tains a large proportion of potassium salts. Thus sheep's dung is rich in them, and in washing sheep's wool salts of potassium pass into the water.

The ash of tree stems, as the already dormant portion of the plant (Chapter VIII., Note 1), contains little potash. For the extraction of potash, which was formerly carried on extensively in the east of Russis (before the discovery of the Stassfurt salt), the ash of grasses, and the green portions of potatoes, buckwheat, \&o., are taken and treated with water (lixiviated), the solution is evaporated, and the residue ignited in order to destroy the organic matter present in the extract. The residue thus obtained is com. posed of raw potash. It is refined by a second dissolution in a small quantity of water, for the potash itself is very soluble in water, whilst the impurities are sparingly soluble. The solution thus obtained is again evaporated, and the residue ignited, and this potash is then called refined potash, or pearlash. This method of treatment cannot give chemically pure potassium carbonate. A certain amount of impurities remain. To obtain chemically pure potassium carbonate, some other salt of potassium is gene. rally taken and purified by crystallisation. Potassium carbonate crystallises with difficulty, and it cannot therefore be purified by this means, whilst other salts, such as the tartrate, acid carbonate, sulphate, or nitrate, \&c., crystallise easily and may thus be directly purified. The tartrate is most freguently emplayed, since it is prepared in large 
may also be obtained from the chloride by a method similar to that by which sodium carbonate is prepared from sodium chloride. ${ }^{8}$ bis There is no difficulty in obtaining any salt of potassium - for example, the sulphate, ${ }^{9}$

quantities (as a sediment from wine) for medicinal use under the name of crearn of tartar. When ignited without access of air, it leaves a mixture of charcosl and potassium carbonate. The charcoal so obtained being in a finely-divided condition, the mixture (called 'black flux'), is sometimes used for reducing metals from their oxides with the aid of heat. A certain quantity of nitre is added to burn the charcoal formed by heating the cream of tartar. Potassium carbonate thus prepared is further purified by converting it into the acid salt, by passing a current of carbonio anhydride through a strong solution. $\mathrm{KHCO}_{3}$ is then formed, which is less soluble than the normal salt (as is aiso the case with the corresponding sodium salts), and therefore crystals of the acid salt separate from the solution on cooling. When ignited, they part with their water and carbonio enhydride, and pure potassium carbonate remains behind. The physical properties of potassium carbonate distinguish it sufficiently from sodium carbonate; it is obtained from solutions as a powdery white mass, having an alkaline taste and reaction, and, as a rule, shows only traces of crystallisation. It also attracto the moisture of the air with great energy. The crystals do not contain water, but absorb it from the air, deliquescing into i saturated solution. It melts at a red heat $\left(1045^{\circ}\right)$, and at a still higher temperature is even converted into vapour, as has been observed at glass works where it is employed. It is very soluble. At the ordinary temperature, water dissolves an equal weight of the salt. Crystals containing two equivalents of water separate from such a saturated solntion when strongly cooled (Morel obtained $\mathrm{K}_{2} \mathrm{CO}_{3} 3 \mathrm{H}_{2} \mathrm{O}$ in well-formed crystals at $+10^{\circ}$ ). There is no necessity to describe its reactions, because they are all analogous to those of sodium carbonate. When manufactured sodium carbonate was but little known, the consumption of potassium carbonate was very considerable, and even now washing 'soda is frequently replaced for household purposes by 'ley'-i.e. an aqueous solution obtained from ashes. It contain potassium carbonate, which acts like the sodium salt in washing tissues, linen, \&c.

A mixture of potassium and sodinm carbonates fuses with much greater ease than the separate salts, and a mixture of their solutions gives well-crystallised saltg-for instance (Marguerite's salt), $\mathrm{K}_{2} \mathrm{CO}_{3}, 6 \mathrm{H}_{2} \mathrm{O}, 2 \mathrm{Ne}_{2} \mathrm{CO}_{3}, 6 \mathrm{H}_{2} \mathrm{O}$. 'Crystallisation also occurs in other multiplo proportions of $\mathrm{K}$ and $\mathrm{Na}$ (in the above case 1:2, bat 1:1 and 1:8 are known), and always with $6 \mathrm{~mol} . \mathrm{H}_{2} \mathrm{O}$. This is evidently a combination by similarity, as in alloys, solutions, \&c.

8 bis About 25,000 tons of potash annually are now prepared from $\mathrm{KCl}$ by this method at Stassfurt.

- Potassium sulphate, $\mathrm{K}_{2} \mathrm{SO}_{4}$, crystallises from its solutions in an anhydrous condi. tion, in which respect it differs from the corresponding sodium salt, just as potassium carbonate differs from sodium carbonate. In general, it must be observed that the majority of sodium salts combine mure easily with water of crystallisation than the potassium salts. The solubility of potassium sulphate does not show the same peculiarities as that of sodium sulphate, because it does not combine with water of crystallisation; at the ordinary temperature 100 parts of water dissolve about 10 parts of the salt, at $0^{\circ} 8.3$ parts, and at $100^{\circ}$ about 26 parts. The acid sulphate, $\mathrm{KHSO}_{4}$, obtained easily by heating crystals of the normal salt with sulphuric acia, is frequently employed in chemical practice. On heating the mixture of acid and salt, fumes of sulphuric acid are at first given off; when they cease to be evolved, the acid salt is contained in the residue. At a higher temperature (of above $600^{\circ}$ ) the acid salt parts with all the acid contained in it, the normal salt being re-formed. The definite composition of this acid salt, and the ease with which it decomposes, render it exceedingly valuable for certain chemical trans. formations accomplished by means of sulphuric acid at a high temperature, because it is possible to take, in the form of this salt, a strictly definite quantity of sulphurio acid, 


\section{bromide, and iodide ${ }^{10}$ - by the action of the corresponding acid on KCT and especially on the carboriate, whilst the hydroxide, caustic potash, $\mathrm{KHO}$, which is in many respects analogous to caustic soda, is easily ob-}

and to cause it tô act on a given substance at a high temperature, which it is often necessary to do, more especially in chemical analysis. In this case, the acid salt acts in exactly the same manner as sulphurio acid itself, but the latter is inefficient at temperatures above $400^{\circ}$, because it all evaporates, while at that temperature the acid salt.still remains in a fused state, and acts with the elements of sulphurio acid on the substance taken. Hence by its means the boiling-point of sulphurio acid is raised. Thus the acid potassium sulphate is employed, where for conversion of certain oxides, 6ach as those of iron, dluminium, and chromium, into salts, a high temperatare is wequired.

Weber, by heating potassium sulphate with an excess of sulphurio acid at $100^{\circ}$, observed the formation of a lower stratum, which was found to contain a definite compound containing eight equivalents of $\mathrm{SO}_{3}$ per equivalent of $\mathrm{K}_{2} \mathrm{O}$. The salts of rubidium, crsium, and thallium give a similar result, but those of sodium and lithium do not. (See Notie 1.)

10 The bromide and iodide of potassium are nsed, like the corresponding sodinm compounds, in medicine and photography. Potassiun' iodide is easily obtained in a pure state by saturating a solution of hydriodic acid with caustio potash. In praotioe, how. ever, this method is rarely had recourse to, other more simple processes being employed althongh they do not give so pure a product. They aim at the direct formation of hydriodic acid in the liquid in the presence of potassium hydroxide or carbonate, Thus iodine is thrown into a solution of pure potash, and hydrogen sulphide passod through the mixture, the iodine being thus converted into hydriodio acid. Or a solution. is prepared from phosphorus, iodine, and water, contrining hydriodio and phosphorio acid; lime is then added to this solution, when calcium iodide is obtained in solution, and calcium phosphate as a precipitate. The solution of calcium iodide gives, with potassium carbonate, insoluble calcium carbonate and a solution of potassium iodide. If iodine is added to a slightly-heated solution of caustia potash (free from carbonato-that is, freshly prepared), so long as the solution. is not coloured from the presence of an excess of iodine, there is formed (as in the action of chlorine on a solution of caustio potash) a mixture of potassium iodide.and iodate. On evaporating the solution thus obtained and igniting the residue, the iodate is destroyed and converted into iodide, the oxygen being disengaged, and potassium jodide only is left behind. On dissolving the residue in water and then evaporating, cubical crystals of the anhydrous salt are obtained, which are soluble in water and alcohol, and on fusion give an alkaline reaction, owing to the fact that when ignited a portion of the salt decomposes, forming potassium oxide. The neutral salt may be obtained by adding hydriodio acid to this alkaline salt until it gives an acid reaction. It is best to add some finely-divided charcoal to the mixture of iodate and iodide before igniting it, as this facilitates the evolution of the oxygen from the iodate. The iodate may also be converted into iodide by the action. of certain reducing agents, such as zino amalgam, which when boiled with a solution containing an iodate converts it into iodide. Potassinm iodide may also be prepared by mixing a solution of ferrous iodide (it is best if the solution contain an excess of iodine) and potassium carbonate, in which case ferrous carbonate $\mathrm{FeCO}_{3}$, is precipitated (with an excess of iodine the precipitate is granular, and contains $\cdot a$ compound of the suboxide and oxide of iron), while potassium iodide remains in solution. Ferrous iodide, $\mathrm{FeI}_{2}$, is obtained by the direct action of iodine on iron in water. Potassium iodide considerably lowers the temperature (by $24^{\circ}$ ), when it dissolves in water, 100 parts of the salt dissolve in $73^{\circ} 5$ parts of water at $12.5^{\circ}$, in 70 parts at $18^{\circ}$, whilst the saturated solntion which boils at $120^{\circ}$ contains 100 parts of salt per 45 parts of water. Solutions of potassium iodide dissolve a considerable mount of iodine; strong solutions even dissolving as much or more iodine than ther contain as potassium iodide (see Note 3 bis'and Chapter XI., Note 64). 
tained by means of lime in exactly the same manner in which sodiun hydroxide is prepared from sodium carbonate. ${ }^{1.1}$ Therefore, in order to complete our knowledge of the alkali metals, we will only describe two salts of potassium which are of practical importance, and whose analogues have not been described in the preceding chapter, potassium cyanide and potassium nitrate.

Potassium cyanide; which presents in its chemical relatipns a certain analogy with the halogen salts of potassium, is not only formed according to the equation, $\mathrm{KHO}+\mathrm{HCN}=\mathrm{H}_{2} \mathrm{O}+\mathrm{KCN}$, but also whenever a nitrogenous carbon compound-for instance, animal matter-is heated in the presence of metallic potassium, or of a compound of potassium, and even when a mixture of potash and carbon is heated in a stream of nitrogen. Potassium cyanide is obtained from yellow prussiate, which has been already mentioned in Chapter IX., and whose preparation on a large scale will be described in Chapter XXII. If the jellow prussiate be ground to a powder and dried, so that it loses its water of crystallisation, it then melts at a red heat, and decomposes into carbide of iron, nitrogen, and potassium cyanide, $\mathrm{FeK}_{4} \mathrm{C}_{6} \mathrm{~N}_{6}$ $=4 \mathrm{KCN}+\mathrm{FeC}_{2}+\mathrm{N}_{2}$. After the decomposition it is found that the yellow salt has been converted into a white mass of potassium cyanide. The carbide of iron formed collects at the bottom of the vessel. If the mass thus obtained be treated with water, the potassium cyanide is partially decomposed by the water, but if it be treated with alcohol, then the cyanide is dissolved, and on cooling separates in a crystalline form. ${ }^{12}$ A solution: of potassium cyanide has a powerfully alkaline

11 Carstio potash is not only formed by the action of lime on allute solntions of potassium carbonate (as sodium hydroxide is prepared from sodium carbonate), but by igniting potassium nitrate with finely-divided copper (8ee Note 15), and also by mixing solntions of potassium sulphate (or even of alum, $\mathrm{KAl}_{2} \mathrm{O}_{8}$ ) and barinm hydroxide, $\mathrm{BaH}_{2} \mathrm{O}_{2}$. It is sometimes purified by dissolving it in alcohol (the impurities, for example, patassium sulphate and carbonate, are not dissolved) and then evaporating the alcohol.

The specific gravity of potassium hydroxide is 2.04 , but that of its solutions (see Chapter XII., Note 18) at $15^{\circ} \mathrm{S}=9,992+90^{\circ} 4 p+0.28 p^{y}$ (here $p^{2}$ is + , and for sodium hydroxide it.is -). Strong solutions, when cooled, yield a.crystallo-hydrate, $\mathrm{KHO}, 4 \mathrm{H}_{2} \mathrm{O}_{2}$ which dissolves in water, producing cold (like $2 \mathrm{NaHO}, 7 \mathrm{H}_{2} \mathrm{O}$ ), whilst potassium hydroxide in solution develops a considerable amount of heat.

12 When the yellow prussiate is beated to redness, all the cyanogen which was in combination with the iron is decomposed into vitrogen, which is evolved as gais, and carbon, which combines with the iron. In order to avoid this, potassium carbonate is added to the yellow prussiate, while it is being fused. A mixture of 8 parts of anhydrous yellow prussiate and 3 . parts of pure potassium carbonate is generally taken. Double decomposition then takes place, resulting in the formation of ferrous garbonate and potassium cyanide. But by this method, as by the first, a pure salt is not obtained, because a portion of the potassium cyanide is oxidised at the expense of the iron carbonate and forms potassium cyanate, $\mathrm{FeCO}_{3}+\mathrm{KCN}=\mathrm{CO}_{2}+\mathrm{F} \theta+\mathrm{KCNO}$; and the, potassium cyanide very easily forms oxide, which acts on the sides of the vessel in. which the mixture is heated (to avoid, this iron vessels should be used). By adding, 
reaction, a smell.like that of bitter almonds, peculiar to prussio acid, and acts as a.most powerful poison. Although exceedingly stable in a fused state, pótassium cyanide easily changes when in solution. Prussic acid is so very feebly energetic that even water decomposes potassium cyanide. A solution of the salt, even without access of air, easily turns brown and decompases; and when heated evolvès ammonia and forms potassium formate; this is easily comprehensible from the representation of the cyanogen compounds which was developed in Chapter IX., $\mathrm{KCN}+2 \mathrm{H}_{2} \mathrm{O}=\mathrm{CHKO}_{2}+\mathrm{NH}_{3}$. Furthermore, as carbonic anhydride acts on potassium cyanide with evolution of prussic acid, and as potassium cyanate, which is also unstable, is formed by the action of air, it will be easily seen that solutions of potassium cyanide are very unstable. Potassium cyanide, containing as it does carbon and potassium, is a substance which can act in a very vigorously reducing manner, especially when fused; it is therefore used as a powerful reducing agent at a red heat. ${ }^{13}$ The property of potassium cyanide of giving double salts with other cyanides is very clearly shown by the fact that many metals dissolve in a solution of potassium cyanide, with the evolution of hydrogen. For example, iron, copper, and zino act in this manner. Thus-

$$
4 \mathrm{KCN}+2 \mathrm{H}_{2} \mathrm{O}+\mathrm{Zn}=\mathrm{K}_{2} \mathrm{ZnC}_{4} \mathrm{~N}_{4}+2 \mathrm{KHO}+\mathrm{H}_{2} \text {. }
$$

one part of charcoil powder to the mixture of 8 parts of anhydrous yellow prussiate and 8 parts of potassium carbonate a mass is obtained which is free from cyanate, because the carbon absorbs the oxygen, but in that case it is impossible to obtain a colourless potassium cyanide by simple fusion, although this may be easily done by dissolving it in alcohol. Cyanide of potassium may also be obtained from potassium thiocyanate, which is formed from ammonium thiocyanate obtained by the action of ammonia upon bisalphide of carbon (see works upon Organic Chemistry). Potassium cyanide is now prepared in large quantities from yellow prussiate for gilding and silvering. When fused in large quantities the action of the oxygen of the air is limited, and with great care the operation may be successfully conducted, and therefore, on a large scale, very pure salt is some. times obtained. When slowly cooled, the fused salt separates in cubical crystals like potassium chloride.

Pure $\mathrm{KCN}$ is obtained by passing CNH gas into an alcoholio solution of $\mathrm{KHO}$. The large amount of potassium cyanide which is now required for the extraction of gold from its ores, 18 being replaced by a mixture (Rossler and Gasslaker, 1892) of KCN and $\mathrm{NaCN}$, prepared by heating powdered and dried yellow prussiate with metallio sodium: $\mathrm{K}_{4} \mathrm{Fe}(\mathrm{CN})_{6}+2 \mathrm{Na}=4 \mathrm{KCN}+2 \mathrm{NaCN}+\mathrm{Fe}$. This method offers two advantages over the above methods: (1) the whole of the cyanide is obtained, and does not decompose with the formation of $\mathrm{N}_{2}$; and (2) no cyanates are formed, as is the case. when carbonate of potash is heated with the prussiate.

13 A considerable quantity of potassium cyanide is used in the arts, more particularly for the preparation of metallic solutions which are decomposed by the action of a galvanio current; thus it is very frequently employed in electro-silvering and gilding. An alkaline solution is prepared, which is moderately stable owing to the fact that potassium cyanide in the form of certain double salts - that is, combined with other cyanides-is far more stable than when alone (yellow prussiate, which contains potassium cyanide in combination with ferrous cyanide, is an example of this): 
Gold and silver are soluble in potassium cyanide in the presence of air, in which casc the hydrogen, which would otherwise be evolved in the reaction, combines with the oxygen of the air, forming water (Eissler, MacLaurin, 1893), for example, $4 \mathrm{Au}+4 \mathrm{KCN}+\mathrm{O}+\mathrm{H}_{2} \mathrm{O}=2 \mathrm{AuKC}_{2} \mathrm{~N}_{2}$ $+2 \mathrm{KHO}$, which is taken advantage of for extracting gold from its ores (Chapter XXIV.). ${ }^{13}$ bls Platinum, mercury, and tin are not dis. solved in a solution of potassium cyanide, even with access of air.

Potassium nitrate, or common nitre or saltpetre, $\mathrm{KNO}_{2}$, is chiefly used as a comporient part of gunpowder, in which it cannot be replaced by the sodium salt, because the latter is deliquescent. It is necessary that the nitre in gunpowder should be perfectly pure, as even small traces of sodium, magnesium, and calcium salts, especially chlorides, render the nitre and the gunpowder capable of attracting moisture Nitre may easily be obtained pure, owing to its great disposition to form crystals both large and small, which aids its separation from other salts. The considerable differences between the solubility of nitre at different temperatures aids this crystallisation. A solution of nitre saturated at its boiling point $\left(116^{\circ}\right)$ contains 335 parts of nitre to 100 parts of water, whilst at the ordinary temperature - for instance, $20^{\circ}$ the solution is only able to retain 32 parts of the salt. Therefore, in the preparation and refining of nitre, its solution, saturated at the boiling point, is cooled, and nearly all the nitre is obtained in the form of crystals. If the solution be quietly and slowly cooled in large quantities then large crystals are formed, but if it be rapidly cooled and agitated then small crystals are obtained. In this manner, if not all, at all events the majority, of the impurities present in small quantities remain in the-mother liquor. If an unsaturated solution of nitre be rapidly cooled, so as to prevent the formation of large crystals (in whose crevices the mother liquor, together with the impurities, would remain), the very minute crystals of nitre known as saltpetre flour are obtained.

Common nitre occurs in nature, but only in small quantities in admixture with other nitrates, and especially with sodium, magnesium, and calcium nitrates. Such a mixture of salts of nitric acid is formed in nature in fertile earth, and in those localities where, as in the soil, nitrogenous organic remains are decomposed in the presence of alkalis or alkaline bases with free access of air. This method of the formation of nitrates requires moisture, besides the free access of air, and takes place principally during warm weather. ${ }^{14}$ In warm countries, and in

13 blo A dilute solution of $\mathrm{KCN}$ is taken, not containing more than 1 per cent. $\mathrm{KCN}$. MacLaurin explains this by the fact that strong solutions dissolve gold less rapidly, owing to their dissolving less air, whose oxygen is necessary for the reaction.

14 Besides which Schloessing and Mutintz, by employing rimilar methods to Pasteux, thowed that the formation of nitre in the decomposition of nitrogenous substancos is 
temperate climates during the summer mọths, fertile soils produice a small quantity of nitre. In this respect India is especially known as affording a considerable supply of nitre extracted from the soil. The nitre-bearing soil after the rainy season sometimes becomes covered during the summer with crystals of nitre, formed by the evaporation of the water in which it was previously dissolved. This soil is collected, subjected to repeated lixiviations, and treated for nitre as will be presently described In temperate climates nitrates are obtained from the lime rubbish of demolished buildings which have stood for many years, and especially from those portions which have been in contact with the ground. The conditions there are very favourable for the formation of nitre, because the lime used as a cement in buildings contains the base necessary for the formation of nitrates, while the excrement, urine, and animal refuse are sources of nitrogen. By the methodical lixiviation of this kind of rubbish a solution of nitrogenous salts is formed similar to that obtained by the lixiviation of fertile soil. A similar solution is also obtained by the lixiviation of the so called nitre plantations. They are composed of manure interlaid with brush-wood, and strewn over with ashes, lime, and other alkaline rubbish. These nitre plantations are set up in those localities where the manure is not required for the fertilisation of the soil, as, for example, in the south-eastern 'black earth" Governments of Russia. The same process of oxidation of nitrogenous matter freely exposed to air and moisture during the warm season in the presence of alkalis takes place in nitre plantations as in fertile soil and in the walls of buildings. From all these sources there is obtained a solution containing various salts of nitric acid mixed with soluhle organic matter. The simplest method of treating this impure solution of nitre is to add a solution of potassium carbonate, or to simply treat it with ashes containing this substance. The potassium carbonate enters into double decomposition with the calcium and magnesium salts, forming insoluble carbonates of these bases and leaving the nitre in solution. Thus, for instance, $\mathrm{K}_{2} \mathrm{CO}_{3}+\mathrm{Ca}\left(\mathrm{NO}_{3}\right)_{2}=2 \mathrm{KNO}_{3}+\mathrm{CaCO}_{3}$. Both calcium and magnesium carbonates are insoluble, and therefore after treatment with potassium carbonate the solution no longer contains salts of these motals but only the salts of sodium and potassium together with organic matter. The latter partially separates on heating in an insoluble form, and is entirely destroyed by heating the nitre to a low red beat, The nitre thus obtained is easily purified by

accomplished by the aid of peculiar micro-organisms (ferments), without which the simultaneous action of the other necessary conditions (alkalis, moisture, a temperature of $87^{\circ}$, air ${ }_{3}$ and nitrogenous substances) cannot givive nitre. 
repeated crystallisation. The greater part of the nitre used for making gunpowder is now obtained from the sodium salt Chili saltpetre or cubic nitre, which occurs in nature, as already mentioned. The conversion of this salt into common nitre is also carried on by means of a double decomposition. This is done either by adding potassium carbonate (when, on mixing the strong and hot solutions, sodium carbonate is directly obtained as a precipitate), or, as is now most frequent, potassium chloride. When a mixture of strong solutions of potassium chloride and sodium nitrate is evaporated, sodium chloride first separates, because this salt, which is formed by the double decomposition $\mathrm{KCl}+\mathrm{NaNO}_{3}=\mathrm{KNO}_{3}+\mathrm{NaCl}$, is almost equally soluble in hot and cold water; on cooling, therefore, a large amount of potassium nitrate separates from the saturated solution, while the sodium chloride remains dissolved. The nitre is ultinately purified by recrystallisation and by washing with a saturated solution of nitre, which cannot dissolve a further quantity of nitre but only the impurities.

Nitre is a colourless salt having a peculiar cool taste. It crystal. lises easily in long striated six-sided rhombic prisms terminating in rhombic pyranids. Its crystals (sp. gr. 1.93) do not contain water, but their cavities generally contain a certain quantity of the solution from which they have crystallised. For this reason in refining nitre, the production of large crystals is preverited, saltpetre flour being prepared. At a low red heat $\left(339^{\circ}\right)$ nitre melts to a colourless liquid. ${ }^{14}$ bis Potassium nitrate at the ordinary temperature and in a solid form is inactive and stable, but at a high temperature it acts as a powerful oxidising agent, giving up a considerable amount of oxygen to substances

14 ble Before fusing, the crystals of potassium nitrate change their form, and take the same form as sodium nitrate-that is, they change into rhombohedra. Nitre crýstallises from hot solutions, and in general under the influence of a rise of temperature, in a different form from that given at the ordinary or lower temperatures. Fnsed nitre solidifies to a radiated crystalline mass; but it does not exhibit this structure if metallio chlorides be present, so that this method may, be taken advantage of to determine the degree of purity of nitre.

Carnelley and Thomson (1888) determined the fusing point of mixtures of potassium and sodium nitrates. The first salt fuses at $339^{\circ}$ and the second at $316^{\circ}$, and if $p$ be the percentage amount of potassium nitrate, then the results obtained were-

$\begin{array}{ccccccccc}p=10 & 20 & 30 & 40 & 50 & 60 & 70 & 80 & 90 \\ 298^{\circ} & 283^{\circ} & 268^{\circ} & 242^{\circ} & 231^{\circ} & 231^{\circ} & 242^{\circ} & 284^{\circ} & 806^{\circ}\end{array}$

which confirms Shaffgotsch's observation (1857) that the lowest fusing point (about 281') is given by mixing molecular quantities $\left(p=54^{\circ} 8\right)$ of the salts-that is, in the formation of the alloy, $\mathrm{KNO}_{3}, \mathrm{NaNO}_{3}$.

A somewhat similar result was discovered by the same observers for the solubility of mixtures of these salts at $20^{\circ}$ in 100 parts of water. Thus, if $p$ be the weight of potassium nitrate mixed with $100-p$ parts by weight of sodium nitrate taken for solution, 
nixed with it. ${ }^{15}$ When thrown on to incandescent charcoal it brings about its rapid combustion, and a mechanical mixture of powdered charcoal and nitre ignites when brought into contact with. a red-hot substance, and continues to burn by itself. In this action, ritrogen is evolved, and the oxygen oxidises the charcoal, in consequence of which potassium carbonate and carbonic anhydride are formed: $4 \mathrm{KNO}_{3}+5 \mathrm{C}=2 \mathrm{~K}_{2} \mathrm{CO}_{3}+3 \mathrm{CO}_{2}+2 \mathrm{~N}_{2}$. This phenomenon depends on the fact that oxygen in combining with carbon evolves more heat than it does in combining with nitrogen. Hence, when once the combustion has been started at the expense of the nitre, it is able to go on without requiring the aid of external heat. A similar oxidation or combustion at the expense of the contained oxygen takes place when nitre is heated with-different combustible substances If a mixture of sulphur and nitre be thrown upon a red-hot surface, the sulphur burns, forming potassium sulphate and sulphurous anhydride. In this case, also, the nitrogen of the nitre is evolved as gas $\cdot 2 \mathrm{KNO}_{3}+2 \mathrm{~S}=\mathrm{K}_{2} \mathrm{SO}_{4}+\mathrm{N}_{2}$ $+\mathrm{SO}_{2}$. A similar phenomenon occurs when nitre is heated with many metals. The oxidation of those metals which are able to form acid oxides with an excess of oxygen is especially remarkable. In this case they remain in combination with potassium oxide as potassium salts. Mánganese, antimony, arsenic, iron, chromium, \&c. are instances of this.kind. These elements, like carbon and sulphur, displace free nitrogen. The lower oxides of these metals when fused with nitre pass into the higher oxides. Organic substances are also oxidised when heated with nitre-that is, they burn at the expense of the nitre. It will be readily understood from this that nitre is frequently used in practical chemistry and the arts as an oxidising agent at high temper-

and $c$ be the quantity of the mixed salts which dissolves in 100, the solubility of sodium nitrate being 85 , and of potassium nitrate 84 , parts in 100 parts of water, then-

$\begin{array}{rrrrrrrrr}p=10 & 20 & 80 & 40 & 50 & 60 & 70 & 80 & 90 \\ c=110 & 186 & 186 & 188 & 106 & 81 & 78 & 54 & 41\end{array}$

The maximum solubility proved not to correspond with the most fusible mtrture, but : to one much richer in sodium nitrate.

Both these phenomena show that in homogeneous liquid mixtures the chemical forces that act between substances are the same as those that determine the molecular weights of substances, even when the mixtare consists of snch analogous substances as potas. sium and sodium nitrates, between which there is no direct chemical interchange. It is instructive to note also that the maximum solubility does not correspond with the minimum fusing point, which naturally depends on the fact that in solution a third substance, namely. water, plays a part, although an attraction between the salts, like that which exists between sodium and potassium carbonates (Note 8), also partially acts.

15 Fused nitre, with a further rise of temperature, disengages oxygen and then nitro gen. The nitrite $\mathrm{KNO}_{2}$ is first formed and then potassium oxide. The admixture of certain metals-for example, of finely-divided copper - aids the last decomposition: The exygen in this case naturally passes over to the metal. 
atures. Its application in gunpowder is based on this property; gun. powder consists of a mechanical mixture of finely-ground sulphur, nitre, and charcoal. The relative proportion of these substances varies according to the destination of the powder and to the kind of charcoal employed (a friable, incompletely-burnt charcoal, containing therefore hydrogen and oxygen, is employed). Gases are formed in its combustion, chiefly nitrogen and carbonic anhydride, which create a considerable pressure if their escape be in any way impeded. This action of gunpowder may be expressed by the equation : $2 \mathrm{KNO}_{3}+3 \mathrm{C}+\mathrm{S}=\mathrm{K}_{2} \mathrm{~S}+3 \mathrm{CO}_{2}+\mathrm{N}_{2}$.

It is found by this equation that gunpowder should contain thirty. six parts of charcoal (13.3 p.c.), and thirty-two parts (11.9 p.c.) of sulphur, to 202 parts $(74.8$ p.c.) of nitre, which is very near to its actual composition. ${ }^{16}$

16 In Chine, where the manufacture of gunpowder has long been carried on, $75 \cdot 7$ parts of nitre, 14.4 of charcoal, and $\mathbf{9 . 9}$ of sulphur are used. Ordinary powder for sporting purposes oontains 80 parts of nitre, 12 of charcoal, and 8 of sulphur, whilst the gunpowder used in heavy ordnance contains 75 of nitre, 15 of charcoal, and 10 of sulphur Gunpowder explodes when heated to $300^{\circ}$, when struck, or by contact with a spark. A compact or finely-divided mass of gunpowder burns slowly and has but little disruptive action, because it burns gradually. To act properly the gunpowder must have a definite rate of combustion, so that the pressure should increase during the passage of the projectile along the barrel of the fire-arm. This is done by making the powder in-large granules or in the shape of six-sided prisms with holes through them (prismatio powder).

The prodncts of combustion are of two kinds - (1) gases which produce the pressure and are the cause of the dynamical action of gunpowder, and (2) a solid residue, usually of a black colour owing to its containing unburnt particles of charcoal. Besides charcoal, the residue generally contains potassium sulphide, $\mathrm{K}_{2} \mathrm{~S}$, and a whole series of other salts-for instance, carbonate and sulphate. It is apparent from this that the combustion of gunpowder is not so simple as it appears to be from the above.formula, and hence the weight of the residue is also greater than indicated by that formula. According to the formula, 270 parts of gunpowder give 110 parts of residno-that is, 100 parts of powder give 87.4 parts of residue, $\mathrm{K}_{2} \mathrm{~S}$, whilst in reality the weight of the residue varies from 40 p.o. to 70 p.c. (generally 52 p.o.). This difference depends on the fact that 80 much oxygen (of the nitre) remains in the residue, and it is evident that if the residue varies the composition of the gases evolved by the powder will vary also, and therefore the entire process will be different in different cases. The difference in the composition of the gases and residue depends, as the researches of Gay-Lussac, Shishkoff and Bun. een, Nobel and Abel, Federoff, Debus, \&o., show, on the conditions under which the combustion of the powder proceeds. When gunpowder burns in an open space, the gaseous products which are formed do not remain in contact with the residue, and then a considerable portion of the charcoal entering into the composition of the powder remains unburnt, because the charcoal burns after the sulphur at the expense of the oxygen of the nitre. In this extreme case the commencement of the combustion of the gunpowder may be expressed by the equation, $2 \mathrm{KNO}_{3}+3 \mathrm{C}+\mathrm{S}=2 \mathrm{C}+\mathrm{K}_{2} \mathrm{SO}_{4}+\mathrm{CO}_{2}+\mathrm{N}_{2}$. The residue in a blank cartridge often consists of a mixture of $\mathrm{C}, \mathrm{K}_{2} \mathrm{SO}_{4}, \mathrm{~K}_{2} \mathrm{CO}_{3}$, and $\mathrm{K}_{2} \mathrm{~S}_{2} \mathrm{O}_{3}$. If the combustion of the gunpowder be impeded-if it take place in \& cartridge in the barrel of a gun - the quantity of potassium sulphate will first be diminished, then the amount of sulphite, whilst the amount of carbonic anhydride in the gases and the amount of potassium sulphide in the residue will increase. The quantity of charcoal entering into the action will then be also increased, and bence the amount in the residue 
Metallic potassium was obtained like sodium; first by the action of a galvanic current, then by reduction of the hydroxide by means of metallic iron, and lastly, hy the action of charcoal on the carbonate at a high temperature. The behaviour of metallic potassium differs, how. ever; from that of sodium, because it easily combines with carbonio oxide, forming an explosive and inflammable mass. ${ }^{17}$

Potassium is quite as volatile as sodium, if not more so. At the ordinary temperature potassium is even softer than sodium ; its freshlycut surfaces present a whiter colour than sodium, but, like the latter, and with even greater ease, it oxidises in moist air. It is brittle at low temperatures, but is quite soft at $25^{\circ}$, and melts at $58^{\circ}$ At a low red heat $\left(667^{\circ}\right.$, Perkin) it distils without change, forming a green vapour, whose density, ${ }^{18}$ according to $\mathrm{A}$. Scott (1887), is equal to 19 (if that of

will decrease. Under these circumstances the weight of the residue will be less-for example, $4 \mathrm{~K}_{8} \mathrm{CO}_{8}+4 \mathrm{~S}=\mathrm{K}_{2} \mathrm{SO}_{4}+8 \mathrm{~K}_{2} \mathrm{~S}+4 \mathrm{CO}_{2}$. Besides which, carbonic oxide has been found in the gases, and potassium bisulphide, $\mathrm{K}_{2} \mathrm{~S}_{2}$, in the residue of gunpowder. The amount of potassium sulphide, $\mathrm{K}_{2} \mathrm{~S}$, increases with the completeness of the combustion, and is formed in the residue at the expense of the potassium sulphite. In recent times the knowledge of the action of gunpowder and other explosives has made much progress, and has developed into a vast province of artillery science, which, guided by the discoveries of chemistry, has worked out a 'smokeless powder which burns without leaving a residue, and does not therefore give any 'powder smoke' (to hinder the rapidity of firing and aiming), and at the same time disengages a greater volume of gas and conequently gives (under proper conditions of combustion) the possibility of communicating to the charge a greater initial velocity, and therefore greater distance, force, and accursoy of aim. Such 'smolseless powdex' is prepared either from the varieties of nitro-oellulose (Chapter VI, Note 87) or from a mixture of them with nitro-glycerine (ibid). In burning they give, besides steam and nitrogen, generally a large amount of oxide of carbon (this is a very serions drawback in all the present forms of smokeless powder, because carbonic oxide is poisonous), and also $\mathrm{CO}_{2}, \mathrm{H}_{2}$, \&a.

17 The substances obtained in this case are mentioned in Chapter IX., Note 81.

16 A. Scott (1887) determined the vapour densities of many of the alkali elements and their compounds in a platinum vessel heated in a furnace and previously filled with nitrogen. But these, the first data concerning a subject of great importance, have not jet boen suffioiently fully described, nor have they received as much attention as could be desired. Taking the density of hydrogen as unity, Scott found the vapour densities of the following substances to be-

\begin{tabular}{|c|c|c|c|c|}
\hline $\mathrm{Na}$ & 12. & $(11 \cdot 5)$ & $\mathbf{K I}$ & $92(84)$. \\
\hline $\mathbf{K}$ & 19 & $(19 \cdot 5)$. & $\mathbf{R b C l}$ & $70(60)$. \\
\hline $\mathrm{CsCl}$ & $89^{\circ}$ & $(84 \cdot 8)$. & $\mathrm{CaI}$ & 188 (180). \\
\hline $\mathrm{FeCl}_{5}$ & 68 & & $\mathrm{AgCl}$ & $80(71 \cdot 7)$ \\
\hline
\end{tabular}

In brackets are given the densities corresponding with the formulw, acoonding to Avogadro-Gerhardt's law. This figure is not given for $\mathrm{FeCl}_{3}$, because in all probabllity under these conditions (the temperature st.which it was determined) a portion of the $\mathrm{WeCl}_{3}$ was decomposed. If it was not decomposed, then a density 81 would correapond with the formula $\mathrm{FeCl}_{3}$, and if the decomposition were $\mathrm{Fe}_{2} \mathrm{Cl}_{6}=2 \mathrm{FeCl}_{2}+\mathrm{Cl}_{2}$, then the density should be 54. With regard to the silver chloride, there is reason to think that the platinum decomposed this salt. The majority of Scott's results so closely correspond with the formulio that a better concord cannot be expected in sucb determinations. V. Moyer (1887) gives 98 as the density of $\mathrm{KI}$. 
hydrogen $=1$ ). This shows that the molecule of potassium (like that of sodium, mercury, and zinc) contains but one atom. This is also the case with many other metals, judging by recent researches. ${ }^{19}$ The specific gravity of potassium at $15^{\circ}$ is 0.87 , and is therefore less than that of sodium, as is also the case with all its compounds. ${ }^{20}$ Potassium decomposes water with great ease at the ordinary temperature, evolving 45,000 heat units per atomic weight in grams. The heat evolved is sufficient to inflame the hydrogen, the flame being coloured violet from the presence of particles of potassium. ${ }^{21}$

With regard to the relation of potassium to hydrogen and oxygen, it is closely analogous to sodium in this respect. Thus, with hydrogen it forms potassium hydride, $\mathrm{K}_{2} \mathrm{H}$ (between $200^{\circ}$ and $411^{\circ}$ ), and with oxygen it gives a suboxide $\mathrm{K}_{4} \mathrm{O}$, oxide $\mathrm{K}_{2} \mathrm{O}$, and peroxide, only more oxygen enters into the composition of the latter than in sodium peroxide; potassium peroxide contains $\mathrm{KO}_{2}$, but it is probable that in the combustion of potassium an oxide $\mathrm{KO}$ is also formed. Potassium, like sodium, is soluble in mercury. ${ }^{22}$ In a word, the relation between sodium and potassium is as close as that between chlorine and bromine; or, better still, between fluorine and chlorine, as the atomic weight of

29. The molecules of non-metals are more complex-for instance, $\mathrm{H}_{2}, \mathrm{O}_{3}, \mathrm{Cl}_{2}$, \& . But arsenic, whose superficial appearance recalls that of metals, but whose chemical properties approach more nearly to the non-metals, has a complex molecule containing $\mathrm{As}_{4}$.

${ }^{20} \mathrm{As}$ the atomic weight of potassium is greater than that of sodium, the volumes of the molecules, or the quotients of the molecular weight by the specific gravity; for potassium compounds are greater than those of sodium compounds, because both the denominator and numerator of the fraction increase. We cite for comparison the volumes of the corresponding compounds-

\begin{tabular}{|c|c|c|}
\hline $\mathrm{NQ}$ & NaHO 18 & $\mathrm{NaCl} 28$ \\
\hline K 45 & KHO 27 & $\mathbf{K C l} \quad 39$ \\
\hline
\end{tabular}

21 The same precautions must be taken in decomposing wster by potassium as have to be observed with sodium (Chapter II., Note 8).

It must be observed that potassium decomposes carbonic anhydride and carbonio. oxide when heated, the carbon being liberated and the oxygen taken up by the metal, whilst on the other hand charcoal takes up oxygen from potassium, as is seen from the preparation of potassium by heating potash with charcoal, hence the reaction $\mathrm{K}_{2} \mathrm{O}+\mathrm{O}$ $=\mathrm{K}_{2}+\mathrm{CO}$ is reversible and the relation is the same in this case as between hydrogen and zino.

29 Potassium forms alloys with sodium in all proportions. The alloys containing 1 and 8 equivalents of potassium to one equivalent of sodium are liquids, like mercury at the ordinary temperature. Joannis, by determining the amount of heat developed by these alloys in decomposing water, found the evolution for $\mathrm{Na}_{2} \mathrm{~K}, \mathrm{NaK}, \mathrm{NaK}_{2}$, and $\mathrm{NaK}_{5}$ to be $44^{\circ}, 44^{\circ} 1,43.8$ and $44^{\circ} 4$ thousand heat units respectively (for $\mathrm{Na} 42 \cdot 6$ and for $\mathrm{K} 45^{\circ} 4$ ). The formstion of the alloy $\mathrm{NaK}_{2}$ is therefore accompanied by the development of heat, whilst the other alloys may be regarded as solutions of potassium or sodium in this alloy. In any case a fall of the temperature of fusion is evident in this instance as in the alloys of nitre (Note 14). The liquid alloy $\mathrm{NaK}_{2}$ is now used for filling thermometers employed for temperatures above $860^{\circ}$, when mercury boils. 
sodium, 23, is as much greater than that of fluorine, 19, as that of potassium, 39 , is greater than that of chlorine, 35.5 .

The resemblance between potassium and sodium is so great that their compounds can only be easily distinguished in the form of certain of their salts. For instance, the acid potassium tartrate, $\mathrm{C}_{4} \mathrm{H}_{5} \mathrm{KO}_{6}$ (cream of tartar), is distinguished by its sparing solubility in water and in alcohol, and in a solution of tartaric acid, whilst the corresponding sodium salt is easily soluble. Therefore, if a solution of tartaric acid be added in considerable excess to the solutions of the majority of potassium salts, a precipitate of the sparingly-soluble acid salt is formed, which does not occur with salts of sodium. The chlorides $\mathrm{KCl}$ and $\mathrm{NaCl}$ in solutions easily give double salts $\mathrm{K}_{2} \mathrm{PtCl}_{6}$ and $\mathrm{Na}_{2} \mathrm{PtCl}_{6}$, with platinic chloride, $\mathrm{PtCl}_{4}$, and the solubility of these salts is very different, especially in a mixture of alcohol and ether. The sodium salt is easily soluble, whilst the potassium salt is insoluble or almost so, and therefore the reaction with platinic chloride is that most often used for the separation of potassium from sodium, as is more fully described in works on analytical chemistry.

It is possible to discover the least traces of these metals in admixture together, by means of their properrty of imparting different colours to a flame. The presence of a salt of sodium. in a flame is recognised by a brilliant yellow coloration, and a pure potassium salt colours a colourless flame violet. However, in the presence of a sodium salt the pale violet coloration given by a potassium salt is quite undistinguishable, and it is at tirst sight impossible in this case to discover the patassium salt in the presence of that of sodium. But by decomposing the light given by a flame coloured by these metals or a mixture of them, by means of a prism, they are both easily distinguishable, because the yellow light emitted by the sodium salt depends on a group of light rays having a definite index of refraction which corresponds with the jellow portion of the solar spectrum, having the index of refraction of the Fraunhofer line (strictly speaking, group of lines) D, whilst the salts of potassium give a light from which these rays are entirely absent, but which contain rays of a red and violet colour. Therefore, if a potassium salt occur in a Hlame, on decomposing the light (after passing it through a narrow slit) by means of a prism, there will be seen red and violet bands of light situated at a considerable distance from each other; whilst if a sodium salt be present a yellow line will also appear. If both metals simultaneously occur in a flame and emit light, the spectrum lines corresponding to the potassium and the sodium will appear simultaneously. 
For convenience in carrying on this kind of testing, spectroscopea (fig. 72) are constructed, ${ }^{23}$ consisting of a refracting prism and three tubes placed in the plane of the refracting angle of the prism. One of the tubes, $\mathrm{C}$, has a vertical slit at the end, giving access to the light to be tested, which then passes into the tube (collimator), containing a lens which gives the rays a parallel direction. The rays of light having passed through the slit, and having become parallel, are refracted and dispersed in the prism, and the spectrum formed is observed through the eye-piece of the other telescope B. The third tube D contains a horizontal transparent scale (at the outer end) which is divided into equal divisions: The light from a source such as a gas burner or candle placed before this tube, passes through the scale, and is reflected on that face of the prism which stands before the telescope B, so that the image of the scale is seen through this telescope simultaneously with the spectrum given by the rays passing through the slit of the tube C. In

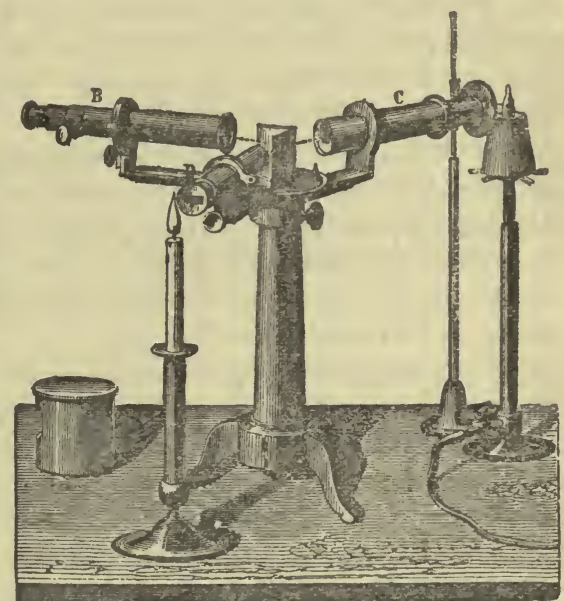

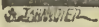

FIG. 72.-Spectroscope. The prism and table are covered with an opaque cover. The spectrum obtained from the flame coloured by a substance introduced on the wire is vieved through B. A light is placed before the scale D in order to illuminate the image of the scale reflected through $B$ by the side of the prism.

this manner the image of the scale and the spectrum given by the source of light under investigation are seen simultaneously. If the

23 For accurate measurements and comparative researches more complicated spectroscopes are required which give a greater dispersion, and are furnished for this purpose with several prisms - for example, in Browning's spectroscopo the light passes throngh six prisms, and then, having undergone an internal total reflection, passes through the npper portion of the same six prisms, and again by an internal total reflection passes into the ocular tube. With such a powerful dispersion the relative position of the spectral lines may be determined with accuracy. For the absolute and exact determination of the wave lengths it is particularly important that the spectroscope should be furnished with diffraction gratings. The construction of spectroscopes destined for special purposes (for example, for investigating the light of stars, or for determining the absorption spectra in microscopio preparations, \&c.) is exceedingly varied. Details of the subject must be looked for in works on physics and on spectrum analysis. Among the latter the best known for their completeness and merit are those of Roscoe, Kayser, Vogel, and Lecog de Boisbaudran. 
sun's rays be directed through the slit of the tube C, then the ob. server looking through the eye-piece of B will see the solar spectrum, and (if the aperture of the slit be narrow and the apparatus correctly adjusted) the dark Fraunhofer lines in it. ${ }^{24}$ Small-sized spectroscopes are usually so adjusted that (looking through B) the violet portion of the spectrum is seen to the right and the red portion to the left, and the Fraunhofer line $\mathrm{D}$ (in the bright yellow portion of the spectrum) is situated on the 50th division of the scale.25 If the light emitted by an incandescent solid-for example, the Drummond light-be passed through the spectroscope, then all the colours of the solar spectrum are seen, but not the Fraunhofer lines. To observe the result given by a flame coloured by various salts a Bunsen gas burner (or the pale flame of hydrogen gas issuing from a platinum orifice) giving so pale a flame that its spectrum will be practically invisible is placed before the slit. If any compound of sodium be placed in the flame of the gas burner (for which purpose a platinum wire on whose end sndium chloride is fused is fixed to the stand), then the flame is coloured yellow, and on looking through the spectroscope the observer will see a bright yellow line falling upon the 50th division of the scale, which is seen together with the spectrum in the telescope. No yellow lines of other refractive index, nor any rays of any other colour, will be seen, and, therefore, the spectrum corresponding with sodium compounds consists of yellow rays of that index of refraction which belong to the Fraunhofer (black) line D of the solar spectrum. If a potassium salt be introduced into the flame instead of a sodium salt, then two bands will be seen which are much feebler than the bright sodium band-namely, one red line near the Fraunhofer line A and another violet line. Besides which, a pale, almost eontinuous, spectrum will be

24 The arrangement of all the parts of the apparatus so as to give the clearest possible vision and accuracy of observation must evidently precede every kind of spectroscopio determination. Details concerning the practical use of the spectroscope must be looked for in special works on the subject. In this trea ise the reader is supposed to have a certain knowledge of the physical data respecting the refraction of light, and its. dispersion and diffraction, and the theory of light, which allows of the determination of the length of the wares of light in absolute measure on the basis of observations with diffraction gratings, the distance between whose divisions may be easily measured in fractions of a millimetre; by such means it is possible to determine the wave-length of any given ray of light.

${ }_{25}$ In order to give an idea of the size of the scale, we may observe that the ordinary spectrum eztends from the zero of the scale (where the red portion is situated) to the 170th division (where the end of the visible violet portion of the spectrum is situated), and that the Fraunhofer line A (the extreme prominent line in the red) corresponds with the 17 th division of the scale; the Fraunhofer line $F$ (at the beginning of the blue, near the green colour) is situated on the 90 th division, and the line $\mathrm{G}$, which is clearly seen in the beginning of the violet portion of the spectrum, corresponds with the 127 th division of the scale. 
observed in the central portions of the scale. If a mixture of sodium and potassium salts be now introduced into the flame, three lines will be seen simultaneously-namely, the red and pale violet lines of potassium and the yellow line of sodium. In this manner it is possible, by the aid of the spectroscope, to determine the relation between the spectra of metals and known portions of the solar spectrum. The con. tinuity of the latter is.interrupted by dark lines (that is, by an absence of light of a definite index of refraction), termed the Fraunhofer lines of the solar spectrum. It has been shown by careful observations (by Fraunhofer, Brewster, Foucault, Angström, Kirchhoff, Cornu, Lockyer, Dewar, and others) that there exists an exact agreement between the spectra of certain metals and certain of the Fraunhofer lines. Thus the bright yellow sodium line exactly corresponds with the dark Fraunhofer line $\mathrm{D}$ of the solar spectrum. A similar agreement is observed in the case of many other metals. This is not an approximate or chance correlation. In fact, if a spectroscope having a large number of refracting prisms and a high magnifying power be used, it is seen that the dark line $D$ of the solar spectrum consists of an entire system of closely adjacent but definitely situatea fine and wide (sharp, distinct) dark lines, ${ }^{26}$ and an exactly similar group of bright lines is obtained when the yellow sodium line is examined through the same apparatus, so that each bright sodium line exactly corresponds with a dark line in the solar spectrum. ${ }^{26}$ bis This conformity of the bright lines formed by sodium with the dark lines of the solar spectrum cannot be accidental. This conclusion is further confirmed by the fact that the bright lines of other metals correspond with dark lines of the solar spectrum. Thus, for example, a series of sparks passing between the iron electrodes of a Ruhmkorff coil gives 450 very distinct lines characterising this metal. All these 450 bright lines, constituting the whole spectrum corre: sponding with iron, are repeated, as Kirchhoff showed, in the solar spectrum as dark Fraunhofer lines which occur in exactiy the same situations as the bright lines in the iron spectrum, just as the sodium lines correspond with the band D in the solar spectrum. Many observers have in this manner studied the solar spectrum and the spectra of different metals simultaneously, and discovered in the former lines which

26 The two most distinct lines of $D$, or of sodium, have wave-lengths of 589.5 and 588.9 millionths of a millimeter, besides which fainter and fainter lines are seen whose wave. lengths in millionths of a millimeter are $588^{\circ} 7$ and $588^{\circ} 1,616^{\circ} 0$ and $615^{\circ} 4,515.5$ and $515^{\circ} 2$, $498 \cdot 8$ and $498^{\circ} 2$, \&c., according to Liveing and Dewar.

86 bls In the ordinary spectroscopes which are usually employed in chemical research, one yellow band, which does not split up into thinner lines, is seen instead of the system of sodium lines, owing to the small dispersive power of the prism and the width of the slit of the object tube. 
correspond not only with sodium and iron, but also with many other metals. ${ }^{27}$ The spectra of such elements as hydrogen, oxygen, nitrogen, and other gases may be observed in the so-called Geissler's tubes-that is, in glass tubes containing rarefied gases, through which the discharge of a Ruhmkorff's coil is passed. Thus hydrogen gives a spectrum composed of three lines-a red line corresponding with the Fraunhofer line C, a green line corresponding with the line F, and a violet line corresponding with one of the lines between $G$ and $H$. Of these rays the red is the brightest, and therefore the general colour of luminous hydrogen (with an electric discharge through a Geissler tube) is reddish.

The correlation of the Fraunhofer lines with the spectra of metals depends on the phenomenon of the so-called reversal of the spectrum. This phenomenon consists in this, that instead of the bright spectrum corresponding with a metal, under certain circumstances a similar dark

27 The most accurate investigations made in this respect are carried on with spectra obtained by diffraction, becanse in this case the position of the dark and bright lines does not depend on the index of refraction of the material of the prism, nor on the dispersive power of the apparatus. The best-that is, the most general and accurate-method of expressing the results of such determinations consists in determining the lengths of the waves corresponding to the rays of a definite index of refraction. (Sometimes instead of this the fraction of 1 divided by the square of the wave-length is given.) We will express this wave-length in millionth parts of a millimetre (the ten-millionth parts are already doubtful, and fall within the limits of error). In order to illustrate the relation between the wave-lengths and the positions of the lines of the spectrum, we will cite the wavelengths corresponding with the chief Fraunhofer lines and colours of the spectrum.

Fraunhofer line,

Wave-length

Colour

$$
\begin{aligned}
& \begin{array}{lllllllll}
\text { A } & \text { B } & \text { C } & \text { D } & \text { E } & \text { b } & \text { F } & \text { G } & \text { H }
\end{array} \\
& \underbrace{761.0687 .5656 .6}_{\text {red }} \underbrace{589 \cdot 5-588.9}_{\text {orange }} \underbrace{527.8}_{\text {yellow }} \underbrace{518 \cdot 7}_{\text {green }} \underbrace{486.5 \quad 481.0}_{\text {blue }} \underbrace{397 \cdot 2}_{\text {violet }}
\end{aligned}
$$

In the following table are given the wave-lerigths of the light rays (the longest and most distinct, see later) for certain elements, those in black type being the most clearly defined and distinct lines, which are easily obtained either in the flame of a Bunsen's burner, or in Geissler's tubes, or in general, by an electric discharge. These lines refer to the elements (the lines of compounds are different, as will be afterwards explained, but many compounds are decomposed by the flame or by an electric discharge), and moreover to the elements in an incandescent and rarefied gaseons state, for the spectra sometimes vary considerably with a variation of temperature and pressure.

It may be mentioned that the red colour corresponds with lines having a wave-length of from 780 (with a greater wave-length the lines are hardly visible, and are ultra red) to 650 , the orange from 650 to 590 , the yellow from 590 to 520 , the green from 520 to 490 , the blue from 490 to 420 , and the violet from 420 to 380 millionth parts of a millimetre. Beyond 880 the lines are scarcely visible, and belong to the ultra-violet. For fluorine Moissan found as many as 13 bright lines from 744 to 623 .

In the table (p. 565) which is arranged in conformity with the image of the spectrum as it is seen (the red lines on the left-hand and the violet on the right-hand side), the figures in black type correspond with lines which are so bright and distinctly visiblo that they may easily be made use of, both in determining the relation between the divisions of the scale and the wave-lengths, and in determining the admixture of a given element with another. Brackets join those lines between which several other lines are clearly visible if the dispersive-power of the spectroscope permits distinguishing the neighbouring lines. In 
spectrum in the form of Fraunhofer lines may be obtained, as will be explained directly In order to clearly understand the phenomenon of

the ordinary laboratory spectroscopes with one prism, even with all possible preoision of arrangement and with a brilliancy of light permitting the observations being made with a very narrow aperture, the lines whose wavelengths only differ by $2-3$ millionths of a millimetre, are blurred together; and with a wide aperture a series of lines differing by even as much as 20 millionths of a millimetre appear as one wide line. With a faint light (that is, with a small quantity of light entering into the spectroscope) only the most brilliant lines are clearly visible. The length of the lines does not always correspond with their brilliancy. According to Lnckyer this length is determined by placing the carbon electrodes (between which the incandescent vapours of the metals are formed), not horizontally to the slit (as they are generally placed, to give more light), but vertically to it. Then certain lines appear long and others short. As a rule (Lockyer, Dewar, Cornu), the longest lines are those with which it is easiest to obtain reversed spectra (see later). Conse. quently, these lines are the most characteristic. Only the longest and most brilliant are given in our table, which is composed on the basis of a collection of the data at our dis. posal for bright spectra of tho in. candescent and rarefied vapours of the elements. As the spectra change with great variations of temperature and vapour density (the faint lines become brilliant whilst the bright jines sometimes disappear), which is particularly clear from Ciamician's researches on the halogens, until the method of observation and the theory of the subject are enlarged, particular theoretical importance should not be given to the wave-lengths showing the maximum brilliancy, which only possess a practical significance in the common methods of spectroscopic observations. In

\begin{tabular}{|c|c|}
\hline "10" & | | | 范 \\
\hline 桴 & $\mid 1 \dot{0}$ \\
\hline 茫 & $|1| 1 \mid$ 歺o \\
\hline$M$ & 유: || | \\
\hline คี & 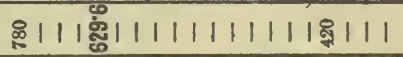 \\
\hline 8 & 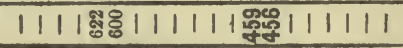 \\
\hline 8 & 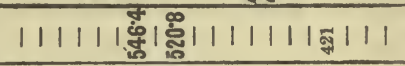 \\
\hline ซี & 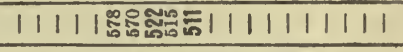 \\
\hline$\stackrel{0}{0}$ & 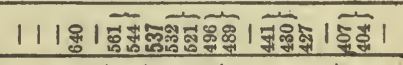 \\
\hline 볼 & 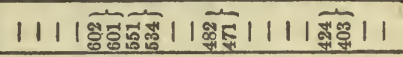 \\
\hline 禹 & 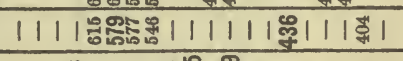 \\
\hline כี & 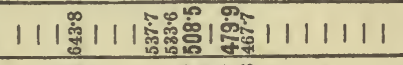 \\
\hline ลี & 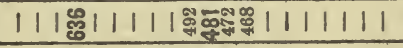 \\
\hline 曋 & 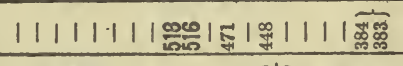 \\
\hline ซึ & 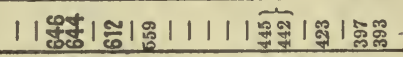 \\
\hline ผู & 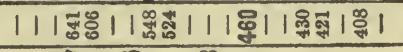 \\
\hline ตึ & 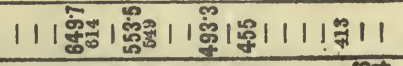 \\
\hline च & 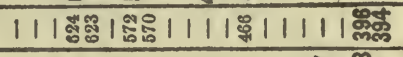 \\
\hline §ึ & $1111111111111111 \% 1 \%$ \\
\hline 홈 & 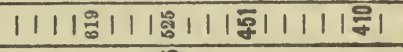 \\
\hline F & 1111111 ! \\
\hline 멿 & $|1|$ 电| \\
\hline मे & 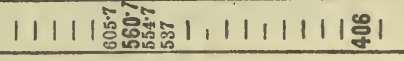 \\
\hline$n$ & 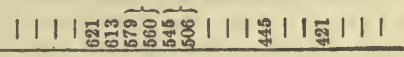 \\
\hline मूल & 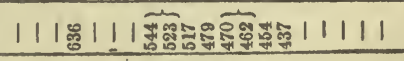 \\
\hline छः & 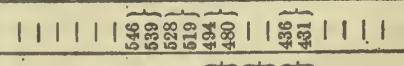 \\
\hline $0^{\infty}$ & 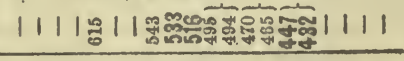 \\
\hline $\mathrm{Ra}^{+1}$ & $8 \% 1181$ \\
\hline
\end{tabular}


reversed spectra, it must be known that when light passes through certain transparent substances these substances retain rays of a certain refrangibility. The colour of solutions is a proof of this. Light which has passed through a yellow solution of a uranium salt contains no violet rays, and after having passed through a red solution of a permanganate, does not contain many rays in the yellow, blue, and green portions of the spectrum. Solutions of copper salts absorb nearly all red rays. Sometimes colourless solutions also absorb rays of certain definite refractive indexes, and give absorptron spectra. Thus solutions of salts of didymium absorb rays of a certain refrangibility, and therefore an impression of black lines is received, ${ }^{28}$ as shown in fig. 73. Many vapours (iodine) and gases (nitric peroxide) give similar

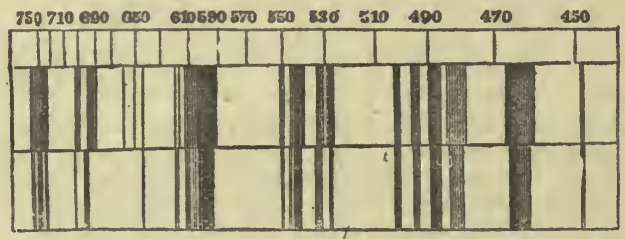

Fia. 73.-Absorption spectrum (Lecoq de Boisbaudran) of salts of aidymium in concentrated and dilute sólutions.

spectra. Light which has passed through a deep layer of aqueous vapour, oxygen, or nitrogen also gives an absorption spectrum. For this reason the peculiar (winter) dark lines discovered by Brewster are bbserved in sunlight, especially in the evening and morning, when the sun's rays pass through the atmosphere (containing these substances) by a longer path than at mid.day. It is evident that the Fruunhofer

general the spectra of metals are simpler than those of the halogens, and the latter are variable; at an increased pressure all spectral lines become broader.

${ }_{28}$ The method of observing absorption spectra consists in taking a continuous spectrum of white light (one which does not show either dark lines or particularly bright luminous bands-for instance, the light of a candle, lamp, or other source). The collimator (that is, the tube with the slit) is directed towards this light, and then all the colours of the spectrum are visible in the ocular tube. A transparent absorptive medium - for instance, a solution or tube containing a gas-is then placed between the source of light and the apparatus (or anywhere inside the apparatus itself in the path of the rays). In this case either the entire spectrum is uniformly fainter, or absorption bands appear on the bright field of the continnous spectrum in definite positions along it. These bands have different lengths and positions, and distinctness and intensity of absorption, according to the properties of the absorptive medium. Like the luminous spectra given by incandescent gases and vapours, the absorption spectra of a number of substances have already been studied, and some with great precision-as, for example, the spectrum of the brown vapours of nitrogen dioxide by Hasselberg (at Pulkowa), the spectra of colouring matters (Eder and others), especially of those applied to orthot. 
lines may be ascribed to the absorption of certain rays of light in its passage from the luminous mass of the sun to the earth. The remarkable progress made in all spectroscopic research dates from the investigations made by Kirchhoff (1859) on the relation between absorption spectra and the spectra of luminous incandescent gases. It had already been observed long before (by Fraunhofer, Foucault, Angström) that the bright spectrum of the sodium flame gives two bright lines which are in exactly the same position as two black lines known as $\mathrm{D}$ in the solar spectrum, which evidently belong to an absorption spectrum. When Kirchhoff caused diffused sunlight to fall upon the slit of a spectroscope, and placed a sodium flame before it, a perfect superposition was observed-the bright sodium lines completely covered the black lines $\mathrm{D}$ of the solar spectrum. When further the continuous spectrum of a Drummond light showed the black line D on placing a sodium flame between it and the slit of the spectroscope-that is, when the Fraunhofer line of the solar spectrum was artificially produced-then there was no doubt that its appearance in the solar spectrum was due to the light passing somewhere through incandescent vapours of sodium. Hence a new theory of reversed spectra ${ }^{29}$ arose-that is,

chromatic photography, the spectra of blood, chlorophyll (the green constituent of leaves), and other similar substances, all the more carefully as by the aid of their spectra the presence of these substances may be discovered in small quantities (even in microscopical quantities, by the aid of special appliances on the microscope); and the ehanges they undergo investigated.

The absorption spectra, abtained at the ordinary temperature and proper to substances in all physical states, offer a most extensive but as yet little studied field, both

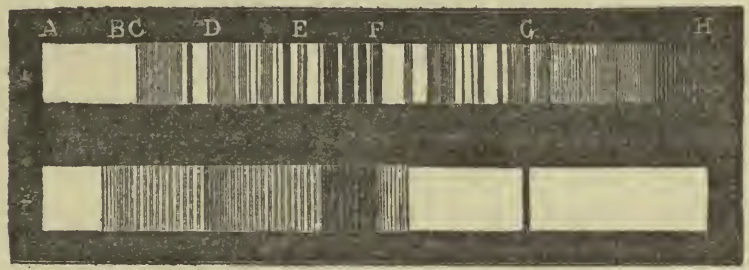

FiG. 74.-Absorption spectra of nitrogen dioxide and iodine.

for the general theory of spectroscopy, and for gaining an insight into the structure of substances. The investigation of colouring matters has already shown that in certain cases a definite change of composition and structure entails not only a definite change of the colours but also a displacement of the absorption bands by a definite number of wave-lengths.

${ }^{29}$ A number of methods have been invented to demonstrate the reversibility of spectra; among these methods we will cite two which are very easily carried out. In Bunsen's method sodium chloride is put into an apparatus for evolving hydrogen (the spray of the salt is then carried off by the hydrogen and colours the flame with the 
of the relation between the waves of light emitted and absorbed by a substance under given conditions of temperature: this is expressed by Kirchhoff's law, discovered by a careful analysis of the phenomena. This law may be formulated in an elementary way as follows : At a given temperature the relation between the intensity of the light emitted (of a definite wave-length) and the absorptive capacity with respect to the samie colour (of the same wave-length) is a constant quantity. ${ }^{30}$ As a black dull surface emits and also absorbs a considerable quantity of heat rays whilst a polished metallic surface both absorbs and emits but few, so a flame coloured by sodium emits a considerable quantity of yellow rays of a definite refrangibility, and has the property of absorbing a considerable quantity of the rays of the same refractive index. In general, the medium which emits definite rays also absorbs them.

Thus the bright spectral rays characteristic of a given metal may be reversed-that is, converted into dark lines-by passing light which gives a continuous spectrum through a space containing the heated vapours of the given metal. A similar phenomenon to that thus arti. ficially produced is observed in sunlight, which shows dark lines characteristic of known metals-that is, the Fraunhofer lines form an absorption spectrum or depend on a reversed spectrum ; it being presupposed that the sun itself, like all known sources of artificial light, gives a continuous spectrum without Fraunhofer lines. ${ }^{31}$ We must

yellow sodium colour), and the hydrogen is ignited in two burners-in one large one with a wide flame giving a bright yellow sodium light, and in another with a small fine orifice whose flame is pale : this flame will throw a dark patch on the large bright flame. In Ladoffisk's method the front tube (p. 561) is unscrewed from a spectroscope directed towards the light of a lamp (a continuous spectrum), and the flame of a spirit lamp coloured by a small quantity of $\mathrm{NaCl}$ is placed between the tube and the prism; a black band corresponding to sodium will then be seen on looking through the ocular tube. This experiment is always successful if only there be the requisite relation between the strength of light of the two lamps.

30 The absorptive capacity is the relation between the intensity of the light (of a given wave-length) falling upon and retained by a substance. Bunsen and Roscoe showed by direct experinent that this ratio is a constant quantity for every substance. If $A$ stand for this ratio for a given substance at a given temperature-for instance, for a flame coloured by sodium - and $E$ be the intensity of the light of the same wave-length emitted at the same temperature by the same substance, then Kirchhoff's law, the explanation and deduction of which must be looked for in text-books of physics, states that the fraction $A / E$ is a constant quantity depending on the nature of a substance (as $A$ depends on it) and determined by the temperature and wave-length.

31 Heated metals begin to emit light (only visible in the dark) at about $420^{\circ}$ (vary. ing with the metal). On further heating, solids first emit red, then yellow, and lastly white light. Compressed or heavy gases (see Chapter III., Note 44), when strongly heated, also emit white light. Heated liquids (for example, molten steel or platinum) also give a white compound light. This is readily understood. In a dense mass of matter the collisions of the molecules and atoms are so frequent that waves of only a few definite lengths cannot appear; the reverse is possible in rarefied gases or vapours. 
imagine that the sun, owing to the high temperature which is proper to it, emits a brilliant light which gives a continuous spectrum, and that this light, before reaching our eyes, passes through a space full of the vapours of different-metals and their compounds. As the earth's atmosphere ${ }^{32}$ contains very little, or no, metallic vapours, and as they cannot be sup-

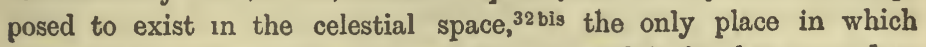
the existence of such vapours can be admitted is in the atmosphere surrounding the sun itself. As the cause of the sun's luminosity must be looked for in its high temperature, the existence of an atmosphere containing metallio vapours is readily understood, because at that high temperature such metals as sodium, and even iron, are separated from their compounds and converted into vapour. The sun must be imagined as surrounded by an atmosphere of incandescent vaporous and gaseous matter, ${ }^{33}$ including those elements whose reversed spectra correspond with the Fraunhofer lines-namely, sodium, iron, hydrogen, lithium, calcium, magnesium, \&c. Thus in spectrum analysis we find a means of determining the composition of the inaccessible heavenly luminaries, and much has been done in this respect since Kirchhoff's theory was formulated. By observations on the spectra of many heavenly bodies, changes have been discovered going on in them, ${ }^{34}$ and

53 Brewster, as is mentioned above, first distinguished the atmospheric, cosmical Fraunhofer lines from the solar lines. Janssen showed that the spectrum of the atmosphere contains lines which depend on the absorption produced by aqueous vapour. Egoreff, Olszewski, Janssen, and Liveing and Dewar showed by a series of experiments that the oxygen of the atmosphere gives rise to certain lines of the solar spectrum, especially the line A. Liveing and Dewar took a layer of 165 c.m. of oxygen compressed under a pressure of 85 atmospheres, and determined its absorption spectrum, and found that, besides the Frounhofer lines $A$ and $B$, it contained the following groups: 630-622, $581-568,535,480-475$. The same lines were found for liquid oxygen.

$32 \mathrm{bls}$ If the material of the whole heavenly space formed the absorbent medium, the spectra of the stars would be the same as the solar spectrum; but Huyghens, Lockyer, and others showed not only that this is the case for only a few stars, but that the majority of stars give spectra of a different character with dark and bright lines and bands.

33 Eruptions, like our volcanic eruptions, but on an incomparably larger scale, are of frequent occurrence on the sun. They are seen as protuberances visible during a total eclipse of the sun, in the form of vaporous masses on the edge of the solar dise and emitting a faint light. These protuberances of the sun are now observed at all times by means of the spectroscope (Lockyer's method), because they contain luminous vapours (giving bright likes) of hydrogen and other elements.

34 The great interest and vastness of astro-physical observations cancerning the sun, comets, stars, nebulæ, \&c., render this new province of natural science very important, and necessitate referring the reader to special works on the subject.

The most important astro-physical data since the time of Kellner are those referring to the displacement of the lines of the spectrum. Just as a musical note changes its pitch with the approach or withdrawal of the resonant object or the ear, so the pitch of the luminous note or wave-length of the light varies if the luminous (or absorbent) vapour: and the earth from which we observe it approach or recede from each other; this 
many of the elements known to is have been found with certainty in them. ${ }^{35}$ From this it must be concluded that the same elements which exist on the earth eccur throughout the whole universe, and that at that degree of heat which is proper to the sun those simple.substances which we accept as the elements in chemistry are still undecomposed and remain unchanged. A high temperature forms one of those conditions under which compounds most easily decompose; and if sodium or a similar element were a compound, in all probability it would be decomposed into component parts at the high temperature of the sun. This may indeed be concluded from the fact that in ordinary spectroscopic experiments the spectra obtained often belong to the metals and not to the compounds taken; this depends on the decomposition of these compounds in the heat of the fane. If

expresses itself in a visible displacement of the spectral lines. The solar eruptions even give broken lines in the spectrum, because the rapidly moving eruptive masses of vapour and gases either travel in the direction of the eye or fall back towards the sun. As the earth travels with the solar system among the stars, so it is possible to determine the direction and velocity with which the sun travels in space by the displacement of the spectral lines and light of the stars. The changes proceeding on the sun in its mass, which must be pronounced as vaporous, and in its atmosphere, are now studied by means of the spectroscope. For this purpose, many special astro-physical observatories now exist where these investigations are carried on.

We may remark that if the observer or luminous object moves with a velocity $\pm v$, the ray, whose wave-length is $\lambda$, has an apparent wave-length $\lambda \frac{n \neq v}{n}$, where $n$ is the velocity of light. Thus Tolon, Huyghens, and others proved that the star Aldebaran approaches the solar system with a velocity of 30 kilometres per second, while Arcturus is rsceding with a velocity of $\mathbf{4 5}$ kilometres. The majority of stars give a distinct hylurogen spectrum, besides which nebulø also give the spectrum of nitrogen. Lockyer classes the stars from their spectra, according to their period of formation, showing that some stars are in a period of increasing temperature (of formation or aggregation), whilst others are in a period of cooling. Altogether, in the astro-physical investigation of the spectra of heavenly bodies we find one of the most interesting subjects of recent science.

55 Spectrum analysis has proved the indubitable existence in the sun and stars of a number of elements known in chemistry. Huyghens, Secchi, Lockyer, and others have furnished a large amount of material upon this subject. $\boldsymbol{\Lambda}$ compilation of existing information on it has been given by. Prof. S. A. Kleiber, in the Journal of the Russian Physico-chemical Society for 1885 (vol. xviii. p. 146). Besides which, a peculiar Glement called helium has been discovered, which is characterised by a line (whose wavelength is 587.5 , situated near $\mathrm{D}$ ), which is seen very brightly in the projections (pro. tuberances) and spots of the sun, but which does not belong to any known element, and is not reproducible as a reversed, dark line. This may be a right conclusion-that is to say, it is pessible that an element may be discovered to which the spectrum of helium corresponds-but it may be that the helium line belongs to one of the known elements, because spectra vary in the brilliancy and position of their lines with changes of temperature and pressure. Thus, for instance, Lockyer could only see the line 423, at the very end of the calcium spectrum, at comparatively low temperatures, whilst the lines 397 and 393 appear at a higher temperature, and at a still higher temperature the line 428 becomes quite invisible. 
common salt be introduced into the flame of a gas-burner, a portion of it is decomposed, first forming, in all probability, with water, hydrochloric acid and sodium hydroxide, and the latter then becoming partially decomposed by the hydrocarbons, giving metallic sodium, whose incandescent vapour emits light of a definite refrangibility. This conclusion is arrived at from the following experiment :- If hydrochloric acid gas be introduced into a flame coloured by sodium it is observed that the sodium spectrum disappears, owing to the fact that metallic sodium cannot remain in the flame in the presence of an excess of hydrochlorio acid: The same thing takes place on the addition of sal-ammoniac, which in the heat of the flame gives hydrochloric acid. If a porcelain tube containing sodium chloride (or sodium hydroxide or carbonate), and closed at both ends by glass plates, be so powerfully heated that the salt volatilises, then the sodium spectrum is not observable; but if the

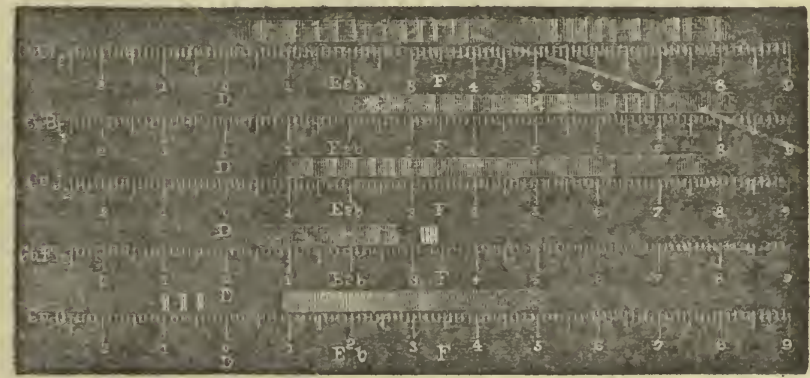

FIG. 75.-Bright spectra of copper compounds.

salt be replaced by sodium, then either the bright line or the absorption spectra is obtained, according to whether the light emitted by the incandescent vapour be observed, or light passing through the tube. Thus the above spectrum is not given by sodium chloride or other sodiurn compound, but is proper to the metal sodium itself. This is also the case with other analogous metals. The chlorides and other halogen compounds of barium, calcium, copper, \&c., give independent spectra which differ from those of the metals. If barium chloride be introduced into a flame, it gives a mixed spectrum belonging to metallic . barium and barium chloride. If besides barium chloride, hydrochloric acid or sal-ammoniac be introduced into the flame, then the spectrum of the metal disappears, and that of the chloride remains, which differs distinctly from the spectrum of barium fluoride, barium bromide, or barium iodide. A certain common resemblance and certain common 
lines are observed in the spectra of two different compounds of one and the same element obtained in the above-described manner, and also in the spectrum of the metal, but they all have their peculiarities. The independent spectra of the compounds of copper are easily observed (fig. 75). Thus certain compounds which exist in a state of vapour, and are luminous at a high temperature, give their independent spectra. In the majority of cases the spectra of compounds are composed of indistinct luminous lines and complete bright bands, whilst metallio elements generally give a few clearly-defined spectral lines. ${ }^{36}$ There is

36 Spectroscopic observations are still further complicated by the fact that one and the same substance gives different spectra at different temperatures. This is especially the crse with gases whose spectra are obtained by an electric discharge in tubes. Pliicker, Wiillner, Schuster, and others showed that at low temperatures and pressures the spectra of iodine, sulphur, nitrogen, oxygen, \&c. are quite different from the spectra of the same elements at high temperatures and pressures. This may either depend on the fact that the elements change their molecular structure with a change of temperature, just as ozone is converted into oxygen (for instance, from $\mathrm{N}_{2}$ molecules are obtained containing only one atom of nitrogen), or else it may be because at low temperature certain rays have a greater relative intensity than those which appear at higher tem. peratures. If we suppose that the molecules of a gas are in continual motion, with a velocity dependent on the temperature, then it must be adinitted that they often strike against each other and rebound, and thus communicate peculiar motions to each other and the supposed ether, which express themselves in luminiferous phenomena. A rise of the temperature or an increase in the density of a gas must have an influence on the

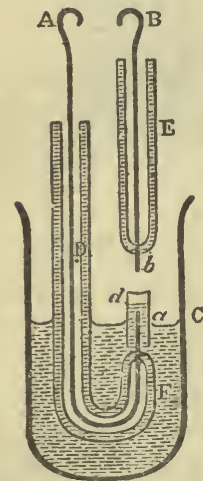

FIG. 76. - Method of showing the spec. trum of sukstances in solution. collision of its molecules and luminiferous motions thus produced, and this may.be the cause of the difference of the spectra under these circumstances. It has been shown by direct experiment that gases compressed by pressure, when the collision of the molecules must be frequent and varied, exhibit a more complex spectrum on the passage of an electric spark than rarefied gases, and that even a continuous spectrum appears. In order to show the variability of the spectrum according to the circumstances under which it proceeds, it may be mentioned that potassium sulphate fused on a platinum wire gives, on the passage of a series of sparks, a distinct aystem of lines, 583-578, whilst when a series of sparks is passed throngh a solution of this salt this system of lines is faint, and when Roscoe and Schuster observed the absorp. tion spectrum of the vapour of metallic potassium (which is green) they remarked a number of lines of the same intensity as the above system in the red, orange, and yellow portions.

The spectra of solutions are best observed by means of Lecog de Boisbaudran's arrangement, shown in fig. 76. A bent capillary tube, D F, inside which a platinum wire, A $a$ (from 0.3 to $0.5 \mathrm{~mm}$. in diameter) is fused, is immersed in a narrow cylinder, $C$ (in which it is firmly held by a cork). The projecting end, $a$, of the wire is covered by a fine capillary tube, $d$, which extends 1-2 $\mathrm{mm}$. beyond the wire. Another straight capillary tube, $\mathrm{E}$, with a platinum wire, $\mathrm{B} b$, about $1 \mathrm{~mm}$. in diameter (a finer wire soon becomes hot), is held (by a cork or in a stand) above the end of the tube, D. If the wire $\mathrm{A}$ be now connected with the positive, and the wire $B$ with the negative terminal of a. Ruhmkorff's coil (if the wires be connected in the opposite order, the spectrum of air is obtained), a series of sparks rapidly following each other appear between $a$ and $b$. and. 
no reason for supposing that the spectrum of a compound is equal to the sum of the spectra of its elements-that is, every compound which is not decomposed by heat has its own proper spectrum. This is best proved by absorption spectra, which are essentially only reversed spectra observed at low temperatures. If every salt of sodium, lithium, and potassium gives one and the same spectrum, this must be ascribed to the presence in the flame of the free metals liberated by the decomposition of their salts. Therefore the phenomena of the spectrum are deternined by molecules, and not by atoms-that is, the molecules of the metal sodium, and not its atoms, produce those particular vibrations which determine the spectrum of a sodium salt. Where there is no free metallic sodium there is no sodium spectrum.

Spectrum analysis has not only endowed science with a knowledge of the composition of - distant heavenly bodies (of the sun, stars, nebulæ, comets, \&c.), but has also given a new method for studying the matter of the earth's surface. With its help Bunsen discovered two new elements belonging to the group of the alkali metals, and thallium, indium, and gallium were afterwards discovered by the same means. The spectroscope is employed in the study of rare metals (which in solution often give distinct absorption spectra), of dyes, and of many organic substances, \&c. ${ }^{37}$ With respect to the metals which are analogous to sodium, they all give similar very volatile

their light may be examined by placing the apparatus in front of the slit of a spectro. scope. The variations to which a spectrum is liable may easily be observed by increasing the distance between the wires, altering the direotion of the current or strength of the solution, \&c.

37 The importance of the spectroscope for the parpose of clemical research was already shown by Gladstone in 1856, but it did not become an accessory to the laboratory until after the discoveries of Kirchhoff and Bunsen. It may be hoped that in time spectroscopic researches will meet certain wants of the theoretical (philosophical) side of chemistry, but as yet all that has been done in this respect can only be regarded as attempts which have not yet led to any trustworthy conclusions. Thus many investigators, by collating the wave-lengths of all the light vibrations excited by a given element, endeavour to find the law governing their mutual relations; others (especially Hartley and Ciamician), by comparing the spectra of analogous elements (for instance, chlorine, bromine, and iodine), have succeeded in noticing definite features of resemblance in them, whilst others (Grïnwald) search for relations between the spectra of compounds and their component elements, \&c.; but-owing to the multiplicity of the spectral lines proper to many elements, and (especially in the ultra-red and ultra-violet ends of the specirum) the existence of lines which are undistinguishable owing to their faintness, and also owing to the comparative novelty of spectroscopic research-this subject cannot be considered as in any way perfected. Nevertheless, in certain instances there is evidently some relationship between the wave-lengths of all the spectral lines formed by a given element. Thus, in the hydrogen spectrum the wave-length $=364.542 \mathrm{~m}^{2} /\left(\mathrm{m}^{2}-4\right)$, if $m$ varies as a series of whole numbers from 3 to 15 (Walmer, Hagebach, and others). For example, when $m=3$, the wave-length of one of the brightest lines of the hydrogen spectrum is obtained $\left(656^{\circ} 2\right)$, when $m=7$, nne of the visible violet lines (396.8), and when $m$ is greater than 9, the ultra-violet lines of the hydrogen spectrum. 
salts and such very characteristic spectra that the least traces of them ${ }^{30}$ are discovered with great ease by means of the spectroscope. For instance, lithium gives a very brilliant red coloration to a flame and a very bright red spectral line (wave-length, 670 millionths mm.), which indicates the presence of this metal in admixture with compounds of other alkali inetals.

Lithium, $\mathrm{Li}$, is, like potassium and sodium, somewhat widely spread in siliceous rocks, but only occurs in small quantities and as mere traces in considerable masses of potassium and sodium salts. Only a very few rather rare minerals contain more than traces of it, ${ }^{39}$ for example, spodumene and lithia mica. Many compounds of lithium are in all respects closely analogous to the corresponding compounds of sodium

38 in order to show the degree of sensitiveness of spectroscopic reactions the following observation of Dr. Bence Jones may be cited: If a solution of 3 grains of a lithium salt be injected under the skin of a guinea.pig, after the lapse of four minutes, lithium can be discovered in the bile and liquids of the eye, and, after ten minutes, in all parts of the animal.

5y Thus spodumene contains up to 6 p.c. of lithium oxide, and petolite, and lepidolite or lithia mica, about 3 p.o. of lithium oxide. This mica is met with in certain granites in a somewhat considerable quantity, and is therefore most frequently employed for the preparation of lithium compounds. The treatment of lepidolite is carried on on a large scale, becanse certain salts of lithium are employed in medicine as a remedy for certain diseases (stone, gouty affections), as they have the power of dissolving the insoluble nric acid which is then deposited. Lepidolite, which is unacted on by acids in its natural state, decomposes under the action of strong hydrochloric acid after it has been fused. After being subjected to the action of the hydrochloric acid for several hours all the silica is obtained in an insoluble form, whilst the metallic oxides pass into solution as chlorides. This solution is mixed with nitric acid to convert the ferrous salts into ferric, and sodium carbonate is then added until the liquid becomes neutral; by which means a precipitate is formed of the oxides of iron, alumina, magnesia, \&c., as insoluble oxides and carbonates. The solution (with an excess of water) then contains the chlorides of the alkaline metals $\mathrm{KCl}, \mathrm{NaCl}, \mathrm{LiCl}$, which do not give a precipitate with sodium carbonate in a dilute solution. It is then evaporated, and a strong solution of sodium carbonate added. This precipitates lithium carbonate, which, although solublo in water, is much less so than sodium carbonate, and therefore the latter precipitates lithium from strong solutions as carbonate, $2 \mathrm{LiCl}+\mathrm{Na}_{2} \mathrm{CO}_{3}=2 \mathrm{NaCl}+\mathrm{Li}_{2} \mathrm{CO}_{3}$. Lithium carbonate, which resembles sodium carbonate in many respects, is a substance which is very slightly soluble in cold water and is only moderately soluble in boiling water. In this respect lithium forms a transition between the metals of the alkalis and other metals, especially those of the alkaline earths (magnesium, barium), whose carbonates are only sparingly soluble. Oxide of lithium, $\mathrm{Li}_{2} \mathrm{O}$, may be obtained by heating lithium carbonate with charcoal. Lithinm oxide in dissolving gives (per gram-molecule) 26,000 heat units; but the combination of $\mathrm{Li}_{2}$ with $\mathrm{O}$ evolves 140,000 chlories-that is, more thian $\mathrm{Na}_{2} \mathrm{O}$ (100,000 calories) and $\mathrm{K}_{2} \mathrm{O}$ ( 97,000 calories), as shown by Beketoff (1887). Oeuvrard (1892) heated lithinm to redness in nitrogen, and observed the absorption of $\mathrm{N}$ and formation of $\mathrm{Ji}_{3} \mathrm{~N}$, like $\mathrm{Na}_{3} \mathrm{~N}$ (see Chapter XII. Note 50 ).

$\mathrm{LiCl}, \mathrm{LiBr}$, and $\mathrm{LiI}$ form crystallo-hydrates with $\mathrm{H}_{2} \mathrm{O}, 2 \mathrm{H}_{2} \mathrm{O}$, and $3 \mathrm{H}_{2} \mathrm{O}$. As a rule, $\mathrm{LiBr} 2 \mathrm{H}_{2} \mathrm{O}$ crystallises out, but Bogorodsky (1894) showed that a solution containing $\mathrm{LiBr}+3^{\circ} 7 \mathrm{H}_{2} \mathrm{O}$, cooled to $-62^{\circ}$, separates out crystals $\mathrm{LiBr} 3 \mathrm{H}_{2} \mathrm{O}$, which decompose at $+4^{\circ}$ with the separation of $\mathrm{H}_{2} \mathrm{O}$. $\mathrm{LiF}$ is bot slightly soluble (in 800 parts) in water (and still less so in a solution of $\mathrm{NH}_{4} \mathrm{~F}$ ). 
and potassium; but the carbonate is sparingly soluble in cold water, which fact is taken advantage of for separating lithium from potassium and sodium. This salt, $\mathrm{Li}_{2} \mathrm{CO}_{3}$, is easily converted into the other compounds of lithium. Thus, for instance, the lithium hydroxide, LiHO, is obtained in exactly the same way as caustic soda, by the action of lime on the carbonate, and it is soluble in water and crystallises (from its solution in alcohol) as $\mathrm{LiHO}_{2} \mathrm{H}_{2} \mathrm{O}$. Metallic lithium is obtained by the action of a galvanic current on fused lithium chloride; for this purpose a cast-iron crucible, furnished with a stout cover, is filled with lithium chloride, heated until the latter fuses, and a strong galvanic current is then passed through the molten mass. The positive pole (fig. 77) consists of a dense carbon rod $\mathrm{C}$ (surrounded by a porcelain tube $P$ fixed in an iron tube $\mathrm{BB}$ ), and the negative pole of an iron wire, on which the metal is deposited after the current has passed through the molten mass for a certain length of time. Chlorine is evolved at the positive pole. When a somewhat considerable quantity of the metal has accumulated on the wire it is withdrawn, the metal is collected from it, and the experiment is

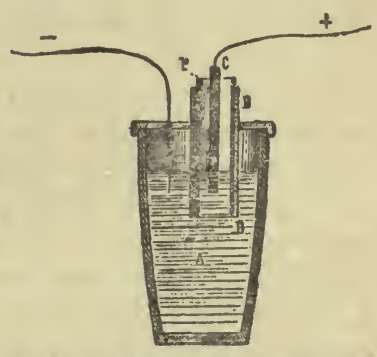

F1G. 77.-Preparation of lithium by the action of a galvanic current on fused lithium chloride.

then carried on as before. ${ }^{39}$ bis Lithium is the lightest of all metals, its specific gravity is 0.59 , owing to which it floats even on naphtha; it melts at $180^{\circ}$, but does not volatilise at a red heat. Its appearance recalls that of sodium, and, like it, it has a yellow tint. At $200^{\circ}$ it burns in air with a very bright flame, forming lithium oxide. In decomposing water it does not ignite the hydrogen. The characteristic test for lithiun compounds is the red coloration which they impart to a colourless flame.40

Bunsen in 1860 tried to determine by means of the spectroscope

so bis Guintz (1898) recommends adding $\mathrm{KCl}$ to the $\mathrm{LiCl}$ in preparing $\mathrm{Ll}$ by this method, and to act with a current of 10 amperres at 20 volts, and not to heat above $450^{\circ}$, so as to avoid the formation of $\mathrm{Li}_{2} \mathrm{Cl}$.

40 In determining the presence of lithinm in a given compound, it is best to treat the material under investigation with acid (in the case of mineral silicon compounds bydrofluoric acid must be taken), and to treat the residue with sulphuric acid, evaporate to dryness, and extract with alcohol, which dissolves a certain amount of the lithium sulphate. It is easy to discover lithium in such an alcoholio solution by means of the coloration imparted to the flame on burning it, and in case of doubt by investigating its light in a spectroscope, because lithium gives a red line, which is very characteristic and is found as a dark line in the solar spectrum. Lithium was first discovered in 1817 in petolite by Arfredson. 
whether any other as yet unknown metals might not occur in different natural products together with lithium, potassium, and sodium, and he soon discoviered two new alkali metals showing independent spectra. They are named after the characteristic coloration which they impart to the flame. One which gives a red and violet band is named rubidium, from rubidius (dark red), and the other is called coesium, because it colours a pale flame sky blue, which depends on its containing bright blue rays, which appear in the spectrum of cæsium as two blue bands (table on p. 565). Both metals accompany sodium, potassium, and lithium, but in small quantities; rubidium occurs in larger quantity than crsium. The amount of the oxides of cæsium and rubidium in lepidolite does not generally exceed one-half per cent. Rubidium has also been found in the ashes of many plants, while the Stassfurt carnallite (the mother-liquor obtained after having been treated for $\mathrm{KCl}$ ) forms an abundant source for rubidium and álso partly for cæsiun. Rubidium also occurs, although in very small quantities, in the majority of mineral waters. In a very few cases crsium is not accompanied by rubidium; thus, in a certain granite on the Isle of Elba, cæsium has been discovered, but not rubidium. This granite contains a very rare mineral called pollux, which containș as much as 4 per cent. of cæsium oxide. Guided by the spectroscope, and aided by the fact that the double salts of platinic chloride and rubidium and cæsium chlorides are still less soluble in water than the corresponding potassium salt, $\mathrm{K}_{2} \mathrm{PtCl}_{6}{ }^{41}$ Bunsen' succeeded in separating both metals from each other and from potassium, and demonstrated the great resemblance

41 The salts of the majority of metals are preclpitated as carbonates on the addition of ammonium carbonate-for instance, the salts of calcium, iron, \&c. The alkalis whose carbonates are soluble are not, however, precipitated in this case. On evaporating the resultant solution and igniting the residue (to remove the ammonium salts), we obtain salts of the alkali metals. They may be separated by adding hydrochloric acid together with a solution of platinic chloride. The chlorides of lithium and sodium give easily soluble double salts with platinic chloride, whilst the chlorides of potassium, rubidium, and cæsium form double salts which are sparingly soluble. A hundred parts of water at $0^{\circ}$ dissolve 0.74 part of the potassium platinochloride; the corresponding rubidium platinochloride is only dissolved to the amount of 0.134 part, and the cesium salt, 0.024 part; at $100^{\circ} 5.13$ parts of potassium platinochloride, $\mathrm{K}_{2} \mathrm{PtCl}_{6}$, are dissolved, 0.634 part of rubidium platinochloride, and 0.177 part of cæsium platinochloride. From this it is clear how the salts of rubidium and crsium may be isolated. The separation of cæsium from rubidium by this method is very tedious. It can be better effected by taking advantage of the difference of the solubility of their carbonates in alcohol; cæsium carbonate, $\mathrm{Cs}_{2} \mathrm{CO}_{3}$, is soluble in alcohol, whilst the corresponding salts of rubidium and potassium are almost insoluble. Setterberg separated these metals as alums, but the best method, that given by Scharples, is founded on the fact that from a mixture of the chlorides of potassium, sodium, cæsium, and rubidium in the pre. sence of hydrochlorio acid, stannic chloride precipitates a double salt of cæsium, which is very slightly soluble. The salts of $\mathrm{Rb}$ and $\mathrm{Cs}$ are closely analogous to those of potassium. 
they bear to each other. The isolated metals, ${ }^{42}$ rubidium and crsium, have respectively the specific gravities 1.52 and 2.366 , and melting points $39^{\circ}$ and $27^{\circ}$ as N.N. Beketoff showed (1894), he having obtained cæsium by heating $\mathrm{CsAlO}_{2}$ with $\mathrm{Mg}$ ( ${ }^{42}$ bis $)$.

Judging by the properties of the free metals, and of their corre. sponding and even very complex compounds, lithium, sodium, potassium, rubidium, and cæsium present an indubitable chemical resem. blance. The fact that the metals easily decompose water, and that their

42 Bunsen obtained rubidium by distilling a mixture of the tartrate with soot, and Beketoff (1888) by heating the hydroxide with aluminium, $2 \mathrm{RbHO}+\mathrm{Al}=\mathrm{RbAlO}_{2}+\mathrm{H}_{2}+\mathrm{Rb}$. By the action of 85 grams of rubidium on water, 94,000 heat units are evolved. Setterberg obtained cæsium (1882) by the electrolysis of a fused mixture of cyanide of cæsium and of barium. Winkler (1890) showed that metallic magnesium reduces the hydrates and sarbonates of $\mathrm{Rb}$ and $\mathrm{Cs}$ like the other alkaline metals. N. N. Beketoff obtained them with aluminium (see following note).

42 bls Beketoff (1888) showed that metallio aluminium reduces the hydrates of the alkaline metals at a red heat (they should be perfectly dry) with the formation of aluminates (Chapter XVII.), $\mathrm{RAlO}{ }_{2}$-for example, $2 \mathrm{KHO}+\mathrm{Al}=\mathrm{KAlO}_{2}+\mathrm{K}+\mathrm{H}_{2}$. It is evident that in this case only half of the alkaline metal is obtained free. On the other hand, K. Winkler (1889) showed that magnesium powder is also able to reduce the alkaline metals from their hydrates and carbonates. N. N. Beketoff and Tscherbacheff (1894) prepared cæsium upon this principle by heating its aluminate $\mathrm{CsAlO}_{2}$ with magnesium powder. In this case aluminate of magnesium is formed, and the whole of the cessium is obtrined as metal: $2 \mathrm{CsAlO}_{2}+\mathrm{Mg}=\mathrm{MgOAl}_{2} \mathrm{O}_{3}+2 \mathrm{Cs}$. A certain excess of alumina was taken (in order to obtain a less hygroscopic mass of aluminate), and magnesium powder (in order to decompose the last traces of water); the $\mathrm{CsAlO}_{2}$ was prepared by the precipitation of crsium -alums by caustio baryta, and evaporating the resultant solution. We may add that N.N. Beketoff (1887) prepared oxide of potassium, $\mathrm{K}_{2} \mathrm{O}$, by heating the peroxide, $\mathrm{KO}$, in the vapour of potassium (disengaged from its alloy with silver), and showed that in dissolving in an excess of water it evolves (for the above. given molecular weight) 67,400 calories (while $2 \mathrm{KHO}$ in dissolving in water evolves 24,920 cal.; so that $\mathrm{K}_{2} \mathrm{O}+\mathrm{H}_{2} \mathrm{O}$ gives 42,480 cal.), whence (knowing that $\mathrm{K}_{2}+\mathrm{O}+\mathrm{H}_{2} \mathrm{O}$ in an excess of water evolves 164,500 ) it follows that $K_{2}+O$ evolves 97,100 cal. This quantity is somewhat less than that $(100,260 \mathrm{cal}$.) which corresponds to sodium, and the energy of the action of potassium npon water is explained by the fact that $\mathrm{K}_{2} \mathrm{O}$ evolves more heat than $\mathrm{Na}_{2} \mathrm{O}$ in combining with water (see Chapter II. Note 9). Just as hydrogen displaces half the $\mathrm{Na}$ from $\mathrm{Na}_{2} \mathrm{O}$ forming $\mathrm{NaHO}$, so also $\mathrm{N}$. N. Beketoff found from experiment and thermo-chemical reasonings that hydrogen-displaces half the potassium from $\mathrm{K}_{2} \mathrm{O}$, forming $\mathrm{KHO}$ and evolving 7,190 calories. Oxide of lithium, $\mathrm{Li}_{2} \mathrm{O}$, which is easily. formed by igniting $\mathrm{Li}_{2} \mathrm{CO}_{3}$ with carbon (when $\mathrm{Li}_{2} \mathrm{O}+2 \mathrm{CO}$ is formed), disengages 26,000 cals. with an excess of water, while the, reaction $\mathrm{Li}_{2}+\mathrm{O}$ gives 114,000 cals. and the reaction $\mathrm{Li}_{2}+\mathrm{H}_{2} \mathrm{O}$ gives only 13,000 cals., and metallic lithium cannot be liberated from oxide of lithium with hydrogen (nor with carbon). Thus in the series $\mathrm{Li}, \mathrm{Na}, \mathrm{K}$, the formation of $\mathrm{R}_{2} \mathrm{O}$ gives most heat with $\mathrm{Li}$ and least with $\mathrm{K}$, while the formation of $\mathrm{RCl}$ evolves most heat with $\mathrm{K}(105,000 \mathrm{cals}$. $)$ and least of all with $\mathrm{Li}(93,500 \mathrm{cals}$.$) .$ Rubidium, in forming $\mathrm{Rb}_{2} \mathrm{O}$, gives 94,000 cals. (Beketoff). Cæsium, in acting upon an excess of water, evolves 51,500 cals., and the reaction $\mathrm{Cs}_{2}+\mathrm{O}$ evolves about 100,000 cals. -i.e. more than $\mathrm{K}$ and $\mathrm{Rb}$, and almost as much as $\mathrm{Na}$-and oxide of crsium reacts with hydrogen (according to the equation $\mathrm{Cs}_{2} \mathrm{O}+\mathrm{H}=\mathrm{Cs} \mathrm{HO}+\mathrm{Cs}$ ) more easily than any of the oxides of the alkali metals, and this reaction takes place at the ordinary temperature (the hydrogen is absorbed), as Beketoff showed (1893). He also obtained a mixed oxide, $\mathrm{AgCsO}$, which was easily formed in the presence of silver, and absorbed hydrogen with the formation of CsHO. 
hydroxides $\mathrm{RHO}$ and carbonates $\mathrm{R}_{2} \mathrm{CO}_{3}$ are soluble in water, whilst the hydroxides and carbonates of nearly all other metals are insoluble, shows that these metals form a natural group of alkali meta?s. The halogens and the alkali metals form, by their character, the two extremes of the elements. Many of the other elements are metals approaching the alkali metals, both in their capacity of forming salts and in not forming acid compounds, but are not so energetic as the alkali metals, that is, they form less energetic bases. Such are the common metals, silver, iron, copper, \&c. Some other elements, in the character of their compounds, approach the halogens, and, like them, combine with hydrogen, but these compounds do not show the energetic property of the halogen acids; in a free state they easily combine with metals, but they do not then form such saline compounds as the halogens do-in a word, the halogen properties are less sharply defined in them than in the halogens themselves. Sulphur, phosphorus, arsenic, \&c. belong to this order of elements. The clearest distinction of the properties of the halogens and alkali metals is expressed in the fact that the former give acids and do not form bases, whilst the latter, on the contrary, only give bases. The first are true acid elements, the latter clearly-defined basic or metallic elements. On combining together, the halogens form, in a chemical sense, unstable compounds, and the alkali metals alloys in which the character of the metals remains unaltered, just as in the compound ICl the character of the halogens remains undisguised; thus both classes of elements on combining with members of their own class form non-characteristic compounds, which have the properties of their components. On the other hand, the halogens on combining with the alkali metals form compounds which are, in all respects, stable, and in which the original characters of the halogens and alkali metals have entirely disappeared. The formation of such compounds is accompanied by evolution of a large amount of heat, and by an entire change of both the physical, and chemical properties of the substances originally taken. The alloy of sodium and potassium, although liquid at the ordinary temperature, is perfectly metallic, like both its components. The compound of sodium and chlorine has neither the appearance nor the properties of the original elements; sodium chloride melts at a higher temperature, and is more difficultly volatile, than either sodium or chlorine.

With all these qualitative differences there is, however, an important quantitative resemblance between the halogens and the alkali metals. This. resemblance is clearly expressed by stating that both orders of elements belong to those which are univalent with respect to hydrogen. It is thus correct to say that both the above-named orders of ele. 
ments replace hydrogen atom for atom. Chlorine is able to take the place of hydrogen by metalepsis, and the alkali metals take the place of hydrogen in water and acids. As it is possible to consecutively re. place every equivalent of hydrogen in a hydrocarbon by chlorine, so it is possible in an acid containing several equivalents of hydrogen to replace the hydrogen consecutively equivalent after equivalent by an alkali metal; hence an atom of these elements is analogous to an atom of hydrogen, which is taken, in all cases, as the unit for the comparison of the other elements. In ammonia, and in water, chlorine and sodium are able to bring about a direct replacement. According to the law of substitution, the formation of sodium chloride, $\mathrm{NaCl}$, at once shows the equivalence of the atoms of the alkali metals and the halogens. The halogens and hydrogen and the alkali metals combine with such elements as oxygen, and it is easily proved that in such compounds one atom of oxygen is able to retain two atoms of the halogens, of hydrogen, and of the alkali metals. For this purpose it is enough to compare the compounds $\mathrm{KHO}, \mathrm{K}_{2} \mathrm{O}, \mathrm{HClO}$, and $\mathrm{Cl}_{2} \mathrm{O}$, with water. It must not be forgotten, however, that the halogens give, with oxygen, besides compounds of the type $\mathrm{R}_{2} \mathrm{O}$, higher acid grades of oxidation, which the alkali metals and hydrogen are not capable of forming. We shall soon see that these relations are also subject to a special law, showing a gradual transition of the properties of the elements from the alkali metals to the halogens. ${ }^{43}$

The atomic weights of the alkali metals, lithium 7, sodium 23, potassium 39 , rubidium 85 , and cæsium 133 , show that here, as in the class of halogens, the elements may be arranged according to their atomic weights in order to compare the properties of the analogous compounds of the members of this group. Thus, for example, the platinochlorides of lithium and sodium are soluble in water; those

45 We may here observe that the halogens, and especially iodine, may play the part of metals (hence iodine is more easily replaced by metals than the other halogens, and it approaches nearer to the metals in its physical properties than the other halogens). Schiitzenberger obtained a compound $\mathrm{C}_{2} \mathrm{H}_{3} \mathrm{O}(\mathrm{OCl})$, which he called chlorine acetate, by acting on acetic anhydride, $\left(\mathrm{C}_{2} \mathrm{H}_{3} \mathrm{O}\right)_{2} \mathrm{O}$, with chlorine monoxide, $\mathrm{Cl}_{2} \mathrm{O}$. With iodine this compound gives off chlorine and forms iodine acetate, $\mathrm{C}_{2} \mathrm{H}_{3} \mathrm{O}(\mathrm{OI})$, which also is formed by the action of iodine chloride on sodium acetate, $\mathrm{C}_{2} \mathrm{H}_{3} \mathrm{O}(\mathrm{ONa})$. These compounds are evidently nothing else than mixed anhydrides of hypochlorous and hypoiodous acids, or the products of the substitution of hydrogen in RHO by a halogen (see Chapter XI., Notes 29 and 78 bis). Such compounds are very unstable, decompose with an explosion when heated, and are changed by the action of water and of many other reagents, which is in sccordance with the fact that they contain very closely allied elements, as does $\mathrm{Cl}_{2} \mathrm{O}$ itself, or ICl or KNa. By the action of chlorine monoxide on a mixture of iodine and acetic anhydride, Schuitzenberger also obtained the compound $\mathrm{I}\left(\mathrm{C}_{2} \mathrm{H}_{3} \mathrm{O}_{2}\right)_{3}$, which is analogous to $\mathrm{ICl}_{3}$, because the groun $\mathrm{C}_{2} \mathrm{H}_{3} \mathrm{O}_{2}$ is, like $\mathrm{Cl}$, a halogen, forming salts with the metals. Similar properties are found in iodosobenzene (Chapter XI., Note 79). 
of potassium, rubidium, and cæsium sparingly soluble, and the greater the atomic weight of the metal the less soluble is the salt. In other cases the reverse is observed-the greater the atomic weight the more soluble are the corresponding salts. The variation of properties with the variation in atomic weights even shows itself in the metals themselves; thus lithium volatilises with difficulty, whilst sodium is obtained by distillation, potassium volatilises more. easily than sodium, and rubidium and crsium as we have seen, are still more volatile. 


\section{CHAPTER XIV}

\section{THE VALENCY AND SPECIFIC HEAT OF THE METALS. MAGNESIOM. CALCIUM, STRONTIUM, BARIUM, AND BERTLLIUM}

Ir is easy by investigating the composition of corresponding com. pounds, to establish the equivalent weights of the metals compared with hydrogen-that is, the quantity which replaces one part by weight of hydrogen. If a metal decomposes acids directly, with the evolution of hydrogen, the equivalent weight of the metal may be determined by taking a definite weight of it and measuring the volume of hydrogen evolved by its action on an excess of acid; it is then easy to calculate the weight of the hydrogen from its volume. ${ }^{1}$ The same result may be arrived at by determining the composition of the normal salts of the metal ; for instance, by finding the weight of metal which combines with 35.5 parts of chlorine or 80 parts of bromine. ${ }^{2}$ The equivalent of a metal may be also ascertained by simultaneously (i.e. in one circuit) decomposing an acid and a fused salt of a given metal by an electric current and determining the relation between the amounts of hydrogen and metal separated, because, according to Faraday's law, electrolytes (conductors of the second order) are always decomposed in equivalent quantities. $^{2}$ bis The equivalent of a metal may even be found by simply

1 Under favourable circumstances (by taking all the requisite precautions), the weight of the equivalent may be accurately determined by this method. Thus Reynolds and Ramsay (1887) determined the equivalent of zinc to be 32.7 by this method (from the average of 29 experiments), whilst by other methods it has been fixed (by different observers) between 32.55 and 33.95 .

The differences in their equivalents may be demonstrated by taking equal weights of different metals, and collecting the hydrogen evolved by them (under the action of an acid or alkali).

2 The most accurate determinations of this kind were carried on by Stas, and will be described in Chapter XXIV.

$2 \mathrm{bis}$ The amount of electricity in one coulomb according to the present nomenclature of electrical units (see Works on Physics and Electro-technology) disengages 0.00001036 gram of hydrogen, 0.00112 gram of silver, 0.0003263 gram of copper from the salts of the oxide, and 0.0006526 gram from the salts of the suboxide, \&c. These amounts stand in the same ratio as the equivalents, i.e. as the quantities replaced by one part by weight of hydrogen. The intimate bond which is becoming more and more marked existing between the electrolytic and purely chemical relations of substances (especially in solutions) and the application of electrolysis to the preparation of numerous substances 
determining the relation between its weight and that of its salt. giving oxide, as by this we know the quantity of the metal which combines with 8 parts by weight of oxygen, and this will be the equivalent, because 8 parts of oxygen combine with 1 part by weight of hydrogen. One method is verified by another, and all the processes for the accurate determination of equivalents require the greatest care to avoid the absorption of moisture, further oxidation, volatility, and other accidental influences which affect exact weighings. The description of the methods necessary for the attainment of exact results belongs to the province of analytical chemistry.

For univalent metals, like those of the alkalis, the weight of the equivalent is equal to the weight of the atom. For bivalent metals the atomic weight is equal to the weight of two equivalents, for $n$-valent metals it is equal to the weight of $n$ equivalents. Thus aluminium, $\mathrm{Al}=27$, is trivalent, that is, its equivalent $=9$; magnesium, $\mathrm{Mg}=24$, is bivalent, and its equivalent $=12$. Therefore, if potassium or sodium, or in general a univalent metal, $\mathrm{M}$, give compounds $\mathrm{M}_{2} \mathrm{O}, \mathrm{MHO}$, $\mathrm{MCl}, \mathrm{MNO}_{3}, \mathrm{M}_{2} \mathrm{SO}_{4}$, \&c., and in general $\mathrm{MX}$, then for bivalent metals like magnesium or calcium the corresponding compounds will be $\mathrm{MgO}, \mathrm{Mg}(\mathrm{HO})_{2}, \mathrm{MgCl}_{2}, \mathrm{Mg}\left(\mathrm{NO}_{3}\right)_{2}, \mathrm{MgSO}_{4}$, \&c., or in general $\mathrm{MX}_{2}$.

By what are we to be guided in ascribing to some metals univalency and to others bi-, ter-, quadri-, n-valency? What obliges us to make this difference? Why are not all metals given the same valency-for instance, why is not magnesium considered as univalent?

on a large scale, together with the employment of electricity for obtaining high temperatures, \&c., makes me regret that the plan and dimensions of this book, and the impossibility of giving a concise and objective exposition of the necessary electrical facts, prevent $\mathrm{my}$ entering upon this province of knowledge, although I consider it my duty to recommend its study to all those who desire to take part in the furthen develop. ment of our science.

There is only one side of the subject respecting the direct correlation between therma chemical data and electro-motive force, which I think right to mention here, as it justifies the general conception, enunciated by Faraday, that the galvanic current is an aspect of the transference of chemical motion or reaction along the conductors.

From experiments conducted by Favre, Thomsen, Garni, Berthelot, Cheltzoff, and others, upon the amount of heat evolved in a closed circuit, it follows that the electro. motive force of the current or its capacity to do a certain work, $\mathrm{E}$, is proportional to the whole amount of heat, $Q$, disengaged by the reaction forming the source of the ourrent. If $E$ be expressed in volts, and $Q$ in thousands of units of heat referred to equivalent weights, then $\mathrm{E}=0.0436 \mathrm{Q}$. For example in a Daniells battery $\mathrm{E}=1.09$ both by experiment and theory, because in it there takes place the decomposition of $\mathrm{CuSO}_{4}$ into $\mathrm{Cu}+\mathrm{O}$ together with the formation of $\mathrm{Zn}+\mathrm{O}$ and $\mathrm{ZnO}+\mathrm{SO}_{3} \mathrm{Aq}$, and these reactions correspond to $Q=25.06$ thousand units of heat. So also in all other primary batteries (e.g. Bunsen's, Poggendorff's, \&c.) and secondary ones (for instance, those acting acoording to the reaction $\mathrm{Pb}+\mathrm{H}_{2} \mathrm{SO}_{4}+\mathrm{PbO}_{2}$, as Cheltzofis showed) $\mathrm{E}=0.0436 \mathrm{Q}$. 
If this be done, taking $\mathrm{Mg}=12$ (and not 24 as now), not only is a simplicity of expression of the composition of all the compounds of magnesium attained, but we also gain the advantage that their composition will be the same as those of the corresponding compounds of sodium and potassium. These combinations were so expressed formerly -why has this since been changed?

These questions could only be answered after the establishment of the idea of multiples of the atomic weights as the minimum quantities of certain elements combining with others to form compounds-in a word, since the time of the establishment of Avogadro-Gerhardt's law (Chapter VII.). By taking such an element as arsenic, which has many volatile compounds, it is easy to determine the density of these compounds, and therefore to establish their molecular weights, and hence to find the indubitable atomic weight, exactly as for oxygen, nitrogen, chlorine, carbon, \&cc. It appears that $A s=75$, and its compounds correspond, like the compounds of nitrogen, with the forms $\mathrm{AsX}_{3}$, and $\mathrm{AsX}_{5}$; for example, $\mathrm{AsH}_{3}, \mathrm{AsCl}_{3}, \mathrm{AsFl}_{5}, \mathrm{As}_{2} \mathrm{O}_{5}$, \&c. It is evident that we are here dealing with a metal (or rather element) of two valencies, which moreover is never univalent, but tri- or quinquivalent. This example alone is sufficient for the recognition of the existence of polyvalent atoms anong the metals. And as antimony and bismuth are closely analogous to arsenic in all their compounds, (just as potassium is analogous to rubidium and cæsium); so, although very few volatile compounds of bismuth are known, it was necessary to ascribe to them formulæ corresponding with those ascribed to arsenic.

As we shall see in describing them, there are also many analogous metals among the bivalent elements, some of which also give volatile compounds. For example, zinc, which is itself volatile, gives several volatile compounds (for instance, zinc ethyl, $\mathrm{ZnC}_{4} \mathrm{H}_{10}$, which boils at $118^{\circ}$, vapour density $\left.=61.3\right)$, and in the molecules of all these com. pounds there is never less than 65 parts of zinc, which is equivalent to $\mathrm{H}_{2}$, because 65 parts of zinc displace 2 parts by weight of hydrogen ; so that zinc is just such an example of the bivalent metals as oxygen, whose equiralent $=8$ (because $\mathrm{H}_{2}$ is replaced by $\mathrm{O}=16$ ), is a representative of the bivalent elements, or as arsenic is of the tri- and quinqui-valent elements. And, as we shall afterwards see, magnesium is in many respects closely analogous to zinc, which fact obliges us to regard magnesium as a bivalent metal.

Such metals as mercury and copper, which are able to give not one but two bases, are of particular importance for distinguishing univalent and bivalent metals. Thus copper gives the suboxide $\mathrm{Cu}_{2} \mathrm{O}$ and the 
oxide $\mathrm{CuO}$ - that is, the compounds $\mathrm{CuX}$ corresponding with the sub. oxide are analogous (in the quantitative relations, by their composition) to $\mathrm{NaX}$ or $\mathrm{AgX}$, and the compounds of the oxide $\mathrm{CuX}_{2}$, to $\mathrm{MgX}_{2}$, $\mathrm{ZnX}_{2}$, and in general to the bivalent metals. It is clear that in such examples we must make a distinction between atomic weights and equivalents.

In this manner the valency, that is, the number of equivalents entering into the atom of the metals may in many cases be established by means of comparatively fow volatile metallic compounds, with the aid of a search into their analogies (concerning which see Chapter XV.). The law of specific heats discovered by Dulong and Petit has frequently been applied to the same purpose ${ }^{3}$ in the history of chemistry, especially since the development given to this law by the researches of Regnault, and since Cannizzaro (1860) showed the agreement between the deductions of this law and the consequences arising from ArogadroGerhardt's law.

Dulong and Petit, having determined the specific heat of a number of solid elementary substances, observed that as the atomic weights of the elements increase, their specific heats decrease, and that the product

3 The chief means by which we determine the valency of the elements, or what multiple of the equivalent should be ascribed to the atom, are: (1) The law of AvogadroGexhardt. This method is the most general and trustworthy, and has already been applied to a great number of elements. (2) The different grades of oxidation and their isomorphism or analogy in general; for example, $F_{\theta}=56$ because the suboxide (ferrou's oxide) is isomorphous with magnesium oxide, \&c., and the oxide (ferric oxide) contains half as much oxygen again as the suboxide. Berzelins, Marignac, and others took advantage of this method for determining the oomposition of the compounds of many elements. (8) The specific heat, according to Dulong and Petit's law. Regnault, and more especially Cannizzaro, used this method to distinguish univalent from bivalent metals. (4) The periodio law (see Chapter XV.) has served as a means for the determination of the atomic weights of cerrium, uranium, yttrium, \&o., and more especially of gallium, soandium, and germanium. The correction of the results of one method by those of others is generally had recourse to, and is quite necessary, because, phenomena of dissooiation, polymerisation, \&a., may complicate the individual determinations by each mothod.

It will be well to observe that a number of other methods, especially from the province of those physicel properties which are clearly dependent on the magnitude of the atom (or.equivalent) or of the molechule, may lead to the same result. I may point out, for instance, that even the specifio gravity of solutions of the metallic chlorides may serve lor this purpose. Thus, if beryllinm be taken as trivalent-that is; if the composition of Its chloride be taken as $\mathrm{BeCl}_{3}$. (or a polymeride of it), then the speoific gravity of colutions of bergllium ohloride, will not fit into the series of the other metallio chlorides. But by asoribing to it an atomio weight $\mathrm{B}_{\theta}=7$, or taking $\mathrm{B}_{\theta}$ as bivalent, and the composition of its ohloride as $\mathrm{BeCl}_{2}$, we arrive at the general rule given in Chapter VII., Note 28. Thus W. G. Burdakoff determined in my laboratory that the speciflo gravity at $15^{\circ} / 4^{\circ}$ of the solution $\mathrm{BeCl}_{2}+200 \mathrm{H}_{2} \mathrm{O}=1.0138$ - that is, greater than the corresponding solution $\mathrm{XOl}+800 \mathrm{H}_{2} \mathrm{O}(-1.0121)$, and less than the solution $\mathrm{MgCl}_{2}+200 \mathrm{H}_{2} \mathrm{O}(=1.0208)$, as would follow from the magnitude of the molecular weight $\mathrm{BeCl}_{2}=80$, sinco $\mathrm{KOl}=74 \cdot 5$ and $\mathrm{MgCl}_{2}=95$. 
of the specific heat $Q$ into the atomic weight $A$ is an almost constant quantity. This means that to bring different elements into a known thermal state an equal anount of work is required if atomic quantities of the elements are taken; that is, the amounts of heat expended in heating equal quantities by weight of the elements are far from equal, but are in inverse proportion to the atomic weights. For thermal changes the atom is a unit; all atoms, notwithstanding the difference of weight and nature, are equal. This is the simplest expression of the fact discovered by Dulong and Petit. The specific heat measures that quantity of heat which is required to raise the temperature of one unic of weight of a substance by one degree. If the magnitude of the specific heat of elements be multiplied by the atomic weight, then we obtain the atomic heat-that is, the amount of heat required to raise the temperature of the atomic weight of an element by one degree. It is these products which for the majority of the elements prove to be approximately, if not quite, identical. A complete identity cannot be expected, because the specific heat of one and the same substance varies with the temperature, with its passage from one state into another, and frequently with even a simple mechanical change of density (for instance by hammering), not to speak of allotropic changes, \&c. We will cite several figures ${ }^{4}$ proving the truth of the conclu-

+ The specifio heats here given refer to different limits of temperature, but in the majority of cases between $0^{\circ}$ and $100^{\circ}$; only in the case of bromine the specifio heat is taken (for the solid state) at a temperature below $-7^{\circ}$, according to Regnault's determination. The variation of the specifio heat with a change of temperature is a very complex phenomenon, the consideration of which I think would here be out of place. I will only cite a fow figures as an example. According to Bystrom, the specifio heat of iron at $0^{\circ}=0.1116$, at $100^{\circ}=0.1114$, at $200^{\circ}=0.1188$, at $300^{\circ}=0.1267$, and at $1,400^{\circ}$ $=0.4081$. Between these last limits of temperature a change takes place in iron (a spontaneous heating, recalescence), as we shall see in Chapter XXII. For quartz $\mathrm{SiO}_{2}$ Pionchon gives $Q=0 \cdot 1737+894 t 10^{-0}-27 t^{2} 10^{-0}$ up to $400^{\circ}$, for metallio aluminium (Richards, 1892) at $0^{\circ} 0.222^{\circ}$, at $20^{\circ} 0.224$, at $100^{\circ} 0.232$; consequently, as a rule, the specific heat varies slightly with the temperature. Still more remarkable are H. E. Weber's observations on the great variation of the specifio heat of charcoal, the diamond and boron:

\begin{tabular}{llllll|} 
Wood charcoal & $0^{\circ}$ & $100^{\circ}$ & $200^{\circ}$ & $600^{\circ}$ & $800^{\circ}$ \\
Diamond & 0.15 & 0.23 & 0.29 & 0.44 & 0.46 \\
Boron & 0.10 & 0.19. & 0.22 & 0.44 & 0.45 \\
\hline & 0.22 & 0.29 & 0.85 & - & -
\end{tabular}

These determinations, which have been verifed by Dewar, Le Chatelier (Chapter VIII., Note 18), Moissan, and Gauthier, the latter finding for boron $\mathrm{AQ}=6$ at $400^{\circ}$, are of especial importance as confirming the universality of Dulong and Petit's law, because the elements mentioned above form exceptions to the general rule when the mean specifio heat is taken for temperatures between $0^{\circ}$ and $100^{\circ}$. Thns in the case of the diamond the product of $A \times Q$ at $0^{\circ}=1 \cdot 2$, and for boron $=2 \cdot 4$. But if we take-the specifio heat towards which-there is evidently a tendency with a rise of temperature, we obtain a product approaching to 6 as with other elements. Thus with the diamond and charcoal, it is evident that the specific heat tends towards 0.47 , which multipled by 12 
sions arrived at by Dulong and Petit with respect to solid elenentary bodies.

\begin{tabular}{|c|c|c|c|c|}
\hline & In & $\mathrm{Na}$ & $\mathrm{Mg}$ & \\
\hline $\mathbf{A}=$ & 7 & 23 & 24 & 31 \\
\hline $\mathbf{Q}=$ & 0.9408 & $0 \cdot 2934$ & 0.245 & 0.202 \\
\hline \multirow[t]{2}{*}{$\mathbf{A Q}=$} & $6 \cdot 59$ & 6.75 & $5 \cdot 88$ & $6 \cdot 26$ \\
\hline & $\mathrm{F}_{\theta}$ & $\mathrm{Cu}$ & $\mathrm{Zn}$ & $\mathrm{Br}$ \\
\hline$A=$ & 56 & 63 & 65 & 80 \\
\hline $\mathbf{Q}=$ & 0.112 & 0.093 & 0.093 & 0.0843 \\
\hline $\mathrm{AQ}=$ & $6 \cdot 27$ & $5 \cdot 86$ & 6.04 & $6 \cdot 74$ \\
\hline $\mathbf{A}=$ & $106^{\mathrm{Pd}}$ & $108^{\mathrm{Ag}}$ & $118^{\text {Ṣn }}$ & $127^{I}$ \\
\hline $\mathbf{Q}=$ & 0.0592 & 0.056 & 0.055 & 0.541 \\
\hline $\mathbf{A Q}=$ & $6 \cdot 28$ & 6.05 & $6 \cdot 49$ & $6 \cdot 87$ \\
\hline$A=$ & 196 & $198^{\mathrm{Au}}$ & $200^{\mathrm{Hg}}$ & $206^{\mathrm{Pb}}$ \\
\hline $\mathbf{Q}=$ & 0.0325 & 0.0324 & 0.0333 & 0.0315 \\
\hline $\mathrm{AQ}=$ & 6.37 & $6 \cdot 41$ & $6 \cdot 66$ & $6 \cdot 49$ \\
\hline
\end{tabular}

It is seen from this that the product of the specific heat of the element into the atomic weight is an almost constant quarity, which is nearly 6. Hence it is possible to determine the valency by the specific heats of the metals. Thus, for instance, the specific heats of lithium, sodium, and potassium convince us of the fact that their atomic weights are indeed those which we chose, because by

gives 5.6, the same as for magnesium and aluminium. I may here direct the reader's attention to the fact that for solid elements having a small atomic weight, the epecifio heat varies considerably if we take the average figures for temperatures $0^{\circ}$ to $100^{\circ}$ :

$\begin{array}{rlcc}\mathrm{Li}=7 & \mathrm{~B}_{0}=9 & \mathrm{~B}=11 & \mathrm{C}=12 \\ \mathbf{Q}=0.94 & -0.42 & 0.24 & 0.20 \\ \mathbf{A Q}=6.6 & 8.8 & 2.6 & 2.4\end{array}$

It is therefore clear that the specific heat of beryllium determined at a low temperature cannot serve for establishing its atomicity. On the other hand, the low atomio heat of charcoal, graphite, and the diamond, boron, \&o., may perhaps depend on the complexity of the molecules of these elements. The necessity for acknowledging a great complexity of the molecules of carbon was explained in Chapter VIII. In the case of sulphur the molecule contains at least $S_{6}$ and its atomic heat $=82 \times 0 \cdot 163=5.22$, which is distinotly below the normal. If a large number of atoms of carbon are contained in the molecule of charcoal, this would to a certain extent account for its comparatively small atomic heat. With respect to the specific heat of compounds, it will not be out of place to mention here the conclusion arrived at by Kopp, that the molecular heat (that is, the product of MQ) may be looked on as the sum of the atomic heats of its component elements; but as this rule is not a general one, and can only be applied to give an approximate estimate of the specific heats of substances, I do not think it necessary to go into the details of the conclusions described in Liebig's 'Annalen Supplement-Band,' 1864; which includes a number of detarminations made by Kopp. 
multiplying the specific heats found by experiment by the corresponding atomic weights we obtain the following figures: $\mathrm{Li}, 6.59, \mathrm{Na}, 6.75$ and $K, 6 \cdot 47$. Of the alkaline earth metals the specitic heats have been determined : of magnesium $=0.245$ (Regnault and Kopp), of calcium $=0.170$ (Bunsen); and of barium $=0.05$ (Mendeleetf). If the same composition be ascribed to the compounds of magnesium as to the corresponding compounds of potassium, then the equiralent of mag nesium will be equal to 12 . On multiplying this atomic weight by the specific heat of magnesium, we obtain a figure 2.94 , which is half that which is given by the other solid elements and therefore the atomio weight of magnesium must be taken as equal-to 24 and not to 12 . Then the atomic heat of magnesium $=24 \times 0.245=5.9$; for calcium, giving its compounds a composition $\mathrm{CaX}_{2}$-for example $\mathrm{CaCl}_{2}, \mathrm{CaSO}_{4}$, $\mathrm{CaO}(\mathrm{Ca}=40)$-we obtain an atomic heat $=40 \times 0.17=6.8$, and for barium it is equal to $137 \times 0.05=6.8$; that is, they must be counted as bivalent, or that their atom replaces $\mathrm{H}_{2}, \mathrm{Na}_{2}$, or $\mathrm{K}_{2}$. This conclusion may be confirmed by a method of analogy, as we shall afterwards see. The application of the principle of specific heats to the determination of the magnitudes of the atomio weights of those metals, the ragnitude of whose atomic weights could not be determined by Avogadro-Gerhardt's law, was made about 1860 by the Italian professor Cannizzaro.

Exactly the same conclusions respecting the bivalence of magnesium and its analogues are obtained by comparing the specific heats of their compounds, especially of the halogen compounds as the most simple, with the specific heats of the corresponding alkali compounds. Thus, for instance, the specific heats of magnesium and calcium chlorides, $\mathrm{MgCl}_{2}$ and $\mathrm{CaCl}_{2}$, are 0.194 and 0.164 , and of sodium and potassium chlorides, $\mathrm{NaCl}$ and $\mathrm{KCl}, 0.214$ and 0.172 , and therefore their molecular heats (or the products $\mathrm{QM}$, where $\mathrm{M}$ is the weight of the molecule) are 18.4 and $18.2,12.5$ and 12.8 , and hence the atomic heats (or the quotient of QM by the number of atoms) are all nearly 6, as with the elements. Whilst if, instead of the actual atomic weights $\mathrm{Mg}=24$ and $\mathrm{Ca}=40$, their equivalents 12 and 20 be taken, then the atomic heats of the chlorides of magnesium and calcium would be about 4.6, whilst those of potassium and sodium chlorides are about $6.3 .^{5}$ We

5 It must be remarked that in the case of oxygen (and also hydrogen and carbon) compounds the quotient of $\mathrm{MQ} / n$, where $n$ is the number of atoms in the molecule, is always less than 6 for solids; for example, for $\mathrm{MgO}=5 \cdot 0, \mathrm{CuO}=5 \cdot 1, \mathrm{MnO}_{2}=4 \cdot 6$, ice $(Q=0.504)=3, \mathrm{SiO}_{2}=3 \cdot 5$, \&c. At present it is impossible to say whether this depends on the smaller specifio heat of the atom of oxygen in its solid compounds (Kopp, Note 4) or on some other cause; but, nevertheless, taking into account this decrease depending on the presence of oxygen, a reflection of the atomicity of the elements may to a certain 
must remark, however, that as the specific heat or the amount of heat required to raise the temperature of a unit of weight one degree ${ }^{6}$ is a

extent be seen in the specifio heat of the oxides. Thus for alumina, $\mathrm{Al}_{2} \mathrm{O}_{5}(\mathrm{Q}=0.217)$, $M Q=22 \cdot 3$, and therefore the quotient $M Q / n=4 \cdot 5$, which is nearly that given by magnesium oxide, $\mathrm{MgO}$. But if we ascribe the same composition to alumina, as to magnesia-that is, if aluminium were counted as divalent-we should obtain the figure 8.7, which is much less. In general, in compounds of identical atomic composition and of analogous chemical properties the molecular heats MQ are nearly equal, as many investigators have long remarked. For example, $\mathrm{ZnS}=11.7$ and $\mathrm{HgS}=11.8$; $\mathrm{MgSO}_{4}=27.0$ and $\mathrm{ZnSO}_{4}=28 \cdot 0$, \&c.

- If $W$ be the amount of heat contained in a mass $m$ of a substance at a temperature s, and $d W$ the amount expended in heating it from $t$ to $t+d t$, then the specific heat $Q=d W /(m \times d t)$. The specifio heat not only varies with the composition and complexity of the molecules of a substance, but also with the temperature, pressure, and physical state of a substance. Even for gases the variation of $Q$ with $t$ is to be observed. Thus it is seen from the experiments of Regnault and Wiedemnnn that the specific heat of carbonic anhydride at $0^{\circ}=0.19$, at $100^{\circ}=0.22$, and at $200=0.24$. But the variation of the specific heat of permanent gases with the temperature is, as far as we know, very in. considerable. According to Mallard and $\mathrm{L}_{\theta}$ Chatelier it is $=\frac{0.0006}{\mathrm{M}}$ per $1^{\circ}$, where $\mathrm{M}$ is the molecular weight (for instance, for $\mathrm{O}_{2}, \mathrm{M}=82$ ). Therefore the speciflo heat of those permanent gases which contain two atoms in the molecule $\left(\mathrm{H}_{2}, \mathrm{O}_{2}, \mathrm{~N}_{2}, \mathrm{CO}\right.$, and NO) may be, as is shown by experiment, taken as not varying with the temperature. The constancy of the specific heat of perfect gases forms one of the fundamental propositions of the whole theory of heat and on it depends the determination of temperatures by means of gas-thermometers contsining hydrogen, nitrogen, or air. Le Chatelier (1887), on the basis of existing determinations, concludes that the molecular heat-that is, the product $\mathrm{MQ}-$ of all gases varies in proportion to the temperature, and tends to become equal $(=6.8)$ at the temperature of absolute zero (that is, at $\left.-273^{\circ}\right)$; and therefore $M Q=6 \cdot 8+a(273+t)$, whete $a$ is a constant quantity which increases with the complexity of the gaseous molecule and $Q$ is the specific heat of the gas under a constant pressure. For permanent gases $a$ almost $=0$, and therefore $M Q=6.8$-that is, the atomic heat (if the molecule contains two atoms) $=3 \cdot 4,28$ it is in fact (Chapter IX., Note 17 ble). As regards liquids (as we as the vapours formed by them), the specifie heat always rises with the temperature. Thus for benzene it equals $0.38+0.0014 t$. R. Sohiff (1887) showed that the variation of the specific heat of many organio liquids is proportional to the change of temperature (as in the case of gases, according to Le Chatelier), and reduced these variations into dependence with their composition and absolute boiling point. It is very probable that the theory of liquids will make use of these simple relations which recall the simplicity of the variation of the specifio gravity (Chapter II., Note 34), cohesion, and other properties of liquids with the lemperature. They are all expressed by the linear function of the temperature, $a+b t$, with the same degree of proximity as the property of gases is expressed by the equátion $p v=R t$.

As regards the relation between the specific heats of liquids (or of solids) and of their vapours, the specifio heat of the vapour (and also of the solid) is always less than that of the liquid. For example, benzene vapour 0.22 , liquid 0.88 ; chloroform vapour 0.18 , liquid 0.28 ; steam 0.475 , liquid water 1.0 . But the complexity of the relations existing in specifio heat is seen from the fact that the specifio heat of ice $=0.502$ is less than that of liquid water. According to Regnault, in the case of bromine the speoific heat of the vapour $=0.055$ at $\left(150^{\circ}\right)$, of the liquid $=0.107$ (at $\left.30^{\circ}\right)$, and of solid bromine $=0.084\left(a t-15^{\circ}\right)$. The specifio heat of solid benzoic acid (according to experiment and calculation, Hess, 1888) between $0^{\circ}$ and $100^{\circ}$ is 0.81 , and of liquid benzoic acid 0.50. One of the problems of the present day is the explanation of those complex relations which exist between the composition and such properties as specific heat, latent heat, expansion by heat, compression, internal friction, cohesion, and so forth. They ann 
complex quantity-including not only the increase of the energy of a substance with its rise in temperature, but also the external work of expansion ${ }^{7}$ and the internal work accomplished in the molecules only be connected by a complete theory of liquids, which may now soon be expected, more especially as many sides of the subject have already been partially explained.

7 According to the above reasons the quantity of heat, $Q$, required to raise the tent. perature of one part by weight of a substance by one degree may be expressed by the sum $Q=K+B+D$, where $K$ is the heat actually expended in heating the substance, or what is termed the absolute specific heat, B the amount of heat expended in the internal work accomplished with the rise of temperature, and D the amount of heat ex. pended in external work. In the case of gases the last quantity may be easily detertmined, knowing their coefficient of expansion, which is approximately $=0.00368$. By applying to this case the same argument given at the end of Note 11, Chapter I., we find that one cubio metre of a gas heated $1^{\circ}$ produces an external work of $10338 \times 0.00868$, or 88.02 kilogrammetres, on whioh $88.02,424$ or 0.0897 heat units are expended. This is the heat expended for the external work produced by one cubio metre of a gas, but the specific heat refers to units of weight, and therefore it is necessary in order to know D to reduce the above quantity to $a$ unit of weight. One cubic metre of hydrogen at $0^{\circ}$ and $760 \mathrm{~mm}$. pressure weighs 0.0896 kilo, a gas of molecular weight $M$ has a density M.'2, consequently a cubio metre weighs (at $0^{\circ}$ and $760 \mathrm{~mm}$.) $0.0448 \mathrm{M}$ kilo, and therefore 1 kilogram of the gas occupies a volume 1/0.0448M cubic metres, and henoe the external work $D$ in the heating of 1 kilo of the given gas through $1^{\circ}=0 \cdot 0896,0^{\circ} \cdot 0448 \mathrm{M}$, or $D=2 / \mathrm{M}$.

Taking the magnitude of the internal work $\mathbf{B}$ for gases as negligeable if permanent gases are taken, and therefore supposing $B=0$, we find the specific heat of gases at a constant pressure $Q=K+2 M$, where $K$ is the specifio heat. at a constant volume, or the true specific heat, and $M$ the molecular weight. Hence $K=Q-2 / M$. The magnitude of the specific heat $Q$ is given by direct experiment. According to Regnault's experiments, for oxygen it $=0.2175$, for hydrogen 3.405 , for nitrogen 0.2438 ; the molecular weights of these gases a,re 82,2 , and 28 , and therefore for oxygen $K=0 \cdot 2175-0 \cdot 0625=0 \cdot 1550$, for hydrogen: $K=8 \cdot 4050-1 \cdot 000=2 \cdot 4050$, and for nitrogen $K=0 \cdot 2438-0 \cdot 0714=0 \cdot 1724$. These true specifio heats of elements are in inverse proportion to their atomio weights that is, their product by the atomic weight is a constant quantity. In fact, for oxygen this product $=0 \cdot 155 \times 16=2 \cdot 48$, for hydrogen $2 \cdot 40$, for nitrogen $0 \cdot 7724 \times 14=2 \cdot 414$, and therefore if $\mathbf{A}$ stand for the atomio weight we obtain the expression $\mathbf{K} \times \mathbf{A}=\mathbf{a}$ constaint, which may be taken as 2.45. This is the true expression of Dulong and Petit's law, because $K$ is the true specific heat and $A$ the weight of the atom. It should be remarked, moreover, that the product of the observed specific heat $Q$ into $A$ is also a constant guantity (for oxygen $=8 \cdot 48$, for hydrogen $=3 \cdot 40$ ), because the external work $D$ is also inversely proportional to the atomic weight.

In the case of gases we distinguish the specific heat at a constant pressure of (wo designated this quantity above by $Q$ ), and at a constant volume $c$. It is evident that the relation between the two specific heats, $k$, judging. from the above, is the ratio of $\mathbf{Q}$ to $\mathrm{K}$, or equal to the ratio of $2 \cdot 45 n+2$ to $2 \cdot 45 n$. 'When $n=1$ this ratio $k=1 \cdot 8$; when $n=2, k=1 \cdot 4$, when $n=3, k=1 \cdot 3$, and with an excoedingly large number $n$, of atoms in the - molecule, $k=1$. That is, the ratio between the specifio heats decreases from 1.8 to 1.0 as the number of atoms, $n$, contained in the molecule increases. This deduotion is verified to a certain extent by direct experiment. For such gases as hydrogen, oxygen, nitrogen, carbonio oxide, air, and others in which $n=2$, the magnitude of $k$ is determined by methods described in works on physies (for example, by the ohange of cemperature with an alteration of pressure. by the velocity of sound, 80.) and is 'found in reality to be nearly 1.4, and for such gases as carbonio anhydride, nitric dioxide, and others it is nearly 1.8. Kundt and Warburg (1875), by means of the approximato method mentionod in Note 29, Chapter VII., determined $k$ for mercury vapour when $n=1$, and found it to bo $=1 \cdot 67$ - that is, a larger quantity than for atr, as would be expected from the abore.

It may be admitted that the true atomic heat of gases $=2 \cdot 48$, only under the condition 
causing them to decompose according to the rise of temperature ${ }^{8}$ therefore it: is impossible to expect in the magnitude of the specific heat the great simplicity of relation to composition which we see, for instance, in the density of gaseous substances. Hence, although the specific heat is one of the important means for determining the atomicity of the elements, still the mainstay for a true judgment of atomicity is only given by Avogadro-Gerhardt's law, i.e. this other method can only be accessory or preliminary, and when possible recourse should be had to the determination of the vapour density.

Among the bivalent metals the first place, with respect to their distribution in nature, is occupied by magnesium and calcium, just as sodium and potassium stand first amongst the univalent metals. The relation which exists between the atomic weights of these four metals confirms the above comparison. In fact, the combining weight of magnesium is equal to 24 , and of calcium 40 ; whilst the combining weights of sodium and potassium are 23 and 39 -that is, the latter

that they are distant from a liquid state, and do not undergo a chemical change when heated-that is, when no internal work is produced in them $(B=0)$. Therefore this work may to a certain extent be judged by the observed specific heat. Thus, for instance, for chlorine $(Q=0.12$, Regnault; $k=1.88$, according to Straker and Martin, and therefore $\mathrm{K}=0.09, \mathrm{MK}=6 \cdot 4$ ), the atomic heat (8.2) is much greater than for other gases containing two atoms in a molecule, and it must be assumed, therefore, that when it is heated some great internal work is accomplishied.

In order to generalise the facts concerning the speoific heat of gases and solids, it appears to me possible to accept the following general proposition: the atomic heat (that is, $\mathrm{AQ}$ or $\mathrm{QM} / n$, where $\mathrm{M}$ is the molecular weight and $n$ the number of molecules) is smaller (in solids it attains its highest value 6.8 and in gases 3.4 ), the more complex the molecule (i.e. the greater the number $(n)$ of atoms forming $i t$ ) and so much smaller, up to a certain point (in similar physical states) the smaller the mean atomic weight $M / n$.

8 As an example, it will be sufficient to refer to the specific heat of nitrogen tetroxide, $\mathrm{N}_{2} \mathrm{O}_{4}$, which, when heated, gradually passes into $\mathrm{NO}_{2}$-that is, chemical work of decom. position proceeds, which consumes heat. Spealking generally, specifio heat is a complex quantity, in which it is clear that thermal data (for instance, the heat of reaction) alone cannot give an idea either of chemical or of physical changes-individually, but always depend on an association of the one and the other. If a substance be heated from $t_{0}$ to $t_{1}$ it cannot but suffer a chemical change (that is, the state of the atoms in the molecules changes more or less in one way or another) if dissociation sets in at a temperature $t_{1}$. Even in the case of the elements whose molecules contain only one atom, a true chemical change is possible with a rise of temperature, because more heat is evolved in chemical reactions than that quantity which participates in purely physical changes. One gram of hydrogen (specific heat $=3.4$ at a constant pressure) cooled to the temperature of absolute zero will evolve altogether about one thousand units of heat, 8 grams of oxygen half this amount, whilst in combining together they evolve in the formation of 9 grams of water more than thirty times as much heat. Hence the store of chemical energy (that is, of the motion of the atoms, vortex, or other) is much greater than the physical store proper to the molecules, but it is the change accomplished by the former that is the canse of chemical transformations. Here we evidently touch on those limits of existing knowledge beyond which the teaching of science does not yet allow us to pass. Many new scientific discoveries have still to be made before this is possible. 
are one unit less than the former. ${ }^{9}$ They all belong to the number of light metals, as they have but a small specific gravity, in which respect they differ from the ordinary, generally known heavy, or ore, metals (for instance, iron, copper, silver, and lead), which are distinguished by a much greater specific gravity. There is no doubt that their low specific gravity has a significance, not only as a simple point of distinction, but also as- a property which determines the fundamental properties of these metals. Indeed, all the light metals have a series of points of resem blance with the metals of the alkalis; thus both magnesium and calcium, like the metals of the alkalis, decompose water (without the addition of acids), although not so easily as the latter metals. The process of the decomposition is essentially one and the same; for example, $\mathrm{Ca}+2 \mathrm{H}_{2} \mathrm{O}=\mathrm{CaH}_{2} \mathrm{O}_{2}+\mathrm{H}_{2}$-that is, hydiogen is liberated and a hydroxide of the metal formed. These hydroxides are bases which neutralise nearly all acids. However, the hydroxides $\mathrm{RH}_{2} \mathrm{O}_{2}$ of calcium and magnesium are in no respect so energetic as the hydroxides of the true metals of the alkalis; thus when heated they lose water, are not so soluble, develop less heat with acids, and form various salts, which are less stable and more easily decomposed by heat than the corresponding salts of sodium and potassium. Thus calcium and magnesium carbonates easily part with carbonic anhydride when ignited; the nitrates are also very easily decomposed by heat, calcium and magnesium oxides, $\mathrm{CaO}$ and $\mathrm{MgO}$, being left behind. The chlorides of magnesium and calcium, when heated with water, evolve hydrogen chloride, forming the corresponding hydroxides, and when ignited the oxides themselves. All these points are evidence of a weakening of the alkaline properties.

These metals have been termed the metals of the alkaline earths, because they, like the alkali metals, form energetic bases. They are called alkaline earths because they are met with in nature in a state of combination, forming the insoluble mass of the earth, and because as oxides, $\mathrm{RO}$, they themselves have an earthy appearance. Not a few salts of these metals are known which are insoluble in water, whilst the corresponding salts of the alkali metals are generally soluble-for example, the carbonates, phosphates, borates, and other salts of the alkaline earth metals are nearly insoluble. This enables us to separate the metals of the alkaline earths from the metals of the alkalis. For this purpose a solution of ammonium carbonate is added to a mixed solution of salts of both kinds of metals, when by a double decomposition the insoluble carbonates of the metals of the alkaline earths are formed

2 As if $\mathrm{NaH}=\mathrm{Mg}$ and $\mathrm{KEH} \equiv \mathrm{Cs}$, which is in accordance with thoir valency. $\mathrm{KH}$ includes two monovalent elements, and is a bivalent group like $\mathrm{Ca}$. 
and fall as a precipitate, whilst the metals of the alkalis remain in solution: $\mathrm{RX}_{2}+\mathrm{Na}_{2} \mathrm{CO}_{3}=\mathrm{RCO}_{3}+2 \mathrm{NaX}$.

We may here remark that the oxides of the metals of the alkaline earths are frequently called by special names : $\mathrm{MgO}$ is called magnesia or bitter earth ; $\mathrm{CaO}$, lime; $\mathrm{SrO}$, strontia ; and $\mathrm{BaO}$, baryta.

In the primary rocks the oxides of calcium and magnesium are combined with silica, sometimes in variable quantities, so that in some cases the lime predominates and in other cases the magnesium. The two oxides, being analogous to each other, replace each other in equivalent quantities. The various forms of augite, hornblende, or amphibole, and of similar minerals, which enter into the composition of uearly all rocks, contain lime and magnesia and silica. The majority of the primary rocks also contain alumina, potash, and soda. These rocks, under the action of water (containing carbonic acid) and air, give up lime and magnesia to the water, and therefore they are contained in all kinds of water, and especially in sea-water. The carbonates $\mathrm{CaCO}_{3}$ and $\mathrm{MgCO}_{3}$, frequently met with in nature, are soluble in an excess of water saturated with carbonic anlydride, ${ }^{10}$ and therefore many natural waters contain these salts, and are able to yield them when evaporated. However, one kilogram of water saturated with carbonio anhydride does.not dissolve more than three grams of calcium carbonate. By gradually expelling the carbonic anhydride from such water, an insoluble precipitate of calcium carbonate separates out. It may confidently be stated that the formation of the very widely distributed strata of calcium and magnesium carbonates was of this nature, because these strata are of a sedimentary character-that is, such as would be exhibited by a gradually accumulating deposit on the bottom of the sea, and, moreover, frequently containing the remains of marine plants, and animals, shells, \&c. It is very probable that the presence of these organisms in the sea has played the chief part in the precipitation of the carbonates from the sea water, because the plants absorb $\mathrm{CO}_{2}$, and many of the organisms $\mathrm{CaCO}_{3}$, and after death give deposits of carbonate of lime; for instance, chalk, which is almost entirely composed of the minute remains of the calcareous shields of such organisms. These deposits of calcium and magnesium carbonates are the most important sources of these metals. Lime generally predominates, because it is present in rocks and running water in greater quantity than magnesia, and in this case these sedimentary rocks are

10 Sodium carbonate and other carbonates of the alkalis give acid salts which are les. soluble than the normal; here, on the contrary, with an excess of carbonic anbydride, a salt is formed which is more soluble than the normal, but this acid salt is more unstable than sodium hydrogen carbonate, $\mathrm{NaHCO}_{3}$. 
termed limestone. Some common flagstonès used for paving, \&c., and chalk may be taken as examples of this kind of formation. Those limestones in which a considerable portion of the calcium is replaced by magnesium are termed dolomites. The dolomites are distinguished by their hardness, and by their not parting with the whole of their carbonic anhydride so easily as the limestones under the action of acids. Dolomites "sometimes contain an equal number of molecules of calcium aarbonate and magnesium carbonate, and they also sometimes appear in a crystalline form, which is easily intelligible, because calcium carbonate itself is exceedingly common in this form in nature, and is.then known as calc spar, whilst natural crystalline magnesium carbonate is termed magnesite. The formation of the crystalline varieties of the insoluble carbonates is explained by the possibility of a slow deposition from solutions containing carbonic acid. Besides which (Chapter X.) calcium and magnesium sulphates are obtained from sea water, and therefore they are met with both as deposits and in springs. It must be observed that magnesium is held in considerable quantities in sea water, because the sulphate and chloride of magnesium are very soluble in water, whilst calcium sulphate is but little soluble, and is used in the formation of shells; and therefore if the occurrence of considerable deposits of magnesium sulphate cannot be expected in nature, still, on the other hand, one would expect (and they do actually occur) large masses of calcium sulphate or gypsum, $\mathrm{CaSO}_{4}, 2 \mathrm{H}_{2} \mathrm{O}$. Gypsum sometimes forms strata of immense size, which extend over many hectometres-for example, in Russia on the Volga, and in the Donetz and Baltic provinces.

Lime and magnesia also, but in much smaller quantities (only to the amount of several fractions of a per cent. and rarely more), enter into the composition of every fertile soil, and without these bases the soil is unable to support vegetation. Lime is particularly important in this respect, and its presence in a larger quantity generally improves the ${ }^{\circ}$ harvest, although purely calçareoús soils are as a rule infertile. For this reason the soil is fertilised both with linp ${ }^{12}$ itself and with

11 The formation of dolomite may be explained, if only we imagine that a solution of - magnesium salt acts on calcium carbonate. Magnesium carbonate may be formed by double decomposition, and it must be supposed that this process oases at a certain limit (Chapter XII), when we shall obtain a mixture of the carbonates of caloinm ind magnesium. Haitinger heated a mixture of calcium carbonate, $\mathrm{CaCO}_{3}$, with a solu. tion of an equivalent quantity of magnesium sulphate, $\mathrm{MgSO}_{4}$, in a closed tube at $200^{\circ}$. and then a portion of the magnesia wotually passed into the state of magnesium carbonate, $\mathrm{M}_{\mathrm{gCO}}$, and a portion of the lime was converted into gypsum, $\mathrm{CaSO}_{4}$. Lubavin (1892) showed that $\mathrm{MgCO}_{3}$ is more soluble than $\mathrm{CaCO}_{3}$ in salt water, which is of some: ignificance in explaining the composition of sea water.

18 The undoubted action of lime in increasing the fertility of coils-if not in every 
marl - that is, with clay mixed with a certain quantity of calcium car bonate, strata of which are found nearly everywhere

From the soil the lime and magnesia (in a smaller quantity) pass into the substance of plants, where they occur as salts. Certain of these salts separate in the interior of plants in a crystalline form-for example, calcium oxalate. The lime occurring in plants serves as the source for the formation of the various calcareous secretions which are so common in animals of all classes The bones of the highest animal orders, the shells of mollusca, the covering of the sea-urchin, and similar solid secretions of sea animals, contain calcium salts, namely, the shells mainly calcium carbonate, and the bones mainly calcium phosphate. Certain limestones are almost entirely formed of such deposits. Odessa is situated on a limestone of this kind. composed of shells. Thus magnesium and calcium occur throughout the entire realm of nature, but calcium predominates.

As lime and magnesia form bases which are in many respects analogous, they were not distinguished from each other for a long time. Magnesia was obtained for the first time in the seventeenth century from Italy, and used as a medicine, and it was only in the last century that Black, Bergmann, and others distinguished magnesia from lime.

Metallic magnesrum (and calcium also) is not obtained by heating magnesium oxide or the carbonate with charcoal, as the alkali metals are obtained, ${ }^{13}$ but is liberated by the action of a galvanic current on fused magnesium chloride (best mixed with potassium chloride), Davy and Bussy obtained metallic magnesium by acting on magnesium

case, at all events, with ordınary soils which have long been under corn-is based not so much on the need of plants for the lime itself as on those chemical and physical changes which it produces in the soil, as a particularly powerful base which aids the alteration of the mineral and organic elements of the soil.

13 Sodium and potassium only decompose magnesium oxide at a white heat and very feebly, probably for two reasons. In the first place, because the reaction $\mathrm{Mg}+\mathrm{O}$ deve. lops more heat (about 140 thousand calories) than $\mathrm{K}_{2}+\mathrm{O}$ or $\mathrm{Na}_{2}+\mathrm{O}$ (about 100 thousand calories); and, in the second place, because magnesia is not fusible at the heat of a furnace and cannot act on the charcoal, sodium, or potassium-that is, it does not pass into that mobile state which is necessary for reaction. The first reason alone is not sufficient to explain the absence of the reaction between charcoal and magnesia, because iron and charcoal in combining with oxygen evolve less heat than sodium or potassium, yet, nevertheless, they can displace them. With respect to magnesium chloride, it acts on sodium and potassium, not only because their combination with chlonne evolves more heat than the combination of chlorine and magnesium $\left(\mathrm{Mg}+\mathrm{Cl}_{2}\right.$ gives 150 and $\mathrm{Na}_{2}+\mathrm{Cl}_{2}$ about 195 thousand calories), but also because a fusion, both of the magnesium chloride and of the double salt, takes place under the action of heat. It is probable, however, that a reverse reaction will take place. A reverse reaction might probably be expected, and Winkler (1890) showed that Mg reduces the oxides of the alkali metals (Chapter XIII, Note 42). 
chloride with the vapours of potassium. At the present time (Deville's process) magnesium is prepared in rather considerable quantities by a similar process, only the potassium is replaced by sodium. Anhydrous magnesium chloride, together with sodium chloride and calcium fluoride, is fused in a close crucible. The latter substances only serve to facilitate the formation of a fusible mass before and after the reaction, which is indispensable in order to prevent the access and action of air One part of finely divided sodium to five parts of magnesium chloride is thrown into the strongly heated molten mass, and after stirring the reaction proceeds very quickly, and magnesium separates, $\mathrm{MgCl}_{2}+\mathrm{Na}_{2}$ $=\mathrm{Mg}+2 \mathrm{NaCl}$. In working on a large scale, the powdery metallic magnesium is then subjected to distillation at a white heat. The distillation of the magnesium is necessary, because the undistilled metal is not homogeneous ${ }^{14}$ and burns unevenly : the metal is prepared for the purpose of illumination. Magnesium is a white metal, like silver ; it is not soft like the alkali metals, but is, on the contrary, hard like the majority of the ordinary metals. This follows from the fact that it melts at a somewhat high temperature-namely, about $500^{\circ}$-and boils at about $1000^{\circ}$ It is malleable and ductile, like the generality of metals, so that it can be drawn into wires and rolled into ribbon; it is most frequently used for lighting purposes in the latter form. Unlike the alkali metals, magnesium does not decompose the atmospheric moisture at the ordinary temperature, so that it is almost unacted on by air ; it is not even acted on by water at the ordinary temperature, so that it may be washed to free it from sodium chloride. Magnesium only decomposes water with the evolution of hydrogen at the boiling point of water, ${ }^{15}$ and more rapidly at still higher temperatures. This is explained by the fact that in decomposing water magnesium forms an insoluble hydroxide, $\mathrm{MgH}_{2} \mathrm{O}_{2}$, which covers the metal and hinders the further action of the water. Magnesium eisily displaces hydrogen from acids, frrming magnesium salts. When ignited it burns, not only in oxygen but in air (and even in carbonic anhydride), forming a white powder of magnesium oxide, or magnesia ; in burning it emits a white and exceedingly brilliant light. The strength of this light naturally depends on the fact that magnesium (24 parts by weight) in burning

14 Commercial magnesium generally contains a certain amount of magnesium nitride (Deville and Caron), $\mathrm{Mg}_{5} \mathrm{~N}_{2}$-that is, a product of substitution of ammonia which is directly formed (as is easily shown by experiment) when magnesium is heated in nitrogen. It is a yellowish green powder, which gives ammonia and magnesia with water, and cyanogen when heated with carbonic anhydride. Pashkoffsky (1893) showed that $\mathbf{M g}_{3} \mathbf{N}_{2}$ is easily formed and is the sole product when $\mathrm{Mg}$ is heated to redness in a ourrent of $\mathrm{NH}_{3}$. Perfectly pure magnesium may be obtained by the action of a galvanic current.

15 Hydrogen peroxide (Weltzien) dissolves magnesium. The reaction has not been investigated 
evolves about 140 thousand heat units, and that the product of com. bustion, $\mathrm{MgO}$, is infusible by heat; so that the vapour of the burning magnesium contains an ignited powder of non-volatile and infusible magnesia, and consequently presents all the conditions for the production of a brilliant light. The light emitted by burning magnesium contains many rays which act chemically, and are situated in the violet and ultra-violet parts of the spectrum. For this reason burning magnesium may be employed for producing photographic images. ${ }^{16}$

Owing to its great affinity for oxygen, magnesium reduces many metals (zinc, iron, bismuth, antimony, cadmium, tin, lead, copper, silver, and others) from solutions of their salts at the ordinary temperature, ${ }^{17}$ and at a red heat finely divided magnesium takes up the oxygen from silica, alumina, boric anhydride, \&c.; so that silicon and similar elements may be obtained by directly heating a mixture of powdered silica and magnesium in an infusible glass tube. ${ }^{18}$

The affinity of magnesium for the halogens is much more feeble than for oxygen, ${ }^{19}$ as is at once evident from the fact that a solution of iodine acts feebly on magnesium; still magnesium burns in the vapours of iodine, bromine, and chlorine. The character of magnesium is also seen in the fact that all its salts, especially in the presence of water, are decomposable at a comparatively moderate temperature, the elements of the acid being evolved, and the magnesium oxide, which is non-volatile and unchangeable by heat, being left. This naturally refers to those acids which are themselves volatilised by heat. Even magnesium sulphate is completely decomposed at the temperature at which iron melts, oxide of magnesium remaining behind. This decomposition of magnesium salts by heat proceeds

16 A special form of apparatus is used for burning magnesium. It is a clockwork arrangement in which a cylinder rotates, round which a ribbon or wire of magnesium is wound. The wire is subjected to a uniform unwinding and burning as the cylinder rotates, and in this manner the combustion may continue uniform for a certain time. The same is attained in special lamps, by cansing a mixture of sand. and finely divided magnesium to fall from a funnel-shaped reservoir on to the flame. In photography it is best to blow finely divided magnesium into a colourless (spirit or gas) flame, and for instantaneons photography to light a cartridge of a mixture of magnesium and chlorate of potassium by means of a spark from a Ruhmkorff' 8 coil (D Mendeléeff, 1889).

17 According to the observations of Maack. Comaille, Böttger, and othera. The reduction by heat mentioned further on was pointed out by Geuther, Phipson, Parkinson and Gattermann.

18 This action of metallic magnesium in all probability depends, althongh only partially (see Note 13), on its volatility, and on the fact that, in combining with a given quantity of oxygen, it evolves more heat than aluminium, silicon, potassium. and other elements.

19 Davy, on heating magnesia in chlorine, concluded that there was a complete sub. stitution, because the volume of the oxygen was half the volume of the chlorine; it is probable, however, that owing to the formation of chlorine oxide (Chapter XI., Note 30) the decomposition is not complete and is limited by a reverse reaetion. 
much more easily than that of calcium salts. For example, magnesium carbonate is totally decomposed at $170^{\circ}$, magnesium oxide being left behind. This magnesia, or magnesium oxide, is met with both in an anbydrous and hydrated state in nature (the anhydrous magnesia as the mineral periclase, $\mathrm{MgO}$, and the hydrated magnesia as brucite, $\mathrm{MgH}_{2} \mathrm{O}_{2}$ ). Magnesia is a well-known medicine (calcined magnesia-magnesia usta). It is a white, extremely fine, and very voluminous powder, of specific gravity 3.4 ; it is infusible by heat, and only shrinks or shrivels in an oxyhydrogen flame. After long contact the anhydrous magnesia combines with water, although very slowly, forming the hydroxide $\mathrm{Mg}(\mathrm{HO})_{2}$, which, however, parts with its water with great ease when heated even below a red heat, and again yields anhydrous magnesia. - This hydroxide is obtained directly as a gelatinous amorphous substance when a soluble alkali is mixed with a solution of any magnesium salt, $\mathrm{MgCl}_{2}+2 \mathrm{KHO}=\mathrm{Mg}(\mathrm{HO})_{2}+2 \mathrm{KCl}$. This decomposition is complete, and nearly all the magnesium passes into the precipitate; and this clearly shows the almost perfect insolubility of magnesia in water. Water dissolves a scarcely per. ceptible quantity of magnesium hydroxide-namely, one part is dis. solved by 55,000 parts of water. Such a solution, however, has an alkaline reaction, and gives, with a salt of phosphoric acid, a precipitate of magnesium phosphate, which is still more insoluble. Magnesia is not only dissolved by acids, forming salts, but it also dis. places certain other bases-for example, ammonia from amrnonium salts when boiled; and the hydroxide also absorbs carbonic anhy. dride from the air. The magnesium salts, like those of calcium, potas. sium, and sodium, are colourless if they are formed from colourless acids. Those which are soluble have a bitter taste, whence magnesia has been termed bitter-earth. In comparison with the alkalis magnesia is a feeble base, inasmuch as it forms somewhat unstable salts, easily gives basic salts, forms acid salts with difficulty, and is able to give double salts with the salts of the alkalis; which facts are characteristic of feeble bases, as we shall see in becoming acquainted with the different metals.

The power of magnesium salts to form double and basic salts is very frequently shown in reactions, and is specially marked as regards ammonium salts. If saturated solutions of magnesium and ammonium sulphates are mixed together, a crystalline double salt $\mathrm{Mr} g\left(\mathrm{NH}_{4}\right)_{2}\left(\mathrm{SO}_{4}\right)_{2}, 6 \mathrm{H}_{2} \mathrm{O},{ }^{20}$ is immediately precipitated. A strong

20 Even a solution of ammonium chloride gives, this salt with magnesium sulphate. Its sp. gr. is $1.72 ; 100$ parts of water at $0^{\circ}$ dissolve 9 , at $20^{\circ} 17.9$ parts of the anhydrous salt. At about $130^{\circ}$ it loses all its water. 
solution of ordinary ammonium carbonate dissolves magnesium oxide or carbonate, and precipitates crystals of a double salt, $\mathrm{Mg}\left(\mathrm{NH}_{4}\right)_{2}\left(\mathrm{CO}_{3}\right)_{2}, 4 \mathrm{H}_{2} \mathrm{O}$, from which water extracts the ammonium carbonate. With an excess of an ammonium salt the double salt passes into solution, ${ }^{21}$ and therefore if a solution contain a magnesium salo and an excess of an ammonium salt-for instance, sal-ammoniacthen sodium carbonate will no longer precipitate magnesium carbonate. A mixture of solutions of magnesium and ammonium chlorides, on evaporation or refrigeration, gives a double salt, $\mathrm{Mg}\left(\mathrm{NH}_{4}\right) \mathrm{Cl}_{3}, 6 \mathrm{H}_{2} \mathrm{O}^{22}$ The salts of potassium, like those of ammonium, are able to enter into combination with the magnesium salts. ${ }^{23}$ For instance, the double salt, $\mathrm{MgKCl}_{3}, 6 \mathrm{H}_{2} \mathrm{O}$, which is known as carnallite, ${ }^{24}$ and occurs in the salt mines of Stassfurt, may be formed by freezing a saturated solution of potassium chloride with an excess of magnesium chloride. A saturated solution of magnesium sulphate dissolves potassium sulphate, and solid magnesium sulphate is soluble in a saturated solution of potassium sulphate. A double salt; $\mathrm{K}_{2} \mathrm{Mg}\left(\mathrm{SO}_{4}\right)_{2}, 6 \mathrm{H}_{2} \mathrm{O}$, which closely resembles the above-mentioned ammonium salt, crystallises from these solutions. ${ }^{25}$

21 This is an example of equilibrium and of the influence of mass; the double salt is decomposed by water, but if instead of water we take a solution of that soluble part which is formed in the decomposition of the double salt, then the latter dissolves as a whole.

22 If an excess of ammonia be added to a solution of magnesium chloride, only half the magnesium is thrown down in the precipitate, $2 \mathrm{MgCl}_{2}+2 \mathrm{NH}_{4} \cdot \mathrm{OH}=\mathrm{Mg}(\mathrm{OH})_{2}$ $+\mathrm{Mg} \cdot \mathrm{NH}_{4} \mathrm{Cl}_{3}+\mathrm{NH}_{4} \mathrm{Cl}$. A solution of ammonium chloride reacts with magnesia, evolving ammonia and forming a solution of the same salt, $\mathrm{MgO}+3 \mathrm{NH}_{4} \mathrm{Cl}=\mathrm{MgNH}_{4} \mathrm{Cl}_{3}+\mathrm{H}_{2} \mathrm{O}$ $+2 \mathrm{NH}_{3}$.

Among.the double'salts of ammonium and magnesium, the phosphate. $\mathrm{MgNH}_{4} \mathrm{PO}_{4}, 6 \mathrm{H}_{2} \mathrm{O}$, is almost insoluble in water $(0.07 \mathrm{gram}$ is soluble in a litre), even in the presence of ammonia. Magnesia is very frequently precipitated as this salt from solntions in which it is held by ammonium salts. As lime is not retained in solution by the presence of ammonium salts, but is precipitated nevertheless by sodium carbonate, \& c., it is very easy to separate calcium from magnesium by taking advantage of these properties.

${ }_{23}$ In order to see the nature and cause of formation of double salts, it is sufficient (although this does not embrace the whole essence of the matter) to consider that one of the metals of such salts (for instance, potassium) easily gives acid salts, and the other (in this instance, magnesium) basic salts ; the properties of distinctly basio elements prodominate in the former, whilst in the latter these properties are enfeebled, and the salts formed by them bear the character of acids-for example, the salts of aluminium or magnesium act in many cases like acids. By their mutual combination these two opposite properties of the salts.are both satisfied

24 Carnallite has been mentioned in Chapter X. (Note 4) and in Chapter XIII. These deposits also contain much kainile, $\mathrm{KMgCl}\left(\mathrm{SO}_{4}\right), 8 \mathrm{H}_{2} \mathrm{O}$ (sp. gr. $2 \cdot 13 ; 100$ parts of water dissolve 79.6 parts at $18^{\circ}$ ). This donble salt contains two metals and two haloids. Feit (1889) also obtained a bromide corresponding to carnallite.

2s The component parts of certain double salts diffuse at different rates, and as the diffused solution contains a different proportion of the component salts than the wolution taken of the donble salt, it shows that such salts are decomposed by water. According to Riudorff, the double salts, like carnallite, $\mathrm{MgK}_{2}\left(\mathrm{SO}_{4}\right)_{2}, 6 \mathrm{H}_{2} \mathrm{O}$, and the alums, all belong to this order (1888). But such salts as tartar emetio, tho double oxalates, and 


\section{The nearest analogues of magnesium are eble to give exactly similar double salts, both in crystalline form (monoclinic system) and com.}

double cyanides are not separated by diffusion, which in all probability depends both on the relative rate of the diffusion of the component salts and on the degree of affinity acting between them. Those complex states of equilibrium which exist between water, the individual salts MX and NY, and the double salt MNXY, have been already partially analysed (as will be shown hereafter) in that case when the system is heterogeneous (that is, when something separates out in a solid state from the liquid solution), bub in the case of equilibria in a homogeneous liquid medium (in a solution) the phenomenon is not so clear, because it concerns that very theory of solution which cannot yet be considered as established (Chapter I., Note 9, and others). As regards the heterogeneous decomposition of donble salts, it has long been known that sach salts as carnallite and $\mathrm{K}_{2} \mathrm{Mg}\left(\mathrm{SO}_{4}\right)_{2}$ give up the more soluble salt if an insufficient quantity of water for their complete solution be taken. The complete saturation of 100 parts of water requires at $0^{\circ} 14 \cdot 1$, at $20^{\circ} 25$, and at $60^{\circ} 50^{\circ} 2$ parts of the latter double salt (anhydrous), while 100 parts of water dissolve 27 parts of magnesium sulphate at $0^{\circ}, 36$ parts at $20^{\circ}$, and 55 parts at $60^{\circ}$, of the anhydrous salt taken. Of all the states of equilibrium exhibited by double salts the most fully investigated as yet is the system containing water, sodium sulphate, magnesium sulphate, and their double salt, $\mathrm{Na}_{2} \mathrm{Mg}\left(\mathrm{SO}_{4}\right)_{2}$, which crystallises with 4 and $6 \mathrm{~mol}$. $\mathrm{OH}_{2}$. The first crystallo-hydrate, $\mathrm{MgNa}_{2}\left(\mathrm{SO}_{4}\right)_{2}, 4 \mathrm{H}_{2} \mathrm{O}$, occurs at Stassfurt, and as a sedimentary deposit in many of the salt lakes near Astrakhan; and is. therefore called astrakhanite. The specific gravity of the monoclinic prisms of this salt is $2 \cdot 22$. If this salt, in a finely divided state, be mixed with the necessary quantity of water (according to the equation $\mathrm{MgN}_{2}\left(\mathrm{SO}_{4}\right)_{2}, 4 \mathrm{H}_{2} \mathrm{O}+13 \mathrm{H}_{2} \mathrm{O}=\mathrm{Na}_{2} \mathrm{SO}_{42}, 10 \mathrm{H}_{2} \mathrm{O}+\mathrm{MgSO}_{4}, 7 \mathrm{H}_{2} \mathrm{O}$ ), the mixture solidifies like plaster of Paris into a homogeneous mass if the temperature be below $22^{\circ}$ (Van't Hoff und Van Deventer, 1886; Bakhuis Roozeboom, 1887); but if the temperature be above this transition-point the water and double selt do not react on each other; that is, they do not solidify or give a mixture of sodium and magnesium sulphates. If a mixture (in equivalent quantities) of solutions of these salts be evaporated, and crystals of astrakhanite and of the individual salts capable of proceeding from it be added to the concentrated solution to avoid the possibility of a supersaturated solution, then at temperatures above $22^{\circ}$ astrakhanite is exclusively formed (this is the method of its production), but at lower temperatures the individual salts are alone produced. If equivalent amounts of Glauber's salt and magnesium sulphate be mixed together in a solid state, there is no change at temperaturea below $22^{\circ}$, but at higher temperatures astrakhanite and water are formed. The volume occupied by $\mathrm{Na}_{2} \mathrm{SO}_{4}, 10 \mathrm{H}_{2} \mathrm{O}$ in grams $=322 / 1 \cdot 46=220^{\circ} 5$ cubic centimetres, and by $. \mathrm{MgSO}_{4}, 7 \mathrm{H}_{2} \mathrm{O}=246 / 1 \cdot 68=146.4$ c.c.; hence their mixture in equivalent quantities occupies a volume of 366.9 c.c. The volume of astrakhanite $=334 / 2 \cdot 22=150 \cdot 5$ c.c., and the volume of $13 \mathrm{H}_{2} \mathrm{O}=234$ c.c., hence their sum $=380^{\circ} 5$ c.c., and therefore it is easy to follow the formation of the astrakhanite in a suitable apparatus (a kind of thermometer containing oil and a powdered mixture of sodium and magnesium sulphates), and to see by the variation in volume that below $22^{\circ}$ it remains unchanged, and at higher temperatures proceeds the more quickly the higher the temperature. At the transition temperature the solubility of astrakhanite and of the mixture of the component salts is one and the same, whilst at higher temperatures a solution which is saturated for a mixture of the individual salts would be supersaturated for astrakhanite, and at lower temperatures the solution of astrakhanite will be supersaturated for the component salts, as has been -shown with especial detail by Karsten, Deacon, and others. Roozeboom showed that there are two limits to the composition of the solutions which can exist for a double salt; these limits are respectively obtained by dissolving a mixture of the double salt with each of its component simple salts. Van't Hoff demonstrated, besides this, that the tendency towards the formation of double salts has a distinct inflnence on the progress of double decomposition, for at temperatures above $31^{\circ}$ the nixture $2 \mathrm{MgSO}_{4}, 7 \mathrm{H}_{2} \mathrm{O}+2 \mathrm{NaCl}$ passes into $\mathrm{MgNa}_{2}\left(\mathrm{SO}_{4}\right)_{2}, 4 \mathrm{H}_{2} \mathrm{O}+\mathrm{MgCl}_{2}, \mathrm{CH}_{2} \mathrm{O}+4 \mathrm{H}_{2} \mathrm{O}$, whilst below $31^{\circ}$ there is not this 
position; they, like this salt (see Chapter XV.), are easily able (at $140^{\circ}$ ) to part with all their water of crystallisation, and correspond with the salts of sulphuric acid, whose type may be taken as magnesium sulphate, $\mathrm{MgSO}_{4}{ }^{26}$ It occurs at Stassfurt as kieserite, $\mathrm{MgSO}_{4}, \mathrm{H}_{2} \mathrm{O}$, and generally separates from solutions as a heptahydrated salt, $\mathrm{MgSO}_{4}, 7 \mathrm{H}_{2} \mathrm{O}$, and from supersaturated solutions as a hexahydrated salt, $\mathrm{MgSO}_{4}, 6 \mathrm{H}_{2} \mathrm{O}$; at temperatures below $0^{\circ}$ it crystallises out as a dodecaliydrated salt, $\mathrm{MgSO}_{4}, 12 \mathrm{H}_{2} \mathrm{O}$, and a solution of the com. position $\mathrm{MgSO}_{4}, 2 \mathrm{H}_{2} \mathrm{O}$ solidifies completely at $-5^{\circ} .{ }^{27}$ Thus between

double decomposition, but it proceeds in the opposite directiou, as may be demonstrated by the above-described methods. Van der Heyd obtained a potassium astrakhanite, $\mathrm{K}_{2} \mathrm{SO}_{4} \mathrm{MgSO}_{4}, 4 \mathrm{H}_{2} \mathrm{O}$, from solutions of the component salts at $100^{\circ}$

From these experiments on double salts we see that there is as close a dependence between the temperature and the formation of substances as there is between the temperature and a clange of state. It is a case of Deville's principles of dissociation, extended in the direction of the passage of a solid into a liquid. On the other hand, we see here how essential a role water plays in the formation of compounds, and how the affinity for water of crystallisation is essentially arialogous to the affinity between salts, and hence also to the affinity of rcids for bases, because the formation of double salts does not differ in any essential point (except the degree of affinity-that is, from a quantititive aspect) from the formation of salts themselves. When sodium hydroxide with nitric acid gives sodium nitrate and water the phenomenon is essentially the same as in the formation of astraklisnite from the salts $\mathrm{Na}_{2} \mathrm{SO}_{4}, 10 \mathrm{H}_{2} \mathrm{O}$ and $\mathrm{MgSO}_{4}, 7 \mathrm{H}_{2} \mathrm{O}$. Water is disengaged in both cases, and hence the volumes are altered.

26 This salt, and especially its erystallo-hydrate with $7 \mathrm{H}_{2} \mathrm{O}$, is generally known as Epsom salts. It has long been used as a purgative. It is easily obtained from magnesia and sulphurio acid, and it separates on the evaporation of sea water and of many saline springs. When carbonic anhydride is obtained by the action of sulphuric acid on magnesite, magnesium sulphate remains in solution. When dolomite-that is, a mixture of magnesium and calcium carbonates-is subjected to the action of a solution of hydrochloric acid until about half of the salt remains, the calcium carbonate is mostly dise solved and magnesium carbonate is left, which by treatment with sulphurio acid gives a solution of magnesium sulphate.

${ }^{27}$ The anhydrous salt, $\mathrm{MgSO}_{4}$ (sp. gi. 2.61), attracts moisture $\left(7 \mathrm{~mol} . \mathrm{H}_{2} \mathrm{O}\right.$ ) from moist air; when heated in steam or hydrogen chloride it gives sulphuric acid, and when heated with carbon it is denomposed according to the equation $2 \mathrm{MgSO}_{4}+\mathrm{C}=2 \mathrm{SO}_{2}+\mathrm{CO}_{2}$ $+2 \mathrm{MgO}$. The monohydrated salt (kieserite), $\mathrm{MgSO}_{4}, \mathrm{H}_{2} \mathrm{O}$ (sp. gr. 2.56), dissolves in water with difficulty; it is formed by heating the other crystallo-hydrates to $185^{\circ}$. The hexahydrated salt is dimorphouis. If a solution, saturated at the boiling-point, be prepared, and cooled -without access of crystals of the heptahydrated salt, then $4 \mathrm{MSO}_{4}, 6 \mathrm{H}_{2} \mathrm{O}$ crystallises out in monoclinic prisms (Loewel, Marignac), which are quite as unstable as the salt, $\mathrm{Na}_{2} \mathrm{SO}_{4}, 7 \mathrm{H}_{2} \mathrm{O}$; but if prismatic crystals of the cubio system of the copper-nickel salts of the composition $\mathrm{MSO}_{4}, 6 \mathrm{H}_{2} \mathrm{O}$ be added, then crystals of $\mathrm{MgSO}_{4}, 6 \mathrm{H}_{2} \mathrm{O}$ are deposited on them as prisms of the cubic system (Lecoq de Boisbau. dran). The commori cryetallo-hydrate, $\mathrm{MgSO}_{4}, 7 \mathrm{H}_{2} \mathrm{O}$, Epeom salts, belongs to the rhombio eystem, and is obtained by crystallisation below $80^{\circ}$. It specific gravity is 1.69 . In a vacuum, or at $100^{\circ}$, it loses $5 \mathrm{H}_{2} \mathrm{O}$, at $132^{\circ} \mathrm{CH}_{2} \mathrm{O}$, and at $210^{\circ}$ all the $7 \mathrm{H}_{2} \mathrm{O}$ (Graham). If erystals of ferrous or cobaltio sulphate be placed in a saturated solution, hexagonal crystals of the heptahydrated salt are formed (Leoog de Boisbaudran); they present an unstable state of equilibrium, and soon become cloudy, probably owing to their trans. formation into the more stable common form. Fritzsche, by cooling saturated solutions below $0^{\circ}$, obtained a mixture of crystals of ice and of a dodecabydrated salt, which easily 
water and magnesium sulphate there may exist several definite and more or less stable degrees of equilibrium; the double salt $\mathrm{MgSO}_{4} \mathrm{~K}_{2} \mathrm{SO}_{4}, 6 \mathrm{H}_{2} \mathrm{O}$ may be regarded as one of these equilibrated mystems, the more so since it contains $6 \mathrm{H}_{2} \mathrm{O}$, whilst $\mathrm{MgSO}_{4}$ forms its most stable system with $7 \mathrm{H}_{2} \mathrm{O}$, and the double salt may be considered as this crystallo-hydrate in which one molecule of water is replaced by the molecule $\mathrm{K}_{2} \mathrm{SO}_{4}{ }^{28}$

The power of forming basic salts is a very remarkable peculiarity of magnesia and other feeble bases, and especially of those corresponding with polyvalent metals. The very powerful bases corresponding with univalent metals-like potassium and sodium--do not form basic salts, and, indeed, are more prone to give acid salts, whilst magnesium easily and frequently forms basic salts, especially with feeble acids, although there are some oxides-as, for example, copper and lead oxides-which still more frequently give basic salts. If a cold solution of magnesium sulphate be mixed with a solution of sodium carbonate there is formed a gelatinous precipitate of a basic salt,

split up at temperatures above $0^{\circ}$. Guthrie showed that dilute solntions of magnesium sulphate, when refrigerated, separate ice until the solution attains a composition $\mathrm{MgSO}_{4}, 24 \mathrm{H}_{2} \mathrm{O}$, which will completely freeze into a crystallo-hydrate at $-5 \cdot 3^{\circ}$. According to Coppet and Ruidorff, the temperature of the formation of ice falls by $0.673^{\circ}$ for every part by weight of the heptahydrated salt per 100 of water. This figure gives (Chapter I., Note 49) $i=1$ for both the heptahydrated and the anhydrous selt, from which it is evident that it is impossible to judge the state of combination in which a dissolved substance occurs by the temperature of the formation of ice.

The solubility of the different crystallo-hydrates of magnesium sulphate, according to Loewel, also varies, like those of sodium sulphate or carbonate (see Chapter XII., Notes 7 and 18). At $0^{\circ} 100$ parts of water dissolves $40.75 \mathrm{MgSO}_{4}$ in the presence of the hexahydrated salt, $34.67 \mathrm{MgSO}_{4}$ in the presence of the hexagonal heptahydrated salt, and only 26 parts of $\mathrm{MgSO}_{4}$ in the presence of the ordinary heptahydrated salt-that is, solutions giving the remaining crystallo-hydrates will be supersaturated for the ordinary heptahydrated salt.

All this shows how many diverse aspects of more or less stable equilibria may exist between water and a substance dissolved in it; this has already been enlarged on in Chapter I.

Carefully purified magnesium aulphate in its aquoous solution gives, according to Stcherbakoff, an alkaline reaction with litmus, and an acid reaction with phenol. phthalein.

The specifio gravity of solutions of certain salts of magnesium and calcium roduced $1015^{\circ} / 4^{\circ}$ (see my work oited, Chapter I., Note 19 ), are, if water at $4^{\circ}=10,000$,

$$
\begin{aligned}
& \mathrm{MgSO}_{4}: 8=9,992+99 \cdot 89 p+0.558 p^{2} \\
& \mathrm{MgCl}_{2}: 8=9,992+81 \cdot 81 p+0.872 p^{2} \\
& \mathrm{CaCl}_{2}: 8=9,992+80 \cdot 24 p+0.476 p^{2}
\end{aligned}
$$

Graham even distinguished the last equivalent of the water of crystallisation of the heptahydrated salt as that which is replaced by other salts, pointing out that double anlts like $\mathrm{K}_{\mathrm{GK}} ;\left(\mathrm{SO}_{4}\right)_{2}, 6 \mathrm{H}_{2} \mathrm{O}$ lose all their water at $185^{\circ}$, whilst $\mathrm{MgSO}_{4}, 7 \mathrm{H}_{2} \mathrm{O}$ only part with $6 \mathrm{H}_{2} \mathrm{O}$. 
$\mathrm{Mg}(\mathrm{HO})_{2}, 4 \mathrm{MgCO}_{3}, 9 \mathrm{H}_{2} \mathrm{O}$; but all the magnesia is not precipitated in this case, as a portion of it remains in solution as an acid double salt. If sodium carbonate be added to a boiling solution of magnesium sulphate a precipitate of a still more basic salt is formed, $4 \mathrm{MgSO}_{4}$ $+4 \mathrm{Na}_{2} \mathrm{CO}_{3}+4 \mathrm{H}_{2} \mathrm{O}=4 \mathrm{Na}_{2} \mathrm{SO}_{4}+\mathrm{CO}_{2}+\mathrm{Mg}(\mathrm{OH})_{2}, 3 \mathrm{MgCO}_{3}, 3 \mathrm{H}_{2} \mathrm{O}$. This basic salt forms the ordinary drug magnesia (magnesia alba), in the form of light porous lumps. Other basic salts are formed under certain modifications of temperature and conditions of decomposition. But the normal salt, $\mathrm{MgCO}_{3}$, which occurs in nature as magnesito in the form of rhombohedra of specific gravity $3 \cdot 056$, cannot be obtained by such a method of precipitation. In fact, the formation of the different basic salts shows the power of water to decompose the normal salt. It is possible, however, to obtain this salt both in an anhydrous and hydrated state. A solution of inagnesium carbonate in water containing carbonic acid is taken for this purpose. The reason for this is easily understood-carbonic anhydride is one of the products of the decomposition of magnesium carbonate in the presence of water. If this solution be left to evaporate spontaneously the normal salt separates in a hydrated form, but in the evaporation of a heated solution, through which a strean of carbonic anhydride is passed, the anhydrous salt is formed as a crystalline mass, which remains unaltered in the air, like the natural mineral. ${ }^{29}$ The decomposing influence. of water on the salts of magnesium, which is directly dependent on the feeble basic properties of magnesia, ${ }^{30}$ is inost clearly seen in magnesium chloride, $\mathrm{MgCl}_{2}$. This salt is contained ${ }^{31}$ in the last mother-liquors of the evaporation of sea-water. On cooling a sufficiently concentrated solution, the crystallo-hydrate, $\mathrm{MgCl}_{2}, 6 \mathrm{H}_{2} \mathrm{O}$, separates; ${ }^{32}$ but if it be

29 The crystalline form of the anhydrous salt obtained in this manner is not the same as that of the natural salt. The former gives rhombohedra, like those in which calcium carbonate appears as calc spar, whilst the natural salt appears as rhombic prisms, like those sometimes presented by the same carbonate as aragonite, which will shortly be described.

so Magnesium sulphate enters into certain reactions which are proper to sulphurio acid itself. Thus, for instance, if a carefully prepared mixture of equivalent quantities of hydrated magnesium sulphate and sodium chloride be heated to redness, the evolution of hydrochloric acid is observed just as in the action of sulphurio acid on common salt, $\mathrm{MgSO}_{4}+2 \mathrm{NaCl}+\mathrm{H}_{2} \mathrm{O}=\mathrm{Na}_{2} \mathrm{SO}_{4}+\mathrm{MgO}+2 \mathrm{HCl}$. Magnesium sulphate acts in a similar manner on nitrates, with the evolution of nitric acid. A mixture of it with common salt and manganese peroxide gives chlorine. Sulphuric acid is sometimes replaced by magnesium sulphate in galvanic batteries-for example, in the well-known Meidinger battery. In the above-mentioned reactions we see a striking example of the similarity of the reactions of acids and salts, especially of, salts which cuntain such feeble bases as magnesia.

31 As sea-water contains many salts, $\mathrm{MCl}$ and $\mathrm{MgX}_{2}$, it follows, according to Berthollet's teaching, that $\mathrm{MgCl}_{2}$ is also present.

33 As the crystallo-hydrates of the salts of sodium often. contain $10 \mathrm{H}_{2} \mathrm{O}, 80$ many of the salts of magnesium contain $6 \mathrm{H}_{2} \mathrm{O}$. 
further heated (above $106^{\circ}$ ) to remove the water, then hydrochloric acid passes off together with the latter, so that there ultimately remains magnesia with a small quantity of magnesium chloride. ${ }^{33}$ From what has been said it is evident that anhydrous magnesium chloride cannot be obtained by simple evaporation. But if sal-ammoniac or sodiun chloride be added to a solution of magnesium chloride, then the evolution of hydrochloric acid does not take place, and after complete evaporation the residue is perfectly soluble in water. This renders it possible to obtain anhydrous magnesium chloride from its aqueous solution. Indeed the mixture with sal-ammoniac (in excess) may be dried (the residue consists of an anhydrous double salt, $\mathrm{MgCl}_{2}, 2 \mathrm{NH}_{4} \mathrm{Cl}$ ) and then ignited $\left(460^{\circ}\right)$, when the sal-ammoniac is converted into vapour and a fused mass of anhydrous magnesium chloride remains behind. The anhydrous chloride evolves a very considerable amount of heat on the addition of water, which shows the great affinity the salt has for water. ${ }^{34}$ Anhydrous magnesium chloride is not only obtained by the above method, but is also formed by the direct combination of chlorine and magnesium, and by the action of chlorine on magnesium oxide, oxygen being evolved; this proceeds still more easily by heatiny magnesia with charcoal in a stream of chlorine, when the charcoal serveș to take up the oxygen. This latter method is also employed for the preparation of chlorides which are formed in an anhydrous condition with still greater difficulty than magnesium chloride. Anhydrous magnesium chloride forms a colourless, transparent mass, composed of flexiblo crystalline plates of a pearly lustre. It fuses at a low red heat $\left(708^{\circ}\right)$ into a colourless liquid, remains uncluanged in a dry state, but under the action of moisture is partially decomposed even at the ordinary temperature, with formation of hydrochloric acid. When heated in the presence of oxygen (air) it gives chlorine and the basic salt, which

33 This decomposition is most simply defined as the result of the two reverse reactions, $\mathrm{MgCl}_{2}+\mathrm{H}_{2} \mathrm{O}=\mathrm{MgO}+2 \mathrm{HCl}$ and $\mathrm{MgO}+2 \mathrm{HCl}=\mathrm{MgCl}_{2}+\mathrm{H}_{2} \mathrm{O}$, or as a distribution between $\mathrm{O}$ and $\mathrm{Cl}_{2}$ on the one hand and $\mathrm{H}_{2}$ and $\mathrm{Mg}$ on the other. (With $\mathrm{O}, \mathrm{MgCl}_{2}$ gives chlorine, see Chapter X., Note 33, and Chapter II., Note 3 bis and others, where the reactions and applications of $\mathrm{MgCl}_{2}$ are given.) It is then clear that, sccording to Berthollet's doctrine, the mass of the hydrochlorio acid converts the magnesium oxide into chloride, and the mass of the water converts the magnesium chloride into oxide. The crystallo-hydrate, $\mathrm{MgCl}_{2}, 6 \mathrm{H}_{2} \mathrm{O}$, forms the limit of the reversibility. But an intermediate state of equilibrium may exist in the form of basic salts. On mixing ignited magnesia with a solution of magnesium chloride of specific gravity about $1 \cdot 2$, a solid mass is obtained which is scarcely decomposed by water at the ordinary temperature (see Chapter XVI., Note 4). A sinilar means is employed for cementing sawdust into a solid mass, called cylolite, used for flooring, \&c.

We may remark that $\mathrm{MgBr}_{2}$ cryetallises not only with $6 \mathrm{H}_{2} \mathrm{O}$ (temperature of fusion $152^{\circ}$ ), but also with $10 \mathrm{H}_{2} \mathrm{O}$ (temperature of fusion $+12^{\circ}$, formed at $-18^{\circ}$. Panfiloff, 1804).

84 According to Thomsen, the combination of $\mathrm{MgCl}_{2}$ with $6 \mathrm{H}_{2} \mathrm{O}$ evolves 33,000 calories, and its solution in an excess of water 36,000 . 
is formed with even greater facility under the action of heat in the presence of steam, when $\mathrm{HCl}$ is formed, according to the equation $2 \mathrm{MgCl}_{2}+\mathrm{H}_{2} \mathrm{O}=\mathrm{MgOMgCl}_{2}+2 \mathrm{HCl}^{34}$ bis

Calcium (or the metal of lime) and its compounds in many respects present a great resemblance to magnesium compounds, but are also clearly distinguished from them by many properties. ${ }^{35}$ In general, calcium stands to magnesium in the same relation as potassium occupies in respect to sodium. Davy obtained metallic calcium, like potassium, as an amalgam by the action of a galvanic current; but neither charcoal nor iron decomposes calcium oxide, and even sodium decom. poses calcium chloride ${ }^{36}$ with difficulty. But a galvanic current easily decomposes calcium chloride, and metallic sodium somewhat easily decomposes calcium iodide when heated. As in the case of hydrogen, potassium, and magnesium, the affinity of iodine for caleium is feebler than that of chlorine (and oxygen), and therefore it is not surprising that calcium iodide may be subjected to that decomposition, which the chloride and oxide undergo with difficulty. ${ }^{37}$ Metallic calcium is of a yellow colour, and has a considerable lustre, which it preserves in dry air. Its specific gravity is 1.58. Calcium is distinguished by its great ductility; it melts at a red heat and then burns in the air with a very brilliant flame; the brilliancy is due to the formation of finely divided infusible calcium oxide. Judging from the fact that calcium in burning gives a very large flame, it is probable that this

34 bis Hence $\mathrm{MgCl}_{2}$ may be employed for the preparation of chlorine and hydrochlorio acid (Chapters X. and XI.). In general magnesium chloride, which is obtained in large guantities from sea water and Stassfurt carnallite, may find numerous practical uses.

35. There are many other methods of separating calcium from magnesium besides that mentioned above (Note 22). Among them it will be sufficient to mention the behaviour of these bases towards a solution of sugar; hydrated lime is exceedingly soluble in an aqueous solution of sugar, whilst magnesia is but little soluble. All the lime may be extracted from dolomite by burning it, slaking the mixture of oxides thus obtained, and adding a 10 p.c. solution of sugar. Carbonic anhydride precipitates calcium carbonate from this solution. The addition of sugar (molasses) to the lime used for building purposes powerfully increases the binding power of the mortar, as I have myself found. I have been told that in the East (India, Japan) the addition of sugar to cement has long been practised.

36 Moreover Caron obtained an alloy of calcium and zine by fusing calcium chloride with zinc and sodium. The zinc distilled from this alloy at a white heat, leaving calcium behind (Note 50 ).

37 Calcium iodide may be prepared by saturating lime with hydriodic acid. It is a very soluble salt (at $20^{\circ}$ one part of the salt requires 0.49 part and at $43^{\circ} 0.85$ part of water for solution), is deliquescent. in the air, and resembles calcium chloride in many respects. It changes but little when evaporated, and like calcium chloride fuses when heated, and therefore all the water may be driven off by heat. If anhydrous calcium iodide be heated with an equivalent quantity of sodium in a closely covered iron crucible, sodium iodide and metallic calcium are formed (Liés-Bodart). Dumas advises carrying on this reaction in a closed space under pressure. 
metal is volatile. Calcium decomposes water at the ordinary tem. perature, and is oxidised in moist air, but not so rapidly as sodium. In burning, it gives its oxide or lime, $\mathrm{CaO}$, a substance which is familiar to every one, and of which we have already frequently had occasion to speak. This oxide is not met with in nature in a free state, because it is an energetic base which everywhere encounters acid substances forming salts with them. It is generally combined with silica, or occurs as calcium carbonate or sulphate. The carbonate and nitrate are decomposed, at a red heat, with the formation of lime. As a rule, the carbonate, which is so frequently met with in nature, serves as the source of the calcium oxide, both commercial and pure. When heated, calcium carbonate dissociates: $\mathrm{CaCO}_{3}=\mathrm{CaO}+\mathrm{CO}_{2}$. In practice the decomposition is conducted at a bright red heat, in the presence of steam, or a current of a foreign gas, in heaps or in special kilns. ${ }^{38}$

Calcium oxide-that is, quicklime-is a substance (sp. gr. 3.15)

33 Kilns which act either intermittently or continuously are built for this purpose. Those of the first kind are filled with alternate layers of fuel and limestone; the fuel is lighted, and the heat developed by its combustion serves for decomposing the limestone. When the process is completed the kiln is allowed to cool somewhat, the lime raked out, and the same process repeated. In the continuously acting furnaces, con. structed like that shown in fig. 78, the kiln itself only contains limestone, and there are lateral hearths for burning the fuel, whose flame passes through the limestene and serves for its decomposition. Such furnaces are able to work continuously, because the unburnt limestone may be charged from above and the burnt lime raked out from below. It is not every limestone that is suitable for the preparation of lime, because many contain impurities, principally clay, dolomite, and sand. Such limestones when burnt either fuse partially or give an impure lime, called poor lime in distinction from that obtained from purer limestone, which is called rich lime. The latter kind is charac. terised by its disintegrating into a fine powder when treated with water, and is suitable for the majority of uses to which lime is applied, and for which the poor lime is sometimes quite unfit. However, certain kinds of poor lime (as we shall see in Chapter XVIII., Note 25) are used in the preparation of hydraulio cements, which solidify into a hard mass under water,

In order to obtain perfectly pure lime it is necessary to take the purest possible materials. In the laboratory, marble or shells are used for this purpose as a pure form of caloium carbonate. They are first burnt in a furnawe, then put in a crucible and moistened with a small quantity of water, and finally strongly ignited, by which means a pure lime is obtained. Pure lime may be more rapidly prepared by taking calcium nitrate, $\mathrm{CaN}_{2} \mathrm{O}_{6}$, which is easily obtained by dissolving limestone in nitric acid. The solution obtained is boiled with a small quantity of lime in order to precipitate the foreign oxides which are.insoluble in water. The oxides of iron, aluminium, \&c., are precipitated by this means. The salt is then crystallised and ignited: $\mathrm{Carr}_{2} \mathrm{O}_{6}$ $-\mathrm{CaO}+2 \mathrm{NO}_{2}+\mathrm{O}$.

In the decomposition of calcium carbonate the lime preserves the form of the lumps subjected to ignition; this is one of the signs distinguishing quicklime when it is freshly burnt and unaltered by air. It attracts moisture from the air and then disintegrates to a powder; if left long exposed in the air, it also attracts carbonic anhydride and increases in volume; it does not entirely pass into carbonate, but forms a compound of the latter with caustic lime. 
which is unaffected by heat, ${ }^{39}$ and may therefore serve as a fire. resisting material, and was employed by Deville for the construction of furnaces in which platinum was melted, and silver volatilised by the action of the heat evolved by the combustion of detonating gas. The hydrated lime, slaked lime, or calcium hydroxide, $\mathrm{CaH}_{2} \mathrm{O}_{2}$ (specific gravity 2.07) is a most common alkaline substance, employed largely
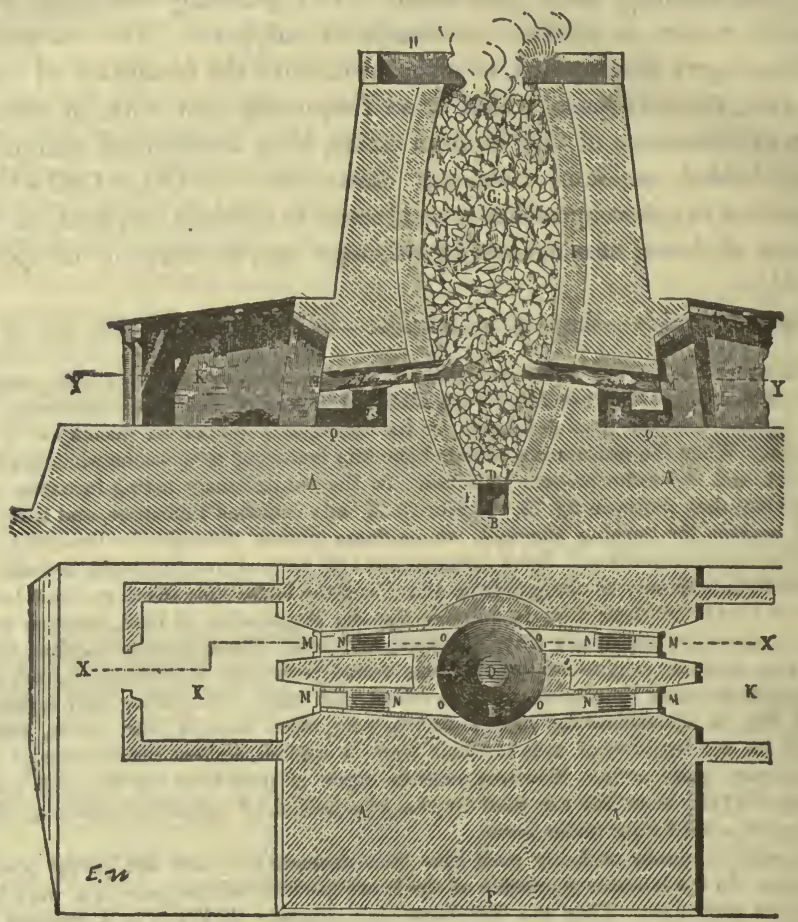

F10. 78. - Continually-acting kiln for burning lime. The lime is charged from above and caloined by four lateral grates, $\mathrm{R}, \mathrm{M}$. D, fire-bars. $\mathrm{B}$, space for withdrawing the burnt lime $\mathrm{K}$, stoke. house. $\mathrm{M}$, fire grate. $\mathrm{Q}, \mathrm{R}$, under,grate.

in building for making mortars or cements, in which case its bind. ing property is mainly due to the absorption of carbonic anhydride. ${ }^{10}$

89 Lime, when raised to white heat in the vapour of potassium, gives calcium, and In chlorine it gives off oxygen. Sulphur, phosphorus, \&c, when heated with lime, are absorbed by it.

40 The greater quantity of lime is used in making mortar for binding bricks or stones logether, in the form of lime or cement, or the so-called slaked lime. For this purpose the lime is mixed with water and sand, which serves. to separate the particles of lime 
Lime, like other alkalis, acts on many animal and vegetable substances, and for this reason has many practical uses-for example, for removing fats, and in agriculture for accelerating the decomposition of organic substances in the so-called composts or accumu. lations of vegetable and animal remains used for fertilising land. Calcium hydroxide easily loses its water at a moderate heat $\left(530^{\circ}\right)$, but it does not part with water at $100^{\circ}$. When mixed with water, line forms a pasty mass known as slaked lime and in a more dilute form as milk of lime, because when shaken up in water it remains suspended in it for a long time and presents the appearance of a milky liquid. But, besides this, lime is directly soluble in water, not to any considerable extent, but still in such a quantity that lime water is precipitated by carbonic anhydride, and has clearly distinguishable alkaline properties. One part of lime requires at the ordinary temperature about 800 parts of water for solution. At $100^{\circ}$ it requires about 1500 parts of water, and therefore lime-water becomes cloudy when boiled. If lime-water be evaporated in a vacuum, calcium hydroxide separates in six-sided crystals ${ }^{41}$ If lime-water be mixed with hydrogen peroxide minute crystals of calcium peroxide, $\mathrm{CaO}_{2}, 8 \mathrm{H}_{2} \mathrm{O}$, separate; this compound is very un. stable and, like barium peroxide, is decomposed by heat. Lime, as a powerful base, combines with all acids, and in this respect presents a transition from the true alkalis to magnesia. Many of the salts of

from each other. If only lime paste were put between two bricks they would not hold firnly together, because after the water had evaporated the lime would occupy a smaller space than before, and therefore cracks and powder would form in its mass, so that it would not at all produce that complete cementation of the bricks which it is desired to attain. Pieces of stone-that is, sand-mixed with the lime hinder this process of disintegration, because the lime binds together the individual grains of sand mixed with it, and forms one concrete mass, in consequence of a process which proceeds after the desiccation or removal of the water. The process of the solidification of lime, taken as slaked lime, consists first in the direct evaporation of the water and crystallisation of the hydrate, so that the lime binds the stones and sand mixed with it, just as glue binds two pieces of wood. But this preliminary binding action of lime is feeble (as is seen by direct experi. ment) unless there be further alteration of the lime leading to the formation of carbonates, silicates, and other salts of calcium which are distinguished by their great cohesiveness. With the progress of time the cement is partially subjected to the action of the carbonic anlyydride in the air, owing to which calcium carbonate is formed, but not more than half the lime is thus converted into carbonate. Besides which, the lime partially acts on the silica of the bricks, and it is owing to these new combinations simultaneously forming in the cement that it'gradually becomes stronger and stronger. Hence the binding action of the lime becomes stronger with the lapse of time. This is the reason (and not, as is sometimes said, because the ancients knew how to build stronger than we do) why build. ings which have stood for centuries possess a very strongly binding cement. Hydraulic cements will be described later (Chapter XVIII., Note 25).

41 Professor Glinka measured the transparent bright crystals of calcium hydroxide which are formed in common hydraulic (Portland) cement. 
calcium (the carbonate, phosphate, borate, and oxalate) are insoluble in water; besides which the sulphate is only sparingly soluble. As a more energetic base than magnesia, lime forms salts, $\mathrm{CaX}_{2}$, which are distinguished by their stability in comparison with the salts $\mathrm{MgX}_{2}$; neither does lime so easily form basic and double salts as magnesia.

Anhydrous lime does not absorb dry carbonic anhydride at the ordinary temperature. This was already known by Scheele, and Prof. Schuliachenke showed that there is no absorption even at $360^{\circ}$ It only proceeds at a red heat, ${ }^{42}$ and then only leads to the formation of a mixture of calcium oxide and carbonate (Rose). But if the lime be slaked or dissolved, the absorption of carbonic anhydride proceeds rapidly and completely. These phenomena are connected with the dissociation of calcium carbonate, studied by Debray (1867) under the influence of the conceptions of dissociation introduced into science by Henri Saint-Claire Deville. Just as there is no vapour tension for non-volatile substances, so there is no dissociation tension of carbonic anhydride for calcium carbonate at the ordinary

42 The act of heating brings the substance into that state of internal motion which is required for reaction. It should be considered that by the act of heating not only is the bond between the parts, or cohesion of the molecules, altered (generally diminished), not only is the motion or store of energy of the whole molecule increased, but also that in all probability the motion of the atoms themselves in molecules undergoes a change. The same kind of change is accomplished by the act of solution, or of combination in general, judging from the fact that a dissolved or combined substance-for instance, lime with water-reacts on carbonic anhydride as it does under the action of heat. For the comprehension of chemical phenomena it is exceedingly useful to recognise clearly this parallelism. Rose's observation on the formation (by the slow diffusion of solutions of calcium chloride and sodium carbonate) of aragonite from dilute, and of calc spar from strong, solutions is easily understood from this point of view. As aragonite is always formed from hot solutions, it appears that dilution with water acts like heat. The following experiment of Kiihlmann is particularly instructive in this sense. Anhydrous (perfectly dry) barium oxide does not react with monohydrated sulphuric acid, $\mathrm{H}_{2} \mathrm{SO}_{4}$ (containing neither free water nor anhydride, $\mathrm{SO}_{3}$ ). But if either an incandescent object or a moist substance is brought into contact with the mixture a violent reaction immediately begins (it is essentially the same as combustion), and the whole mass reacts.

The influence of solution on the process of reaction is instructively illustrated by the following experiment. Lime, or barium oxide, is placed in a flask or retort having an upper orifice and connected with a tube immersed in mercury. A funnel furnished with a stopcock and filled with water is fixed into the upper orifice of the retort, which is then filled with dry carbonic anhydride. There is no absorption. When a constant temperature is arrived at, the unslaked oxide is made to absorb all the carbonic anhydride by carefully admitting water. A vacuum is formed, as is seen by the mercury rising in the neck of the retort. With water the absorption 'goes on to the end, whilst under the action of heat there remains the dissociating tension of the carbonic anhydride. Furthermore, we here see that, with a certain resemblance, there is also a distinction, depending on the fact that at low temperatures calcium carbonate does not dissociate; this determines the complete absorption of the carbonic anhydride in the aqueous solution. 
temperature. Just as every volatile substance has a maximum possible vapour tension for every temperature, so also calcium carbonate has its corresponding dissociation tension; this at $770^{\circ}$ (the boiling point of cadmium) is about $85 \mathrm{~mm}$. (of the mercury column), and at $930^{\circ}$ (the boiling point of $\mathrm{Zn}$ ) it is about $520 \mathrm{~mm}$. As, if the tension be greater, there will be no evaporation, so also there will be nu decomposition. Debray took crvstals of calc spar, and could not observe the least change in them at the boiling point of zinc $\left(930^{\circ}\right)$ in an atmosphere of carbonic anhydride taken at the atmospheric pressure $(760 \mathrm{~mm}$.), whilst on the other hand calcium carbonate may be completely decomposed at a much lower temperature if the tension of the carbonic anhydride be kept below the dissociation tension, which may be done either by directly pumping away the gas with an air-pump, or by mixing it with some other gas - that is, by diminishing the partial pressure of the carbonic anhydride, ${ }^{43}$ just as an object may be dried at the ordinary temperature by removing the aqueous vapour or by carrying it off in a stream of another gas. Thus it is possible to obtain calcium carbonate from lime and carbonic anhydride at a certain temperature above that at which dissociation begins, and conversely to decompose calcium. carbonate at the same temperature into lime and carbonic anhydride. ${ }^{44}$ At the ordinary temperature the reaction of the first order (combination) cannot proceed because the second (decomposition, dissociation)

43 Experience has shown that by moistening partially-burnt lime with water and reheating it, it is easy to drive off the last traces of carbonic anhydride from it, and that, in general, by blowing air or steam through the lime, and even by using moist fuel, it.is possible to accelerate the decomposition of the calcium carbonate. The partial pressare is decreased by these means.

44 Before the introduction of Deville's theory of dissociation, the modus operandi of decompositions like that under consideration was understood in the sense that decomposition starts at a certain temperature, and that it is accelerated by a rise of temperature, bnt it was not considered possible that combination could proceed at the same temperature as that at which decomposition goes on. Berthollet and Deville introduced the conception. of equilibrium into chemical science, and elucidated the question of reversible reactions. Naturally the subject is still far from being clear-the questions of the rate and completeness of reaction, of contact, \&c., still intrude themselves-but an important step has been made in chemical mechanics, and we have started on a new path which promises further progress, towards which much has been done not only by Deville himself, but more especially by the French chemists Debray, Troost, Lemoine, Hautefeuille, Le Chatelier, and others. Among othor things those investigators have shown the close resemblance between the phenomena of evaporation and dissociation, and pointed out that the amount of heat absorbed by a dissociating substance may be calculated according to the law of the variation of dissociation-pressure, in exactly the same manner as it is possible to calculate the latent heat of the evaporation of water, knowing the variation of the tension with the temperature, on the basis of the second law of the mechanical theory of heat. Details of this subject must be looked for in special works on physical chemistry. One and the same conception of the mechanical theory of heat is applicable to dissociation and evaporation. 
cannot take place, and thus all the most important phenomena with respect to the behaviour of lime towards carbonic anhydride are explained by starting from one common basis. ${ }^{45}$

Calcium carbonate, $\mathrm{CaCO}_{\text {, }}$, is sometimes met with in nature in a crystalline form, and it forms an example of the phenomenon terned dimorphism-that is, it appears in two crystallime forms. When it exhibits combinations of forms belonging to the hexagonal system (sixsided prisms, rhombohedra, \&c.) it is called calc spar. Calc spar has a specific gravity of $2 \cdot 7$, and is further characterised by a distinct cleavage along the planes of the fundamental rhombohedron having an angle of $105^{\circ}$. Perfectly transparent Iceland spar presents a clear example of double refraction (for which reason it is frequently employed in physical apparatus). The other form of calcium carbonate occurs in crystals belonging to the rhombic system, and it.is then called aragonite; its specific gravity is 3.0 . If calcium carbonate be artificially produced by slow crystallisation at the ordinary temperature, it appears in the rhombohedral form, but if the crystallisation be aided by heat it then appears as aragonite. It may therefore be supposed that calc spar presents the form corresponding with a low temperature, and aragonite. with a higher temperature during crystallisation. ${ }^{46}$

43 But the question as to the formation of a basic calcium carbouate with a rise of temperature still remains unlecided. The presence of water complicates all the relations between lime and carbonic anhydride, all the more as the existence of an attraction between calcium carbonate and water is seen from its being able to give a crystallohydrate, $\mathrm{CaCO}_{3}, 5 \mathrm{H}_{2} \mathrm{O}$ (Pelouze), which crystallises in rhombic prisms of sp. gr. about $1 \cdot 77$ and loses its water at $20^{\circ}$. These crystals are obtained when a solution of lime in sugar and water is left long exposed to the air and slowly attracts carbonic anhydride from it, and also by the evaporation of such a solution at a temperature of about $3^{\circ}$. On the other hand, it is probable that an acid salt, $\mathrm{CaH}_{2}\left(\mathrm{CO}_{3}\right)_{2}$, is formed in an aqueous solution, not only because water containing carbonic acid dissolves calcium carbonate, but more especially in view of the researches of Schloesing (1872), which showed that at $16^{\circ}$ a litre of water in an atmosphere of carbonic anhydride (p pressure 0.984 atmo. sphere) dissolves 1.086 gram of calcium carbonate and 1.778 gram of carbonic anhydride, which corresponds with the formation of calcium hydrogen carbonate, and the solution of carbonic anhydride in the remaining water. Caro showed that a litre of water is able to dissolve as much as $3 \mathrm{grams}$ of calcium carbonate if the pressure be increased to 4 and more atmospheres. The calcium carbonate is precipitated when the carbonic anhydride passes off in the air or in a current of another gas; this also takes place in many natural springs. Tufa, stalactites, and other like formations from waters containing calcium carbonate and carbonic acid in solution are formed in this manner. The solubility of calcium carbonate itself at the ordinary temperature does not exceed 13 milligrams per litre of water.

46 Dimorphous bodies differ from true isomers and polymers in that they do not differ in their chemical reactions, which are determined by a difference in the distribu. tion (motion) of the atoms in the molecules, and therefore dimorphism is usually. ascribed to a difference in the distribution of similar molecules, building up a crystal. Although such a hypothesis is quite admissible in the spirit of the atomic and molecular theory, yet, as in such a redistribution of the molecules a perfect conservation of the distribution of the atoms in them cannot be imagined, and in every effort of chemical 
Calcium sulphate in combination with two equivalents of water, $\mathrm{CaSO}_{4}, 2 \mathrm{H}_{2} \mathrm{O}$, is very widely distributed in nature, and is known as gypsum. Gypsum loses one and a half and two equivalents of water at a moderate temperature, ${ }^{47}$ and anhydrous or burnt gypsum is then obtained, which is also known as plaster of Paris, and is employed in large quantities for modelling. ${ }^{48}$ This use depends on the fact that burnt and finely-divided and sifted gypsum forms a paste when mixed with water ; after a certain time this paste becomes slightly heated and solidifies, owing to the fact that the anhydrous calcium sulphate, $\mathrm{CaSO}_{4}$, again combines with water. When the plaster of Paris and water are first made into a paste they form a mechanical mixture, but when the mass solidifies, then a compound of the calcium sulphate with two molecules of water is produced; and this may be regarded as derived from $\mathrm{S}(\mathrm{OH})_{6}$ by the substitution of two atoms of hydrogen by one atom of bivalent calcium. Natural gypsum sometimes appears as perfectly colourless, or variegated, marble-like, masses, and sometimes in perfectly colourless crystals, selenite, of specific gravity 2.33. The semi-transparent gypsum, or alabaster, is often carved into small statues. Besides

reaction there must take place a certain motion among the atoms; so in my opinion there is no firm basis for distinguishing dimorphism from the general conception of isomerism, under which the cases of those organic bodies which are dextro and lævo rotatory (with respect to polarised light) have recently been brought with such brilliant success. When calcium carbonate separates out from solutions, it has at first a gelatinous aprearance, which leads to the supposition that this salt appears in a colloidal state. It only crystallises with the progress of time. The colloidal state of calcium carbonate is particularly clear from the following observations made by Prof. Famintzin, who showed that when it separates from solutions it is obtained under certain conditions in the form of grains having the peculiar paste-like structure proper to starch, which fact has not only an independent interest, but presents an example of a mineral substance being obtained in a form until then only known in the organic substances elaborated in plants. This shows that the forms (cells, vessels, \&c.) in which vegetable and animal substances occur in organisms do not present in themselves anything peculiar to organisms, but are only the result of those particular conditions in which these substances are formed. Traube and afterwards Monnier and Vogt (1882) obtained formations which, under the microscope, were in every respect identical in appearance with regetable cells, by means of a similar slow formation of precipitates (by reacting on sulphates of different metals with sodium silicate or carbonate).

47 According to $\mathrm{Le}$ Chatelier (1888), $1 \frac{1}{\mathrm{H}_{2} \mathrm{O}}$ is lost at $120^{\circ}$-that is, $\mathrm{H}_{2} \mathrm{O}, 2 \mathrm{CaSO}_{4}$ is formed, but at $194^{\circ}$ all the water is expelled. According to Shenstone and Cundall (1888) gypsum begins to lose water at $70^{\circ}$ in dry air. The semi-hydrated compound $\mathrm{H}_{2} \mathrm{O}, 2 \mathrm{CaSO}_{4}$ is also formed when gypsum is heated with water in a closed vessel at $150^{\circ}$ (Hoppe-Seyler).

48 For stucco-work it is usual to add lime and sand, as the mass is then harder and does not solidify so quickly. For imitating marble, glue is added to the plaster, and the mass ia polished when thoronghly dry. Re burnt gypsum cannot be used over again, as that which has once solidified is, like the natural anhydride, not able to recombine with water. It is evident that the structure of the molecules in the crystallised mass, or in general in any dense mass, exerts an influence on the chemical action, which is more particularly cvident in metals in their different forms (powder, crystalline, rolled, \&c.) 
which an anhydrous calcium sulphate, $\mathrm{CaSO}_{4}$, called anhydrite (specific gravity 2.97), occurs in nature. It sometimes occurs along with gypsum. It is no longer capable of combining directly with water, and differs in this respect from the anhydrous salt obtained by gently ignit. ing gypsum. If gypsum be very strongly heated it shrinks and loses its power of combining with water. 48 bis One part of calcium sulphate requires at $0^{\circ} 525$ parts of water for solution, at $38^{\circ} 466$ parts, and at $100^{\circ}$ 571 parts of water. The maximum solubility of gypsum is at about $36^{\circ}$, which is nearly the same temperature as that at which sodium sulphate is nost soluble. ${ }^{49}$

As lime is a more energetic base than magnesia, so calcium chloride, $\mathrm{CaCl}_{2}$, is not so easily decomposed by water, and its solutions only disengage a small quantity of hydrochloric acid when evaporated, and when the evaporation is conducted in a stream of hydrochloric acid it easily gives an anhydrous salt which fuses at $719^{\circ}$; otherwise an aqueous solution yields a crystallo-hydrate, $\mathrm{CaCl}_{2}, 6 \mathrm{H}_{2} \mathrm{O}$, which melts at $30^{\circ} 50$

48 bls According to MacColeb, gypsum dehydrated at $200^{\circ}$ has a specific gravity 2.577, and heated to its point of fusion, 2.654. Potilitzin (1894) also admits the two above-named modifications of anhydrous gypsum, which, moreover, always contain the semi-hydrated hydrate (Note 47), and he explains by their relation to water the phenomena observed in the solidification of a mixture of burnt gypsum and water.

49 As Marignac showed, gypsum, especially when desicated at $120^{\circ}$, easily gives supersaturated solutions with respect to $\mathrm{CaSO}_{4}, 2 \mathrm{H}_{2} \mathrm{O}$, which contain as much as 1 part of $\mathrm{CaSO}_{4}$ to 110 parts of water. Boiling dilute hydrochloric acid dissolves gypsum, forming calcium chloride. The behaviour of gypsum towards the alkaline carbonates has been described in Chapter $\mathbf{X}$. Alcohol precipitates gypsum from its aqueous solutions, because, like the sulphates in general, it is sparingly soluble in alcohol. Gypsum, like all the sulphates, when heated with charcoal, gives up its oxygen, forming the sulphide, CaS.

Calcium sulphate, like magnesium sulphate, is capable of forming double salts, but with difficulty, and they are chemically less stable. They contain, as is always the case with double salts, less water of crystallisation than the component salts. Rose, Struvé, and others obtained the salt $\mathrm{CaK}_{2}\left(\mathrm{SO}_{4}\right)_{2}, \mathrm{H}_{2} \mathrm{O}$; a mixture of gypsum with an equivalent amount of potassium sulphate and water solidifies into a homogeneous mass. Fritzsche obtained the corresponding sodium salt in a hydrated and anhydrous state, by heating a mixture of gypsum with a saturated solution of sodium sulphate. The anhydrous salt occurs in nature as glauberite. Fritzsche also obtained gaylussite, $\mathrm{Na}_{2} \mathrm{Ca}\left(\mathrm{CO}_{3}\right)_{2}, 5 \mathrm{H}_{2} \mathrm{O}$, by pouring a saturated solution of sodium carbonate on to freshly-precipitated calcinm carbonate. Calcium also forms basic salts, but only a few. Veeren (1892) obtained $\mathrm{Ca}\left(\mathrm{NO}_{3}\right)_{2} \mathrm{Ca}(\mathrm{OH})_{2}, 2 \frac{1}{2} \mathrm{H}_{2} \mathrm{O}$ by leaving powdered caustic lime in a saturated solution of $\mathrm{Ca}\left(\mathrm{NO}_{3}\right)_{2}$ until it solidified. This salt is decomposed by water.

50 Calcium chloride has a specific gravity $2 \cdot 20$, or, when fused, $2 \cdot 12$, and the sp. gr. of the crystallised salt $\mathrm{CaCl}_{2}, 6 \mathrm{H}_{2} \mathrm{O}$ is 1.69 . If the volume of the crystals at $0^{\circ}=1$, then at $29^{\circ}$ it is 1.020 ; and the volume of the fused mass at the same temperature is $1 \cdot 118$ (Kopp) (specific gravity of solutions, see Note 27). The solution containing 50 p.c. $\mathrm{CaCl}_{2}$ boils at $130^{\circ}, 70$ p.c. at $158^{\circ}$. Superheated steam decomposes calcium chloride with more diffculty than magnesium chloride and with greater ease than barium chloride (Kuhnheim). Sodium does not decompose fused calcium chloride even on prolonged heating (LiésBodart), but an alloy of sodium with zino, lead, and bismuth decomposes it, forming an 
Just as for potassium, $\mathrm{K}=39$ (and sodium, $\mathrm{Na}=23$ ), there are the near analogues, $\mathrm{Rb}=85$ and $\mathrm{Cs}=133$, and also another, $\mathrm{Li}=7$, so

alloy of calcium with one of the above-named metals (Caron). The zinc alloy may be obtained with as much as 15 p.c. of calcium. Calcium chloride is soluble in alcohol and absorbs ammonia.

A gram molecular weight of calcium chloride in dissolving in an excess of water evolves 18,723 calories, and in dissolving in alcohol 17,555 units of heat, according to Pickering.

Roozeboom made detailed researches on the crystallo-hydrates of calcium chloride (1889), and found that $\mathrm{CaCl}_{2,}, 6 \mathrm{H}_{2} \mathrm{O}$ melts at $30^{\circ} \cdot 2$, and is formed at low temperatures from solutions containing not more than 103 parts of calcium chloride per 100 parts of water; if the amount of salt (always to 100 parts of water) reaches 120 parts, then tabular crystals of $\mathrm{CaCl}_{2}, 4 \mathrm{H}_{2} \mathrm{O} \beta$ are formed, which at temperatures above $38.4^{\circ}$ are converted into the crystallo-hydrates $\mathrm{CaCl}_{2}, 2 \mathrm{H}_{2} \mathrm{O}$, whilst at temperatures below $18^{\circ}$ the $\beta$ variety passes into the more stable $\mathrm{CaCl}_{2}, 4 \mathrm{H}_{2} \mathrm{O} \alpha$, which process is aided by mechanical friction. Hence, as is the case with magnesium sulphate (Note 27), one and the same crystallo. hydrate appears in two forms-the $\beta$, which is easily produced but is unstable, and the $\alpha$, which is stable. The solubility of the above-mentioned hydrates of chloride of calcium, or amount of calcium chloride per 100 parts of water, is as follows :-

\begin{tabular}{|c|c|c|c|c|c|}
\hline & $0^{\circ}$ & $20^{\circ}$ & $30^{\circ}$ & $40^{\circ}$ & $60^{\circ}$ \\
\hline $\mathrm{CaCl}_{2}, 6 \mathrm{H}_{2} \mathrm{O}$ & 60 & 75 & 100 & & $2 \cdot 8)$ \\
\hline $\mathrm{CaCl}_{2}, 4 \mathrm{H}_{2} \mathrm{O} \alpha$ & - & 90 & 101 & 117 , & \\
\hline $\mathrm{CaCl}_{2}, 4 \mathrm{H}_{2} \mathrm{O} \boldsymbol{\beta}$ & - & 104 & 114 & $-\}$ & \\
\hline $\mathrm{CaCl}_{2}, 2 \mathrm{H}_{2} \mathrm{O}$ & - & - & $(308 \cdot 3)$ & 128 & 137 \\
\hline
\end{tabular}

The amount of calcium chloride to 100 parts of water in the crystallo-liydrate is given in brackets. The point of intersection of the curves of solubility lies at about $80^{\circ}$ for the first two salts and about $45^{\circ}$ for the salts with $4 \mathrm{H}_{2} \mathrm{O}$ and $2 \mathrm{H}_{2} \mathrm{O}$. The crystals $\mathrm{CaCl}_{2}, 2 \mathrm{H}_{2} \mathrm{O}$ may, however, be obtained (Ditte) at the ordinary temperature from solutions containing hydrochloric acid. The vapour tension of this crystallo-hydrate equals the atmospheric at $165^{\circ}$, and therefore the crystals may be dried in an atmosphere of steam and obtained without a mother liquor, whose vapour tension is greater. This crystallo-hydrate decomposes at about $175^{\circ}$ into $\mathrm{CaCl}_{2}, \mathrm{H}_{2} \mathrm{O}$ and a solution; this is easily brought about in a closed vessel when the pressure is greater than the atmosphere. This crystallo-hydrate is destroyed at temperatures above $260^{\circ}$, anhydrous calcium chloride being formed.

Neglecting the unstable modification $\mathrm{CaCl}_{2}, 4 \mathrm{H}_{2} \mathrm{O}$, we will give the temperatures $t$ at which the passage of one hydrate into another takes place and at which the solution $\mathrm{CaCl}_{2}+n \mathrm{H}_{2} \mathrm{O}$, the two solids $\mathrm{A}$ and $\mathrm{B}$ and aqueous vapour, whose tension is given as $p$ in millimetres, are able to exist together in stable equilibrium, according to Roozeboom's determinations :

\begin{tabular}{ccclc}
$t$ & $n$ & \multicolumn{1}{c}{$\mathbf{A}$} & \multicolumn{1}{c}{$\mathrm{B}$} & $p$ \\
$-55^{\circ}$ & 14.5 & \multicolumn{1}{c}{$\mathrm{ice}$} & $\mathrm{CaCl}_{2}, 6 \mathrm{H}_{2} \mathrm{O}$ & 0 \\
$+29 \cdot 8^{\circ}$ & $6 \cdot 1$ & $\mathrm{CaCl}_{2}, 6 \mathrm{H}_{2} \mathrm{O}$ & $\mathrm{CaCl}_{2}, 4 \mathrm{H}_{2} \mathrm{O}$ & 6.8 \\
$45 \cdot 3^{\circ}$ & $4 \cdot 7$ & $\mathrm{CaCl}_{2}, 4 \mathrm{H}_{2} \mathrm{O}$ & $\mathrm{CaCl}_{2}, 2 \mathrm{H}_{2} \mathrm{O}$ & 11.8 \\
$175 \cdot 5^{\circ}$ & $2 \cdot 1$ & $\mathrm{CaCl}_{2}, 2 \mathrm{H}_{2} \mathrm{O}$ & $\mathrm{CaCl}_{2}, \mathrm{H}_{2} \mathrm{O}$ & 842 \\
$260^{\circ}$ & 1.8 & $\mathrm{CaCl}_{2}, \mathrm{H}_{2} \mathrm{O}$ & $\mathrm{CaCl}_{2}$ & Several atmospheres
\end{tabular}

Solutions of calcium chloride may serve as a convenient example for the study of the supersaturated state, which in this case easily occurs, because different hydrates are formed. Thus at $25^{\circ}$ solutions containing more than 83 parts of anhydrous calcium chloride per 100 of water will be supersaturated for the hydrate $\mathrm{CaCl}_{2}, 6 \mathrm{H}_{2} \mathrm{O}$.

On the other hand, Hammerl showed that solutions of calcium chloride, when frozen, deposit ice if they contain less than 43. parts of salt per 100 of water, and if more the 
in exactly the same manner for calcium, $\mathrm{Ca}=40$ (and magnesium, $\mathrm{Mg}=24$ ), there is another analogue of lighter atomic weight, beryllium, $\mathrm{Be}=9$, besides the near analogues strontium, $\mathrm{Sr}=87$, and barium, $\mathrm{Ba}=137$. As rubidium and cæsium are more rarely met with in nature than potassium, so also strontium and barium are rarer than calcium (in the same way that bromine and iodine are rarer than chlorine). Since they exhibit many points of resemblance with calcium, strontium and barium may be characterised after a very short acquaintance with their chief compounds ; this shows the important advantages gained by distributing the elements according to their natural groups, to which matter we shall turn our attention in the next chapter.

Among the compounds of barium met with in nature the commonest is the sulphate, $\mathrm{BaSO}_{4}$, which forms anhydrous crystals of the rhombic system, which are identical in their erystalline form with anhydrite, and generally occur as transparent and semi-transparent masses of tabular erystals having a high specific gravity, namely $4 \cdot 45$, for which reason this salt bears the name of heavy spar or barytes. Analogous to it is celestine, $\mathrm{SrSO}_{4}$, which is, however, more rarely met with. Heavy spar frequently forms the gangue separated on dressing metallic ores from the vein stuff; this mineral is the. source of all other barium compounds; for the carbonate, although more easily transformed into the other compounds (because acids act directly on it, evolving carbonic anhydride), is a comparatively rare mineral $\left(\mathrm{BaCO}_{3}\right.$ forms the mineral witherite; $\mathrm{SrCO}_{3}$, strontianite; both are rare, the latter is found at Etna). The treatment of barium sulphate is rendered difficult from the fact that it is insoluble both in water and acids, and has therefore to be treated by a method of reduction. ${ }^{51}$ Like sodium sulphate and calcium sulphate, heavy spar when heated with charcoal parts with its oxygen and forms barium sulphide, BaS. For this purpose a pasty mixture of powdered heavy spar, charcoal, and tar is subjected to the action of a strong heat, when $\mathrm{BaSO}_{4}$ $+4 \mathrm{C}=\mathrm{BaS}+4 \mathrm{CO}$. The residue is then treated with water, in which the barium sulphide is soluble. ${ }^{52}$ When boiled with hydrochloric acid,

crystallo-hydrate $\mathrm{CaCl}_{2}, 6 \mathrm{H}_{2} \mathrm{O}$ separates, and that a solution of the above composition $\left(\mathrm{CaCl}_{2}, 14 \mathrm{H}_{2} \mathrm{O}\right.$ requires 44.0 parts calcium chloride per 100 of water) solidifies as a cryohydrate at about $-55^{\circ}$.

31 The action of barium sulphate on sodium and potassium carbonates is given on p. 487.

52 Barium sulphide is decomposed by water, $\mathrm{BaS}+2 \mathrm{H}_{2} \mathrm{O}=\mathrm{H}_{2} \mathrm{~S}+\mathrm{Ba}(\mathrm{OH})_{2}$ (the reaction is reversible), but both substances are soluble in water, and their separation is complicated by the fact that barium sulphide absorbs oxygen and gives insoluble barium sulphate. The hydrogen sulphide is sometimes removed from the solution by boiling with the oxides of copper or zinc. If sugur be added to a solution of barium sulphide, barium saccharate is precipitated on heating; it is decomposed by carbonic anhy- 
barium chloride, $\mathrm{BaCl}_{2}$, is obtained in solution, and the sulphur is disengaged as gaseous sulphuretted hydrogen, $\mathrm{BaS}+2 \mathrm{HCl}=\mathrm{BaCl}_{2}+\mathrm{H}_{2} \mathrm{~S}$. In this manner barium sulphate is converted into barium chloride, ${ }^{53}$ and the latter by double decomposition with strong nitric acid or nitre gives the less soluble barium nitrate, $\mathrm{Ba}\left(\mathrm{NO}_{3}\right)_{2},{ }^{54}$ or with sodium

dride, so that barium carbonate is formed. An equivalent mixture of sodium sulphate with barium or strontium sulphates when ignited with charcoal gives a mixture of sodium sulphide and barium or strontium sulphide, and if this mixture be dissolved in water and the solution evaporated, barium or strontium hydroxide crystallises out on cooling, and sodium hydrosulphide, NaHS, is obtained in solution. The hydroxides $\mathrm{BaH}_{2} \mathrm{O}_{2}$ and $\mathrm{SrH}_{2} \mathrm{O}_{2}$ are prepared on a large scale, being applied to many reactions; for example, strontium hydroxide is prepared for sugar works for extracting crystallisable sugar from molasses.

We may remark that Boussingault, by igniting barium sulphate in hydrochloric acid gas, obtained a complete decomposition, with the formation of barium chloride. Attention should also be turned to the fact that Grouven, by heating a mixture of charcoal and strontium sulphate with magnesium and potassium sulphates, showed the easy decom. posability depending on the formation of double salts, such as $\mathrm{SrS}, \mathrm{K}_{2} \mathrm{~S}$, which are easily soluble in water, and give a precipitate af strontium carbonate with .carbonic anhydride. In such examples as these we see that the force which binds double salts may play a part in directing the course of reactions, and the number of double salts of silica on the earth's surface shows that nature takes advantage of these forces in her chemical processes. It is worthy of remark that Buchner (1893), by mixing a 40 per cent. solution of barium acetate with a 60 per cent. solution of sulphate of alumina, obtained a thick glutihous mass, which only gave a precipitate of $\mathrm{BaSO}_{4}$ after being diluted with water.

55 Barium sulphate is sometimes converted into barium chloride in the following manner: finely-ground barium sulphate is heated with coal and manganese chloride (the residue from the manufacture of chlorine). The mass becomes semi-liquid, and when it evolves carbonic oxide the heating is stopped. The following double decom. positions proceed during this operation: first the carbon takes up the oxygen from the barium sulphate, and gives sulphide, BaS, which enters into double decomposition with the chloride of manganese, $\mathrm{MnCl}_{2}$, forming manganese sulphide, $\mathrm{MnS}$, which is insoluble in water, and soluble barium chloride. This solution is easily obtained pure because mnny foreign impurities, such as iron, remain in the insoluble portion with the manganese. The solution of barium chloride is chiefly used for the preparation of barium sulphate, which is precipitated by sulphuric acid, by which means barium sulphate is re-formed as a powder. This salt is characterised by tho fact that it is unacted on by the majority of chemical reagents, is insoluble in water, and is not dissolved by acids. Owing to this, artificial barium sulphate forms a permanent white paint which is used instead of (and mixed with) white lead, and has been termed 'blane fixé' or 'permanent white.

The solution of one part of calcium chloride at $20^{\circ}$ requires 1.36 part of water, the solution of one part of strontium chloride requires 1.88 part of water at the same tem. perature, and the solution of barium chloride 2.88 parts of water. The solubility of the bromides and-iodides varies in the same proportion. The chlorides of barium and stron. tium crystallise out from solution with great ease in combination with water; they form $\mathrm{BaCl}_{2}, 2 \mathrm{H}_{2} \mathrm{O}$ and $\mathrm{SrCl}_{2}, 6 \mathrm{H}_{2} \mathrm{O}$. The latter (which separates out at $40^{\circ}$ ) resembles the salts of $\mathrm{Ca}$ and $\mathrm{Mg}$ in composition, and Étard (1892) obtained $\mathrm{SrCl}_{2}, 2 \mathrm{H}_{2} \mathrm{O}$ from solutions at $90-130^{2}$. We may also observe that the crystallo-hydrates $\mathrm{BaBr}_{2}, \mathrm{H}_{2} \mathrm{O}$ and $\mathrm{BaI}_{2}, 7 \mathrm{H}_{2} \mathrm{O}$ are known.

54 The nitrates $\mathrm{Sr}\left(\mathrm{NO}_{3}\right)_{2}$ (in the cold its solutions give a crystallo-hydrate containing $\left.4 \mathrm{H}_{2} \mathrm{O}\right)$ and $\mathrm{Ba}\left(\mathrm{NO}_{3}\right)_{2}$ are so very sparingly soluble in water that they separate in considerable quantities when $a$ solution of sodium nitrate is added to a strong solution of eithes 
carbonate a precipitate of barium carbonate, $\mathrm{BaCO}_{3}$. Both these salts are able to give barium oxide, or baryta, $\mathrm{BaO}$, and the hydroxide, $\mathrm{Ba}(\mathrm{HO})_{2}$, which differs from lime by its great solubility in water, ${ }^{55}$ and by the ease with which it forms a crystallo-hydrate, $\mathrm{BaH}_{2} \mathrm{O}_{2}, 8 \mathrm{H}_{2} \mathrm{O}$, from its solutions. Owing to its solubility, baryta is frequently employed in manufactures and in practical chemistry as an alkali which has the very important property that it may be always entirely removed from solution by the addition of sulphuric acid, which entirely separates it as the insoluble barium sulphate, $\mathrm{BaSO}_{4}$. It may also be removed whilst it remains in an alkaline state (for example, the excess which may remain when it is used for saturating acids) by means of carbonic anhydride, which also completely precipitates baryta as a sparingly soluble, colourless, and powdery carbonate. Both these reactions show that baryta has such properties as would very greatly extend its use were its compounds as widely distributed as those of sodium and calcium, and were its soluble compounds not poisonous. Barium nitrate is directly decomposed by the action of heat, barium oxide being left behind. The same takes place with barium carbonate, especially that form of it precipitated from solutions, and when mixed with charcoal or ignited in an atmosphere of steam. Barium oxide combines with water with the development of a $\operatorname{larg} \theta$ amount of heat, and the resultant hydroxide is very stable in its retention of the water, although it parts with it when strongly ignited. ${ }^{55}$ bis With oxygen the anhydrous oxide gives, as already mentioned in

barium or strontium chloride. They are obtained by the action of nitric acid on the carbon. ates or oxides. 100 parts of water at $15^{\circ}$ dissolve 6.5 parts of strontium nitrate and 8.2 parts of barium nitrate, whilst more than 300 parts of calcium nitrate are soluble at the same temperature. Strontium nitrate communicates a crimson coloration to the flame of burning substances, and is therefore frequently used for Bengal fire, fireworks, and signal lights, for which purpose the salts of lithium are still better fitted. Calcium nitrate is exceedingly hygroscopic. Barium nitrate, on the contrary, does not show this property in the least degree, and in this respect it resembles potassium nitrate, and is therefore used instead of the latter for the preparation of a gunpowder which is called 'saxifragin powder' (76 parts of barium nitrate, 2 parts of nitre, and 22 parts of charcoal).

55 The dissociation of the crystallo-hydrate of baryta is given in Chapter I., Note 65. 100 parts of water dissolve

$\begin{array}{llcccc} & 0^{\circ} & 20^{\circ} & 40^{\circ} & 60^{\circ} & 80^{\circ} \\ \mathrm{BaO} & 1.5 & 8.5 & 7.4 & 18.8 & 90.8 \\ \mathrm{SrO} & 0.3 & 0.7 & 1.4 & 3 & 9\end{array}$

Supersaturated solutions are easily formed.

The anhydrous oxide. $\mathrm{BaO}$ fuses in the oxyhydrogen flame. When ignited in the vapour of potassium, the latter takes up the oxygen; whilst in chlorine, oxygen is separated and barium chloride formed.

55 bis Brugellmann, by heating $\mathrm{BaH}_{2} \mathrm{O}_{2}$ in a graphite or clay crucible, obtained $\mathrm{BaO}$ in needles, sp. gr. 5.32 , and hy heating in a platinum crucible-in crystals belonging to 
Chapters III. and IV, a peroxide, $\mathrm{BaO}_{2}{ }^{56}$ Neither calcium nor strontium oxides are able to give such a peroxide directly, but they form peroxides under the action of hydrogen peroxide.

Barium oxide is decomposed when heated with potassium; fused barium chloride is decomposed, as Davy showed, by the action of a galvanic current, forming metallic barium ; and Crookes (1862) obtained an amalgam of barium from which the mercury could easily be driven off, by heating sodium amalgam in a saturated solution of barium chloride. Strontium is obtained by the same processes. Both metals are soluble in mercury, and seem to be non-volatile or only very slightly volatile. They are both heavier than water; the specific gravity of barium is $3 \cdot 6$, and of strontium $2 \cdot 5$. They both decompose water at the ordinary temperature, like the metals of the alkalis.

Barium and strontium as saline elements are characterised by their powerful basic properties, so that they form acid salts with difficulty, and scarcely form basic salts. On comparing them together and with calcium, it is evident that the alkaline properties in this group (as in the group potassium, rubidium, cæsium) increase with the atomic weight, and this succession clearly shows itself in many of their corresponding compounds. Thus, for instance, the solubility of the hydroxides $\mathrm{RH}_{2} \mathrm{O}_{2}$ and the specific gravity ${ }^{57}$ rise in passing from calcium to strontium and barium, while the solubility of the sulphates

the cubical system, sp. gr. $5 \cdot 74$. SrO is obtained in the latter form from the nitrate. The following are the specific gravities of the oxides from different sources :-

$\begin{array}{cccr} & \mathrm{MgO} & \mathrm{CaO} & . \mathrm{SrO} \\ \text { from } \mathrm{RN}_{2} \mathrm{O}_{6} & 3 \cdot 38 & 3 \cdot 25 & 4 \cdot 75 \\ \text { " } \mathrm{RCO}_{3} & 3 \cdot 48 & \mathbf{3} 26 & 4 \cdot 45 \\ \Rightarrow \mathrm{RH}_{2} \mathrm{O}_{2} & 3 \cdot 41 & \mathbf{8} 25 & 4.57\end{array}$

56 The property of barium oxide of absorbing oxygen when heated, and giving the peroxide, $\mathrm{BaO}_{2}$, is very characteristic for this oxide (see Chapter III., Note 7). It only belongs to the anhydrous oxide. The hydroxide does not absorb oxygen. Peroxides of calciun and strontium may be obtained by means of hydrogen peroxide. Barium peraxide is insoluble in water, but is able to form a hydrate with it, and also to combine with hydrogen peroxide, forming a very unstable compound having the composition $\mathrm{BaH}_{2} \mathrm{O}_{4}$ (obtained by Professor Schöne), which in course of time evolves oxygen (Chapter IV., Note 21).

57 Even in solutions a gradual progression in the increase of the specific gravity shows itself, not only for equivalent solutions (for instance, $\mathrm{RCl}_{2}+200 \mathrm{H}_{2} \mathrm{O}$ ), but even with an equal percentage composition, as is seen from the curves giving the specific gravity (water $4^{\circ}=10,000$ ) at $15^{\circ}$ (for barium chloride, according to Bourdiakoff's determinetions):

$$
\begin{aligned}
& \mathrm{BeCl}_{2}: \mathrm{S}=9,992+67 \cdot 21 p+0 \cdot 111 p^{2} \\
& \mathrm{CaCl}_{2}: \mathrm{S}=9,992+80 \cdot 24 p+0 \cdot 476 p^{2} \\
& \mathrm{SrCl}_{2}: \mathrm{S}=9,992+85 \cdot 57 p+0 \cdot 733 p^{2} \\
& \mathrm{BaCl}_{2}: \mathrm{S}=9,992+86 \cdot 56 p+0 \cdot 813 p^{2}
\end{aligned}
$$


decreases, ${ }^{58}$ and therefore in the case of magnesium and beryllium, as metals whose atomic weights are still less, we should expect the solu. bility of the sulphates to be greater, and this is in reality the case.

Just as in the series of the alkali metals we saw the metals potassium, rubidium, and crsium approaching near to each other in their properties, and allied to them two metals having smaller combining weights-namely, sodium, and the lightest of all, lithium, which all exhibited certain peculiar characteristic properties-so also in the case of the metals of the alkaline earths we find, besides calcium, barium, and strontium, the metal magnesium and also beryllium or glucinum. In respect to the magnitude of its atomic weight, this last occupies the same position in the series of the metals of the alkaline earths as lithium does in the series of the alkali metals, for the combining weight of beryllium, $\mathrm{Be}$ or $\mathrm{Gl}=9$. This combining weight is greater than that of lithium (7), as the combining weight of magnesium (24) is greater than that of sodium (23), and as that of calcium (40) is greater than that of potassium (39), \&c. ${ }^{59}$ Beryllium was so named because it occurs in the mineral beryl. The metal is also called glucinum (from the Greek word $\gamma \lambda v \kappa v$ 's, 'sweet'), because its salts have a sweet taste. It occurs in beryl, aquamarine, the emerald, and other minerals, which are generally of a green colour ; they are sometimes found in considerable masses, but as a rule are comparatively rare and, as transparent crystals, form precious stones. The composition of beryl and of the emerald is as follows : $\mathrm{Al}_{2} \mathrm{O}_{3}, 3 \mathrm{BeO}, 6 \mathrm{SiO}_{2}$. The Siberian and Brazilian beryls are the best known. The specific gravity of beryl is about $2 \cdot 7$. Beryllium oxide, from the feebleness of its basic properties, presents

58 One part of calcium sulphate at the ordinary temperature requires about 500 parts of water for solution, strontium sulplate about 7,000 parts, barium sulphate about 400,000 parts, whilst beryllium sulphate is easily soluble in water.

59 We refer beryllium to the class of the bivalent metals of the alkaline earths-that is, we ascribe to its oxide the formula $\mathrm{BeO}$, and do not consider it as trivalent ( $\mathrm{Be}=13.5$, Chapter VII., Note 21), although that view has been upheld by many chemists. The true atomic composition of beryllium oxide was first given by the Russian ehemist, Avdéeff (1819), in his researches on the compounds of this metal. He compared the compounds of beryllium to those of mngnesium, and refuted the notion prevalent at the time, of the resemblance between the oxides of beryllium and aluminium, by proving that beryllium sulphate presents a greater resemblance to magnesium sulphate than to aluminium sulphate. It was especially noticed that the analogues of alumina give alums, whilst beryllium oxide, although it is a feeble base, easily giving, like magnesia, basic and double salts, does not form true alums. The establishment of the periodic system of the elements (1869), considered in the following chapter, immediately indicated that Avdeeff's view corresponded with the truth-that is, that beryllium is bivalent, which therefore necessitated the denial of its trivalency. This scientific controversy. resulted in a long series of researches (1870-80) concerning this element, and ended in Nilson and Pettersson-two of the chief advocates of the trivalency of beryllium-deter. mining the vapour density of $\mathrm{BeCl}_{2}(=40$, Chapter VII., Note 21), which gave an undoubted proof of its bivalency (see also Note 8 ). 
an analogy to aluminium oxide in the same way that lithium oxide is analogous to magnesium oxide. ${ }^{60}$ Owing to its rare occurrence in nature, to the absence of any especially distinct individual properties, and to the possibility of foretelling them to a certain extent on the basis of the periodic system of the elements given in the following chapter, and owing to the brevity of this treatise, we will not discuss at any length the compounds of beryllium, and will only observe that their individuality was pointed out in 1798 by Vauquelin, and that metallic beryllium was obtained by Wöhler and Bussy. Wöhler obtained metallic beryllium (like magnesium) by acting on beryllium chloride, $\mathrm{BeCl}_{2}$, with potassium (it is best prepared by fusing $\mathrm{K}_{2} \mathrm{BeF}_{4}$ with $\mathrm{Na}$ ). Metallic beryllium has a specific gravity 1.64 (Nilson and Pettersson). It is very infusible, melting at nearly the same temperature as silver, which it resembles in its white colour and lustre. It is characterised by the fact that it is very difficultly oxidised, and even in the oxidising flame of the blowpipe is only superficially covered by a coating of oxide; it does not burn in pure oxygen, and does not decompose water at the ordinary temperature or

60 Beryllium oxide, like aluminium oxide, is precipitated from solutions of its salts by alkalis as a gelatinous hydroxide, $\mathrm{BeH}_{2} \mathrm{O}_{2}$, which, like alumina, is soluble in an excess of caustic potash or soda. This reaction may be taken advantage of for distinguishing and separating beryllium from aluminium, because when the alkaline solution is diluted with water and boiled, beryllium hydroxide is precipitated, whilst the alumina remains in solution. The solubility of the beryllium oxide at once clearly indicates its feeble basic properties, and, as it were, separates this oxide from the class of the alkaline earths. But on arranging the oxides of the above-described metals of the alkaline earths accord. ing to their decreasing atomic weights we have the series

$$
\mathrm{BaO}, \mathrm{SrO}, \mathrm{CaO}, \mathrm{MgO}, \mathrm{BeO} \text {, }
$$

in which the basic properties and solubility of the oxides consecutively and distinctly decrease until we reach a point when, had we not known of the existence of the beryllium oxide, we should expect to find in its place an oxide insoluble in water and of feeble basio properties. If an alcoholic solution of caustic potash be saturated with the hydrate of $\mathrm{BeO}$, and evaporated under the receiver of an air pump, it forms silky crystals $\mathrm{BeK}_{2} \mathrm{O}_{2}$.

Another characteristic of the salts of beryllium is that they give with aqueous am. monia a gelatinous precipitate which is soluble in an excess of ammonium carbonate like the precipitate of magnesia; in this beryllium oxide differs from the oxide of aluminium. Beryllium oxide easily forms a carbonate which is insoluble in water, and resembles magnesium carbonate in many respects. Beryllium sulphate is distinguished by its considerable solubility in water-thus, at the ordinary temperature it dissolves in an equal weight of water ; it crystallises out from its solutions in well-formed crystals, which do not change in the air, and contain $\mathrm{BeSO}_{4}, 4 \mathrm{H}_{2} \mathrm{O}$. When ignited it leaves beryllium oxide, but this oxide, after prolonged ignition, is re-dissolved by sulphuric acid, whilst aluminium sulphate, after a similar treatment, leaves aluminium oxide, which is no longer soluble in acids. With a few exceptions, the salts of beryllium crystallise with great difficulty, and to a considerable extent resemble the salts of magnesium; thus, for instance, beryllium chloride is analogous to magnesium chloride. It is volatile in an anhydrous state, and in a hydrated state it decomposes, with the evolution of hydro. chloric acid. 
at a red heat, but gaseous hydrochloric acid is decomposed by it when slightly heated, with evolution of hydrogen and development of a considerable amount of heat. Even dilute hydrochloric acid acts in the same manner at the ordinary temperature. Beryllium also acts easily on sulphuric acid, but it is remarkable that neither dilute nor strong nitric acid acts on beryllium, which seems especially able to resist oxidising agents. Potassium hydroxide acts on beryllium as on aluminium, hydrogen being disengaged and the metal dissolved, but ammonia has no action on it. These properties of metallic beryllium seem to isolate it from the series of the other metals described in this chapter, but if we compare the properties of calcium, magnesium, and beryllium we shall see that magnesium occupies a position intermediate between the other two. Whilst calcium decomposes water with great ease, magnesium does so with difficulty, and beryllium not at all. The peculiarities of beryllium among the metals of the alkaline earths recall the fact that in the series of the halogens we saw that fluorine differed from the other halogens in many of its properties and had the smallest atomic weight. The same is the case with regard to beryllium among the other metals of the alkaline earths.

In addition to the above characteristics of the compounds of the metals of the alkaline earths, we must add that they, like the alkali metals, combine with nitrogen and hydrogen, and while sodium nitride (obtained by igniting the amide of sodium, Chapter XII., Note 44 bis) and lithium nitride (obtained by heating lithium in nitrogen, Chapter XIII., Note 39 ) have the composition $R_{3} N$, so the nitrides of magnesium (Note 14), calcium, strontium, and barium. have the composition $\mathrm{R}_{3} \mathrm{~N}_{2}$, for example, $\mathrm{Ba}_{3} \mathrm{~N}_{2}$, as might be expected from the diatomicity of the metals of the alkaline earths and from the relation of the nitrides to ammonia, which is obtained from all of these compounds by the action of water. The nitrides of $\mathrm{Ca}, \mathrm{Sr}$, and $\mathrm{Ba}$ are formed directly (Maquenne, 1892) by heating the metals in nitrogen. They all have the appearance of an amorphous powder of dark colour; as regards their reactions, it is known that besides disengaging ammonia with water, they form cyanides when heated with carbonic oxide; for instance, $\mathrm{Ba}_{3} \mathrm{~N}_{2}+2 \mathrm{CO}=\mathrm{Ba}(\mathrm{CN})_{2}+2 \mathrm{BaO}^{61}$

The metals of the alkaline earths, just like $\mathrm{Na}$ and $\mathrm{K}$, absorb hydrogen under certain conditions, and form pulverulent easily oxidisable metallic hydrides, whose composition corresponds exactly to that of $\mathrm{Na}_{2} \mathrm{H}$ and $\mathrm{K}_{2} \mathrm{H}$, with the substitution of $\mathrm{K}_{2}$ and $\mathrm{Na}_{2}$ by the atoms

61 Thus in the nitrides of the metals we have substances by means of which we can easily obtain from the nitrogen of the air, not only ammonia, but also with the aid of $\mathrm{CO}$, by synthesis, $₫$ whole series of complex carbon and nitrogen compounds. 
$\mathrm{Be}, \mathrm{Mg}, \mathrm{Ca}, \mathrm{Sr}$, and $\mathrm{Ba}$. The hydrides of the metals of the alkaline earths were discovered by C. Winkler (1891) in investigating the reducibility of these metals by magnesium. In reducing their oxides by heating them with magnesium powder in a stream of hydrogen, Winkler observed that the hydrogen was absorbed (but very slowly), i.e. at the moment of their separation all the metals of the alkaline earths cornbine with hydrogen. This absorptive power increases in passing from $\mathrm{Be}$ to $\mathrm{Mg}, \mathrm{Ca}, \mathrm{Sr}$, and $\mathrm{Ba}$, and the resultant hydrides retain the combined hydrogen 62 when heated, so that these hydrides are distinguished for their considerable stability under heat, but they oxidise very easily. ${ }^{63}$

Thus the analogies and correlation of the metals of these two groups are now clearly marked, not only-in their behaviour towards oxygen, chlorine, acids, \&c., but also in their capability of combining with nitrogen and hydrogen.

63 As the hydrides of calcium, magnesium, \&c. are very stable under the action of heat, and these metals and hydrogen occur in the sun, it is likely that the formation of their hydrides may take place there. (Private communication from Prof. Winkler, 1894.) It is probable that in the free metals of the alkaline earths hitherto obtained a portion was frequently in combination with nitrogen and hydrogen.

63 Thus, for instance, a mixture of 56 parts of $\mathrm{CaO}$ and 24 parts of magnesium powder is heated in an iron pipe (placed over a row of gas burners as in the combustion furnace used for organic analysis) in a stream of hydrogen. After being heated for $\frac{1}{2}$ hour the mixture is found to absorb hydrogen (it no longer passes over the mixture, but is retained by it). The product, which is light grey, and slightly coherent, disengages a mass of hydrogen when water is poured over it, and burns when heated in air. The resultant mass contains 33 per cent. $\mathrm{CaH}$, about 28 per cent. $\mathrm{CaO}$, and about 38 per cent. $\mathrm{MgO}$. Neither $\mathrm{CaH}$ nor any other $\mathrm{MH}$ has yet been obtained in a pure state.

The acetylene derivatives of the metals of the alkaline earths $\mathrm{C}_{2} \mathrm{M}$ (Chapter VIII., Note 12 bis), for instance, $\mathrm{C}_{2} \mathrm{Ba}$, obtained by Maquenne and Moissan, belong to the same class of analogous compounds. It must here be remarked that the oxides $\mathrm{MO}$ of the metals of the alkaline earths, although not reducible by carbon at a furnace heat, yet under the action of the heat attained in electrical furnaces, not only give up their oxygen to carbon (probably partly owing to the action of the current), but also combine with carbon. The resultant compounds, $\mathrm{C}_{2} \mathrm{M}$, evolve acetylene, $\mathrm{C}_{2} \mathrm{H}_{2}$, with $\mathrm{HCl}_{2}$ just as $\mathrm{N}_{2} \mathrm{M}_{3}$ give ammonia. We may remark moreover that the series of compounds of the metals of the alkaline earths with hydrogen, nitrogen and carbon is a discovery of recent years, and that probably further research will give rise to similar unexpected compounds, and by extending our knowledge of their reactions prove to be of great interest. 

<smiles>C=C</smiles> 
This book is due on the last DATE stamped below.

To renew by phone, call 459-2050.

Books not returned or renewed within 14 days

after due date are subject to billing.

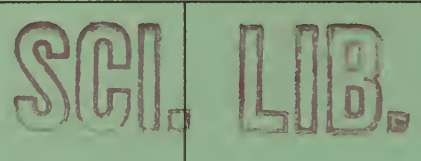




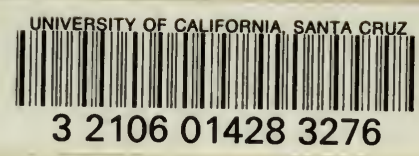


(5.) -

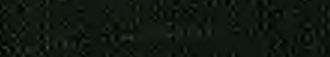

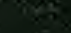

.

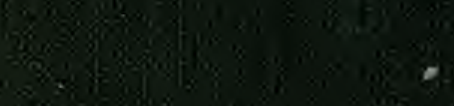

